Andreas Poppe

Auswirkungen der

Einführung einer

konsolidierten

Körperschaftsteuer-

Bemessungsgrundlage in der Europäischen Union 
Andreas Poppe

\section{Auswirkungen der Einführung einer konsolidierten Körperschaftsteuer-Bemessungsgrundlage in der Europäischen Union}

Das Nebeneinander der 27 europäischen Steuersysteme verzerrt Standort-, Investitions- und Finanzierungsentscheidungen und behindert die grenzüberschreitende Geschäftstätigkeit von Unternehmen im Binnenmarkt. Diese Hindernisse beabsichtigt die Europäische Union durch die Einführung der konsolidierten Körperschaftsteuer-Bemessungsgrundlage zu beseitigen. Dieses Konzept sieht innerhalb eines Konzerns vor, die Bemessungsgrundlage aller Konzernunternehmen nach einheitlichen Standards zu ermitteln, sie anschließend zu konsolidieren und den Konzerngewinn dann auf die einzelnen Steuerhoheiten mittels einer Formel aufzuteilen. Ziel der Arbeit ist es, die mit diesem Systemwechsel verbundenen Auswirkungen auf die statutarischen Konzernstrukturen sowie auf das Steueraufkommen der Mitgliedstaaten anhand empirischer Analysen abzuschätzen.

Andreas Poppe, 1977 in Bremen geboren; 1998-2002 Studium der Betriebswirtschaftslehre an der European Business School (EBS) in OestrichWinkel, 2000 an der San Diego State University (USA), 2001 am UNITEC Institute of Technology (Neuseeland); 2003-2006 Mitarbeiter am Institut für deutsche und internationale Besteuerung der Georg-August-Universität Göttingen. 
Auswirkungen der Einführung einer konsolidierten KörperschaftsteuerBemessungsgrundlage in der Europăischen Union 


\title{
cege-Schriften
}

Centrum für Europa-, Governance- und Entwicklungsforschung (cege)

Georg-August-Universität Göttingen

Center for European, Governance and Economic Development Research (cege)

\section{Band 14}

\author{
Herausgegeben von \\ Kilian Bizer, Stephan von Cramon-Taubadel, \\ Andreas Oestreicher, Renate Ohr, \\ Gerhard Rübel, Robert Schwager,
}

Verantwortlicher Herausgeber für diesen Band:

Andreos Oestreicher

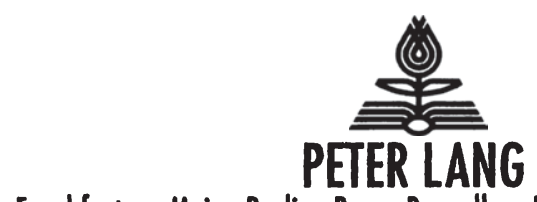

Frankfurt am Main - Berlin - Bern - Bruxelles - Now York - Oxford - Wien 


\section{Andreas Poppe}

\section{Auswirkungen der Einführung einer konsolidierten Körperschaftstever- Bemessungsgrundlage in der Europäischen Union}

Eine empirische Analyse der Konzernstrukturen und des Steveraufkommens

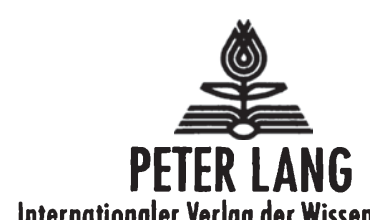

Internationaler Verlag der Wissenschaften

Andreas Poppe - 978-3-631-75688-1

Downloaded from PubFactory at 01/11/2019 02:57:41AM 


\title{
Blbliografische Information der Deutschen Nationalbibliothek Die Deutsche Nationalbibliothek verzeichnet diese Publikation in der Deutschen Nationalbibliografie; detaillierte bibliografische Daten sind im Internet über <http://www.d-nb.de> abrufbar.
}

Open Access: The online version of this publication is published on www.peterlang.com and www.econstor.eu under the international Creative Commons License CC-BY 4.0. Learn more on how you can use and share this work: http://creativecommons.org/licenses/ by/4.0.

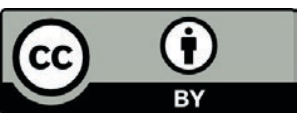

This book is available Open Access thanks to the kind support of ZBW - Leibniz-Informationszentrum Wirtschaft.

Zugl.: Göttingen, Univ., Diss., 2007

Gedruckt auf alterungsbestãndigem, säurefreiem Papier.

\author{
D 7 \\ ISSN 1617-741X \\ ISBN 978-3-631-57228-3 \\ ISBN 978-3-631-75688-1 (eBook) \\ (c) Peter Lang GmbH \\ Internationaler Verlag der Wissenschaften \\ Frankfurt am Main 2008 \\ Alle Rechte vorbehalten.
}

Das Werk einschließlich aller seiner Teile ist urheberrechtlich geschützt. Jede Verwertung außerhalb der engen Grenzen des

Uneberrechtsgesetzes ist ohne Zustimmung des Verlages unzulässig und strafbar. Das gilt insbesondere für Vervielfältigungen, Übersetzungen, Mikroverfilmungen und die Einspeicherung und Verarbeitung in elektronischen Systemen.

Printed in Germany 123457

www.peterlang.de 


\section{Vorwort}

In der Europäischen Union werden Kapitalgesellschaften grundsätzlich nach dem Trennungsprinzip besteuert. Danach bezieht sich die Besteuerung grundsätzlich auf das einzelne zivilrechtlich selbstständige Unternehmen. Jede Gesellschaft hat ihr Einkommen und Vermögen auch dann einzeln zu ermitteln, wenn sie als Konzerngesellschaft Teil einer wirtschaftlichen Einheit ist. Diese Orientierung am Trennungsprinzip ist allerdings nicht ohne Ausnahme. So sind in vielen Ländern unter bestimmten Voraussetzungen Verluste zwischen den Gesellschaften einer wirtschaftlichen Einheit verrechenbar. Zum Teil ist die Besteuerung eines Konzerns auf konsolidierter Basis möglich. Bis auf wenige Fälle sind diese Elemente einer Konzernbesteuerung jedoch national begrenzt. Das hat zur Folge, dass

- Unternehmen verpflichtet sind, ihre Gewinne aus grenzüberschreitenden Transaktionen nach den unterschiedlichen Vorschriften von bis zu 27 Mitgliedstaaten zu ermitteln und zwischen den Unternehmen der beteiligten Mitgliedstaaten einzeln aufzuteilen,

- Gewinnausschüttungen im Konzern steuerbar sind und

- ein Verlustausgleich zwischen den verschiedenen Teilen eines Unternehmens grundsätzlich nur innerhalb der Besteuerungsgrenzen eines Mitgliedstaates möglich ist.

Ein Teil dieser Probleme wurde zwar in den vergangenen Jahren durch gezielte Maßnahmen gemindert. Daneben werden die Mitgliedstaaten zunehmend durch Urteile des EuGH verpflichtet, Grundfreiheiten missachtende Regelungen der nationalen Steuergesetze abzubauen. Die Europäische Kommission zielt jedoch in ihrer Strategie auf eine umfassende Lösung ab und propagiert die Entwicklung einer gemeinsamen konsolidierten Körperschaftsteuerbemessungsgrundlage („CCCTB“). Diese Besteuerung von Konzerngesellschaften auf Basis einer konsolidierten Bemessungsgrundlage setzt voraus, dass

- das zu versteuernde Einkommen dieser Gesellschaften nach europaweit einheitlichen Regelungen ermittelt,

- zum gemeinsamen Einkommen des grenzüberschreitend tätigen Konzerns zusammengefasst und

- auf die am diesem Einkommen beteiligten Gesellschaften nach bestimmten Aufteilungsmaßstäben verteilt wird.

In der Praxis bedeutet bereits die Einigung auf einheitliche Regelungen für die Ermittlung des zu versteuernden Einkommens eine Herausforderung. Gleichwohl gibt es Perspektiven. In jedem Fall werden die Mitgliedstaaten ihre Zustimmung zu einer Besteuerung nach einer СССТB nur geben können, wenn sich abschätzen lässt, welche Wirkungen die Einführung einer CCCTB für das Steueraufkommen dieser Staaten haben wird. Bisher ist diese Folgenabschätzung jedoch nicht abschließend geklärt. Daher ist es das Ziel der vor- 
liegenden Arbeit von Herr Dr. Poppe, zu dieser Klärung beizutragen und die möglichen Wirkungen zu analysieren, die mit der Einführung einer CCCTB in Europa verbunden wären. $\mathrm{Zu}$ diesem $\mathrm{Zweck}$ bezieht er sich auf Jahresabschlussdaten, die er auf Basis von Beteiligungsinformationen, Wirtschaftszweigklassifikationen und anderen Einzelangaben mit großer Sorgfalt in zum Teil sehr aufwendigen Schritten zu einer auf Konzerne bezogene umfassende Datenbasis aufbereitet. Mit seiner sorgfältigen Aufbereitung unternehmensbezogener Daten für Konzerne in Europa gewinnt er nicht nur eine tragfähig Basis für die sich anschließende Analyse der möglichen Aufkommenswirkungen. Es gelingt ihm auch eine bisher nicht bekannte Beschreibung der rechtlichen Konzernstrukturen in Europa, deren Ausgestaltung er zum Teil mit Hilfe von Methoden der deskriptiven und induktiven Statistik auf einen steuerlichen Einfluss zurückführen kann. Zentraler Gegenstand seiner Arbeit sind aber die möglichen Aufkommenswirkungen, die mit der Einführung einer СССТВ für die beteiligten Mitgliedstaaten der EU verbunden sein können. Seine Ergebnisse beruhen auf komparativ-statischen Mikrosimulationsrechnungen für einen Basisfall, alternative Mindestbeteiligungshöhen und Aufteilungsfaktoren und schließen auch eine Identifikation der verschiedenen Einzeleffekte, die sich mit der Einführung einer CCCTB verbinden, ein.

Die Arbeit von Herrn Dr. Poppe, die im Rahmen eines umfassenderen Forschungsprojekts durch die Deutsche Forschungsgemeinschaft gefördert und von der Wirtschaftswissenschaftlichen Fakultät der Georg-August-Universität Göttingen als Dissertation angenommen wurde, leistet einen wichtigen Beitrag zur Forschung auf dem Gebiet der Konzernbesteuerung in Europa. Sie ist in ihren Ergebnissen nicht nur für Wissenschaftler, die Dienststellen der Europäischen Kommission oder betroffene Fachministerien in den EU Mitgliedstaaten von Interesse, sondern ist auch für künftige Arbeiten im Bereich der unternehmensbezogenen Steuerwirkungsanalyse methodisch von Bedeutung. Die beschriebene Aufbereitung von Datenbankinformationen einschließlich der Abbildung von Konzernstrukturen stellt nicht nur für die hier vorliegende Arbeit, sondern auch für eine Vielzahl folgender Forschungsvorhaben eine Pionierleistung dar. Ich bin überzeugt, dass die vorliegende Arbeit sowohl für Wissenschaftler als auch Fachleute, die sich in ihrer Arbeit mit Fragen der Konzernbesteuerung befassen, von erheblichem Nutzen sein wird. 


\section{Danksagung}

Das Verfassen einer Dissertation stellt zwar formal eine Eigenleistung dar, die aber ohne die umfangreiche Unterstützung vieler Personen nicht möglich ist. An erster Stelle gilt mein Dank meinem Doktorvater Herrn Prof. Dr. Andreas Oestreicher für die Betreuung dieser Arbeit. In einer Vielzahl von Gesprächen hat er mir wertvolle Denkanstöße gegeben, die ich stets zu schätzen wusste. Daneben danke ich auch Herrn Prof. Dr. Waldemar Toporowski für die zügige Übernahme des Zweitgutachtens sowie Herrn Prof. Dr. Robert Schwager für die Leitung der Disputation.

Meinen langjährigen Mitstreitern am Lehrstuhl möchte ich für ihre unermüdliche Diskussionsbereitschaft $\mathrm{zu}$ wissenschaftlichen Fragestellungen danken. Mein besonderer Dank gilt hier Reinald Koch und Christian Hundeshagen, die mir stets mit wertvollen Anmerkungen zur Seite standen. Danken möchte ich auch Heike Riesselmann, Matthias Duensing und Christoph Vormoor, die wesentlich dazu beigetragen haben, dass die Zeiten am Lehrstuhl und jenseits des Oeconomicums unvergessen bleiben werden. $\mathrm{Zu}$ dem positiven Arbeitsklima beigetragen haben auch die studentischen Hilfskräfte des Lehrstuhls. In diesem Zusammenhang bedanke ich mich bei Bastian Rehr auch für die ausführlichen Diskussionen rund um den Fußball.

Meinen Freunden bin ich dankbar für die gelungenen Abwechslungen zum teilweise einsamen Alltag eines Wissenschaftlers. Tom Laband ließ mich bei unseren unzähligen Tischtennismatches selbst in den stressigen Phasen auf andere Gedanken kommen. Dies gilt auch für Nick Dose während der vielen gemeinsamen Stunden auf dem Wasser. Raimo Reese danke ich für die unvergessenen gemeinsamen Studienjahre sowie seine positive Sicht der Dinge beim Verfassen einer Dissertation. Für stete Aufmunterungen bin ich auch Kio Otto, Philipp Dedem und Julian Scheuble dankbar.

Nicht zuletzt gilt mein Dank meinen Eltern und meiner Schwester Carola für den notwendigen Rückhalt und die immerwährende Unterstützung, die sie mir nicht nur in den letzten vier Jahren der Promotion entgegengebracht haben. Ohne sie wäre alles so nicht möglich gewesen. Ihnen ist diese Arbeit gewidmet.

Mein größter Dank neben meiner Familie gebührt Marie-Christin Tromm, da sie mir während der gesamten Arbeit ihr Ohr und Herz lieh. Sie hat stets an den Erfolg des Projekts geglaubt, gewusst, mich in schwierigen Phasen zu motivieren und mir besonders in dem Nerven aufreibenden letzten Jahr der Promotion das nötige Verständnis entgegengebracht. 
Andreas Poppe - 978-3-631-75688-1

Downloaded from PubFactory at 01/11/2019 02:57:41AM

via free access 


\section{Inhaltsverzeichnis}

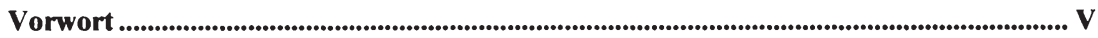

Danksagung.................................................................................................................................................... VII

Abkürzungsverzeichnis................................................................................................................................XIII

Abbildungsverzeichnis .............................................................................................................XIX

Tabellenverzeichnis ......................................................................................................XXIII

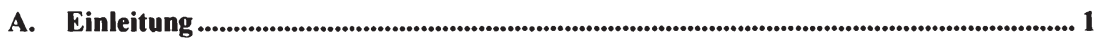

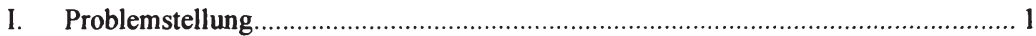

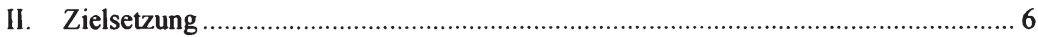

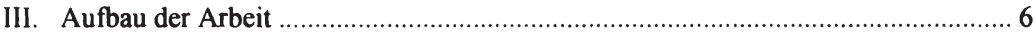

B. Besteuerung von Konzernen in den Mitgliedstaaten der Europäischen Union .......... 9

I. Alternative Besteuerungskonzeptionen für Unternehmensgruppen.......................... 9

II. Besteuerung von Konzernen nach dem Prinzip der selbstständigen Einheit ............ 11

III. Gruppenbesteuerungssysteme in der Europäischen Union .................................... 14

1. Reichweite der Gruppenbesteuerungssysteme ............................................ 14

2. Anwendungsvoraussetzungen der Gruppenbesteuerungssysteme ……........... 17

IV. Unternehmensteuerliche Hindernisse bei grenzüberschreitender Geschäftstätigkeit in der Europäischen Union ..................................................... 21

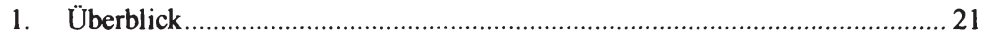

2. Verlustverrechnung ................................................................................ 22

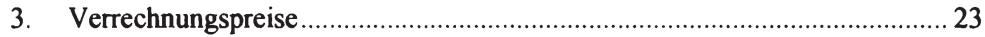

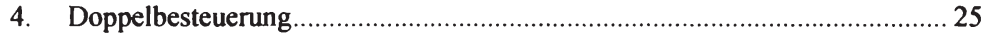

C. Konsolidierte Unternehmensbesteuerung in der Europäischen Union...................... 27

I. Vorschläge zur Beseitigung der steuerlichen Hindernisse im Binnenmarkt........... 27

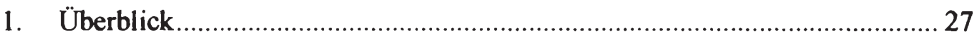

2. Darstellung der gezielten Lösungsansătze ……………………………….... 28

3. Darstellung der umfassenden Lösungsansätze ................................................. 30

4. Bewertung der umfassenden Lösungsansätze ……………………………..... 36

a) Grad der Beseitigung der unternehmenssteuerlichen Hindernisse.............. 36

b) Politische Durchsetzbarkeit der umfassenden Lösungsansätze.................... 40

c) Zusammen fassende Würdigung der umfassenden Lösungsansätze............ 42

II. Einheitliche (konsolidierte) Körperschaftsteuer-Bemessungsgrundlage .................. 44

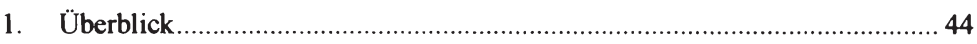

2. Abgrenzung des Konsolidierungskreises ...................................................... 45

a) Sachliche Abgrenzungskriterien 


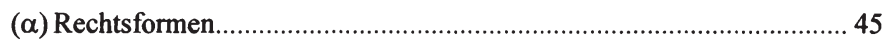

(ß) Wirtschaftliche Abgrenzungskriterien ................................................. 47

( $\gamma$ ) Rechtliche Abgrenzungskriterien ......................................................50

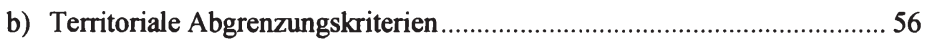

3. Ermittlung der einheitlichen Bemessungsgrundlage ...................................... 61

a) Vereinheitlichung der steuerlichen Gewinnermittlungsvorschriften in

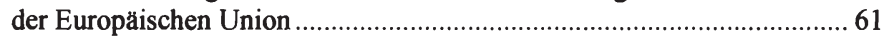

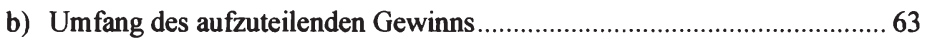

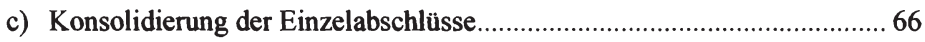

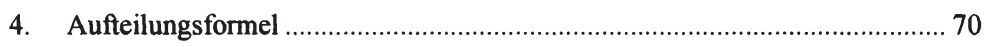

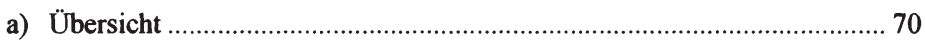

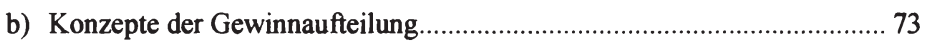

( $\alpha$ ) Gewinn als Indikator furr die Inanspruchnahme offentlicher

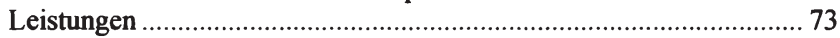

( $\beta$ ) Makroökonomische und industrieweite Größen als Aufteilungsmaßstab....................................................................... 74

( $\gamma$ ) Gewinn als Verzinsung des eingesetzten Kapitals ............................... 75

(ס) Aufteilung nach dem Ursprung des Gewinns....................................... 78

c) Bewertung der Aufteilungsfaktoren ...................................................... 79

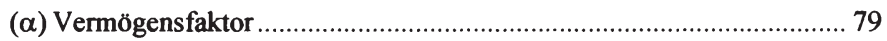

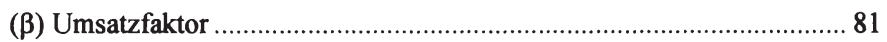

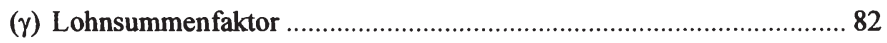

III. Forschungsbedarf im Rahmen der empirischen Analyse ....................................... 83

D. Methodik der empirischen Analyse...................................................................................... 85

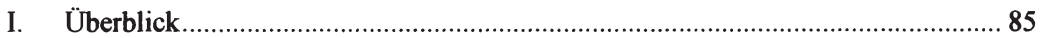

II. Anforderungen an die Datenquelle und Auswahl der Datenbank ............................ 85

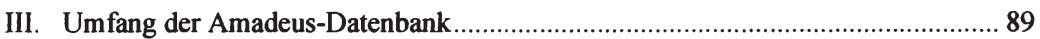

IV. Aufbereitung der Unternehmenseinzeldaten ......................................................... 92

1. Beteiligungsinformationen ........................................................................ 92

2. Wirtschaftszweigklassifikationen der Unternehmen........................................96

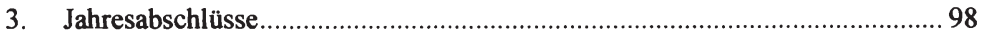

a) Auswahl und Beschreibung der Datenbasis ............................................ 98

b) Ergänzung von Jahresabschlussinformationen......................................... 104

( $\alpha$ ) Vorüberlegungen zur Methodik ....................................................... 104

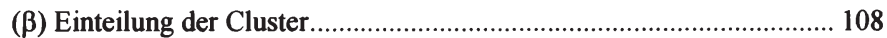

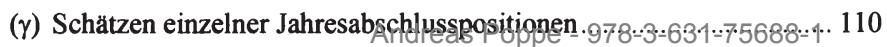




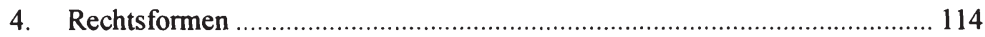

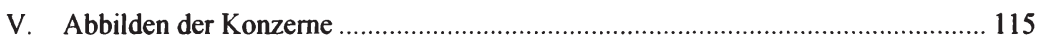

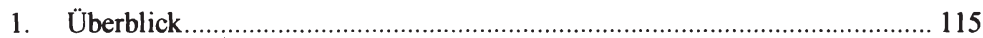

2. Vergleichskonzern................................................................................ 116

a) Definition von Konzern- und Konzernmuttergesellschaften................... 116

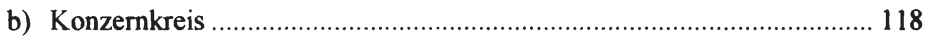

c) Bestimmung der Konzernbranche ...................................................... 119

3. Abbilden der steuerlichen Konzernkreise ............................................. 121

a) Nationale Gruppenbesteuerungssysteme ............................................ 121

b) Steuerlicher Konsolidierungskreis der konsolidierten Körperschaftsteuer-Bemessungsgrundlage ..................................... 125

E. Analyse der Konzernstrukturen in Europa .......................................................... 127

I. Beschreibung der Konzerne in Europa ............................................................. 127

II. Analyse der Konzernstruktur unter steuerplanerischen Gesichtspunkten ............. 136

1. Einsatz von Holdinggesellschaften ...................................................... 136

a) Eingrenzung des Untersuchungsgegenstandes ................................. 136

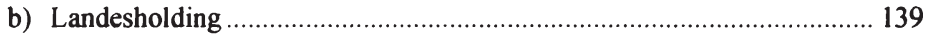

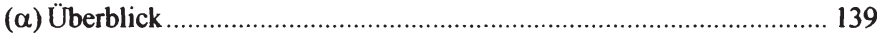

( $\beta$ ) Motive für den Einsatz von Landesholdings ................................. 139

(aa) Anwendung von Gruppenbesteuerungssystemen.................... 139

(bb) Teilwertabschreibungen von Beteiligungsbuchwerten ............ 143

(cc) Erhöhung des zulässigen Verhältnisses der GesellschafterFremdfinanzierung zum Eigenkapital .

$(\gamma)$ Identifizierung von Landesholdings in der Amadeus-Datenbank und Beschreibung der Datenlage ................................................... 145

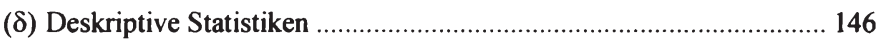

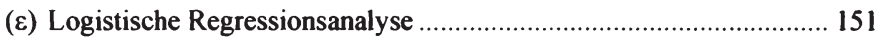

2. Einsatz von Konzernfinanzierungsgesellschaften .................................. 157

a) Steuerliche Vorteile der Funktionsverlagerung und ihre Grenzen.......... 157

b) Identifizierung der Finanzierungsgesellschaften................................ 160

F. Quantitative Auswirkungen der Einführung der konsolidierten Besteuerung...... 163

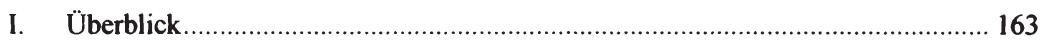

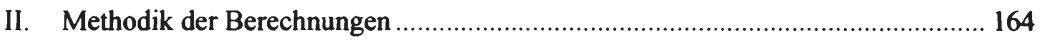

1. Ermittlung der konsolidierten Bemessungsgrundlage ................................ 164

a) Ermittlung des aufzuteilenden Gewinns ............................................ 164

b) Konsolidierung der Einzelabschlüsse.................................................. 166 
c) Einperiodige Betrachtungsweise ....................................................... 168

2. Aufteilung des Gesamterfolgs.................................................................. 169

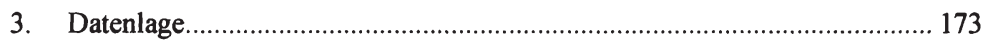

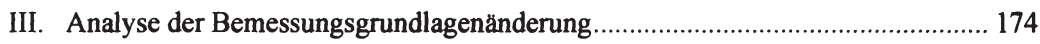

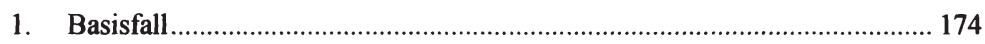

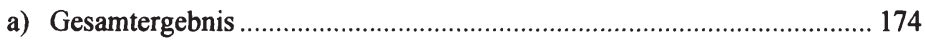

b) Zerlegung der Aufkommenswirkung in ihre Einzeleffekte..................... 176

$(\alpha)$ Beschreibung der Einzeleffekte................................................... 176

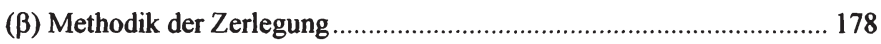

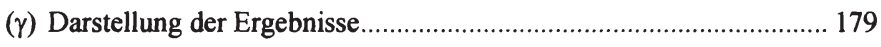

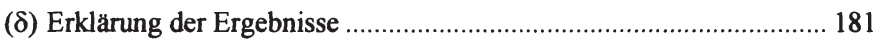

c) Berücksichtigung einer pauschalen Verlustverrechnung ........................ 189

d) Operativer Gewinn als aufzuteilende Bemessungsgrundlage .................. 193

e) Vergleich mit auf anderen Datenbasen beruhenden empirischen

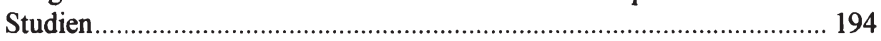

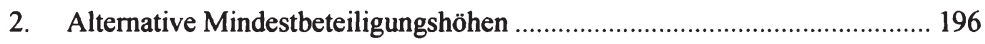

3. Alternative mikroökonomische Aufteilungsformeln ............................... 198

a) Alternative Gewichtung der Formelfaktoren ..................................... 198

b) Alternativer Aufteilungsfaktor ............................................................ 202

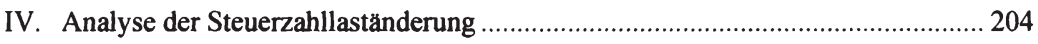

G. Zusammenfassung der zentralen Untersuchungsergebnisse................................... 209

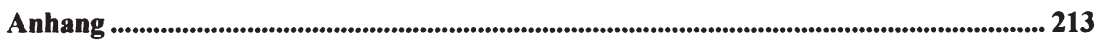

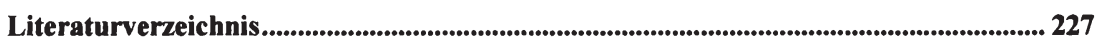

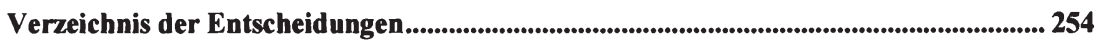

Verzeichnis der Drucksachen, Richtlinien, Verordnungen............................................. 256 


\section{Abkürzungsverzeichnis}

a. auch

Abl. Amtsblatt

a. n. g. andere nicht genannte

Anm. Anmerkung

AnwBl. Anwaltsblatt

AStG Außensteuergesetz

AT Österreich

ATECO ..... Classificazione delle attività economiche

Aufl. Auflage

AWV Außenwirtschaftsverordnung

$\mathrm{BB}$ Betriebs-Berater

$\mathrm{BE}$ Belgien

BFH Bundesfinanzhof

BFuP Betriebswirtschaftliche Forschung und Praxis

BIFD Bulletin for International Fiscal Documentation

BIK Bedrijfsindeling kamers van koophandel

BIP Bruttoinlandsprodukt

BMF Bundesministerium der Finanzen

BMG Bemessungsgrundlage

BR-Drs. ..... Bundesrat-Drucksache

BStB1 Bundessteuerblatt

CCCTB ..... Common Consolidated Corporate Tax Base

CESifo ....... Center for Economic Studies und Institut für Wirtschaftsforschung

CNAE ....... Clasificación Nacional de Actividades Económicas

CTJ . Canadian Tax Journal

CUP comparable uncontrolled price

CY Zypern

$\mathrm{CZ}$ Tschechische Republik

DB Der Betrieb

DBW Die Betriebswirtschaft

DE Deutschland

Destatis Statistisches Bundesamt Deutschland 
DK ............ Dänemark

DM ............ Deutsche Mark

DStR ......... Deutsches Steuerrecht

DSWR ....... Datenverarbeitung, Steuern, Wirtschaft, Recht

EATR ........ effective average tax rate

EE ............. Estland

EG ............ Europäische Gemeinschaft

EGV ......... Vertrag zur Gründung der Europäischen Gemeinschaft

EK ............. Eigenkapital

EMTR ....... effective marginal tax rate

ES ............. Spanien

ET ….........European Taxation

EU ............. Europäische Union

EuGH ........ Europäischer Gerichtshof

EUR ........... Euro

EUV ......... Vertrag über die Europäische Union

EuZW ....... Europäische Zeitung für Wirtschaftsrecht

EZB ........... Europäische Zentralbank

FA ............. Finanzarchiv

FA …......... Formula Apportionment

FI ............... Finnland

FJ .............. Finanz-Journal

FK ............ Fremdkapital

FN ............. Fußnote

FR ............. Frankreich

GB ............ Großbritannien

GE ............. Geldeinheiten

GewStG .... Gewerbesteuergesetz

GFJ ........... Global Finance Journal

$\mathrm{GmbH}$....... Gesellschaft mit beschränkter Haftung

GmbHR .... GmbH-Rundschau

GR Griechenland

GuV Gewinn-und-Verlust-Rechnung 
HFR .......... Höchstrichterliche Finanzrechtsprechung

HLR .......... Harvard Law Review

Hrsg. ......... Herausgeber

$\mathrm{HU}$............ Ungarn

IAS ........... International Accounting Standards

IE .............. Irland

IFRS .......... International Financial Reporting Standards

IFSC .......... International Financial Services Centre

int............... international

IRZ ........... Zeitschrift für internationale Rechnungslegung

ISIC ........... International Classification of all Economic Activities

IStR ........... Internationales Steuerrecht

IT .............. Italien

ITPF .......... International Tax and Public Finance

ITPJ ........... International Transfer Pricing Journal

i. V. m. ...... in Verbindung mit

JoPE .......... Journal of Public Economics

k. A. .......... keine Angaben

Kfz. ........... Kraftfahrzeug

KG ............ Kommanditgesellschaft

KKS .......... Kaufkraftstandards

KMU ......... kleinere und mittlere Unternehmen

KStG ......... Körperschaftsteuergesetz

Lfg. ........... Lieferung

LT ............. Litauen

LU .............. Luxemburg

LV ............. Lettland

Mio. ........... Million

Mrd. .......... Milliarde

MT ............ Malta

MTR ......... Mutter-Tochter-Richtlinie

m. w. N. .... mit weiteren Nachweisen Andreas Poppe - 978-3-631-75688-1 
n. a. not available

NACE ...... Nomenclature statistique des Activités économiques dans la Communauté Européenne

NAF .......... nomenclature d'activités française

NAFTA ..... North American Free Trade Agreement

NAICS ...... North American Industry Classification System

NL ............ Niederlande

nn .............. not null

Nr. ............ Nummer

NTJ .......... National Tax Journal

NV ............ nicht veröffentlicht

o. A. .......... ohne Angabe

OECD ....... Organisation for Economic Cooperation and Development

o. O. ......... ohne Ortsangabe

OTA ......... Office of Tax Analysis

ÖVwGH .... Österreichischer Verwaltungsgerichtshof

PL ............ Polen

PT ............ Portugal

Rs. ............ Rechtssache

Rz. ........... Randziffer

SA ........... Separate Accounting

SE ............. Schweden

SI .............. Slowenien

SIC ............ Standard Industrial Classification

SK ............. Slowakei

SNI ........... Standard för svensk näringsgrensindelning

STN .......... State Tax Notes

StuW ........ Steuern und Wirtschaft

SWI ........... Steuern und Wirtschaft International

TEUR ........ Tausend Euro

TLR .......... Tax Law Review

TN ............. Tax Notes 
TNI .......... Tax Notes International

TPITP ....... Tax Planning International Transfer Pricing

UDITPA ... Uniform Division of Income for Purposes Act

UNCTAD . United Nations Conference on Trade and Development

UNICE ...... Union of Industrial and Employers' Confederations of Europe

UNO ......... United Nation Organization

URL .......... Uniform Resource Locator

US/U.S. ..... United States

USA .......... United States of America

u. U. .......... unter Umständen

v.

vom

WIRO ....... Wirtschaft und Recht in Osteuropa

WPg .......... Die Wirtschaftsprüfung

WuS .......... Wirtschaft und Statistik

WZ ........... Wirtschaftszweigklassifikation

ZEW ......... Zentrum für Europäische Wirtschaftsforschung 
Andreas Poppe - 978-3-631-75688-1

Downloaded from PubFactory at 01/11/2019 02:57:41AM

via free access 


\section{Abbildungsverzeichnis}

Abbildung 1: Behandlung von Minderheitsgesellschaftern.............................54

Abbildung 2: Entwicklung der Anzahl der Konsolidierungskreise in Abhängigkeit von der Mindestbeteiligungsquote....................... 55

Abbildung 3: Besteuerungsprinzipien bei der formelhaften Gewinnaufteilung.

Abbildung 4: Entwicklung der Anzahl der US-Bundesstaaten, die dem Umsatz innerhalb der Aufteilungsformel ein höheres Gewicht beimessen

Abbildung 5: Anpassungen der Beteiligungsinformationen - Beispiel ......... 94

Abbildung 6: Verteilung der Beteiligungshöhen (Anzahl > 5.000) ............... 95

Abbildung 7: Häufigkeit des Wechsels der Ertragslage der Unternehmen zwischen 1997 und 2003.......................................................... 100

Abbildung 8: Bilanz in der Amadeus-Datenbank ........................................... 101

Abbildung 9: Gewinn-und-Verlust-Rechnung in der AmadeusDatenbank

Abbildung 10: Clusterbildung zum Ergänzen von Jahresabschlussinformationen

Abbildung 11: Steuerliche Konzernkreise als Teilmenge des Vergleichskonzerns................................................................ 116

Abbildung 12: Definition der Konzernmuttergesellschaft.............................. 117

Abbildung 13: Absetzen direkt gehaltener eigener Anteile ............................ 118

Abbildung 14: Absetzen indirekt gehaltener eigener Anteile........................... 118

Abbildung 15: Kreisbeteiligungen .............................................................. 119

Abbildung 16: Sprungkonsolidierung ........................................................... 119

Abbildung 17: Verteilung der Bemessungsgrundlage auf die Konzerne -

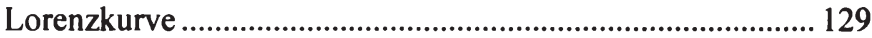

Abbildung 18: Klassifizierung von Holdinggesellschaften ........................... 138

Abbildung 19: Anteil der Teilkonzerne mit Landesholding nach Ländern .... 147

Abbildung 20: Anteil der Teilkonzerne mit Landesholding in Abhängigkeit von der Anzahl der Tochtergesellschaften....... 150

Abbildung 21: Integration von Finanzierungs- und Koordinationszentren in den Konzernaufbau ........................................................... 158

Abbildung 22: Abbildung des Aufteilungsfaktors Umsatz............................ 170 
$\mathrm{XX}$

Abbildung 23: Aufkommenswirkung der Einführung einer konsolidierten Körperschaftsteuer-Bemessungsgrundlage - Basisfall 175

Abbildung 24: Zerlegung des Aufkommenseffekts der Einführung einer konsolidierten Körperschaftsteuer-Bemessungsgrundlage in drei Einzeleffekte

Abbildung 25: Aufkommenswirkung der Einführung einer konsolidierten Körperschaftsteuer-Bemessungsgrundlage - Zerlegung des Basisfalls in Einzeleffekte

Abbildung 26: Gegenüberstellung des Formeleffekts und der potenziellen Gewinnverlagerung

Abbildung 27: Aufkommenswirkung der Einführung einer konsolidierten Körperschaftsteuer-Bemessungsgrundlage Berücksichtigung einer pauschalen Verlustverrechnung 190

Abbildung 28: Änderung des Aufkommenseffekts der Einführung einer konsolidierten Körperschaftsteuer-Bemessungsgrundlage unter Berücksichtigung einer pauschalen Verlustverrechnung gegenüber dem effektiven Gewinnsteuersatz

Abbildung 29: Vergleich der Untersuchungsergebnisse des Basisfalls mit Berechnungen auf der Basis der Direktinvestitionsstatistik ... 196

Abbildung 30: Entwicklung der gesamten konsolidierten Körperschaftsteuer-Bemessungsgrundlage in v. H. SA in Abhängigkeit von der Mindestbeteiligungsquote

Abbildung 31: Aufkommenswirkung der Einführung einer konsolidierten Körperschaftsteuer-Bemessungsgrundlage bei alternativen Mindestbeteiligungsquoten in v. H. SA

Abbildung 32: Aufkommenseffekt der Einführung einer konsolidierten Körperschaftsteuer-Bemessungsgrundlage auf der Basis des Vermögens, der Lohnsumme und des Umsatzes in v. H. SA und Abweichung vom Basisfall in Prozentpunkten 200

Abbildung 33: Durchschnittliche quadrierte Abweichung des Aufkommenseffekts der Einführung einer konsolidierten Körperschaftsteuer-Bemessungsgrundlage in Abhängigkeit von der Gewichtung der Formelfaktoren in v. H. SA 201

Abbildung 34: Lohnniveau und Arbeitsproduktivität in der Europäischen Union 
Abbildung 35: Gegenüberstellung des Jahresverdienst und der Veränderung des Steueraufkommens der konsolidierten Körperschaftsteuer-Bemessungsgrundlage nach Ersatz der Lohnsumme durch die Mitarbeiteranzahl sowie die Abbildung des Aufkommenseffekts gegenüber der SA BMG in v. $\mathrm{H}$.

Abbildung 36: Relative Steueraufkommensdifferenz auf der Grundlage des Quellen- und Wohnsitzprinzips und Vergleich mit dem nominalen Gewinnsteuersatz. 
Andreas Poppe - 978-3-631-75688-1

Downloaded from PubFactory at 01/11/2019 02:57:41AM

via free access 


\section{Tabellenverzeichnis}

Tabelle 1: Vermeidung der Doppelbesteuerung von Dividenden .............. 12

Tabelle 2: $\quad$ Abschreibung von Beteiligungsbuchwerten .............................. 13

Tabelle 3: Gruppenbesteuerungssysteme in der Europäischen Union ...... 15

Tabelle 4: $\quad$ Veranlagungstechnik der Gruppenbesteuerungssysteme ......... 16

Tabelle 5: $\quad$ Reichweite der wirtschaftlichen Einheit der

Gruppenbesteuerungssysteme.............................................. 16

Tabelle 6: Territoriale Reichweite der Gruppenbesteuerungssysteme ...... 17

Tabelle 7: $\quad$ Qualifizierende Rechtsformen der

Gruppenbesteuerungssysteme

Tabelle 8: $\quad$ Höhe der Mindestbeteiligung der

Gruppenbesteuerungssysteme.

Tabelle 9: $\quad$ Vergleich der umfassenden Lösungsansätze ............................ 35

Tabelle 10: Unternehmensdatenbanken im Vergleich.................................88

Tabelle 11: Anzahl der Unternehmen in der Amadeus-Datenbank nach

Mitgliedstaaten.....

Tabelle 12: Relativer Anteil der Kapitalgesellschaften in der Amadeus-

Datenbank

Tabelle 13: Anpassung der Angaben der Beteiligungshöhe in der Amadeus-Datenbank

Tabelle 14: Aufsummierte bekannte und zuordnungsfähige Anteile der

Anteilseigner in v. H. je Gesellschaft .....

Tabelle 15: Anteil der Unternehmen ohne Angaben zum

Wirtschaftszweig

Tabelle 16: Jahr des Jahresabschlusses nach Ländern................................. 99

Tabelle 17: Anteil der Unternehmen in der Amadeus-Datenbank mit

Daten

Tabelle 18: Konsolidierte Jahresabschlüsse in der Datenbank nach

Ländern

Tabelle 19: Verzerrung der Aufkommenswirkung der konsolidierten

Körperschaftsteuer-Bemessungsgrundlage durch die

Vernachlässigung von Unternehmen ohne

Jahresabschlussangaben

Tabelle 20: Anteil der im Anhang der Mutter-Tochter-Richtline aufgeführten Rechtsformen in der Datenbank. 
Tabelle 21: Mindestbeteiligungsquote im Veranlagungszeitraum $2003 \ldots 122$

Tabelle 22: Berechnung der Beteiligungsquote......................................... 123

Tabelle 23: Positionen von Buchungen aus einem

Gewinnabführungsvertrag in der Gewinn-und-Verlust-

Rechnung

Tabelle 24: Anzahl der Organgesellschaften in Deutschland und

Österreich

Tabelle 25: Verteilung der Konzerne und Konzerngesellschaften nach

Ländern

Tabelle 26: Größe der Konzerne gemessen an der Anzahl der

Gesellschaften in v. H.

Tabelle 27: Summe der von anderen Konzerngesellschaften direkt an einer Konzerngesellschaft gehaltenen Beteiligung in v. H. ... 130

Tabelle 28: Länge der Beteiligungsketten ................................................... 130

Tabelle 29: Anzahl der Grenzübertritte ................................................. 131

Tabelle 30: Verteilung der Konzernbranche............................................. 133

Tabelle 31: Gesellschaften nach Funktionen ............................................ 134

Tabelle 32: Über welche Mitgliedstaaten fließen Direktinvestitionen nach Europa?.

Tabelle 33: Durchschnittlich direkte Höhe der Beteiligung und Mindestbeteiligungsquote.

Tabelle 34: Verteilung der Landesholding in Abhängigkeit von der Verfügbarkeit der Gruppenbesteuerungssysteme und einer möglichen Verlustverrechnung innerhalb der letzten vier Jahre

Tabelle 35: Verteilung der Landesholdings in Abhängigkeit von einer möglichen Teilwertabschreibung von

Beteiligungsbuchwerten

Tabelle 36: Verteilung der Landesholdings in Abhängigkeit von besonderen „thin-capitalization rules“ für Holdinggesellschaften.

Tabelle 37: Güte der Anpassung des logistischen Regressionsmodells .... 153

Tabelle 38: Ergebnisse der Regressionsanalyse - Landesholding (7415)

Tabelle 39: Konzernfinanzierungsgesellschaften ...................................... 161

Tabelle 40: $\quad$ Verschuldungsgrad im Konzern ............................................ 162 
Tabelle 41: Variable Parameter bei der Analyse des Aufkommenseffekts der konsolidierten Körperschaftsteuer-Bemessungsgrundlage............................. 163

Tabelle 42: Anteil der Dividenden am Finanzergebnis .............................. 164

Tabelle 43: $\quad$ Anpassung der Gewinn-und-Verlust-Rechnung bei Organträgern und Organgesellschaften

Tabelle 44: Direkte Beteiligung der Organträger an den

Organgesellschaften

Tabelle 45: Unternehmen mit Daten in v. H. nach Anzahl der Konzerngesellschaften

Tabelle 46: Aufkommenswirkung der Einführung einer konsolidierten Körperschaftsteuer-Bemessungsgrundlage - Basisfall

Tabelle 47: Effektive steuerliche Tarifbelastung 2003 in der Europäischen Union in v. $\mathrm{H}$.

Tabelle 48: International vertikal integrierte Konzerne.

Tabelle 49: Lieferungs- und Leistungsbeziehung zwischen den Mitgliedstaaten in v. $\mathrm{H}$.

Tabelle 50: Zusammenführung der Einzeleffekte der

Gewinnverlagerung.

Tabelle 51: Vergleich des Formeleffekts mit dem Indikator für potenzielle Gewinnverlagerung.

Tabelle 52: Aufkommenswirkung der Einführung einer konsolidierten Körperschaftsteuer-Bemessungsgrundlage Berücksichtigung einer pauschalen Verlustverrechnung

Tabelle 53: Vergleich der Rangfolgen des Aufkommenseffekts der Einführung einer konsolidierten KörperschaftsteuerBemessungsgrundlage bei direkter Zurechnung des Finanzergebnisses mit dem Basisfall.

Tabelle 54: Meldeangaben der Direktinvestitionsstatistik

Tabelle 55: Rechtsformen in der Datenbank

Tabelle 56: Angepasste NACE-Abschnitte und -Abteilungen in der Datenbank

Tabelle 57: Anpassen der Aktivseite der Bilanz......................................... 219

Tabelle 58: Anpassen der Passivseite der Bilanz........................................ 220

Tabelle 59: Anpassen der Gewinn-und-Verlust-Rechnung. 
Tabelle 60: Länge der Beteiligungsketten - Vergleich der AmadeusDatenbank mit der Direktinvestitionsstatistik der Deutschen Bundesbank

Tabelle 61: Vergleich des Aufkommenseffekts der Einführung einer konsolidierten Körperschaftsteuer-Bemessungsgrundlage für die Formelfaktoren Vermögen, Lohnsumme und Umsatz

Tabelle 62: Durchschnittliche quadrierte Abweichung des Aufkommenseffekts der Einführung einer konsolidierten Körperschaftsteuer-Bemessungsgrundlage in Abhängigkeit von der Gewichtung der Formelfaktoren in v. H. SA

Tabelle 63: Lohnniveau und Arbeitsproduktivität in der Europäischen Union

Tabelle 64: Vergleich des Aufkommenseffekts der Einführung einer konsolidierten Körperschaftsteuer-Bemessungsgrundlage bei Verwendung der Lohnsumme und Mitarbeiteranzahl als Aufteilungsfaktor.

Tabelle 65: Vergleich des Aufkommenseffekts der Einführung einer konsolidierten Körperschaftsteuer-Bemessungsgrundlage bei Anwendung der Anrechnungsmethode und der Freistellungsmethode 


\section{A. Einleitung}

\section{Problemstellung}

Die Europäische Gemeinschaft bildet das Herzstück der Europäischen Union. Als Europäische Wirtschaftsgemeinschaft 1957 gegründet, ${ }^{1}$ verfolgt sie vor allem wirtschaftliche Interessen, die gemäß Art. 2 EGV in der Errichtung eines Gemeinsamen Marktes, einer Wirtschafts- und Währungsunion sowie der Durchführung der in den Artikeln 3 und 4 genannten gemeinsamen Politiken und Maßnahmen bestehen. ${ }^{2}$ Mit der Einführung des gemeinsamen Binnenmarktes zum 31. 12. 1992, ${ }^{3}$ der den freien Waren-, Personen-, Dienstleistungsund Kapitalverkehr zwischen den Mitgliedstaaten sicherstellt, und der Europäischen Währungsunion, die am 1. 1. 1999 in Kraft trat, konnten wesentliche Ziele des Vertragswerks bereits umgesetzt werden. ${ }^{4}$ Dem enormen Wandel der wirtschaftlichen Rahmenbedingungen folgte die Steuerrechtsordnung der Mitgliedstaaten nur zögerlich. ${ }^{5}$ Ursächlich hierfür ist der fehlende direkte, gesetzlich verankerte Harmonisierungsauftrag. Dieser kann bei den direkten Steuern im Gegensatz zu den indirekten nur aus der nicht steuerspezifischen Rechtsangleichungsgrundnorm des Art. 94 EGV abgeleitet werden. ${ }^{6}$ Verbindliche Rechtsakte setzen dabei einen einstimmigen Beschluss voraus, wodurch die Angleichung der direkten Steuern ein Akt politischer Willensbildung wird. Dieser konnte bisher nur bruchstückhaft vollzogen werden, ${ }^{7}$ da nicht alle Mit-

1 Die Europäische Wirtschaftsgemeinschaft wurde 1957 durch den Abschluss der Römischen Verträge von den sechs Mitgliedern der Montanunion - Frankreich, Italien, Deutschland, Belgien, den Niederlanden und Luxemburg - unterzeichnet.

2 Ziel ist es, ,in der ganzen Gemeinschaft eine harmonische, ausgewogene und nachhaltige Entwicklung des Wirtschaftslebens, ein hohes Beschäftigungsniveau und ein hohes Maß an sozialem Schutz, die Gleichstellung von Männern und Frauen, ein beständiges, nichtinflationäres Wachstum, einen hohen Grad von Wettbewerbsfähigkeit und Konvergenz der Wirtschaftsleistungen, ein hohes $\mathrm{Ma} ß$ an Umweltschutz und Verbesserung der Umweltqualität, die Hebung der Lebenshaltung und der Lebensqualität, den wirtschaftlichen und sozialen Zusammenhalt und die Solidarität zwischen den Mitgliedstaaten zu fördern." Art. 2 EGV.

3 Art. 14 EGV.

4 Art. 3 Abs. 1 c EGV; Art 14 EGV.

5 Vgl. Spengel, C., Unternehmensbesteuerung, 2003, S. 2.

6 Eine Ausnahme bildet der in seiner Bedeutung zweitrangige Art. 293 EGV (zweiter Teilstrich), der die Beseitigung der Doppelbesteuerung innerhalb der Gemeinschaft sicherstellen soll. Vgl. zu den Rechtsgrundlagen der Harmonisierung der direkten Steuern Hey, J., Harmonisierung, 1997, S. 80-98; Beiser, R./Pülzl, P., in: Weber/Wimmer, Verfassungsstaat, 2005, S. 43-54.

7 Die wesentlichen Richtlinien, die bislang im materiell-steuerlichen Bereich verabschiedet wurden, sind die Fusionsrichtlinie (Richtlinie 90/434/EWG des Rates v. 23. 7. 1990, Abl. 
gliedstaaten von einer Angleichung der Rechtsvorschriften profitieren. ${ }^{8}$ Zudem zählen die Mitgliedstaaten die Finanzhoheit zum Kernbereich ihrer Souveränität, welche sie nicht bereit sind, ohne Weiteres aufzugeben. Denn Steuerpolitik ist besonders im Bereich der Unternehmenssteuer auch Standortpolitik und damit ein wichtiger Wettbewerbsfaktor. ${ }^{9}$ So stehen bis heute die Strukturen der nationalen Steuerrechtsordnungen weitestgehend unkoordiniert nebeneinander. ${ }^{10}$

Dabei fehlte es in der Vergangenheit nicht an Bemühungen zur Harmonisierung der Unternehmensbesteuerung. Vielmehr sind die Initiativen, ${ }^{11}$ die direkten Steuern zu vereinheitlichen, so alt wie die Europäische Gemeinschaft selbst. Zuletzt hat eine Gruppe von Experten im Auftrag der Europäischen Kommission in einer im Jahr 2001 veröffentlichten Studie die Unternehmensbesteuerung in der Europäischen Union umfangreich analysiert und dabei unter anderem die steuerlichen Hindernisse, die derzeit einer grenzüberschreitenden wirtschaftlichen Tätigkeit im Binnenmarkt entgegenstehen, aufgelistet. ${ }^{12}$ In dieser sogenannten Binnenmarktstudie kommen sie zu dem Schluss, dass die eigentliche Ursache der zusätzlichen Steuer- und Befolgungskosten die Koexistenz von 25 Steuersystemen mit den dazugehörigen Doppelbesteuerungsabkommen ist. ${ }^{13}$ Zudem hat der EuGH z. B. in den Rechtssachen Safir, Verkooijen und Saint-

Nr. L 225, S. 1-5), die Mutter-Tochter-Richtlinie (Richtlinie 90/435/EWG des Rates v. 23. 7. 1990, Abl. Nr. L 225 v. 20. 8. 1990, S. 6-9) und eine Richtlinie über eine gemeinsame Steuerregelung für Zahlungen von Zinsen und Lizenzgebühren (Richtlinie 2003/49/EG des Rates v. 3. 6. 2003 Abl. Nr. L 157 v. 26.06.2003, S. 49-54).

8 Beispielsweise existieren in Irland spezielle Steuerregime für Finanzierungsgesellschaften, deren steuerlicher Vorteil mit Einführung einer harmonisierten Unternehmensbesteuerung größtenteils verloren geht. Vgl. $\mathrm{zu}$ den Irish Financial Service Centers Scheffler, W., in: Bank/Schiller, Finanzintermediation, 2004, S. 507-528; Burwitz, G., Konzernfinanzierungsgesellschaften, 2005.

9 Vgl. Fuest, W., Steuerharmonisierung, 2006, S. 5.

10 Mitte 2001 lagen dem Rat 16 ausgearbeitete Richtlinienvorschläge vor, davon einige seit Anfang der 90er Jahre. Vgl. Kilches, R., in; FJ, 2001, S. 175. Für eine Übersicht über die Aktivitäten der Europäischen Kommission im Bereich der direkten Steuern zwischen 1999 und 2004 vgl. Europäische Kommission, Activities, 2004, Anhang.

1 Zu nennen sind hier insbesondere der Neumark-Bericht (1962), das van den TempelGutachten (1969), der Werner-Stufenplan (1971), der Richtlinien-Vorschlag zur Harmonisierung der Körperschaftsteuersysteme (1975), die Forderung nach der Angleichung der Körperschaftsteuersysteme (1984) und der Ruding-Bericht (1992). Für eine detaillierte Darstellung der Unternehmenssteuerpolitik der Europäischen Union im Zeitablauf vgl. Treisch, C., Unternehmensbesteuerung, 2004, S. 13-23; Hey, J., Harmonisierung, 1997, S. 67-76.

12 Europäische Kommission, Unternehmensbesteuerung, 2001.

13 Da die Binnenmarktstudie im Jahr 2001 veröffentlicht wurde, beziehen sich deren Angaben stets auf 15 Mitgliedstaaten. Vgl. Europäische Kommission, Unternehmensbesteuerung, 2001, S. 18-19. 
Gobain festgestellt, dass Steuersysteme, die grenzüberschreitende Tätigkeiten oder Investitionen behindern, mit den Grundfreiheiten des EG-Vertrags unvereinbar sind. ${ }^{14}$ Vor diesem Hintergrund strebt die Europäische Kommission langfristig die Umsetzung eines umfassenden Lösungsansatzes an, wobei sich ihre Bemühungen auf die Einführung einer konsolidierten KörperschaftsteuerBemessungsgrundlage konzentrieren. ${ }^{15} \mathrm{Z}$ war existieren durchaus auch kritische Stimmen gegenüber der Harmonisierung der Unternehmensbesteuerung, ${ }^{16}$ doch verbleibt bei dem angestrebten Lösungsvorschlag die Steuersatzautonomie bei den Mitgliedstaaten, wodurch gleichzeitig das Subsidiaritätsprinzip gewahrt bleibt. ${ }^{17}$

Die äußeren Rahmenbedingungen für die Realisierung einer harmonisierten Unternehmensbesteuerung erscheinen derzeit besser als je zuvor. Die fortschreitende Verwirklichung des gemeinsamen Binnenmarktes hat den Harmonisierungsbemühungen im Bereich der direkten Steuern neuen Schwung verliehen. So schlug die Europäische Kommission im Jahr 2005 einen Neubeginn für die Lissabon-Strategie mit den Schwerpunkten Wachstum und Arbeitsplätze vor $^{18}$ und bekräftigte ihr Vorhaben, für Unternehmen, die im Binnenmarkt agieren, eine gemeinsame Steuerbemessungsgrundlage zur Verfügung zu stellen. ${ }^{19}$ Auch die Zunahme der Entscheidungen des EuGHs im Bereich der direkten Steuern fördert den Harmonisierungsprozess. Zwar bilden sie derzeit noch ein Kontrastprogramm zu den politischen Bemühungen um die Steuerharmonisierung, doch verdeutlicht der negative Integrationsmechanismus ${ }^{20}$ den Mitgliedstaaten zunehmend, dass ihre nationalen Steuergesetze den Anforderungen des Gemeinsamen Binnenmarktes nicht mehr gerecht werden. ${ }^{21}$ Zudem hat die Europäische Union mit dem Vertrag von Amsterdam den rechtlichen Rahmen dafür geschaffen, eine Harmonisierung durch das Institut der verstärkten Zusammenarbeit auch nur für eine Teilgruppe der Mitgliedstaaten zu

14 EuGH v. 21. 9. 1999, Rs. C-307/97 - Saint-Gobain, in: EuGHE, 1999, I-6161; v. 28.4. 1998, Rs. C-118/96 - Safir, in: EuGHE, 1998, I-1897; v. 6. 6. 2000, Rs. C-35/98 Verkooijen, in: $E u G H E, 2000$, I-4071.

$15 \mathrm{Vgl}$. Europäische Kommission, Binnenmarkt, 2003, S. 4.

16 Für Argumente gegen eine umfangreiche Steuerharmonisierung in der Europäischen Union vgl. Treisch, C., Unternehmensbesteuerung, 2004, S. 6-8 m. w. N.

17 Spengel, C.; in: Lüdicke, Steuerrecht, 2004, S. 135.

18 Vgl. Europäische Kommission, Neubeginn, 2005; dieselbe, Lissabon-Programm, 2005.

19 Vgl. Europăische Kommission, Herausforderungen, 2005, S. 5-6. Bis 2008 beabsichtigt die Kommission, einen Vorschlag für einen Gemeinschaftsrechtsakt für eine einheitliche konsolidierte Körperschaftsteuer-Bemessungsgrundlage vorzulegen.

Vgl. English, J., in: StuW, 2003, S. 91.

21 Vgl. Beiser, R./Pülzl, P., in: Weber/Wimmer, Verfassungsstaat, 2005, S. 61. 
realisieren. ${ }^{22}$ Selbst wenn ein solcher Vorstoß im Bereich der Steuern teilweise kritisch gesehen wird, ${ }^{23}$ ermöglicht er doch, geplante Regelungen zunächst für einen kleinen Teilnehmerkreis umzusetzen. Außerdem treibt die Währungsunion den Integrationsprozess der Europäischen Union weiter voran, da Preise für Waren, Dienstleistungen und Zinsen eine höhere Transparenz erreicht haben und somit der bislang Standortunterschiede ausgleichende Wechselkursmechanismus entfallen ist. ${ }^{24}$ Zudem bedarf es einer harmonisierten Unternehmensbesteuerung, um die Attraktivität und Effektivität der Europäischen Gesellschaft (SE) voll zu entfalten. Ohne diese steuerlichen Maßnahmen bietet die Gründung einer SE keine nennenswerten Vorteile. ${ }^{25}$ Darüber hinaus ist durch die Aufnahme weiterer zwölf Mitgliedstaaten zum 1. 5. $2004^{26}$ und zum 1. 1. $2007^{27}$ die Anzahl der parallel existierenden Steuersysteme innerhalb der Europäischen Union auf 27 angestiegen, was den Handlungsbedarf im Bereich der direkten Steuern weiter verschärft. Schließlich treten durch den stetig zunehmenden internationalen Geschäftsverkehr, der grenzüberschreitendes Handeln eher zur Regel als zur Ausnahme werden lässt, und die veränderten wirtschaftlichen Rahmenbedingungen durch die Informations- und Kommunikationstechnologie die steuerlichen Probleme bei grenzüberschreitender Tätigkeit wie z. B. die Funktionsverlagerung, die Verrechnungspreisgestaltung oder die Verlustverrechnungsproblematik verstärkt in den Vordergrund. ${ }^{28}$

Diese Ausgangslage und die Notwendigkeit einer Harmonisierung der direkten Steuern ist der Europäischen Kommission bewusst. Bereits seit 2004 existiert

Art. 40 und Art 43 EUV. Vgl. hierzu a. Beiser, R./Pülzl, P., in: Weber/Wimmer, Verfassungsstaat, 2005, S. 50-54.

23

Die Vorbehalte werden insbesondere von weniger integrationswilligen Mitgliedstaaten hervorgebracht, die zu vermeiden versuchen, bei späterer Teilnahme vor vollendeten Tatsachen zu stehen. Vgl. Selling, H.-J., in: IStR, 2000, S. 424. Kritisch auch Cerioni, L., in: ET, 2006, S. 187-196.

24 Cordewener spricht in diesem Zusammenhang von dem sogenannten "Riff-Effekt“, der steuerliche Einflüsse auf den Wirtschaftsverkehr im Binnenmarkt in den Vordergrund treten lässt, je mehr außersteuerliche Handelshindernisse beseitigt werden. Vgl. Cordewener, A., Grundfreiheiten, 2002, S. 30-31.

Europäische Kommission, Unternehmensbesteuerung, 2001, S. 545-546; Spengel, C.; in: Lüdicke, Steuerrecht, 2004, S. 135.

26 Estland, Lettland, Litauen, Malta, Polen, Slowakei, Slowenien, Tschechische Republik, Ungarn und Zypern sind zum 1. 5. 2005 der Europäischen Union beigetreten.

Bulgarien und Rumänien sind zum 1. 1. 2007 der Europäischen Union beigetreten.

Die Zahl der multinationalen Unternehmen hat sich seit den 60er Jahren bis Ende der 90er Jahre fast versechsfacht. Damit verbunden ist eine Verdopplung des Anteils am Welt-BIP, das von multinationalen Unternehmen erwirtschaftet wird. Vgl. Europäische Kommission, Unternehmensbesteuerung, 2001, S. 20-21. Zu den Veränderungen der Organisationsstruktur von verbundenen Unternehmen durch die Informations- und Kommunikationstechnologie vgl. Schäfer, A./Spengel, C., ICT, 2003, S. 2-5. 
die Arbeitsgruppe „Gemeinsame konsolidierte Körperschaftsteuer-Bemessungsgrundlage“, deren Aufgabe es ist, die „konsolidierte Steuerbemessungsgrundlage für in der EU tätige Unternehmen unter technischen Gesichtspunkten zu prüfen. Die Arbeitsgruppe erörtert die Besteuerungsgrundsätze, die grundlegenden Strukturelemente einer einheitlichen (konsolidierten) Körperschaftsteuer-Bemessungsgrundlage und andere technische Fragen wie etwa das Verfahren der ,Aufteilung' einer solchen Bemessungsgrundlage zwischen den Mitgliedstaaten.“29 Neben den Veröffentlichungen der Arbeitsgruppe zum Stand ihrer Arbeit ist auch im Schriftum eine Vielzahl theoretischer Abhandlungen erschienen. ${ }^{30}$ Empirische Fragestellungen wurden dabei fast immer ausgeblendet oder nur sehr rudimentär behandelt. ${ }^{31}$ Dass bislang wissenschaftlich fundierte Erkenntnisse auf der Basis von empirischen Analysen über die Auswirkung einer Einführung einer konsolidierten Körperschaftsteuer-Bemessungsgrundlage fehlen, zeigt nicht zuletzt der Hinweis der Europäischen Kommission vom 13. 12. 2006, selbst die Folgen der Implementierung abschätzen zu wollen. ${ }^{32}$

29 Europäische Kommission, Arbeitsleitlinien, 2004, S. 2.

30 Vgl. für viele Oestreicher, A., in: StuW, 2002, S. 342-356; Gammie, M./Radaelli, C.M./Klemm, A., Reform, 2001; Hellerstein, W./McLure Jr., C.E., in: ITPF, 2004, S. 199220; McLure Jr., C.E., in: TNI, 2004, S. 45-69; Mintz, J.M., in: ITPF, 2004, S. 221-234; Scheffler, W., in: Oestreicher, Konzernbesteuerung, 2005, S. 305-331; Spengel. C./Braunagel, R.U., in: StuW, 2006, S. 34-49; Weiner, J.M., Company, 2006.

31 Fuest/Hemmelgarn/Ramb haben auf Basis der Daten der Direktinvestitionsstatistik der Deutschen Bundesbank die Aufkommenswirkungen der Einführung einer konsolidierten Körperschaftsteuer-Bemessungsgrundlage analysiert. Vgl. Fuest, C./Hemmelgarn, T./Ramb, F., Apportionment, 2006. Devereux und Loretz haben eine vergleichbare Analyse auf der Basis der Orbis Datenbank des Anbieters Bureau van Dijk durchgeführt. Vgl. Devereux, M.P./Loretz, S., Apportionment, 2007.

32 Vgl. Europäische Kommission, Mechanismen, 2006, S. 3-4. „Die Einzelheiten zu dieser Folgenabschătzung sind noch nicht abschließend geklärt, ein Element könnte jedoch eine Analyse sein, wie sich eine Umstellung auf eine neue Aufteilungsmethode für die ,EUSteuerbemessungsgrundlage ‘ auf die Körperschaftsteuer-Bemessungsgrundlagen der Mitgliedstaaten auswirken würde. Da keine Daten zur Steuerbemessungsgrundlage öffentlich verfügbar sind, ist es bislang nicht möglich, eine umfassende Analyse durchzuführen, bei der die derzeitige Aufteilung der Steuerbemessungsgrundlagen der Konzerne nach Mitgliedstaat mit der Aufteilung der Steuerbemessungsgrundlagen der Konzerne nach Mitgliedstaat im Rahmen eines Aufteilungsmechanismus verglichen wird. Zu diesem Zweck möchte die Kommission wissen, ob die Mitgliedstaaten bereit sind, die furr die Durchführung einer solchen Folgenabschätzung erforderlichen Steuerdaten zur Verfügung zu stellen. Ohne diese Daten würde die Folgenabschätzung auf Grundlage der öffentlich verfügbaren Rechnungslegungsdaten anhand der derzeitigen Verteilung der handelsrechtlichen Gewinne stellvertretend für die derzeitige Verteilung der KörperschaftsteuerBemessungsgrundlagen durchgeführt werden." Europăische Kommission, Mechanismus, 2006, S. 3-4. 


\section{Zielsetzung}

Um die Auswirkungen der Einführung der konsolidierten KörperschaftsteuerBemessungsgrundlage auf die Konzernstrukturen und das Steueraufkommen empirisch beurteilen zu können, bedarf es der Kenntnis über die mögliche Umsetzung dieses Lösungsvorschlags. Daneben muss eine geeignete Datenbasis gefunden werden, mit deren Hilfe die offenen Fragen beantwortet werden können. Vor diesem Hintergrund besteht die Zielsetzung dieser Arbeit zunächst darin, die konsolidierte Körperschaftsteuer-Bemessungsgrundlage zu präzisieren. Ergebnis kann aber kein konkreter Lösungsansatz sein. Vielmehr soll der derzeitige Stand der Diskussion im Schriftum und auf der Ebene der Europäischen Kommission wiedergegeben und beurteilt werden. Zudem sollen mögliche Alternativen der Ausgestaltung einer einheitlichen (konsolidierten) Körperschaftsteuer-Bemessungsgrundlage identifiziert werden, die im Rahmen der empirischen Analyse bewertet werden können. Diese Zielsetzung erscheint besonders vor dem Hintergrund sinnvoll, dass die konkrete Ausgestaltung der konsolidierten Bemessungsgrundlage einen politischen Prozess darstellt, dessen Ausgang noch offen ist. Nicht Bestandteil dieser Arbeit ist die Vereinheitlichung der Gewinnermittlungsvorschriften.

Neben diesem theoretischen Gerüst muss für die empirischen Auswertungen eine geeignete Datenbasis gefunden und entsprechend den Anforderungen aufbereitet werden. Sind diese Voraussetzungen erfüllt, kann sich den zwei thematischen Schwerpunkten dieser Arbeit, der Auswirkung der Einführung der konsolidierten Körperschaftsteuer-Bemessungsgrundlage auf die Konzernstrukturen und das Steueraufkommen, gewidmet werden. Die Konzernstrukturanalyse soll insbesondere statutarische Strukturen identifizieren, die das Resultat steuerplanerischer Gestaltungen sind. Denn mit der Einführung der konsolidierten Bemessungsgrundlage wird diesen steuerplanerischen Konstruktionen vielfach die Existenzgrundlage genommen, sodass eine Anpassung der Konzernstrukturen erwartet werden kann. Daneben sind die Aufkommenswirkungen der Einführung der konsolidierten Bemessungsgrundlage im Rahmen einer komparativ-statischen Mikrosimulation zu quantifizieren. Entsprechend den theoretischen Vorarbeiten sollen dabei unterschiedliche Alternativen einer konsolidierten Körperschaftsteuer-Bemessungsgrundlage ceteris paribus miteinander verglichen werden.

\section{Aufbau der Arbeit}

Um die gesteckten Ziele zu erreichen, bietet sich folgender, sechsteiliger Aufbau der Arbeit an: 
Nach der Einleitung (Kapitel $A$ ) werden in Kapitel $B$ die Grundstrukturen der Ertragsbesteuerung von verbundenen Unternehmen innerhalb der 25 EUMitgliedstaaten erarbeitet und systematisiert. ${ }^{33}$ Dieser qualitative Teil der Arbeit dient der Darstellung der für die quantitativen Analysen relevanten gesetzlichen Regelungen. Somit beschränkt er sich auf die Grundstrukturen der Konzernbesteuerung im Ertragsteuerrecht. Außerdem werden die wesentlichen, damit einhergehenden unternehmenssteuerlichen Probleme und Hindernisse bei einer grenzüberschreitenden Geschäftstätigkeit in der Europäischen Union herausgearbeitet.

Die dargelegten Hindernisse in Kapitel B stehen dem Ziel der Europäischen Union, der Verwirklichung eines Gemeinsamen Binnenmarktes, entgegen. Die als Reaktion darauf angestellten Reformüberlegungen sind Bestandteil des Kapitels $C$. Zunächst werden die wesentlichen Lösungsansätze zur Beseitigung der Hindernisse vorgestellt und hinsichtlich ihrer politischen Durchsetzbarkeit und ihres Potenzials, die derzeit bestehenden Probleme zu beseitigen, bewertet (Abschnitt C.I). Als vielversprechendster Ansatz gilt sowohl im Schrifttum als auch für die Europäische Kommission die einheitliche (konsolidierte) Körperschaftsteuer-Bemessungsgrundlage. Dieser Reformvorschlag ist zentrales Thema dieser Arbeit und wird ausführlich in Kapitel C.II beschrieben. Die Darstellung gliedert sich in drei Teile. Zunächst ist der Konsolidierungskreis abzugrenzen, d.h. es sind die Kriterien festzulegen, anhand derer die Mitglieder eines steuerlichen Konzernkreises ausgewählt werden. Anschließend wird die Ermittlung der gemeinsamen Bemessungsgrundlage erörtert. Dabei wird nur am Rande auf die Vereinheitlichung der Gewinnermittlungsvorschriften eingegangen, da deren Berücksichtigung den Rahmen dieser Arbeit sprengen würde und diese außerdem bei den empirischen Berechnungen vernachlässigt werden. Im dritten Schritt werden mögliche Mechanismen zur Aufteilung der gemeinsamen Bemessungsgrundlage auf die einzelnen Konzerneinheiten vorgestellt. Da die einheitliche (konsolidierte) Körperschaftsteuer-Bemessungsgrundlage hohe Ähnlichkeiten mit der US-amerikanischen „unitary taxation“ aufweist, wird vielfach auf die amerikanischen Regelungen, die damit verbundenen Erfahrungen und die dazugehörige wissenschaftliche Diskussion zurückgegriffen. Kapitel $\mathrm{C}$ schließt mit dem Aufzeigen des empirischen Forschungsbedarfs hinsichtlich der Ausgestaltung der einheitlichen (konsolidierten) Körperschaftsteuer-Bemessungsgrundlage.

Die Auswahl und Aufbereitung der Datenbasis für die empirischen Analysen sind Bestandteil von Kapitel D. Zunächst werden die Anforderungen, die an eine Datenbasis für die angestrebten Analysen gestellt werden, in Abschnitt D.II erarbeitet und eine geeignete Datenbasis ausgewählt. Im folgenden Abschnitt D.III

33 Die am 1. Januar 2007 beigetretenen Mitgliedstaaten Bulgarien und Rumänien werden in der Arbeit nicht berücksichtigt. 
wird die selektierte Amadeus-Datenbank des Anbieters Bureau van Dijk vorgestellt und auf die Repräsentativität der darin enthaltenen Unternehmen hin erörtert. Abschnitt D.IV und D.V legen die Aufbereitung der unternehmensbezogenen Einzeldaten dar und beschreiben die Abbildung der Konzernstrukturen auf der Basis der zur Verfügung stehenden Beteiligungsinformationen.

Kapitel $E$ befasst sich mit den Konzernstrukturen der multinationalen Unternehmen. Im ersten Abschnitt werden die vorgefundenen Konzernstrukturen beschrieben. Dabei stehen weniger steuerliche Aspekte im Vordergrund als vielmehr eine allgemeine Darstellung des Aufbaus von Konzernen. Im Abschnitt E.II erfolgt dann die Analyse der Konzernstrukturen unter steuerlichen Gesichtspunkten. Dazu werden die in Kapitel D ermittelten Konzernstrukturen mit den in Kapitel B dargelegten steuerrechtlichen Regelungen in einen sachlogischen Zusammenhang gebracht. Zur Komplexitätsreduktion werden dabei nur typische, zu steuerplanerischen Zwecken gegründete Gesellschaften analysiert und deren Existenz ökonometrisch erklärt. Im Einzelnen geht es in diesem Zusammenhang um den Einsatz von Holding- und Finanzierungsgesellschaften.

Kapitel $F$ analysiert schließlich die quantitativen Auswirkungen der Einführung der einheitlichen (konsolidierten) Körperschaftsteuer-Bemessungsgrundlage. Grundlage hierfür bilden erneut die in Kapitel D abgebildeten Konzerne sowie das in Kapitel B und C beschriebene bestehende bzw. vorgesehene steuerrechtliche Regelwerk. Nach der Erläuterung der Methodik der Berechnung in Abschnitt F.II werden im darauffolgenden Teil die Aufkommenswirkung der Einführung einer einheitlichen Bemessungsgrundlage mittels einer statischen Mikrosimulation ermittelt, die den Berechnungen zugrunde liegenden Prämissen analysiert und ein Vergleich mit anderen existierenden empirischen Studien angestellt. Daran anschließend werden ceteris paribus einzelne Parameter der einheitlichen Körperschaftsteuer-Bemessungsgrundlage wie die Mindestbeteiligungsquote und Elemente der Aufteilungsformel variiert und in Abschnitt F.IV die Änderung der Steuerzahllast nach Maßgabe einer kapitalimport- und exportneutralen Besteuerung ermittelt.

Die Arbeit schließt mit einer Zusammenfassung der zentralen Untersuchungsergebnisse, die in Kapitel $G$ wiedergegeben werden. 


\section{B. Besteuerung von Konzernen in den Mitgliedstaaten der Europäischen Union}

\section{Alternative Besteuerungskonzeptionen für Unternehmensgruppen}

Die Besteuerung von Kapitalgesellschaften erfolgt in allen europäischen Mitgliedstaaten nach dem Trennungsprinzip. Dieses unterscheidet zwischen der Ebene der Gesellschaft und der der Gesellschafter. ${ }^{34}$ Dabei stehen sich Körperschaft und Anteilseigner grundsätzlich wie zwei Dritte gegenüber. Das Steuerrecht knüpft damit an die zivilrechtlichen Wertungen an und akzeptiert die eigene Rechtsfähigkeit von juristischen Personen auch zum Zwecke der Besteuerung. ${ }^{35}$ Dies gilt auch, wenn der Anteilseigner wiederum eine Kapitalgesellschaft ist, also zwei oder mehrere Unternehmen miteinander verbunden sind. Damit wird dem Konzern als wirtschaftliche Zusammenfassung rechtlich selbstständiger Einheiten in den europäischen Rechtsordnungen keine eigene Rechtspersönlichkeit zugesprochen (Trennungstheorie) ${ }^{36}$ Der Trennungstheorie steht die Einheitstheorie gegenüber. Sie erkennt die rechtliche Verbundenheit der Konzerngesellschaften an und begreift eine Unternehmensgruppe als eine wirtschaftliche Einheit. ${ }^{37}$ Die einzelnen juristischen Personen werden weder objektiv noch subjektiv steuerpflichtig. Steuersubjekt ist allein die Konzernunternehmung. ${ }^{38}$ Alle Rechtsbeziehungen zwischen den Konzerngesellschaften sind dementsprechend innerbetriebliche Vorgänge, die das Konzernergebnis nicht beeinflussen. ${ }^{39}$ Die uneingeschränkte Übernahme der Einheitstheorie für die Konzernbesteuerung ist jedoch nur bedingt möglich. Sind Minderheitsgesellschafter an einzelnen Konzerngesellschaften beteiligt oder wird der Konzern in mehreren Steuerhoheiten tätig, ist eine verursachungsgerechte Aufteilung des Gesamtergebnisses auf die einzelnen Gesellschaften unausweichlich. ${ }^{40}$

34 Vgl. Spengel, C., Unternehmensbesteuerung, 2003, S. 13; Weiner, J.M., in: TNI, 2000, S. 1115.

35 Vgl. Scheffler, W., Unternehmen, 2006, S. 165.

36 Vgl. Scheuchzer, M., Konzernbesteuerung, 1994, S. 28.

37 Vgl. Hübl, L., in: DStZ, 1965, S. 21.

38 Vgl. Scheuchzer, M., Konzernbesteuerung, 1994, S. 37.

39 Oestreicher, A., in: Oestreicher, Konzernbesteuerung, 2005, S. 20.

40 Vgl. Scheffler, W., in: $D B W, 1991$, S. 712. Theoretisch möglich wäre die Umsetzung der Einheitstheorie innerhalb der Europăischen Union, wenn dem Konzept der europäischen Körperschafsteuer gefolgt werden würde und zudem eine Mindestbeteiligung von 100 v. H. zur Abgrenzung des Konzerns gefordert würde. Für eine kurze Darstellung der europäischen Körperschaftsteuer vgl. Kapitel C.I.3. 
Dementsprechend folgen die derzeitige Konzernbesteuerung in den Mitgliedstaaten $^{41}$ und die von der Europäischen Kommission vorgeschlagenen Reformkonzepte $^{42}$ der Trennungstheorie. Damit stellt sich zugleich die Frage, wie die Gesamtbemessungsgrundlage auf die einzelnen Konzerneinheiten aufzuteilen ist. Grundsätzlich kann zwischen dem Prinzip der selbstständigen Einheit (,separate entity theory“) und dem Prinzip des einheitlichen Unternehmens (,unitary entity theory“) unterschieden werden. ${ }^{43}$ Ersteres betrachtet die Tochtergesellschaften eines Konzerns als eigenständige Wirtschaftseinheiten, die ihren Gewinn losgelöst vom Gesamterfolg des Konzerns zu ermitteln und zu versteuern haben. Die Abgrenzung der Gewinne zwischen verbundenen Unternehmen hat derart zu erfolgen, als stünden sich die Konzerngesellschaften wie fremde Dritte gegenüber. Das Prinzip des einheitlichen Unternehmens erkennt dagegen die wirtschaftliche Verbundenheit der Konzerngesellschaften an. Ausgangspunkt der Besteuerung bildet stets der konsolidierte Konzernerfolg, der mittels eines Schlüssels auf die einzelnen Konzerngesellschaften aufgeteilt wird. Jede Konzerngesellschaft erhält anteilig den Gewinn zugewiesen, der ihrem Beitrag zum Konzernerfolg entspricht. Somit erfolgt die Gewinnabgrenzung nicht mehr direkt durch die separate Gewinnermittlung, sondern indirekt.

Alle EU-Mitgliedstaaten schreiben derzeit die Ermittlung der Bemessungsgrundlage nach dem Prinzip der selbstständigen Einheit vor. Die wirtschaftliche Verbundenheit der Konzerngesellschaften findet zunächst keine Berück-

4I Der Einheitstheorie am nächsten kommt die niederländische Gruppenbesteuerung, die von einer fiktiven Fusion der Konzerngesellschaften ausgeht (Art. 15 Abs. 1 S. 1 Wet Vpb). Das System der steuerlichen Einheit betrifft aber nur die Ermittlung des Steuerobjekts im Konzern. Die Steuersubjekteigenschaft der Konzerngesellschaften bleibt weiterhin bestehen. Vgl. Sievert, E., Konzernbesteuerung, 2006, S. 145; Grotherr, S., in: StuW, 1996, S. 361 .

In Bezug auf die deutsche körperschaftsteuerliche Organschaft hat der BFH immer wieder auf die Zurechnungstheorie verwiesen. Vgl. BFH v. 9. 9. 1986, VIII R 20/85, BFH/NV 1987, S. 442; v. 14. 4. 1992, VIII R 149/86, BStBI II 1992, S. 817. Sie ist in diesem Zusammenhang aber als Methodik der Gewinnermittlung im Konzern und nicht als eigenständige Theorie der Konzernbesteuerung zu verstehen. Vgl. Scheuchzer, M., Konzernbesteuerung, 1994, S. 27. So auch Jurkat, der die verschiedenen Theorien zusammenfasst und mit Ausnahme der Bilanzierungstheorie zwischen der Einheitstheorie und dem Theorienkreis, der die subjektive Steuerpflicht des Organs bejaht, differenziert. Vgl. Jurkat, W., Organschaft, 1975, S. 53.

Für eine kurze Darstellung der vier verschiedenen Reformvorschläge vgl. Kapitel C.I.3.

Das Prinzip des einheitlichen Unternehmens ist nicht mit der Einheitstheorie zu verwechseln. Aufgrund der Trennungstheorie bleibt bei Anwendung des Prinzips des einheitlichen Unternehmens die Steuersubjekteigenschaft der Konzerngesellschaften erhalten. Für eine detaillierte Darstellung und Analyse dieser beiden Prinzipien vgl. Oestreicher, A., Konzerngewinnabgrenzung, 1999. 
sichtigung. Demgegenüber folgen die vorgebrachten Reformkonzepte dem Prinzip des einheitlichen Unternehmens.

Inhalt des folgenden Kapitels ist eine kurze Darstellung der geltenden, auf dem Prinzip der selbstständigen Einheit basierenden Konzernbesteuerung und der damit verbunden unternehmenssteuerlichen Hindernisse in Europa. Die zur Beseitigung der steuerlichen Hürden vorgebrachten Reformvorschläge sind Gegenstand des Kapitels C.

\section{Besteuerung von Konzernen nach dem Prinzip der selbstständigen Einheit}

Nach dem Prinzip der selbstständigen Einheit ermittelt jede Konzerngesellschaft ihren Gewinn separat, ungeachtet der bestehenden Beherrschungs- und Abhängigkeitsverhältnisse und der daraus resultierenden wirtschaftlichen Einheit eines Konzerns. ${ }^{44}$ Damit wird die wirtschaftliche Einheit rechtlich selbstständiger Konzerngesellschaften negiert. Die isolierte Betrachtung der einzelnen juristischen Personen führt zu einer Verschiebung der Grenze zwischen Unternehmungs- und Marktbereich, ${ }^{45}$ indem innerhalb der wirtschaftlichen Einheit ein Markt fingiert wird. Da aber der Mechanismus der Preisbildung zwischen den verbundenen Einheiten aufgrund des fehlenden Interessengegensatzes zwischen Anbieter und Nachfrager zum Erliegen kommt, kann ein Gleichgewichtspreis nicht ermittelt werden. Stattdessen werden die Bedingungen, die unabhängige dritte Marktpartner den konzerninternen Transaktionen zugrunde legen würden, als Maßstab für die Aufteilung des Konzernerfolgs herangezogen (,dealing at arm's length“).

Zur Umsetzung des Fremdvergleichsgrundsatzes hat sich eine Vielzahl von Methoden herausgebildet. ${ }^{46}$ Grundsätzlich lassen sich die Standardmethoden von den Gewinnmethoden unterscheiden. Letztere können zudem in transaktionsbezogene und globale Gewinnmethoden untergliedert werden. ${ }^{47}$ Welche Methoden im Einzelfall zur Anwendung kommen, ist nicht eindeutig geregelt. Die globalen Gewinnmethoden erachtet die OECD prinzipiell als ungeeignet, da sie nicht mit dem Fremdvergleichsgrundsatz konform gehen. ${ }^{48}$ Bei den übrigen Methoden stehen insbesondere die drei Standardmethoden gleichrangig neben-

44 Zur Diskussion, inwieweit eine Mehrheitsbeteiligung bereits eine wirtschaftliche Einheit darstellt, vgl. Kapitel C.II.2.

$45 \mathrm{Vgl}$. Scheuchzer, M., Konzernbesteuerung, 1994, S. 29.

46 Dieser Fremdvergleichsgrundsatz findet sich auch in Art. 7 Abs. 2, Art. 9 Abs. 1 OECD-MA und $\S 1$ Abs. 1 AStG wieder.

$47 \mathrm{Vgl}$. hierzu die ÏJbersicht in Jacobs, O.H. (Hrsg.), Unternehmensbesteuerung, 2002, S. 936; Kromer, C., in: Kessler/Kröner/Köhler, Konzernsteuerrecht, 2004, S. 289.

Vgl. OECD, Guidelines, 2001, Tz. 3.1, Tz. 3.74. 
einander. Jedoch wird das Wahlrecht faktisch durch die zugrunde liegende Transaktion bereits eingeschränkt. ${ }^{49}$

Mit der steuerlichen Betrachtungsweise eines Konzerns als eine Gruppe unabhängiger Unternehmen sind verschiedene steuerliche Konsequenzen verbunden. Erstens bedingt eine fehlende Konsolidierung, dass Gewinnausschüttungen zwischen Konzerngesellschaften nicht eliminiert werden. Sie werden auf der Ebene der empfangenden Gesellschaften grundsätzlich als steuerbares Einkommen erfasst. Da Gewinnausschüttungen eine Nachsteuergröße darstellen, für die die ausschüttende Gesellschaft bereits Körperschaftsteuer entrichtet hat, führt eine erneute Erfassung der Dividenden bei der empfangenden Gesellschaft zu einer wirtschaftlichen Doppelbesteuerung. Zur Vermeidung werden Gewinnausschüttungen innerhalb Europas gemäß der Mutter-Tochter-Richtline ${ }^{50}$ bei der empfangenden Gesellschaft freigestellt oder aber die im Ausland entrichtete Steuer im Inland angerechnet (Tabelle 1). Eine Doppelbesteuerung von Gewinnausschüttungen und Dividenden ist damit weitestgehend ausgeschlossen.

Tabelle 1: Vermeidung der Doppelbesteuerung von Dividenden

\begin{tabular}{|l|l|}
\hline Freistellung 100 v. H. & $\begin{array}{l}\text { Österreich, Zypern, Tschechien, Dänemark Estland, Finnland, } \\
\text { Griechenland, Ungarn, Irland (national), Lettland, Litauen, } \\
\text { Luxemburg, Niederlande, Polen, Portugal, Schweden, Slowenien, } \\
\text { Slowakei, Großbritannien (national) }\end{array}$ \\
\hline Freistellung 95 v. H. & Belgien, Frankreich, Deutschland, Italien \\
\hline Anrechnung & $\begin{array}{l}\text { Irland (international), Malta, Spanien, } \\
\text { Großbritannien (international) }\end{array}$ \\
\hline
\end{tabular}

In Anlehnung an: Endres, D. et al., Taxable Income, 2006, S. 85

Zweitens ist eine Verlustverrechnung zwischen den Konzerngesellschaften nicht möglich. Lediglich bei Veräußerung, Tausch oder Liquidation können Verluste in das Ergebnis der Muttergesellschaft einbezogen werden. ${ }^{52}$ Darüber hinaus

49 Im Allgemeinen liefert bei einem Seriengeschäft (Warenverkehr) die Preisvergleichsmethode zuverlässigere Ergebnisse, während zur Bewertung von konzerninternen Dienstleistungen oder nicht marktgängigen Produkten die Kostenaufschlagmethode bzw. Wiederverkaufspreismethode Vorrang haben. Vgl. Jacobs, O.H. (Hrsg.), Unternehmensbesteuerung, 2002, S. 924.

50 Richtlinie 90/435/EWG des Rates v. 23. 7. 1990, Abl. Nr. L 225 v. 20. 8. 1990, S. 6-9.

51 Die Mitgliedstaaten können die mit der Beteiligung zusammenhängenden Kosten vom steuerlichen Gewinn der Muttergesellschaft abziehen. Werden diese pauschal festgesetzt, darf der Pauschalbetrag 5 v. H. des Gewinns nicht übersteigen. Dies entspricht dann einer Freistellung der Dividenden von 95 v. H. Art. 4 Abs. 2 Richtlinie 90/435/EWG des Rates v. 23. 7. 1990, Abl. Nr. L 225 v. 20. 8. 1990, S. 6-9.

52 Vgl. Oestreicher, A., in: Oestreicher, Konzernbesteuerung, 2005, S. 5. 
erlauben einige Mitgliedstaaten, Buchwertabschreibungen für Investitionen in eine Tochtergesellschaft steuerlich geltend $\mathrm{zu}$ machen, wenn eine Wertminderung der Beteiligung eintritt (Tabelle 2).

Tabelle 2: $\quad$ Abschreibung von Beteiligungsbuchwerten

\begin{tabular}{|c|c|}
\hline Buchwertabschreibung verpflichtend & $\begin{array}{l}\text { Österreich }{ }^{1,2}, \text { Tschechien, Estland, Frankreich }^{3} \text {, } \\
\text { Ungarn, Spanien }\end{array}$ \\
\hline $\begin{array}{l}\text { Buchwertabschreibung verboten oder } \\
\text { nicht steuerwirksam }\end{array}$ & $\begin{array}{l}\text { Belgien, Zypern, Dänemark, Finnland, Griechenland, } \\
\text { Irland, Lettland, Litauen, Malta, Polen, Portugal, } \\
\text { Slowenien, Slowakei, Schweden, Großbritannien, } \\
\text { Niederlande, Deutschland, Italien, Luxemburg }\end{array}$ \\
\hline \multicolumn{2}{|c|}{$\begin{array}{l}\text { Gilt nur für dauerhafte Wertminderungen. } \\
2 \text { Für Beteiligungen, die im Ausland gelegen sind, ist die Abschreibung nicht steuerwirksam. } \\
3 \text { Für Investitionen in dividendenberechtigte Aktien sind Abschreibungen nicht steuerwirk- } \\
\text { sam; Verluste können jedoch mit Wertsteigerungen verrechnet werden. } \\
{ }^{4} \text { Beschränkungen für nicht börsennotierte Beteiligungen }\end{array}$} \\
\hline
\end{tabular}

In Anlehnung an: Endres, D. et al., Taxable Income, 2006, S. 85

Drittens bedingt die fremdvergleichskonforme Gewinnabgrenzung, dass Zwischengewinne aus konzerninternen Lieferungen und Leistungen realisiert werden.

Sowohl die fehlende Verlustverrechnung als auch die nicht vorhandene Zwischenergebniseliminierung können eine Besteuerung nach sich ziehen, obwohl die wirtschaftliche Einheit kein entsprechendes Ergebnis erzielt. Unter der Prämisse, dass Gesellschaften langfristig Gewinne erzielen, handelt es sich hierbei lediglich um einen temporären Effekt, denn zum einen besteht die Möglichkeit eines interperiodischen Verlustausgleichs, ${ }^{53}$ zum anderen wird bei einer Lieferung oder Leistung des Konzerns gegenüber einem fremden Dritten nur die Wertschöpfung besteuert, die die letzte Konzerneinheit erbracht hat. Die vorgezogene Besteuerung erhöht jedoch aufgrund des negativen Zinseffekts die Steuerbelastung von Konzernen, womit der temporäre Effekt auch einen definitiven Charakter besitzt. Zur Vermeidung bzw. Abmilderung dieses Ergebnisses stellt die Mehrzahl der Mitgliedstaaten verbundenen Unternehmen spezielle Besteuerungsregime zur Verfügung. Diese Gruppenbesteuerungssysteme stehen jedoch nicht im Widerspruch zu der oben dargelegten Trennungstheorie der Konzernbesteuerung. Vielmehr müssen sie als Ergänzung verstanden werden, die die nicht systemimmanente Berücksichtigung der wirtschaftlichen Verbundenheit von Unternehmen teilweise beseitigt. Auf

53 Einige Mitgliedstaaten beschränken den Verlustvortrag zeitlich. So dürfen in Spanien Verluste nur 15 Jahre, in Finnland zehn Jahre, in Portugal sechs Jahre und in Tschechien, Griechenland, Italien, Lettland, Litauen, Polen, der Slowakei und Slowenien nur fünf Jahre vorgetragen werden. Vgl. Endres, D. et al., Taxable Income, 2006, S. 81. 
welche Art und Weise, in welchem Umfang und unter welchen Voraussetzungen die einzelnen Gruppenbesteuerungssysteme dies erreichen, wird im folgenden Kapitel erörtert.

\section{Gruppenbesteuerungssysteme in der Europäischen Union}

\section{Reichweite der Gruppenbesteuerungssysteme}

Die Berücksichtigung der wirtschaftlichen Einheit zivilrechtlich selbstständiger Konzerngesellschaften erfolgt in der Mehrheit der Mitgliedstaaten anhand spezieller Besteuerungsregime. Zwar hat die separate Gewinnermittlung der einzelnen Körperschaften weiterhin Bestand, doch können im Rahmen der Besteuerung Gewinne und Verluste verrechnet werden. Darüber hinaus sehen einige Mitgliedstaaten eine Zwischenergebniseliminierung vor, durch die die Besteuerung von nicht realisierten Gewinnen der wirtschaftlichen Einheit vermieden wird. Die einzelnen nationalen Regelungen unterscheiden sich teilweise erheblich voneinander. ${ }^{54}$ Dennoch soll anstelle einer detaillierten Darstellung der jeweiligen gesetzlichen Regelungen eine systematische Gegenüberstellung der Gruppenbesteuerungssysteme treten. ${ }^{55}$

Sieben Mitgliedstaaten verfügen über kein spezielles Besteuerungsregime für Unternehmensgruppen (Tabelle 3). Hierzu zählen Belgien, Estland, Griechenland, Litauen, die Slowakei, die Tschechische Republik und Ungarn. Eine Besonderheit stellt in diesem Zusammenhang Estland dar. Das estnische Steuersystem kennt keine Körperschaftsteuer auf den Jahresüberschuss im herkömmlichen Sinn, sondern besteuert ausschließlich die Gewinnausschüttungen an die Anteilseigner. ${ }^{56} \mathrm{Da}$ Ausschüttungen erst nach einer Verlustkompensation denkbar sind, ist eine Konsolidierung systemimmanent nicht notwendig. ${ }^{57}$

54 Die folgenden Ausführungen berücksichtigen den Rechtsstand des Jahres 2006. Die Daten der empirischen Untersuchung stammen überwiegend aus dem Jahr 2003. Folgerichtig basieren auch die Berechnungen und Auswertungen auf den damals geltenden gesetzlichen Regelungen, sodass Abweichungen gegenüber der hier dargelegten Rechtslage unausweichlich sind. Für eine ausführliche Darstellung der einzelnen Regelungen vgl. Oestreicher, A. et al., Weiterentwicklung, 2006, S. 71-102.

55 Die für die Darstellung notwendigen Daten beruhen überwiegend auf einer von den Universitäten Erlangen-Nürnberg, Göttingen und Mannheim mit der Unterstützung von PricewaterhouseCoopers durchgeführten Befragung. Vgl. Endres, D. et al., Taxable Income, 2006.

56 Lehis, L., in: IStR, 2001, S. 707-709; Hahner, A., in: WIRO, 2002, S. 145. Für eine allgemeine Übersicht über das estnische Steuersystem vgl. Sepp, J./Wrobel, R., in: Hasse/Schenk/Graf Wass von Czege, Wettbewerb, 2002, S. 69-75.

57 Vgl. Endres, D. et al., Verlustberücksichtigung, 2006, S. 19. 
Tabelle 3: Gruppenbesteuerungssysteme in der Europäischen Union

\begin{tabular}{|l|l|}
\hline $\begin{array}{l}\text { Kein Gruppenbesteuerungssystem } \\
\text { verfügbar }\end{array}$ & $\begin{array}{l}\text { Belgien, Estland, Griechenland, Litauen, Slowakei, } \\
\text { Tschechien, Ungarn }\end{array}$ \\
\hline Gruppenbesteuerungssystem verfügbar & $\begin{array}{l}\text { Dänemark, Deutschland, Finnland, Frankreich, } \\
\text { Großbritannien, Irland, Italien, Lettland } \\
\text { Luxemburg, Malta, Niederlande, Österreich, } \\
\text { Polen, Portugal, Slowenien, Schweden, Spanien, } \\
\text { Zypern }\end{array}$ \\
\hline
\end{tabular}

In Anlehnung an: Endres, D. et al., Taxable Income, 2006, S. 85

Die Anwendung der in den übrigen 18 Mitgliedstaaten existierenden Gruppenbesteuerungsvorschriften erfolgt für gewöhnlich auf Antrag und ist freiwillig. Lediglich in Dänemark ${ }^{58}$ und auf Malta ist die Veranlagung als Unternehmensgruppe obligatorisch, sobald die Voraussetzungen dazu erfüllt werden. ${ }^{59}$

Die Gruppenbesteuerungssysteme in der Europäischen Union unterscheiden sich sowohl hinsichtlich des Umfangs, in dem der wirtschaftlichen Einheit Rechnung getragen wird, als auch in Bezug auf das Verfahren, mit dem die vorgesehenen steuerlichen Wirkungen erreicht werden sollen. Dabei bedingt das angewandte Verfahren bereits teilweise die Reichweite des Gruppenbesteuerungssystems. So geht z. B. eine Vollkonsolidierung der Einzelabschlüsse stets mit einer umfassenden Berücksichtigung der wirtschaftlichen Einheit einher.

Nach der Form der Veranlagung kann zwischen Systemen mit Einzelveranlagung und Zusammenveranlagung differenziert werden (Tabelle 4). Im Rahmen der Einzelveranlagung wird nicht das gesamte Einkommen der Tochtergesellschaft der Muttergesellschaft zugerechnet, sondern es findet ausschließlich ein Verlustausgleich statt. Die Verrechnung von Verlusten zwischen den Mitgliedern der Gruppe kann dabei durch die Übertragung von Verlusten auf eine Kapitalgesellschaft erfolgen. Das Gruppenbesteuerungssystem von Großbritannien, Irland, Lettland, Malta und Zypern sieht ein derartiges ,group relief" vor. Das gleiche Ergebnis wird auch mit dem in Finnland und Schweden angewandten ,group contribution“-Konzept erreicht. Anstelle von Verlustzuweisungen können dort ertragreiche Konzerngesellschaften Ausgleichszahlungen leisten, die bei der zahlenden Gesellschaft als Aufwand und bei der empfangenden Gesellschaft als Ertrag verbucht werden.

Dänemark, Deutschland, Frankreich, Italien, Luxemburg, Österreich, Polen, Portugal, Slowenien und Spanien folgen der Zurechnungsmethode. Hierbei werden die ertragsteuerlichen Bemessungsgrundlagen zunächst auf der Basis der einzelnen Konzerngesellschaften ermittelt und auf der Ebene der Obergesellschaft zusammengeführt. Im Vergleich zur Einzelveranlagungsmethode erfolgt

58 Vgl. Kessler, W./Daller, R., in: $I S t R, 2005$, S. 2*.

59 Vgl. Endres, D. et al., Taxable Income, 2006, S. 783-786. 
der Ausgleich von Verlusten und Gewinnen immer auf der Ebene der Obergesellschaft.

Das niederländische Gruppenbesteuerungssystem geht über eine Zurechnung der Ergebnisse der Tochtergesellschaften hinaus, indem es die Eigenständigkeit der Konzerngesellschaften im Rahmen der Ermittlung des Steuerobjekts negiert. Das konsolidierte Ergebnis der Gruppe wird auf der Grundlage eines steuerlichen Konzernabschlusses ermittelt und bei der Obergesellschaft besteuert. ${ }^{60}$

Tabelle 4: Veranlagungstechnik der Gruppenbesteuerungssysteme

\begin{tabular}{|l|l|l|}
\hline \multirow{2}{*}{$\begin{array}{l}\text { Einzel- } \\
\text { veranlagung }\end{array}$} & Group Relief & Großbritannien, Irland, Lettland, Malta, Zypern \\
\cline { 2 - 3 } & Group Contribution & Finnland, Schweden \\
\hline \multirow{2}{*}{$\begin{array}{l}\text { Zusammen- } \\
\text { veranlagung }\end{array}$} & Zurechnungsmethode & $\begin{array}{l}\text { Dänemark, Deutschland, Frankreich, Italien, } \\
\text { Luxemburg, Österreich, Polen, Portugal, Slowenien, } \\
\text { Spanien }\end{array}$ \\
\cline { 2 - 3 } & Vollkonsolidierung & Niederlande \\
\hline
\end{tabular}

In Anlehnung an: Endres, D. et al., Verlustberücksichtigung 2006, S. 19; Endres, D. et al., Taxable Income, 2006, S. 91

Die Reichweite der Gruppenbesteuerungssysteme und damit das Ausmaß, in dem die wirtschaftliche Verbundenheit der Konzerngesellschaften berücksichtigt wird, lässt sich in drei Gruppen unterteilen (Tabelle 5).

Tabelle 5: Reichweite der wirtschaftlichen Einheit der Gruppenbesteuerungssysteme

\begin{tabular}{|l|l|}
\hline Vollkonsolidierung & Niederlande \\
\hline $\begin{array}{l}\text { Verlustverrechnung mit } \\
\text { Zwischenergebniseliminierung }\end{array}$ & Frankreich, Irland, Italien, Malta, Spanien, Großbritannien \\
\hline Verlustverrechnung & $\begin{array}{l}\text { Dänemark, Deutschland, Finnland, Lettland, Luxemburg, } \\
\text { Österreich, Polen, Portugal, Schweden, Slowenien, Zypern }\end{array}$ \\
\hline
\end{tabular}

In Anlehnung an: Endres, D. et al., Taxable Income, 2006, S. 93

Die Mehrzahl der Mitgliedstaaten erlaubt verbundenen Unternehmen lediglich, Gewinne und Verluste miteinander zu verrechnen. Eine Zwischenergebniseliminierung erfolgt in diesen Ländern nicht, sodass Gewinne aus dem konzerninternen Lieferungs- und Leistungsverkehr zu versteuern sind. In Frankreich, Irland, Italien, Malta, Spanien und Großbritannien können neben der Verrechnung von Verlusten diese Zwischenergebnisse teilweise oder sogar vollständig neutralisiert werden. Ob das durch eine Buchwertverknüpfung erreicht wird oder nachträglich die Neutralisierung erfolgt, ist in den sechs Mitgliedstaaten unterschiedlich geregelt. Die dritte Gruppe umfasst nur das Gruppen-

60 Die Steuersubjekteigenschaft der einzelnen Konzerngesellschaften bleibt formal bestehen. Vgl. FN 41. 
besteuerungssystem der Niederlande, das entsprechend der Veranlagungstechnik eine Vollkonsolidierung der steuerlichen Einzelabschlüsse vorsieht. Im Gegensatz zur Verlustverrechnung mit Neutralisierung der Zwischenergebnisse werden im Rahmen einer originären Gesamtergebnisermittlung die Ergebnisse sowie die Vermögenswerte und die Verbindlichkeiten der Konzerngesellschaften konsolidiert. ${ }^{61}$

\section{Anwendungsvoraussetzungen der Gruppenbesteuerungssysteme}

Die wesentlichen Voraussetzungen für die Inanspruchnahme einer Gruppenbesteuerung lassen sich grob in drei Gruppen unterteilen. Es existieren neben territorialen Kriterien, Anforderungen an die Rechtsform, unter der die Konzerngesellschaften firmieren dürfen, als auch Ansprüche an die Beteiligungsverhältnisse zwischen den Gesellschaften. Die jeweiligen Ausprägungen dieser Anwendungsvoraussetzungen weichen unter den Mitgliedstaaten teilweise erheblich voneinander ab, sodass die zuvor dargestellte Reichweite der wirtschaftlichen Einheit zusätzlich durch den Umfang der sich qualifizierenden Gesellschaften bestimmt wird.

Tabelle 6: Territoriale Reichweite der Gruppenbesteuerungssysteme

\begin{tabular}{|c|c|}
\hline Ausländische Gesellschaften & $\begin{array}{l}\text { Österreich, Dänemark, Frankreich, Italien, } \\
\text { Großbritannien }{ }^{2}\end{array}$ \\
\hline $\begin{array}{l}\text { Inländische Betriebsstätte einer aus- } \\
\text { ländischen Muttergesellschaft }\end{array}$ & $\begin{array}{l}\text { Österreich, Dänemark, Finnland, Frankreich, } \\
\text { Deutschland, Irland, Italien', Luxemburg, Nieder- } \\
\text { lande, Schweden, Spanien, Großbritannien }\end{array}$ \\
\hline $\begin{array}{l}\text { Inländische Schwestergesellschaften } \\
\text { einer ausländischen Muttergesellschaft }\end{array}$ & $\begin{array}{l}\text { Zypern, Irland, Italien', Lettland, Malta, } \\
\text { Schweden, Großbritannien }\end{array}$ \\
\hline \multicolumn{2}{|c|}{$\begin{array}{l}\text { Vielfach hängen die grenzüberschreitenden Regelungen vom Sitz der ausländischen Gesell- } \\
\text { schaft ab. Diese Übersicht geht stets davon aus, dass die ausländischen Gesellschaften ihren } \\
\text { Sitz in der Europäischen Union haben. } \\
1 \text { Nur in Bezug auf die nationale Gruppenbesteuerung } \\
2 \text { Nur endgültig nicht verrechenbare Verluste gemäß dem EuGH-Urteil Marks \& Spencer }\end{array}$} \\
\hline
\end{tabular}

In Anlehnung an: Endres, D. et al., Taxable Income, 2006, S. 89

Die Mehrheit der Mitgliedstaaten erlaubt nur Gesellschaften mit Sitz im Inland, in den Genuss der Gruppenbesteuerung zu kommen (Tabelle 6). Dabei können in Österreich, Dänemark, Finnland, Frankreich, Deutschland, Irland, Italien, Luxemburg, den Niederlanden, Schweden, Spanien und Großbritannien auch Betriebsstätten ausländischer Gesellschaften Teil der steuerlichen Unternehmensgruppe sein. Zypern, Irland, Italien, Lettland, Malta, Schweden und Großbritannien erkennen zumindest die rechtliche Struktur des Konzerns im Ausland an, sodass Schwestergesellschaften, deren gemeinsame Muttergesell-

61 Vgl. Sievert, E., Konzernbesteuerung, 2006, S. 145-147. 
schaft ihren Sitz im Ausland hat, im Inland als eine Unternehmensgruppe behandelt werden. In den übrigen Mitgliedstaaten muss demgegenüber stets eine nationale Obergesellschaft vorhanden sein.

Ein grenzüberschreitendes Gruppenbesteuerungssystem existiert lediglich in Dänemark, Frankreich, Italien und Österreich. ${ }^{62}$ Dort können unter teilweise sehr restriktiven Bedingungen auch ausländische Tochterunternehmen bei der Berechnung der Steuerlast der nationalen Unternehmensgruppe berücksichtigt werden. ${ }^{63}$ So gilt in Dänemark im Vergleich zu Frankreich und Italien ein besonders restriktives ,all-in-all-out"-Prinzip, das bei Anwendung der grenzüberschreitenden Konsolidierung den Einbezug des gesamten ausländischen Konzerns, einschließlich der ausländischen Spitzeneinheit und der Zwischengesellschaften ober- und unterhalb der dänischen Konzerngesellschaften, verlangt. Zudem sind auch alle ausländischen Konzerngesellschaften verpflichtet, ihren Gewinn nach dänischem Recht zu ermitteln. Die Anwendung der grenzüberschreitenden Gruppenbesteuerung ist für zehn Jahre bindend, und ein vorzeitiges Ausscheiden führt zu einer Nachversteuerung der bis dahin verrechneten Verluste. ${ }^{64}$ Damit sind die Rahmenbedingungen für die Konzerngesellschaften derart ungünstig, dass wohl nur wenige Unternehmen für eine grenzüberschreitende Gruppenbesteuerung optieren. ${ }^{65}$

Formal besteht auch in Frankreich für Obergesellschaften mit dem „régime du bénéfice consolidé" die Möglichkeit, die gesamten Ergebnisse ihrer in Frankreich oder im Ausland gelegenen Tochtergesellschaften und Betriebsstätten in ihr Ergebnis einzubeziehen. Da aber für die Anwendung dieses Regimes eine Ministererlaubnis erforderlich ist und diese nur sehr restriktiv gewährt wird, machten Anfang 2004 nur elf französische Konzerne von diesem System Gebrauch. $^{66}$

Italien hat zum 1. Januar 2004 erstmalig ein Gruppenbesteuerungssystem eingeführt. Mit der „consolidato mondiale“ besteht auch die Möglichkeit, weltweit

62 Vgl. Endres, D. et al., Taxable Income, 2006, S. 89.

63 Dänemark hat zum 15. 12. 2004 den Anwendungsbereich der internationalen Gruppenbesteuerung drastisch eingeschränkt, um die durch missbrăuchliche Gestaltungen hervorgerufene Belastung des Haushalts in Grenzen zu halten und die Senkung des Körperschaftsteuersatzes um 2 v. H. gegenzufinanzieren. Vgl. Kessler, W./Daller, R., in: IStR, 2005, S. 2*; Ottosen, A./Nørremark, M., in: BIFD, 2005, S. 471. Für eine Darstellung des zuvor geltenden Gruppenbesteuerungssystems vgl. Watrin, C./Sievert, E./Strom, C., in: $F R, 2004$, S. 4-5.

64 Vgl. Bjørnholm, N./Becker-Christensen, A., in: ET, 2006, S. 49.

65 Vgl. Kessler, W./Daller, R., in: IStR, 2005, S. 2*.

66 Vgl. Scheunemann, M., Konzernbesteuerung, 2005, S. 279-280; Richard, J., in: intertax, 2003, S. 30-31. 
die Ergebnisse der Tochtergesellschaften quotal zu berücksichtigen. ${ }^{67}$ Die Inanspruchnahme der internationalen Gruppenbesteuerung erfordert die Zustimmung der Finanzbehörden ${ }^{68}$ und folgt dem ,all-in-all-out“-Prinzip. Darüber hinaus verlangt der italienische Gesetzgeber eine Mindestbestandsfrist von fünf Jahren und die Aufstellung der Jahresabschlüsse aller beteiligten Tochtergesellschaften nach italienischem Recht.

Zuletzt hat Österreich mit Wirkung zum 1. Januar 2005 die geltenden Organschaftsregelungen abgeschafft und ein neues Gruppenbesteuerungssystem eingeführt, ${ }^{69}$ das nicht länger die wirtschaftliche und organisatorische Eingliederung sowie das Vorliegen eines Gewinnabführungsvertrags voraussetzt. Darüber hinaus dürfen erstmals auch ausländische Kapitalgesellschaften Gruppenmitglieder sein, soweit sie unmittelbar mit in Österreich unbeschränkt steuerpflichtigen Gruppenmitgliedern oder mit dem Gruppenträger finanziell

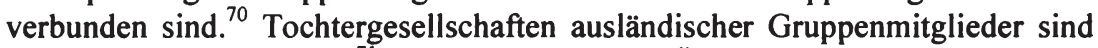
demnach ausgeschlossen. ${ }^{71}$ Damit grenzt auch Österreich die Reichweite der grenzüberschreitenden Verlustverrechnung ein, um eine Erosion des Steuersubstrats zu vermeiden.

Die Rechtsformen, unter denen die Gesellschaften firmieren, stellen das zweite Abgrenzungskriterium der Konsolidierungskreise dar.

Tabelle 7: Qualifizierende Rechtsformen der Gruppenbesteuerungssysteme

\begin{tabular}{|l|c|c|}
\hline & Obergesellschaft & Tochtergesellschaft \\
\hline $\begin{array}{l}\text { Körperschaftsteuer- } \\
\text { subjekte }\end{array}$ & $\begin{array}{c}\text { Alle Mitgliedstaaten mit } \\
\text { Gruppenbesteuerungssystem }\end{array}$ & $\begin{array}{c}\text { Alle Mitgliedstaaten mit } \\
\text { Gruppenbesteuerungssystem }\end{array}$ \\
\hline $\begin{array}{l}\text { Einkommensteuer- } \\
\text { subjekte }\end{array}$ & Deutschland, Lettland & - \\
\hline
\end{tabular}

In Anlehnung an: Endres, D. et al., Taxable Income, 2006, S. 762-765

67 Vgl. Romani, B./Stmad, O./Grabbe, C., in: IStR, 2004, S. 159-160; Dörr, I., in: DSWR, 2004, S. 248-249.

68 Dabei handelt es sich nicht um einen reinen Formalakt, denn die Finanzverwaltung kann ihre Zustimmung an eine erhöhte Mitwirkung der Konzerngesellschaften knüpfen. Vgl. Sievert, E., Konzernbesteuerung, 2006, S. 168.

$69 \mathrm{Vgl}$. für einen Überblick über das österreichische Gruppenbesteuerungssystem Hirschler, K./Schindler, C., in: IStR, 2004, S. 508-512; Sievert, E., Konzernbesteuerung, 2006, S. 195-206.

70 Hirschler, K./Schindler, C., in: IStR, 2004, S. 508.

7 Durch steuerplanerische Gestaltungen wie z. B. die „Cross-Over-Kaskade“ können unter bestimmten Voraussetzungen auch Gesellschaften auf tieferer ausländischer Ebene in das österreichische Gruppenbesteuerungssystem integriert werden. Vgl. Stefaner, M./ Weninger, P., in: SWI, 2005, S. 133-141. 
Die Anwendung der Gruppenbesteuerung ist in der Mehrzahl der Mitgliedstaaten den Körperschaftsteuersubjekten vorbehalten (Tabelle 7). Ausschließlich in Deutschland und Lettland können auch Einkommensteuersubjekte als Obergesellschaft Bestandteil der Gruppe sein. Deutschland, Österreich, Finnland, die Niederlande, Polen, Slowenien, Spanien und Schweden beschränken den Kreis der qualifizierenden Gruppenträger oder Gruppengesellschaften auf bestimmte Körperschaftsteuersubjekte. ${ }^{72}$ Daneben existieren in den einzelnen Ländern weitere Voraussetzungen, die z. B. an die Aktivität ${ }^{73}$ oder den Körperschaftsteuertarif $^{74}$ anknüpfen.

Tabelle 8: Höhe der Mindestbeteiligung der Gruppenbesteuerungssysteme

\begin{tabular}{|l|l|}
\hline 100 v. H. & Slowenien \\
\hline$>95$ v. H. & Frankreich (national), Luxemburg, Niederlande, Polen \\
\hline$>90$ v. H. & Finnland, Lettland, Portugal, Schweden \\
\hline$>75$ v. H. & Zypern, Irland, Spanien, Großbritannien \\
\hline$>50$ v. H. / 51 v. H. & $\begin{array}{l}\text { Österreich, Dänemark, Frankreich (international), Deutschland, } \\
\text { Italien, Malta }\end{array}$ \\
\hline
\end{tabular}

In Anlehnung an: Endres, D. et al., Taxable Income, 2006, S. 86

Neben der territorialen Eingrenzung und den Rechtsformvoraussetzungen stellt jedes Konzernbesteuerungssystem Anforderungen an die Beteiligungsstruktur der Unternehmensgruppe. Alle Mitgliedstaaten, die ein Gruppenbesteuerungssystem implementiert haben, schreiben eine Mindestbeteiligungsquote der Obergesellschaft an den einzubeziehenden Tochtergesellschaften vor. Diese liegt in der Hälfte der europäischen Länder bei mindestens $90 \mathrm{v} . \mathrm{H}$. (Tabelle 8). ${ }^{75}$

Als Bezugsgröße der Mindestbeteiligungsquote kommt der Anteil an Stimmrechten, am Beteiligungskapital, an Gewinnausschüttungen und Liquidationserlösen sowie eine Kombination aller drei Anteile in Frage. ${ }^{76}$ Schließlich ist auch die Berechnung der Beteiligungsquote in den europäischen Ländern abweichend geregelt. Lettland, Polen und Slowenien berücksichtigen keine indirekten Beteiligungen. Die Obergesellschaft muss mit der geforderten Mindestbeteiligungsquote unmittelbar beteiligt sein. In den übrigen Mitgliedstaaten

72 Für eine detaillierte Übersicht vgl. Endres, D. et al., Taxable Income, 2006, S. 762-765; Sievert, E., Konzernbesteuerung, 2006, S. 231-234.

73 Von der Anwendung der Gruppenbesteuerung ausgeschlossen sind z. B. in Deutschland Lebens- und Krankenversicherungen ( $\S 14 \mathrm{Abs} .2 \mathrm{KStG})$ und in Finnland Banken und Versicherungen sowie Unternehmen ohne aktive Tätigkeit.

74 In Großbritannien, Luxemburg und Polen müssen die qualifizierten Gesellschaften dem regulären Körperschaftsteuertarif unterliegen.

75 Vgl. Sievert, E., Konzernbesteuerung, 2006, S. 234.

76 Vgl. Endres, D. et al., Taxable Income, 2006, S. 86. 
werden indirekte Beteiligungen grundsätzlich bei der Ermittlung der Mindestbeteiligungsquote berücksichtigt. Dabei erfolgt stets die Addition der mittelbaren und unmittelbaren Beteiligungen. Um indirekte Beteiligungen einbeziehen zu können, muss die Mindestbeteiligungsquote in Deutschland, Frankreich, Portugal und Spanien auch gegenüber der vermittelnden Gesellschaft erfüllt werden. In Portugal und Spanien muss das erforderliche Beteiligungsverhältnis darüber hinaus auch multiplikativ durchgerechnet zwischen der Muttergesellschaft und der indirekten Beteiligung bestehen. ${ }^{77}$

\section{Unternehmensteuerliche Hindernisse bei grenzüberschreitender Geschäftstätigkeit in der Europäischen Union}

\section{1. Überblick}

Die primäre Ursache der steuerlichen Hindernisse bei grenzüberschreitender Geschäftstätigkeit liegt in der Koexistenz von 25 verschiedenen Steuersystemen. Damit existieren im europäischen Binnenmarkt zugleich 25 eigenständige Steuergebiete. Diese Aufteilung des Binnenmarktes in einzelne Steuerhoheiten, verbunden mit dem Prinzip der selbstständigen Einheit, ist für die Unternehmen mit verschiedenen steuerlichen Konsequenzen verknüpft: ${ }^{78}$

1. Die Unternehmen müssen alle Transaktionen entsprechend dem Fremdvergleichsgrundsatz bewerten und abrechnen.

2. Die Mitgliedstaaten gewähren nur in geringem Umfang die Verrechnung von Verlusten über die Grenze hinweg, da ihnen auch ein Zugriff auf die Gewinne verwehrt bleibt.

3. Kollidierende Steueransprüche können eine Doppelbesteuerung der Gewinne nach sich ziehen.

4. Umstrukturierungsvorgänge können Mitgliedstaaten den steuerlichen Zugriff auf bisher unrealisierte Gewinne entziehen, sodass die einzelnen Steuerhoheiten diese zum Zeitpunkt der Umstrukturierung besteuern. ${ }^{79}$

5. Grenzüberschreitend tätige Konzerne müssen die Vorschriften aller Mitgliedstaaten beachten, in denen sie einen steuerlichen Anknüpfungspunkt vorweisen. Dadurch entstehen den Konzernen erhebliche Befolgungskosten, die zusätzlich durch das Bemühen, die bestehenden steuerlichen Hindernisse

77 Vgl. Endres, D. et al., Verlustberücksichtigung 2006, S. 70.

79 Da sich die vorliegende Arbeit auf die laufende Besteuerung von Konzernen in Europa beschränkt, werden Umstrukturierungsvorgänge nicht weiter beachtet. 
durch steuerplanerische Maßnahmen zu vermeiden oder zu minimieren, ansteigen. $^{80}$

Im Folgenden werden die drei wesentlichen unternehmensteuerlichen Hindernisse (die fehlende grenzüberschreitende Verlustverrechnung, die Bestimmung fremdvergleichskonformer Verrechnungspreise und die Doppelbesteuerung von Gewinnen) näher betrachtet. ${ }^{81}$

\section{Verlustverrechnung}

Die Möglichkeit eines umfassenden grenzüberschreitenden Verlustausgleichs existiert innerhalb der Europäischen Union nicht. Zwar sehen mittlerweile vier Länder entsprechende Regelungen vor, doch ist deren Ausgestaltung derart restriktiv, dass nur wenige Konzerne hiervon profitieren dürften. In der Mehrzahl der Länder ist eine Verlustverrechnung zwischen Tochterkapitalgesellschaften weiterhin auf das Inland begrenzt. Sieben europäische Länder kennen sogar kein Gruppenbesteuerungssystem. Das Fehlen einer grenzüberschreitenden Verlustverrechnung stellt nach Ansicht des Expertenpanels eines der zentralen Probleme dar, die vorrangig gelöst werden müssen. ${ }^{82}$ Dies bestätigt auch eine Umfrage des Verbandes der Schwedischen Industrie. ${ }^{83}$ Danach konnten 96 v. H. der Gesellschaften eines grenzüberschreitend tätigen Konzerns erlittene Verluste nur eingeschränkt oder gar nicht verrechnen, was in $56 \mathrm{v} . \mathrm{H}$. der Fälle zumindest in gewissem Maße zu einer endgültigen Doppelbesteuerung führte. Die derzeitige Rechtslage in der Europäischen Union hat zur Folge, dass unternehmerische Entscheidungen beeinflusst werden, indem z. B. die Nutzung eines eingeschränkten Verlustausgleichs spezifische Unternehmensstrukturen erfordert. ${ }^{84}$ Zudem werden Inlandsinvestitionen begünstigt. Das Fehlen eines

80 Schätzungen beziffern die Befolgungskosten auf 2-4 v. H. der Steuereinnahmen, wobei ein positiver Zusammenhang mit dem Umfang des Auslandsgeschäfts und ein negativer Zusammenhang mit der Unternehmensgröße besteht. Vgl. Europäische Kommission, Unternehmensbesteuerung, 2001, S. 799-803, m. w. N.; Blumenthal, M./Slemrod, J., in: ITPF, 1995, S. 39-45.

81 Darüber hinaus untersucht die Kommission in ihrer Studie die Belange kleiner und mittlerer Unternehmen, sieht aber grundsätzlich keinen Anlass, diese Gruppe gesondert zu berücksichtigen. Gleichwohl weist sie auf den negativen Zusammenhang zwischen Anteil der Befolgungskosten und der Unternehmensgröße hin und plädiert für vereinfachte Regelungsvorschriften. Vgl. Europäische Kommission, Unternehmensbesteuerung, 2001, S. 497.

82 Vgl. Europäische Kommission, Unternehmensbesteuerung, 2001, S. 347; UNICE, Memorandum, 2000, S. 5.

83 Vgl. die Veröffentlichung der Umfrage in Lodin, S.-O./Gammie, M., Home, 2001, S. 7476.

84 Zum Beispiel kann das Einrichten einer Auslandsbetriebsstătte anstelle einer Kapitalgesellschaft vorteilhaft sein, um Verluste grenzüberschreitend ausgleichen zu können. Mit 
umfassenden grenzüberschreitenden Ausgleichs der Verluste verletzt damit das Grundrecht der Niederlassungsfreiheit und steht im Widerspruch zum Konzept des gemeinsamen Binnenmarktes. ${ }^{85}$

\section{Verrechnungspreise}

Die direkte Gewinnabgrenzung mittels fremdvergleichskonformer Verrechnungspreise ist primär kein Hindernis für die grenzüberschreitende Unternehmenstätigkeit in der Europäischen Union. Vielmehr stellt sie ein kohärentes und schlüssiges Konzept zur korrekten Zuordnung der Unternehmensgewinne auf Steuerhoheiten dar. ${ }^{86}$ Dennoch wird die Verrechnungspreisproblematik hier separat behandelt, da sie Ursache für viele Doppelbesteuerungstatbestände und unverhältnismäßig hohe Befolgungskosten ist und ihre Bedeutung im Rahmen des stetig wachsenden konzerninternen Handels von Gütern und Dienstleistungen stark zugenommen hat. ${ }^{87}$

Die OECD-Leitlinien weisen ausdrücklich darauf hin, dass die Ermittlung von Verrechnungspreisen keine exakte Wissenschaft ist, sondern stets ein Ermessensspielraum verbleibt. Ein einzelner Fremdvergleichspreis kann demnach nicht immer exakt ermittelt werden. Vielmehr liefern die Methoden eine Bandbreite plausibler Ergebnisse, weswegen das Festlegen von Verrechnungspreisen eines ausgeprägten Urteilsvermögens bedarf. ${ }^{88}$ Der Ermessensspielraum bei der Bestimmung von fremdvergleichskonformen Verrechnungspreisen, gepaart mit den Interessengegensätzen zwischen den nationalen Finanzverwaltungen und zwischen den Finanzverwaltungen und den Unternehmen, führt vielfach zu einer Doppelbesteuerung der Gewinne. ${ }^{89}$ So sind die Unternehmen bestrebt, ihre Ge-

Ausnahme von Deutschland, Frankreich, Griechenland, Luxemburg, Polen und der Slowakei berücksichtigen alle Mitgliedstaaten Betriebsstättenverluste entweder durch Anwendung der Anrechnungsmethode oder, bei Anwendung der Freistellungsmethode, durch eine temporäre Verlustverrechnung. Vgl. zu der Verlustverrechnung bei Auslandsbetriebsstätten Endres, D. et al., Verlustberücksichtigung 2006, S. 16-18. Finanzierung der Steuerlast aufkommen, da eine Steuerstundung regelmäßig nicht erfolgt.

Finanzierung der Steuerlast aufkommen, da eine Steuerstundung regelmäßig nicht erfolgt.
So waren am 31. 12. 2004 noch 24 Verständigungsverfahren nach dem EU-Schiedsüber-
einkommen anhängig, deren Anträge vor 2000 eingegangen sind. Vgl. Europäische
Kommission, Schiedsübereinkommen, 2005, S. 11 .

Finanzierung der Steuerlast aufkommen, da eine Steuerstundung regelmäßig nicht erfolgt.
So waren am 31. 12. 2004 noch 24 Verständigungsverfahren nach dem EU-Schiedsüber-
einkommen anhängig, deren Anträge vor 2000 eingegangen sind. Vgl. Europăische
Kommission, Schiedsübereinkommen, 2005, S. 11 .

Finanzierung der Steuerlast aufkommen, da eine Steuerstundung regelmäßig nicht erfolgt.
So waren am 31. 12. 2004 noch 24 Verständigungsverfahren nach dem EU-Schiedsüber-
einkommen anhängig, deren Anträge vor 2000 eingegangen sind. Vgl. Europăische
Kommission, Schiedsübereinkommen, 2005, S. 11 . 
winne in dem Land auszuweisen, das diese niedriger besteuert. ${ }^{90}$ Zielsetzung der Finanzverwaltung ist hingegen, das nationale Steuersubstrat zu sichern. Um den Gestaltungsspielraum der Unternehmen einzuschränken und die Festsetzung der Verrechnungspreise besser nachzuvollziehen, verlangt die Mehrzahl der Mitgliedstaaten von den Unternehmen, die Ermittlung der Verrechnungspreise ausführlich zu dokumentieren, was zusätzliche Befolgungskosten verursacht. ${ }^{91}$

Mit dem EU-Schiedsübereinkommen besteht auf europäischer Ebene bereits ein Streitbeilegungsmechanismus, der Doppelbesteuerung infolge von Gewinnberichtigungen einzelner Mitgliedstaaten vermeiden soll. ${ }^{92}$ Administrative und technische Schwierigkeiten veranlassten die Unternehmen jedoch häufig dazu, von einem Verständigungsverfahren abzusehen. ${ }^{93}$ Zur Verbesserung der Situation wurde, basierend auf den Vorschlägen der Binnenmarktstudie, ein EUVerrechnungspreisforum eingerichtet. ${ }^{94}$ Dieses verabschiedete am 27. Juni 2006 zwei Verhaltenkodizes, die eine wirksamere und einheitlichere Anwendung des Schiedsübereinkommens ${ }^{95}$ und eine standardisierte Verrechnungspreisdokumentation $^{96}$ gewähren sollen. ${ }^{97}$ Selbst wenn damit Doppelbesteuerungen im

Eine Umfrage von Ernst \& Young revidiert das Bild, dass Unternehmen mittels Verrechnungspreisen systematische Gewinne verlagern. Vgl. Ernst \& Young, Pricing, 1999, S. 12-13. Dem stehen empirische Untersuchungen der Wissenschaft gegenüber, die Gewinnverlagerungen der Unternehmen statistisch nachweisen konnten. Vgl. z. B. Bartelsman, E./Beetsma, R., in: JoPE, 2003, S. 2237-2240.

91 Für einen Vergleich der nationalen Dokumentationsvorschriften mit dem EU Verhaltenskodex vgl. Schnorberger, S./Rosenkranz, J./Garcia, M., in: intertax, 2006, S. 305-313, 406-417. Über $82 \mathrm{v}$. H. der großen Unternehmen sehen insbesondere Verrechnungspreise und die damit verbundenen Dokumentationsvorschriften als wesentlichen Treiber der Befolgungskosten an. Vgl. Europăische Kommission, Survey, 2004, S. 3-4. Vgl. a. FN 80. Vgl. Übereinkommen 90/436/EWG, Abl. Nr. L 225 v. 20. 8. 1990, S. 10-24.

Der Umfrage von Ernst \& Young zufolge führten Verrechnungspreiskorrekturen bei 42 v. H. der Unternehmen zu Doppelbesteuerung. Gleichzeitig liegt die Erfolgsquote der Verständigungsverfahren bei ca. $85 \mathrm{v}$. H. Beide Zahlen verdeutlichen, dass in vielen Făllen kein Gebrauch vom Schiedsübereinkommen gemacht wurde.

94 Das Forum wurde durch die Kommission im Juni 2002 formell errichtet. Es setzt sich zusammen aus einem Experten der Steuerverwaltung jedes Mitgliedstaates und zehn Experten der Wirtschaft. Zu der Tätigkeit des EU-Verrechnungspreisforums vgl. Europäische Kommission; Verrechnungspreisforum, 2004, S. 18-28; dieselbe, Verhaltenskodex, 2005, S. 3-12.

$95 \mathrm{Vgl}$. Verhaltenskodex zur wirksamen Durchführung des Übereinkommens über die Beseitigung der Doppelbesteuerung im Falle von Gewinnberichtigungen zwischen verbundenen Unternehmen, Abl. Nr. C 176 v. 28. 7. 2006, S. 8-12. in der Europäischen Union, Abl. Nr. C 176 v. 28. 7. 2006, S. 1-7.

97 Vgl. a. Europäische Kommission, Verrechnungspreisforum, 2004, S. 30-44; dieselbe, Verhaltenskodex, 2005, S. 13-23. 
europäischen Binnenmarkt vermieden werden können und die Befolgungskosten sinken, richtet sich diese Maßnahme nur gegen die Symptome. Die Ursache dieses unternehmenssteuerlichen Hindernisses, die Ermittlung von fremdvergleichskonformen Verrechnungspreisen, bleibt jedoch weiterhin bestehen.

\section{Doppelbesteuerung}

Artikel 293 EGV schreibt den Mitgliedstaaten ausdrücklich vor, die Vermeidung der Doppelbesteuerung sicherzustellen. Dennoch ist das Netz an Doppelbesteuerungsabkommen in der Europäischen Union weiterhin lückenhaft. ${ }^{98}$ Die Anzahl der Doppelbesteuerungsabkommen der 25 Mitgliedstaaten beläuft sich auf 277. Damit fehlen 23 Doppelbesteuerungsabkommen. ${ }^{99}$ Zudem werden die bestehenden Abkommen den Anforderungen des gemeinsamen Binnenmarktes immer weniger gerecht. $\mathrm{Zu}$ nennen ist hier beispielsweise die fehlende Verpflichtung der zuständigen Behörden zu einer Einigung in Fällen von Doppelbesteuerung. Auch werden die bilateralen Abkommen nur unzureichend den im EG-Vertrag verankerten Gleichbehandlungsgrundsätzen gerecht.

Als ein Hauptanliegen der Unternehmen identifizierte die Binnenmarktstudie die Vermeidung der wirtschaftlichen Doppelbesteuerung ausgeschütteter Gewinne und Dividenden. Zwar hatte die Europäische Kommission bereits 1990 die Mutter-Tochter-Richtlinie ${ }^{100}$ verabschiedet, doch war deren Anwendungsbereich eingeschränkt. Zum einem profitierte eine Vielzahl der Gesellschaften nicht von der Richtlinie, da sie entweder nicht unter einer der im Anhang aufgelisteten Rechtsform firmierten oder keine Beteiligung an der Tochtergesellschaft über 25 v. H. bestand. Zum anderen war die Anwendung der Richtlinie unklar, wenn die Gesellschaftsanteile einer Betriebsstätte zuzurechnen waren. Zur Beseitigung dieser Hindernisse beschloss der EU-Ministerrat 2003 eine Erweiterung der Richtlinie. $^{101}$ Diese schließt jetzt alle körperschaftsteuerpflichtigen Gesellschaften ein. ${ }^{102}$ Dazu zählen erstmalig auch Personengesellschaften, die automatisch der Körperschaftsteuer unterliegen. ${ }^{103}$ Zudem wird die erforderliche

98 Die Mitgliedstaaten sehen zwar auch unilaterale Maßnahmen zur Vermeidung der Doppelbesteuerung vor, doch erfolgt nicht immer eine vollständige Entlastung der Steuerpflichtigen. Zudem sollen Doppelbesteuerungsabkommen dazu beitragen, Quellensteuern zu vermeiden. Vgl. Europäische Kommission, Unternehmensbesteuerung, 2001, S. 396.

$99 \mathrm{Vgl}$. Kommission der Europäische Gemeinschaften, Treaties, 2005, Annex B.

${ }^{100}$ Richtlinie 90/435/EWG des Rates vom 23. 7. 1990, Abl. Nr. L 225 v. 20. 8. 1990, S. 6-9.

101 Vgl. Richtlinie 2003/123/EG des Rates vom 22. 12. 2003, Abl. Nr. L 007 v. 3. 1. 2004, S. 41-44.

102 Vgl. Bullinger, P., in: IStR, 2004, S. 406-412. Zur Umsetzung in nationales Recht vgl. Hörnschemeyer, A., in: $G m b H R, 2005$, S. 1397-1409.

${ }^{103}$ Für eine Auflistung französischer Personengesellschaften, die die Option für eine Besteuerung durch die Körperschaftsteuer haben, vgl. Kußmaul, H./Schäfer, R., in: IStR, 
Mindestbeteiligung bis zum Jahr 2009 auf zehn v. H. abgesenkt. ${ }^{104}$ Schließlich erfasst die Richtlinie nun ausdrücklich Dreieckssachverhalte, bei denen Betriebsstätten relevante Beteiligungen besitzen. ${ }^{105}$ Damit wird weitestgehend eine Doppelbesteuerung von Gewinnausschüttungen vermieden. Dazu stehen den Mitgliedstaaten weiterhin die Anrechnungsmethode und die Freistellungsmethode zur Verfügung. Dem Vorschlag der Binnenmarktstudie, dieses Wahlrecht zugunsten einer kapitalimportneutralen Besteuerung aufzugeben ${ }^{106}$, wurde nicht gefolgt. ${ }^{107}$

2000, S. 162-164. In Spanien unterliegen Personengesellschaften mit Ausnahme der BGB-Gesellschaft der Körperschaftsteuer. Vgl. Lüdemann, P./Hruschka, F., in: IStR, 2000, S. 25.

104 Das Absenken der Mindestbeteiligungshöhe erfolgt in drei Schritten. Zum 1. 1. 2005 und 2007 wurde die Mindestbeteiligung abgesenkt und entspricht derzeit 15 v. H. Zum 1. 1. 2009 reduziert sich die geforderte Beteiligungshöhe um weitere fünf Prozentpunkte. Vgl. Artikel 3 Abs. 1a Richtlinie 90/435/EWG des Rates v. 23. 7. 1990, Abl. Nr. L 225 v. 20. 8. 1990, S. 6-9.

$105 \mathrm{Vgl}$. Zanotti, E., in: ET, 2005, S. 493-505.

$106 \mathrm{Vgl}$. Europäische Kommission, Unternehmensbesteuerung, 2001, S. 337.

107 Vgl. Artikel 4 Abs. 1 Richtlinie 90/435/EWG des Rates v. 23. 7. 1990, Abl. Nr. L 225 v. 20. 8. 1990, S. 6-9. 


\section{Konsolidierte Unternehmensbesteuerung in der Europäischen Union}

\section{Vorschläge zur Beseitigung der steuerlichen Hindernisse im Binnenmarkt}

\section{1. Überblick}

Die Europäische Kommission unterscheidet in ihrer Studie zu Unternehmensbesteuerung im Binnenmarkt im Hinblick auf die Beseitigung der steuerlichen Hindernisse $^{108}$ zwischen zwei möglichen Lösungskonzepten. Zum einen könnte für jedes Problem eine spezifische Lösung entwickelt werden. Als gezielte Maßnahme wäre z. B. vorstellbar, die grenzüberschreitende Verlustverrechnung durch eine entsprechende Richtlinie zu ermöglichen. Demgegenüber sind die umfassenden Lösungsansätze darauf ausgerichtet, auf eine kohärente Weise die Hindernisse $\mathrm{zu}$ reduzieren bzw. zu beseitigen. ${ }^{109}$ Dabei konkurrieren beide Optionen nicht miteinander, sondern ergänzen sich. Langfristig werden die unternehmenssteuerlichen Hindernisse nur mit umfassenden Lösungsansätzen zu beseitigen sein. ${ }^{110}$ Jedoch entfalten sie ihre Wirkung nicht für alle Gesellschaften. So bedarf es z. B. im Zusammenhang mit Personengesellschaften gezielter Maßnahmen. ${ }^{11}$ Auch einige Wirtschaftszweige könnten von vornherein von der Anwendung ausgeschlossen sein. Im Fall einer Umsetzung der umfassenden Lösungsansätze auf freiwilliger Basis könnten Unternehmen zudem für eine Besteuerung auf Grundlage des bestehenden Steuersystems optieren. Schließlich muss die simultane Einführung einer einheitlichen Regelung in allen 25 Mitgliedstaaten der Europäischen Union in näherer Zukunft als unwahrscheinlich erachtet werden. ${ }^{112}$ Vor diesem Hintergrund ist eine zweigleisige

${ }^{108}$ Für eine umfassende Darstellung der unternehmenssteuerlichen Hindernisse innerhalb des europäischen Binnenmarkts vgl. Kapitel B.IV.

109 Vgl. Europäische Kommission, Unternehmensbesteuerung, 2001, S. 423; Oestreicher, A., in: StuW, 2002, S. 346-348.

110 Vgl. Spengel, C./Braunagel, R.U.; in: StuW, 2006, S. 45; Spengel, C.; in: Lüdicke, Steuerrecht, 2004, S. 125.

III Soweit sich die grenzüberschreitenden Hindernisse ausschließlich aus der Definition und der daraus resultierenden steuerlichen Behandlung dieser Rechtsformen ergeben, kann auch keine umfassende Maßnahme die damit zusammenhängenden Probleme lösen. Vgl. Europäische Kommission, Unternehmensbesteuerung, 2001, S. 527.

112 Die Europäische Kommission befürwortet in diesem Zusammenhang Mechanismen der „verstärkten Zusammenarbeit" (Artikel 43-45 EUV und Artikel I-43, III-322-329 Entwurf des Verfassungsvertrags) einzelner Mitgliedstaaten, sollte keine Einigung unter allen 25 Mitgliedstaaten erzielt werden können. Vgl. Europäische Kommission, Binnenmarkt, 2003, S. 30. 
Herangehensweise geboten, ${ }^{113}$ die die Umsetzung eines umfassenden Lösungsansatzes zwingend erforderlich macht. Dieses wird besonders durch die Probleme verdeutlicht, die aus der Koexistenz von 25 Steuersystemen herrühren. ${ }^{114}$

\section{Darstellung der gezielten Lösungsansätze}

Zur kurz- und mittelfristigen Beseitigung der steuerlichen Hindernisse listet die Europäische Kommission eine Vielzahl von gezielten Abhilfemaßnahmen auf, deren Umsetzung teilweise bereits erfolgte. ${ }^{115}$

1. Im Bereich der Dividendenbesteuerung wurden durch die Verabschiedung der Richtlinie 2003/123/EG ${ }^{116}$ zur Änderung der Mutter-Tochter-Richtlinie ${ }^{117}$ die wesentlichen Vorschläge umgesetzt. ${ }^{118}$ Eine Doppelbesteuerung von Gewinnausschüttungen ist damit innerhalb der Europäischen Union weitestgehend ausgeschlossen. ${ }^{119}$

2. Zur Ermöglichung der grenzüberschreitenden Verlustverrechnung plädiert die Europäische Kommission für eine Überarbeitung und Verbesserung des Richtlinienvorschlags von 1991. ${ }^{120}$ Obwohl für 2004 diesbezüglich eine einschlägige Kommissionsinitiative geplant war, ${ }^{121}$ konnten bis heute keine Fortschritte verzeichnet werden. Diese Zurückhaltung beruht zum einen auf der Erfahrung aus dem Scheitern des Verlustverrechnungsrichtlinienvorschlags von 1991. ${ }^{122}$ Zum anderen waren sowohl am EuGH ${ }^{123}$ als auch an

113 So auch Mors, M., in: TNI, 2002, S. 157.

114 Vgl. hierzu Kapitel B.IV.

$115 \mathrm{Vgl}$. Europäische Kommission, Unternehmensbesteuerung, 2001, S. 23-26, 423-504; Spengel, C.; in: Lüdicke, Steuerrecht, 2004, S. 125; Wehrheim, M., in: IStR, 2003, S. 16-17.

116 Richtlinie 2003/123/EG des Rates v. 22. 12. 2003, Abl. Nr. L 007 v. 3. 1. 2004, S. 41-44.

117 Richtlinie 90/435/EWG des Rates v. 23. 7. 1990, Abl. Nr. L 225 v. 20. 8. 1990, S. 6-9.

118 Vgl. Bullinger, P., in: IStR, 2004, S. 406-412. Zur Umsetzung in nationales Recht vgl. Hörnschemeyer, A., in: GmbHR, 2005, S. 1397-1409. Vgl. a. Kapitel B.IV.4.

119 Für verbleibende Anwendungsprobleme vgl. Bullinger, P., in: IStR, 2004, S. 409-412.

120 Vorschlag einer Richtlinie des Rats v. 28. 11. 1990, KOM (90) 595 endg., Abl. Nr. C 53 v. 28. 2. 1991, S. 30.

$121 \mathrm{Vgl}$. Europäische Kommission, Binnenmarkt, 2003, S. 10.

122 Die Kommission begründet den Rückzug mit der Verfolgung einer neuen Konzeption, deren Vorschlag in Vorbereitung ist. Vgl. Europäische Kommission, Vorschläge, 2001, S. 23.

123 Vgl. EuGH v. 14. 12. 2000, Rs. C-141/99 - AMID, in: EuGHE, 2000, I-11619; v. 12. 9. 2002, Rs. C-431/01 - Mertens, in: EuGHE, 2002, I-7073; v. 15. 5. 1997, Rs. C-250/95 Futura/Singer, in: EuGHE, 1997, I-2471; v. 13. 12. 2005, Rs. C-446/03 - Marks \& 
innerstaatlichen Gerichten ${ }^{124}$ Verfahren anhängig, deren Ausgang eine Kommissionsinitiative maßgeblich beeinflusst. Das inzwischen ergangene Urteil zur Rechtssache Marks \& Spencer ${ }^{125}$ hat in diesem Bereich besonderes Aufsehen erregt. Die Befürchtungen der Mitgliedstaaten, eine umfassende Verlustberücksichtigung über die Grenze hinweg zu ermöglichen, haben sich nicht bestätigt. Im Ergebnis müssen nur Verluste grenzüberschreitend verrechnet werden können, deren Nutzung andernfalls endgültig ausgeschlossen wäre, wobei die Nachweispflicht bei der Muttergesellschaft liegt. Obwohl durch das Urteil der Reformdruck geringer als erwartet ausfällt und zunächst nur unilaterale Anpassungen der Gruppenbesteuerungssysteme erforderlich sind, wurde noch im Jahr 2006 eine erste Mitteilung zur grenzüberschreitenden Verlustverrechnung veröffentlicht. ${ }^{126}$

3. Auf Empfehlung der Binnenmarktstudie wurde bereits ein EU-Forum für Verrechnungspreisfragen eingerichtet, dessen Aufgabe unter anderem der Entwurf einer Richtlinie zur Aktualisierung und Verbesserung der Schiedsübereinkommen sein sollte. Anstelle der Richtlinie wurden zwei Verhaltenskodizes verabschiedet, die aber lediglich eine politische Verpflichtung darstellen und die Rechte und Pflichten der Mitgliedstaaten sowie die jeweiligen Zuständigkeiten der Mitgliedstaaten und der Gemeinschaft unberührt lassen. ${ }^{127}$

Weitere gezielte Maßnahmen, den Hindernissen im Binnenmarkt entgegenzuwirken, sind

4. die Vermittlung von Orientierungshilfen zur einheitlichen Anwendung des EU-Steuerrechts und die verstärkte Überprüfung der Umsetzung von EUSteuervorschriften in innerstaatliches Recht,

5. die Ausweitung und Verbesserung der Fusionsrichtlinie ${ }^{128}$,

Spencer, in: $I S t R$, 2006, S. 19-23; v. 21. 2. 2006, Rs. C-152/03 - Ritter-Coulais, in: HFR, 2006, S. 525.

124 Vgl. Ö VwGH v. 25. 9. 2001, Rs. Nr. 99/14/0217, siehe hierzu Lang, M. et al., in: $S W I$, 2002, S. 428-440.

125 Vgl. EuGH v. 13. 12. 2005, Rs. C-446/03 - Marks \& Spencer, in: IStR, 2006, S. 19-23; Balmes, A./Brück, M./Ribbrock, M., in: BB, 2006, S. 186-189. Zu den Auswirkungen auf die deutsche Organschaft vgl. Herzig, N./Wagner, T., in: DStR, 2006, S. 9-11. Maiterth analysiert das Urteil aus einer ökonomischen Sicht und kommt zu dem Schluss, dass eine Verrechnung der Verluste im Ansässigkeitsstaat der Muttergesellschaft nicht gerechtfertigt sei. Vgl. Maiterth, R., in: DStR, 2006, S. 915-919.

$126 \mathrm{Vgl}$. Europäische Kommission, Verluste, 2006.

127 Vgl. Kapitel B.IV.3.

128 Richtlinie 90/434/EWG des Rates v. 23. 7. 1990, Abl. Nr. L 225, v. 20. 8. 1990, S. 1-5. Mit der Verabschiedung der Richtlinie 2005/19/EG des Rates v. 17. 2. 2005, Abl. Nr. L 58 , v. 4. 3. 2005, S. 19-27 wurde weitgehend dem Vorschlag zur Änderung der Richtlinie 
6. die Anpassung des OECD-Modells zur Vermeidung von Doppelbesteuerungen an die Anforderungen des EG-Vertrags.

\section{Darstellung der umfassenden Lösungsansätze}

Die umfassenden Maßnahmen zielen andererseits auf einen ganzheitlichen Ansatz $a b$, der systembedingt die steuerlichen Hindernisse im Binnenmarkt reduziert oder sogar vollständig beseitigt. Ausgangspunkt ist die Überlegung, dass die Mehrheit der Probleme bei grenzüberschreitenden Unternehmenstätigkeiten auf die Koexistenz 25 unterschiedlicher Steuersysteme zurückzuführen ist. So sind bei der Gewinnabgrenzung, dem Verlustausgleich oder bei Umstrukturierungsmaßnahmen stets die nationalen Steuergrenzen zu akzeptieren. Vor diesem Hintergrund ist die Grundvoraussetzung für einen umfassenden Lösungsansatz ein einheitliches körperschaftsteuerliches Regelwerk. ${ }^{129}$ Aufgrund des verminderten administrativen Aufwands sänken die Befolgungskosten der Unternehmen. Sollen hingegen auch grenzüberschreitend Verluste ausgeglichen, steuerliche Wirkungen konzerninterner Leistungstransaktionen ausgeschaltet und Umstrukturierungsmaßnahmen steuerneutral ermöglicht werden, bedarf es eines konsolidierten steuerlichen Abschlusses. ${ }^{130}$ Hierzu eignet sich eine Vielzahl theoretischer Lösungsansätze. ${ }^{131}$ Im Folgenden werden lediglich vier Alternativen diskutiert, die auch von der EU-Kommission vorgeschlagen werden. ${ }^{132}$ Im Einzelnen handelt es sich um folgende Ansätze: ${ }^{133}$

90/434/EWG des Rates vom 23. 7. 1990, KOM (2003) 613 endg. v. 17. 10. 2003 gefolgt. Vgl. a. Kinzl, U.-P., in: $B B, 2005$, S. 971-975; Benecke, A., in: IStR, 2005, S. 606-612 und 641-648.

129 Vgl. Oestreicher, A., in: StuW, 2002, S. 347.

${ }^{130}$ Ein Summenabschluss löst nur bedingt alle Probleme, da sich weiterhin Zinseffekte im Rahmen der Gestaltung der Verrechnungspreise und der Umstrukturierungsmaßnahmen ergeben können.

$131 \mathrm{Zu}$ den in der Literatur diskutierten Lösungsansätzen, die hier nicht dargestellt werden, zählen unter anderem das „European Tax Allocation System“ (vgl. Hernler, J., in: ET, 2004, S. 246-251; derselbe, in: $D B, 2003$, S. 60-65) und eine harmonisierte Dual Income Tax in Europa (vgl. Cnossen, S., in: FA, 2001, S. 536-543). Auch die Abschaffung der Körperschaftsteuer, die lediglich $3,5 \mathrm{v}$. H. des europäischen Bruttoinlandsprodukts beträgt, wird als eine Alternative diskutiert. Vgl. Gammie, M./Radelli, C.M./Klemm, A., Reform, 2001, S. 37.

132 Vgl. Europäische Kommission, Unternehmensbesteuerung, 2001, S. 505-568.

133 Vgl. Mintz, J.M., in: CESifo Forum, 2002, 1, S. 6-7; derselbe, in: ITPF, 2004, S. 226-227; Oestreicher, A., in: StuW, 2002, S. 347-348. Für einen ökonomischen Vergleich der Besteuerung im Sitzland und der einheitlichen (konsolidierten) Bemessungsgrundlage vgl. Mintz, J.M./Weiner, J.M., in: ITPF, 2003, S. 699-704. 


\section{Besteuerung im Sitzland}

2. Einheitliche (konsolidierte) Bemessungsgrundlage

3. Europäische Körperschaftsteuer

4. Obligatorische einheitliche „harmonisierte Bemessungsgrundlage“.

Unabhängig vom konkreten Ansatz erfolgt die Berechnung des steuerpflichtigen Gewinns immer in drei Schritten. Erstens ermitteln alle Konzerngesellschaften innerhalb eines Konzerns ihre Gewinne separat nach einheitlichen Vorschriften. Zweitens werden die Einzelergebnisse zum konsolidierten Ergebnis zusammengefasst, und drittens wird die Konzernbemessungsgrundlage anhand eines Schlüssels auf die einzelnen Steuerhoheiten aufgeteilt. ${ }^{134}$

Der Grundgedanke der Besteuerung im Sitzland beruht auf der gegenseitigen Anerkennung der nationalen Steuervorschriften. ${ }^{135}$ Innerhalb eines multinationalen Konzerns würde der Konzerngewinn stets nach den steuerlichen Vorschriften des Sitzlandes der Muttergesellschaft ermittelt werden. Damit existierten zwar weiterhin 25 unterschiedliche Steuersysteme in der Europäischen Union, innerhalb eines Konzerns käme aber nur das Steuergesetz des Sitzlandes der Muttergesellschaft zur Anwendung, unabhängig davon, in welchem europäischen Land sich die Konzerngesellschaften befänden. ${ }^{136} \mathrm{Die}$ Anwendung der Sitzlandbesteuerung wäre fakultativ, dann aber für alle qualifizierten Konzerngesellschaften bindend (kein „cherry-picking“). ${ }^{137}$ Im Ergebnis würden alle Gliedgesellschaften so behandelt, als läge ihr Sitz im Land des Stammhauses. Damit erfolgte auch die Verlustverrechnung der rechtlich selbstständigen Einheiten nach Maßgabe des im Sitzland der Muttergesellschaft geltenden Gruppenbesteuerungssystems. ${ }^{138}$ Verzerrungen aufgrund von Verrechnungspreisgestaltungen wären nur noch in Abhängigkeit von dem maß-

134 Vgl. Spengel, C., in: Lüdicke, Steuerrecht, 2004, S. 133-134. Fällt die Europäische Körperschaftsteuer ausschließlich der Europäischen Union zu, entfallt hierbei die Aufteilung der Bemessungsgrundlage bzw. der Steuereinnahmen.

135 Der Ansatz der Besteuerung im Sitzland geht auf die „Stockholmer Gruppe“ zurück, der unter anderem die Professoren Lodin und Gammie angehören. Vgl. für eine ausführliche Abhandlung Lodin, S.-O./Gammie, M., in: ET, 1999, S. 288-294; dieselben, Home, 2001.

${ }^{136}$ Für eine kritische Analyse der Besteuerung im Sitzland vgl. Giannini, S., in: CESifo Forum, 2002, 1, S. 25-27; Mintz, J.M./Weiner, J.M., in: ITPF, 2003, S. 699-704; Schön, W., in: $E T, 2002$, S. 284-285.

137 Vgl. Lodin, S.-O./Gammie, M., in: ET, 1999, S. 292; dieselben, Home, 2001, S. 23; Europäische Kommission, Unternehmensbesteuerung, 2001, S. 514.

138 Vgl. Lodin, S.-O./Gammie, M., in: ET, 1999, S. 292; dieselben, Home, 2001, S. 26, 32; Europäische Kommission, Unternehmensbesteuerung, 2001, S. 506. 
gebenden Gruppenbesteuerungssystem möglich. ${ }^{139}$ Würden die Einzelabschlüsse wie z. B. in den Niederlanden voll konsolidiert ${ }^{140}$, wären die derzeitigen Probleme bei der Festsetzung konzerninterner Verrechnungspreise behoben. Erfolgte hingegen keine Zwischenergebniseliminierung, bestünde für die Unternehmen ein Anreiz, Gewinne möglichst spät zu realisieren, um von den damit verbundenen Zinseffekten zu profitieren. Eine Gewinnverlagerung auf Basis der Gestaltung von Verrechnungspreisen wäre nicht mehr möglich, da die Aufteilung der Bemessungsgrundlage zwischen den Mitgliedstaaten eines Konzerns mittels einer Formel erfolgte. ${ }^{141}$ Die Sitzlandbesteuerung würde bewirken, dass innerhalb eines Landes zwei konkurrierende Unternehmen verschiedenen Steuersystemen unterworfen sein könnten. ${ }^{142}$ Daraus würden Wettbewerbsverzerrungen resultieren, denen die Unternehmen mit Anpassungsmaßnahmen begegnen würden. So könnten Unternehmen bei stark unterschiedlichen Bemessungsgrundlagen oder Gruppenbesteuerungssystemen durch „countryshopping" ihre Steuerlast reduzieren. ${ }^{143}$ Gleichzeitig wären die Mitgliedstaaten bestrebt, ihre Steuersätze zu senken und die Bemessungsgrundlage zu verjüngen, da die resultierenden Steuerausfälle wesentlich durch die europäischen Partnerländer mitgetragen werden würden. ${ }^{144}$ Langfristig könnte nur eine weitgehende Angleichung der Steuervorschriften diese Probleme lösen. Dafür spricht auch der administrative Aufwand, den die Sitzlandbesteuerung implizieren würde: Denn entweder müsste jede Finanzverwaltung 25 Steuervorschriften beherrschen oder aber die Finanzverwaltung im Sitzland der Muttergesellschaft wäre verantwortlich für alle Tochtergesellschaften der nationalen Konzerne. Ob jedoch die Mitgliedstaaten die Erhebung von Steuern in ihrem territorialen Bereich anderen Mitgliedstaaten übertragen würden, ist höchst zweifelhaft. ${ }^{145}$ Ungeachtet der dargelegten Vorbehalte erwägt die Europäische Kommission, die Sitzlandbesteuerung im Rahmen eines Pilotprojekts für

139 Vgl. Lodin, S.-O./Gammie, M., in: ET, 1999, S. 292; dieselben, Home, 2001, S. 33-34; Europäische Kommission, Unternehmensbesteuerung, 2001, S. 507.

140 Vgl. Endres, D., in: Herzig, Organschaft, 2003, S. 461-482. Siehe a. Kapitel B.III.

${ }^{141}$ Eine ausführliche Diskussion über die Ausgestaltung einer Aufteilungsformel findet sich in Kapitel C.II.4.

142 Vgl. Weiner, J.M., in: CESifo Forum, 2002, 1, S. 13; Schön, W., in: ET, 2002, S. 285; Brügelmann, R., in: ifo Schnelldienst, 2004, 11, S. 7.

143 Vgl. Mintz, J.M., in: CESifo Forum, 2002, 1, S. 7; Sánchez, G.E./Fluxà, J.F., in: ET, 2006, S. 203-206.

144 Vgl. Weichenrieder, A., in: ifo Schnelldienst, 2002, 2, S. 11; Oestreicher, A., in: StuW, 2002, S. 347.

145 Vgl. Mintz, J.M., in: CESifo Forum, 2002, 1, S. 7; Rädler, A., in: TNI, 2002, S. 157. 
kleinere und mittlere Unternehmen ${ }^{146}$ zunächst für einen auf fünf Jahre befristeten Zeitraum einzuführen. ${ }^{147}$

Die einheitliche (konsolidierte) Bemessungsgrundlage entspricht vom Grundkonzept her der Besteuerung im Sitzland, jedoch mit dem gravierenden Unterschied, dass anstelle des Steuersystems des Sitzlandes der Konzernmutter eine harmonisierte Bemessungsgrundlage tritt. Nicht mehr die Steuervorschrift des Sitzlandes der Muttergesellschaft ist für alle Konzerngesellschaften maßgebend, sondern eine fakultative, zusätzliche EU-Bemessungsgrundlage. Auf dieser Grundlage stellen alle Konzerngesellschaften ihre Einzelabschlüsse auf, die dann zu einem konsolidierten Abschluss zusammengefasst werden. AnschlieBend wird das konsolidierte Ergebnis mittels einer Formel den entsprechenden Steuerhoheiten zugeteilt und dort mit dem jeweiligen Steuersatz des Mitgliedstaates belegt. Verantwortlich für die Anwendung der neuen europäischen Steuervorschriften innerhalb eines Konzerns wäre der Mitgliedstaat der Muttergesellschaft. Dabei ist die Bestimmung des Sitzlandes weitaus weniger problematisch, da hieraus keine primären Belastungsunterschiede für die Gliedgesellschaften resultieren. Sekundär können sich dennoch steuerliche Standortvorteile einzelner Mitgliedstaaten ergeben, wenn nämlich die Besteuerung durch nationale Rechtsordnung, Auslegungen und gerichtliche Entscheidungen im Sitzland der Muttergesellschaft beeinflusst werden. Vor diesem Hintergrund befürwortet die EU-Kommission, alle steuerrelevanten Optionen auf EU-Ebene zu regeln. ${ }^{148}$ Im Ergebnis hätten die existierenden Steuervorschriften der 25 Mitgliedstaaten weiterhin Bestand, und die Unternehmen in der Europäischen Union könnten zwischen der derzeitigen Besteuerung und der einheitlichen (konsolidierten) Bemessungsgrundlage frei wählen.

Die Europäische Körperschaftsteuer geht über den Vorschlag der einheitlichen (konsolidierten) Bemessungsgrundlage hinaus. Neben der harmonisierten Bemessungsgrundlage sieht dieses Konzept ein einheitliches Steuergesetz in der

146 Gemäß der allgemein üblichen Definition der EU zählen zu den kleinen und mittleren Unternehmen Gesellschaften mit weniger als 250 Beschäftigten, einem Jahresumsatz von höchstens 50 Mio. Euro und/oder einer Jahresbilanzsumme von bis zu 43 Mio. Euro. Vgl. Empfehlung der Kommission v. 6. 5. 2003 (2003/361/EC), Abl. Nr. L 124 v. 20. 5. 2003, S. 36-42.

147 Vgl. Europäische Kommission, Pilotprojekt, 2004; dieselbe, Sitzlandbesteuerung, 2005, S. 9-14, für eine detaillierte Beschreibung des möglichen Pilotprojekts vgl. S. 15-27; Diemer, R., in: DSWR, 2003, S. 151-153; Diemer, R./Mors, M., in: ITPJ, 2006, S. 111-116; Thömmes, O./Rasch, S./Koch, T., in: ITPJ, 2006, S. 121-123; Cerioni, L., in: ET, 2006, S. 13-20.

$148 \mathrm{Vgl}$. Europäische Kommission, Unternehmensbesteuerung, 2001, S. 520-521. 
Europäischen Union vor. ${ }^{149}$ Dies impliziert, dass unabhängig vom Sitzland der Gesellschaften neben einer einheitlichen Bemessungsgrundlage auch ein einheitlicher Körperschaftsteuertarif angewendet wird. Die administrative Verwaltung kann hierbei sowohl auf europäischer Ebene als auch auf der Ebene der einzelnen Mitgliedstaaten erfolgen. Im Zusammenhang damit wird angestrebt, dass die Einnahmen aus dieser Steuer anteilig oder vollständig der Europäischen Union zufallen. ${ }^{150}$ Über den Bedarf der Europäischen Union hinaus erzielte Einnahmen flössen zurück an die Mitgliedstaaten, wobei die Aufteilung auf der Basis von makroökonomischen Faktoren erfolgen sollte, ${ }^{151}$ um die der direkten Gewinnabgrenzung inhärenten Probleme zu vermeiden. ${ }^{152}$ Die Anwendung der Europäischen Körperschaftsteuer wäre für die Unternehmen fakultativ; daneben hätten die nationalen Körperschaftsteuersysteme weiterhin Bestand. Eine problematische Folge der Europäischen Körperschaftsteuer jedoch sind die damit verbundenen Anreizwirkungen. Die Mitgliedstaaten wären bestrebt, ihre nationalen Steuersysteme derart zu gestalten, dass sich möglichst wenige Unternehmen für die Europäische Körperschaftsteuer entscheiden. Außerdem wäre die effiziente Steuerdurchsetzung gefährdet, da die Einnahmen der EU zuflössen und die Verwaltung auf nationaler Ebene verbliebe. ${ }^{153}$

Der umfassendste Harmonisierungsansatz ist die obligatorische einheitliche „,harmonisierte Bemessungsgrundlage“. Dieser Ansatz sieht eine verpflichtende Anwendung eines einheitlichen Körperschaftsteuerkonzepts innerhalb der Europäischen Union vor, welches an die Stelle der 25 bestehenden Steuersysteme tritt. ${ }^{154}$ Damit geht dieser Lösungsvorschlag deutlich über die Harmonisierungsbestrebungen der vorherigen Maßnahmen hinaus. Innerhalb der Europäischen Union wäre lediglich ein Körperschaftsteuergesetz maßgebend, dessen Anwendung sowohl für rein nationale als auch internationale Körperschaftsteuersubjekte verpflichtend wäre. Eine harmonisierte Bemessungsgrund-

149 Vgl. Plasschaert, S., in: ET, 1997, S. 10-13; derselbe, in: ET, 2002, S. 326-345; derselbe, Income Tax, 2002, S. 1-23; Europäische Kommission, Unternehmensbesteuerung, 2001, S. 509-511.

${ }^{150}$ Vgl. Plasschaert, S., in: ET, 1997, S. 10; derselbe, Income Tax, 2002, S. 18. Kritisch hierzu Fuest, C., Coordination, 2002, S. 10-11.

151 Vgl. hierzu Kapitel C.II.4.b)( $\beta)$.

152 Vgl. Plasschaert, S., Income Tax, 2002, S. 14. Für eine Diskussion über mögliche eigenstăndige EU-Steuern vgl. derselbe, in: ET, 2004, S. 470-479.

153 Vgl. Fuest, C./Huber, B., in: Vierteljahrhefte zur Wirtschaftsforschung, 2003, S. 387. Eine empirische Untersuchung derartiger Anreizprobleme zeigt für Deutschland, dass die Abschaffung des horizontalen Finanzausgleichs das Steueraufkommen der Bundesländer ceteris paribus um $15 \mathrm{v}$. H. steigern würde. Vgl. hierzu Baretti, C./Huber, B./Lichtblau, $\mathrm{K}$., in: ITPF, 2002, S. 631-649.

154 Vgl. Europäische Kommission, Unternehmensbesteuerung, 2001, S. 510-551. 
lage würde die Konsolidierung der Einzelabschlüsse beinhalten. Die Aufteilung der Bemessungsgrundlage zwischen den Steuerhoheiten erfolgt auch hier anhand einer Formel. ${ }^{155}$

Tabelle 9 fasst die Unterschiede und Gemeinsamkeiten der verschiedenen Ansätze in einer Übersicht zusammen.

Tabelle 9: $\quad$ Vergleich der umfassenden Lösungsansätze

\begin{tabular}{|c|c|c|c|c|}
\hline & $\begin{array}{l}\text { Besteuerung im } \\
\text { Sitzland }\end{array}$ & СССТВ & $\begin{array}{l}\text { Europäische } \\
\text { Körperschaft- } \\
\text { steuer }\end{array}$ & $\begin{array}{l}\text { Obligatorische } \\
\text { Harmonisienung }\end{array}$ \\
\hline Grundlage & $\begin{array}{l}\text { Gegenseitige An- } \\
\text { erkennung }\end{array}$ & Harmonisierung & Harmonisierung & Harmonisierung \\
\hline \begin{tabular}{|l|} 
Steuerrecht \\
\end{tabular} & Bestehendes & Neues & Neues & Neues \\
\hline Anwendung & Fakultativ & Fakultativ & $\begin{array}{l}\text { Fakultativ oder } \\
\text { obligatorisch }\end{array}$ & Obligatorisch \\
\hline $\begin{array}{l}\text { Teilnahme } \\
\text { (im Prinzip) }\end{array}$ & $\begin{array}{l}\text { Alle/bestimmte } \\
\text { Unternehmen }\end{array}$ & $\begin{array}{l}\text { Alle/bestimmte } \\
\text { Unternehmen }\end{array}$ & $\begin{array}{l}\text { Alle/bestimmte } \\
\text { Unternehmen }\end{array}$ & $\begin{array}{c}\text { Alle Unter- } \\
\text { nehmen }\end{array}$ \\
\hline Einführung & $\begin{array}{l}\text { Gegebenenfalls } \\
\text { außerhalb des } \\
\text { institutionellen } \\
\text { Rahmens der EU }\end{array}$ & $\begin{array}{l}\text { Innerhalb des } \\
\text { institutionellen } \\
\text { Rahmens der EU }\end{array}$ & $\begin{array}{l}\text { Innerhalb des } \\
\text { institutionellen } \\
\text { Rahmens der EU }\end{array}$ & $\begin{array}{c}\text { Innerhalb des } \\
\text { institutionellen } \\
\text { Rahmens der } \\
\text { EU }\end{array}$ \\
\hline \begin{tabular}{|l|} 
Anzahl der \\
Steuersysteme \\
\end{tabular} & 25 derzeitige & $\begin{array}{l}25 \text { derzeitige und } \\
\text { ein neues }\end{array}$ & $\begin{array}{c}25 \text { derzeitige und } \\
\text { ein neues }\end{array}$ & Eines \\
\hline \begin{tabular}{|l|} 
Gleich- \\
behandlung \\
aller \\
Teilnehmer \\
\end{tabular} & $\begin{array}{l}\text { Nein - potenziell } \\
25 \text { Sitzländer }\end{array}$ & $\mathrm{Ja}$ & $\mathrm{Ja}$ & $\mathrm{Ja}$ \\
\hline $\begin{array}{l}\text { Verlust- } \\
\text { konsolidierung }\end{array}$ & $\begin{array}{c}\text { In Abhängigkeit } \\
\text { des Gruppen- } \\
\text { besteuerungssyste } \\
\text { ms des Sitzstaates }\end{array}$ & $\mathbf{J a}$ & $\mathrm{Ja}$ & Ja \\
\hline $\begin{array}{l}\text { Verrechnungs- } \\
\text { preisgestaltung } \\
\text { in der EU }\end{array}$ & $\begin{array}{c}\text { Zwischen den } \\
\text { Teilnehmern ge- } \\
\text { klärt }\end{array}$ & $\begin{array}{c}\text { Zwischen den } \\
\text { Teilnehmern ge- } \\
\text { klärt }\end{array}$ & $\begin{array}{c}\text { Zwischen den } \\
\text { Teilnehmern ge- } \\
\text { klärt }\end{array}$ & Geklärt \\
\hline $\begin{array}{l}\text { Steuersatz vom } \\
\text { Mitgliedstaat } \\
\text { festgelegt }\end{array}$ & $\mathrm{Ja}$ & $\begin{array}{c}\text { Ja - Möglichkeit } \\
\text { eines „,speziellen“ } \\
\text { Steuersatzes }\end{array}$ & EU-Satz & $\mathbf{J a}$ \\
\hline $\begin{array}{l}\text { Aufteilungs- } \\
\text { formel }\end{array}$ & $\mathrm{Ja}$ & $\mathrm{Ja}$ & Vorstellbar & $\mathrm{Ja}$ \\
\hline
\end{tabular}

In Anlehnung an: Europäische Kommission, Unternehmensbesteuerung, 2001, S. 514

${ }^{155}$ Für eine detaillierte Analyse unterschiedlicher Aufteilungsmechanismen vgl. Kapitel C.II.4.b). 


\section{Bewertung der umfassenden Lösungsansätze}

\section{a) Grad der Beseitigung der unternehmenssteuerlichen Hindernisse}

Die Mehrzahl der unternehmenssteuerlichen Hindernisse geht auf die Koexistenz von 25 Steuersystemen zurück. ${ }^{156}$ Setzten die umfassenden Lösungsansätze eine Harmonisierung der Bemessungsgrundlage voraus, fielen die entsprechenden Bewertungen sehr ähnlich aus. Um redundante Ausführungen zu vermeiden, wird deshalb zunächst lediglich zwischen der Besteuerung im Sitzland und den Ansätzen mit einer harmonisierten Bemessungsgrundlage unterschieden. Als Bewertungsmaßstab kommt der Grad der Reduzierung unternehmenssteuerlicher Hindernisse zum Tragen. Die Ausführungen beschränken sich auf Darstellungen im Bereich der Befolgungskosten, der grenzüberschreitenden Verlustverrechnung, der Dividendenbesteuerung und der Verrechnungspreisgestaltung. ${ }^{157}$ Ergänzend muss die politische Durchsetzbarkeit der einzelnen Lösungsansätze bewertet werden.

Eine Vereinheitlichung der Steuersysteme senkt grundsätzlich die Befolgungskosten, da sich die Konzerne nur noch mit einem Regelwerk konfrontiert sehen. ${ }^{158}$ Das Ausmaß, in dem die Aufwendungen sinken, hängt maßgeblich von dem Umfang des Konsolidierungskreises ab. So schließt z. B. eine hohe Mindestbeteiligungsquote als Voraussetzung der Ergebniskonsolidierung innerhalb eines Konzerns viele Tochtergesellschaften aus, die weiterhin das nationale Besteuerungsrecht ihres Sitzlandes zu befolgen hätten. Die harmonisierten Ansätze sind hier der Sitzlandbesteuerung überlegen, da auch die ausgeschlossenen Gesellschaften, soweit ihre Rechtsform dies erlaubt, für eine Anwendung des harmonisierten Regelwerks optieren können, auch wenn eine Konsolidierung der Ergebnisse ausgeschlossen ist. ${ }^{159}$ Demgegenüber sind die Auswirkungen

156 Vgl. Kapitel C.I.3.

157 Für eine kritische Bewertung der Lösungsansätze in Bezug auf die übrigen unternehmenssteuerliche Hindernisse vgl. Europäische Kommission, Unternehmensbesteuerung, 2001, S. 424-535.

${ }^{158}$ Vgl. Krause-Junk, G. in: ifo Schnelldienst, 2002, 2, S. 4. Ist die Anwendung des umfassenden Lösungsansatzes fakultativ, könnte für die Unternehmen ein Anreiz bestehen, den steuerlichen Gewinn sowohl nach dem harmonisierten als auch nach dem alten Steuersystem zu ermitteln, um den für sie günstigeren auszuwählen. Vgl. Gammie, M./Radelli, C.M./Klemm, A., Reform, 2001, S. 40.

159 Inwieweit auch rein national tătige Gesellschaften im Fall einer harmonisierten Besteuerung diese anwenden dürfen, ist ungeklärt, wobei ein Ausschluss dieser Gesellschaften zu erheblichen Verzerrungen zwischen nationalen und grenzüberschreitend tätigen Gesellschaften führt. Vgl. Giannini, S., in: CESifo Forum, 2002, 1, S. 27. Auch Schön spricht sich für eine Anwendung eines harmonisierten Ansatzes für rein national 
aufseiten der Finanzverwaltung ambivalent. Zum einen reduziert sich die Arbeitsbelastung durch den weitestgehend entfallenden Arbeitsbereich der Verrechnungspreise. Zum anderen entstehen durch die Aufteilung der Bemessungsgrundlage neue Aufgabenfelder. ${ }^{160}$ Die Zuständigkeit der nationalen Finanzverwaltungen für ausländische Gesellschaften im Rahmen der Sitzlandbesteuerung erhöht die Befolgungskosten in Ländern, in denen vermehrt Konzernmuttergesellschaften angesiedelt sind. Erwartungsgemäß sollten sich Mehr- und Minderbelastung ausgleichen, sofern die unterschiedlichen Belastungen der Konzerne diese nicht zu einer Verlegung des Sitzes der Muttergesellschaft bewegen. Die Umsetzung eines harmonisierten Ansatzes erfordert von den nationalen Finanzverwaltungen sowohl die Kenntnis des nationalen, bereits existierenden als auch die des neuen Regelwerks. Lediglich im Rahmen einer obligatorischen Harmonisierung und einer europäischen Körperschaftsteuer mit zentraler Verwaltung würde sich für die nationalen Finanzverwaltungen eine eindeutige Abnahme des Arbeitspensums einstellen.

Unabhängig von den einzelnen Vorschlägen hängt das Ausmaß der Reduktion der Befolgungskosten von der Anzahl der teilnehmenden Mitgliedstaaten ab. ${ }^{161}$ Beschränkt sich der Teilnehmerkreis auf einige wenige Staaten, bedeutet dies für eine Vielzahl der Konzerne, dass sie gar nicht oder nur eingeschränkt von den umfassenden Maßnahmen profitieren, woraus sich unweigerlich Verzerrungen ergeben.

Sowohl die Besteuerung im Sitzland als auch ein harmonisierter Ansatz ermöglichen einen grenzüberschreitenden Verlustausgleich. Ist dieser bei einer Harmonisierung der Bemessungsgrundlage durch die Konsolidierung der Einzelergebnisse systemimmanent, erfolgt die Verlustverrechnung bei der Sitzlandbesteuerung nach Maßgabe des im Sitzland der Muttergesellschaft geltenden Gruppenbesteuerungssystems. ${ }^{162}$ Diese Systeme unterscheiden sich innerhalb der Europäischen Union jedoch erheblich voneinander. So reicht die

tätige Unternehmen aus, äußert aber zugleich seine Bedenken hinsichtlich des Subsidiaritätsprinzips. Vgl. Schön, W., in: ET, 2002, S. 280-281.

$160 \mathrm{Vgl}$. Europäische Kommission, Unternehmensbesteuerung, 2001, S. 519.

161 Gegen eine einheitliche Bemessungsgrundlage sprechen sich derzeit die Tschechische Republik, Estland, Irland, die Slowakische Republik und Großbritannien aus. Vgl. Cerioni, L., in: ET 2006, S. 187.

162 Die Dauer des Verlustvortrags und -rücktrags sollte hingegen ausschließlich durch die Regeln des Steuerrechts des Sitzstaates der Gesellschaften bestimmt werden. Vgl. Westberg, B., in: ET, 2002, S. 325. Zu den einzelnen bestehenden Gruppenbesteuerungssystemen in den Mitgliedstaaten der EU vgl. Kapitel B.III. 
Spannweite von gar keinem Gruppenbesteuerungssystem ${ }^{163}$ bis hin zu einer vollständigen Konsolidierung der Einzelabschlüsse ${ }^{164}$. Neben der Ausgestaltung der Gruppenbesteuerungssysteme unterscheiden sich auch die für eine Anwendung $\mathrm{zu}$ erfüllenden Voraussetzungen voneinander. Die qualifizierende Mindestbeteiligungsquote reicht z. B. von $50 \mathrm{v} . \mathrm{H}^{165}$ bis zu $100 \mathrm{v} . \mathrm{H} .{ }^{166}$ All dies führt zu erheblichen Verzerrungen. Im Extremfall würden zukünftig Konzerne bei Anwendung der Sitzlandbesteuerung weniger Verluste als bisher verrechnen können. ${ }^{167}$ Ein Konzern mit Sitz in Griechenland und zwei Tochtergesellschaften in Deutschland hätte nach bestehendem Recht die Möglichkeit, die Gewinne und Verluste der deutschen Gesellschaften durch das Instrument der Organschaft zu verrechnen. ${ }^{168}$ Die Einführung der Sitzlandbesteuerung würde aber bedeuten, dass aufgrund des fehlenden griechischen Gruppenbesteuerungssystems auch zwischen den deutschen Gesellschaften keine Verlustverrechnung mehr möglich wäre.

Probleme bei der Dividendenbesteuerung wären innerhalb des qualifizierenden Konzerns gelöst. Bei den harmonisierten Ansätzen wären Dividenden im Zuge der Konsolidierung zu neutralisieren; im Fall der Besteuerung nach dem Sitzland würden Dividendenzahlungen als inländische Einkünfte behandelt. Weiterhin ungeklärt bliebe die Behandlung von grenzüberschreitenden Ausschüttungen an nicht qualifizierte Anteilseigner in einen Staat mit Anrechnungsverfahren. Hier kann grenzüberschreitend auch bedeuten, dass der Minderheitsgesellschafter zwar im gleichen Staat wie die ausschüttende Gesellschaft sitzt, diese aber im Fall der Besteuerung im Sitzland ihren Abschluss nach den

${ }^{163} \mathrm{Zu}$ diesen Ländern gehören Belgien, Estland, Griechenland, Litauen, Slowakei, Tschechien und Ungarn. Vgl. Endres, D. et al., Verlustberücksichtigung, 2006, S. 19.

164 Eine vollständige Konsolidierung der Einzelabschlüsse sieht ausschließlich das niederländische Steuerrecht vor.

${ }^{165} \mathrm{Zu}$ diesen Ländern gehören Deutschland, Italien, Malta und Österreich. In Deutschland ist zu beachten, dass für die Begründung einer Organschaft neben der finanziellen Eingliederung der Organgesellschaft auch ein Ergebnisabführungsvertrag abgeschlossen werden muss. Empirische Untersuchungen zeigen, dass sich diese Anforderung negativ auf die Begründung einer Organschaft auswirkt. Vgl. hierzu Oestreicher, A./Koch, R., Organschaft, 2007.

166 Nach der Absenkung der Mindestbeteiligungsvoraussetzung in Dänemark zum 1. 1. 2005 setzt in der Europäischen Union nur noch Slowenien eine Beteiligung von $100 \mathrm{v}$. H. voraus.

167 Vgl. Europäische Kommission, Unternehmensbesteuerung, 2001, S. 522.

168 Das Instrument der Organschaft erlaubt nicht die Verrechnung von Verlusten zwischen Schwestergesellschaften. Durch die Gründung einer Landesholding oder einer Betriebsstätte, die die Beteiligungen an den deutschen Gesellschaften halten, könnten die Verluste dennoch verrechnet werden. 
Steuervorschriften eines anderen Mitgliedstaates aufstellt. Vermutlich wäre für Ausschüttungen eines ausländischen Tochterunternehmens an Minderheitsaktionäre mit Sitz im Land der Muttergesellschaft das dort geltende Steuerrecht maßgebend. Dividendenausschüttungen der ausländischen Tochtergesellschaft an Minderheitsaktionäre im Inland bzw. in einem anderen Mitgliedstaat wären hingegen nach den Vorschriften des Sitzlandes der Tochtergesellschaft abzuwickeln. ${ }^{169}$

Eine konsolidierte Steuerbemessungsgrundlage löst das Problem der Verrechnungspreisgestaltung bei Lieferung und Leistungen zwischen den einbezogenen Konzerngesellschaften vollständig. ${ }^{170}$ Unverändert bleibt die Situation gegenüber nicht in den Konzernkreis aufgenommenen Konzerngesellschaften, da hier weiterhin das nationale Recht bei der Bestimmung von Verrechnungspreisen Anwendung findet. ${ }^{171}$ Aber auch innerhalb des europäischen Binnenmarktes bleibt das Problem bei Beziehungen zu nicht qualifizierten Gesellschaften bestehen. ${ }^{172}$ Sollte ein „cherry-picking“ in Bezug auf die in den Konsolidierungskreis aufzunehmenden Gesellschaften nicht erlaubt sein, könnten die Konzerne die Voraussetzungen für die Inanspruchnahme einer konsolidierten Besteuerung einzelner Gesellschaften auch bewusst nicht erfüllen, um so wieder über Verrechnungspreisgestaltungen Gewinne ins niedrig besteuerte Ausland verlagern zu können. Denkbar ist auch, dass im Rahmen der Harmonisierung Zwischenergebnisse nicht eliminiert werden. Zwar ist dann eine Gewinnverlagerung nicht möglich, da die Bemessungsgrundlage mittels einer Formel den Steuerhoheiten zugeteilt wird, doch wären die Konzerne bestrebt, den Erfolg möglichst spät auszuweisen, um Zinseffekte nutzen zu können. Besonders bei der Einführung der Sitzlandbesteuerung wäre diese zeitliche Komponente von Bedeutung, da lediglich das Gruppenbesteuerungssystem der Niederlande im nationalen Fall eine Zwischenergebniseliminierung vorsieht. Abschließend sei angemerkt, dass bei weiterhin abweichenden Steuersätzen in den jeweiligen Mitgliedstaaten unter Anwendung der indirekten Gewinnabgrenzung die Unternehmen zukünftig anstelle der Verrechnungspreise die für

169 Vgl. Europäische Kommission, Unternehmensbesteuerung, 2001, S. 523.

170 Vgl. Spengel, C./Braunagel, R., in: StuW, 2006, S. 47-48; Westberg, B., in: ET, 2002, S. 326. Erfolgt die Aufteilung der konsolidierten Bemessungsgrundlage nach dem Beitrag der Wertschöpfung, beeinflussen die internen Verrechnungspreise weiterhin die Höhe des Steueraufkommens, wenn auch in abgeschwächter Form. Vgl. McLure Jr., C.E., in: CESifo Forum, 2005, 1, S. 35-36.

171 Grundsätzlich ist auch eine weltweite formelhafte Gewinnaufteilung denkbar, doch verneint die OECD diese Form der Gewinnabgrenzung im multinationalen Konzern. Vgl. OECD, Guidelines, 2001, Anm. 3.74.

172 Vgl. Mintz, J.M., in: CESifo Forum, 2002, 1, S. 8. 
eine Aufteilung der Bemessungsgrundlage relevanten Größen beeinflussen werden, da die formelhafte Gewinnaufteilung wie eine Steuer auf die Formelfaktoren wirkt. ${ }^{173}$ An die Stelle von Verrechnungspreisgestaltungen tritt dann die Formelfaktorengestaltung, wobei die reale Verlagerung von Vermögen und Lohnsummen wohl mit erheblich mehr Aufwand verbunden ist. ${ }^{174}$

\section{b) Politische Durchsetzbarkeit der umfassenden Lösungsansätze}

Die politische Durchsetzbarkeit der angestrebten Lösungsansätze wird maßgeblich durch den Rechtsrahmen der Europäischen Union mitbestimmt. Im Bereich der direkten Steuern besteht, anders als bei den indirekten Steuern, ${ }^{175}$ kein konkreter Harmonisierungsauftrag. Als Rechtsgrundlage für die Angleichung der direkten Steuern kommt vorwiegend Art. 94 EGV in Betracht. ${ }^{176}$ Danach sind die Rechts- und Verwaltungsvorschriften der Mitgliedstaaten anzugleichen, „die sich unmittelbar auf die Errichtung oder das Funktionieren des Gemeinsamen Marktes auswirken“. ${ }^{177}$ Zum Erlass der Richtlinien ist ein einstimmiger Ratsbeschluss erforderlich (Art. 94, 95 Abs. 2 EGV). ${ }^{178}$

Dementsprechend erscheint die Akzeptanz von Ansätzen unrealistisch, die eine zwingende Teilnahme aller Mitgliedstaaten voraussetzen. Die Einführung einer obligatorischen harmonisierten Bemessungsgrundlage muss damit derzeit als

173 Vgl. McLure Jr., C.E., in: Aaron/Boskin, Economics, 1980, S. 328; Weiner, J.M., in: CESifo Forum, 2002, 1, S. 16; Wellisch, D., in: FA, 2004, S. 25. Siehe a. Kapitel C.II.4.a).

$174 \mathrm{Vgl}$. für die Darstellung von Ansatzpunkten zur Steuerplanung bei einer formelhaften Gewinnaufteilung Scheffler, W., in: Oestreicher, Konzernbesteuerung, 2005, S. 317-328.

175 Der Harmonisierungsauftrag im Bereich der indirekten Steuern basiert auf dem Verbot der steuerlichen Diskriminierung bei der Einfuhr (Art. 90 EGV). Davon betroffen sind vor allem die Umsatzsteuer und die besonderen Verbrauchssteuern. Vgl. zu den Einzelheiten Bieber, R./Epiney, A./Haag, M., Union, 2005, S. 459-467. Für eine ausführliche Darstellung vgl. Hagen, A., indirekte Steuern, 2000.

176 Vgl. Jacobs, O.H. (Hrsg.), Unternehmensbesteuerung, 2002, S. 96-97. Neben Art. 94 EGV erlaubt Art 96 Abs. 2 EGV den Erlass von Richtlinien mit qualifizierter Mehrheit, soweit erhebliche Wettbewerbsverzerrungen von der Kommission festgestellt werden, die auch nach Beratung mit den betreffenden Mitgliedstaaten nicht abgestellt werden können. Bisher wurde von diesem Recht kein Gebrauch gemacht. Vgl. hierzu Kellersmann, D./Treisch, C., Europäische Unternehmensbesteuerung, 2001, S. 77-78. Ferner kann zur Harmonisierung der direkten Steuern die Vertragsabrundungskompetenz nach Art. 308 EGV herangezogen werden. Vgl. hierzu Oppermann, T., Europarecht, 2005, Rz. 17/32 i. V. m. Rz. $6 / 58$.

177 Art. 94 EGV. In der fehlenden Gemeinschaftskompetenz auf dem Gebiet der direkten Steuern sieht Lehner den wesentlichen Kritikpunkt der umfassenden Lösungsvorschläge. Vgl. Lehner, M., in: ifo Schnelldienst, 2002, 2, S. 8.

178 Für eine ausführliche Diskussion der Rechtfertigung der Angleichung der Unternehmensbesteuerung nach Art. 94, Art. 95 (ex Art. 100, Art. 100a) vgl. Hey, J., Harmonisierung, 1997, S. 84-98. 
politisch nicht durchsetzbar erachtet werden. ${ }^{179}$ Ähnliches gilt für eine Europäische Körperschaftsteuer. Zwar ist grundsätzlich eine Umsetzung durch eine Ländergruppe denkbar, doch widerspricht sie dem Grundgedanken des Lösungsansatzes, welcher der Europäischen Union als supranationaler Regierung ein eigenes Besteuerungsrecht zuteilt. ${ }^{180}$ Den beiden verbleibenden Ansätzen, der Besteuerung im Sitzland und der einheitlichen (konsolidierten) Körperschaftsteuer-Bemessungsgrundlage, ist hingegen die fakultative Teilnahme der Mitgliedstaaten und der Unternehmen gemein. Damit kann anfänglich auch eine Einführung eines Lösungsansatzes innerhalb einer kleineren Anzahl von Mitgliedstaaten realisiert werden. Der Ausschluss einiger Mitgliedstaaten bedeutet aber, dass eine Rechtsangleichung nach Art. 94 EGV nicht möglich ist ${ }^{181}$, sodass eine Umsetzung entweder auf Ebene der EU durch das Instrument der verstärkten Zusammenarbeit oder auf bilateraler Ebene durch den Mechanismus des Doppelbesteuerungsabkommens erfolgen muss. Da die Sitzlandbesteuerung auf der gegenseitigen Anerkennung der Steuersysteme beruht, sollte nach Auffassung der Kommission das Pilotprojekt durch ein Protokoll zur Ergänzung der Doppelbesteuerungsabkommen der teilnehmenden Staaten realisiert werden. ${ }^{182}$ Die einheitliche (konsolidierte) Körperschaftsteuer-Bemessungsgrundlage hingegen ergänzt die bestehenden Steuersysteme um ein weiteres. Entsprechend bedarf es für ihre Umsetzung Initiativen auf der Ebene der Europäischen Union, deren Legitimation auf der Basis einer „verstärkten Zusammenarbeit" gemäß Art. 43-45 EUV erfolgen kann. ${ }^{183}$ Dieses Instrument ermöglicht es acht oder mehr Mitgliedstaaten, eine Zusammenarbeit zu vereinbaren und dafür auf die Ressourcen der Europäischen Union zurückzugreifen. ${ }^{184}$ Dass eine Beschränkung auf einen kleinen Kreis von Mitgliedstaaten die Einführung einer konsolidierten Bemessungsgrundlage nicht zwangsweise erleichtert, zeigt die deutsche Haltung gegenüber dieser Strategie. So befürchtet z. B. der Finanzausschuss bei einer Initiative von nur acht Mitgliedstaaten einen unfairen Steuerwettbewerb und eine Erosion des Steueraufkommens, sodass er

179 Vgl. Mintz, J., in: ITPF, 2004, S. 227.

180 Vgl. McLure Jr., C.E., in: CESifo Forum, 2005, 1, S. 33.

181 Vgl. Art. 249 EGV.

${ }^{182} \mathrm{Vgl}$. Diemer, R./Mors, M., in: ITPJ, 2006, S. 115; Europäische Kommission, Sitzlandbesteuerung, 2005, S. 23.

183 Vgl. Europäische Kommission, Non-Paper, 2004a; McLure Jr., C.E., in: CESifo Forum, 2005, 1, S. 33; Hrehorovska, L., in: intertax, 2006, S. 160.

184 Art. 43 EUV. Für eine umfassende Darstellung der Anwendungsvoraussetzungen der verstärkten Zusammenarbeit vgl. Derpa, U., Zusammenarbeit, 2003, S. 171-199. In Bezug auf die Einführung der einheitlichen (konsolidierten) Besteuerung vgl. Cerioni, L., in: ET, 2006, S. 187-196. 
dem Bundesrat erhöhte Anstrengungen empfiehlt, die Anzahl der teilnehmenden Mitgliedstaaten zu erhöhen. ${ }^{185}$

\section{c) Zusammenfassende Würdigung der umfassenden Lösungsansätze}

Die Beurteilung der vier potenziellen Lösungsansätze zeigt, dass die obligatorische einheitliche Bemessungsgrundlage die steuerlichen Hindernisse innerhalb der Europäischen Union am effektivsten abbaut bzw. beseitigt. Rein ökonomisch betrachtet wäre die Einführung dieses Vorschlags sinnvoll, da er neben den Vorteilen einer konsolidierten Bemessungsgrundlage auch deren Anwendung für sämtliche Kapitalgesellschaften innerhalb der Europäischen Union vorschreibt. ${ }^{186}$ Auf diese Weise wären jegliche Verzerrungen, die aus dem Nebeneinander von 25 Steuersystemen herrühren, in der Europäischen Union beseitigt. Die Frage nach der politischen Durchsetzbarkeit ist derzeit wohl zu verneinen, wobei dieser Lösungsvorschlag auch über das ursprüngliche Ziel der Studie hinausgeht, die lediglich eine Beseitigung der bestehenden Investitionshindernisse für die grenzüberschreitende Wirtschaftstätigkeit multinational agierender Unternehmen im Binnenmarkt vorsah. ${ }^{187}$ Entsprechend nahm die Kommission dieses Modell auch nur der Vollständigkeit halber in die Binnenmarktstudie auf. ${ }^{188}$

Die europäische Körperschaftsteuer weist starke Parallelen zur einheitlichen (konsolidierten) Bemessungsgrundlage auf, erfordert jedoch einen stärkeren politischen Willen. So muss neben der Festlegung eines einheitlichen Körperschaftsteuersatzes ${ }^{189}$ möglicherweise auch der institutionelle Rahmen ${ }^{190}$ für die Administration der Steuer auf der Ebene der Europäischen Union geschaffen werden. Die damit verbundenen Schwierigkeiten lassen eine Umsetzung in näherer Zukunft unwahrscheinlich erscheinen. ${ }^{191}$

Eine Sonderstellung nimmt die Besteuerung im Sitzland ein. Zwar gilt sie offiziell als umfassender Lösungsansatz, der eine realistische und wirksame Option für den Umgang mit den spezifischen Problemen der KMU bei der Befolgung der Steuervorschriften im Binnenmarkt darstellt. Gleichzeitig weist

185 Vgl. BR-Drs. 281/1/06 v. 6. 6. 2006.

186 Vgl. Mintz, J., in: ITPF, 2004, S. 227; Schön, W., in: ITPF, 2002, S. 281.

187 Vgl. Westberg, B., in: ET, 2002, S. 330; Oestreicher, A., in: StuW, 2002, S. 352.

$188 \mathrm{Vgl}$. Europäische Kommission, Unternehmensbesteuerung, 2001, S. 505.

189 Vgl. Ruding, O., in: ET, 2002, S. 5.

190 Vgl. Mors, M., in: CESifo Forum, 2002, 1, S. 23; Oestreicher, A., in: StuW, 2002, S. 352.

191 Herr Kovács, EU Kommissar für Steuern, sieht die Finanzierung der Europäischen Union über direkte Steuern derzeit als nicht konsensfăhig an. Vgl. Rickman, J., in: TNI, 2006, S. 959. 
selbst die Kommission auf die systematischen Defizite ${ }^{192}$ dieses Vorschlags hin und spricht ihm, wie auch seine Autoren, lediglich einen temporären Charakter $\mathrm{zu}^{193}$

Im Ergebnis wird vonseiten der Wirtschaft ${ }^{194}$, in der Literatur ${ }^{195}$ und seitens der Europäischen Kommission ${ }^{196}$ die einheitliche (konsolidierte) Körperschaftsteuer-Bemessungsgrundlage langfristig als der erfolgversprechendste Ansatz betrachtet. ${ }^{197}$ Durch das Fortbestehen der 25 nationalen Steuervorschriften erscheint die politische Umsetzung als ein ambitioniertes, aber erreichbares Ziel. ${ }^{198}$ Wesentliche Zweifel richten sich nicht speziell gegen die einheitliche (konsolidierte) Körperschaftsteuer-Bemessungsgrundlage. Vielmehr werden in der wissenschaftlichen Diskussion allgemein die mit einer harmonisierten Besteuerung verbundenen Wohlfahrtsgewinne in Frage gestellt. ${ }^{199}$ Denn durch den

192 Besonders kritisch wird die Mischung aus kapitalimport- und kapitalexportneutraler Besteuerung gesehen. Vgl. hierzu McLure Jr., C.E., in: CESifo Forum, 2005, 1, S. 36-37; Gammie, M./Radaelli, C.M./Klemm, A., Reform, 2001, S. 44-46; Lodin, S.O./Gammie, M., Home, 2001, S. 64.

193 Europäische Kommission, Non-Paper, 2004b; Lodin, S.O./Gammie, M., Home, 2001, S. 20.

194 Gemäß einer Umfrage von Bravenec aus dem Jahr 2000/2001 befürworten 88,9 v. H. der Befragten eine Koordination der Gewinnermittlung in Europa. Eine formelhafte Gewinnaufteilung lehnen nur 37 v. H. ab. Vgl. Bravenec, L., in: ET, 2001, S. 428-429; derselbe, in: $E T, 2000$, S. 463.

195 Vgl. für viele Oestreicher, A., in: StuW, 2002, S. 353; Giannini, S., in: CESifo Forum, 2002, 1, S. 26-29; Ruding, O., in: ET, 2002, S. 5; derselbe, in: EC Tax Review, 2005, S. 3; Gammie, M./Radaelli, C.M./Klemm, A., Reform, 2001, S. 54; UNICE, Memorandum, 2000, S. 11; Spengel, C. in: Lüdicke, Steuerrecht, 2004, S. 155; derselbe, in: ifo Schnelldienst, 2004, 13, S. 6. Darüber hinaus wird die Akzeptanz der einheitlichen (konsolidierten) Körperschaftsteuer-Bemessungsgrundlage in der wissenschaftlichen Diskussion deutlich, indem insbesondere jüngere Veröffentlichungen sich ausschließlich diesem Vorschlag widmen. Vgl. Weiner, J.M., Company, 2006, S. 33-107; Cerioni, L., in: ET, 2006, S. 187-196; Mintz, J.M./Weiner, J.M., in: ITPF, 2003, S. 695-711; Mintz, J.M., in: ITPF, 2004, S. 227.

196 Auch die Europäische Kommission hat ihre Meinung konkretisiert. Im Jahr 2002 favorisierte sie noch keine von den vier umfassenden Maßnahmen. Vgl. Mors, M., in: CESifo Forum, 2002, 1, S. 23. In ihrer Mitteilung vom 24. 11. 2003 wird ausschließlich nur noch die Sitzlandbesteuerung und die einheitliche (konsolidierte) KörperschaftsteuerBemessungsgrundlage erwähnt, wobei letztere langfristig das angestrebte Ziel darstellt. Vgl. Europäische Kommission, Binnenmarkt, 2003, S. 12-15, 22-27.

197 Vgl. Weiner, J.M., in: CESifo Forum, 2002, 1, S. 12; McLure, Jr. C.E., in: CESifo Forum, 2005, 1, S. 33; Cnossen, S., in: FA, 2001, S. 533; Europäische Kommission, Binnenmarkt, 2003, S. 12-25.

198 Vgl. Ruding, O., in: ET, 2002, S. 5.

199 Vgl. hierzu Cnossen, S., in: FA, 2001, S. 535. Mintz, J.M., in: CESifo Forum, 2002, 1, S. 8; Fuest, C./Huber, B., in: Vierteljahrhefte zur Wirtschaftsforschung, 2003, S. 89; 
Verzicht der umfassenden Lösungsansätze auf eine Vereinheitlichung der Steuersätze geht wohl der wichtigste Vorteil der steuerlichen Koordinierung, die Beseitigung der Verzerrung der Realinvestitionen durch die Besteuerung, verloren. ${ }^{200}$ Berechnungen von „Copenhagen Economics“ zeigen jedoch, dass allein die Harmonisierung der Bemessungsgrundlage ohne eine konsolidierte Besteuerung sich positiv auf das Bruttoinlandsprodukt und die Wohlfahrt auswirkt. $^{201}$

\section{Einheitliche (konsolidierte) Körperschaftsteuer-Bemessungsgrundlage}

\section{1. Überblick}

Auch wenn das Modell der einheitlichen (konsolidierten) KörperschaftsteuerBemessungsgrundlage in seinen Grundzügen bekannt ist, verbleibt eine Vielzahl offener Fragen hinsichtlich der konkreten Ausgestaltung des Lösungsansatzes. ${ }^{202}$ Auf der Ebene der Europäischen Union richteten sich die Anstrengungen der vergangenen Jahre vornehmlich auf die Schaffung einer einheitlichen Bemessungsgrundlage. ${ }^{203}$ Erst in jüngerer Vergangenheit wurden mögliche Gestaltungsalternativen einer Konzernbesteuerung erörtert. Auch in der Literatur sind Beiträge rar, die sich mit der Konsolidierungstechnik, dem Umfang des Konsolidierungskreises sowie der Ausgestaltung des Aufteilungsmaßstabs auseinandersetzen. Dabei liegt in der Festlegung dieser Kriterien, insbesondere der Definition des Aufteilungsmaßstabes, die politische Brisanz. ${ }^{204}$ Ziel des folgen-

Osterweil, E., in: ET, 2002, S. 274; Gassner, W., in: ET, 2002, S. 320-321; Weiner, J.M., in: TNI, 2002, S. 519.

${ }^{200}$ Vgl. Fuest, C./Huber, B., in: Vierteljahrhefte zur Wirtschaftsforschung, 2003, S. 89; Mintz, J.M., in: CESifo Forum, 2002, 1, S. 33; Fuest, C., Coordination, 2002, S. 12-13.

201 Die Zuwăchse der Studie werden insoweit unterschătzt, als keine Anpassungen hinsichtlich der Abnahme der Befolgungskosten berücksichtigt wurden. Vgl. Jensen, J./Svensson, P., Cooperation, 2004, S. 22-27, 38-39.

202 Auch Weichenrieder betont den Forschungsauftrag der Binnenmarktstudie. Vgl. Weichenrieder, A., in: ifo Schnelldienst, 2002, 2, S. 11.

${ }^{203}$ Die EU-Kommission hat ihre Arbeiten bzgl. der Konsolidierung und der Aufteilung der Bemessungsgrundlage 2006 begonnen. Ferner sollten die Untergruppen „Abschreibung“, „Rücklagen, Rückstellungen und Schulden“ und „steuerbares Einkommen“ und „internationale Aspekte“ ihre Arbeit bis Ende 2006 abgeschlossen haben. Vgl. Europăische Kommission, Umsetzung, 2006, S. 7, 15-19.

204 So weist Radulescu richtigerweise auf die enormen Schwierigkeiten bei der Wahl eines adäquaten einheitlichen Aufteilungsschlüssels hin. Vgl. Radulescu, D.M., in: ifo Schnelldienst, 2002, 2, S. 15-16. Auch Hey betont die enormen Schwierigkeiten, sich innerhalb der EU auf einen Zerlegungsschlüssel für das Steueraufkommen einigen zu müssen, und 
den Kapitels ist es daher, den derzeitigen Stand der Diskussion im Schrifttum und auf der Ebene der Europäischen Kommission wiederzugeben und zu erweitern. Daneben werden mögliche Fragestellungen, wie z. B. die Festsetzung der Mindestbeteiligungsquote, aufgezeigt, zu deren Lösung die empirische Analyse der Konzernstrukturen und die Quantifizierung der Auswirkungen der Einführung einer konsolidierten Steuerbemessungsgrundlage in der Europäischen Union beitragen. Die Ausgestaltung der Gewinnermittlungsvorschriften ist nicht Bestandteil des folgenden Abschnitts. Insbesondere zu der Option, die IFRS als Ausgangspunkt für eine steuerliche Gewinnermittlung zu nutzen, ist im Schrifttum bereits eine Vielzahl von Beiträgen erschienen.

\section{Abgrenzung des Konsolidierungskreises}

\section{a) Sachliche Abgrenzungskriterien}

\section{(a) Rechtsformen}

Die Forderung nach einem gerechten Steuersystem zielt vordergründig auf eine Gleichbehandlung ab, die sich aus Art. 3 GG ableitet. Nach herrschender Meinung ist diese durch ein die Entscheidungen des Steuerpflichtigen nicht beeinflussendes, also neutrales Steuersystem gewährleistet. ${ }^{205}$ Auch die Rechtsformneutralität kann diesem allgemeinen Postulat der Entscheidungsneutralität subsumiert werden. ${ }^{206}$ Auf europäischer Ebene ergibt sich die Rechtsformneutralität aus der Niederlassungsfreiheit, ${ }^{207}$ wobei das Gebot zur Gleichbehandlung nur für körperschaftsteuerliche Investoren abgeleitet werden kann. ${ }^{208}$

Um eine neutrale Unternehmensbesteuerung innerhalb der Europäischen Union sicherzustellen, müssen sowohl Kapitalgesellschaften als auch Personengesellschaften in den Genuss der einheitlichen (konsolidierten) Körperschaftsteuer-

geht deshalb von reduzierten Chancen aus, dass es tatsächlich zu weiteren, von allen 25 Mitgliedstaaten konsentierten Harmonisierungsfortschritten kommt. Vgl. Hey, J., in: StuW, 2004, S. 206.

205 Vgl. Oestreicher, A., in: Oestreicher, Konzernbesteuerung, 2005, S. 12-13.

206 Vgl. zu den Neutralitätspostulaten König, R./Wosnitza, M., in: Steuerwirkungslehre, 2004, S. 142-144. Kritisch zum Postulat der Rechtsformneutralität Wagner, F., in: StuW, 2006, S. 102.

207 So macht der EuGH bereits in seinem Urteil zur Rechtssache Avoir-Fiscal deutlich, dass durch diskriminierende steuerliche Bestimmungen die freie Wahl der Rechtsform nicht eingeschränkt werden darf. EuGH v. 28. 1. 1986, Rs. 270/83 - Avoir-Fiscal, in: EuGHE 1986, Rn. 22. Jedoch macht Lang deutlich, dass der Art. 43 EGV sich nicht zu einem absolut wirkenden Gebot der Rechtsformneutralität verselbstständigt hat. Vgl. Lang, M., in: IStR, 2006, S. 397-402.

208 Vgl. Jacobs, O.H. (Hrsg.), Unternehmensbesteuerung, 2002, S. 196. 
Bemessungsgrundlage kommen. Zugleich lassen sich damit Gestaltungsoptionen verhindern, durch die Unternehmen über die Wahl der Rechtsform ihren Einbezug in den Konsolidierungskreis bewusst steuern könnten. Schließlich würde ein Ausschluss von Personengesellschaften verstärkt Länder wie z. B. Deutschland benachteiligen, in denen ein wesentlicher Anteil der Gesellschaften als Personengesellschaft firmiert und sogar multinationale Unternehmensgruppen sich derartiger Rechtsformen bedienen. ${ }^{209}$ Trotz dieser klaren steuersystematischen Vorgaben erwägt die Europäische Union, Personengesellschaften und Kapitalgesellschaften, die transparent besteuert werden, von der gemeinsamen (konsolidierten) Körperschaftsteuer-Bemessungsgrundlage auszuschließen, da die steuerliche Behandlung solcher Gesellschaften viele ungelöste Fragen aufwirft. ${ }^{210}$ Denn zum einen bedeutet eine Ausweitung der Harmonisierungsbestrebungen auf Einkommensteuersubjekte einen weiteren Eingriff in die Fiskalautonomie der Mitgliedstaaten. ${ }^{211}$ Zum anderen würde eine Berücksichtigung von Kapital- und Personengesellschaften rechtsformabhängige Besteuerungsunterschiede in die Konzernbesteuerung verlagern. Dieses Problem wäre zwar zu umgehen, indem die Einbeziehung einer Personengesellschaft in den Konsolidierungskreis davon abhängig gemacht wird, dass diese zuvor zur Körperschaftsteuer optiert. Da aber innerhalb der Europäischen Union ein derartiges "Check-the-Box"-Wahlrecht in den nationalen Steuersystemen die Ausnahme darstellt ${ }^{212}$ und ein zusätzlicher Verlust der Fiskalautonomie für die Mitgliedstaaten indiskutabel wäre, erscheint eine Beschränkung auf Kapitalgesellschaften trotz der methodischen Einwände sinnvoll. Konkret könnte auf die erweiterte Liste der im Anhang der Mutter-Tochter-Richtlinie aufgeführten Rechtsformen abgestellt werden, wobei abzuwägen wäre, inwieweit auch die dort aufgeführten, der Körperschaftsteuer unterliegenden Personengesellschaften $\mathrm{zu}$ berücksichtigen sind. ${ }^{213}$

209 Vgl. Schön, W., in: ET, 2002, S. 280. Gemäß der Gewerbesteuerstatistik 2001 beträgt der Anteil der Kapitalgesellschaften unter den Gewerbebetrieben lediglich 12,2 v. H., was aber gleichzeitig einem Anteil von 50,4 v. H. des kumulierten Steuermessbetrags entspricht. Beispiele für multinationale Unternehmensgruppen, deren Dachgesellschaft eine Personengesellschaft ist, sind die Dr. August Oetker KG oder die Tengelmann Warenhandelsgesellschaft KG.

210 Vgl. Europäische Kommission, Persönlicher Anwendungsbereich, 2006, S. 5.

$211 \mathrm{Vgl}$. Schön, W., in: ET, 2002, S. 280.

212 In Frankreich können beispielsweise Personengesellschaften zur „impôt sur les sociétés“ optieren. Vgl. Frank, R., Option, 2002, S. 152-159. Auch für Deutschland wurde ein derartiges Modell im Rahmen der Unternehmenssteuerreform diskutiert. Vgl. Herzig, N., in: $D B$, 2006. S. 3. Kritisch dazu Lang, J., in: GmbHR, 2000, S. 459-462 m. w. N.; Tipke, K., Steuerrechtsordnung, 2003, S. 1202 m. w. N.

213 Vgl. die Übersicht im Anhang der Richtlinie 90/435/EWG des Rates v. 23. 7. 1990, Abl. Nr. L 225 v. 20. 8. 1990, S. 6-9. Zu den Problemen einer expliziten Liste und anderen 


\section{(B) Wirtschaftliche Abgrenzungskriterien}

Nachdem im vorherigen Abschnitt erörtert wurde, welche Rechtsformen Bestandteil eines Konsolidierungskreises sein könnten, werden nun die Anforderungen an die Beziehungen der Unternehmen innerhalb eines Konsolidierungskreises diskutiert. Grundsätzlich sollen nur die rechtlichen Einheiten zum Konsolidierungskreis zählen, die gemeinsam eine wirtschaftliche Einheit bilden, wobei sich zwei Ansätze zur Bestimmung des Konsolidierungskreises unterscheiden lassen. Einerseits kann der Konsolidierungskreis ausschließlich auf der Basis rechtlicher Merkmale wie der Höhe des Beteiligungsbesitzes oder des Stimmrechtsanteils abgegrenzt werden. ${ }^{214}$ Kritiker sehen in den genannten Merkmalen zwar ein Indiz für eine wirtschaftliche Einheit, befürworten aber eine weitergehende Prüfung. Für sie stehen die wirtschaftlichen Gegebenheiten wie die Integration betrieblicher Funktionen und eine zentrale Führung im Vordergrund. Dementsprechend wird der Konsolidierungskreis andererseits nach wirtschaftlichen Gesichtspunkten bestimmt.

Methodisch korrekt ist der ökonomische Ansatz. ${ }^{215}$ Eine Vielzahl rechtlich selbstständiger Subjekte soll nur dann gemeinsam veranlagt werden, wenn diese eine wirtschaftliche Einheit bilden. Herrscht soweit noch Einigkeit, so differieren bereits bei der Definition einer wirtschaftlichen Einheit die Meinungen. Denn objektiv überprüfbare Kriterien festzulegen, erscheint schwierig bzw. unmöglich. ${ }^{216}$ Wenn auch hiermit bereits ein entscheidender Einwand gegen die Anwendung wirtschaftlicher Abgrenzungskriterien hervorgebracht wird, sollen dennoch im Folgenden unterschiedliche Definitionen eines „unitary business“ vorgestellt werden, die entweder von US-amerikanischen Gerichtshöfen entwickelt wurden oder Bestandteil der diesbezüglichen wissenschaftlichen Diskussion sind.

Eine exakte Definition des „unitary business" existiert in den Vereinigten Staaten nicht ${ }^{217}$ und wird auch ausdrücklich abgelehnt. ${ }^{218}$ Stattdessen haben sich

Alternativen, den Anwendungsbereich festzulegen, vgl. Europäische Kommission, Persönlicher Anwendungsbereich, 2006, S. 3-4.

214 In der Literatur wird vielfach unter der rechtlichen Definition des Konsolidierungskreises nur die Abgrenzung anhand des Beteiligungskapitals verstanden. Da sich die Höhe des Beteiligungskapitals und die Stimmrechtsanteile einander in der Regel entsprechen, werden beide Merkmale den rechtlichen Abgrenzungskriterien subsumiert.

215 Vgl. Jacobs, O.H./Spengel, C./Schäfer, A., in: intertax, 2004, S. 277-278.

${ }^{216} \mathrm{Vgl}$. Corrigan, E., in: TN, 1980, S. 510; Hellerstein, W./McLure Jr., C.E., in: ITPF, 2004, S. 204-205; Schäfer, A./Spengel, C., ICT, 2003, S. 21.

217 Grundsätzlich finden sich die Leitlinien der Besteuerung durch die Staaten im Uniform Division of Income for Purposes Act, der aber keine Informationen hinsichtlich der Abgrenzung eines Unitary Business enthält. 
im Laufe der Jahre drei wesentliche Prüfverfahren der Gerichte herauskristallisiert. ${ }^{219}$

1. Der „Three Unity Test“ des kalifornischen Supreme Court ${ }^{220}$ erfordert einheitliche Beteiligungsverhältnisse, einheitliches Handeln und eine einheitliche Führung. Eine wirtschaftliche Einheit wird dann angenommen, wenn die Muttergesellschaft über die einfache Stimmrechtsmehrheit verfügt, zentrale Betriebsabteilungen für Marketing oder Einkauf existieren und eine zentrale Führungsinstanz für die strategische Planung verantwortlich ist. ${ }^{221}$

2. Der „Contribution or Dependency Test ${ }^{4222}$ ist eine Weiterentwicklung des „Three Unity Test“, der im Wesentlichen die Vorschläge von Altman und Keesling übernimmt. ${ }^{223}$ Deren Meinung nach zeichnet sich eine wirtschaftliche Einheit durch die wechselseitige Abhängigkeit und gegenseitige Förderung von betrieblichen Prozessen aus. ${ }^{224}$

3. Der US Supreme Court stellte erstmals in seiner Entscheidung Mobil Oil v. Commissioner of Taxes ${ }^{225}$ einen Kriterienkatalog zur Definition einer wirtschaftlichen Einheit auf. Diese wird bei einer zentralen Unternehmensführung, der funktionellen Eingliederung der Konzerngesellschaften und dem Erzielen von Skaleneffekten angenommen. ${ }^{226}$ Wenn auch mit abweichender Gewichtung der Einzelaspekte, hat sich das Gericht seitdem immer auf diese berufen, wenn zu klären war, ob eine wirtschaftliche Einheit vorliegt. ${ }^{227}$

218 Vgl. McLure Jr., C.E., in: TNI, 2004, S. 55; McLure Jr., C.E./Weiner, J.M., in: Cnossen, Capital, 2000, S. 286.

219 Vgl. hierzu a. die Analyse von Bökelmann, J., Gewinnzurechnung, 1997, S. 102-112. Eine vergleichende Übersicht findet sich in Oestreicher, A., Konzerngewinnabgrenzung, 1999, S. 139.

${ }^{220}$ Butler Brothers v. McColgan, 17 Cal. 2d 664, 1941, 111 P. 2d 334, California, 1941.

221 Vgl. hierzu ausführlich Riecker, A., Körperschaftbesteuerung, 1996, S. 191-194; Weiner, J.M., Implementing, 1999, S. 31; dieselbe, in: TNI, 1996, S. 2134; Miller, B.F., in: McLure, State, 1984, S. 140-141.

222 Edison California Stores v. McColgan, 30 Cal. 2d 480, 1947, 183 P 2d 16, California, 1947.

${ }^{223}$ Vgl. Altman, G.T./Keesling, F.M., Income, 1946; Weiner, J.M., Implementing, 1999, S. 31-23.

224 Vgl. Oestreicher, A., Konzerngewinnabgrenzung, 1999, S. 138; Riecker, A., Körperschaftbesteuerung, 1996, S. 190-191; McLure Jr., C.E./Weiner, J.M., in: Cnossen, Capital, 2000, S. 265; Weiner, J.M., in: TNI, 1996, S. 2145.

225 Mobil Oil v. Commissioner of Texas of Vermont, 445 U.S. 425, 1980, S. 439.

226 Vgl. McLure Jr., C.E./Weiner, J.M., in: Cnossen, Capital, 2000, S. 265; Oestreicher, A., Konzerngewinnabgrenzung, 1999, S. 138.

227 Asarco Inc. v. Idaho State Tax Commission, 458 U.S. 307, 1982; F.W. Woolworth Corp. v. Taxation and Revenue Department of New Mexico, 458 U.S. 354, 1982, S. 364; Container Corp. of America v. Franchise Tax Board, 463 U.S. 159, 1983, S. 179; Al- 
Im Schriftum wird dem „Three-Stage Test“ von McLure und dem Interdependenzansatz von Hellerstein besondere Aufmerksamkeit geschenkt.

Nach Auffassung von McLure liegt eine wirtschaftliche Einheit dann vor, wenn eine direkte Gewinnabgrenzung mittels fremdvergleichskonformer Verrechnungspreise scheitert. Er prüft in drei Schritten, ob eine direkte Gewinnabgrenzung möglich ist. Dies ist dann nicht der Fall, wenn folgende Tatbestände kumulativ erfüllt sind: ${ }^{228}$

1. Das betreffende Unternehmen ist in ein Gesamtunternehmen finanziell oder organisatorisch eingegliedert.

2. Unternehmensteile sind wirtschaftlich in das Gesamtunternehmen eingegliedert. Als Indizien dafür werden Skalenerträge, vertikale Integration und unternehmensinterner Wertetransfer gesehen.

3. Die festgestellte wirtschaftliche Verflechtung ist wesentlicher Art.

Der Ansatz von Hellerstein sieht eine wirtschaftliche Einheit durch wechselseitige Interdependenzen zwischen den Unternehmenseinheiten begründet. ${ }^{229}$ Diese sind gegeben, wenn mehrere Unternehmenseinheiten wesentlich an einem Wertschöpfungsprozess beteiligt sind, wobei die Definition nur auf primäre Funktionen wie den Handel und die Produktion abstellt. Die Unternehmensbereiche des zentralen Managements, des Rechnungswesens, des Personalwesens oder des Marketings werden bei der Beurteilung explizit ausgeschlossen. Von einer wesentlichen Interdependenz ist auszugehen, wenn über ein Viertel bis ein Drittel der Lieferungen und Leistungen zwischen verbundenen Unternehmen erfolgt. ${ }^{230}$

Unabhängig von dem ausgewählten Testverfahren verbleibt stets ein subjektives Element bei der Bestimmung einer wirtschaftlichen Einheit. ${ }^{231}$ Das wird besonders deutlich durch die Wesentlichkeitsforderung der beiden zuletzt genannten Ansätze. ${ }^{232}$ Auch die vage Konkretisierung von Hellerstein kann dabei lediglich als Anhaltspunkt dienen. Der Supreme Court fasst diese Problematik folgendermaßen zusammen:

lied-Signal, Inc. v. Director, Division of Taxation, 504 U.S. 768, 1992 und zuletzt Barclays Bank PLC v. Franchise Tax Board of California, 512 U.S. 298, 1994. Vgl. a. Riecker, A., Körperschaftbesteuerung, 1996, S. 199-200.

228 McLure Jr., C.E., in: McLure, State, 1984, S. 106-108.

229 Vgl. Hellerstein, J.R., in: NTJ, 1968, S. 501-503.

${ }^{230}$ Hellerstein ging zunächst nur von einer wesentlichen Interdependenz aus und ergänzte seinen Ansatz spăter um diese Schwellenwerte mit dem Ziel, die Administration zu erleichtern. Vgl. Hellerstein, J.R., in: TN, 1982, S. 166.

231 Vgl. Europäische Kommission, Fragen zur Konzernbesteuerung, 2006, S. 5.

232 Vgl. Riecker, A., Körperschaftbesteuerung, 1996, S. 199-200. So auch Weiner, wenn sie die nachträgliche Konkretisierung der Wesentlichkeitsforderung von Hellerstein als Vorteil würdigt. Vgl. Weiner, J.M., Implementing, 1999, S. 32. 
„A final point that needs to be made about the unitary business concept is that it is not, so to speak, unitary: there are variations on the theme, and any number of them are logically consistent with the underlying principals motivating the approach. ${ }^{\text {“ }} 233$

\section{( $\gamma$ ) Rechtliche Abgrenzungskriterien}

Die Überlegung, den Konsolidierungskreis auf der Basis rechtlicher Merkmale abzugrenzen, resultiert aus den Schwierigkeiten, eine wirtschaftliche Einheit objektiv zu definieren. ${ }^{234}$ Demgegenüber ist die Bestimmung des steuerlichen Konsolidierungskreises anhand der Höhe des Beteiligungsbesitzes oder der Anzahl der Stimmrechte leicht und rechtssicher nachprüfbar. ${ }^{235}$ Die größere Transparenz der Abgrenzung erhöht dabei die Steuergerechtigkeit, da spezifische Einzelfallentscheidungen entfallen. Zudem ist mit geringeren Befolgungskosten sowohl für die Unternehmen als auch für die Steuerverwaltung zu rechnen, denn die Objektivität der Abgrenzungsmerkmale erlaubt eine vereinfachte Administration. Sieht man die Rechtfertigung einer konsolidierten Besteuerung auch in den systematischen Einwänden gegenüber einer fremdvergleichskonformen Gewinnabgrenzung bei verbundenen Unternehmen, ist die Beherrschung der Tochtergesellschaften die notwendige Bedingung für das Vorliegen einer wirtschaftlichen Einheit. Denn die Kritik am Grundsatz des Fremdvergleichs richtet sich vornehmlich gegen die fehlende Berücksichtigung der durch die Integration bewirkten Skalenvorteile, Synergieeffekte und Einsparungen von Transaktionskosten. ${ }^{236} \mathrm{Um}$ diese jedoch zu erzielen, ist gemäß der Transaktionskostentheorie eine Beherrschung des verbundenen Unternehmens Grundvoraussetzung, denn erst dann tritt an die Stelle der Koordination über Verträge eine hierarchische Beziehung. ${ }^{237}$ Ist eine Beherrschung der Tochtergesellschaft hingegen nicht gegeben, muss in der Regel auch eine wirtschaftliche Unabhängigkeit angenommen werden, da die Unternehmen bestrebt sind, ausschließlich ihren eigenen steuerlichen Gewinn zu minimieren. ${ }^{238}$ Schließlich stellt die Mehrzahl der Mitgliedstaaten bereits jetzt im Rahmen der nationalen Gruppenbesteuerungssysteme auf die Stimmrechte oder den Be-

233 Container Corp. of America v. Franchise Tax Board, 463 U.S. 159, 1983, S. 167.

${ }^{234}$ Vgl. Corrigan, E., in: TN, 1980, S. 510; Hellerstein, W./McLure Jr., C.E., in: ITPF, 2004, S. 205.

235 Vgl. Weiner, J.M., Group Taxation, 2005, S. 27; McLure Jr., C.E./Weiner, J.M., in: Cnossen, Capital, 2000, S. 265-266; Corrigan, E., in: TN, 1980, S. 510.

236 Vgl. Jacobs, O.H. (Hrsg.), Unternehmensbesteuerung, 2002, S. 875-877 m. w. N.

237 Gemäß der Transaktionskostentheorie liegt die vertikale Integration von Unternehmen in der Einsparung von Transaktionskosten begründet. Vgl. Oestreicher, A., Konzerngewinnabgrenzung, 1999, S. 90-93 m. w. N.

238 Vgl. McLure Jr. C.E., in: McLure, State, 1984, S. 103-104. 
teiligungsbesitz $a b$, sodass diesbezüglich mit einer größeren politischen Akzeptanz zu rechnen ist. ${ }^{239}$

In dem wesentlichen Vorteil dieses Ansatzes, der Objektivität und der damit verbundenen Einfachheit, liegt zugleich eine große Gefahr. Eine gezielte Steuerung der Stimmrechtsanteile bzw. der Beteiligungshöhe ermöglicht es Unternehmen, den Konzernkreis entsprechend ihren Anforderungen zu erweitern bzw. zu verkleinern. ${ }^{240}$ Zum Beispiel könnte eine Konzerngesellschaft mit Sitz in einem Niedrigsteuerland die geforderte Beteiligungsquote bzw. den Stimmrechtsanteil kurzfristig erfüllen, um Verluste verrechnen zu können und auf diese Weise die konsolidierte Bemessungsgrundlage zu schmälern. Die Gewinne dieses Unternehmens hingegen unterlägen weiterhin der niedrigeren Besteuerung, da in den entsprechenden Perioden die Abgrenzungskriterien verfehlt werden würden. ${ }^{241}$

Um diesen Gestaltungsrahmen zu begrenzen, sollten die rechtlichen $\mathrm{Ab}$ grenzungskriterien durch weitere Komponenten ergänzt werden. Denkbar wäre eine zeitliche Komponente, nach welcher eine Konsolidierung erst nach einem festzulegenden Zeitraum möglich wäre bzw. die Unterschreitung der Beteiligungsquote innerhalb dieses Zeithorizonts rückwirkend die einbezogene Gesellschaft aus dem Konsolidierungskreis ausschlösse. Darüber hinaus diente eine Mindestbesitzdauer der Abgrenzung von kurzfristigen Vermögensanlagen, die ihrem Wesen nach nicht Bestandteil einer wirtschaftlichen Einheit sein können. Die Festlegung der Länge des Zeitraums ist eine politische Entscheidung, die so zu treffen ist, dass auf der einen Seite dem Missbrauch vorgebeugt, zugleich aber den Konzernen eine flexible, effiziente und kurzfristige Anpassung ihrer Organisationsstrukturen an die Marktgegebenheiten erlaubt wird. Auch Hellerstein und McLure befürworten, dass weitere Anforderungen an die Definition des Konzernkreises gestellt werden, um Missbrauchstatbeständen vorzubeugen, und plädieren für einen Mindestanteil an Umsatzerlösen, die aus Transaktionen der Gruppenmitglieder untereinander hervorgehen. ${ }^{242}$

239 Vgl. Kapitel B.III.2.

240 Vgl. Lannoo, K./Levin, M., EU Tax, 2002, S. 18; Westberg, B., in: ET, 2002, S. 328; McLure Jr., C.E./Weiner, J.M., in: Cnossen, Capital, 2000, S. 265-266; Hellerstein, W./McLure Jr., C.E., in: ITPF, 2004, S. 205. Auch Schön weist auf die Problematik des sich immerwährend verändernden Konsolidierungskreises durch Anpassung der Beteiligungsquoten hin. Vgl. Schön, W., in: ET, 2002, S. 281.

241 Vgl. Weiner, J.M., Apportionment, 2002, S. 6. Der U.S. Supreme Court führte dieses Argument als Ablehnung sogenannter „bright-line“-Tests an. Siehe Allied-Signal Inc. v. Director, Division of Taxation, 504 U.S. 768, 1992 und Mobil Oil Corp. v. Commissioner of Taxes of Vermont, 445 U.S. 425, 1980.

${ }^{242}$ Vgl. Hellerstein, W./McLure Jr., C.E., in: ITPF, 2004, S. 205. 
Die bisherigen Ausführungen sind implizit von einer Übereinstimmung der Beteiligungskapitalquote mit den Stimmrechtsanteilen ausgegangen. Zwar mag diese Kongruenz dem Regelfall entsprechen, doch sind durch stimmrechtslose Kapitalanteile Differenzen zwischen der Beteiligungsquote und dem Stimmrechtsanteil eines Gesellschafters möglich. Darüber hinaus können andere rechtliche, von den Beteiligungsverhältnissen unabhängige Beherrschungsmöglichkeiten bestehen. Somit ist zu prüfen, ob für die Abgrenzung des Konsolidierungskreises auf die Kapitalbeteiligung oder den Stimmrechtsanteil abzustellen ist und inwieweit weitere Beherrschungsmöglichkeiten in die Beurteilung einfließen sollten.

Betrachtet man die Abgrenzung des Konsolidierungskreises anhand rechtlicher Kriterien als Second-best-Lösung, deren Relevanz durch die fehlende objektive Überprüfbarkeit der First-best-Lösung, der Abgrenzung auf der Basis wirtschaftlicher Integrationsindikatoren, begründet wird, muss auf die Anzahl der Stimmrechte abgestellt werden. Denn zwischen der wirtschaftlichen Integration und der Beteiligungshöhe besteht kein direkter Zusammenhang. Sowohl eine vollständige finanzielle Eingliederung einer Tochtergesellschaft ohne wirtschaftliche Integration ${ }^{243}$ als auch eine wirtschaftliche Einheit bei fehlender einfacher Mehrheitsbeteiligung sind denkbar. ${ }^{244}$ Demgegenüber ist eine mögliche Einflussnahme auf die Geschäftsentscheidungen zumindest die notwendige Bedingung für das Vorliegen einer wirtschaftlichen Einheit. ${ }^{245}$ Wie die Stimmrechtsmehrheit bzw. die Beherrschung der Untergesellschaft erreicht wird, ist vor diesem Hintergrund zunächst unerheblich. Insofern ist grundsätzlich eine Anlehnung an das Control-Konzept der IAS zu begrüßen ${ }^{246}$, das ausschließlich auf die Beherrschung des Tochterunternehmens abstellt, welche gemäß IAS 27.13 auch bei fehlender Stimmrechtsmehrheit vorliegen kann. ${ }^{247}$ Durch die

243 Beispiele hierfür sind Beteiligungen, die als Anlage gehalten werden, oder zwei unterschiedliche Geschäftsbereiche, die unter einer Finanzholding gebündelt werden.

244 Vgl. McLure Jr., C.E./Weiner, J.M., in: Cnossen, Capital, 2000, S. 265-266; Weiner, J.M., Group Taxation, 2005, S. 27-28.

245 McLure drückt diesen Zusammenhang eindeutig aus, indem er sagt: „If the division of income between two companies is to be manipulated $[\ldots]$, it is generally necessary that the two firms be under common control. Where common control is lacking, economic interdependence ordinarily should not lead to a conclusion that a unitary business exists [...]" McLure Jr., C.E., in: McLure, State, 1984, S. 103.

246 Die Anlehnung an das Control-Konzept wäre vorteilhaft, da eine Umsetzung innerhalb der Europäischen Union bereits im Rahmen der 7. EG Richtlinie angewendet wird. Siehe Siebente Richtlinie 83/349/EWG des Rates v. 13. 6. 1983, Abl. Nr. L 193 v. 18. 7. 1983, S. 1-17. Vgl. a. Schön, W., in: ET, 2002, S. 281.

247 Eine Beherrschung liegt ebenfalls vor, sofern die Möglichkeit gegeben ist, (a) über mehr als die Hälfte der Stimmrechte kraft einer mit anderen Anteilseignern abgeschlossenen Vereinbarung zu verfügen, (b) die Finanz- und Geschäftspolitik eines Unternehmens gemäß einer Satzung oder einer Vereinbarung zu bestimmen, (c) die Mehrheit der Mit- 
völlige Loskopplung von der Höhe der Beteiligung könnte selbst dann eine Gesellschaft Teil eines Konsolidierungskreises sein, wenn keine einfache Mehrheitsbeteiligung vorliegt. ${ }^{248}$ Dies hätte aber zur Folge, dass auch Ergebnisse von Tochtergesellschaften konsolidiert würden, deren Anteile und somit auch Gewinnansprüche mehrheitlich Gesellschaftern zuzuordnen wären, die nicht zum Konsolidierungskreis gehören. An einer Mehrheitsbeteiligung ist daher ebenfalls zwingend festzuhalten. Im Ergebnis sollte sowohl die finanzielle Eingliederung als auch die Möglichkeit der Beherrschung gegeben sein, wobei letztere nicht ausschließlich über stimmberechtigte Kapitalanteile zu erreichen ist. ${ }^{249}$

Die erforderliche Höhe der Mindestbeteiligung ist zum einen eine politische Entscheidung, die sich an den bestehenden Vorschriften der Gruppenbesteuerungssysteme im Sinne einer erhöhten Akzeptanz orientieren sollte. Zum anderen ist bei einer hohen Beteiligung verstärkt eine wirtschaftliche Integration zu vermuten, sodass die Wahl der erforderlichen Mindestbeteiligung auch von den übrigen Kriterien zur Abgrenzung des Konsolidierungskreises abhängt. Je mehr wirtschaftliche Indikatoren im Rahmen der Abgrenzung des Konsolidierungskreises berücksichtigt werden, also eine Kombination aus wirtschaftlicher und rechtlicher Abgrenzung erfolgt, desto niedriger kann bzw. sollte die Mindestbeteiligungsquote ausfallen. Grundsätzlich ist jede höhere Mindestbeteiligungsquote neben der einfachen Mehrheitsbeteiligung denkbar, wobei mit einer qualifizierten Mehrheitsbeteiligung ( $\geq 75 \mathrm{v}$. H.) und einer vollständigen finanziellen Eingliederung (100 v. H.) weitere Vorteile verbunden sind. Die qualifizierte Mehrheitsbeteiligung bietet sich unter dem Aspekt der Beherrschung an, da sie dem Gesellschafter zumindest im deutschen Gesellschaftsrecht die Einflussnahme auf sämtliche Geschäftsentscheidungen und Entscheidungen mit konstitutivem Charakter ermöglicht. ${ }^{250}$ Außerdem entspricht sie der derzeitigen durchschnittlichen Mindestbeteiligungsquote der nationalen Gruppensteuerungssysteme in der Europäischen Union, sodass von einer breiten Akzeptanz dieses Schwellenwertes auszugehen ist. ${ }^{251}$ Eine vollständige finan-

glieder der Geschäftsführungs- und/oder Aufsichtsorgane zu ernennen oder abzuberufen, wobei die Verfügungsgewalt über das andere Unternehmen bei diesen Organen liegt, oder (d) die Mehrheit der Stimmen bei Sitzungen der Geschäftsführungs- und/oder Aufsichtsorgane oder eines gleichwertigen Leitungsgremiums zu bestimmen, wobei die Verfügungsgewalt über das andere Unternehmen bei diesen Organen liegt. Vgl. IAS 27.13.

Vgl. Schön, W., in: ET, 2002, S. 281.

$249 \mathrm{Zu}$ dem gleichen Schluss kommt auch die Kommission. Vgl. Europäische Kommission, Comments, 2006, S. 2-3.

250 Eine qualifizierte Mindestbeteiligungsquote fordern auch Lodin/Gammie im Rahmen der Sitzlandbesteuenung. Vgl. Lodin, S.-O./Gammie, M., in: ET, 1999, S. 292.

251 Vgl. hierzu Tabelle $8, \frac{1+4 \times 0,95+4 \times 0,9+4 \times 0,75+5 \times 0,5}{18}=0,772$. 
zielle Eingliederung bietet den Vorteil, dass die problematische Behandlung von Minderheitsgesellschaftern entfällt. Dies sei an folgendem Beispiel illustriert:

\begin{tabular}{l} 
Abbildung 1: Behandlung von Minderheitsgesellschaftern \\
\cline { 2 - 10 } \\
\cline { 2 - 9 }
\end{tabular}

Quelle: eigene Darstellung

\section{Beispiel 1:}

Das Unternehmen A ist zu $70 \mathrm{v}$. H. am Unternehmen B beteiligt. Die verbleibenden $30 \mathrm{v} . \mathrm{H}$. am Unternehmen B hält der Minderheitsgesellschafter D. Auch an Unternehmen A sind mehrere Minderheitsgesellschafter beteiligt, wobei hier exemplarisch nur der Anteilseigener $\mathrm{C}$ betrachtet wird. Als Aufteilungsfaktor im Rahmen der formelhaften Gewinnaufteilung dient ausschließlich das Vermögen, das bei beiden Gesellschaften gleich groß ist. Unternehmen A erleidet einen Verlust von $-50 \mathrm{GE}$, und Unternehmen B erzielt zugleich einen Gewinn von $100 \mathrm{GE}$.

Gemäß dem Seperate Accounting muss Unternehmen A in der laufenden Periode keine Steuern entrichten und weist einen Verlustvortrag in Höhe von $50 \mathrm{GE}$ aus. Da dieser die zukünttigen steuerlichen Gewinne der Gesellschaft mindert, entspricht er abgezinst einer Negativsteuer, von der Anteilseigener $C$ gemäß seiner Beteiligung in Höhe von 2,056 GE profitiert. ${ }^{253}$ Der Überschuss des Unternehmens B unterliegt in der laufenden Periode der Körperschaftsteuer von $25 \mathrm{v}$. H., die zu $30 \mathrm{v}$. H. wirtschaftlich von D zu tragen ist. ${ }^{254}$

Die formelhafte Gewinnaufteilung hat zur Folge, dass die reale Steuerbelastung durch den Zinseffekt der vorzeitigen Verlustverrechnung sinkt. Dadurch profitieren aber sowohl der Minderheitsgesellschafter C als auch D. Obwohl das Unternehmen A Verluste erwirtschaftet, muss es in der laufenden Periode Steuern entrichten, die auch der Minderheitsgesellschafter C wirtschaftlich zu tragen hat. Es lässt sich zwar dagegen anführen, dass $\mathrm{C}$ eigentlich eine $\mathrm{Be}-$ teiligung an einer wirtschaftlichen Einheit hält und auch im umgekehrten Fall von Verlustzuweisungen profitieren würde. Rechtlich ist dem aber nicht zuzustimmen, da $\mathrm{C}$ ausschließlich an der juristischen Person A beteiligt ist. Außerdem ergeben sich aufgrund der zeitlichen Verschiebung Zinseffekte, die einen Minderheitsgesellschafter je nach Ertragslage benachteiligen oder bevorzugen. Auch kann man argumentieren, dass die direkte Gewinnabgrenzung nicht der Realität entspricht und deswegen auch die auf der Basis des Fremdvergleichsgrundsatzes ermittelten Überschüsse ein falsches Bild der Ertragslage der Unternehmen widerspiegeln. Dem ist aber entgegenzuhalten, dass die indirekte Methode systembedingt lediglich

252 Die Bewertung des Verlustvortrags erfolgt auf der Basis empirischer Erhebungen. Vgl. hierzu Abschnitt F.II.1.c).

$\begin{array}{ll}253 & -50 \times 0,25 \times 0,2 \times\left(\frac{1}{1,05}\right)^{4}=-2,056 . \\ & 254 \\ & 100 \times 0,25 \times 0,3=7,5 .\end{array}$ 
eine pauschale Zuordnung der Gewinne vornimmt, sodass die zugewiesenen Gewinne nur im Ausnahmefall der Wertschöpfung entsprechen.

Als potenzielle Lösung käme eine anteilige Konsolidierung in Frage, ${ }^{255}$ doch widerspräche diese Vorgehensweise der Grundidee des integrierten Unternehmens. ${ }^{256}$ Auch eine Ausgleichszahlung an die Minderheitsgesellschafter entwickelt eine ähnliche Wirkung, da sie implizit eine anteilige Verlustnutzung bewirkt. Auch wenn vor diesem Hintergrund eine vollständige Eingliederung als Mindestbeteiligungsquote attraktiv erscheint, ist sie ökonomisch nicht zu rechtfertigen. Darüber hinaus besteht die Gefahr einer Zerteilung wirtschaftlicher Einheiten. Dies ließe die Reform der Unternehmensbesteuerung teilweise obsolet werden, denn neben der eingeschränkten Verlustverrechnungsmöglichkeit könnten weiterhin Gewinne durch Verrechnungspreisgestaltungen zwischen zwei Konsolidierungskreisen verlagert werden. ${ }^{257}$ Abbildung 2 zeigt die Entwicklung der Konsolidierungskreise in Abhängigkeit von der Mindestbeteiligungsquote. Erhöht sich die Mindestbeteiligung um fünf Prozentpunkte, steigt durchschnittlich die Anzahl der Konsolidierungskreise grenzüberschreitend tätiger Konzerne um zehn Prozentpunkte.

Abbildung 2: Entwicklung der Anzahl der Konsolidierungskreise in Abhängigkeit von der Mindestbeteiligungsquote

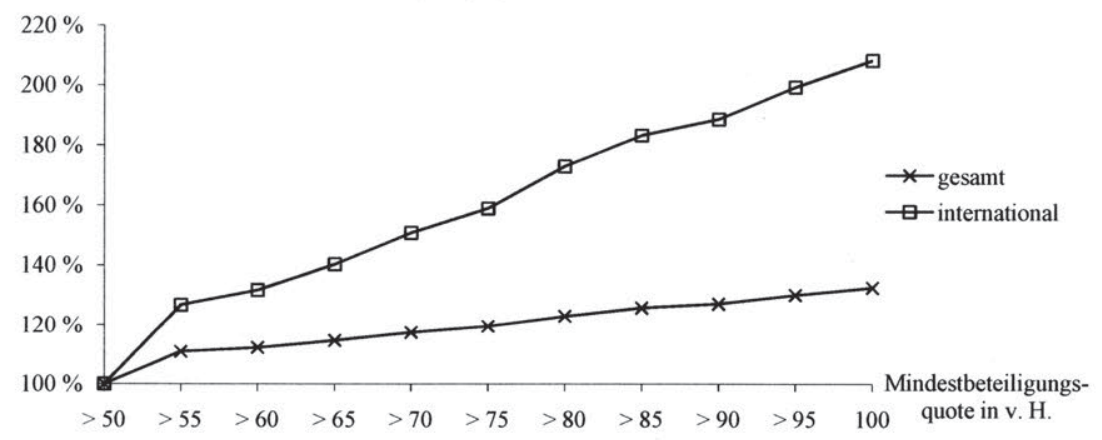

Quelle: eigene Darstellung

Bei der Berechnung der Stimmrechtsanteile oder der Höhe der Beteiligung sind grundsätzlich auch indirekte Beteiligungsbeziehungen zu berücksichtigen. Da vordergründig die Möglichkeit der Einflussnahme auf die Geschäftsent-

\footnotetext{
255 Vgl. Hellerstein, W./McLure Jr., C.E., in: ITPF, 2004, S. 205.

256 Auch die Kommission lehnt eine anteilige Konsolidierung aufgrund der hohen Komplexität ab. Vgl. Europäische Kommission, Comments, 2006, S. 2.

257 Vgl. Weiner, J.M., Apportionment, 2002, S. 6.
} 
scheidungen gegeben sein muss, sollte die Zielgröße additiv berechnet werden. ${ }^{258}$

Die Anwendung der konsolidierten einheitlichen Bemessungsgrundlage ist grundsätzlich fakultativ. Um den steuerplanerischen Gestaltungsspielraum der Unternehmen einzuschränken, ${ }^{259}$ sollten bei Anwendung dieser Regelungen stets alle qualifizierten Konzerngesellschaften miteinbezogen werden (alles oder nichts). ${ }^{260}$

\section{b) Territoriale Abgrenzungskriterien}

Überlegungen zur territorialen Abgrenzung der gemeinsamen (konsolidierten) Körperschaftsteuer-Bemessungsgrundlagen beinhalten zwei Aspekte. Zum einen ist die Frage zu erörtern, welche Gesellschaften bzw. Betriebsstätten Teil des Konsolidierungskreises sind und inwieweit außerhalb des Konsolidierungskreises belegene Konzerneinheiten bei der Abgrenzung zu berücksichtigen sind. Zum anderen ist zu klären, welche Einkommensbestandteile die gemeinsame Bemessungsgrundlage umfasst und wie außereuropäische Einkünfte europäischer Gesellschaften und Einkünfte außereuropäischer Gesellschaften aus europäischen Quellen zu behandeln sind. Auch wenn die Überlegungen zu beiden Fragestellungen eng miteinander verzahnt sind, soll in diesem Abschnitt zunächst auf erstere eingegangen werden. Überlegungen zum Umfang der Bemessungsgrundlage finden sich in Kapitel C.II.3.b).

Grundsätzlich gibt es zwei Ansätze, welche Einzelabschlüsse in das konsolidierte Ergebnis einfließen sollen. Entweder werden nur die Ergebnisse der nach sachlichen Abgrenzungskriterien qualifizierten Gesellschaften (,at water's edge") berücksichtigt, oder die Einzelabschlüsse aller weltweiten Konzernunternehmen sind Bestandteil des konsolidierten Abschlusses. ${ }^{261}$ Letztere Vorgehensweise würde aus Sicht der europäischen Gesellschaften auch gegenüber außereuropäischen Unternehmen eine direkte Gewinnabgrenzung auf der Basis fremdvergleichskonformer Verrechnungspreise durch eine weltweite

258 Bei dieser Berechnung ergibt sich die durchgerechnete indirekte Beteiligung nicht aus der Multiplikation der verknüpfenden Beteiligungen, sondern aus der vollen Zurechnung der Beteiligung, sofern die Obergesellschaft in Höhe der Mindestbeteiligung an der vermittelnden Gesellschaft beteiligt ist. Vgl. a. Europäische Kommission, Comments, 2006, S. 2.

259 Durch eine gezielte Auswahl von Konzerngesellschaften könnten nur Verluste in ein Hochsteuerland hineingetragen werden. Die ausländischen Gewinne hingegen unterlägen weiterhin dem niedrigen Steuersatz im Ausland.

260 Vgl. Europäische Kommission, Fragen zur Konzernbesteuerung, 2006, S. 6-7.

261 Vgl. Weiner, J.M., Company, 2006, S. 70. 
formelhafte Gewinnaufteilung ersetzen. ${ }^{262}$ Damit einher geht aber zunächst ein einseitiger Vertragsbruch der Mitgliedstaaten hinsichtlich der Doppelbesteuerungsabkommen. Grenzüberschreitende Transaktionen zwischen verbundenen Unternehmen sind wie zwischen fremden Dritten abzuwickeln. Dieser Fremdvergleichsgrundsatz findet sich in Art. 9 Abs. 1 des Musterabkommens von OECD und UN ${ }^{263}$ Zwar wäre theoretisch eine aufwendige Neuverhandlung der Doppelbesteuerungsabkommen denkbar; ${ }^{264}$ sie schafft aber nur Abhilfe, wenn auch der Drittstaat die globale Gewinnabgrenzung akzeptiert. Das Nebeneinander von direkter und indirekter Gewinnabgrenzung führt andernfalls in der Mehrzahl der Fälle zu Doppel- und Minderbesteuerungen. ${ }^{265}$ Grenzen nämlich Gesellschaften mit Sitz in einem Drittstaat ihren Gewinn weiterhin direkt anhand eines fremdvergleichskonformen Verrechnungspreises ab und wendet gleichzeitig die komplementäre Gesellschaft der Transaktion eine indirekte Gewinnabgrenzung anhand einer Formel an, so führen beide Methoden in der Regel zu unterschiedlichen Ergebnissen. Es lässt sich theoretisch zeigen, dass die direkte und die indirekte Methode einander entsprechen. ${ }^{266}$ In der Praxis allerdings sind die restriktiven Annahmen der Beweisführung nicht vorzufinden, ${ }^{267}$ sodass eine Übereinstimmung beider Methoden zufälliger Natur ist. ${ }^{268}$ Erfolgt die formelhafte Gewinnaufteilung gemäß dem in den USA weit verbreiteten „supply-demand approach“, der auch der Nachfrageseite einen Besteuerungsanspruch einräumt, ist selbst die theoretische Übereinstimmung beider Methoden nicht mehr gegeben. Geht eine weltweite Konsolidierung darüber hinaus mit einer entsprechenden Verlustverrechnung einher, könnten

262 Für eine ausführliche Beurteilung der Anwendbarkeit der globalen Gewinnaufteilung siehe Bökelmann, J., Gewinnzurechnung, 1997, S. 245-278.

${ }^{263}$ Eine globale Gewinnaufteilung wird von der OECD sogar ausdrücklich abgelehnt. Vgl. OECD, Guidelines, 2001, Anm. 3.74.

$264 \mathrm{Vgl}$. Hellerstein, W./McLure Jr., C.E., in: ITPF, 2004, S. 206. Es ist jedoch zu beachten, dass auch im Rahmen eines ,at water's edge“-Ansatzes Doppelbesteuerungsabkommen zu überarbeiten wären, wenn auch in einem geringeren Umfang. Vgl. hierzu Weiner, J.M., in: CESifo Forum, 2002, 1, S. 19.

265 Vgl. Gammie, M./Radaelli, C.M./Klemm, A., Reform, 2001, S. 47; Carlson, G.N./Galper, H., in: McLure, State, 1984, S. 23; Luttermann C., in: IStR, 1994, S. 490.

266 Vgl. Musgrave, P.B., in: McLure, State, 1984, S. 238-240.

267 Voraussetzungen sind, dass sowohl die Nachfrage als auch die Produktionsfunktionen voneinander unabhängig sind. In diesem Fall führt aber auch die direkte Gewinnabgrenzung mittels fremdvergleichskonformer Verrechnungspreise zu einem befriedigenden Ergebnis.

${ }^{268}$ Shackford und Slemrod haben für US-amerikanische multinationale Unternehmen einen Zuwachs der Steuerverbindlichkeit auf Basis empirischer Daten in Höhe von 38 v. H. ermittelt, sollte die USA weltweit die Gewinne indirekt mittels einer Drei-Faktoren-Formel abgrenzen. Vgl. Shackelford, D./Slemrod, J., in: ITPF, 1998, S. 53. 
Verluste aus Drittländern dauerhaft nach Europa transferiert werden. ${ }^{269}$ So wird sowohl von der Europäischen Kommission ${ }^{270}$ als auch im Schrifttum ${ }^{271}$ das „water's edge principle“ befürwortet und nur Gesellschaften oder Betriebsstätten mit Sitz in den teilnehmenden europäischen Mitgliedstaaten können Teil eines Konsolidierungskreises werden. ${ }^{272}$ Gegenüber nicht qualifizierten Gesellschaften erfolgt die Gewinnabgrenzung weiterhin auf der Basis fremdvergleichskonformer Verrechnungspreise. ${ }^{273}$ Diese territoriale Einschränkung ist auch vor dem Hintergrund zur rechtfertigen, dass der EG-Vertrag die Schaffung eines gemeinsamen Marktes innerhalb der geografischen Grenzen der Europäischen Union vorsieht und auch nur die dort bestehenden Wettbewerbsverzerrungen beseitigen möchte. ${ }^{274}$

Um in den Genuss der einheitlichen (konsolidierten) KörperschaftsteuerBemessungsgrundlage zu kommen, ist grundsätzlich die Ansässigkeit eines körperschaftsteuerpflichtigen Unternehmens oder das Vorliegen einer Betriebsstätte im Gemeinschaftsgebiet notwendig. Das erfordert eine einheitliche Definition der Ansässigkeit innerhalb der Europäischen Union. So könnte entsprechend den Bestimmungen der Mutter-Tochter-Richtlinie verfahren werden. Danach gilt eine Gesellschaft in dem Mitgliedstaat als ansässig, in dem sie nach dem jeweiligen Steuerrecht ihren Sitz hat und nicht aufgrund eines Doppelbesteuerungsabkommens dieses Staates als außerhalb der Gemeinschaft ansässig

${ }^{269}$ Erwirtschaftet z. B. eine Schweizer Konzerngesellschaft einer deutschen Unternehmensgruppe langfristig Verluste und wird insolvent, so schmälern die Verluste das konsolidierte Ergebnis und damit die Bemessungsgrundlage der europäischen Mitgliedstaaten, in denen der Konzern außerdem eine Betriebsstätte oder Tochterkapitalgesellschaft unterhält.

${ }^{270}$ Vgl. Europäische Kommission, Umsetzung, 2006, S. 19.

271 Vgl. Hellerstein, W./McLure Jr., C.E., in: ITPF, 2004, S. 206; Gammie, M./Radaelli, C.M./Klemm, A., Reform, 2001, S. 47; Weiner, J.M., Apportionment, 2002, S. 10.

${ }^{272}$ Auch die amerikanischen Bundesstaaten grenzen mittlerweile den Konsolidierungskreis ,at water's edge“ ab. Widerstand seitens der US-Bundesregierung, wichtiger Handelspartner der USA und multinationaler Konzerne zwangen die entsprechenden Bundesstaaten, die globale Gewinnaufteilung abzuschaffen, obwohl der US Supreme Court in der Rechtssache Barclays zum wiederholten Mal die Verfassungsmäßigkeit dieser Regelungen festgestellt hat. Siehe Barclays Bank PLC. v. Franchise Tax Board, 512 U.S. 298, 1994. Vgl. a. Hellerstein, W.McLure Jr., C.E., in: BIFD, 2004, S. 93-94; Oestreicher, A., Konzerngewinnabgrenzung, 1999, S. 134-135.

${ }^{273}$ Zum gleichen Ergebnis kommt McDaniel, der die Einführung einer formelhaften Gewinnaufteilung zwischen den Mitgliedstaaten der NAFTA analysiert. Vgl. McDaniel, P.R., in: $T L R$, 1994, S. 726-728. Zur Problematik der Einkommensabgrenzung vgl. Kapitel C.II.3.b).

${ }^{274}$ Vgl. Riecker, A., Körperschaftbesteuerung, 1996, S. 359. 
betrachtet wird. ${ }^{275}$ Die Kriterien für das Vorliegen einer Betriebsstätte sowie die Bestimmung von deren Ansässigkeit erfolgt in Art. 2 Abs. 2 der MTR. ${ }^{276}$ Abweichend von dieser Definition sollten feste Geschäftseinrichtungen auch dann als Betriebsstätten gelten, wenn dadurch die Tätigkeit einer außerhalb der Gemeinschaft ansässigen Gesellschaft in einem Mitgliedstaat ausgeübt wird.

Nachdem die territorialen Grenzen des Konsolidierungskreises abgesteckt wurden, ist weiterhin unklar, inwieweit gebietsfremde Gesellschaften bei der Beurteilung der sachlichen Voraussetzung des Konsolidierungskreises zu berücksichtigen sind. Im Einzelnen ist zu erörtern, ob

1. europäische Teilkonzerne einer in einem Drittstaat belegenen Obergesellschaft,

2. Schwestergesellschaften einer in einem Drittstaat belegenen Obergesellschaft und

3. indirekt über das EU-Ausland gehaltene Konzerngesellschaften

sich für eine einheitliche (konsolidierte) Körperschaftsteuer-Bemessungsgrundlage qualifizieren. Grundsätzlich besteht kein Grund, europäischen Teilkonzernen die Möglichkeit der konsolidierten Gewinnermittlung zu versagen. ${ }^{277}$ Dadurch würde der Anwendungsbereich stark eingeschränkt, sodass eine Vielzahl europäischer Gesellschaften von den Vorteilen der Einführung der konsolidierten Besteuerung ausgenommen wäre. ${ }^{278}$ Das wiederum riefe starke Verzerrungen innerhalb der Europäischen Union hervor, sodass eine Gleichbehandlung europäischer Konzerne und Teilkonzerne mit einer Obergesellschaft in einem Drittstaat geboten ist. Daneben würde das Versagen der Anwendung der einheitlichen konsolidierten Körperschaftsteuer-Bemessungsgrundlage steuerplanerische Gestaltungsmöglichkeit eröffnen, da durch die Gründung einer Holdinggesellschaft in einem Drittstaat, die die Anteile an Betriebsstätten bzw. verbundenen Unternehmen in der Europäischen Union hält, international tätige Unternehmen die Konsolidierung mit indirekter Gewinnabgrenzung umgehen

275 Art. 2 Abs. 1 b) Richtlinie 90/435/EWG des Rates v. 23. 7. 1990, Abl. Nr. L 225 v. 20. 8. 1990, S. 6-9.

276 Die konkrete Aufnahme von Betriebsstätten in die Mutter-Tochter-Richtlinie erfolgte erst mit der Richtlinie 2003/123/EG des Rates v. 22. 12. 2003, Abl. Nr. L 007 v. 3. 1. 2004, S. 41-44.

277 Auch die Europäische Kommission sieht ausdrücklich für außerhalb der Europäischen Union beherrschte Konzerne die Möglichkeit der Anwendung einer konsolidierten Besteuerung vor. Vgl. Europäische Kommission, Fragen zur Konzernbesteuerung, 2006, S. 6.

${ }^{278}$ Die empirische Analyse ergab, dass über 8 v. H. der identifizierten Konzerne europäische Teilkonzerne sind, wobei darin nur Konzerne enthalten sind, an denen ein Anteilseigener direkt zu über $50 \mathrm{v}$. H. beteiligt ist. 
könnten. ${ }^{279}$ Abkommensrechtliche Bedenken bestehen insoweit nicht, als dass die Möglichkeit der Bildung von Teilkonzernen den Anwendungsrahmen vergleichbarer innergemeinschaftlicher Sachverhalte übertrifft, sofern das „allin-all-out"-Prinzip angewendet wird. ${ }^{280}$ Entsprechendes gilt für europäische Teilkonzerne, deren oberste Einheit eine Betriebsstätte einer im Drittstaat ansässigen Gesellschaft ist.

Schwieriger ist die Abwägung, ob zwei Schwestergesellschaften einer in einem Drittstaat ansässigen Obergesellschaft zur Konsolidierung ihrer Einzelabschlüsse berechtigt sein sollten. Zum einen gehören sie zu derselben wirtschaftlichen Einheit; zum anderen hat die beide Unternehmen beherrschende Gesellschaft ihren Sitz außerhalb des territorialen Bereichs der Gemeinschaft. Ausschlaggebend könnte in diesem Zusammenhang die sachliche Abgrenzung des Konsolidierungskreises sein. Eine rein rechtliche Abgrenzung des Konsolidierungskreises würde die Konsolidierung zwischen den Schwestergesellschaften verneinen, da eine Schwestergesellschaft weder einen Beteiligungsbesitz noch Stimmrechtsanteile an der anderen Schwestergesellschaft vorzuweisen hat. Geht es hingegen um die Zusammenveranlagung von wirtschaftlichen Einheiten, wäre eine Konsolidierung zwischen den beiden Tochtergesellschaften die logische Konsequenz. Es sei jedoch angemerkt, dass auch im Falle einer rein rechtlichen Abgrenzung des Konsolidierungskreises das $\mathrm{Zu}$ sammenfassen zweier Schwestergesellschaften durch die Gründung einer Europaholding zu einem Teilkonzern, der wiederum zur Konsolidierung berechtigt wäre, nur einen geringen steuerplanerischen Aufwand darstellt. Diese Überlegungen sind entsprechend auf die in der Europäischen Union belegenen Betriebsstätten ausländischer Gesellschaften anzuwenden.

Die eben skizzierte Argumentation lässt sich auch auf Sachverhalte übertragen, bei denen eine europäische Muttergesellschaft an einer Gesellschaft innerhalb der Gemeinschaft beteiligt ist, wobei die für eine Konsolidierung qualifizierende Mehrheit nur über eine Gesellschaft mit Sitz in einem Drittstaat erreicht wird. Grundsätzlich darf die vermittelnde Gesellschaft nicht in den Konsolidierungskreis miteinbezogen werden; sie ist aber im Rahmen der Abgrenzung des Konsolidierungskreises $\mathrm{zu}$ berücksichtigen, sofern die Zusammenfassung wirtschaftlicher Einheiten vorrangiges Ziel der konsolidierten Besteuerung ist.

279 Eine derartige steuerplanerische Gestaltung ist nur sinnvoll, wenn die Anwendung der einheitlichen konsolidierten Bemessungsgrundlage nach dem „all-in-all-out“ Prinzip erfolgt, d. h. ein „cherry-picking“ nicht möglich ist.

${ }^{280}$ Würde Teilkonzernen ausländischer Gesellschaften hingegen die Konsolidierung der Einzelabschlüsse versagt bleiben, obwohl europäische Konzerne auch nur für Teilkonzerne die einheitliche konsolidierte Bemessungsgrundlage anwenden könnten, verstieße diese Vorgehensweise wahrscheinlich gegen das Diskriminierungsverbot des Art. 24 OECD-MA. 
Damit wäre eine Zusammenveranlagung der europäischen Mutter- und Tochtergesellschaft gegeben, obwohl die Mutter direkt weniger Anteile, als nach Maßgabe der Mindestbeteiligungsquote erforderlich ist, hält.

\section{Ermittlung der einheitlichen Bemessungsgrundlage}

\section{a) Vereinheitlichung der steuerlichen Gewinnermittlungsvorschriften in der Europäischen Union}

Die Einführung der einheitlichen (konsolidierten) Bemessungsgrundlage bedarf zugleich einer gemeinsamen Gewinnermittlungsvorschrift in der Europäischen Union. Ein zweistufiges Vorgehen, bei dem zunächst eine gemeinsame $\mathrm{Be}-$ messungsgrundlage ohne Konsolidierung geschaffen wird, lehnt die Kommission ausdrücklich ab, da das Problem des fehlenden grenzüberschreitenden Verlustausgleichs und die Schwierigkeiten in Verbindung mit den Verrechnungspreisen ungelöst blieben. Zudem könnte eine Konsolidierung langfristig erschwert werden, wenn die Vorschläge nicht unmittelbar auf eine konsolidierte Bemessungsgrundlage ausgerichtet wären. ${ }^{281}$

Als mögliche Ausgangsbasis werden sowohl vonseiten der EU-Kommission ${ }^{282}$ als auch im Schrifttum ${ }^{283}$ die IFRS diskutiert. Die Überlegung, sich dieser Rechnungslegungsstandards als konzeptionelles Hilfsmittel zu bedienen, beruht auf der IAS-Verordnung ${ }^{284}$, die ab 2005 kapitalmarktorientierte Mutterunternehmen in der Europäischen Union zur Aufstellung eines Konzernabschlusses nach IFRS verpflichtet. ${ }^{285}$ Eine Anlehnung an dieses Regelwerk wäre eine erhebliche Vereinfachung und ließe den Arbeitsaufwand der Unternehmen sinken. ${ }^{286}$ Außerdem bestehen bereits mit den IFRS von allen Mitgliedstaaten akzeptierte Regeln der handelsrechtlichen Gewinnermittlung. Sich dieses Konsenses zwischen den Mitgliedstaaten zu bedienen erscheint vor dem Hintergrund sinnvoll, dass eine Angleichung der steuerlichen Gewinnermittlung in der

$281 \mathrm{Vgl}$. Europäische Kommission, Fragen zur Konzernbesteuerung, 2006, S. 2.

${ }^{282} \mathrm{Vgl}$. Europäische Kommission, Unternehmensbesteuerung, 2001, S. 507-509 und 544-545.

${ }^{283}$ Vgl. Schneider, D., in: $B B, 2003$, S. 299-304; Schön, W., in: ET, 2004, S. 426-440; Spengel, C., in: $I S t R, 2003$, S. 29-36, 67-72; derselbe, in: $D B, 2006$, S. 681-687; Oestreicher, A., in: Hebig et al., Entwicklungsaspekte, 2006, S. 283-292; Gammie, M. et al., Achieving, 2005, S. 23-52.

284 Verordnung (EG) Nr. 1606/2002 des europäischen Parlaments und des Rates v. 19.7. 2002, Abl. Nr. L 243, v. 11. 9. 2002, S. 1-4.

${ }^{285} \mathrm{Vgl}$. Spengel, C., in: $I S t R, 2003$, S. 29-31; derselbe, in: $D B, 2006$, S. 681; Scheffler, W., in: Endres et al., Wandel, 2005, S. 105. Davon betroffen sind ca 7.000 Unternehmen in der Europäischen Union.

286 Vgl. Oestreicher, A., in: Hebig et al., Entwicklungsaspekte, 2006, S. 283. 
Vergangenheit an den zu großen Unterschieden zwischen den nationalen Vorschriften gescheitert ist. ${ }^{287}$ Letztlich fühlt sich die Kommission in ihrem Vorhaben aufgrund der im Jahr 2003 durchgeführten Konsultation zur Implikation der IAS für die Schaffung einer konsolidierten Steuerbemessungsgrundlage bestärkt. $^{288}$

Eine uneingeschränkte Übernahme der IFRS für eine steuerliche Gewinnermittlung wird grundsätzlich mit dem Hinweis auf die unterschiedlichen Zielsetzungen der handels- und steuerrechtlichen Rechnungslegung abgelehnt. ${ }^{289}$ Aber auch eine Maßgeblichkeit der IFRS findet wenig Fürsprecher, ${ }^{290}$ da erstens die IFRS ein recht schnelllebiges Regelsystem darstellen und zweitens selbst im Rahmen des Endorsement-Verfahrens keine parlamentarische Instanz an der Rechtsentwicklung beteiligt ist. ${ }^{291}$ Vielmehr werden die IFRS als ,starting point" einer harmonisierten Bemessungsgrundlage betrachtet. ${ }^{292}$ Entsprechend beschränkt sich die gegenwärtige Diskussion auf die Frage, in welchem Umfang sich die steuerliche Gewinnermittlung an den internationalen Rechnungslegungsstandards orientieren soll. Herzig hält eine Übernahme der IFRS-Regelungen nur im Einzelfall für möglich, da den informationsgeprägten Rechnungslegungsgrundsätzen nach IFRS insofern nicht gefolgt werden kann, als die in der

Vgl. Schön, W., in: ET, 2004, S. 440; Schreiber, U., in: StuW, 2004, S. 224. Bereits 1988 bereitete die Europäische Kommission einen Vorschlag zur Vereinheitlichung der Gewinnermittlung vor, der aber nicht weiter verfolgt wurde. Vgl. hierzu Kuiper, G.W., in: $E T, 1988$, S. 319-329. Für eine ausführliche Gegenüberstellung der Gewinnermittlungsvorschriften in den 25 Mitgliedstaaten vgl. Endres, D. et al., Taxable Income, 2006.

288 Vgl. Europäische Kommission, Konsultationspapier, 2003; dieselbe, Ergebnisse, 2003; Schön, W., in: ET, 2004, S. 428.

${ }^{289}$ Vgl. Oestreicher, A., in: Hebig et al., Entwicklungsaspekte, 2006, S. 2; Esterer, F., in: Endres et al., Wandel, 2005, S. 126; Schneider, D., in: BB, 2003, S. 304.

290 Vgl. Herzig, in: $W P g, 2005$, S. 214; derselbe, in: IStR, 2006, S. 558-560; derselbe, in: Endres et al., Wandel, 2005, S. 134; Schön, W., in: ET, 2004, S. 440. Für eine Maßgeblichkeit hingegen spricht sich beispielsweise der Bund der Wirtschaftsakademiker aus. Vgl. Arbeitskreis "Steuern und Revision“ im Bund der Wirtschaftsakademiker (BWA) e. V., in: DStR, 2004, S. 1267-1678. Auch Dietel kommt in seiner Analyse zu dem Schluss, dass die steuerliche Gewinnermittlung grundsätzlich den IAS/IFRS folgt. Lediglich den Grundsatz der Einmalbesteuerung sieht er stellenweise durchbrochen. Vgl. Dietel, M., Gewinnermittlung, 2004, S. 263-264. Vgl. für eine ausfuhrliche Analyse a. Oestreicher, A./Spengel, C., Maßgeblichkeit, 1999.

291 Vgl. Herzig, N., in: IStR, 2006, S. 559; derselbe, in: Hebig et al., Entwicklungsaspekte, 2006, S. 272-274; Schreiber, U., in: StuW, 2004, S. 225.

292 Vgl. hierfür beispielhaft Schön, W., in: ET, 2004, S. 440; Jacobs, O.H. et al., in: intertax, 2005, S. 414; Oestreicher, A., in: Hebig et al., Entwicklungsaspekte, 2006, S. 285; Levin, M., Harmonising, 2003, S. 7; Herzig, N., IAS/IFRS, 2004, S. 465; Schreiber, U., in: StuW, 2004, S. 225; Schneider, D., in: BB, 2003, S. 304; Gammie, M. et al., Achieving, 2005, S. 60 . 
Steuerbilanz relativ stärker gewichteten Objektivierungsregeln dem entgegenstehen. ${ }^{293}$ Demgegenüber sehen Oestreicher und Spengel zwischen IFRS und den Zielen der steuerlichen Gewinnermittlung keinen grundlegenden Gegensatz. Ihrer Ansicht nach weisen die IFRS, trotz der primären Ausrichtung auf die Informationsinteressen der Kapitalgeber, eine breite Basis objektivierter Gewinnermittlungsregeln auf. ${ }^{294}$ Einigkeit herrscht insoweit, als dass die „Marktbewertung" nicht im Einklang mit den Besteuerungsprinzipien steht und Beschränkungen des Verlustausgleichs aufzuheben sind. ${ }^{295}$

Parallel zur wissenschaftlichen Diskussion hat die Europäische Kommission bereits eine Arbeitsgruppe eingesetzt, welche die Definition einer einheitlichen konsolidierten Steuerbemessungsgrundlage für in der Europäischen Union tätige Unternehmen unter technischen Gesichtspunkten prüfen soll. ${ }^{296}$ Sie verwendet explizit die internationalen Rechnungslegungsstandards als Hilfsmittel für die Konzipierung der Bemessungsgrundlage. ${ }^{297}$

\section{b) Umfang des aufzuteilenden Gewinns}

Steht fest, welche Gesellschaften zu einer wirtschaftlichen Einheit zählen, und ist der Gewinn jeder Konzerneinheit bekannt, ist im Weiteren der Umfang des aufzuteilenden Erfolgs zu bestimmen. Dabei ist erstens zu klären, ob Gewinnbestandteile, und wenn ja, welche von ihnen im Rahmen der formelhaften Gewinnaufteilung einer Gesellschaft weiterhin direkt zugeordnet werden sollen, und zweitens, wie eine Doppelbesteuerung ausländischer Einkünfte vermieden werden kann.

Die Beantwortung der ersten Frage hängt unmittelbar mit dem Ziel der indirekten Gewinnaufteilung und der Definition einer wirtschaftlichen Einheit zusammen. Soll eine indirekte Gewinnabgrenzung nur dann vorgenommen werden, wenn die direkte Abgrenzung scheitert, wäre eine Differenzierung zwischen aufzuteilendem und direkt zuzurechnendem Gewinn korrekt. ${ }^{298}$ Dies bewirkt eine Besteuerung dieser Einkommensbestandteile nach dem Quellen-

293 Vgl. Herzig, N., IAS/IFRS, 2004, S. 60.

294 Vgl. Spengel, C., in: $D B, 2006$, S. 687; derselbe, in: IStR, 2003, S. 72; Oestreicher, A., in: Hebig et al, Entwicklungsaspekte, 2006, S. 291.

295 Vgl. Spengel, C., in: DB, 2006, S. 687; Oestreicher, A., in: Hebig et al., Entwicklungsaspekte, 2006, S. 291.

296 Der Fortschritt der Arbeitsgruppe „Gemeinsame konsolidierte KörperschaftsteuerBemessungsgrundlage" kann auf der Internetseite der Generaldirektion Steuern und Zollunion der Europäischen Kommission eingesehen werden.

297 Vgl. Europäische Kommission, Umsetzung, 2006, S. 5.

$298 \mathrm{Vgl}$. hierzu die Abgrenzung der wirtschaftlichen Einheit gemäß dem Three-Stage Test von McLure in Abschnitt C.II.2.a)( $(\beta)$. 
prinzip. ${ }^{299}$ So wären z. B. Mieteinkünfte nicht Bestandteil der Aufteilungsmasse, da sie dem Belegenheitsstaat der Immobilie direkt zugeordnet werden könnten. ${ }^{300}$ Mag das „Heraustrennen“ spezifischer Einkommensbestandteile zunächst sinnvoll erscheinen, so ist es dennoch im Rahmen der konsolidierten Körperschaftsteuer-Bemessungsgrundlage nicht zu empfehlen: ${ }^{301}$

Erstens erfordert die Zerlegung des Einkommens in aufteilbare und zuordenbare Bestandteile eine exakte Definition derselben. Dass eine solche nicht leicht aufzustellen ist, zeigen die Erfahrungen in den USA. ${ }^{302}$ Die Abgrenzung von „business" und „non-business income“ war Bestandteil zahlreicher Gerichtsverfahren. Zweitens eröffnet eine derartige Differenzierung immer Spielraum für Steuergestaltungen. ${ }^{303}$ Drittens bleiben bei einer direkten Zuordnung die bestehenden unternehmenssteuerlichen Hindernisse in der Europäischen Union bestehen. Ein grenzüberschreitender Verlustausgleich wäre nicht mehr automatisch gewährleistet, und durch die Gestaltung von Verrechnungspreisen könnten weiterhin Einkommensbestandteile verlagert werden. Letztlich werden die zuordenbaren Gewinnbestandteile eine untergeordnete Rolle spielen, sodass auch pragmatische Gesichtspunkte gegen eine Differenzierung sprechen. ${ }^{304}$

In Bezug auf ausländische Einkünfte gibt die Mehrzahl der Mitgliedstaaten der Besteuerung des Welteinkommens den Vorzug vor dem Territorialprinzip, sodass auch die Kommission dieses Konzept favorisiert. ${ }^{305}$ Zur Vermeidung der Doppelbesteuerung kommen sowohl die Freistellungs- als auch die Anrechnungsmethode in Frage. ${ }^{306}$ Das wirft die Frage auf, ob einer kapitalimportoder kapitalexportneutralen Besteuerung der Vorzug zu gewähren sei. Aus steuersystematischer Sicht wird in jüngeren Veröffentlichungen eine kapital-

299 Vgl. Hellerstein, W./McLure Jr., C.E., in: ITPF, 2004, S. 202.

300 Weitere Bestandteile des sogenannten ,non-business income“ in den USA sind Pachteinnahmen, Lizenzgebühren und Gewinne bzw. Verluste aus der Verpachtung, der Überlassung und dem Verkauf von Grundbesitz. Siehe Sec. 1 e) i. V. m. a) UDITPA.

301 Vgl. Hellerstein, W.McLure Jr., C.E., in: ITPF, 2004, S. 202-203; Hellerstein, W., in: STN, 2001, S. 725-739.

302 Aus diesem Grund folgen einige US-Bundesstaaten nicht weiter den Vorgaben des UDITPA, sondern teilen alle Gewinne mittels einer Formel auf. Vgl. Weiner, J.M., in: TNI, 1996, S. 2120-2121.

303 Auch Mazerov weist auf die Ausweichmaßnahmen der Unternehmen hin und befürwortet vor diesem Hintergrund eine möglichst breite Definition des aufzuteilenden Gewinns. Vgl. Mazerov, M., Loopholes, 2003, S. 9-11; kritisch Fox, W.F./Murray. M.N./Luna, L., in: NTJ, 2005, S. 150-152.

304 Vgl. Hellerstein, W.McLure Jr., C.E., in: ITPF, 2004, S. 203.

$305 \mathrm{Vgl}$. Europäische Kommission, Internationale Aspekte, 2006, S. 4.

${ }^{306}$ Vgl. Weiner, J.M., Group Taxation, 2005, S. 36. 
exportneutrale Besteuerung befürwortet, ${ }^{307}$ da die Argumente für eine kapitalimportneutrale Besteuerung zunehmend an Gewicht verlieren. Denn letztere wird stets über die relative Leistungsfähigkeit gerechtfertigt. Dazu müssen jedoch die Bedingungen einer festen wirtschaftlichen Verknüpfung mit dem besteuernden Ausland und unterschiedliche Wettbewerbsverhältnisse im In- und Ausland kumulativ erfüllt sein. Durch die verstärkt prozessorientierten Geschäfts- und Organisationsstrukturen und die damit verbundene Zunahme der grenzüberschreitenden Lieferungs- und Leistungsbeziehungen sind jedoch die einzelnen Märkte nur noch schwierig voneinander zu separieren. Zudem ist das Vorliegen eines steuerlichen Anknüpfungspunktes in einem Staat kein eindeutiges Indiz mehr für eine feste wirtschaftliche Verknüpfung mit der dort geltenden Organisationsstruktur. Schließlich ist mit einer Freistellung der Dividenden noch längst keine kapitalimportneutrale Besteuerung erreicht, denn finanziert z. B. der Anteilseigner die ausländische Tochtergesellschaft über ein Darlehen, unterliegen die Zinserträge im Inland regelmäßig der Besteuerung und reduzieren die Bemessungsgrundlage im Ausland. Damit hat der Konzern faktisch ein Wahlrecht, wo der ökonomische Gewinn besteuert werden soll, was sich negativ auf die Sicherung des inländischen Steueraufkommens auswirkt und die Forderung nach einem neutralen Steuersystem erheblich verletzt. ${ }^{308}$

Die Anwendung der Anrechnungsmethode stellt sich im Rahmen einer einheitlichen konsolidierten Bemessungsgrundlage jedoch als sehr komplex heraus. Denn es erscheint nicht gerecht, die ausländische Steuer nur in einem Staat anzurechnen, sodass auch sie anhand eines Schlüssels auf die einzelnen Sitzstaaten der Konzerntochtergesellschaften aufzuteilen ist. Bei abweichenden Steuersätzen innerhalb der EU führt eine Aufteilung des Anrechnungsbetrags aber zwangsweise zu einem anderen Ergebnis. Darüber hinaus muss auch der Anrechnungshöchstbetrag für jeden Mitgliedstaat separat ermittelt werden. Schließlich soll die einheitliche konsolidierte Bemessungsgrundlage ausschließlich die Ermittlung und Aufteilung der Bemessungsgrundlage regeln. Die Anrechnungsmethode setzt aber an den Steuerbeträgen an und beeinflusst damit indirekt die Berechnung der Steuerschuld, was ihre Anwendung problematisch macht. $^{309}$

Bezogen auf das letzte Argument wäre die Freistellungsmethode der Anrechnungsmethode überlegen. Zudem ist ihre Anwendung deutlich einfacher, ${ }^{310}$

307 Vgl. Spengel, C., Unternehmensbesteuerung, 2003, S. 364; Jacobs, O.H. (Hrsg.), Unternehmensbesteuerung, 2002, S. 27-35; Homburg, S., in: Endres et al., Wandel, 2005, S. 23-27. Früher anderer Auffassung Jacobs, vgl. Jacobs, O.H. (Hrsg.), Besteuerung, 1983, S. 25-26.

308 Vgl. Homburg, S., in: Endres et al., Wandel, 2005, S. 23-24.

309 Vgl. Europäische Kommission, Räumlicher Anwendungsbereich, 2006, S. 6.

${ }^{310}$ Vgl. Hellerstein, W.McLure Jr., C.E., in: ITPF, 2004, S. 207. 
da sich durch die Freistellung der ausländischen Einkünfte vor der Aufteilung der Bemessungsgrundlage auch die Diskussion über die Eignung der Formel in Bezug auf ausländische Einkünfte erübrigt. Jedoch birgt dieses Konzept stets die Gefahr der Steuergestaltungen, die von niedrigen Steuersätzen in Drittländern profitieren. $^{311}$ Trotz der systematischen Vorbehalte sprechen sich Hellerstein und McLure für eine Freistellung der im Ausland erzielten Einkünfte aus. ${ }^{312}$

\section{c) Konsolidierung der Einzelabschlüsse}

Steht der Jahresüberschuss der einzelnen Konzerngesellschaften fest, sind anschließend die Einzelabschlüsse zu einer gemeinsamen Bemessungsgrundlage zusammenzuführen. Grundsätzlich ist von einer Summenbilanz durch Addition der Einzelabschlüsse bis hin zu einer vollständigen Konsolidierung und einer damit zusammenhängenden Kapital-, Schulden-, Aufwands- und Ertragskonsolidierung sowie einer Zwischenergebniseliminierung jede Art von Konsolidierung denkbar. Vorrangiges Ziel der konsolidierten Bemessungsgrundlage ist es nicht, zu Informationszwecken einen Konzernabschluss aufzustellen, der die rechtlich selbstständigen Tochtergesellschaften entsprechend der wirtschaftlichen Einheit als ein Unternehmen präsentiert. Vielmehr soll die Gesamtbemessungsgrundlage des Konzerns ermittelt werden. Bezogen auf diese Zielsetzung unterscheiden sich ein Summenabschluss und ein vollständig konsolidierter Konzernabschluss nur hinsichtlich der Periodisierung der Erträge. Gegenüber der direkten Methode, die mittels transaktionsbezogener Verrechnungspreise auf der Basis des Fremdvergleichsgrundsatzes die Gewinne abgrenzt, weist die indirekte Methode unabhängig von ihrer konkreten Ausgestaltung theoretisch wesentliche Vorteile auf: ${ }^{313}$

1. Durch die Konsolidierung der Einzelabschlüsse erfolgt automatisch eine Verrechnung der Gewinne und Verluste innerhalb des Konzerns. Somit ist erstens eine umfangreiche, grenzüberschreitende Verlustverrechnung möglich. Aber auch zwischen nationalen Gruppengesellschaften ergeben sich Vorteile, da eine Konsolidierung der Ergebnisse vielfach den nationalen Gruppenbesteuerungssystemen überlegen ist. So ist es unerheblich, welche Gesellschaften einen Gewinn bzw. Verlust erzielen, denn die Verlustverrechnung ist auch zwischen Schwestergesellschaften garantiert. ${ }^{314}$ Eine mehr-

311 Vgl. Hellerstein, W./McLure Jr., C.E., in: ITPF, 2004, S. 207.

312 Vgl. Hellerstein, W./McLure Jr., C.E., in: ITPF, 2004, S. 207. Bzgl. der Freistellung von Dividenden vgl. a. McLure, Jr., C.E., in: TN, 1986, S. 975-986.

313 Vgl. Spengel, C./Braunnagel, R., in: StuW, 2006, S. 48-49. Zur Beseitigung der unternehmenssteuerlichen Hindernisse siehe ausführlich Abschnitt C.I.4.a).

314 Vgl. Scheffler, W., in: BFuP, S. 158. 
fache Nutzung von Verlusten wird grundsätzlich unterbunden, indem der Verlust nur einmal innerhalb eines Konzerns festgestellt wird. ${ }^{315}$

2. Beteiligungsaufwendungen mindern die Konzernbemessungsgrundlage unabhängig davon, auf welcher Ebene im Konzern sie anfallen.

3. Die formelhafte Gewinnaufteilung trennt die Zuordnung der Bemessungsgrundlage von der Gewinnermittlung ab. Damit partizipieren alle Konzerngesellschaften anteilig an sämtlichen Aufwendungen und Erträgen. Steuerplanerischen Gestaltungen wird somit ihre Wirkungskraft entzogen.

Welche Konsolidierungsschritte im Einzelnen vorzunehmen sind, wird im Folgenden erörtert werden. Dabei ist zu beachten, dass die Konsolidierung der Einzelbeschlüsse ausschließlich der Ermittlung einer gemeinsamen Bemessungsgrundlage dient und insofern ausschließlich ergebniswirksame Konsolidierungsmaßnahmen notwendig sind.

Gemäß IFRS 3 wird als Kapitalkonsolidierungsmethode ausschließlich die Erwerbsmethode angewendet. ${ }^{316}$ Diesem Verfahren liegt die Fiktion des Erwerbs der einzelnen Vermögensgegenstände zum Zeitpunkt der erstmaligen Einbeziehung des Tochterunternehmens zugrunde. ${ }^{317}$ Die dabei aufgedeckten stillen Reserven sowie ein etwaiger Geschäfts- und Firmenwert werden in den Folgejahren ertragswirksam abgeschrieben. Im Ergebnis wird auf Ebene des Konzerns ein ,asset deal" fingiert. Für den Verkäufer kommt hingegen ausschließlich die steuerliche Behandlung eines ,share deal“ in Frage. Somit erzielt der Verkäufer regelmäßig steuerfreie Einnahmen, denen im gleichen Umfang ertragswirksames Abschreibungspotenzial aus der Kapitalkonsolidierung gegenübersteht. Konsequenterweise müsste ein späterer Verkauf der Beteiligung steuerpflichtig sein. Zwar handelt es sich dabei rein rechtlich um einen ,share deal“, aber in Anlehnung an obige Fiktion wäre eine Teilbetriebsveräußerung zu unterstellen. Im Ergebnis wird also die Versteuerung gestundet und der damit verbundene Zinseffekt vom Staat getragen. Denkbar ist auch, die stillen Reserven und den Geschäfts- und Firmenwert erfolgsneutral abzuschreiben oder gar keine Kapitalkonsolidierung vorzunehmen. Sollen jedoch ein Unternehmenszusammenschluss durch Übertragung der Anteile am Eigenkapital eines Unternehmens („share deal") und durch Übertragung des Reinvermögens (,,asset deal") steuerlich gleich behandelt werden, ist eine Kapitalkonsolidierung zwingend notwendig. Sie verfolgt konsequent den Ansatz der wirtschaftlichen Einheit und behandelt einen Konzern steuerlich wie ein einzelnes Unternehmen.

Im Rahmen der Schuldenkonsolidierung werden Ansprüche und Verpflichtungen, die zwischen den Konzerngesellschaften bestehen, gegeneinander auf-

\footnotetext{
315 Vgl. Herzig, N./Wager, T., in: $D B, 2005$, S. 9.

316 IAS 27.22 und IFRS 3.

317 Vgl. Küting, K./Weber, C.-P., Konzernabschluss, 2006, S. 231.
} 
gerechnet. Dies geschieht erfolgsneutral, soweit sich die korrespondierenden Wertgrößen in gleicher Höhe gegenüberstehen. ${ }^{318}$ Ist dieses nicht der Fall und ist die Differenz auf eine erfolgswirksame Buchung zurückzuführen, ${ }^{319}$ muss auch die Eliminierung der Differenz erfolgswirksam erfolgen. Um den Einfluss der Finanzierungsvorgänge innerhalb des Konzerns auszublenden, ist eine Schuldenkonsolidierung nicht zwingend erforderlich. Bereits im Summenabschluss heben sich Zinsertrag und Zinszahlungen gegenseitig auf, und wegen der indirekten Gewinnaufteilung ist es unerheblich, in welchem Mitgliedstaat der Aufwand bzw. der Ertrag entsteht. ${ }^{320}$

Aufgabe der Aufwands- und Ertragskonsolidierung ist zum einen die erfolgsneutrale Verrechnung bzw. Umgliederung von Aufwendungen und Erträgen in der Konzern-Gewinn-und-Verlust-Rechnung; zum anderen werden konzerninterne Gewinnausschüttungen sowie $\mathrm{Zu}$ - und Abschreibungen auf Anteile an konsolidierten Unternehmen erfolgswirksam korrigiert. Die ertragswirksame Aufwands- und Ertragseliminierung entspricht damit der notwendigen Freistellung konzerninterner Repatriierungen von Gewinnen, die eine Mehrfachbesteuerung des Konzernergebnisses auf den verschiedenen Konzernebenen (Kaskadeneffekt) verhindern soll. ${ }^{321}$

Der Idee des einheitlichen Unternehmens folgend, dürfen Gewinne erst realisiert werden, wenn sie gegenüber einem fremden Dritten erzielt werden. Konzerninterne Zwischenergebnisse sind folglich ertragswirksam zu eliminieren. Durch die Zwischenergebniseliminierung verlieren Verrechnungspreisgestaltungen endgültig ihre Wirkung. Würden hingegen die Einzelabschlüsse addiert werden, bestünde zwar kein Anreiz mehr, die Gewinne durch Verrechnungspreisgestaltungen zwischen den Mitgliedstaaten zu verlagern, doch wären die Konzerne bestrebt, ihren Erfolg möglichst spät zu realisieren, um von dem dadurch entstandenen Steuerstundungseffekt zu profitieren. Verschieben sich in den Folgejahren die Aufteilungsfaktoren, kann es im Vergleich zum Ausgangsjahr auch zu einer geringeren Konzernsteuerbelastung zum Zeitpunkt der Gewinnrealisation kommen. Damit tritt ein dauerhafter Steuerspareffekt ein. Ein

318 Vgl. Küting, K./Weber, C.-P., Konzernabschluss, 2006, S. 385.

${ }^{319}$ Beispiele für echte erfolgswirksame Aufrechnungsdifferenzen sind Rückstellungen und Forderungen, die auf der Basis der Niederstwertvorschriften teilweise oder vollständig abgeschrieben wurden, Kreditgewăhrungen mit Abschlag (Disagio) und Währungsumrechnungsdifferenzen.

${ }^{320}$ So sind wohl auch Spengel/Braunagel zu verstehen, wenn sie eine Kapital-, Forderungsund Schuldenkonsolidierung zur Ausschaltung der konzerninternen Finanzierungsvorgänge fordern und im gleichen Zuge vom aufzuteilenden Nettoergebnis sprechen. Vgl. Spengel, C./Braunnagel, R., in: StuW, 2006, S. 48.

321 Vgl. Spengel, C./Braunnagel, R., in: StuW, 2006, S. 48. Sie fordern explizit eine Freistellung der Dividenden einbezogener Gesellschaften, obwohl sie bei ihren Überlegungen vom Nettoergebnis des Konzerns ausgehen. 
vertikal integrierter Konzern wäre bei einer reinen Addition der Einzelabschlüsse also bestrebt, die stillen Reserven erst bei der Lieferung oder Leistung an einen fremden Dritten aufzulösen, wenn die Prozesskette eine Durchlaufzeit von mehr als einer Periode besitzt. Die Zwischenergebniseliminierung bindet hingegen die stillen Reserven unabhängig vom vereinbarten Verrechnungspreis in der gemeinsamen Bemessungsgrundlage. Eine Diskussion über die Höhe der aufzulösenden stillen Reserven kann bereits dem Grunde nach nicht entstehen. ${ }^{322}$

Im Ergebnis ist festzuhalten, dass eine Eliminierung der Zwischengewinne zwingend notwendig ist, um die dargelegten unternehmenssteuerlichen Hindernisse in der Europäischen Union zu beseitigen. Darüber hinaus ist eine vollständige Konsolidierung wünschenswert, da dann die Besteuerung einer wirtschaftlichen Einheit derjenigen eines einzelnen Rechtsträgers entspricht. Es sei jedoch angemerkt, dass dieses Ergebnis nicht Zielsetzung einer konsolidierten Besteuerung ist und mit einem hohen administrativen Aufwand verbunden ist. Folglich erwägt die Europäische Kommission, lediglich konzerninterne Transaktionen zu eliminieren, und unterscheidet dabei zwei möglich Alternativen: ${ }^{323}$ Entweder werden sämtliche konzerninternen Lieferungen und Leistungen bei der Ermittlung der Bemessungsgrundlagen außer Acht gelassen, oder aber interne Transaktionen werden zu ihren Kosten erfasst. Beide Vorgehensweisen führen zum gleichen Ergebnis. Alternative II hinterlässt jedoch durch die Berücksichtigung der Lieferung und Leistung bei jedem einzelnen Unternehmen einen „Prüfpfad“. Dieser erscheint besonders dann hilfreich, wenn fremdvergleichskonforme Daten z. B. beim Verkauf einer Beteiligung vorgelegt werden müssen. ${ }^{324}$ Beispiel 2 verdeutlicht beide Vorgehensweisen.

\section{Beispiel 2:}

Von Unternehmen A gekaufte Waren $(100 \mathrm{GE})$ werden über B an C veräußert. Schließlich verkauft $C$ die Waren an einen fremden Dritten für $120 \mathrm{GE}$.

\begin{tabular}{|l|c|c|c|c|c|c|}
\hline Unternehmen & \multicolumn{2}{c|}{ A } & \multicolumn{2}{c|}{ B } & \multicolumn{2}{c|}{ C } \\
\hline & Kosten & Einnahmen & Kosten & Einnahmen & Kosten & Einnahmen \\
\hline Alternative I & 100 & - & - & - & - & 120 \\
Alternative II & 100 & 100 & 100 & 100 & 100 & 120 \\
\hline
\end{tabular}

Quelle: eigene Darstellung

322 Vgl. Scheffler, W., in: Oestreicher, Konzernbesteuerung, 2005, S. 322-324.
323 Vgl. Europäische Kommission, Fragen zur Konzernbesteuerung, 2006, S. 9.
324 Vgl. Europäische Kommission, Fragen zur Konzernbesteuerung, 2006, S. 9. 


\section{Aufteilungsformel}

\section{a) Übersicht}

Im zweiten Schritt der Steuerberechnung wird der zuvor durch einen Betriebsvermögensvergleich ermittelte Gesamterfolg des Konzerns ${ }^{325}(\pi)$ anhand einer Formel auf die einzelnen Mitgliedstaaten der Europäischen Union bzw. die dort belegenen Konzerneinheiten aufgeteilt. Grundsätzlich werden dabei Kennzahlen $(x, y, z)$ einzelner Gliedgesellschaften ins Verhältnis zu den Gesamtgrößen des Konzerns gesetzt. Die Gewichtung $(\alpha)$ der in der Formel enthaltenen Faktoren kann je nach zugrunde liegender Zielsetzung differieren. Die Steuerlast $(T)$ eines Unternehmens in Staat (i) berechnet sich gemäß folgender Formel ${ }^{326}$.

$$
T_{i}=t_{i} \pi_{i}=t_{i}\left[\alpha^{x} \frac{x_{i}}{x}+\alpha^{y} \frac{y_{i}}{y}+\alpha^{z} \frac{z_{i}}{z}\right] \times \pi
$$

Da die in der Formel enthaltenen Faktoren für die Aufteilung der Bemessungsgrundlage verantwortlich sind, wirkt die formelhafte Gewinnaufteilung wie eine Steuer auf dieselben. ${ }^{327}$ Lediglich die absolute Höhe der Steuerlast hängt von dem ermittelten Gewinn ab. Die Definition der Formel impliziert somit auch die Bestimmung der Anknüpfungsmerkmale einer konsolidierten Gewinnbesteuerung. ${ }^{328} \mathrm{Im}$ Wesentlichen handelt es sich hierbei um die Frage der ökonomischen Konsistenz einer Besteuerung sowie um die juristische Betrachtung internationaler Eigentumsrechte. ${ }^{329}$ Grundsätzlich lassen sich drei Konzepte unterscheiden. ${ }^{330}$ Der Aufteilung des Gewinns nach makroökonomischen bzw. industrieweiten Größen liegt keine fundierte Theorie zugrunde. Dieser Ansatz ist vielmehr als pauschaler Lösungsansatz zur Verhinderung von Steuergestaltungsmaßnahmen zu sehen. Der äquivalenztheoretische Ansatz sieht das Besteu-

\footnotetext{
${ }^{325}$ Vgl. hierzu die Ausführungen in Kapitel C.II.3.

326 Soll eine Doppelbesteuerung von Gewinnen vermieden werden, so müssen die Gewichtungsfaktoren über alle Staaten hinweg gleich sein $\left(\alpha_{i}^{x / y / z}=\alpha_{i}^{x / y / z} \forall i\right)$ und in Summe eins ergeben $\left(\alpha^{\mathrm{x}}+\alpha^{\mathrm{y}}+\alpha^{\mathrm{z}}=1\right)$.

327 Vgl. McLure Jr., C.E., in: Aaron/Boskin, Economics, 1980, S. 328; Weiner, J.M., in: CESifo Forum, 2002, 1, S. 16; Wellisch, D., in: FA, 2003, S. 25. Grodon und Wilson zeigen, welche Effekte eine Besteuerung der Faktoren Umsatz, Vermögen, Lohnsumme auf die Investitionstätigkeit, die Beschäftigung und den Umsatz innerhalb eines Staates haben. Vgl. Gordon, R./Wilson, J.D., in: Econometrica, 1986, S. 1357-1373.

${ }^{328}$ Das Vorliegen einer wirtschaftlichen Größe in einem Land ist jedoch keine hinreichende Bedingung für die Entstehung einer Steuer. Zusätzlich müssen die traditionellen Anknüpfungspunkte wie die physische Präsenz (Betriebsstätte) oder die Ansässigkeit eines verbundenen Unternehmens vorliegen. Vgl. Wellisch, D., in: StuW, 2004, S. 269; Wildasin, D.E., in: CTJ, 2000, S. 429-440.

329 Vgl. Musgrave, P.B., in: Public Finance, 1972, S. 389.

330 Vgl. Musgrave, P.B., in: McLure, State, 1984, S. 230-232.
} 
erungsrecht in der Inanspruchnahme öffentlicher Leistungen begründet. ${ }^{331}$ Eine Aufteilung des Gewinns erfolgt in Abhängigkeit des Umfangs dieser Inanspruchnahme. Demgegenüber knüpft der Besteuerungsanspruch der übrigen Überlegungen nicht an den gewonnenen Nutzen aus öffentlichen Leistungen, sondern an die Leistungsfähigkeit des Unternehmens an. ${ }^{332}$ Der Konzernerfolg wird nach dem Beitrag der einzelnen Gliedgesellschaften zum Konzernerfolg auf die Staaten aufgeteilt. Über die Definition dieses Beitrags existieren differierende Annahmen. Erstens kann das Konzernergebnis als Verzinsung des investierten Kapitals betrachtet werden. ${ }^{333}$ Eine Aufteilung gemäß den Vermögenswerten wäre die logische Konsequenz dieses kapitalmarkttheoretischen Ansatzes. Demgegenüber betrachten sowohl der „,supply“- als auch der „,supplydemand approach" den Gewinn als Ergebnis der Wertschöpfung, ${ }^{334}$ wobei sich diese Ansätze hinsichtlich des Ursprungs der Wertschöpfung unterscheiden. Da Unternehmen in der Europäischen Union im Rahmen der Umsatzsteuer bereits den Mehrwert ermitteln, wird im Rahmen der konsolidierten Steuerbemessungsgrundlage in der Europäischen Union auch eine Aufteilung auf der Basis dieser Daten diskutiert. ${ }^{335}$ Bei näherer Betrachtung wird deutlich, dass eine Verteilung sowohl gemäß den Produktionsfaktoren Arbeit und Kapital als auch anhand des Mehrwerts Parallelen aufweist, sodass letztere Aufteilungsformel auch dem

331 Vgl. Musgrave, P.B., in: Public Finance, 1972, S. 400; Oestreicher, A., Konzerngewinnabgrenzung, 1999, S. 173-174.

332 Vgl. zum Stand der Diskussion, ob Körperschaften eine selbstständige Leistungsfähigkeit besitzen, Tipke, K., Steuerrechtsordnung, 2003, S. 1169-1175.

${ }^{333}$ Vgl. Higinbotham, H.N. et al., in: TLR, 1987, S. 362-367; Hellerstein und McLure zerlegen den Konzernerfolg in den normalen Ertrag des Kapitals und den okonomischen Gewinn und untersuchen für die jeweilige Teilmenge eine optimale Aufteilungsformel. Vgl. Hellerstein, W./McLure Jr., C.E., in: ITPF, 2004, S. 209-210.

334 Vgl. Oestreicher, A., Konzerngewinnabgrenzung, 1999, S. 179; siehe a. Musgrave, P.B., in: McLure, State, 1984, S. 234.

335 Vgl. Lodin, S.O./Gammie, M., Home, 2001, S. 47-50; Wellisch, D., in: StuW, 2004, S. 273-274; Hellerstein, W./McLure Jr., C.E., in: ITPF, 2004, S. 213-217. AgúndezGarcia weist jedoch darauf hin, dass der im Rahmen der Mehrwertsteuer ermittelte Mehrwert nicht geeignet ist, als Aufteilungsschlüssel der konsolidierten Bemessungsgrundlage zu dienen. Vgl. Agúndez-Garcia, A., Apportionment, 2006, S. 69-70. Der Mehrwert kann sowohl indirekt durch Substraktion des Input vom Output als auch direkt durch Addition der Löhne, Zinsen und des Gewinns berechnet werden. Theoretisch führen beide Methoden zum gleichen Ergebnis. Insbesondere die direkte Berechnung verdeutlicht das Defizit dieses Ansatzes. So ist der Gewinn bereits für die Berechnung des Mehrwerts zu ermitteln, wodurch, wenn auch in abgeschwächter Form, die existierenden Probleme im Rahmen der direkten Gewinnabgrenzung bei einer formelhaften Gewinnaufteilung anhand des Mehrwerts bestehen bleiben. Aus diesem Grund wird dieser Ansatz im weiteren Verlauf der Arbeit vernachlässigt. 
„supply approach“ subsumiert wird. ${ }^{336}$ Abbildung 3 fasst die Besteuerungsprinzipien im Rahmen einer formelhaften Gewinnaufteilung zusammen.

Abbildung 3: Besteuerungsprinzipien bei der formelhaften Gewinnaufteilung

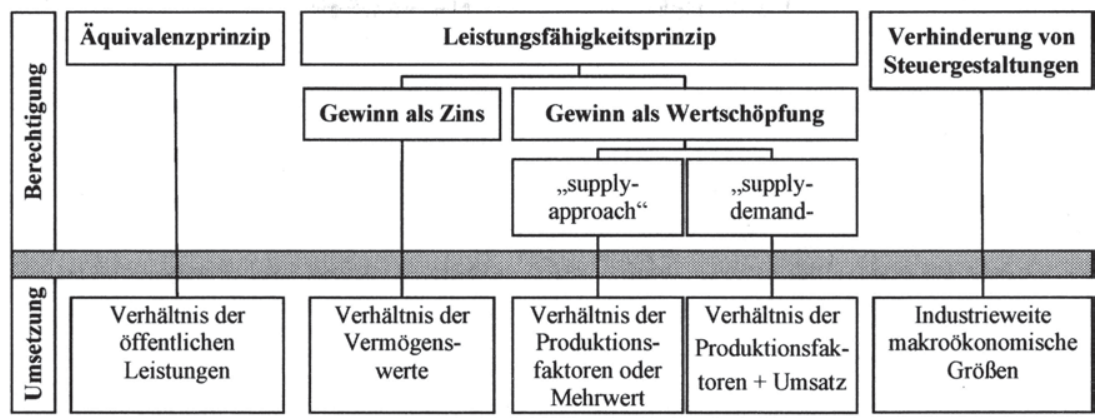

In Anlehnung an: Musgrave, P.B., in: McLure, State, 1984, S. 231

Unabhängig von dem verfolgten Ansatz ist eine präzise geografische Zuordnung der Bemessungsgrundlage nicht möglich. Eine formelhafte Gewinnaufteilung liefert stets nur eine Approximation der real in den Staaten angefallenen $\mathrm{Ge}-$ winne. ${ }^{337}$ Diese Feststellung war eine der Beweggründe, weswegen der RudingAusschuss eine pauschale Aufteilung der Gewinne anhand einer Formel ablehnte. ${ }^{338}$ Dabei wird jedoch übersehen, dass auch die Gewinnabgrenzung nach dem Prinzip der selbstständigen Einheit eine korrekte Gewinnzuordnung nicht erreicht. ${ }^{339}$ Es existieren sowohl praktische Probleme bei der Bestimmung angemessener Verrechnungspreise ${ }^{340}$ als auch methodische Vorbehalte gegenüber dem Grundsatz des Fremdvergleichs ${ }^{341}$. Insbesondere das Negieren von Skalen-

${ }^{336}$ Dies trifft vor allem dann zu, wenn bei der Berechnung des Mehrwerts dem „origin-based approach“ gefolgt wird. Vgl. Agúndez-García, A., Apportionment, 2006, S. 74.

337 Vgl. Weiner, J.M., in: ifo Studien, 2002, S. 523; dieselbe, Apportionment, 2002, S. 3; Hellerstein, W.McLure Jr. C.E., in: ITPF, 2004, S. 204; Container Corp. of America v. Franchise Tax Board, 463 U.S. 159, 1983.

338 Vgl. Europäische Kommission, Sachverständigenausschuß, 1992, S. 137.

339 Vgl. Li, J., in: CTJ, 2002, S. 855-856. Reinhardt zeigt, dass bei unvollkommenen Märkten ausschließlich zwei Arten der geschäftsfallbezogenen Gewinnzerlegung, die Restgewinnanalyse und die Methode der vergleichbaren Gewinnaufteilung, eine verursachungsgerechte Gewinnzurechnung gewährleisten. Dieses Resultat basiert auf der Trennung von Erfolgsermittlung und Erfolgsallokation im Rahmen der Gewinnaufteilung. Vgl. Reinhardt, F., Erfolgsabgrenzung, 2003, S. 217-221; siehe a. Vormoor, C., Unternehmensdatenbanken, 2005, S. 38.

${ }^{340} \mathrm{Vgl}$. für eine kritische Beurteilung des Prinzips der selbstständigen Einheit Oestreicher, A., Konzerngewinnabgrenzung, 1999, S. 75-124.

${ }^{341} \mathrm{Zu}$ den methodischen Vorbehalten gegenüber dem Grundsatz des Fremdvergleichs vgl. Jacobs, O.H. (Hrsg.), Unternehmensbesteuerung, 2002, S. 875-877. 
vorteilen, Synergieeffekten und Einsparungen von Transaktionskosten zwischen verbundenen Unternehmen lässt die Angemessenheit von fremdvergleichskonformen Verrechnungspreisen zumindest fraglich erscheinen.

\section{b) Konzepte der Gewinnaufteilung}

\section{(a) Gewinn als Indikator für die Inanspruchnahme öffentlicher Leistungen}

Der Erhebung von Steuern für die Inanspruchnahme von öffentlichen Leistungen liegt das Äquivalenzprinzip ${ }^{342}$ zugrunde. Die einzelnen Staaten sollen für ihre Bereitstellung der zur Gewinnerzielung benötigten Mittel wie z. B. der Infrastruktur und den natürlichen Ressourcen entschädigt werden. Die in der Aufteilungsformel enthaltenen Faktoren sind derart zu wählen, dass die durch die Konzerngesellschaften verursachten Kosten der Staaten abgebildet werden. Grundsätzlich ist eine Messung der Inanspruchnahme öffentlicher Leistungen nicht zweifelsfrei möglich. Um dennoch eine Verbindung zwischen Umfang und Wert der öffentlichen Leistung und einer Unternehmenskennzahl herzustellen, können die Aufwendungen der Konzerngesellschaften herangezogen werden. Dieser Vorgehensweise liegt die Annahme zugrunde, dass öffentliche Leistungen die Aufwendung aller Unternehmen im gleichen relativen $\mathrm{Ma} ß$ reduzieren. ${ }^{343}$ Selbst wenn entsprechende Faktoren und die Gewichtung derselben im Hinblick auf den Äquivalenzgedanken gewählt werden könnten, so dient weiterhin der Gewinn als Steuerbemessungsgrundlage. D. h. konkret, dass zwei Unternehmen mit identischen Aufwendungen unterschiedlich hohe Steuerzahlungen entrichten müssten, nur weil eines der beiden effizienter produziert. Dies widerspricht jedoch der obigen Annahme, dass eine Beziehung zwischen den Aufwendungen eines Unternehmens und den in Anspruch genommenen öffentlichen Leistungen besteht. ${ }^{344}$ Dieser Widerspruch, d. h. der fehlende $\mathrm{Zu}$ sammenhang zwischen der Entwicklung des Gewinns auf der einen Seite und der Nutzung öffentlicher Leistungen auf der anderen Seite, ist auch ein wesentlicher Grund, weshalb das Äquivalenzprinzip als Rechtfertigung der Erhebung einer separaten Körperschaftsteuer vielfach abgelehnt wird. ${ }^{345}$ Damit wäre auch

${ }^{342}$ Das Äquivalenzprinzip baut auf der Vertragstheorie oder auch auf der Assekuranztheorie auf, wonach Steuern das Entgelt für staatliche Leistungen darstellen. Vgl. Hey, J., Harmonisierung, 1997, S. 256.

343 Vgl. Musgrave R.A./Musgrave, P.B., in: Bird/Head, Fiscal, 1972, S. 70.

$344 \mathrm{Vgl}$. Oestreicher, A., Konzerngewinnabgrenzung, 1999, S. 173-174; Musgrave, P.B., in: McLure, State, 1984, S. 229-230; dieselbe, in: Public Finance, 1972, S. 400.

345 Vgl. Hey, J., Harmonisierung, 1997, S. 262; Schipporeit, E., in: StuW, 1980, S. 193; Steuerreformkommission, Gutachten, 1971, KST IV, Tz. 58; Vodrazka, K., in: StuW, 1971, S. 240; Wagner, A., Gebührenlehre, 1890, S. 214; Schneider, D., in: StuW, 1975, S. 107; derselbe, in: Neumark, Finanzwissenschaft, 1980, S. 529-546; derselbe, Grund- 
eine Gewinnzuordnung unter der Berücksichtigung des Äquivalenzgedankens systematisch nicht korrekt. In den Vereinigten Staaten hingegen basierte die Einführung der Körperschaftsteuer zunächst ausschließlich auf dem Äquivalenzprinzip. ${ }^{346}$ Somit ist es nicht verwunderlich, dass auch bei der Begründung der Aufteilungsformel auf das Aufwiegen staatlicher Leistungen verwiesen wird. $^{347}$

\section{(B) Makroökonomische und industrieweite Größen als Aufteilungsmaßstab}

Bei der Verwendung makroökonomischer bzw. industrieller Größen fließen ausschließlich nicht unternehmensbezogene Größen in die Aufteilungsformel ein. Die Verteilung des Konzernerfolgs erfolgt damit unabhängig vom Umfang der Aktivitäten einer Konzerngesellschaft in einem Staat. Sobald ein verbundenes Unternehmen die räumlichen Anknüpfungskriterien in einem Land erfüllt, kann es erheblich zur Steuer herangezogen werden, auch wenn der Konzern nur untergeordnete Interessen in dem Staat verfolgt. ${ }^{348}$ Voraussetzung dafür ist lediglich, dass der Umfang der makroökonomischen oder industriellen Größen in dem entsprechenden Staat im Vergleich zu den übrigen Staaten stark ausgeprägt ist. Diese Vorgehensweise widerspricht den internationalen Besteuerungsprinzipien, die sich im Territorialprinzip widerspiegeln, da der Gewinn unabhängig von seiner Entstehung der Steuer unterworfen wird. Besonders bei großen Differenzen der Körperschaftsteuersätze ist für Unternehmen eine vom Ort der Gewinnerstehung unabhängige Besteuerung der Unternehmensgewinne inakzeptabel. Auf diese Problematik weist auch die Europäische Kommission hin, sodass die Anwendung einer makroökonomischen Aufteilungsformel wohl ausgeschlossen werden kann. ${ }^{349}$

Der Grund, warum diese Option der Formelausgestaltung dennoch in der Literatur - wenn auch nur am Rande - diskutiert wird, ist die fast vollständige

züge, 1994, S. 175. Konträrer Meinung hingegen sind Redig, K./Müller, W., Steuerlehre, 1999, S. 321, die nach wie vor im Äquivalenzprinzip die üblichste Begründung der Körperschaftsteuer sehen und die Rechtfertigungsversuche von Schneider stellenweise als unüblich bezeichnen.

346 Dort wird die Körperschaftsteuer als ,a tax upon the doing business with the advantage which inhere in the pecularities of corporate or joint stock organizations" gesehen. Flint v. Stone Tracy Co, 220 U.S. 107, 1911.

347 Vgl. House of Representatives, Report, 1964, S. 159; General Motors Corp. v. District of Columbia, 380 U.S. 553, 1965.

348 Vgl. Wellisch, D., in: StuW, 2004, S. 271; Eggert, W./Haufler, A., Coordination, 2006, S. 17; Hellerstein, W./McLure Jr., C.E., in: ITPF, 2004, S. 211; Mintz, J., in: FA, 1999, S. 407.

349 Vgl. Europäische Kommission, Mechanismus, 2006, S. 5. 
Loskopplung der Steuerermittlung von steuerplanerischen Gestaltungsmaßnahmen der Unternehmen. ${ }^{350}$ Es bedarf schon einer marktbeherrschenden Stellung eines Konzerns in einem Land, um durch unternehmenspolitische Entscheidungen Einfluss auf die in die Formel einfließenden Größen zu nehmen. Somit garantiert dieser Ansatz im besonderen Maße die Neutralitätseigenschaft der Besteuerung. Jedoch existiert dieser Zustand nur kurzfristig unter Vernachlässigung der dynamischen Anpassungsmaßnahmen seitens der Unternehmen. Langfristig werden die Konzerne ihre Struktur derart ausrichten, dass Anknüpfungspunkte in Staaten vermieden werden, die die Steuerzahllast der Konzerne erhöhen. Anstelle einer in den Konsolidierungskreis einbezogenen Tochtergesellschaft oder einer Betriebsstätte tritt dann ein Direktgeschäft oder eine Gesellschaft, die nicht im Rahmen der Konsolidierung veranlagt wird. Die hier beschriebenen Anpassungen wären hinfällig, wenn sich die Steuersätze zwischen den Mitgliedstaaten der Europäischen Union nur geringfügig voneinander unterschieden. Jedoch bestünde dann auch kein Grund mehr, makroökonomische Faktoren zu benutzen. Der Anreiz zur Gewinnverlagerung wäre gering, und die Effekte der Formelaufteilung beschränkten sich auf die grenzüberschreitende Verlustverrechnung.

\section{( $\gamma$ ) Gewinn als Verzinsung des eingesetzten Kapitals}

Der Ansatz, den Konzernerfolg anhand des geografischen Standorts der eingesetzten Vermögenswerte auf die einzelnen Gebietskörperschaften aufzuteilen, beruht auf der Vorstellung, dass der Gewinn der Verzinsung des investierten Kapitals entspricht. ${ }^{351}$ Analog zum Arbeitsortprinzip ${ }^{352}$, das Arbeitseinkommen in dem Land besteuert, in dem die Arbeit erbracht worden ist, soll der Gewinn am Ort des investierten Kapitals, also am Ort der Belegenheit der Wirtschaftsgüter besteuert werden. ${ }^{353}$ Dazu ist der Konzernerfolg im Verhältnis der ökonomischen Werte der Wirtschaftsgüter auf die Gliedgesellschaften aufzuteilen. Einer derartigen Verteilung liegt die implizite Annahme zugrunde, dass sich

350 Vgl. Eggert, W./Haufler, A., Coordination, S. 17; Sørensen, P., in: ITPF, 2004, S. 96.

${ }^{351}$ Im Rahmen der Verrechnungspreisbestimmung wird diese Methode vielfach als „(asset-base) profit split" oder „capital employed method" bezeichnet. Dabei ist ihre Anwendung auf einzelne Geschäftsfälle beschränkt, sodass sie lediglich eine Ergänzung der direkten Methode durch Bestandteile der indirekten Methode darstellt. Vgl. Jacobs, O.H. (Hrsg.), Unternehmensbesteuerung, 2002, S. 941. Für eine beispielhafte Steuerberechnung mittels der ,asset-base profit split method“" siehe Eden, L., Multinationals, 1998, S. 46.

352 Nach dem Arbeitsortprinzip sind Einkünfte aus einem bestehenden Arbeitsverhältnis grundsătzlich in dem Staat auszuweisen, in dem die Tătigkeit ausgeübt wird. Vgl. Jacobs, O.H. (Hrsg.), Unternehmensbesteuerung, 2002, S. 1265.

353 Vgl. Musgrave, P.B., in: Public Finance, 1972, S. 400; Gammie, M./Radaelli, C.M./Klemm, A., Reform, 2001, S. 49; Harvard Note, in: HLR, 1976, S. 1207. 
erstens die Produktivitäten des Kapitals an allen Standorten entsprechen ${ }^{354}$ und zweitens der Marktwert der eingesetzten Vermögenswerte bekannt ist. ${ }^{355}$ Bei näherer Betrachtung zeigt sich, dass weder die Annahme einheitlicher Renditen haltbar ist noch der ökonomische Wert der Vermögensgegenstände einwandfrei bestimmt werden kann. ${ }^{356}$

Zunächst ist festzustellen, dass eine formelhafte Gewinnaufteilung in Abhängigkeit vom Wert des investierten Kapitals nur dann zu einem befriedigenden Ergebnis führt, wenn das Verhältnis zwischen Gewinn und Kapital an allen Standorten gleich ist, also alle Konzerngesellschaften dieselbe Produktivität aufweisen. Um diese Annahme zu überprüfen, wird ein vollkommener Markt mit freier Faktormobilität, abnehmenden Skalenerträgen, unbegrenzt schneller Reaktionsfähigkeit von Anbietern und Nachfragern, vollständiger Information und ohne Zugangsbeschränkungen betrachtet. Unter dem Postulat der Gewinnmaximierung muss ein unabhängiges Unternehmen langfristig seine Kapitalkosten $r$ erwirtschaften. Unterschreiten die Gewinne die Opportunitätskosten des Kapitals, scheidet das Unternehmen aus dem Markt aus, da eine Anlage am Kapitalmarkt zum Zinssatz $r$ gewinnmaximal ist. Gleichzeitig können keine die Kapitalkosten übersteigenden Gewinne erzielt werden, da bei freiem Marktzutritt neue Wettbewerber so lange um die Überrenditen konkurrieren, bis Kapitalkosten und Marktzins wieder einander entsprechen. ${ }^{357}$

Auch ein integrierter Konzern erwirtschaftet als Gesamtgebilde immer nur die Kapitalkosten. Überrenditen werden wieder durch den Marktzutritt konkurrierender Konzerne aufgebraucht, und eine Verzinsung des Kapitals unter dem Marktzins erfordert erneut eine Investition am Kapitalmarkt zum Zinssatz $r{ }^{358}$ Innerhalb eines integrierten Konzerns fehlt jedoch der Marktmechanismus, der die Verzinsung des Kapitals dem Marktzins entsprechen lässt. Theoretisch könnten damit die Kapitalkosten einzelner Konzerngesellschaften vom Marktzinssatz abweichen, da ausschließlich der Konzernverbund in Summe die Kapitalkosten verdienen muss. Jedoch können Überrenditen einer Konzerngesellschaft (A) nur erzielt werden, wenn zeitgleich eine andere Konzern-

354 Untersuchungen zur aggregierten Kapitalproduktivităt in Deutschland, Japan und den USA zeigen, dass sich die Kapitalproduktivităt in den einzelnen Ländern erheblich voneinander unterscheidet. So lag die Kapitalproduktivităt in Deutschland für den Zeitraum von 1970 bis 1993 durchschnittlich 35 v. H. unter jener der USA. Vgl. McKinsey Global Institute, Productivity, 1996, Chapter 2, S. 1.

355 Siehe hierzu Kapitel C.II.4.c)( $\alpha)$.

356 Die dargelegte Argumentationskette wurde übernommen von Oestreicher. Vgl. Oestreicher, A., Konzerngewinnabgrenzung, 1999, S. 174-179.

357 Vgl. Klemm, R.J./Dwyer, D.W./Brewer, T.L., in: GFJ, 1995, S. 115; Oestreicher, A., Konzerngewinnabgrenzung, 1999, S. 175.

358 Vgl. Higinbotham, H.N. et al., in: TLR, 1987, S. 363-364. 
gesellschaft (B) weniger als die Kosten des Kapitals erwirtschaftet. In diesem Fall lässt sich jedoch das Konzernergebnis steigern, indem das Kapital innerhalb des Konzerns von B nach A umverteilt wird ${ }^{359}$ oder aber Gesellschaft B liquidiert und das freigesetzte Kapital am Kapitalmarkt investiert wird. ${ }^{360}$ Beide Anpassungsreaktionen garantieren, dass auf jeder Konzernstufe die Kapitalkosten erwirtschaftet werden. In einem vollkommenen Markt entspricht somit der Gewinn einer Konzerngesellschaft immer den Opportunitätskosten des investierten Kapitals. ${ }^{361}$

In der Realität ist kein Markt vollkommen, und es existieren sehr wohl $\mathrm{Zu}$ gangsbeschränkungen, immobile Produktionsfaktoren, unvollständige Information und Verzögerungen bei den Anpassungsreaktionen der Marktteilnehmer. Dies bedeutet zugleich, dass die Gewinne der Konzerngesellschaften von den Kapitalkosten abweichen können. Dennoch lässt eine Aufgabe der restriktiven Annahmen des vollkommenen Marktes den Ansatz der Aufteilung des Konzerngewinns anhand des investierten Kapitals nicht zwangsläufig scheitern. So können die Differenzen bei den Kapitalkosten durch die unterschiedliche $\mathrm{Ge}$ wichtung des investierten Kapitals beseitigt werden. ${ }^{362}$ Wesentlich problematischer ist jedoch die implizite Annahme, dass die Quelle des Gewinns ausschließlich im investierten Kapital liegt. Zumindest in der kurzfristigen Perspektive setzt sich der Gewinn aus drei Bestandteilen zusammen: ${ }^{363}$ der Verzinsung des Kapitals, einer Risikoprämie und der ökonomischen Rente. Besteht bei den ersten beiden Bestandteilen des Gewinns noch ein Zusammenhang mit dem Standort des eingesetzten Kapitals, ist er bei der ökonomischen Rente nicht mehr zu erkennen. Monopolistische bzw. monopsonistische Gewinne haben ihren Ursprung für gewöhnlich in der Ausgestaltung der Produkt- und Faktormärkte und stehen nicht in Beziehung zu dem investierten Kapital. Um auch diesem Gewinnbestandteil Rechnung zu tragen, erscheint es sinnvoll, weitere Faktoren in die Formel aufzunehmen. ${ }^{364}$

359 Vgl. Higinbotham, H.N. et al., in: TLR, 1987, S. 363-364.

360 Vgl. Klemm, R.J./Dwyer, D.W./Brewer, T.L., in: GFJ, 1995, S. 115.

${ }^{361}$ Dieses Ergebnis ist insofern interessant, da es sich unter gegebenen Voraussetzungen auch bei der direkten Gewinnabgrenzung mittels der Preisvergleichsmethode einstellt. Vgl. Klemm, R.J./Dwyer, D.W./Brewer, T.L., in: GFJ, 1995, S. 115.

${ }^{362}$ Vor dem Hintergrund unterschiedlicher Kapitalproduktivităten aufgrund immobiler Produktionsfaktoren befürwortet Musgrave, dass bei der Aufteilung nicht ausschließlich auf das Kapital, sondern auch auf das Verhältnis von Kapital zu Arbeit abgestellt wird. Vgl. Musgrave, P.B., in: Public Finance, 1972, S. 400; Vgl. a. Higinbotham, H.N. et al., in: $T L R, 1987$, S. 375.

363 Vgl. Musgrave, P.B., in: Public Finance, 1972, S. 400; Oestreicher, A., Konzerngewinnabgrenzung, 1999, S. 177.

364 Vgl. Oestreicher, A., Konzerngewinnabgrenzung, 1999, S. 177. 


\section{(8) Aufteilung nach dem Ursprung des Gewinns}

Grundgedanke der folgenden Ansätze ist es, den Gewinn an seinem Entstehungsort $\mathrm{zu}$ besteuern. Wo der Ursprung des Gewinns liegt, darüber existieren zwei unterschiedliche Auffassungen. Eine eindeutige ökonomische Empfehlung, einem dieser Ansätze zu folgen, besteht nicht. ${ }^{365}$

Der „supply approach“ betrachtet den Gewinn als am Einsatzort der Produktionsfaktoren erwirtschaftet. ${ }^{366}$ Eine Aufteilungsformel, die den Gewinn entsprechend den eingesetzten Vermögenswerten (Kapital, Boden) und der Lohnsumme (Arbeit) aufteilt, wird diesem Ansatz gerecht. ${ }^{367}$ Der Faktor Arbeit ist jedoch bereits in Abhängigkeit von der Ausgestaltung des Faktormarktes ${ }^{368}$ durch Löhne und Gehälter vergütet worden. Der darüber hinaus erzielte Gewinn muss aber dem Kapital zugerechnet werden, da dieses die Eigentumsrechte besitzt. ${ }^{369}$ Zum gleichen Ergebnis kommen auch Hellerstein und McLure: „It does not seem that there is a good case for basing apportionment on costs of labor; after all, corporate income is a return to capital, not labor". ${ }^{370}$ Aus diesem Grund wird in der Literatur dem „supply approach“ auch vielfach die Aufteilung des Gewinns gemäß dem investierten Kapital subsumiert. ${ }^{371}$

Der „supply-demand approach“ hingegen betrachtet den Gewinn als Ergebnis des Zusammentreffens von Angebot und Nachfrage. ${ }^{372}$ Sowohl dem Staat, in dem die Güter und Leistungen produziert und erbracht werden, als auch dem Staat, in dem diese abgesetzt werden, steht ein Besteuerungsrecht zu. Dabei wird die Angebotsseite durch das Vermögen und die Lohnsumme ${ }^{373}$ in der Auf-

365 Vgl. Musgrave, P.B., in: McLure, State, 1984, S. 235.

366 Vgl. Gammie, M./Radaelli, C.M./Klemm, A., Reform, 2001, S. 49; Oestreicher, A., Konzerngewinnabgrenzung, 1999, S. 179.

$367 \mathrm{Vgl}$. McLure Jr., C.E./Weiner, J., in: Cnossen, Capital, 2000, S. 267.

${ }^{368}$ Musgrave sieht dagegen die Lohnsumme als Faktor der Zuordnung monopsonistischer Gewinne auf dem Arbeitsmarkt. Vgl. Musgrave, P.B., in: Public Finance, 1972, S. 400.

${ }^{369}$ Dies verdeutlicht, dass die Wertschöpfung als Indikator für die Aufteilung des Gewinns nicht unumstritten ist. Vgl. Harvard Note, in: $H L R, 1976, \mathrm{~S} .1207$.

370 Hellerstein, W.McLure Jr., C.E., in: ITPF, 2004, S. 210.

371 Vgl. Hellerstein, W.McLure Jr., C.E., in: ITPF, 2004, S. 209. Sørensen betont die Verzerrungen, die durch die Hinzunahme des Lohnfaktors hervorgerufen werden und spricht sich auch für eine Aufteilung gemäß dem investierten Kapital aus. Vgl. Sørensen, P., in: ITPF, 2004, S. 95-96.

372 Vgl. Musgrave, P.B., in: McLure, State, 1984, S. 234; Harvard Note, in: HLR, 1976, S. 1207.

373 Hinsichtlich der Aufnahme der Lohnsumme gelten auch hier die bereits dargelegten Vorbehalte. Hellerstein und McLure interpretieren Musgrave (Musgrave, P.B., in: McLure, State, 1984, S. 234) derart, dass sie unter dem „supply-demand approach“ nur eine Auf- 
teilungsformel repräsentiert. Demgegenüber trägt die Aufnahme der Außenumsätze in die Aufteilungsformel der Nachfrageseite Rechnung. Gleichzeitig existiert damit ein Faktor in der Aufteilungsformel, der die Ausgestaltung der Gütermärkte miteinbezieht und somit die in Abschnitt C.II.4.b)( $\gamma)$ dargelegten Vorbehalte hinsichtlich der Aufteilung der ökonomischen Renten teilweise ausräumt.

Die Aufteilungsformel, die den Gewinn entsprechend den gleichgewichteten Faktoren Vermögen, Lohnsumme und Umsatz auf die einzelnen Steuerhoheiten verteilt, wird allgemein als Massachusetts-Formula bezeichnet. Sie hat Einzug in den Uniform Division of Income for Tax Purposes Act gehalten, ${ }^{374}$ der die Grundlage der Einkommensabgrenzung zwischen den einzelnen Bundesstaaten der USA bildet.

\section{c) Bewertung der Aufteilungsfaktoren}

\section{(a) Vermögensfaktor}

Welches Vermögen in den Formelfaktor einfließt, hängt mit der Wahl des aufzuteilenden Gewinns zusammen. Werden Einkommensbestandteile wie z. B. Patenteinnahmen direkt zugerechnet, wie es in den USA der Fall ist, sollten auch die korrespondierenden Vermögensgegenstände nicht in die Formel eingehen. Zur Vermeidung steuergestalterischer Maßnahmen sollten geleaste Vermögensgegenstände bei der Messung des Vermögensfaktors berücksichtigt werden. Die Ermittlung der Vermögenswerte ist jedoch in der Praxis nicht unproblematisch. Grundsätzlich sind für die Bewertung des Kapitals die Marktwerte maßgebend. Da der Marktwert des investierten Kapitals nur bei börsennotierten Gesellschaften bekannt ist, werden im Rahmen der Aufteilung nicht die Passivseite der Bilanz, sondern die eingesetzten Vermögenswerte herangezogen. Jedoch sind auch hierfür zunächst die Marktwerte zu ermitteln. Dabei kann die Bewertung sowohl nach dem Ertragswert- oder Substanzwertverfahren als auch durch den Ansatz vergleichbarer Marktpreise erfolgen. ${ }^{375}$ Letzterer ist lediglich für einen eingeschränkten Teil der Vermögenswerte praktikabel, da regelmäßig das Kriterium der Vergleichbarkeit nicht erfüllt ist. Das Ertragswertverfahren umgeht diese Problematik, indem die Bewertung anstelle eines Vergleichs anhand der abdiskontierten zukünftigen Gewinne erfolgt. Wäre aber die geografische Zuordnung der Einkommensüberschüsse bekannt, könnten diese als Bemessungsgrundlage herangezogen werden, und eine Aufteilung wäre obsolet. ${ }^{376}$

teilung des Gewinns gemäß dem Vermögen und den Umsätzen, nicht aber anhand der Lohnsumme verstehen. Vgl. Hellerstein, W.McLure Jr., C.E., in: ITPF, 2004, S. 209.

374 Siehe Sec. 9 UDITPA.

$375 \mathrm{Vgl}$. Oestreicher, A., Konzerngewinnabgrenzung, 1999, S. 178.

376 Vgl. McLure Jr., C.E./Weiner, J., in: Cnossen, Capital, 2000, S. 269. 
Damit verbleibt einzig eine Bewertung der Wirtschaftsgüter zu (fiktiven) Wiederherstellungskosten. Zur Ermittlung sind sowohl für die Abschreibungen als auch für zwischenzeitliche Wertsteigerungen die Höhe und der Zeitpunkt festzulegen. ${ }^{377}$ Für ein verbundenes Unternehmen besteht damit stets der Anreiz, in Staaten mit einem niedrigen Steuersatz die Vermögensgegenstände über- und in Hochsteuerländern unterzubewerten. Vor diesem Hintergrund erscheint eine objektive Ermittlung des ökonomischen Werts im Rahmen der formelhaften Gewinnaufteilung unwahrscheinlich.

Liegt der Gewinnaufteilung hingegen nicht der Gedanke der Kapitalverzinsung, sondern jener der Wertschöpfung zugrunde, kommt neben dem bestandsorientierten Ansatz auch noch ein kostenorientierter Ansatz in Frage. ${ }^{378}$ Dabei ist der Verbrauch der Produktionsfaktoren zu Marktwerten zu ermitteln, was allerdings in der Praxis im Vergleich zum bestandsorientierten Ansatz ähnliche Probleme hervorruft. Beide Verfahren führen nur bei gleicher wirtschaftlicher Nutzungsdauer zu identischen Ergebnissen. Unterscheiden sich die Nutzungsdauern hingegen, erhält, bei gleichen Marktwerten der Vermögensgegenstände, die Steuerhoheit, in der die Vermögensgegenstände mit der kürzeren Nutzungsdauer belegen sind, mehr Steuersubstrat zugewiesen. ${ }^{379}$

Da eine objektive Ermittlung von Marktwerten unmöglich erscheint und mit einem hohen administrativen Aufwand verbunden wäre, treten in den USA an die Stelle des ökonomischen Werts die historischen Anschaffungskosten. ${ }^{380}$ Besonders problematisch ist in diesem Zusammenhang die Bewertung der selbst geschaffenen immateriellen Vermögensgegenstände, da nur ein Ertragswertverfahren zur Anwendung kommen kann und damit die Möglichkeit der Gewinnverschiebung durch Verrechnungspreisgestaltung gegeben ist. Darüber hinaus ist bei immateriellen Vermögensgegenständen die Zuordnung zu einer Steuerhoheit schwierig. ${ }^{381}$ Trotz dieser Vorbehalte plant die Europäische Kommission immaterielle Vermögensgegenstände im Rahmen der Gewinnaufteilung zu berück-

\footnotetext{
377 Vgl. Oestreicher, A., Konzerngewinnabgrenzung, 1999, S. 178.

378 Vgl. Musgrave, P.B., in: McLure, State, 1984, S. 214; Hellerstein, W.McLure Jr., C.E., in: ITPF, 2004, S. 209.

379 Vgl. hierzu das Beispiel in Oestreicher, A., Konzerngewinnabgrenzung, 1999, S. 192.

${ }^{380}$ Siehe Sec. 11 UDITPA. Vgl. a. Weiner, J.M., in: TNI, 1996, S. 2124. Eine Ausnahme bildet der US-Bundesstaat New York, der „versucht“, Marktwerte bei der Bewertung des Vermögens heranzuziehen.

381 Vgl. Sørensen, P., in: ITPF, 2004, S. 97; McLure Jr., C.E./Weiner, J., in: Cnossen, Capital, 2000, S. 269-270. Einkommen aus Patenten und Lizenzen zählt in den USA zum „non-business income“, welches direkt den einzelnen Bundesstaaten zugerechnet wird. Dementsprechend gehen immaterielle Vermögensgegenstände auch nicht in die Bewertung des Formelfaktors Vermögen mit ein.
} 
sichtigen, da sie mittlerweile einen der potenziell wichtigsten Faktoren für die Gewinngenerierung darstellen. ${ }^{382}$

Auch bei materiellen Vermögensgegenständen treten durch die Bewertung $\mathrm{zu}$ historischen Anschaffungskosten aufgrund abweichender Kapitalbindungsdauern Verzerrungen auf. ${ }^{383}$ Aus diesem Grund verzichtet Kanada auf den Faktor Vermögen in der Aufteilungsformel. ${ }^{384}$

\section{(B) Umsatzfaktor}

Die Umsätze gehen stets mit ihren aktuellen Erträgen aus den Lieferungen und Leistungen in die Aufteilungsformel ein. Jedoch bleiben, im Vergleich zum Ansatz im Jahresabschluss einer Gesellschaft, Umsätze teilweise unberücksichtigt bzw. werden einer anderen Steuerhoheit im Zuge der Aufteilung zugerechnet. Die Aufnahme der Umsätze in die Aufteilungsformel dient der Berücksichtigung der Nachfrageseite. ${ }^{385}$ Damit dürfen nur Umsatzerlöse in die Aufteilungsformel einfließen, die gegenüber einem fremden Dritten erzielt werden. Innenumsätze, die aus internen Lieferungs- und Leistungstransaktionen resultieren, finden keine Berücksichtigung. Die verbleibenden Außenumsätze sind gemäß dem Bestimmungslandprinzip der Steuerhoheit der Nachfrageseite zuzuordnen. Verkauft eine Konzerngesellschaft mit Sitz in Land A eine Lieferung oder Leistung an einen fremden Dritten in Land B, erhält das Land B einen Besteuerungsanspruch. Das Vorliegen von Umsatzerlösen in einem Land ist aber keine hinreichende, sondern nur eine notwendige Bedingung für die $\mathrm{Zu}$ ordnung von Besteuerungsrechten. Daneben muss der Konzern auch einen steuerlichen Anknüpfungspunkt in Land B vorweisen. Ist dieser nicht gegeben, greift in einigen Bundesstaaten der USA die sogenannte „throwback rule“, die wieder dem Ursprungsland der Lieferung und Leistung das Besteuerungsrecht zuordnet. ${ }^{386}$ Dadurch wird die Entstehung von weißen Einkünften verhindert. Erhielte nämlich ein Staat Steuersubstrat zugewiesen, das er aufgrund der fehlenden Anknüpfung eines Konzerns nicht besteuern dürfte, unterlägen diese Gewinne keiner Besteuerung. ${ }^{387}$ Gleichzeitig resultiert aus der „throwback rule“

382 Vgl. Europäische Kommission, Machanismus, 2006, S. 8.

383 Vgl. McLure Jr., C.E./Weiner, J., in: Cnossen, Capital, 2000, S. 269. Vgl. a. das Beispiel von McLure Jr., C.E., in: McLure, State, 1984, S. 252. Oestreicher, A., Konzerngewinnabgrenzung, 1999, S. 179.

384 Vgl. Smith, E.H., in: CTJ, 1976, zitiert nach Weiner, J.M., Implementing, 1999, S. 24. "[There is] no desire to get into the complications and controversies of a three-factor formula involving capital assets in the province."

Vgl. Kapitel C.II.4.b)(ס).

386 Siehe Sec. 16 b UDITPA.

387 Vgl. McLure Jr., C.E./Weiner, J., in: Cnossen, Capital, 2000, S. 270-271. Zum Vorliegen eines Anknüpfungspunktes vgl. Abschnitt C.II.2. 
eine zufällige Zuordnung der Besteuerungsansprüche, da die Nachfrageseite lediglich dann im Rahmen der formelhaften Gewinnaufteilung berücksichtigt wird, wenn der die Lieferung und Leistung erbringende Konzern im Land des fremden Dritten auch einen Anknüpfungspunkt vorweisen kann. ${ }^{388}$

Vor dem Hintergrund, dass der „supply-demand approach“ sowohl die Angebotsseite als auch die Nachfrageseite der Wertschöpfung repräsentieren soll, ist eine Gleichgewichtung der drei Aufteilungsfaktoren inkonsequent. ${ }^{389}$ Entsprechend sind in den USA vermehrt Bundesstaaten dazu übergegangen, die Außenumsätze in der Aufteilungsformel stärker zu gewichten (Abbildung 4). ${ }^{390}$

Abbildung 4: Entwicklung der Anzahl der US-Bundesstaaten, die dem Umsatz innerhalb der Aufteilungsformel ein höheres Gewicht beimessen

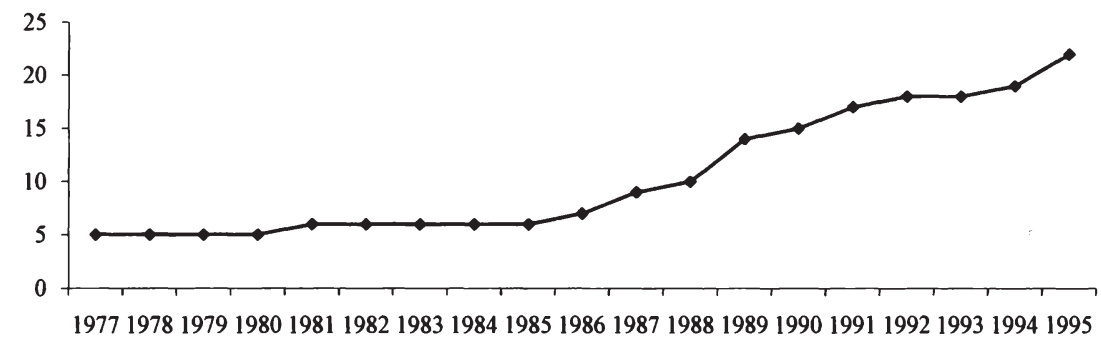

Quelle: Goolsbee, A./Maydew, E.L., in: JoPE, 2000, S. 131

\section{( $\gamma$ ) Lohnsummenfaktor}

Bestehen hinsichtlich der Berücksichtigung der Lohnsumme in der Aufteilungsformel offensichtlich methodische Einwände, so ist die Bewertung dieses Formelfaktors weniger problembelastet. ${ }^{391}$ Grundsätzlich fallen unter die Verrechnungsgröße Lohn sämtliche Formen der Vergütung für persönliche Dienstleistungen. In den USA zählen dazu Löhne, Gehälter, Kommissionen,

${ }^{388}$ Vgl. Fox, W.F.Murray. M.N./Luna, L., in: NTJ, 2005, S. 153-155; Oestreicher, A., Konzerngewinnabgrenzung, 1999, S. 184.

389 Vgl. Oestreicher, A., Konzerngewinnabgrenzung, 1999, S. 182.

390 Ein Abweichen von der Massachusetts-Formula war erst durch das Urteil des Supreme Court in der Rechtssache Moorman Manufacturing Company vs. Bair möglich (Moorman Manufacturing Company vs. Bair, 437 U.S. 267, 1978). Neben dem methodischen Einwand, dass der Umsatzfaktor nur zu $33 \mathrm{v}$. H. in die Aufteilungsformel einfließt, ist auch die Wirkung der formelhaften Gewinnaufteilung ausschlaggebend für eine Erhöhung des Gewichts des Umsatzfaktors. So entspricht eine formelhafte Gewinnaufteilung einer Besteuerung der in die Formel einfließenden Größen. Um die negativen Auswirkungen hinsichtlich der Investitionstätigkeit und der Schaffung von Arbeitsplätzen zu mindern, sind viele Staaten zu einer stärkeren Gewichtung des Umsatzfaktors übergegangen.

Vgl. Hellerstein, W.McLure Jr., C.E., in: ITPF, 2004, S. 211. 
Provisionen und weitere Zuwendungen an die Mitarbeiter (z. B. Pensionen). ${ }^{392}$ Aber auch Spesen und andere besteuerbare Lohnnebenleistungen (bestimmte Sachbezüge) sind Bestandteil dieses Formelfaktors. ${ }^{393}$ Analog zur Berücksichtigung geleaster Vermögensgegenstände sollten auch Zahlungen an unabhängige Kontraktoren bei der Bestimmung der Lohnsumme berücksichtigt werden. ${ }^{394}$ Neben der Frage, welche Bestandteile der Mitarbeitervergütungen in die Lohnsumme einfließen sollten, ist bei der Bewertung auch die Maßeinheit entscheidend. Mit der einfachen Berücksichtigung der Lohnsumme geht die Befürchtung einher, dass die Lohnsatzunterschiede zwischen den Staaten zu Verzerrungen bei der Aufteilung der Bemessungsgrundlage führen. So wird vermutet, dass Ländern mit einem hohen Lohnniveau zu viel Steuersubstrat zugewiesen wird. Folglich erwägen McLure und Weiner anstelle der Lohnsumme einen Rückgriff auf die Anzahl der Mitarbeiter als alternativen Aufteilungsfaktor, um das unterschiedliche Lohnniveau in den einzelnen Ländern auszublenden. ${ }^{395}$ Sie weisen aber zugleich auf die damit einhergehenden konzeptionellen Vorbehalte und potenziellen Verzerrungen hin. Denn den Gewinn in Abhängigkeit von der Anzahl der Mitarbeiter auf die Steuerhoheiten aufzuteilen unterstellt implizit die gleiche Produktivität des Faktors Arbeit in allen Mitgliedstaaten. Oestreicher hingegen zeigt durch einen exemplarischen Vergleich der Lohnstückkosten in sechs Ländern, dass die bestehenden Lohnsatzunterschiede nicht zu einer wesentlichen Benachteiligung der Staaten führen, die ein niedriges Lohnniveau vorweisen. ${ }^{396}$

\section{Forschungsbedarf im Rahmen der empirischen Analyse}

Die Überlegungen zur Gestaltung der einheitlichen (konsolidierten) Körperschaftsteuer-Bemessungsgrundlage verdeutlichen, dass sich auf der Basis theoretischer Analysen zwar Empfehlungen formulieren lassen, aber nur empirische Untersuchungen vermögen die Wirkung der Einführung einer harmonisierten Unternehmensbesteuerung in der Europäischen Union zu belegen.

Um Rückwirkungen auf die Konzernstruktur beurteilen zu können, muss der Aufbau der Konzerne in Europa näher untersucht werden. Dabei sind vor allem

392 Siehe Sec. 1 c UDITPA.

393

Vgl. Riecker, A., Körperschaftbesteuerung, 1996, S. 258.

394 Die Multistate Tax Commission der US-Bundesstaaten empfiehlt jedoch weder Zahlungen an unabhängige Kontraktoren noch Zuwendungen an Mitarbeiter, die ausschließlich in einem Staat tătig werden, in dem das Unternehmen nicht körperschaftsteuerpflichtig ist, zu berücksichtigen. Vgl. Multistate Tax Commission, Compact, 2003, Sec. IV, 13-14.

$395 \mathrm{Vgl}$. McLure Jr., C.E./Weiner, J., in: Cnossen, Capital, 2000, S. 270.

396 Vgl. Oestreicher, A., Konzerngewinnabgrenzung, 1999, S. 184-190. 
Konzernstrukturelemente zu identifizieren, deren Existenz primär auf den bestehenden steuerrechtlichen Rahmen zurückzuführen ist. Die Ergebnisse bilden den Grundstein für weiterführende dynamische Mikrosimulationsmodelle. Ein Rückgriff auf bestehende Untersuchungen ist nicht möglich, da sich die empirische betriebswirtschaftliche Konzernforschung in der Vergangenheit insbesondere mit der Konzernführung und der Konzernorganisation beschäftigte ${ }^{397}$ und der rechtliche Konzernaufbau nur am Rande analysiert wurde. ${ }^{398}$

Die Aufkommenswirkungen der Einführung einer einheitlichen (konsolidierten) Körperschaftsteuer-Bemessungsgrundlage werden mittels einer statischen Mikrosimulationsanalyse abgeschätzt. Insbesondere dort, wo die theoretischen Analysen einen Ermessensspielraum offen lassen, unterstützen die empirischen Berechnungen entsprechende Akteure in ihrer Entscheidungsfindung. So ist beispielsweise die Festlegung der Gewichtung der einzelnen Aufteilungsfaktoren oder aber die Bestimmung der Höhe der Mindestbeteiligungsquote letztlich eine politische Entscheidung, die sich nur bedingt auf der Basis fundierter theoretischer Überlegungen treffen lässt.

Um die oben genannten empirischen Fragestellungen bearbeiten zu können, muss eine geeignete Datenbasis zur Verfügung stehen. Im folgenden Kapitel werden deren Auswahl und Aufbereitung detailliert beschrieben. Vielfach müssen aufgrund der eingeschränkten Datenlage Annahmen getroffen werden. Insofern dient der folgende Abschnitt auch als Grundlage einer korrekten Einschätzung und Interpretation der späteren Ergebnisse.

397 Die konzeptionellen Grundlagen zur Konzernorganisation wurden im Arbeitskreis Dr. Krähe der Schmalenbach-Gesellschaft geleistet. Vgl. Arbeitskreis Professor Dr. Krähe der Schamlenbach-Gesellschaft, Konzernorganisation, 1964. Eine umfangreiche empirische Analyse zur Konzernorganisation findet sich in Hoffmann, F. (Hrsg.), Konzernhandbuch, 1993. Vgl. für eine Übersicht über den Stand der empirischen Konzernorganisationsforschung Matiaske, W./Mellewigt, T., in: Matiaske/Mellewigt, Organisationsforschung, 2000, S. 9-25. Für eine theoretische Darstellung der Parallelen zwischen gesellschaftsrechtlichen und organisatorischen Konzernstrukturen vgl. Bleicher, K., Organisation, 1991, S. 632-644.

$398 \mathrm{Vgl}$. für empirische Befunde zur Rechtsstruktur von Konzernen Weichenrieder, A./Mintz, J.M., Ownerhip, 2006; Lühn, A., Rechtsformwahl, 2004, S. 57-64. Desai, M./Foley. F./Hines Jr. J., in: Hermann/Lipsey, Direct Investment, 2003, S. 61-98. 


\section{Methodik der empirischen Analyse}

\section{I. Überblick}

Die Qualität empirischer Arbeiten wird wesentlich durch zwei Faktoren bestimmt. Erstens muss eine fundierte Datenbasis vorhanden sein, auf deren Grundlage die Analyse erfolgt. Die Anforderungen an eine solche Datenbasis und die Auswahl derselben werden im folgenden Kapitel erläutert. Zweitens müssen die Aufbereitung und ggf. die Ergänzung der Daten plausibel sein. Beide Anforderungen bedingen sich gegenseitig. Je besser und umfangreicher die Datenquelle ist, desto geringer fält die Datenaufbereitung aus und desto weniger Annahmen müssen getroffen werden. Die Sensibilität unternehmensbezogener Einzeldaten bedingt jedoch Informationsdefizite, sodass der Aufbereitung der Datenquelle im Rahmen dieser Arbeit eine wesentliche Bedeutung zukommt. Sie erfordert Pauschalannahmen und Schätzungen, da detailliertere Informationen nicht verfügbar sind. Insofern ist insbesondere die in den folgenden Kapiteln dargestellte Umsetzung der konsolidierten Besteuerung nicht immer deckungsgleich mit den Ergebnissen des Kapitels C.II. Etwaige Abweichungen und die damit verbundenen Verzerrungen werden an entsprechender Stelle erläutert bzw. quantifiziert.

Die Art und der Umfang der empirischen Analyse und des damit verbundenen Datenvolumens erfordern auch einen erheblichen technischen Aufwand. Zunächst müssen sämtliche Daten aus der kommerziellen Datenbank exportiert und in eine neue relationale Datenbank übertragen werden. Dieser Schritt ist notwendig, da die Benutzeroberfläche der ausgewählten Amadeus-Datenbank vornehmlich Suchalgorithmen zur Verfügung stellt. Tief gehende Analysen, komplexe Berechnungen sowie die Veränderung von Daten sind nur sehr eingeschränkt möglich. Aufgrund des Datenumfangs von über 10 Gigabyte und einer damit verbundenen Rechenbelastung bei Ausführung der Prozeduren scheidet eine lokale Desktoplösung ${ }^{399}$ aus. Zum Einsatz kommt ein Microsoft SQL Server als Backend, auf den über Microsoft Access als Frontend zugegriffen wird. Statistische Berechnungen werden mittels der Software SPSS 12 durchgeführt.

\section{Anforderungen an die Datenquelle und Auswahl der Datenbank}

Für eine exakte Abbildung der Steuerbelastung der Konzerne in Europa müssen die Steuerbilanz und der steuerliche Gewinn der einzelnen Konzerngesell-

399 Microsoft Access 2000 kann nur Datenbanken bis zu einer Größe von 2 Gigabyte verwalten. 
schaften bekannt sein. ${ }^{400}$ Darüber hinaus müssen für die Berechnung der konsolidierten Bemessungsgrundlage Informationen über interne Leistungsbeziehungen zwischen den einzelnen Konzernunternehmen vorliegen. Dass eine derart umfangreiche und detaillierte Datenbasis aufgrund des Steuergeheimnisses nicht öffentlich zugänglich ist bzw. gar nicht existiert, ist nachvollziehbar. Somit beschränken sich die folgenden Ausführungen auf die Mindestanforderungen, die an eine Datenbank unter Berücksichtigung der Zielsetzung zu stellen sind.

Folgende fünf Mindestanforderungen muss die Datenbasis erfüllen:

(1) Beteiligungsdaten: Für die Abbildung der Konzernstrukturen müssen alle Beziehungen der Unternehmen untereinander bekannt sein. Die Abgrenzung der steuerlichen Konzernkreise erfolgt nach der Beteiligungshöhe und der Rechtsform, unter der die Gesellschaften firmieren. Beide Informationen müssen in der Datenbasis vorhanden sein. Um eine Zerteilung der Konzerne und damit eine Verzerrung der Ergebnisse zu vermeiden, muss die Datenbasis möglichst breit sein.

(2) Jahreseinzelabschlüsse: Der handelsrechtliche Einzelabschluss der Konzerngesellschaften muss vorhanden sein. Für einen späteren Vergleich der Jahresabschlüsse müssen diese in einem einheitlichen Format vorliegen. Die einzelnen Positionen der Bilanz und der Gewinn-und-Verlust-Rechnung, die Währung, in der die Abschlüsse aufgestellt wurden, und die Einheit müssen länderübergreifend übereinstimmen.

(3) Wirtschaftszweigklassifikation: Für die Auswertung und Identifizierung typischer Konzernstrukturen sind Angaben über die Aktivität der jeweiligen Konzerne notwendig. Eine rein verbale Tätigkeitsbeschreibung erscheint bei der Vielzahl der Unternehmen wenig hilfreich, sodass eine Einordnung der Konzerngesellschaften in ein Wirtschaftszweigklassifikationssystem vorliegen muss.

(4) Identifikationsschlüssel: Die Verknüpfung aller Angaben auf der Ebene des einzelnen Unternehmens muss gewährleistet sein. Diese zunächst triviale Bedingung beschränkt die Auswahl möglicher Datenbanken beträchtlich, da eine Kombination verschiedener Datenquellen fast immer ausgeschlossen oder nur mit erheblichem Aufwand möglich ist. ${ }^{401}$

${ }^{400} \mathrm{Zu}$ wissenschaftlichen Forschungszwecken stellen die Forschungsdatenzentren des Statistischen Bundesamtes und der Statistischen Landesämter auch steuerliche Unternehmensdaten zur Verfügung. Einzeldaten sind aus Gründen der Anonymisierung jedoch nicht zugänglich.

${ }^{401}$ Im Rahmen eines Dienstleistungsauftrags für das Bundesministerium der Finanzen zur Weiterentwicklung der Konzernbesteuerung in Deutschland und Europa wurden die hier gewonnenen Konzernstrukturdaten mit den Steuerbilanzdaten des Statistischen Bundesamtes verknüpft. Dazu wurde zunächst eine Verbindung zwischen der Identifikations- 
(5) Europaweite Datenbank: Die relevanten Unternehmensdaten müssen für alle 25 EU-Mitgliedstaaten vorhanden sein. ${ }^{402}$ Eine Auswahl weniger Länder verzerrt das Ergebnis zwangsläufig, da entweder nur unvollständige Konzerne abgebildet werden oder aber bei einer Beschränkung auf Konzerne aus ausgewählten Mitgliedstaaten die Inbound-Investitionen in diese Länder zum Teil vernachlässigt werden.

Grundsätzlich existiert eine Vielzahl von Anbietern von Unternehmensinformationen. Neben den kommerziellen Angeboten erheben auch öffentliche Institutionen wie z. B. die Deutsche Bundesbank oder das Statistische Amt der Europäischen Gemeinschaften (Eurostat) Unternehmensdaten, welche zu Forschungszwecken genutzt werden können. Die Angebote unterscheiden sich hinsichtlich ihrer Zielsetzung. Ein Großteil der Datenbanken dient der Unterstützung von Marketingaktivitäten und beschränkt sich demnach auf globale Informationen wie Branchen, Adressen und Ansprechpartner. Daneben konzentrieren sich andere Anbieter auf die Bereitstellung von Finanzdaten börsennotierter Unternehmen. Die Statistiken der öffentlichen Institutionen verfügen mehrheitlich nur über aggregierte Daten. Tabelle 10 enthält eine engere Auswahl von Anbietern von Unternehmensinformationen.

Rein nationale Datenbasen sind gemäß den Mindestanforderungen zu vernachlässigen. Die Direktinvestitionsstatistik der Deutschen Bundesbank kann als Zwischenform einer rein nationalen und einer internationalen Datenbank betrachtet werden. Zwar enthält sie Angaben über die einzelnen direkt und indirekt gehaltenen Tochtergesellschaften im Ausland ${ }^{403}$, europäische Konzerne ohne Bezug zu Deutschland werden jedoch vollständig ausgeblendet. Auch die Abbildung deutscher Konzerne erfolgt nur unvollständig. Konzerngesellschaften ohne Direktinvestitionen tauchen gar nicht auf. Halten unterschiedliche Gesellschaften eines Konzerns ein ausländisches Tochterunternehmen, ist es anhand der Direktinvestitionsstatistik nicht möglich, eine Verbindung zwischen diesen beiden Teilkonzernen herzustellen.

Nur die Datenbanken von D\&B und Bureau van Dijk bilden Konzerne europaweit $a b$ und ermöglichen gleichzeitig eine Verknüpfung der Konzernstrukturen mit den Unternehmenseinzeldaten.

nummer des Bureau van Dijk und der Handelsregisternummer hergestellt. Da im Unternehmensregister sowohl die Steuernummern der einzelnen Gesellschaften als auch die Handelsregisternummer hinterlegt sind, konnte dann im zweiten Schritt die Steuernummer ermittelt werden. Vgl. Oestreicher, A. et al., Weiterentwicklung, 2006, S. 428, FN 254.

Eine Übersicht über die 25 Mitgliedstaaten und der innerhalb dieser Arbeit verwendeten Abkürzungen befindet sich in der Tabelle 11.

403 Vgl. zu den Meldevoraussetzungen ausländischer Tochtergesellschaften Tabelle 54 im Anhang. 
Tabelle 10: Unternehmensdatenbanken im Vergleich

Anbieter/Produkt Datenbankbeschreibung

Őffentliche Institutionen:

Bundesbank

1 Mikrodatenbank Direktinvestitionsbestände ${ }^{(1), 2,3,(5)}$

Die Direktinvestitionsstatistik enthält Jahresabschluss- und Beteiligungsdaten aller deutschen Konzernunternehmen und deren Beteiligungen im Ausland. Die Abbildung vollständiger Konzerne ist derzeit noch nicht möglich. ${ }^{404}$

2 Jahresabschlüsse westdeutscher Unternehmen

Die Jahresabschlussdaten basieren auf Angaben, die im Zusammenhang mit dem Rediskontgeschäft eingereicht wurden. Es sind keine Unternehmenseinzeldaten, sondern lediglich nach Branchen untergliederte Verhältniszahlen verfügbar.

Europäische Union

3 Bach-Datenbank ${ }^{(5)}$ Die Bach-Datenbank enthält harmonisierte Jahresabschlussdaten für Unternehmen elf europäischer Mitgliedstaaten. Die Daten liegen aggregiert vor und können nach 23 Branchen, 3 Größenklassen und 94 Jahresabschlusspositionen aufgegliedert werden. Die deutschen Angaben werden aus der Jahresabschlussstatistik der Bundesbank (2) gewonnen.

4 Eurostat - $\quad$ Eurostat hălt aggregierte Statistiken über Input-Variablen (Arbeit/Kapital) und OutUnternehmens- put-Variablen (Umsatz/Wertschöpfung) für die einzelnen EU-Mitgliedstaaten und statistik $^{5}$ Branchen vor. ${ }^{405}$

Kommerzielle Anbieter:

Bureau van Dijk Electronic Publishing GmbH

5 Amadeus 1,2,3,4,5 Die Amadeus-Datenbank hält für ca. 6,15 Mio. aus allen EU-Mitgliedstaaten Jahreseinzelabschlussdaten bereit. Das Ownership-Modul liefert zu den Unternehmen die jeweiligen Beteiligungsinformationen.

Commerzbank AG

6 Wer gehört $\mathrm{zu}$ wem? ${ }^{1,3}$

Die Beteiligungsdatenbank der Commerzbank enthält ca. 12.000 deutsche Unternehmen und ca. 20.000 Anteilseigner.

Dun \& Bradstreet Inc.

7 Family Tree ${ }^{1,3,4,(5)}$ Die Beteiligungsdatenbank enthält über 7,8 Mio. Unternehmensverflechtungen und weltweit 380.000 Unternehmensgruppen.

8 Unternehmens- Die D\&B Datenbank stellt Informationen zur Bewertung der wirtschaftlichen berichte ${ }^{2,3,4,(5)} \quad$ Situation von Unternehmen in neun EU-Mitgliedstaaten zur Verfügung. Eine Verknüpfung mit der Family-Tree-Datenbank ist möglich.

Hoppenstedt Financial Information GmbH

9 Bilanzdatenbank ${ }^{2}$, Für das Jahr 2003 enthält die Datenbank Einzelabschlüsse für 41.144 deutsche Unter3,4 nehmen. Eine Verknüpfung mit der Konzernstrukturdatenbank ist möglich.

10 Konzernstruktur- Die Beteiligungsdatenbank enthält zu den bedeutendsten deutschen und ausländischen datenbank ${ }^{1,4,(5)}$ Konzernen das gesamte aktuelle Beteiligungsportfolio. Derzeit umfasst die Datenbank weltweit ca. 140.000 Unternehmen mit Konzernverflechtungen.

Thomson Financial

12 Worldscope Die Worldscope-Datenbank stellt Jahresabschlussdaten von 40.000 AktiengesellFundamentals ${ }^{2,3,(5)}$ schaften aus weltweit 50 Staaten zur Verfügung.

1-Beteiligungsinformationen 2-Jahrescinzelabschlüse 3-Wirtschaftszweigkdassifikation 4-Identifikationsnummer 5-Europaweite Datenbank

Quelle: eigene Darstellung, Stand: April 2006

404 Die Meldebögen der AWV wurden zum 1. 1. 2006 geändert. Der Meldepflichtige muss neben den Angaben zum Vermögen in fremden Wirtschaftsgebieten auch Auskunft über etwaige Abhăngigkeitsverhältnisse zu anderen deutschen Gesellschaften darlegen. Vgl. Anlage K3 zur AWV.

$405 \mathrm{Vgl}$. zu Definitionen von Merkmalen der strukturellen Unternehmensstatistik Verordnung (EG) Nr. 2700/98 der Kommission v. 17. 12. 1998, Abl. Nr. L 344, v. 18. 12. 1998, S. 49-80. 
Die Amadeus-Datenbank des Anbieters Bureau van Dijk, die größte ihrer Art in Europa, verfügt über sämtliche Unternehmenseinzeldaten in allen $25 \mathrm{EU}$ Mitgliedstaaten (Tabelle 11). Das Zusammenfassen der Bilanzen auf 23 und der Gewinn-und-Verlust-Rechnung auf 24 Positionen erlaubt es, länderübergreifend Bilanzdaten unterschiedlicher Rechnungslegungsstandards miteinander zu vergleichen. Damit erfüllt einzig die Amadeus-Datenbank alle Mindestanforderungen und wird somit als Datenquelle (Stand: Februar 2005, Update 125) für die folgenden empirischen Analysen herangezogen. Zur Verbesserung der Datenlage wird im Rahmen der Datenaufbereitung vereinzelt auf die EurostatUnternehmensstatistik zurückgegriffen.

Tabelle 11: Anzahl der Unternehmen in der Amadeus-Datenbank nach Mitgliedstaaten

\begin{tabular}{|r|r|l|r|}
\hline & Länderkürzel & \multicolumn{1}{|c|}{ Mitgliedstaat } & Anzahl der Unternehmen \\
\hline 1 & AT & Österreich & 98.707 \\
2 & BE & Belgien & 325.109 \\
3 & CY & Zypern & 154 \\
4 & CZ & Tschechische Republik & 15.700 \\
5 & DE & Deutschland & 783.196 \\
6 & DK & Dänemark & 144.226 \\
7 & EE & Estland & 50.033 \\
8 & ES & Spanien & 706.443 \\
9 & FI & Finnland & 69.703 \\
10 & FR & Frankreich & 898.961 \\
11 & GB & Großbritannien & 1.743 .515 \\
12 & GR & Griechenland & 26.769 \\
13 & HU & Ungarn & 190.648 \\
14 & IE & lrland & 118.788 \\
15 & IT & Italien & 281.804 \\
16 & LT & Litauen & 3.164 \\
17 & LU & Luxemburg & 1.444 \\
18 & LV & Lettland & 6.119 \\
19 & MT & Malta & 1.733 \\
20 & NL & Niederlande & 319.766 \\
21 & PL & Polen & 25.968 \\
22 & PT & Portugal & 60.850 \\
23 & SE & Schweden & 247.269 \\
24 & SI & Slowenien & 31.995 \\
25 & SK & Slowakei & 4.070 \\
\hline Europäische Union gesamt & 6.156 .134 \\
\hline
\end{tabular}

Quelle: eigene Darstellung

\section{Umfang der Amadeus-Datenbank}

Obwohl die Amadeus-Datenbank mit mehr als 6,15 Mio. Unternehmen mit Sitz innerhalb der Europäischen Union über eine sehr breite Datenbasis verfügt, stellt sie dennoch keine Vollerhebung dar. Dies ist im Rahmen einer empirischen Analyse zunächst nicht problematisch, sofern eine wirkliche Zufallsstichprobe vorliegt, durch die ein Rückschluss auf die Grundgesamtheit gewährleistet ist. 
Die Amadeus-Datenbank ist allerdings keine Zufallsstichprobe. Sie enthält jedes Unternehmen, über das Informationen verfügbar sind, ${ }^{406}$ soweit keines der folgenden sieben Kriterien erfüllt ist: ${ }^{407}$

1. Der letzte vorhandene Abschluss des Unternehmens stammt aus den Jahren 1997-1999 und früher. In diesem Fall verbleibt das Unternehmen mit seinen Stammdaten in der Datenbank, die Jahresabschlüsse hingegen werden gelöscht. Stammt der letzte bekannte Jahresabschluss aus dem Jahr 1996 oder früher, taucht das Unternehmen nicht in der Datenbank auf.

2. Der Branchencode der Wirtschaftszweigklassifikation NACE Rev. 1.1 ist 6511, 6512, 6522 oder 66*. Zentral-, Universal- und Spezialbanken sowie Versicherungen aller Art sind somit nicht in der Datenbank enthalten. Gleiches gilt für einen aus der NACE-Nomenklatur abgeleiteten nationalen Branchencode. ${ }^{408}$

3. Der Umsatz und die Bilanzsumme übersteigt in Großbritannien, Deutschland und den Niederlanden 450 Mrd. EUR, in Frankreich, Italien und Spanien $200 \mathrm{Mrd}$. EUR und in allen übrigen Mitgliedstaaten 50 Mrd. EUR.

4. Die Anzahl der Mitarbeiter übersteigt 500.000. In diesem Fall wird lediglich die Mitarbeiterzahl auf „n. a.“ gesetzt; das Unternehmen bleibt unverändert in der Datenbank enthalten.

5. Der Umsatz ist unbekannt und die Mitarbeiterzahl übersteigt 100.000 .

6. Der Umsatz pro Mitarbeiter ist kleiner als 300 EUR oder größer als 1 Mrd. EUR.

7. Der Umsatz ist unbekannt, und das Verhältnis von Finanzertrag zu Mitarbeitern oder Finanzergebnis zu Mitarbeitern ist kleiner als 300 EUR.

Somit entscheidet nur das Vorliegen von ausreichend plausiblen und zeitnahen Informationen über die Aufnahme eines Unternehmens in die Datenbank. Rein formell ist ein Rückschluss auf die Grundgesamtheit nicht möglich, da sich die Eigenschaften der Grundgesamtheit und die der Stichprobe systematisch voneinander unterscheiden können. Veröffentlichten z. B. eher profitable Unternehmen ihre Jahresabschlüsse, würde die Ertragslage in der Grundgesamtheit systematisch überschätzt. Um die Aussagekraft der Auswertung beurteilen zu können, soll der Umfang der bewussten Auswahl (Amadeus-Datenbank) an der

406 Der Inhalt der Amadeus-Datenbank beruht auf den Angaben nationaler Datenbankprovider, die vom Bureau van Dijk aufbereitet werden. Der deutsche Datenprovider ist die Creditreform AG.

407 Alle Angaben beziehen sich auf die Amadeus-Datenbank, Stand: Februar 2005, Update 125.

${ }^{408}$ Die Amadeus-Datenbank verfügt für folgende Länder über die nationalen Branchencodes: Italien (ATECO), Niederlande (BIK), Belgien (NACE-BEL), Frankreich, (NAF), Großbritannien (SIC UK), Schweden (SNI), Deutschland (WZ 93), Spanien (CNAE). 
Grundgesamtheit bestimmt werden. Hierzu werden für 17 Mitgliedstaaten der EU die Anzahl der in der Amadeus-Datenbank enthaltenen Unternehmen mit den Einträgen der jeweiligen nationalen Handelsregister verglichen (Tabelle 12).

Tabelle 12: Relativer Anteil der Kapitalgesellschaften in der Amadeus-Datenbank ${ }^{409}$

\begin{tabular}{|c|c|c|c|c|c|c|}
\hline \multirow[b]{2}{*}{ Land } & \multicolumn{3}{|c|}{ Eurostat/Destatis } & \multirow{2}{*}{$\begin{array}{c}\text { Amadeus- } \\
\text { Datenbank Stand } \\
\text { Februar } 2005\end{array}$} & \multirow{2}{*}{$\begin{array}{l}\text { Anteil } \\
\text { in v. } \mathrm{H} .\end{array}$} & \multirow{2}{*}{$\begin{array}{l}\text { Standardabweichung } \\
\text { über die Branchen in } \\
\text { Prozentpunkten }\end{array}$} \\
\hline & Jahr & Anzahl & Erfassungsgrad $^{410}$ & & & \\
\hline $\mathrm{CZ}$ & 2003 & 135.435 & $\sim 99 \%$ & 14.476 & 10,7 & 12,1 \\
\hline$\underset{411}{\mathrm{DE}}$ & 2001 & 650.522 & $100 \%$ & 554.902 & 85,3 & 21,8 \\
\hline DK & 2001 & 74.816 & $\sim 99 \%$ & 88.331 & 118,1 & 14,3 \\
\hline $\mathrm{EE}$ & 2002 & 30.740 & $\sim 99 \%$ & 38.468 & 125,1 & 13,6 \\
\hline ES & 2002 & 959.657 & $\sim 99 \%$ & 674.533 & 70,3 & 6,4 \\
\hline FI & 2003 & 116.244 & $\sim 95 \%$ & 64.659 & 55,6 & 9,4 \\
\hline GB & 2003 & 1.137 .870 & $\sim 750.000$ nicht erfasst & 1.324 .558 & 116,4 & 33,7 \\
\hline $\mathrm{HU}$ & 2002 & 127.789 & $100 \%$ & 80.421 & 62,9 & 19,3 \\
\hline IT & 2002 & 584.093 & sehr gut & 242.261 & 41,5 & 10,7 \\
\hline LT & 2002 & 28.099 & $\sim 80-83 \%$ & 1.923 & 6,8 & 7,9 \\
\hline LU & 2002 & 18.343 & $\sim 99 \%$ & 1.084 & 5,9 & 12,7 \\
\hline LV & 2002 & 27.531 & k.A. & 5.015 & 18,2 & 33,5 \\
\hline $\mathrm{NL}$ & 2001 & 221.653 & $\sim 99 \%$ & 189.925 & 85,7 & 13,6 \\
\hline PT & 2002 & 263.107 & k.A. & 55.757 & 21,2 & 5,9 \\
\hline SE & 2002 & 246.260 & $\sim 99 \%$ & 218.698 & 88,8 & 15,8 \\
\hline SI & 2002 & 28.537 & $100 \%$ & 22.063 & 77,3 & 26,5 \\
\hline SK & 2003 & 65.197 & $\sim 99 \%$ & 3.551 & 5,4 & 4,1 \\
\hline$\Sigma$ & & 4.065 .371 & & 3.025 .723 & 74,4 & 13,4 \\
\hline
\end{tabular}

Quelle: eigene Berechnung auf der Basis der Eurostat-Statistiken zur Unternehmensdemografie, der Körperschaftsteuerstatistik 2001 und der Amadeus-Datenbank

Der Anteil der Unternehmen in der Amadeus-Datenbank beträgt für die hier aufgeführten 17 Länder der EU 74,4 v. H. Besonders in den kleineren neuen Mitgliedstaaten ist die Datenlage schlecht. Deutlich besser ist die Verfügbarkeit von Unternehmensdaten in den großen Volkswirtschaften der EU-15-Staaten. In Dänemark, Estland und Großbritannien übertrifft die Anzahl der in Amadeus hinterlegten Gesellschaften die Beobachtungen der Statistik zur Unternehmensdemografie. Da der Erfassungsgrad der Statistik in Dänemark und Schweden bei ca. $99 \mathrm{v} . \mathrm{H}$. liegt, lässt sich dieses Ergebnis nur mit der zeitlichen Differenz zwischen den Beobachtungen, einer ungenauen Abgrenzung der Rechtsform ${ }^{412}$ oder des Wirtschaftszweigs erklären. Für das Ergebnis in Großbritannien ist zu-

409 Folgende Branchen (NACE) wurden berücksichtigt: C, D, E, F, G, H, I, J ohne J65 und J66, K ohne K7415 (in Deutschland K ohne K741), M, N und O ohne O91.

${ }^{410} \mathrm{Vgl}$. zu den länderspezifischen Hinweisen zur Methodik Eurostat, Unternehmensdemografie, 2006.

411 Die Angaben für Deutschland stammen aus der Körperschaftsteuerstatistik 2001.

412 Für Deutschland werden unter den Kapitalgesellschaften z. B. auch Gesellschaften aufgeführt, die unter der Rechtsform der GmbH \& Co. KG firmieren. 
sätzlich der schlechte Erfassungsgrad der britischen Unternehmen verantwortlich.

Ein Vergleich des Erfassungsgrads der Amadeus-Datenbank und der EurostatStatistik über die Branchen hinweg zeigt, dass zwischen den einzelnen Tätigkeitsbereichen keine wesentlichen Unterschiede bestehen. Eine Verzerrung der Stichprobe durch eine starke Unter- bzw. Überrepräsentativität einzelner Wirtschaftszweige kann damit ausgeschlossen werden. Lediglich für Deutschland, die Niederlande, Spanien und Großbritannien ist eine höhere Standardabweichung zu beobachten, die jedoch aus einzelnen Ausreißern resultiert. So sind in Deutschland die Unternehmen der Energie- und Wasserversorgung mit 43 v. H. stark unterrepräsentiert. In den Niederlanden und Spanien sind Unternehmen des Bergbaus und der Gewinnung von Steinen und Erde übermäßig stark vertreten; Großbritannien weist bei Gesellschaften des Kredit- und Versicherungsgewerbes mit einem Anteil von nur 34 v. H. starke Defizite auf.

Das Vorhandensein eines Unternehmens in der Amadeus-Datenbank ist nicht gleichbedeutend mit der Verfügbarkeit sämtlicher unternehmensspezifischer Daten. Vielfach beschränken sich die Jahresabschlussinformationen auf die Angabe des Umsatzes oder der Bilanzsumme. Auch bei den Beteiligungsbeziehungen fehlen häufig Angaben zu Höhe der Beteiligung. Folglich müssen die Unternehmenseinzeldaten vor einer Auswertung zunächst aufbereitet werden. Die nachfolgenden Abschnitte geben einen Überblick über die Datenlage und erläutern die Vorgehensweise bei der Datenaufbereitung.

\section{Aufbereitung der Unternehmenseinzeldaten}

\section{Beteiligungsinformationen}

Von den 6,15 Mio. europäischen Unternehmen in der Amadeus-Datenbank ${ }^{413}$ werden 1.121.394 Unternehmen nicht ausschließlich von Anteilseignern gehalten, die einer der folgenden Kategorien zuzuordnen sind: „public authorities“, „one or more named individual or families“, „employees/manager/directors“, „public“. Durchschnittlich sind an diesen Unternehmen zwei Anteilseigner beteiligt, sodass in der Datenbank 2.252.231 Beteiligungsbeziehungen hinterlegt sind. Addiert man zu den 1,12 Mio. Tochtergesellschaften die Muttergesellschaften mit Sitz in der Europäischen Union hinzu, die nicht von einer anderen Gesellschaft gehalten werden, stehen 1.415.601 Gesellschaften der AmadeusDatenbank in einem Beteiligungsverhältnis zueinander. In der Datenbank sind grundsätzlich sowohl die Höhe der Beteiligung des direkten Anteilseigners als auch die totale Beteiligungshöhe der obersten Muttergesellschaft hinterlegt.

${ }^{413}$ Die Informationen über die Beteiligungsbeziehungen sind Bestandteil des optionalen Ownership-Moduls der Amadeus-Datenbank. 
Letztere errechnet sich nicht aus den direkten Beteiligungsinformationen, sondern beruht ausschließlich auf Primärangaben. Um Widersprüche innerhalb des Datenmodells auszuschließen, werden im Rahmen der empirischen Untersuchung zur Abbildung der Konzernstrukturen ausschließlich die direkten Beteiligungsangaben berücksichtigt, und soweit notwendig die Gesamtbeteiligung aus diesen Angaben abgeleitet.

Die Informationen zu den Beteiligungsbeziehungen liegen in der Datenbank nur unvollständig vor. Zum einen fehlen Angaben zu den Unternehmensverbindungen, sodass entweder gar keine oder nur lückenhafte Angaben über die Anteilseigner eines Unternehmens verfügbar sind. Zum anderen ist die Höhe der Beteiligung häufig nur ungenau oder gar nicht angegeben. Beides bewirkt, dass sich die Beteiligungen der Anteilseigner an einer Gesellschaft nicht immer auf 100 v. H. summieren (Tabelle 14). Während fehlende Informationen zu den Anteilseignern nicht ergänzt werden können, kann die Beteiligungshöhe auf der Basis der zur Verfügung stehenden Informationen angepasst werden. Tabelle 13 enthält eine Übersicht über die in der Amadeus-Datenbank hinterlegten Beteiligungsinformationen.

Tabelle 13: Anpassung der Angaben der Beteiligungshöhe in der Amadeus-

Datenbank

\begin{tabular}{|c|c|c|c|c|c|c|c|c|c|c|}
\hline \multirow{2}{*}{\multicolumn{4}{|c|}{ Datenbankinformationen }} & \multicolumn{7}{|c|}{ Anpassungsstufen } \\
\hline & & & & \multicolumn{2}{|l|}{ I } & \multirow{2}{*}{ 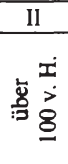 } & \multicolumn{4}{|c|}{ III } \\
\hline 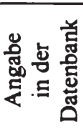 & 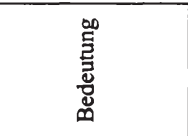 & 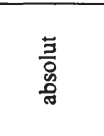 & $\frac{\geqq}{\mathbb{E}}$ & $\begin{array}{l}\text { 品 } \\
\text { 总 } \\
\text { 을 }\end{array}$ & 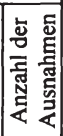 & & 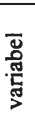 & 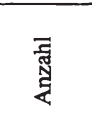 & 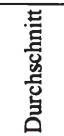 & 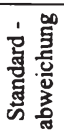 \\
\hline $\mathrm{X}$ & bekannt & 1.023 .445 & $4 \%$ & keine & & $\dot{4}$ & & & & \\
\hline - & unbekannt & 985.690 & $43,8^{\circ}$ & 0,00 & & 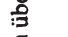 & ja & 790.105 & 32,48 & 35,05 \\
\hline$<\mathrm{X}$ & kleiner $\mathrm{X}$ & 248 & $0,0 \%$ & $X-0,01$ & 18 & 离 & & & & \\
\hline$>\mathrm{X}$ & größer X & 26.377 & $1,2 \%$ & $X+0,01$ & & $I$ & ja & 26.372 & 29,91 & 24,96 \\
\hline CQP1 & 50 v. H. + 1 Aktie & 2 & $0,0 \%$ & 50,01 & & 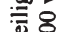 & & & & \\
\hline G & Fehler in der DB & 1 & $0,0 \%$ & 100,00 & & $=$ & & & & \\
\hline MO & mehrheitsbeteiligt & 603 & $0,0 \%$ & 50,01 & & $\frac{\infty}{2}$ & ja & 603 & 36,13 & 13,97 \\
\hline$+1-X$ & $+/-X$ & 58 & $0,0 \%$ & $\mathrm{x}$ & 1 & & & & & \\
\hline NG & unter $1 \mathrm{v} . \mathrm{H}$. & 283 & $0,0 \%$ & 0,01 & & & & & & \\
\hline Wo & über $98 \mathrm{v} . \mathrm{H}$. & 215.524 & $9,6 \%$ & 98,00 & & & & & & \\
\hline Summe & & 2.252 .231 & $100 \%$ & 1.228 .784 & 19 & 24 & & 817.080 & 32,40 & 34,76 \\
\hline
\end{tabular}

Quelle: eigene Berechnung

Bei 45,4 v. H. der Unternehmensverbindungen (X, CQP1, G) ist die Beteiligungshöhe bekannt. Für weitere 9,6 v. H. (WO, MO) kann auf der Basis der Beteiligungsangaben mit Sicherheit auf eine Mehrheitsbeteiligung geschlossen werden. Demgegenüber ist der Umfang der Beteiligung bei $43,8 \mathrm{v}$. H. der Unternehmensbeziehungen unbekannt. Aus den restlichen Angaben kann lediglich geschlossen werden, dass die Beteiligungshöhe einen angegebenen Schwellenwert entweder über- oder unterschreitet. Für die Berechnung der Konzernkreise werden die Beteiligungsinfornationen in đrëi Stufeñ Fuffbéreitet: 
Im ersten Schritt werden alle Angaben in der Datenbank in eine konkrete Zahl übersetzt. Dabei wird stets eine möglichst geringe Abweichung zu den bekannten Angaben gewählt. Ist die Beteiligungshöhe in der Datenbank als ,WO angegeben, bedeutet das, dass der Anteilseigner mindestens mit $98 \mathrm{v} . \mathrm{H}$. an der Tochtergesellschaft beteiligt ist. Entsprechend wird in der Datenbank eine Beteiligung von $98 \mathrm{v}$. H. eingetragen. Um zu verhindern, dass die Beteiligungshöhe nach Anpassung $0 \mathrm{v}$. H. beträgt, wird bei der Angabe $+/-0,0 \mathrm{v}$. H. und $<$ 0,01 die Beteiligungshöhe auf $0,01 \mathrm{v}$. $\mathrm{H}$. gesetzt, sofern dadurch die Gesamtbeteiligung an dem Unternehmen nicht $100 \mathrm{v}$. H. übersteigt.

Der zweite Anpassungsschritt beseitigt Fehler in der Datenbank. Bei 25 Unternehmen beträgt die Gesamtbeteiligung der Anteilseigner weit über $100 \mathrm{v} . \mathrm{H}$.

Im dritten Schritt wird die Beteiligungshöhe von Anteilseignern nach oben korrigiert, sodass in der Summe die Gesamtbeteiligung an den Tochtergesellschaften $100 \mathrm{v} . \mathrm{H}$. entspricht. Variabel sind dabei nur Beteiligungsangaben, deren Höhe entweder gar nicht bekannt ist $(-)$, bei denen lediglich eine Mindestbeteiligungshöhe angegeben ist $(>\mathrm{X})$ oder bei denen die Anteilseigner über eine Mehrheitsbeteiligung verfügen (MO).

Die Anpassung erfolgt anhand der untenstehenden Formel, die garantiert, dass die Gesamtbeteiligung an einem Unternehmen nicht $100 \mathrm{v} . \mathrm{H}$. überschreiten kann. Abbildung 5 verdeutlicht die Vorgehensweise anhand eines Beispiels.

$$
B_{n}=B_{a}+\frac{(100-G)}{A}(2)
$$

wobei: $\quad B_{n} \quad$ Beteiligungshöhe neu

$B_{a} \quad$ Beteiligungshöhe alt

G bekannte Gesamtbeteiligung vor Anpassung

A Anzahl der Anteilseigner, die angepasst werden können

Abbildung 5: Anpassungen der Beteiligungsinformationen - Beispiel

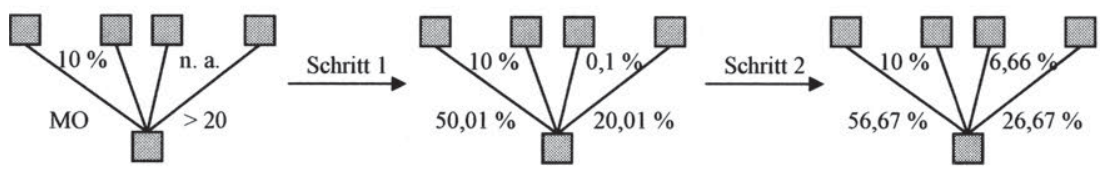

Quelle: eigene Darstellung

Die Tabelle 14 zeigt die aufsummierten bekannten und zuordenbaren Anteile der Anteilseigner in v. H. je Gesellschaft nach dem zweiten und dritten Anpassungsschritt. Sind nach der zweiten Stufe bei 42,48 v. H. der Unternehmen alle Anteilseigner bekannt, steigt der Anteil durch das Anpassen der Beteiligungsinformationen um fast 39 Prozentpunkte auf $81,42 \mathrm{v}$. H. an. Nach der dritten Anpassungsstufe addieren sich bei 93,22 v. H. der Gesellschaften die an ihnen gehaltenen Beteiligungen zu über $90 \mathrm{v}$. H. auf. Berücksichtigt man, dass durchschnittlich ein Unternehmen von zwei Anteilseignern gehalten wird, kann für 
die große Mehrzahl der Unternehmen die Konzernzugehörigkeit sicher festgestellt werden.

Tabelle 14: Aufsummierte bekannte und zuordnungsfähige Anteile der Anteilseigner in v. H. je Gesellschaft

\begin{tabular}{|c|c|c|c|c|c|c|c|c|}
\hline \multirow{3}{*}{$\begin{array}{c}\begin{array}{c}\text { Beteiligungs- } \\
\text { höhe }\end{array} \\
\text { v. H. }\end{array}$} & \multicolumn{4}{|c|}{ nach Stufe I und II } & \multicolumn{4}{|c|}{ nach Stufe III } \\
\hline & \multirow[b]{2}{*}{ absolut } & \multirow{2}{*}{ relativ } & \multicolumn{2}{|c|}{ kumuliert } & \multirow[b]{2}{*}{ absolut } & \multirow[b]{2}{*}{ relativ } & \multicolumn{2}{|c|}{ kumuliert } \\
\hline & & & absolut & relativ & & & absolut & relativ \\
\hline Unbekannt & 235.790 & $21,03 \%$ & 1.121 .394 & $100,00 \%$ & 0 & $0,00 \%$ & 1.121 .394 & $0,00 \%$ \\
\hline$>0$ bis $<10$ & 10.953 & $0,98 \%$ & 885.604 & $78,97 \%$ & 6.947 & $0,62 \%$ & 1.121 .394 & $100,00 \%$ \\
\hline 10 bis $<20$ & 7.453 & $0,66 \%$ & 874.651 & $78,00 \%$ & 5.133 & $0,46 \%$ & 1.114 .447 & $99,38 \%$ \\
\hline 20 bis $<30$ & 7.904 & $0,70 \%$ & 867.198 & $77,33 \%$ & 5.838 & $0,52 \%$ & 1.109 .314 & $98,92 \%$ \\
\hline 30 bis $<40$ & 7.344 & $0,65 \%$ & 859.294 & $76,63 \%$ & 4.889 & $0,44 \%$ & 1.103 .476 & $98,40 \%$ \\
\hline 40 bis $<50$ & 8.647 & $0,77 \%$ & 851.950 & $75,97 \%$ & 6.045 & $0,54 \%$ & 1.098 .587 & $97,97 \%$ \\
\hline 50 bis $<60$ & 56.702 & $5,06 \%$ & 843.303 & $75,20 \%$ & 30.306 & $2,70 \%$ & 1.092 .542 & $97,43 \%$ \\
\hline 60 bis $<70$ & 8.671 & $0,77 \%$ & 786.601 & $70,14 \%$ & 5.655 & $0,50 \%$ & 1.062 .236 & $94,72 \%$ \\
\hline 70 bis $<80$ & 8.762 & $0,78 \%$ & 777.930 & $69,37 \%$ & 5.486 & $0,49 \%$ & 1.056 .581 & $94,22 \%$ \\
\hline 80 bis $<90$ & 9.089 & $0,81 \%$ & 769.168 & $68,59 \%$ & 5.724 & $0,51 \%$ & 1.051 .095 & $93,73 \%$ \\
\hline 90 bis $<100$ & 283.677 & $25,30 \%$ & 760.079 & $67,78 \%$ & 132.367 & $11,80 \%$ & 1.045 .371 & $93,22 \%$ \\
\hline 100 & 476.402 & $42,48 \%$ & 476.402 & $42,48 \%$ & 913.004 & $81,42 \%$ & 913.004 & $81,42 \%$ \\
\hline Summe $^{414}$ & 1.121 .394 & $100 \%$ & 1.121 .394 & $100,00 \%$ & 1.121 .394 & $100 \%$ & 1.121 .394 & $100,00 \%$ \\
\hline
\end{tabular}

Quelle: eigene Berechnungen

Abbildung 6: Verteilung der Beteiligungshöhen (Anzahl > 5.000)

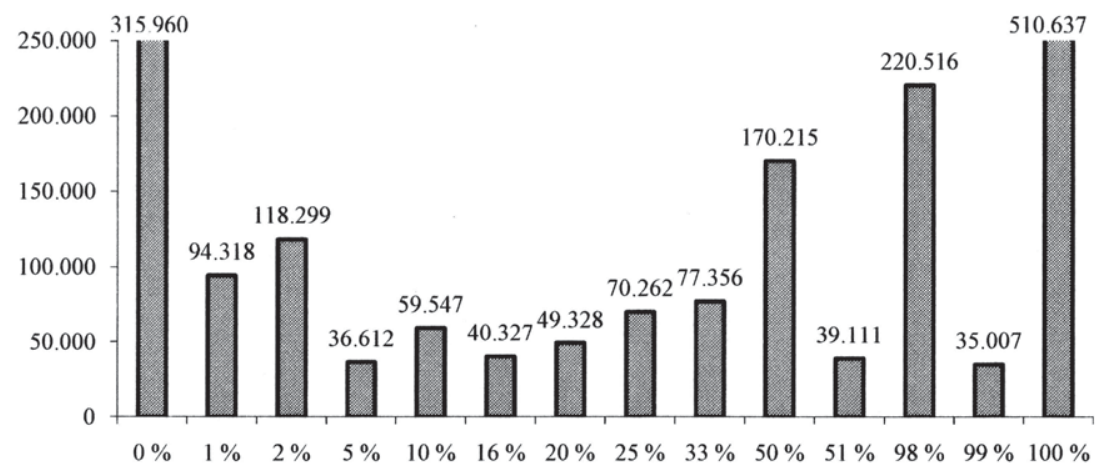

Quelle: eigene Darstellung

Abbildung 6 zeigt die Verteilung der 14 häufigsten Beteiligungshöhen nach der letzten Anpassungsstufe. Über 45 v. H. der Unternehmen werden ausschließlich von einem Anteilseigner gehalten; an $83 \mathrm{v}$. H. der Unternehmen ist ein Gesellschafter direkt mehrheitsbeteiligt. Häufig zu beobachten sind Beteiligungs-

414 In der Datenbank befinden sich 1.141.601 Gesellschaften mit Beteiligungsbeziehungen zu anderen Unternehmen. Von ihnen sind 294.207 Konzernmuttergesellschaften, an denen kein Unternehmen beteiligt ist. Dies erklärt die Summe von 1.121.394 Gliedgesellschaften. 
quoten, die dem Quotienten einer Division mit ganzzahligem Divisor entsprechen. $^{415}$ Die große Anzahl der Unternehmen, die zu $98 \mathrm{v} . \mathrm{H}$. gehalten werden, ist auf die Transformation der originären Beteiligungsangabe ,WO in 98 v. H. zurückzuführen.

\section{Wirtschaftszweigklassifikationen der Unternehmen}

Informationen über die wirtschaftlichen Tätigkeiten der einzelnen Unternehmen sind für die späteren Auswertungen und die Aufbereitung der Datenbasis unumgänglich. Im Rahmen der Konzernstrukturanalyse können die Gesellschaften über das Klassifikationssystem NACE Rev. 1.1 in Produktions-, Dienstleistungs-, Finanzierungs- oder Vertriebsgesellschaften untergliedert werden. Auch für das Auffüllen der Jahresabschlussdaten wird eine Einteilung der Unternehmen in Wirtschaftszweige benötigt, um Verhältniszahlen in Abhängigkeit zu der jeweiligen Tätigkeit der Unternehmen zu berechnen. Schließlich wird auf der Basis der Tätigkeit der einzelnen Konzerngesellschaften die Branchenzugehörigkeit des Gesamtkonzerns ermittelt, wodurch branchenabhängige Auswertungen möglich sind.

Die Amadeus-Datenbank stellt verschiedene Nomenklaturen der Wirtschaftszweige zur Verfügung. Neben den national gebräuchlichen Wirtschaftszweigklassifikationen ${ }^{416}$ sind auch international anerkannte Klassifikationssysteme in der Datenbank hinterlegt. ${ }^{417}$ Um einen Vergleich der Unternehmen untereinander zu gewährleisten, wird im weiteren Verlauf ausschließlich die NACE Rev. $1.1^{418}$ angewendet. Dieser Code eignet sich in dreierlei Hinsicht. Erstens ist

415 Dies mag sowohl auf die Vorgehensweise der Ergänzung der Beteiligungsinformationen als auch auf charakteristische in der Realität zu beobachtende Beteiligungshöhen zurückzuführen sein.

${ }^{416}$ Die nationalen Klassifikationssysteme, die in der Amadeus-Datenbank zur Anwendung kommen, sind: ATECO (Italien, fünf Stellen); BIK (Niederlande, sechs Stellen); NACE-BEL (Belgien, fünf Stellen ); NAF (Frankreich, vier Stellen); SIC UK (Großbritannien, fünf Stellen); SNI (Schweden, fünf Stellen); WZ 93 (Deutschland/Österreich, fünf Stellen); CNAE (Spanien, vier Stellen).

417 Hierzu können die NACE Rev. 1.1 und das NAICS gezählt werden. Ein Rückgriff auf die International Standard Industrial Classification der UNO ist nicht möglich.

418 Das Akronym NACE leitet sich von der französischen Bezeichnung „Nomenclature statistique des Activités économiques dans la Communauté Européenne" ab und bezeichnet die statistische Systematik der Wirtschaftszweige in der Europäischen Gemeinschaft. Diese basiert auf dem International Standard Industrial Classification of all Economic Activities (ISIC Rev. 3) der Vereinten Nationen und wurde zur besseren Darstellung der europäischen Volkswirtschaften den Erfordernissen der Europäischen Union angepasst, ohne dabei die Kompatibilităt zur ISIC aufzugeben. Vgl. Verordnung (EWG) Nr. 3037/90 des Rates v. 9. 10. 1990, Abl. Nr. L 293 v. 24. 10. 1990, S. 1-26 und der Verordnung Nr. 761/93 der Kommission v. 24. 3. 1993, Abl. Nr. L 83 v. 3. 4. 1993, S. 1-24, 
es die Wirtschaftszweigklassifikation der Europäischen Union, die auf die dort vorherrschenden Tätigkeitsfelder der Unternehmen optimal abgestimmt ist. Zweitens orientieren sich alle nationalen Klassifikationssysteme sehr stark an der NACE Rev. 1.1. ${ }^{419}$ Damit ist auch eine Transformation derselben in die NACE Rev. 1.1 ohne größere Anpassungsmaßnahmen möglich. Drittens ist die NACE Rev. 1.1 die mit Abstand am häufigsten hinterlegte Tätigkeitsbeschreibung in der Amadeus-Datenbank.

Tabelle 15: Anteil der Unternehmen ohne Angaben zum Wirtschaftszweig

\begin{tabular}{|c|rrr|}
\hline Land & $\begin{array}{c}\text { Anzahl der } \\
\text { Unternehmen }\end{array}$ & $\begin{array}{c}\text { keine Angaben } \\
\text { vorhanden }\end{array}$ & relativer Anteil \\
\hline AT & 7.663 & 17 & $0,2 \%$ \\
BE & 33.818 & 377 & $1,1 \%$ \\
CY & 29 & 3 & $10,3 \%$ \\
CZ & 1.383 & 0 & $0,0 \%$ \\
DE & 59.710 & 38 & $0,1 \%$ \\
DK & 54.949 & 2.119 & $3,9 \%$ \\
EE & 1.416 & 11 & $0,8 \%$ \\
ES & 45.988 & 145 & $0,3 \%$ \\
FI & 8.266 & 3 & $0,0 \%$ \\
FR & 74.656 & 100 & $0,1 \%$ \\
GB & 296.271 & 75.048 & $25,3 \%$ \\
GR & 2.675 & 0 & $0,0 \%$ \\
HU & 3.009 & 246 & $8,2 \%$ \\
IE & 2.273 & 1.571 & $69,1 \%$ \\
IT & 6.778 & 57 & $0,8 \%$ \\
LT & 225 & 56 & $24,9 \%$ \\
LU & 97 & 18 & $18,6 \%$ \\
LV & 485 & 0 & $0,0 \%$ \\
NL & 162.586 & 33 & $0,0 \%$ \\
PL & 4.002 & 2 & $0,0 \%$ \\
PT & 3.797 & 0 & $0,0 \%$ \\
SE & 63.512 & 9.519 & $15,0 \%$ \\
SI & 46 & 0 & $0,0 \%$ \\
SK & 16 & 1 & $6,3 \%$ \\
\hline Summe & $833.650^{420}$ & 89.364 & $10,7 \%$ \\
\hline
\end{tabular}

Quelle: eigene Berechnung

Berichtigung Abl. Nr. L 159 v. 11. 7. 1995, S. 31. Für eine Übersicht über die Entwicklung der NACE vgl. Mai, H., in: $W u S$, 1991, S. 7-16. Für den gegenwärtigen Revisionsprozess und die Einordnung der NACE in das internationale System von Wirtschaftsklassifikationen vgl. Greulich, M., in: WuS, 2004, S. 381-390.

Derzeit wird die NACE Rev. 1.1 überarbeitet. NACE Rev. 2 wird identisch mit dem ebenfalls neu geordneten ISIC Rev. 4 sein und voraussichtlich erstmalig bei Statistiken mit dem Bezugsjahr 2008 zur Anwendung kommen.

419 Auch die derzeit in Deutschland angewendete Wirtschaftszweigklassifizierung WZ 03 baut auf der durch EG-Verordnungen verbindlich eingeführten NACE Rev. 1.1 auf.

${ }^{420}$ Die Angaben beziehen sich nur auf Unternehmen, die gemäß der Definition des Kapitels D.V.2 als Konzerngesellschaft gelten. 
Wenn keine Informationen über die Tätigkeit eines Unternehmens in Form der NACE Rev. 1.1 vorliegen, werden die nationalen Klassifikationssysteme in die NACE Rev. 1.1 transformiert. Nur für Belgien, Deutschland, England, Frankreich, Italien, die Niederlande, Österreich und Spanien sind Angaben zu den nationalen Branchencodes in der Amadeus-Datenbank hinterlegt. Tabelle 15 fasst die Verfügbarkeit von Wirtschaftszweiginformationen der einzelnen Unternehmen für die Mitgliedstaaten der Europäischen Union zusammen.

Die Untergliederung der NACE Rev. 1.1 nach Abschnitt, Abteilung, Gruppen und Klassen ist für die Datenaufbereitung und -analyse zu detailliert, sodass je nach Anforderung die Unternehmen nach Abschnitten oder Abteilungen untergliedert werden. Die Klasse 7415, Beteiligungsgesellschaften ohne Kapitalanlagegesellschaften/Managementtätigkeiten von Holdinggesellschaften, wird dabei aufgrund ihrer besonderen Bedeutung stets separat behandelt. Außerdem werden einzelne Abteilungen wegen ihrer untergeordneten Bedeutung zusammengefasst bzw. die Abteilung Grundstücks- und Wohnungswesen, Vermietung beweglicher Sachen und Erbringung von unternehmensbezogenen Dienstleistungen in zwei Kategorien aufgesplittet. ${ }^{421}$

\section{Jahresabschlüsse}

\section{a) Auswahl und Beschreibung der Datenbasis}

Die Amadeus-Datenbank wird kontinuierlich aktualisiert. Sobald den nationalen Datenprovidern neue Informationen über ein Unternehmen bekannt sind, werden diese in die Datenbank aufgenommen. Dabei ist es unerheblich, ob ein vollständiger Jahresabschluss oder nur einzelne Bilanzpositionen vorhanden sind. Dies hat zur Folge, dass erstens die letzten verfügbaren Jahresabschlüsse der Unternehmen für unterschiedliche Berichtszeiträume vorliegen und zweitens nicht für alle Jahre ein vollständiger Abschluss hinterlegt ist.

Ziel dieses Kapitels ist es daher, einen zeitnahen Betrachtungszeitraum auszuwählen, der sich mit dem Erhebungszeitraum der Konzernstrukturen in Einklang bringen lässt und der eine möglichst breite Datenbasis bereitstellt. Ferner wird die Vergleichbarkeit der einzelnen Jahresabschlüsse in der Datenbank erörtert und der Umgang mit Konzernabschlüssen in der Datenbank dargelegt. Im Kapitel D.IV.3.b) wird die Ergänzung der fehlenden Jahresabschlussinformationen der ausgewählten Datenbasis durch Schlussweisen und Schätzverfahren beschrieben.

Grundsätzlich wird davon ausgegangen, dass der Stand der Beteiligungsinformationen dem des Jahres des zuletzt in der Datenbank hinterlegten Jahres-

${ }^{421}$ Eine Gegenüberstellung der NACE und der verwendeten Klassifikation befindet sich in Tabelle 56 im Anhang. 
abschlusses entspricht. Da für die Unternehmensbeziehungen keine Mehrjahresdaten vorliegen und über zwei Drittel der Konzernunternehmen ihren letzten Jahresabschluss für das Jahr 2003 in der Datenbank hinterlegt haben, kommt als Betrachtungszeitraum der Analyse vornehmlich das Jahr 2003 in Frage (Tabelle 16). Somit ist sichergestellt, dass für die Mehrzahl der Unternehmen sowohl die Beteiligungsinformationen als auch die Jahresabschlussdaten aus dem gleichen Jahr stammen. Auch die Aktualität der Daten erscheint hinreichend gewährleistet zu sein. Fraglich bleibt nur, ob Unternehmen, die keinen Einzelabschluss in 2003 veröffentlicht haben, vernachlässigt oder aber mit ihren Geschäftsberichten der nachfolgenden oder vorangegangenen Jahren berücksichtigt werden sollen. Da 94 v. H. aller Unternehmen mindestens einen Abschluss für die Jahre 2002 bis 2004 in der Datenbank hinterlegt haben, werden im Folgenden auch Unternehmen mit ihrem Einzelabschluss der Vorjahre oder des Folgejahres in der Analyse berücksichtigt. Nur bei 6 v. H. der Konzernunternehmen ist die zeitliche Differenz des jeweiligen Jahresabschlusses zum Betrachtungszeitraum 2003 größer als ein Jahr. Eine starke konjunkturelle Verzerrung der Jahresabschlussdaten kann somit ausgeschlossen werden.

Tabelle 16: Jahr des Jahresabschlusses nach Ländern ${ }^{422}$

\begin{tabular}{|c|rrrrrr|}
\hline Land & 1999 & 2000 & \multicolumn{1}{c}{2001} & \multicolumn{1}{c}{2002} & \multicolumn{1}{c}{2003} & \multicolumn{1}{c}{2004} \\
\hline FR & $0,1 \%$ & $1,7 \%$ & $3,4 \%$ & $14,8 \%$ & $78,6 \%$ & $1,3 \%$ \\
AT & $0,0 \%$ & $3,1 \%$ & $12,4 \%$ & $17,8 \%$ & $66,7 \%$ & $0,0 \%$ \\
BE & $0,0 \%$ & $0,3 \%$ & $1,0 \%$ & $4,7 \%$ & $85,3 \%$ & $8,7 \%$ \\
CY & $0,0 \%$ & $0,0 \%$ & $0,0 \%$ & $10,3 \%$ & $89,7 \%$ & $0,0 \%$ \\
CZ & $0,0 \%$ & $20,3 \%$ & $12,8 \%$ & $21,5 \%$ & $45,2 \%$ & $0,2 \%$ \\
DE & $0,0 \%$ & $1,3 \%$ & $6,8 \%$ & $13,3 \%$ & $78,5 \%$ & $0,1 \%$ \\
DK & $0,0 \%$ & $0,3 \%$ & $0,5 \%$ & $1,1 \%$ & $64,9 \%$ & $33,2 \%$ \\
EE & $0,0 \%$ & $0,6 \%$ & $2,5 \%$ & $20,9 \%$ & $75,1 \%$ & $0,9 \%$ \\
ES & $0,0 \%$ & $2,8 \%$ & $5,4 \%$ & $25,4 \%$ & $66,4 \%$ & $0,0 \%$ \\
FI & $0,0 \%$ & $0,3 \%$ & $0,6 \%$ & $3,7 \%$ & $89,6 \%$ & $5,8 \%$ \\
GB & $0,1 \%$ & $1,2 \%$ & $3,0 \%$ & $11,5 \%$ & $75,0 \%$ & $9,2 \%$ \\
GR & $0,0 \%$ & $0,3 \%$ & $0,8 \%$ & $1,8 \%$ & $94,2 \%$ & $2,8 \%$ \\
HU & $0,0 \%$ & $14,2 \%$ & $3,1 \%$ & $55,2 \%$ & $27,5 \%$ & $0,0 \%$ \\
IE & $0,1 \%$ & $1,2 \%$ & $8,8 \%$ & $26,2 \%$ & $62,8 \%$ & $0,9 \%$ \\
IT & $0,1 \%$ & $3,9 \%$ & $5,6 \%$ & $24,7 \%$ & $65,6 \%$ & $0,2 \%$ \\
LT & $0,0 \%$ & $8,0 \%$ & $8,9 \%$ & $11,6 \%$ & $69,8 \%$ & $1,8 \%$ \\
LU & $0,0 \%$ & $9,3 \%$ & $19,6 \%$ & $50,5 \%$ & $20,6 \%$ & $0,0 \%$ \\
LV & $0,0 \%$ & $5,4 \%$ & $11,5 \%$ & $29,3 \%$ & $53,0 \%$ & $0,8 \%$ \\
NL & $0,0 \%$ & $2,8 \%$ & $7,9 \%$ & $36,2 \%$ & $52,6 \%$ & $0,5 \%$ \\
PL & $0,0 \%$ & $2,7 \%$ & $13,3 \%$ & $49,2 \%$ & $34,8 \%$ & $0,0 \%$ \\
PT & $0,0 \%$ & $14,9 \%$ & $16,2 \%$ & $23,7 \%$ & $45,2 \%$ & $0,0 \%$ \\
SE & $0,0 \%$ & $1,2 \%$ & $1,6 \%$ & $3,9 \%$ & $75,1 \%$ & $18,2 \%$ \\
SI & $0,0 \%$ & $0,0 \%$ & $0,0 \%$ & $19,6 \%$ & $80,4 \%$ & $0,0 \%$ \\
SK & $0,0 \%$ & $6,3 \%$ & $6,3 \%$ & $31,3 \%$ & $56,3 \%$ & $0,0 \%$ \\
\hline Summe & $0 \%$ & $2 \%$ & $4 \%$ & $17 \%$ & $69 \%$ & $8 \%$ \\
\hline
\end{tabular}

Quelle: eigene Berechnungen

422 Geschäftsberichte, die bis zum 31. März des Folgejahres aufgestellt werden, zählen noch zum Vorjahr. Den Angaben liegen nur Konzerngesellschaften gemäß der Definition des Kapitels D.V.2 zugrunde. 
Auch eine Fehleinschätzung der Ertragslage durch die Verwendung eines von 2003 abweichenden Jahresabschlusses ist nur selten zu erwarten. Die Analyse von Mehrjahresdaten zeigt, dass andauernde kurzfristige Wechsel zwischen positiver und negativer Ertragslage der Unternehmen die Ausnahme darstellen (Abbildung 7). Die Wahrscheinlichkeit eines Vorzeichenwechsels der Ertragslage zwischen zwei Perioden hängt wesentlich von den Vorjahresergebnissen des Unternehmens und der Dauer der vorherigen Ertragslage ab. Je länger ein Unternehmen Gewinne erwirtschaftet, desto unwahrscheinlicher ist ein Verlust im Folgejahr. Weist ein erfolgreiches Unternehmen für das vorangegangene Jahr ausnahmsweise einen Verlust aus, steigt die Wahrscheinlichkeit für einen Fehlbetrag in der Folgeperiode.

Abbildung 7: Häufigkeit des Wechsels der Ertragslage der Unternehmen zwischen 1997 und 2003

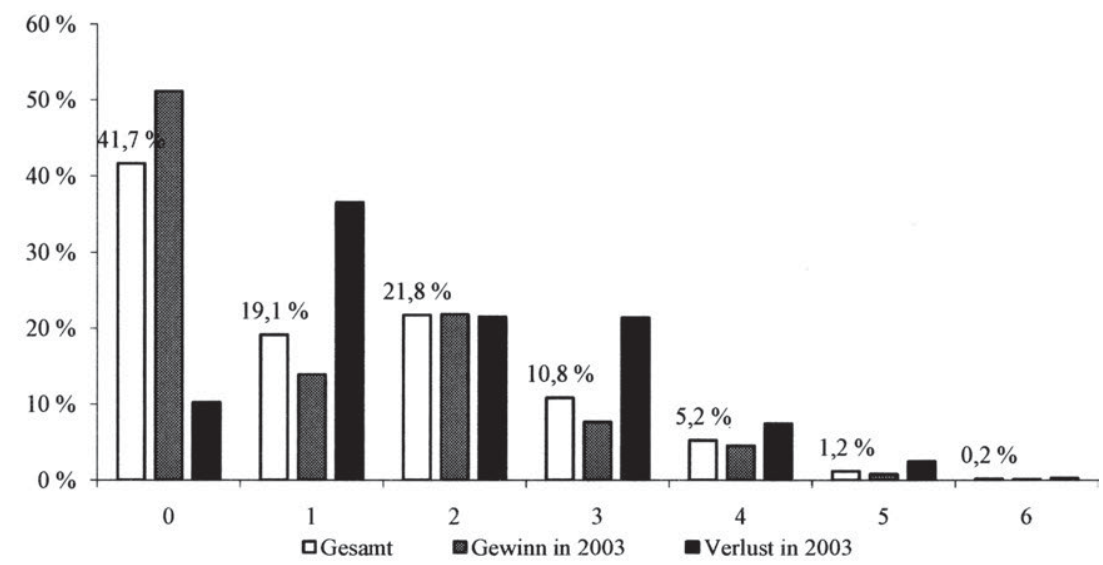

Quelle: eigene Darstellung

Durchschnittlich erfolgt bei 20,5 v. H. der Unternehmen ein Wechsel der Ertragslage zwischen zwei Perioden. ${ }^{423}$ Im Ergebnis sind Verzerrungen durch die Übernahme von Jahresabschlüssen der Vorjahre oder des Folgejahres in das Betrachtungsjahr 2003 nur bei einem geringen Anteil der Unternehmen zu erwarten. Bedenkt man weiterhin, dass dies lediglich für $30 \mathrm{v}$. H. der Unternehmen zutrifft, die zeitliche Differenz zwischen 2003 und dem verwendeten Jahresabschluss gering ist und eine Vernachlässigung dieser Unternehmen im

$4231-\left(0,417 \times \frac{6}{6}+0,191 \times \frac{5}{6}+0,218 \times \frac{4}{6}+0,108 \times \frac{3}{6}+0,052 \times \frac{2}{6}+0,012 \times \frac{1}{6}+0,02 \times \frac{0}{6}\right)=0,205$

Die Berechnung basiert auf dem Gewinn vor Steuern von 135.956 Unternehmen der Jahre 1997 bis 2003. 
Rahmen der Steuerberechnungen auch zu Verzerrungen führen würde, erscheint die hier gewählte Vorgehensweise plausibel.

Abbildung 8: Bilanz in der Amadeus-Datenbank

\begin{tabular}{|l|l|}
\hline Aktiv & \multicolumn{1}{|c|}{ Passiv } \\
\hline Fixed Assets & Shareholders Funds \\
Intangible Fixed Assets & Capital \\
Tangible Fixed Assets & Other Shareholders Funds, incl. Reserves \\
Other Fixed Assets/Financial Fixed Assets & Non-Current Liabilities \\
Current Assets & Long-Term Debt \\
Stocks & Other Non-Current Liabilities/Provisions \\
Debtors & Current Liabilities \\
Other Current Assets & Loans \\
Cash & Creditors \\
& Other Current Liabilities \\
\hline Total Assets & Total Shareholders' Funds and Liabilities \\
\hline
\end{tabular}

Quelle: Amadeus-Datenbank, Bureau van Dijk

Abbildung 9: Gewinn-und-Verlust-Rechnung in der Amadeus-Datenbank

\begin{tabular}{|c|}
\hline $\begin{array}{l}\text { Operating Revenue / Turnover } \\
\text { Sales } \\
\text { Cost of Goods Sold }\end{array}$ \\
\hline $\begin{array}{l}\text { Gross Profit } \\
\text { Other Operating Expenses }\end{array}$ \\
\hline $\begin{array}{l}\text { Operating Profit (Loss) } \\
\text { Financial Revenue } \\
\text { Financial Expenses }\end{array}$ \\
\hline Financial Profit / Loss \\
\hline $\begin{array}{l}\text { Profit (Loss) before Taxation } \\
\text { Taxation } \\
\end{array}$ \\
\hline $\begin{array}{c}\text { Profit (Loss) after Taxation } \\
\text { Extraordinary Revenue } \\
\text { Extraordinary Expenses }\end{array}$ \\
\hline Extraordinary Profit (Loss) \\
\hline Profit (Loss) for Period \\
\hline
\end{tabular}

Quelle: Amadeus-Datenbank, Bureau van Dijk

Im Folgenden rücken die Gliederung und die Vollständigkeit der Daten der hinterlegten Jahresabschlüsse in den Mittelpunkt der Betrachtung. Sowohl die Bilanz (Abbildung 8) als auch die Gewinn-und-Verlust-Rechnung (Abbildung 9) liegen in der Amadeus-Datenbank nur in stark verkürzter und standardisierter Form vor. Dadurch wird eine Vergleichbarkeit aller Jahresabschlüsse in der Datenbank gewährleistet. Die Zuordnung der detaillierten, nationalen Bilanzpositionen zur aggregierten Bilanz und Gewinn-und-Verlust-Rechnung der Amadeus-Datenbank erfolgt mittels sogenannter "correspondence tables“. Da jedoch nicht in allen Mitgliedstaaten ein offizielles, standardisiertes, für alle Gesellschaften gültiges Gliederungsschema für die Aufstellung der Bilanz und der 
Gewinn-und-Verlust-Rechnung existiert, erweist sich die Aggregation der Jahresabschlüsse teilweise als schwierig und ungenau. ${ }^{424}$

Tabelle 17: Anteil der Unternehmen in der Amadeus-Datenbank mit Daten ${ }^{425}$

\begin{tabular}{|c|c|c|c|c|c|c|c|c|c|c|}
\hline \multirow{2}{*}{ Land } & \multicolumn{4}{|c|}{ Bilanz } & \multicolumn{4}{|c|}{ GuV } & \multicolumn{2}{|c|}{ Mitarbeiter } \\
\hline & $\max$ & $\min$. & $\overline{\mathrm{X}}$ & relativ & $\max$ & $\min$. & $\overline{\mathrm{x}}$ & relativ & absolut & relativ \\
\hline AT & 2.537 & 387 & 553 & $7 \%$ & 3.213 & 21 & 591 & $8 \%$ & 4.833 & $63 \%$ \\
\hline $\mathrm{BE}$ & 33.428 & 22.554 & 31.669 & $94 \%$ & 33.192 & 9.459 & 26.249 & $78 \%$ & 17.944 & $53 \%$ \\
\hline CY & 29 & 29 & 29 & $100 \%$ & 29 & 19 & 28 & $96 \%$ & 22 & $76 \%$ \\
\hline $\mathrm{CZ}$ & 925 & 879 & 918 & $66 \%$ & 925 & 391 & 827 & $60 \%$ & 865 & $63 \%$ \\
\hline $\mathrm{DE}$ & 24.235 & 5.164 & 6.674 & $11 \%$ & 34.849 & 901 & 7.148 & $12 \%$ & 45.554 & $76 \%$ \\
\hline DK & 53.296 & 784 & 46.362 & $84 \%$ & 53.296 & 0 & 38.203 & $70 \%$ & 23.507 & $43 \%$ \\
\hline $\mathrm{EE}$ & 1.373 & 1.373 & 1.373 & $97 \%$ & 1.373 & 878 & 1.328 & $94 \%$ & 988 & $70 \%$ \\
\hline ES & 42.292 & 39.024 & 41.618 & $90 \%$ & 41.734 & 32.011 & 39.713 & $86 \%$ & 28.558 & $62 \%$ \\
\hline FI & 8.200 & 4.347 & 7.666 & $93 \%$ & 8.198 & 1.982 & 6.991 & $\%$ & 5.780 & $70 \%$ \\
\hline FR & 71072 & 71.072 & 71.072 & $95 \%$ & 71.072 & 58.948 & 69.676 & $93 \%$ & 58.571 & $78 \%$ \\
\hline GB & 284.179 & 87.193 & 222.871 & $75 \%$ & 105.859 & 0 & 78.641 & $27 \%$ & 57.397 & $19 \%$ \\
\hline GR & 2.645 & 2.645 & 2.645 & $99 \%$ & 2.645 & 0 & 2.029 & $76 \%$ & 2.105 & $79 \%$ \\
\hline $\mathrm{HU}$ & 1.276 & 715 & 1.213 & $40 \%$ & 2.329 & 5 & 944 & $31 \%$ & 1.724 & $57 \%$ \\
\hline IE & 2.094 & 993 & 1.717 & $76 \%$ & 975 & 0 & 606 & $27 \%$ & 740 & $33 \%$ \\
\hline IT & 6.162 & 6.162 & 6.162 & $91 \%$ & 6.162 & 5.714 & 6.122 & $90 \%$ & 6.158 & $91 \%$ \\
\hline LT & 187 & 133 & 181 & $80 \%$ & 186 & 0 & 142 & $63 \%$ & 186 & $83 \%$ \\
\hline LU & 70 & 65 & 69 & $71 \%$ & 68 & 37 & 50 & $51 \%$ & 8 & $8 \%$ \\
\hline LV & 404 & 404 & 404 & $83 \%$ & 404 & 23 & 375 & $77 \%$ & 361 & $74 \%$ \\
\hline NL & 145.148 & 96.028 & 133.733 & $82 \%$ & 15.628 & 2.559 & 11.994 & $7 \%$ & 104.018 & $64 \%$ \\
\hline PL & 3.079 & 1.479 & 2.867 & $72 \%$ & 3.076 & 507 & 2.571 & $64 \%$ & 2.966 & $74 \%$ \\
\hline PT & 2.613 & 1.743 & 2.497 & $66 \%$ & 2.608 & 2.301 & 2.524 & $66 \%$ & 516 & $14 \%$ \\
\hline SE & 59.500 & 33.057 & 54.238 & $85 \%$ & 57.393 & 38 & 44.176 & $70 \%$ & 35.434 & $56 \%$ \\
\hline SI & 43 & 7 & 38 & $84 \%$ & 46 & 0 & 15 & $32 \%$ & 40 & $87 \%$ \\
\hline SK & 14 & 12 & 14 & $86 \%$ & 14 & 2 & 12 & $76 \%$ & 14 & $88 \%$ \\
\hline
\end{tabular}

Quelle: eigene Berechnungen

Zudem unterscheiden sich die Rechnungslegungsvorschriften in den einzelnen Mitgliedstaaten voneinander, sodass selbst bei einer eindeutigen Zuordnung der einzelnen Jahresabschlussgrößen die Vergleichbarkeit über die Grenzen hinweg eingeschränkt ist. Durch das Nebeneinander der IFRS und der nationalen Rechnungslegungsstandards ist selbst innerhalb eines Mitgliedstaates stellenweise nur eine eingeschränkte Vergleichbarkeit gewährleistet. ${ }^{426} \mathrm{Da}$ der den

${ }^{424}$ In Deutschland sind die Gliederungsvorschriften der Bilanz ( $\$ 266 \mathrm{HGB}$ ) nur für große und mittelgroße Kapitalgesellschaften ( $\$ 266$ Abs. 1 HGB) und die der Gewinn-und-Verlust-Rechnung ( $\$ 275$ HGB) nur für große Kapitalgesellschaften ( $\$ 276$ HGB) verpflichtend. Für die übrigen Kapitalgesellschaften existieren Erleichterungen, die das Zusammenfassen bestimmter Posten erlauben. Vgl. hierzu a. ausführlich Oestreicher, A., Handelsbilanzen, 2003, S. 125-153.

425 Die Angaben beziehen sich auf 833.650 Gesellschaften, die gemäß der Konzerndefinition des Kapitels D.V.2 Bestandteil einer Unternehmensgruppe sind.

${ }^{426}$ Dies trifft für Deutschland nur für die Konzernabschlüsse zu. Durch das Kapitalaufnahmeerleichterungsgesetz wurde 1998 der befreiende Konzernabschluss nach § 292a HGB ein- 
Jahresabschlüssen zugrunde liegende Rechnungslegungsstandard unbekannt ist, wird das Problem der Vergleichbarkeit der Jahresabschlüsse im weiteren Verlauf ausgeblendet.

Für die Mehrzahl der in der Amadeus-Datenbank hinterlegten Jahresabschlüsse gilt, dass sie entweder (fast) vollständig sind oder aber nur sehr vereinzelte Angaben zum Eigenkapital, Umsatz oder Gewinn enthalten. Der Anteil an Unternehmen, für die Bilanzinformationen verfügbar sind, unterscheidet sich stark zwischen den Mitgliedstaaten. Besonders deutsche und österreichische aber auch ungarische Unternehmen veröffentlichen ihre Unternehmensdaten nur unzureichend. In Großbritannien, Irland, den Niederlanden und Slowenien ist für die Mehrzahl der Gesellschaften nur eine Bilanz hinterlegt, Angaben zur Gewinn-und-Verlust-Rechnung der Unternehmen fehlen jedoch. Die Anzahl der Mitarbeiter oder die Lohnsumme ist bei britischen, luxemburgischen und portugiesischen Unternehmen in über $80 \mathrm{v}$. H. der Fälle nicht angegeben. Tabelle 17 zeigt die Ergebnisse für die einzelnen Mitgliedstaaten.

Ein geringer Anteil der Unternehmen in der Amadeus-Datenbank verfügt ausschließlich über einen Konzernabschluss (Tabelle 18). Konsolidierte Jahresabschlüsse sind aus der Datenbasis zu entfernen, da andernfalls Sachverhalte falsch, doppelt und/oder verzerrt erfasst würden. Eine Falscherfassung liegt bei Konzernen vor, die Beteiligungen an konsolidierungspflichtigen Tochtergesellschaften mit Sitz außerhalb der Europäischen Union halten. Im Rahmen der konsolidierten Besteuerung gilt gegenüber nicht europäischen Konzerngesellschaften weiterhin der Grundsatz des Fremdvergleichs (,at water's edge“), sodass deren Gewinne und die damit im Zusammenhang stehenden Aufteilungsfaktoren nicht erfasst werden dürfen. ${ }^{427}$ Eine Doppelerfassung existiert bei Sachverhalten, die sowohl im Einzelabschluss der europäischen Tochtergesellschaft als auch im Konzernabschluss der Muttergesellschaft auftauchen. Sitzt die Tochtergesellschaft in einem vom Land der Muttergesellschaft abweichenden Mitgliedstaat, werden Sachverhalte durch die Erfassung im Konzernabschluss der Konzernmuttergesellschaft in einen anderen Mitgliedstaat transferiert. Um diesen Fehler zu vermeiden, werden die Bilanz, die Gewinn-und-Verlust-

geführt, der es börsennotierten Muttergesellschaften gestattete, ihren Konzernabschluss auch nach international anerkannten Rechnungslegungsvorschriften aufzustellen. Vgl. Pellens, B., Fülbier, R.U./Gassen, J., Rechnungslegung, 2004, S. 47-49. Mit Verabschiedung der IAS-Verordung sind ab 2005 kapitalmarktorientierte Unternehmen verpflichtet, die Konzernrechnungslegung nach IFRS vorzunehmen. Vgl. Verordnung (EG) Nr. 1606/2002 des europäischen Parlaments und des Rates v. 19. 7. 2002, Abl. Nr. L 243, v. 11. 9. 2002, S. 1-4. Estland, Malta, Griechenland und Zypern erlaubten bzw. forderten bereits 2003 einen IFRS-Einzelabschluss. Für eine Übersicht über die geplante und bereits erfolgte Umsetzung der in der IAS-Verordnung eingeräumten Wahlrechte in den einzelnen Mitgliedstaaten vgl. Europäische Kommission, Implementation, 2006.

${ }^{427} \mathrm{Vgl}$. hierzu die Erläuterungen des Kapitels C.II.2.b). 
Rechnung und die Anzahl der Mitarbeiter dieser Unternehmen auf null gesetzt. Diese Vorgehensweise unterstellt, dass alle Gesellschaften, die ausschließlich einen Konzernabschluss vorweisen, reine Finanzholdinggesellschaften sind, welche für sich genommen keine Wertschöpfung erbringen und über keine Mitarbeiter verfügen.

Tabelle 18: Konsolidierte Jahresabschlüsse in der Datenbank nach Ländern ${ }^{428}$

\begin{tabular}{|c|rr|}
\hline Land & absolut & \multicolumn{1}{|c|}{ relativ } \\
\hline AT & 15 & $0,20 \%$ \\
CY & 18 & $62,07 \%$ \\
CZ & 2 & $0,14 \%$ \\
DE & 308 & $0,52 \%$ \\
ES & 30 & $0,07 \%$ \\
FR & 9 & $0,01 \%$ \\
GB & 15.601 & $5,27 \%$ \\
IE & 253 & $11,13 \%$ \\
IT & 48 & $0,71 \%$ \\
LT & 42 & $18,67 \%$ \\
LU & 4 & $4,12 \%$ \\
LV & 1 & $0,21 \%$ \\
NL & 7.309 & $4,50 \%$ \\
PL & 8 & $0,20 \%$ \\
PT & 36 & $0,95 \%$ \\
SE & 151 & $0,24 \%$ \\
SK & 2 & $12,50 \%$ \\
\hline Summe & 23.837 & $2,86 \%$ \\
\hline
\end{tabular}

Quelle: eigene Berechnung

\section{b) Ergänzung von Jahresabschlussinformationen}

\section{(a) Vorüberlegungen zur Methodik}

Bei empirischen Untersuchungen stellt sich bei einer derartigen Datenlage immer die Frage, ob die fehlenden Daten ergänzt werden sollen oder ob sich die Analyse lediglich auf die vorhandenen Daten beschränken soll. ${ }^{429}$ Ein gänzliches Vernachlässigen der Unternehmen, die über nur unzureichende Angaben in der Bilanz und der Gewinn-und-Verlust-Rechnung verfügen, wäre fatal, da dadurch gleichzeitig die Konzernstrukturen gekappt würden. Eine zweite Alternative wäre, die Unternehmen als solche bestehen zu lassen, sie jedoch bei der Berechnung der Steuerbelastung zu vernachlässigen. De facto würden alle Jahresabschlusspositionen der Unternehmen, von denen nur unvollständige Angaben

${ }^{428}$ Den Angaben liegen nur Konzerngesellschaften gemåß der Definition des Kapitels D.V.2 zugrunde. Mitgliedstaaten, für deren Unternehmen in der Datenbank kein konsolidierter Jahresabschluss hinterlegt ist, sind nicht aufgeführt.

429 Die Complete Case Analysis setzt eine absolut zufälige Verteilung der fehlenden Daten voraus („Missing Completely At Random“), will man unverzerrte Parameter ermitteln. Vgl. zu den Verfahren der Fallreduktion Göthlich, S., in: Albers et al., Forschung, 2006, S. 137-138. 
verfügbar sind, mit null aufgefüllt bzw. ersetzt werden. Diese Vorgehensweise kann jedoch die Einschätzungen der Auswirkung der Einführung der konsolidierten Besteuerung in Europa verzerren. Ein einfaches Beispiel soll dies verdeutlichen:

\section{Beispiel 2:}

Ein Konzern besteht aus drei produzierenden Unternehmen, wobei zwei in Deutschland und eines in Estland ansässig sind. Aufgrund der sehr restriktiven Datenlage in Deutschland ist nur für eines der beiden deutschen Unternehmen der Jahresabschluss hinterlegt. Des Weiteren wird unterstellt, dass eine formelhafte Gewinnaufteilung eine Verschiebung der Bemessungsgrundlage zugunsten von Deutschland bewirkt.

Tabelle 19: Verzerrung der Aufkommenswirkung der konsolidierten Körperschaftsteuer-Bemessungsgrundlage durch die Vernachlässigung von Unternehmen ohne Jahresabschlussangaben ${ }^{430}$

\begin{tabular}{|c|c|c|c|c|c|c|c|c|}
\hline & Separate & \multicolumn{5}{|c|}{ Formula Apportionment } & \multirow{3}{*}{$\begin{array}{l}\text { Differenz } \\
\text { in } \\
\text { Prozent- } \\
\text { punkten }\end{array}$} \\
\hline & & BMG & Gewinnanteil & \multicolumn{2}{|c|}{ BMG (fiktiv) } & \multicolumn{2}{|c|}{$B M G$ (real) } & \\
\hline \multirow[t]{2}{*}{ DE } & $\begin{array}{l}\mathrm{U} 1 \\
\mathrm{U} 2\end{array}$ & $\begin{array}{l}1.000 \\
1.000\end{array}$ & & absolut & $\begin{array}{c}\text { Ver- } \\
\text { anderung }\end{array}$ & absolut & $\begin{array}{c}\text { Ver- } \\
\text { anderung }\end{array}$ & \\
\hline & $\Sigma$ & $1000 / 2000$ & $57,1 / 72,7$ v. H. & 1.143 & $+14,29 \%$ & 2.182 & $+9,09 \%$ & 5,20 \\
\hline EE & U3 & 1.000 & 42,9/27,3 v. H. & 857 & $-14,29 \%$ & 818 & $-18,18 \%$ & 3,89 \\
\hline \multicolumn{2}{|c|}{$\begin{array}{l}\text { Konzern (fiktiv) } \\
\text { Konzern (real) }\end{array}$} & $\begin{array}{l}2.000 \\
3.000\end{array}$ & & & & & & \\
\hline
\end{tabular}

Quelle: eigene Darstellung

Betrachtet man ausschließlich die Unternehmen, deren Daten in der Datenbank vollständig hinterlegt sind, steigt mit Einführung einer konsolidierten Steuerbemessungsgrundlage in der Europäischen Union die Bemessungsgrundlage in Deutschland um 14,29v. H. Wären für Unternehmen 2 Daten verfügbar gewesen, betrüge die Zunahme der Bemessungsgrundlage in Deutschland lediglich 9,09 v. H. Damit wird die Aufkommenswirkung der konsolidierten Körperschaftsteuer-Bemessungsgrundlage auch von der Datenlage innerhalb der Konzerne mitbestimmt. Zwar gleicht sich der dargestellte Effekt teilweise zwischen den Konzernen aus, aufgrund der unterschiedlichen Datenlage in den jeweiligen Mitgliedstaaten ist jedoch bei Vernachlässigung der Unternehmen ohne vollständige Jahresabschlussangaben ein systematischer Fehler zu erwarten.

Die dritte Alternative besteht in der Ergänzung der in der Amadeus-Datenbank nicht hinterlegten Jahresabschlussgrößen. ${ }^{431}$ Fehlen nur vereinzelte Jahresabschlussposten, können sie teilweise aus den verfügbaren Daten berechnet werden. Ist ein eindeutiger Rückschluss auf die entsprechenden Jahresabschlusspositionen hingegen nicht möglich, müssen diese auf der Basis der für die Ge-

${ }^{430}$ Der relative Gewinnanteil wurde willkürlich festgelegt und basiert auf einer Aufteilungsformel mit nur einem Faktor, dessen Wert für die estnische Gesellschaft 150 und für die beiden deutschen Gesellschaften jeweils 200 betrăgt.

431 Für eine Übersicht über die verschiedenen Imputationsverfahren vgl. Göthlich, S., in: Albers et al., Forschung, 2006, S. 139-144. 
sellschaft verfügbaren Informationen geschätzt werden. Ausgangspunkt dieser Vorgehensweise sind die Erkenntnisse der Jahresabschlussanalyse. Danach sind bei vergleichbaren Unternehmen typische Zusammenhänge innerhalb der Jahresabschlüsse festzustellen. ${ }^{432}$ Die für Unternehmen mit einem vollständigen Jahresabschluss ermittelten Zusammenhänge der einzelnen Jahresabschlusspositionen zueinander können dann auf die übrigen Gesellschaften übertragen werden. Weisen z. B. vergleichbare Unternehmen eine charakteristische Anlagenintensität ${ }^{433}$ auf, kann bei gegebener Bilanzsumme sowohl das Anlagevermögen als auch das Umlaufvermögen geschätzt werden. Denkbar ist auch, dass die Zielvariable von mehr als einer erklärenden Größe abhängt. Diese typischen Beziehungen innerhalb des Jahresabschlusses sind vielfach nur langfristig zu beobachten; kurzfristig können die Unternehmen erheblich davon abweichen. Besonders anschaulich wird dieses Problem, wenn man die Ertragslage eines Unternehmens betrachtet. Langfristig muss jede Gesellschaft einen Gewinn erwirtschaften. Gleichwohl verzeichnen Unternehmen kurzfristig Verluste. Durch das Schätzen der Ertragslage z. B. auf der Basis des eingesetzten Eigenkapitals $^{434}$ würde ein Durchschnittsunternehmen geschaffen werden, welches aller Voraussicht nach einen, wenn auch kleinen, Gewinn erzielt. Eine sinnvolle Abbildung der sich durch die Einführung der konsolidierten Besteuerung ergebenden Verlustverrechnungsmöglichkeiten ist dann nur noch eingeschränkt möglich. Darüber hinaus sind konzernspezifische Gewinnverschiebungen nicht abzubilden. In abgeschwächter Form gelten die vorherigen Überlegungen für alle übrigen Jahresabschlusspositionen. Zwar besitzen Bilanzpositionen und die übrigen Angaben der Gewinn-und-Verlust-Rechnung einen absoluten Nullpunkt, sodass das Vorzeichen der Zielgröße stets korrekt ist, dennoch wird im Ergebnis ein Durchschnittswert ermittelt.

Neben dem der Schätzung inhärenten Problem des Durchschnittsunternehmens bestehen weitere Hindernisse im Rahmen der Ergänzung der fehlenden Jahresabschlussgrößen. Zum einem müssen für eine zielgenaue Bestimmung vergleichbare Unternehmen identifiziert werden. Dies gestaltet sich schwierig, da gerade bei den aufzufüllenden Gesellschaften ein großes Informationsdefizit vorliegt. Insoweit ist nur eine grobe Einteilung möglich. Zum anderen führt eine Schätzung nur zum Erfolg, wenn eine sinnvolle erklärende Größe gegeben ist. Wie oben bereits ausgeführt, ist die Mehrzahl der Jahresabschlüsse entweder

432 So zeigt Jacobs mittels einer Kohonen-Feature Map, dass die Jahresabschlüsse von Unternehmen der gleichen Branche, in diesem Fall Daimler Benz AG, BMW AG und Ford AG, einander ähnlich sind. Vgl. Jacobs, O.H. (Hrsg.), Bilanzanalyse, 1994, S. 261-266.

${ }^{433}$ Die Anlagenintensität ist definiert als $\frac{\text { Anlagevermögen }}{\text { Gesamtvermögen }} \times 100$

434 Die Eigenkapitalrentabilität setzt das ordentliche Ergebnis vor Steuern vom Einkommen und Ertrag ins Verhältnis zum durchschnittlichen Bestand des Eigenkapitals. 
vollständig in der Datenbank hinterlegt, oder es sind nur vereinzelte Angaben zu den Gesellschaften verfügbar. Ist z. B. bei der Ableitung der Mitarbeiteranzahl aus der Lohnsumme mit einem plausiblen Ergebnis zu rechnen, führt die Berechnung derselben ausschließlich auf der Basis der Bilanzsumme zu teilweise irrealen Ergebnissen.

Von den drei Alternativen wie mit Unternehmen verfahren wird, die über unzureichende Jahresabschlussinformationen verfügen, ist die erste Alternative grundsätzlich auszuschließen. Alternative zwei und drei sind in Abhängigkeit von der Datenlage des jeweiligen Unternehmens anzuwenden. Können die fehlenden Jahresabschlussposten aus den verfügbaren Daten berechnet werden, sind plausible Ergebnisse zu erwarten. Ein Schätzen der nicht berechenbaren Jahresabschlusspositionen erscheint vor dem Hintergrund der Verlustverrechnung nur sinnvoll, wenn mindestens eine Gewinngröße in der Gewinn-undVerlust-Rechnung gegeben ist. Daneben soll die Bilanzsumme bekannt sein, da ein unmittelbarer Zusammenhang zwischen der Bilanz und der Erfolgsgröße kurzfristig nicht existiert. Für 389.028 Unternehmen und damit 47 v. H. der Konzerngesellschaften sind in der Datenbank die Bilanzsumme und eine der folgenden Gewinngrößen gegeben: „gross profit", „operating income“, „profit and loss before tax“, „profit and loss after tax" oder „profit and loss for the period“. Bei den übrigen Gesellschaften, die keine Angaben zur Ertragslage und der Bilanzsumme in der Datenbank vorweisen, kommt Alternative zwei zur Anwendung. Diese Konzerngesellschaften bleiben als solche in der Datenbank bestehen, die einzelnen Positionen des Jahresabschlusses werden jedoch auf null gesetzt. Der systematische Fehler, der aus der stark unterschiedlichen Datenlage in den einzelnen Mitgliedstaaten der Europäischen Union hervorgeht, wird abgeschwächt, indem im Rahmen der empirischen Analyse nur Konzerne berücksichtigt werden, bei denen mindestens $50 \mathrm{v} . \mathrm{H}$. der Konzerngesellschaften primär oder geschätzt einen Jahresabschluss vorweisen können. ${ }^{435}$

Für die Berechnung der Steuerzahllast eines Konzerns sind sowohl gemäß der konsolidierten Steuerbemessungsgrundlage als auch nach dem bestehenden System der direkten Gewinnabgrenzung nicht alle Positionen der Bilanz und Gewinn-und-Verlust-Rechnung erforderlich, sodass sich das Ergänzen des Jahresabschlusses auf die erforderlichen Zielgrößen beschränkt. Im Einzelnen betrifft es den Gewinn sowie die Aufteilungsfaktoren der MassachusettsFormula: Vermögen, Umsatz und Lohnsumme. Das Vermögen umfasst nahezu die gesamte Aktivseite der Bilanz, sodass diese für jedes Unternehmen vollständig abgebildet wird. Neben der Lohnsumme wird zusätzlich die Anzahl der Mitarbeiter jedes Unternehmens ermittelt. Aufzuteilende Erfolgsgröße ist sowohl der Gewinn der gewöhnlichen Geschäftstätigkeit als auch der Gewinn

435 Vgl. hierzu Kapitel F.II.3. 
vor Steuern abzüglich der darin enthaltenen steuerfreien Einkommensbestandteile (Dividenden).

\section{(B) Einteilung der Cluster}

Fehlende Angaben des Jahresabschlusses werden bei einer hinreichenden Datenlage mittels Relationen ergänzt, welche auf Basis der Unternehmen ermittelt werden, für die ein vollständiger Jahresabschluss in der Datenbank hinterlegt ist. Um zu verhindern, dass im Ergebnis nur ein Durchschnittsunternehmen abgebildet wird, erfolgt eine Clusterbildung für vergleichbare Unternehmen. Innerhalb eines Clusters werden dann die Relationen zwischen den abhängigen und unabhängigen Jahresabschlusspositionen ermittelt. Dabei wird ein Cluster durch den Sitz, die Tätigkeit und die Größe einer Gesellschaft spezifiziert (Abbildung 10).

Die konsolidierte Körperschaftsteuer-Bemessungsgrundlage entspricht einer Besteuerung der Aufteilungsfaktoren. Lediglich die Höhe der Bemessungsgrundlage wird durch den Konzerngewinn bestimmt. Damit rücken die Aufteilungsfaktoren und deren Verhältnis zum Gewinn in den Mittelpunkt der Betrachtung. Innerhalb der Europäischen Union existiert jedoch ein starkes Gefälle der Arbeits- und Kapitalproduktivität. ${ }^{436}$ Diese Differenz wirkt sich direkt auf die Verteilung der Bemessungsgrundlage des Konzerns aus, sodass auch eine Differenzierung der Cluster nach dem Sitz der Gesellschaften zwingend notwendig ist.

Nicht nur zwischen den Mitgliedstaaten, sondern auch über die Branchen hinweg zeigen sich deutliche Produktivitätsunterschiede. ${ }^{437}$ Vergleicht man z. B. die Arbeitsproduktivität zwischen dem verarbeitenden Gewerbe und dem Dienstleistungssektor, weist letzterer tendenziell eine geringe Arbeitsproduktivität, jedoch eine höhere Kapitalproduktivität auf. Damit erscheint auch eine Differenzierung nach Branchen geboten. Auf welcher Ebene eine Unterteilung der Wirtschaftszweigklassifikationen vorgenommen werden sollte, wird durch

${ }^{436}$ So weist die Europäische Kommission ausdrücklich auf die starke Divergenz der Arbeitsproduktivität innerhalb der Europäischen Union hin. Vgl. Europäische Kommission, in: European Economy, 2003, 6, S. 107-108.

437 Für eine Übersicht über die Kapitalproduktivität in den einzelnen Wirtschaftsbereichen in Deutschland vgl. Schmalwasser, O., in: $W u S, 2001$, S. 353-354. Gemessen in DM je 1.000 DM Kapitalstock reichte die Kapitalproduktivität im Jahr 1998 von 97 in der Land- und Forstwirtschaft und Fischerei bis 1.388 im Baugewerbe. Auch weisen die Wirtschaftszweige eine unterschiedliche Arbeitsproduktivität auf. So blieb die Arbeitsproduktivität je Erwerbstätigen im Euro-Währungsgebiet in den Sektoren verarbeitendes Gewerbe, Handel und Verkehr in den Neunzigerjahren verglichen mit der vorherigen Dekade weitgehend stabil, wohingegen sie im Baugewerbe und im Bereich Finanzierung und Unternehmensdienstleister zurückging und letztendlich negative Zuwachsraten aufwies. Vgl. EZB, in: Monatsbericht, 2004, 7, S. 55-60. 
zwei gegensätzliche Faktoren bestimmt. Zum einem muss innerhalb eines Clusters eine Mehrzahl von Unternehmen vorhanden sein, damit sich einzelne Ausreißer nicht auf die zu berechnenden Relationen auswirken. Zum anderen wird durch eine tiefe Gliederung des Branchencodes die Vergleichbarkeit der Unternehmen erhöht. Da die Differenzierung nach dem Sitzland der Gesellschaften höchste Priorität besitzt, kommt besonders in den Mitgliedstaaten mit einem geringen Anteil an Konzerngesellschaften lediglich eine grobe Unterteilung der Cluster in die unterschiedlichen Wirtschaftszweige in Betracht. Konkret wurde entweder eine Unterteilung in 60 bzw. 19 verschiedene Tätigkeitsbereiche vorgenommen. ${ }^{438}$

Abbildung 10: Clusterbildung zum Ergänzen von Jahresabschlussinformationen

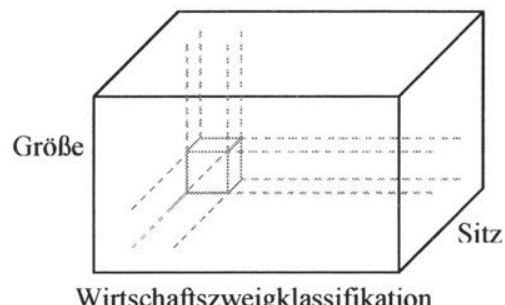

Quelle: eigene Darstellung

Schließlich beeinflusst die Größe der Unternehmen die Zusammensetzung des Jahresabschlusses. Der Grund für diese Annahme sind Lernkurven- und Größendegressionseffekte. Aber auch die Stellung des Unternehmens auf dem relevanten Absatzmarkt wirkt sich auf die Ertragslage aus. ${ }^{439}$ Deshalb kann die Größe eines Unternehmens nicht nur absolut beurteilt werden, sondern muss im Verhältnis zu den übrigen Marktteilnehmern gemessen werden. Aus Gründen der Vereinfachung werden als Marktteilnehmer alle dem Cluster zugehörigen Unternehmen betrachtet. Diese werden in drei gleich große Größenklassen unterteilt. Diese relative Einteilung ist vorteilhaft, da keine quantitativen Grenzen willkürlich festgelegt werden müssen. Wären die Größenklassen hingegen unabhängig von der Eingrenzung nach dem Wirtschaftszweig und dem Sitz definiert worden, resultierte daraus nur teilweise eine weitere Unterteilung. Werden z. B. die absoluten, sich aus einer Gleichverteilung ergebenden Grenzen der Größenklassen deutscher Handelsunternehmen auf kleinere Volkswirtschaften übertragen, ist damit zu rechnen, dass die oberste und unterste Größenklasse unter- bzw. überproportional stark besetzt ist. Darüber hinaus verfälschen

\footnotetext{
${ }^{438}$ Vgl. hierzu die Erläuterungen des Kapitels D.IV.2 und die Tabelle 56 im Anhang.

439 Für empirische Ergebnisse zum Zusammenhang von Marktposition und Rentabilität vgl. Buzzel, R.D./Bradley, T.G., PIMS, 1989, S. 66-75.
} 
Ausreißer bei einer Gleichverteilung der Unternehmen über die Größenklassen hinweg das Ergebnis weniger stark. Die Größe eines Unternehmens wird im Hinblick auf die Datenlage ausschließlich an der Bilanzsumme gemessen. ${ }^{40}$

\section{( $\gamma$ ) Schätzen einzelner Jahresabschlusspositionen}

Die Amadeus-Datenbank berechnet keine Position des Jahresabschlusses selbstständig, sondern gibt ausschließlich die in der Datenbank hinterlegten Werte aus. Fehlt nur ein einzelner Jahresabschlussposten, kann dieser durch Subtraktion der verfügbaren Informationen von der übergeordneten Summe bzw. durch Addition aller verfügbaren Informationen berechnet werden. In der Mehrzahl der Fälle ist bei den fehlenden Bilanzpositionen eine Null zu ergänzen, da die übergeordnete Summe und die verfügbaren Bilanzpositionen bereits übereinstimmen. Eine detaillierte Übersicht über diese Anpassungen kann den Tabellen 57-58 im Anhang entnommen werden. Nach diesen Anpassungsberechnungen weisen über $80 \mathrm{v}$. H. der Konzerngesellschaften eine vollständige Bilanz auf. Eine komplette Gewinn-und-Verlust-Rechnung hingegen besitzen lediglich 40 v. H. der Unternehmen.

Im zweiten Schritt werden die Jahresabschlussposten der verbleibenden Unternehmen mit fehlenden Daten geschätzt. Die hierfür erforderliche Mindestanforderung erfüllen 389.028 Konzerngesellschaften. Im Umkehrschluss fehlen bei $53 \mathrm{v} . \mathrm{H}$. der Unternehmen entweder Angaben zur Bilanzsumme oder zu einer beliebigen Ertragsgröße in der Datenbank. Der Jahresabschluss dieser Unternehmen kann aufgrund der wenigen Angaben, insbesondere zur Ertragslage, nicht durch Schätzverfahren ergänzt werden. Alle Positionen des Jahresabschlusses und die Anzahl der Mitarbeiter als ein potenzieller Aufteilungsfaktor werden gleich null gesetzt.

Bei den Unternehmen, die die Mindestanforderung zum Schätzen der fehlenden Jahresabschlussgrößen erfüllen, ist die Mehrzahl der Bilanzen vollständig. Bei nur 5,5 v. H. der Gesellschaften fehlen einzelne Bilanzpositionen. Betrachtet man nur die für die Aufteilung der Bemessungsgrundlage maßgebliche Aktivseite der Unternehmen, reduziert sich der Anteil an Unternehmen mit unvollständigen Bilanzdaten auf unter 0,04 v. H. Die fehlenden Positionen der Aktivseite der Bilanz werden durch die Multiplikation einer Basisgröße mit einer Verhältniszahl geschätzt. ${ }^{441}$ Dazu werden zunächst für jede konzernverbundene Gesellschaft, die über die entsprechenden Jahresabschlussinformationen verfügt,

440 Die Bilanzsumme ist die einzige kurzfristig ertragsunabhängige Größe, die für alle Unternehmen gegeben sein muss. Vgl. hierzu a. Kapitel D.IV.3.b)( $\alpha$ ).

441 Vgl. für das Regressionsimputationsverfahren ohne Störterm Göthlich, S., in: Albers et al., Forschung, 2006, S. 139-141. 
die Verhältniszahlen berechnet. Anschließend wird innerhalb eines Clusters der Median als repräsentative Verhältniszahl ausgewählt. Existieren innerhalb eines Clusters keine Konzerngesellschaften, auf deren Basis die Verhältniszahl ermittelt werden kann, wird das Cluster erweitert. Dabei kommt zunächst die gröbere Wirtschaftszweigklassifikation zum Tragen, anschließend wird die Differenzierung nach den Größenklassen aufgegeben. Ausgehend von der Bilanzsumme werden im ersten Schritt die Zwischensummen der Anlage- und Umlaufvermögen geschätzt (55 Unternehmen). Im zweiten Schritt werden aus dem Anlage- (53 Unternehmen) und Umlaufvermögen (22 Unternehmen) die Untergrößen abgeleitet.

Einige der 389.028 Gesellschaften weisen für einzelne Bilanzpositionen negative Werte aus. Fließen diese in den Aufteilungsfaktor Vermögen mit ein, führt eine formelhafte Gewinnaufteilung grundsätzlich zu stark verzerrten Ergebnissen. Da die Aufteilungsfaktoren über die Verteilung der Bemessungsgrundlage entscheiden, wirkt die formelhafte Gewinnaufteilung wie eine Steuer auf die Aufteilungsfaktoren. Sind diese für eine oder mehrere Konzerngesellschaften negativ, erhalten sie eine Steuererstattung. ${ }^{442}$ Finanziert wird die Steuererstattung durch die übrigen Konzerngesellschaften, indem diese kumuliert über $100 \mathrm{v}$. H. der Bemessungsgrundlage zugewiesen bekommen. Im Ergebnis werden zwar nur $100 \mathrm{v} . \mathrm{H}$. der Bemessungsgrundlage besteuert, bei internationalen Konzernen tritt jedoch eine starke Verzerrung zwischen den Steuerhoheiten auf. Um dies zu verhindern, wurde der Jahresabschluss von 352 Unternehmen nachträglich mit Nullen aufgefüllt. Damit verbleiben 388.676 Unternehmen mit einer vollständigen Aktivseite der Bilanz in der Datenbank.

Innerhalb der Gewinn-und-Verlust-Rechnung sind sowohl die relevanten Ertragsgrößen (,operating profit and loss“, „profit and loss before tax“) als Bemessungsgrundlage sowie der Umsatz und die Anzahl der Mitarbeiter bzw. die Lohnsumme als Aufteilungsfaktoren durch Schätzen zu ergänzen. Von den 389.028 Konzerngesellschaften fehlen für 1.183 Unternehmen die Angaben zum Gewinn vor Steuern. Dieser kann in der Mehrzahl der Fälle aus dem Gewinn nach Steuern abgeleitet werden. ${ }^{443}$ Dabei wird erneut der Median der Vorsteuer-

${ }^{442}$ Natürlich kann dieser Effekt durch die übrigen Aufteilungsfaktoren kompensiert werden, sodass sich keine Steuererstattung, sondern lediglich eine Reduktion der Steuerzahllast einstellt.

443 Den Vorsteuergewinn durch die Multiplikation des Nachsteuergewinns mit 1-s zu berechnen, liefert ein fehlerhaftes Ergebnis, wenn steuerfreie Gewinnbestandteile wie z. B. Dividenden in dem unbekannten Vorsteuergewinn enthalten sind oder aber das Unternehmen mit Sitz in einem Freistellungsland eine Betriebsstätte unterhält, die in einem Staat mit abweichendem Steuersatz ansässig ist. Darüber hinaus existieren temporäre Effekte aufgrund abweichender Bilanzierungsvorschriften, wie z. B. das Bilanzierungsverbot von Drohverlustrückstellungen in der Steuerbilanz oder die u. U. abweichende Abschreibungsdauer von Firmenwerten. 
Nachsteuer-Relation in den einzelnen Clustern herangezogen. Da negative Einkünfte, soweit keine aktiven latenten Steuern ${ }^{444}$ gebildet werden oder ein Rücktrag der Einkünfte erfolgt, mit einer Steuerlast von null einhergehen, wurde bei der Ermittlung der Verhältniszahlen zwischen Gewinn- und Verlustunternehmen unterschieden. Lediglich in Slowenien kann für keine der 42 in der Datenbank enthaltenen Konzerngesellschaften die Verhältniszahl ermittelt werden, da Informationen über den Gewinn vor Steuern grundsätzlich fehlen. Um diesen dennoch zu ermitteln, wird ausgehend vom Gewinn nach Steuern unter Berücksichtigung des im Jahr 2003 geltenden Körperschaftsteuersatzes auf den Gewinn vor Steuern geschlossen. Bei 16 verbleibenden Unternehmen, die weder den Gewinn nach noch vor Steuern ausweisen, wird letzterer über das Verhältnis des operativen Gewinns zum Gewinn vor Steuern ermittelt.

Der operative Gewinn muss für 3.476 Konzerngesellschaften geschätzt werden. Bei 13 Unternehmen, für die zuvor der Gewinn vor Steuern ermittelt wurde, kann der operative Gewinn einfach durch Subtraktion des Finanzergebnisses vom Gewinn vor Steuern ermittelt werden. Die verbleibenden 3.463 Gesellschaften haben kein Finanzergebnis in der Datenbank hinterlegt. Da dieses ausschließlich auf sehr kleine Gesellschaften zutrifft, wird pauschal ein Finanzergebnis von null unterstellt. Damit entspricht der operative Gewinn für diese Gesellschaften dem Gewinn vor Steuern.

Wesentlich komplexer fällt das Schätzen des Umsatzes aus, da dieser erstens bei 111.940 Gesellschaften zu bestimmen ist, was einem Anteil von über $28 \mathrm{v}$. H. der Konzerngesellschaften mit verfügbaren Jahresabschlüssen entspricht. Zweitens besteht zwischen dem Umsatz und den übrigen Jahresabschlusspositionen kein direkter funktionaler Zusammenhang, sodass die Qualität der Schätzung leidet. Aus diesem Grund wird, soweit kein Rückschluss über andere Gewinn-und-Verlust-Rechnungsinformationen oder historische Daten möglich ist, der Umsatz innerhalb eines Clusters über eine Regressionsfunktion bestimmt.

Zunächst wird auch hier über pauschale Annahmen auf den Umsatz geschlossen. Sind sowohl für den operativen Gewinn als auch für die Herstellkosten Daten verfügbar und lediglich die sonstigen Kosten unbekannt, werden letztere pauschal mit null angesetzt. Damit entspricht der Umsatz dem operativen Gewinn zuzüglich der Herstellkosten. Wird bei Verlustgesellschaften ein negativer Umsatz ermittelt, erweist sich die Pauschalannahme als fehlerhaft und wird verworfen. Im Ergebnis konnte somit für 222 Unternehmen der Umsatz abgeleitet werden.

${ }^{444}$ Nach HGB dürfen latente Steuern auf Verlustvorträge nicht gebildet werden. Der Verlustrücktrag ist innerhalb der Europäischen Union nur in Deutschland, Großbritannien und Irland für ein Jahr und in Frankreich und den Niederlanden für drei Jahre gestattet. Alle übrigen Mitgliedstaaten kennen keinen Verlustrücktrag. 
Anschließend wird der Umsatz aus historischen Daten abgeleitet, indem er gemäß der Entwicklung der Bilanzsumme fortgeschrieben wird. ${ }^{445}$ Folglich muss sowohl der Umsatz als auch die Bilanzsumme in einem der fünf 2003 vorangegangenen Jahre bekannt sein. ${ }^{446}$ Diese Voraussetzung erfüllen 17.861 Konzerngesellschaften.

Für die verbleibenden 94.698 Konzerngesellschaften wird der Umsatz mittels einer linearen multiplen Regressionsfunktion prognostiziert. Als Regressoren werden je nach Verfügbarkeit entweder die Lohnsumme oder die Anzahl der Mitarbeiter und das Umlaufvermögen in die Zielfunktion aufgenommen. Dabei wird für jedes einzelne Cluster eine Regressionsgleichung ermittelt, wobei ein Cluster jetzt nur noch durch das Land und die grobe Wirtschaftszweigklassifikation beschrieben wird. ${ }^{447}$ Kann kein linearer Zusammenhang nachgewiesen werden oder ist sowohl die Lohnsumme als auch die Anzahl der Mitarbeiter nicht in der Datenbank hinterlegt, wird der Umsatz ausschließlich über eine Relation des Umlaufvermögens zum Umsatz aufgefüllt.

Im dritten Schritt wird die Lohnsumme für 136.859 Konzerngesellschaften zunächst auf Basis der Mitarbeiteranzahl, dann gemäß den historischen Daten und schließlich über die Bilanzsumme hergeleitet. Dazu wird für die einzelnen Cluster die Lohnsumme pro Mitarbeiter ermittelt. Liegen hierfür keine Informationen in der Datenbank bereit, wird auf die Lohn- und Gehaltsstatistik der Eurostat-Datenbank zurückgegriffen. Dies betrifft ausschließlich Gesellschaften mit Sitz in Griechenland, Irland und Litauen. Anschließend wird die Lohnsumme über historische Daten hergeleitet, wobei sie gemäß der Entwicklung der Bilanzsumme fortgeschrieben wird. Für die verbleibenden 16.598 Konzerngesellschaften bestimmt sich die Lohnsumme über eine Relation zur Bilanzsumme. Nachdem alle Konzerngesellschaften, welche die Mindestanforderungen erfüllen, über Informationen der Lohnsumme verfügen, wird bei 152.958 Unternehmen die fehlende Anzahl der Mitarbeiter gemäß der Lohnsumme pro Mitarbeiter in den einzelnen Clustern ergänzt.

Im Ergebnis verfügen 388.676 Konzerngesellschaften sowohl über eine vollständige Aktivseite der Bilanz als auch über Informationen zum Umsatz, zum Finanzergebnis, zum operativen Gewinn und dem Gewinn vor Steuern sowie zur Anzahl der Mitarbeiter und der Lohnsumme. Die Bilanz und die Gewinn-und-

${ }^{445} \mathrm{Vgl}$. zu dem Last-Observation/Value-Carried-Forward (LOCF)-Verfahren Göthlich, S., in: Albers et al., Forschung, 2006, S. 140.

446 Um konjunkturelle Verzerrungen zu vermeiden, wurden lediglich die fünf vorhergehenden Berichtszeiträume berücksichtigt.

447 Nur durch eine weiter gefasste Definition der Cluster sind genügend Datensătze für die Ermittlung einer Regressionsfunktion verfügbar. Ziel ist es also nicht mehr, möglichst ein vergleichbares oder eine kleine Gruppe vergleichbarer Unternehmen zu identifizieren, sondern den linearen Zusammenhang zwischen den Größen allgemeiner zu beschreiben. 
Verlust-Rechnung der übrigen 444.974 Konzerngesellschaften weisen für jede Jahresabschlussposition den Wert Null aus.

\section{Rechtsformen}

Im Rahmen der empirischen Analyse sollen sich nur Körperschaftsteuersubjekte für eine konsolidierte Besteuerung qualifizieren können. ${ }^{448} \mathrm{Da}$ die Gesellschaftsrechte zwischen den 25 Mitgliedstaaten der Europäischen Union teilweise erheblich voneinander abweichen und damit die Definition von Mindestanforderungen an die Rechtsform schwierig ist, wird im Folgenden auf die abschließende Aufzählung von Rechtsformen im Anhang der Mutter-TochterRichtlinie $^{449}$ zurückgegriffen.

Tabelle 20: Anteil der im Anhang der Mutter-Tochter-Richtline aufgeführten Rechtsformen in der Datenbank

\begin{tabular}{|c|c|ccc|ccc|}
\hline Land & $\begin{array}{c}\text { o. A. vor } \\
\text { Anpassung }\end{array}$ & $\begin{array}{c}\text { 0. A. nach } \\
\text { Anpassung }\end{array}$ & MTR & Sonstige & $\begin{array}{c}\text { o. A. nach } \\
\text { Anpassung }\end{array}$ & MTR & Sonstige \\
\hline AT & 28 & 20 & 17.615 & 4.565 & $0,1 \%$ & $79,3 \%$ & $20,6 \%$ \\
BE & 151 & 148 & 51.516 & 5 & $0,3 \%$ & $99,7 \%$ & $0,0 \%$ \\
CY & 0 & 0 & 132 & 0 & $0,0 \%$ & $100,0 \%$ & $0,0 \%$ \\
CZ & 0 & 0 & 2.913 & 0 & $0,0 \%$ & $100,0 \%$ & $0,0 \%$ \\
DE & 48 & 1 & 162.284 & 85.764 & $0,0 \%$ & $65,4 \%$ & $34,6 \%$ \\
DK & 0 & 0 & 74.477 & 2.185 & $0,0 \%$ & $97,1 \%$ & $2,9 \%$ \\
EE & 0 & 0 & 2.378 & 1 & $0,0 \%$ & $100,0 \%$ & $0,0 \%$ \\
ES & 950 & 213 & 79.654 & 993 & $0,3 \%$ & $98,5 \%$ & $1,2 \%$ \\
FI & 3 & 3 & 10.213 & 64 & $0,0 \%$ & $99,3 \%$ & $0,6 \%$ \\
FR & 301 & 260 & 108.243 & 10.999 & $0,2 \%$ & $90,6 \%$ & $9,2 \%$ \\
GB & 157 & 0 & 475.052 & 19 & $0,0 \%$ & $100,0 \%$ & $0,0 \%$ \\
GR & 0 & 0 & 5.904 & 0 & $0,0 \%$ & $100,0 \%$ & $0,0 \%$ \\
HU & 16 & 3 & 9.585 & 46 & $0,0 \%$ & $99,5 \%$ & $0,5 \%$ \\
IE & 7.259 & 54 & 24.407 & 881 & $0,2 \%$ & $96,3 \%$ & $3,5 \%$ \\
IT & 477 & 232 & 14.799 & 45 & $1,5 \%$ & $98,2 \%$ & $0,3 \%$ \\
LT & 5 & 5 & 1.217 & 0 & $0,4 \%$ & $99,6 \%$ & $0,0 \%$ \\
LU & 0 & 0 & 131 & 1 & $0,0 \%$ & $99,2 \%$ & $0,8 \%$ \\
LV & 2 & 2 & 1.297 & 15 & $0,2 \%$ & $98,7 \%$ & $1,1 \%$ \\
NL & 41 & 5 & 180.828 & 201 & $0,0 \%$ & $99,9 \%$ & $0,1 \%$ \\
PL & 243 & 179 & 8.588 & 30 & $2,0 \%$ & $97,6 \%$ & $0,3 \%$ \\
PT & 371 & 58 & 9.510 & 20 & $0,6 \%$ & $99,2 \%$ & $0,2 \%$ \\
SE & 0 & 0 & 67.736 & 0 & $0,0 \%$ & $100,0 \%$ & $0,0 \%$ \\
SI & 0 & 0 & 78 & 2 & $0,0 \%$ & $97,5 \%$ & $2,5 \%$ \\
SK & 0 & 0 & 25 & 0 & $0,0 \%$ & $100,0 \%$ & $0,0 \%$ \\
\hline Summe & 10.052 & 1.183 & 1.308 .582 & 105.836 & $0,1 \%$ & $92,4 \%$ & $7,5 \%$ \\
\hline
\end{tabular}

Quelle: eigene Darstellung

448 Vgl. hierzu die Überlegungen in Kapitel C.II.2.a)( $\alpha$ ).

449 Vgl. Richtlinie 90/435/EWG des Rates v. 23. 7. 1990, Abl. Nr. L 225 v. 20. 8. 1990, S. 6-9 und die Richtlinie 2003/123/EG des Rates v. 22. 12. 2003, Abl. Nr. L 007 v. 3. 1. 2004, S. 41-44. Die Änderungsrichtlinie 2003/123/EG umfasst im Wesentlichen drei Elemente: die Absenkung des Beteiligungsschwellenwerts, die Vermeidung der Doppelbesteuerung im Fall von Enkelgesellschaften und die Erweiterung der Liste mit den Gesellschaftsformen, die in den Anwendungsbereich der Richtlinie fallen. 
In der Amadeus-Datenbank sind grundsätzlich Informationen zur Rechtsform der Unternehmen hinterlegt, aber auch hier sind die Angaben nur unvollständig und stellenweise fehlerhaft. Insgesamt führt die Amadeus-Datenbank 172 unterschiedliche Rechtsformen innerhalb der Europäischen Union auf. ${ }^{450}$ Die Mehrzahl der Unternehmen firmiert jedoch unter einer von der Mutter-TochterRichtline anerkannten Rechtsform (Tabelle 20). Lediglich in Deutschland, Frankreich und Österreich ist der Anteil der sonstigen, nicht in der MutterTochter-Richtline enthaltenen Rechtsformen hoch. Das ist hauptsächlich auf die große Bedeutung der Personengesellschaften in diesen Ländern zurückzuführen. Allein für Deutschland enthält die Amadeus-Datenbank fast $80.000 \mathrm{GmbHs}$ \& Co. $\mathrm{KGs}^{451}$, in Österreich sind es 3.600 Gesellschaften dieser Rechtsform. Der Anteil der Gesellschaften, denen keine Rechtsform zugeordnet ist, beträgt 0,7 v. H. Für die Mehrzahl dieser Gesellschaften kann die Rechtsform manuell aus dem Firmennamen abgeleitet werden. Dadurch verringert sich der Anteil der unbestimmten Gesellschaften um 8.869 Unternehmen auf 0,08 v. H. Für die weiteren Auswertungen wurden diese Unternehmen nicht weiter berücksichtigt.

\section{Abbilden der Konzerne}

\section{1. Überblick}

Der „Vergleichskonzern“ bildet die Ausgangsbasis für die Analyse der Konzernstrukturen und die Berechnungen der Bemessungsgrundlagenänderung bei Einführung der konsolidierten Körperschaftsteuer-Bemessungsgrundlage. Bestandteil sind alle Kapitalgesellschaften, an denen direkt oder indirekt eine Mehrheitsbeteiligung besteht. ${ }^{452}$ Damit sind sowohl die nationalen steuerlichen Unternehmensgruppen ${ }^{453}$ als auch die Konzernkreise der einheitlichen konsolidierten Bemessungsgrundlage immer Teilmengen dieses Vergleichskonzerns. Liegt die Mindestbeteiligungsquote der konsolidierten Bemessungsgrundlage bei $50 \mathrm{v}$. H., entspricht dieser Konzernkreis dem Vergleichskonzern.

Diese Vorgehensweise garantiert eine Vergleichbarkeit unterschiedlicher steuerlicher Konzerndefinitionen. Bei der Berechnung der Bemessungsgrundlage

${ }^{450}$ Eine Übersicht über die Einteilung der in der Amadeus-Datenbank aufgeführten Rechtsformen befindet sich in der Tabelle 55 im Anhang.

451 Die Amadeus-Datenbank zählt die GmbH \& Co. KG zu den Kapitalgesellschaften, was wahrscheinlich auf den Suchalgorithmus zurückzuführen ist, der nur nach dem Akronym $\mathrm{GmbH}$ sucht.

452 Für eine exakte Definition vgl. Kapitel D.V.2.

453 Die deutsche Organschaft und das lettische Gruppenbesteuerungssystem erlauben auch Einkommensteuersubjekten Bestandteil einer Unternehmensgruppe zu werden (Tabelle 7). Insofern werden die Gruppenbesteuerungskreise in diesen Ländern nur unvollständig abgebildet. Vgl. hierzu Kapitel B.III. 
werden zwar nur die steuerlichen Konzernkreise berücksichtigt, anschließend erfolgt jedoch die Addition der Einzelergebnisse und ein Gesamtergebnis für den Vergleichskonzern wird ausgewiesen. Damit werden bei Gegenüberstellung verschiedener Konzerndefinitionen immer dieselben Unternehmen miteinander verglichen. Abbildung 11 verdeutlicht die Vorgehensweise an einem Beispiel.

Abbildung 11: Steuerliche Konzernkreise als Teilmenge des Vergleichskonzerns ${ }^{454}$

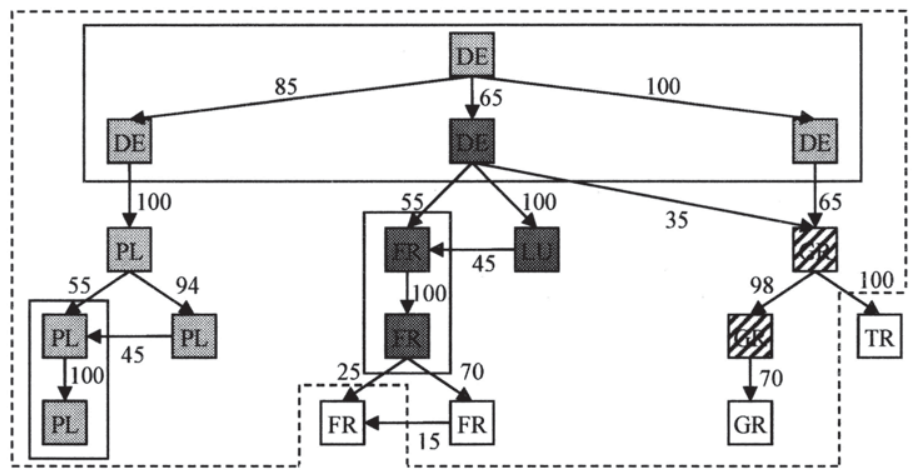

$\square$ Steuerliche Konzernkreise nach geltendem Recht i-j Vergleichskonzern

囚 $\square$ СССТВ Konzerngesellschaften - Mindestbeteiligungsquote 75 v. H.

Quelle: eigene Darstellung

\section{Vergleichskonzern}

\section{a) Definition von Konzern- und Konzernmuttergesellschaften}

Als Konzerngesellschaften gelten ausschließlich Unternehmen mit Sitz in der Europäischen Union, die unter einer im Anhang der Mutter-Tochter-Richtlinie ${ }^{455}$ gelisteten Rechtsform firmieren und die gemäß der Klassifikation der AmadeusDatenbank einer der folgenden Kategorien zuzuordnen sind:

- Foundation

- Industrial Company
- Financial Company

- Keiner Anteilseignerkategorie zugeordnet, jedoch existiert eine Unternehmensidentifikationsnummer

454 Griechenland besitzt derzeit kein Gruppenbesteuerungssystem. Polen besitzt ein Gruppenbesteuerungssystem, das eine Mindestbeteiligungsquote von $95 \mathrm{v}$. H. fordert und indirekte Beteiligungen vernachlässigt. Die deutsche Organschaft verlangt eine Beteiligung von $50 \mathrm{v}$. H. Darüber hinaus muss zwischen dem Organtrăger und der Organgesellschaft ein Gewinnabführungsvertrag abgeschlossen worden sein. Zur Identifizierung des Gewinnabführungsvertrags vgl. Kapitel D.V.3.a).

455 Vgl. Richtlinie 2003/123/EG des Rates v. 22. 12. 2003, Abl. Nr. L 007, v. 3. 1. 2004, S. 41-44. 
Darüber hinaus müssen diese Gesellschaften direkt oder indirekt in einem mehrheitsvermittelnden Beteiligungsverhältnis zu einer anderen Konzerngesellschaft stehen, welche selbst die genannten Anforderungen erfüllt. Ob eine Gesellschaft mehrheitsbeteiligt ist, berechnet sich ausschließlich nach der Höhe der Beteiligung. Abweichende Stimmrechtsanteile können nicht beobachtet werden und sind insofern nicht maßgebend.

Konzernmuttergesellschaften sind Konzerngesellschaften, die mindestens an einer Konzerngesellschaft direkt zu über $50 \mathrm{v}$. H. beteiligt sind und an denen keine andere Konzerngesellschaft direkt oder indirekt mehrheitsbeteiligt ist. Damit werden selbst dann Konzerngesellschaften als Muttergesellschaften qualifiziert, wenn ein Anteilseigner direkt oder indirekt mehrheitlich beteiligt ist, dieser aber seinen Sitz außerhalb der Europäischen Union hat ${ }^{456}$ und/oder einer der folgenden Kategorien (B) angehört:

- Employee/Manager

- Individual(s) or family(ies)

- Other unnamed shareholders

- Public
- Bank

- Mutual \& Pension fund/Trust/Nominee

- Insurance company

- Keiner Anteilseignerkategorie zugeordnet und keine Unternehmensidentifikationsnummer vorhanden

Abbildung 12: Definition der Konzernmuttergesellschaft
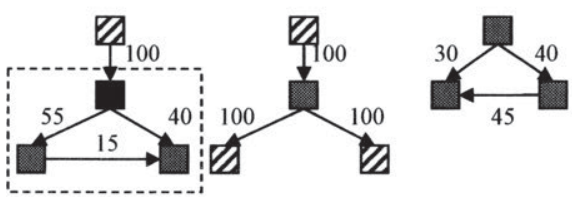

Zeine EU-Gesellschaft:

- Sitz außerhalb der EU

- Rechtsform nicht im Anhang der Mutter-Tochter-Richtlinie

- Kategorie B zugehörig

Konzernmuttergesellschaft

EU-Gesellschaft

Quelle: eigene Darstellung

Banken, Versicherungen und Pensionsfonds erfüllen in zweierlei Hinsicht nicht die Anforderungen an eine Konzerngesellschaft. Erstens muss die Existenz eines „unitary business" stark angezweifelt werden, wenn eine Bank, Versicherung oder ein Fonds mehrheitlich an einem Konzern beteiligt ist. Die Vorstellung, dass alle Konzerne, an denen z. B. die Deutsche Bank AG oder die Allianz AG mehrheitlich beteiligt ist, ihre Verluste verrechnen dürfen, geht sicherlich über das Ziel der konsolidierten Besteuerung hinaus. Zweitens sind die Jahresabschlüsse aufgrund der abweichenden Rechnungslegungsvorschriften nicht mit dem in der Amadeus-Datenbank hinterlegten Gliederungsschema vereinbar,

${ }^{456}$ Vgl. hierzu a. Kapitel C.II.2.b). 
weswegen sie in der Amadeus-Datenbank gar nicht auftauchen. ${ }^{457}$ Abbildung 12 verdeutlicht die Definition von Konzernmuttergesellschaften grafisch.

\section{b) Konzernkreis}

Die Abbildung der Konzernkreise erfolgt gemäß dem Control-Konzept. ${ }^{458}$ Die Berechnung der Beteiligungsquote erfolgt additiv. Mittelbare Beteiligungen sind $\mathrm{zu}$ berücksichtigen, wenn die Beteiligung (direkt/indirekt) an jeder vermittelnden Gesellschaft über 50 v. H. beträgt. Von der Gesamtzahl aller Stimmrechte sind die Stimmrechte aus eigenen Anteilen abzusetzen (Abbildung 13). ${ }^{459}$ Abbildung 13: Absetzen direkt gehaltener eigener Anteile

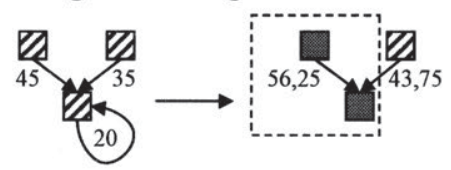

Quelle: eigene Darstellung

Aber auch über mehrere Gesellschaften gehaltene Rückbeteiligungen gilt es zu korrigieren (Abbildung 14). Insgesamt existieren in der Datenbank $10.019 \mathrm{Ge}-$ sellschaften in 4.459 Beziehungskreisen, die indirekt an sich selbst beteiligt sind. Die Mehrzahl (3.840) der Beziehungskreise besteht aus nur zwei Unternehmen, weitere 532 aus drei oder vier Unternehmen, und nur 87 verfügen über mehr als vier Unternehmen.

Abbildung 14: Absetzen indirekt gehaltener eigener Anteile

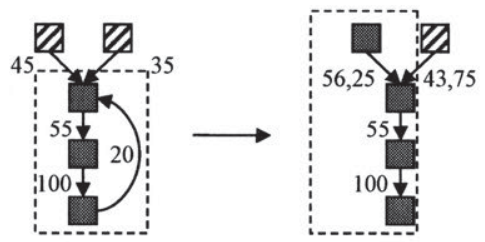

Quelle: eigene Darstellung

Als problematisch erweisen sich Kreisbeziehungen, bei denen die Höhe der Rückbeteiligung $50 \mathrm{v}$. H. übersteigt, da eine eindeutige Definition der Muttergesellschaft nicht mehr möglich ist. Abbildung 15 verdeutlicht die Problematik,

${ }^{457}$ Eine Beschreibung der Kriterien zur Aufnahme in die Amadeus-Datenbank findet sich in Kapitel D.III.

458 Es wird stets von der Übereinstimmung der Beteiligungshöhe und der Stimmrechtsanteile ausgegangen.

$459 \S 16$ III AktG. 
indem der identische Konzern zweimal dargestellt ist, wobei sowohl die Gesellschaft A als auch die Gesellschaft B als Konzernmutter in Frage kommen.

Abbildung 15: Kreisbeteiligungen
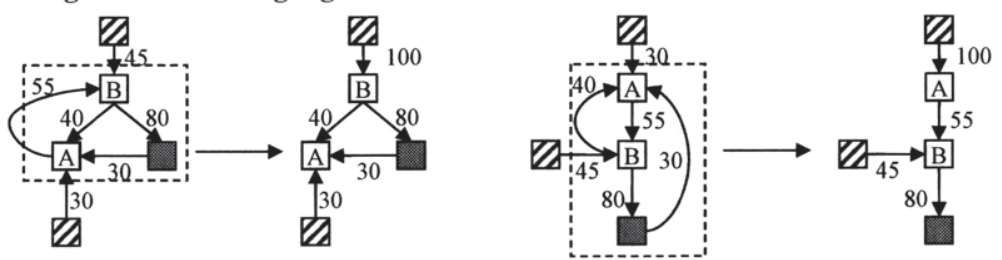

Quelle: eigene Darstellung

Da eine Definition der Muttergesellschaft ohne weitere Informationen nicht möglich ist, werden die betreffenden 383 Konzernkreise mit ihren 811 Konzerngesellschaften von der Untersuchung ausgeschlossen.

Der Konzernkreis wird unterbrochen, sobald eine Konzerngesellschaft zwischengeschaltet wird, die im Rahmen dieser Untersuchung nicht als solche gilt. Selbst wenn die Voraussetzungen des Control-Konzepts erfüllt sind, ist eine Sprungkonsolidierung über die nicht berechtigte Konzerngesellschaft hinweg nicht vorgesehen (Abbildung 16). ${ }^{460}$

Abbildung 16: Sprungkonsolidierung

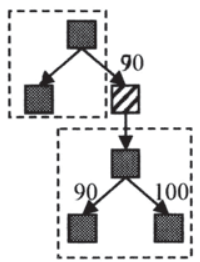
a) Gesellschaft hat ihren Sitz nicht in der EU.
b) Gesellschaft firmiert unter einer nicht im Anhang der MTR aufgeführten Rechtsform.
c) Die Gesellschaft ist eine Bank, Versicherung oder ein Fonds.

Quelle: eigene Darstellung

\section{c) Bestimmung der Konzernbranche}

Die Analyse der Unternehmensgruppen erfolgt auch differenziert nach den Tätigkeitsschwerpunkten der Konzerne. Dazu ist die Wirtschaftszweigklassifikation des Gesamtkonzerns zu ermitteln. Ein Rückschluss vom NACE-Code der Muttergesellschaft auf den Unternehmensverbund ist dabei nicht möglich, da sich dieser stets nur auf die einzelne Gesellschaft bezieht. Folglich ist die Klassifizierung des Gesamtkonzerns aus den einzelnen Tätigkeitsfeldern der Konzerngesellschaften abzuleiten. Dabei wird analog zur Bestimmung der Branche eines

${ }^{460}$ Da die Amadeus-Datenbank über keine oder nur sehr eingeschränkte Beteiligungsinformationen außerhalb der Europäischen Union verfügt, ist diese Vorgehensweise bereits aufgrund der Datenlage zu wăhlen. 
einzelnen Unternehmens, dessen Wertschöpfung aus unterschiedlichen Tätigkeitsbereichen resultiert, verfahren. Grundsätzlich wird eine Gesellschaft stets der Klasse zugeordnet, deren Anteil an der Wertschöpfung $50 \mathrm{v} . \mathrm{H}^{461}$ übersteigt. Erreicht keine der Klassen diesen Schwellenwert, erfolgt die Klassifizierung unter Zuhilfenahme der Top-down-Methode. Diese bestimmt im ersten Schritt den Abschnitt mit der höchsten Wertschöpfung. Innerhalb dieses Abschnitts wird im zweiten Schritt die Abteilung mit dem höchsten Beitrag zur Wertschöpfung definiert. Dem hierarchischen Prinzip folgend wird in zwei weiteren Schritten die Zugehörigkeit zu einer Gruppe und einer Klasse festgelegt. Mittels dieser Vorgehensweise bleibt die Kohärenz der Wirtschaftszweigsystematik gewahrt. Die Klassifizierung auf unterer Ebene stimmt stets mit der Einteilung auf höherer Ebene überein. Im Ergebnis kann damit aber ein Konzern einer Klasse zugeordnet werden, die nicht den höchsten Anteil an der Wertschöpfung aufweist.

Da die Amadeus-Datenbank für die überwiegende Anzahl der Unternehmen über keine Informationen zur Wertschöpfung verfügt, dienen der Umsatz und das Anlagevermögen zu gleichen Teilen als Gewichtungsfaktor. ${ }^{462}$ Durch die Aufnahme dieser zwei Faktoren wird gewährleistet, dass weder Produktionsnoch Handelsgesellschaften die Bestimmung der Konzernbranche überdurchschnittlich beeinflussen. Eine Klassifizierung der Konzerne ausschließlich mittels des Umsatzes lässt Produktionskonzerne leicht als Handelskonzerne erscheinen, sobald der Absatz an Dritte über Vertriebsgesellschaften erfolgt. Die alleinige Berücksichtigung des Anlagevermögens hätte hingegen anlageintensive Gesellschaften überbewertet und Handelskonzerne mit nur einem geringen Produktionsanteil einer Branche des verarbeitenden Gewerbes zugeordnet.

Die Haupttätigkeit von 7 v. H. der Konzerngesellschaften ist das Halten von Beteiligungen. Um zu verhindern, dass sämtliche Holdingkonzerne der Wirtschaftszweigklassifikation „Beteiligungsgesellschaft“463 ${ }^{\star 4 u g e o r d n e t ~ w e r d e n, ~}$ finden diese Gesellschaften bei der Bestimmung der Konzernbranche keine Berücksichtigung. Bei weiteren $28 \mathrm{v}$. H. der Unternehmen fehlen entweder Angaben zum Wirtschaftszweig, ${ }^{464}$ oder es sind keine Informationen über den Umsatz und das Anlagevermögen verfügbar. Diese Konzerngesellschaften können keinen Beitrag zur Ermittlung der Konzernbranche liefern und werden insofern

\footnotetext{
461 Zum Aufbau der Wirtschaftszweigsystematik vgl. Eurostat, NACE, 2002.

${ }^{462}$ Eine Überprüfung der 250 größten deutschen Konzerne ergab, dass eine Gleichgewichtung des Umsatzes und des Anlagevermögens die besten Ergebnisse liefert.

463 NACE Code: KA 74.15.

${ }^{464}$ NACE Code: QA 99.00.
} 
auch vernachlässigt. ${ }^{465}$ Schließlich verfügen $27 \mathrm{v}$. H. der Unternehmen nur über Informationen zu einem der beiden Gewichtungsfaktoren. Die daraus resultierende Verzerrung ist unproblematisch, solange sich dadurch nicht die Rangfolge des Haupttätigkeitsbereichs eines Konzerns ändert. Tendenziell verfügen kleinere Unternehmen über keinen oder einen unvollständigen Jahresabschluss in der Datenbank, sodass der zu erwartende Fehler gering ausfällt. Für die verbleibenden $38 \mathrm{v}$. H. der Konzerngesellschaften liegen alle Informationen zur Bestimmung der Konzernbranche in der Datenbank vor. Aufgrund der dargestellten Datenlage kann für etwa 7.000 Konzerne keine Branche bestimmt werden, da für deren Konzernunternehmen entweder keine Angaben zu den Gewichtungsfaktoren in der Datenbank verfügbar sind, oder die Konzernunternehmen keine Angaben zu ihrer Tätigkeit unterbreitet haben oder aber drittens der Wirtschaftsklassifikation „Beteiligungsgesellschaft“ zuzuordnen sind. Die Verteilung der Konzernbranche für die in der Datenbank abgebildeten Konzerne ist in Tabelle 30 auf Seite 133 aufgeführt.

\section{Abbilden der steuerlichen Konzernkreise}

\section{a) Nationale Gruppenbesteuerungssysteme}

Die teilweise sehr komplexen Regelungen der nationalen Gruppenbesteuerungssysteme erlauben nur eine vereinfachte Umsetzung im Rahmen der empirischen Analyse. ${ }^{466}$ Grundsätzlich wird vermutet, dass die verbundenen Unternehmen zur Anwendung der Gruppenbesteuerungsregelungen optieren, sobald sie die damit einhergehenden Anforderungen erfüllen. Dieser Generalverdacht gilt nicht für Unternehmensgruppen in Deutschland und Österreich. Hier bedarf es zur Begründung einer Organschaft eines Gewinnabführungsvertrages, ${ }^{467}$ an den vor allem auch Haftungsverpflichtungen geknüpft sind. Die Annahme, dass alle nationalen Konzerne, die die Voraussetzung der finanziellen Eingliederung erfüllen, auch eine Organschaft begründen, muss als unrealistisch eingestuft werden. Damit werden die Gruppenbesteuerungssysteme dieser beiden Staaten gesondert behandelt und zunächst zu den Mitgliedstaaten gezählt, die der wirtschaftlichen Einheit von verbundenen Unternehmen nicht gesondert Rechnung tragen.

${ }^{465}$ Keine Angaben zur Wirtschaftszweigklassifikation machen $10,7 \mathrm{v}$. H. der Unternehmen. Vgl. Tabelle 15.

${ }^{466}$ Für eine Darstellung der nationalen Gruppenbesteuerungssysteme in der Europäischen Union vgl. Kapitel B.III.

${ }^{467}$ Hier sei noch einmal darauf hingewiesen, dass Österreich zum 1. 1. 2004 ein neues Gruppenbesteuerungssystem eingeführt hat, das nicht mehr den Abschluss eines Gewinnabführungsvertrags verlangt. 
Die Abgrenzung der nationalen steuerlichen Konzernkreise erfolgt nach Art der finanziellen Verknüpfung, der Rechtsform und dem Sitz der Gesellschaften. Die Einteilung weicht stellenweise von der Darstellung der Rechtslage des Kapitel B.III ab, da die Daten für die empirischen Berechnungen überwiegend aus dem Veranlagungszeitrum 2003 stammen und somit auch die nationalen Gruppenbesteuerungssysteme gemäß dem Rechtsstand 2003 abgebildet werden. ${ }^{468}$

Territorial sind alle Gruppenbesteuerungssysteme auf das jeweilige Inland beschränkt. Damit erfolgt sowohl in Frankreich ${ }^{469}$ als auch in Dänemark ${ }^{470}$, entgegen den gesetzlichen Regelungen, kein grenzüberschreitender Verlustausgleich. In Zypern, Irland, Lettland, Malta, Schweden und Großbritannien jedoch werden bei der Abgrenzung des nationalen Konsolidierungskreises die Beteiligungsverhältnisse im Ausland berücksichtigt. Schwestergesellschaften einer ausländischen Obergesellschaft bilden hier steuerlich eine Einheit (Tabelle 6). Problematisch ist dabei, dass zwei Tochtergesellschaften einer nicht in der Europäischen Union ansässigen Muttergesellschaft ihre Ergebnisse konsolidieren dürfen, die Tochtergesellschaften aber zugleich die europäische Muttergesellschaft von zwei unabhängigen europäischen Teilkonzernen sind. Diese Konstellation verstößt gegen die Teilmengeneigenschaft der Konsolidierungskreise der nationalen Gruppenbesteuerungssysteme gegenüber dem Vergleichskonzern. Um dieses zu verhindern, wird die Konsolidierung nur bei Schwestergesellschaften einer in einem anderen Staat der Europäischen Union ansässigen Muttergesellschaft vollzogen.

Tabelle 21: Mindestbeteiligungsquote im Veranlagungszeitraum 2003

\begin{tabular}{|l|l|}
\hline 100 v. H. & Slowenien, Dänemark \\
\hline$>95$ v. H. & Frankreich, Luxemburg, Niederlande, Polen \\
\hline$>90$ v. H. & Finnland, Lettland, Portugal, Schweden \\
\hline$>75$ v. H. & Zypern, Irland, Spanien, Großbritannien \\
\hline $\begin{array}{l}\text { Deutschland und Österreich werden separat behandelt. Maltesische Gesellschaften sind in } \\
\text { der Datenbank nicht vorhanden. Die übrigen nicht genannten Mitgliedstaaten verfügten 2003 } \\
\text { über kein Gruppenbesteuerungssystem. }\end{array}$ \\
\hline
\end{tabular}

Quelle: eigene Darstellung

${ }^{468}$ Vgl. zur Auswahl des für die empirischen Analyse relevanten Betrachtungszeitraums Kapitel D.IV.3.

${ }^{469}$ Für Frankreich ist diese Einschränkung mit dem sehr kleinen Anwenderkreis des ,régime du bénéfice consolidé" von nur elf französischen Konzernen zu begründen. Vgl. Scheunemann, M., Konzernbesteuerung, 2005, S. 279-280; Richard, J., in: intertax, 2003, S. 30-31.

470 In Dänemark erfolgt die Verrechnung der Auslandsverluste über die Zurechnungsmethode. Empirische Berechnungen haben ergeben, dass deren Aufkommenswirkung nahezu null ist. Vgl. Oestreicher, A. et al., Weiterentwicklung, 2006, S. 413-416. 
Als Rechtsform werden erneut nur die im Anhang der Mutter-Tochter-Richtlinie aufgeführten zugelassen. Dies garantiert, dass die Teilmengeneigenschaft der nationalen Konsolidierungskreise gegenüber dem Vergleichskonzern gewahrt bleibt. Gleichzeitig jedoch führt diese Beschränkung in Lettland und Deutschland $\mathrm{zu}$ leichten Verzerrungen, da deren Gruppenbesteuerungssysteme auch Einkommensteuersubjekte zumindest als Organträger miteinbeziehen (Tabelle 7).

Die geforderte Art der finanziellen Eingliederung wird durch die Mindestbeteiligungsquote (Tabelle 21) und durch die Berechnungsmethode der Beteiligungshöhe (Tabelle 22) konkretisiert.

\section{Tabelle 22: Berechnung der Beteiligungsquote}

\begin{tabular}{|l|l|l|}
\hline \multicolumn{2}{|l|}{ Keine indirekten Beteiligungen } & Polen, Slowenien, Lettland \\
\hline \multirow{2}{*}{$\begin{array}{l}\text { Direkte und } \\
\text { indirekte } \\
\text { Beteiligungen } \\
\text { indirekter Beteiligungen }\end{array}$} & $\begin{array}{l}\text { Mindestbeteiligungsquote ist auf } \\
\text { jeder Konzernstufe zu erreichen }\end{array}$ & $\begin{array}{l}\text { Zypern, Dänemark, Finnland, Groß- } \\
\text { britannien, Irland, Luxemburg, Nieder- } \\
\text { lande, Schweden }\end{array}$ \\
\cline { 2 - 3 } & $\begin{array}{l}\text { Erforderliche Mindestbe- } \\
\text { teiligungsquote muss auch multi- } \\
\text { plikativ durchgerechnet bestehen }\end{array}$ & Portugal, Spanien \\
\hline $\begin{array}{l}\text { Deutschland und Österreich werden separat behandelt. Maltesische Gesellschaften sind in } \\
\text { der Datenbank nicht vorhanden. Die übrigen nicht genannten Mitgliedstaaten verfügten 2003 } \\
\text { über kein Gruppenbesteuerungssystem. }\end{array}$ \\
\hline
\end{tabular}

Quelle: eigene Darstellung

Lettland, Polen und Slowenien berücksichtigen nur direkte Beteiligungen. In den übrigen Mitgliedstaaten werden bei der Ermittlung der Mindestbeteiligungsquote indirekte Beteiligungen grundsätzlich berücksichtigt. In Frankreich, Portugal und Spanien muss die geforderte Mindestbeteiligungsquote auch gegenüber der vermittelnden Gesellschaft erfüllt werden. In Portugal und Spanien muss das erforderliche Beteiligungsverhältnis darüber hinaus auch multiplikativ durchgerechnet zwischen der Muttergesellschaft und der indirekten Beteiligung bestehen.

Die deutsche und österreichische ${ }^{471}$ Organschaft setzt neben der finanziellen Eingliederung einen bestehenden Gewinnabführungsvertrag zwischen der Organgesellschaft und dem Organträger voraus. Darüber hinaus müssen in Österreich die Gruppenmitglieder auch wirtschaftlich und organisatorisch eingegliedert sein. ${ }^{472}$ Inwieweit die Konzerngesellschaften in Deutschland und

471 Die Ausführungen beziehen sich auf die im Veranlagungszeitraum 2003 geltende Rechtslage.

${ }^{472} \mathrm{Vgl}$. Althuber, F./Mang, M., in: IWB, Fach 5, Gruppe 2, 8. 9. 2004, S. 608-609; Hirschler, K./Schnidler, C., in: IStR, 2004, S. 509. 
Österreich diese zusätzlichen Anforderungen erfüllen, lässt sich auf der Basis der Angaben der Amadeus-Datenbank nicht beurteilen. Ein Anteil von nur $2 \mathrm{v}$. H. Organgesellschaften an den unbeschränkt körperschaftsteuerpflichtigen Gesellschaften insgesamt kann aber als Indiz dafür gesehen werden, ${ }^{473}$ dass nur ein geringer Teil der finanziell eingegliederten Tochtergesellschaften auch Organgesellschaften sind. Damit führt eine generelle Organschaftsvermutung zu vielfach falschen Ergebnissen, sodass die einzelnen Organgesellschaften anhand ihrer Gewinn-und-Verlust-Rechnung identifiziert werden müssen.

Tabelle 23: Positionen von Buchungen aus einem Gewinnabführungsvertrag in der Gewinn-und-Verlust-Rechnung

\begin{tabular}{|l|c|c|c|}
\hline & \multicolumn{1}{|c|}{$\begin{array}{c}\text { Gesamtkosten- } \\
\text { verfahren }\end{array}$} & $\begin{array}{c}\text { Umsatzkosten- } \\
\text { verfahren }\end{array}$ & $\begin{array}{c}\text { Ausweis in der } \\
\text { Amadeus- } \\
\text { Datenbank }\end{array}$ \\
\hline $\begin{array}{l}\text { Erträge aus Gewinnabführungs- } \\
\text { verträgen }\end{array}$ & Vor/hinter Posten 9 & Vor/hinter Posten 8 & fipl \\
\hline $\begin{array}{l}\text { Aufwendungen aus } \\
\text { Verlustübernahmen }\end{array}$ & Vor/hinter Posten 13 & Vor/hinter Posten 12 & fipl \\
\hline Erträge aus Verlustübernahme & Vor/hinter Posten 20 & Vor/hinter Posten 19 & extr \\
\hline $\begin{array}{l}\text { Aufgrund von Gewinn- } \\
\text { abführungsverträgen abgeführte } \\
\text { Gewinne }\end{array}$ & Vor/hinter Posten 20 & Vor/hinter Posten 19 & extr \\
\hline
\end{tabular}

In Anlehnung an: Forster, K.H. et al. (Hrsg.), $A D S, 1994, \S 277$ Rz. 65

Der Ausweis der Aufwendungen und Erträge aus Gewinnabführungen erfolgt nach herrschender Meinung und Praxis bei der Organgesellschaft vor Posten 19 bzw. 20 (Tabelle 23). ${ }^{474}$ Diese Posten finden sich in der aggregierten Gewinnund-Verlust-Rechnung der Amadeus-Datenbank im außerordentlichen Ergebnis wieder. Entspricht das außerordentliche Ergebnis dem Gewinn oder Verlust nach Steuern mit umgekehrten Vorzeichen, ist diese Übereinstimmung mit sehr hoher Wahrscheinlichkeit auf die Existenz eines Gewinnabführungsvertrags zurückzuführen. ${ }^{475}$ Somit können 1.310 Organgesellschaften in Deutschland und Österreich identifiziert werden (Tabelle 24). Davon sind 133 Gesellschaften bereits gemäß der Abgrenzung des Vergleichskonzerns eine Konzernmuttergesellschaft. Dass diese Unternehmen trotzdem als Organgesellschaften identi-

${ }^{473}$ Laut Körperschaftsteuerstatistik gab es 2001 in Deutschland 17.185 Organgesellschaften. Vgl. Statistisches Bundesamt, Körperschaftsteuerstatistik, 2005.

474 Vgl. Forster, K.H. et al. (Hrsg.), ADS, 1994, § 277 Rz. 65.

475 Sind Minderheitsgesellschafter an der Organgesellschaft beteiligt, muss das außerordentliche Ergebnis nicht zwangsweise dem Gewinn oder Verlust nach Steuern entsprechen. In diesem Fall kann nicht mehr eindeutig festgestellt werden, ob das außerordentliche Ergebnis auf die Existenz eines Gewinnabführungsvertrags zurückzuführen ist. Damit werden Organgesellschaften mit Minderheitsgesellschaftern, die einen Gewinnanspruch haben, nicht weiter beachtet. 
fiziert werden, hat drei mögliche Gründe: Erstens könnte der Organträger ein Einkommensteuersubjekt sein, ${ }^{476}$ das per definitionem nicht Bestandteil des Vergleichskonzerns ist. ${ }^{477}$ Zweitens könnte der Organträger eine Betriebsstätte eines Unternehmens mit Sitz außerhalb der Europäischen Union sein. ${ }^{478}$ Und drittens könnte der Organträger aufgrund von fehlenden oder unstimmigen Daten nicht in der Amadeus-Datenbank geführt werden. ${ }^{479}$ Weitere 15 Organgesellschaften werden direkt von Unternehmen mit Sitz im europäischen Ausland gehalten. Auch hier liegt die Vermutung einer Betriebsstätte dieser Gesellschaften in Deutschland nahe. Die 148 Organgesellschaften, für die kein Organträger ermittelbar ist, werden im Weiteren nicht beachtet.

Tabelle 24: Anzahl der Organgesellschaften in Deutschland und Österreich

\begin{tabular}{|l|rr|}
\hline & Deutschland & Österreich \\
\hline Identifizierte Organgesellschaften & 1.304 & 6 \\
- Konzernmuttergesellschaften gemäß Vergleichskonzern & 133 & 0 \\
- Nur Anteilseigner mit Sitz im europäischen Ausland & 15 & 0 \\
\hline Organgesellschaften - Organträger bekannt & 1.158 & 6 \\
\hline
\end{tabular}

Quelle: eigene Berechnung

Da der direkte Anteilseigner nicht zwangsweise auch der Organträger sein muss, kann letzterer nicht zweifelsfrei bestimmt werden. Verfügt der direkte Anteilseigner über die geforderte Mindestbeteiligungsquote, wird er im Weiteren als Organträger festgelegt. ${ }^{480}$

Schließlich sind von den 1.164 Organgesellschaften 103 zugleich auch Organträger einer weiteren Organgesellschaft. Um sicherzustellen, dass die Gewinne beider Organgesellschaften dem obersten Organträger im Rahmen der Ermittlung der Bemessungsgrundlage zugerechnet werden, wird beiden Organgesellschaften der oberste Organträger zugeordnet.

\section{b) Steuerlicher Konsolidierungskreis der konsolidierten Körperschaft- steuer-Bemessungsgrundlage}

Eine Mindestbeteiligungsquote von $50 \mathrm{v}$. H. vorausgesetzt, entspricht der steuerliche Konsolidierungskreis der Körperschaftsteuer-Bemessungsgrundlage dem

\footnotetext{
476 Gemäß $§ 14$ Abs. 1 Nr. 2 KStG können auch Einkommensteuersubjekte Organträger sein.

477 Zur Abgrenzung des Vergleichskonzerns vgl. D.V.2.a).

${ }^{478} \S 18 \mathrm{KStG}$ erlaubt den Abschluss einer Organschaft zwischen einer Organgesellschaft und einem ausländischen gewerblichen Unternehmen, das im Inland eine im Handelsregister eingetragene Zweigniederlassung unterhält.

$479 \mathrm{Zu}$ den Aufnahmekriterien der Amadeus-Datenbank vgl. D.III.

${ }^{480}$ Die geforderte Mindestbeteiligungsquote wird lediglich bei einer Organgesellschaft von keinem direkten Anteilseigner erfüllt. Anstelle der direkten Muttergesellschaft gilt hier die Konzernmuttergesellschaft als Organträger.
} 
Vergleichskonzern. ${ }^{481}$ Damit werden im Rahmen der empirischen Analyse folgende Überlegungen des Kapitels C.II.2 umgesetzt: Mitglieder des steuerlichen Konsolidierungskreises können nur Gesellschaften werden, die unter einer im Anhang der Mutter-Tochter-Richtline aufgelisteten Rechtsform firmieren. Personengesellschaften sind damit von der Anwendung der konsolidierten Körperschaftsteuer-Bemessungsgrundlage ausgeschlossen. Die Abgrenzung des Konsolidierungskreises erfolgt ausschließlich nach rechtlichen Kriterien, d. h. nach der Höhe der Beteiligung. Dabei werden sowohl direkt als auch indirekt gehaltene Tochtergesellschaften berücksichtigt, wobei die geforderte Mindestbeteiligung stets auch gegenüber der vermittelnden Gesellschaft bestehen muss. Diese muss außerdem eine qualifizierte Konzerngesellschaft sein. ${ }^{482}$ Territorial wird dem ,at water's edge“-Ansatz gefolgt, der den Konsolidierungskreis auf die Mitgliedstaaten der Europäischen Union begrenzt. ${ }^{483}$ Gegenüber Drittstaaten erfolgt die Gewinnabgrenzung weiterhin direkt mittels fremdvergleichskonformer Verrechnungspreise. Eine Veränderung und Verschiebung der Bemessungsgrundlage kann damit nur innerhalb der Europäischen Union erfolgen, weshalb sich die empirischen Berechnungen auch auf die dort ansässigen Gesellschaften beschränken. ${ }^{484}$

Im Rahmen von Variationsrechnungen wird die Mindestbeteiligungsquote verändert. Sie ist damit die einzige variable Größe des Konsolidierungskreises. Höhere Anforderungen an die Beteiligungsquote lassen dabei den Vergleichskonzern in mehrere steuerliche Konsolidierungskreise zerfallen, was die Verlustverrechnungsmöglichkeiten einschränkt und die Bemessungsgrundlage steigen lässt. ${ }^{485}$

${ }^{481} \mathrm{Vgl}$. für eine ausführliche Darstellung des Vergleichskonzerns und damit des steuerlichen Konsolidierungskreises der konsolidierten Körperschaftsteuer-Bemessungsgrundlage Kapitel D.V.2.

${ }^{482}$ Indirekt, z. B. über eine Personengesellschaft oder eine im nichteuropäischen Ausland ansässige Gesellschaft gehaltene Beteiligungen werden nicht berücksichtigt. Vgl. a. Abbildung 16 .

${ }^{483} \mathrm{Vgl}$. zu den territorialen Abgrenzungskriterien Kapitel C.II.2.b).

${ }^{484}$ Abbildung 11 verdeutlicht die Abgrenzung des steuerlichen Konsolidierungskreises grafisch.

${ }^{485}$ Dies trifft natürlich nur insoweit zu, als dass nicht alle Konzerngesellschaften direkt zu $100 \mathrm{v} . \mathrm{H}$. miteinander verbunden sind und zuvor eine Verlustverrechnung stattfinden konnte, die durch die Anhebung der Mindestbeteiligungsquote verwehrt bleibt. 


\section{E. Analyse der Konzernstrukturen in Europa}

\section{Beschreibung der Konzerne in Europa}

Gemäß der Konzerndefinition in Kapitel D.V.2 können 238.141 Konzerne identifiziert werden, die sich aus 833.650 Konzerngesellschaften zusammensetzen (Tabelle 25).

Tabelle 25: Verteilung der Konzerne und Konzerngesellschaften nach Ländern

\begin{tabular}{|c|rrr|rrr|}
\hline Land & \multicolumn{3}{|c|}{ Konzerne } & \multicolumn{3}{|c|}{ Konzerngesellschaften } \\
& Anzahl & \multicolumn{2}{c|}{ grenzüberschreitend } & Summe & grenzüberschreitend \\
\hline AT & 2.315 & 659 & $28 \%$ & 7.663 & 3.142 & $41 \%$ \\
BE & 11.650 & 1.762 & $15 \%$ & 33.818 & 8.608 & $25 \%$ \\
CY & 16 & 9 & $56 \%$ & 29 & 11 & $38 \%$ \\
CZ & 345 & 68 & $20 \%$ & 1.383 & 711 & $51 \%$ \\
DE & 16.786 & 2.262 & $13 \%$ & 59.710 & 17.688 & $30 \%$ \\
DK & 20.043 & 1.161 & $6 \%$ & 54.949 & 6.789 & $12 \%$ \\
EE & 400 & 66 & $17 \%$ & 1.416 & 624 & $44 \%$ \\
ES & 13.216 & 830 & $6 \%$ & 45.988 & 9.312 & $20 \%$ \\
FI & 2.380 & 317 & $13 \%$ & 8.266 & 2.428 & $29 \%$ \\
FR & 20.245 & 1.658 & $8 \%$ & 74.656 & 20.279 & $27 \%$ \\
GB & 63.250 & 1.863 & $3 \%$ & 296.271 & 54.272 & $18 \%$ \\
GR & 814 & 57 & $7 \%$ & 2.675 & 604 & $23 \%$ \\
HU & 790 & 29 & $4 \%$ & 3.009 & 1.124 & $37 \%$ \\
IE & 877 & 544 & $62 \%$ & 2.273 & 1.340 & $59 \%$ \\
IT & 2.345 & 1.165 & $50 \%$ & 6.778 & 3.908 & $58 \%$ \\
LT & 59 & 11 & $19 \%$ & 225 & 102 & $45 \%$ \\
LU & 30 & 29 & $97 \%$ & 97 & 95 & $98 \%$ \\
LV & 95 & 11 & $12 \%$ & 485 & 293 & $60 \%$ \\
NL & 59.030 & 2.862 & $5 \%$ & 162.586 & 13.384 & $8 \%$ \\
PL & 819 & 54 & $7 \%$ & 4.002 & 1.715 & $43 \%$ \\
PT & 998 & 88 & $9 \%$ & 3.797 & 1.209 & $32 \%$ \\
SE & 21.612 & 1.433 & $7 \%$ & 63.512 & 10.463 & $16 \%$ \\
SI & 23 & 21 & $91 \%$ & 46 & 37 & $80 \%$ \\
SK & 3 & 3 & $100 \%$ & 16 & 16 & $100 \%$ \\
\hline E & 238.141 & 16.962 & $7 \%$ & 833.650 & 158.154 & $19 \%$ \\
\hline
\end{tabular}

Quelle: eigene Berechnungen

Durchschnittlich hält somit eine Konzernmuttergesellschaft 2,5 Tochtergesellschaften. ${ }^{486}$ Nur 7,1 v. H. der Konzerne werden im EU-Ausland mit einer Tochterkapitalgesellschaft aktiv. Mit durchschnittlich 8,3 Gliedgesellschaften sind diese Konzerne erheblich größer als der Durchschnitt aller Unternehmensgruppen. Der Anteil internationaler Konzerne unterscheidet sich zwischen den Mitgliedstaaten und ist in größeren Volkswirtschaften geringer. Zwar ist diese Beobachtung durch die schlechte Datenlage für die kleinen osteuropäischen Mitgliedstaaten verzerrt, entspricht aber den Ergebnissen der Direktinvestitionsforschung, die als Grund für eine Investition im Ausland auch die Erweiterung

$486\left(\frac{833.650-238.141}{238.141}\right)=2,5$. 
des Absatzmarktes anführt. ${ }^{487}$ Die unterschiedliche Größe nationaler und internationaler Konzerne verdeutlicht auch Tabelle 26. Knapp 83 v. H. der nationalen Unternehmensgruppen bestehen aus zwei oder drei Gesellschaften. Bei den vergleichbaren internationalen Konzernen sind es weniger als $50 \mathrm{v} . \mathrm{H}$. Mehr als zehn Gesellschaften hält nur jeder sechste grenzüberschreitende Konzern und gerade mal jeder sechzigste nationale Konzern. Der Vergleich der Verteilung über alle Mitgliedstaaten hinweg zeigt, dass größere Konzerne ihren Sitz vorwiegend in den bedeutenden Volkswirtschaften der Europäischen Union haben. Eine Ausnahme stellt Luxemburg mit einer hohen Anzahl großer Konzerne dar. Sie kann mit dem neben den Niederlanden bevorzugten Standort für EuropaHoldings zusammenhängen. ${ }^{488}$

Tabelle 26: Größe der Konzerne gemessen an der Anzahl der Gesellschaften in v. $H$.

\begin{tabular}{|c|c|c|c|c|c|c|c|c|c|c|c|c|c|c|c|c|c|c|c|c|c|c|c|c|c|c|c|c|c|}
\hline & & 2 & 3 & 4 & & 5 & & 6 & & 7 & & 8 & & 9 & 9 & 10 & & $<1$ & & $<2$ & & $<3$ & & $<5$ & & $<10$ & & $<500$ & $>500$ \\
\hline $\mathrm{AT}$ & 47 & 69 & 2017 & 9 & 5 & 6 & 3 & 5 & 2 & 2 & & 2 & 0 & 1 & 0 & 1 & 0 & 3 & 1 & & & 1 & 0 & & 0 & 1 & & & \\
\hline $\mathrm{BE}$ & 37 & 74 & 1915 & & 5 & 8 & 2 & 5 & 1 & 3 & 1 & 3 & 0 & 2 & 0 & 2 & 0 & 5 & 0 & 2 & 0 & 2 & 0 & 1 & 0 & 1 & 0 & 0 & \\
\hline$Y$ & 56 & 57 & 2229 & & 14 & & & & & & & & & & & & & 11 & & & & & & & & & & & \\
\hline $\mathrm{CZ}$ & 66 & 78 & 1514 & 9 & 5 & 7 & 0 & 1 & 1 & & & & 0 & & 0 & & 0 & & 0 & & & & & & & 1 & & & \\
\hline $\mathrm{E}$ & 37 & 68 & 17 & 11 & 6 & 6 & 3 & 5 & 2 & 4 & 1 & 3 & 1 & 2 & 0 & 2 & 0 & 5 & 1 & 2 & 0 & 3 & 0 & 2 & 0 & 1 & 0 & 10 & \\
\hline DK & 25 & 70 & 1918 & 14 & 6 & 9 & 2 & 5 & 1 & 5 & 1 & 4 & 0 & 2 & 0 & 2 & 0 & 6 & 0 & 3 & 0 & 2 & 0 & 2 & 0 & 1 & 0 & 0 & \\
\hline $\mathrm{EE}$ & 79 & 78 & 1114 & 6 & 3 & & 3 & 3 & 1 & & 0 & & & & & & & 2 & 0 & & & & & & & & & & \\
\hline ES & 28 & 66 & 1617 & 12 & 7 & 8 & 4 & 5 & 2 & 3 & 1 & 3 & 1 & 3 & 1 & 3 & 0 & 7 & 1 & 3 & 0 & 4 & 0 & 3 & 0 & 2 & 0 & 10 & \\
\hline $\mathrm{FI}$ & 30 & 67 & 1418 & 14 & 6 & 10 & 3 & 4 & 2 & 5 & 1 & 11 & 1 & 1 & 1 & 3 & 0 & 6 & 1 & 3 & 0 & 4 & 0 & 3 & 0 & 3 & 0 & 0 & \\
\hline FR & 28 & 63 & 15 & 9 & 8 & 8 & 4 & 6 & 2 & 5 & 1 & 3 & 1 & 2 & 0 & 3 & 0 & 7 & 1 & 4 & 0 & 4 & 0 & 3 & 0 & 2 & 0 & 2 & 0 \\
\hline GB & 25 & 56 & 1019 & 7 & 8 & 6 & 5 & 5 & 3 & 4 & 2 & 3 & 1 & 3 & 1 & 2 & 1 & 7 & 2 & 5 & 1 & 6 & 1 & 6 & 0 & 6 & 0 & $\begin{array}{ll}5 & 0\end{array}$ & 0 \\
\hline GR & 30 & 70 & 14151 & 14 & 7 & 2 & 3 & 5 & 2 & 9 & 1 & 5 & 0 & 4 & 1 & 4 & 0 & 11 & 0 & & 0 & 2 & & 2 & 0 & & & & \\
\hline $\mathrm{HU}$ & 59 & 78 & 1013 & 7 & 4 & & 1 & 3 & 1 & 3 & 0 & 3 & 0 & & 0 & 7 & 0 & 3 & 1 & 3 & 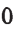 & & 0 & & & & & & \\
\hline IE & 65 & 68 & 1517 & 7 & 8 & 4 & 3 & 3 & 2 & 1 & 0 & 1 & 1 & 1 & 0 & 0 & 0 & 1 & 1 & 0 & & 1 & 1 & 0 & & 1 & & 0 & \\
\hline IT & 58 & 79 & 1713 & 9 & 4 & 4 & 1 & 2 & 1 & 1 & 1 & 2 & 0 & 1 & 0 & 1 & 0 & 2 & 0 & 0 & 0 & 0 & 0 & 1 & 0 & 1 & & 0 & \\
\hline LT & 73 & 75 & 9151 & 18 & 4 & & 2 & & 2 & & & & & & & & & & 2 & & & & & & & & & & \\
\hline LU & 34 & 100 & 21 & 10 & & 7 & & 3 & & 3 & & & & 3 & & 3 & & 3 & & 3 & & 3 & & & & & & 3 & \\
\hline LV & 73 & 81 & 1811 & 9 & 7 & & 1 & & & & & & & & & & & & & & & & & & & & & & \\
\hline NL & 25 & 65 & 16201 & 13 & 8 & 10 & 3 & 7 & 1 & 5 & 1 & 4 & 0 & 3 & 0 & 2 & 0 & 7 & 0 & 3 & 0 & 2 & 0 & 2 & 0 & & 0 & 0 & \\
\hline PL & 46 & 67 & 1915 & 7 & 6 & 6 & 4 & & 2 & & 2 & 6 & 1 & 7 & 1 & & 1 & 4 & 1 & 4 & 1 & 2 & 0 & & 0 & & & & \\
\hline PT & 35 & 66 & 15171 & 11 & 6 & 9 & 3 & 3 & 4 & 2 & 2 & 3 & 1 & 5 & 1 & 1 & 1 & 2 & 1 & 6 & 0 & 3 & & 2 & & & & 1 & \\
\hline SE & 18 & & 20171 & 12 & 6 & 8 & 3 & 6 & 1 & 4 & 1 & 4 & 0 & 3 & 0 & 2 & 0 & 7 & 0 & 4 & 0 & 5 & 0 & 3 & 0 & & 0 & 1 & \\
\hline SI & 76 & 50 & 10 & 14 & & & & & & & 50 & & & & & & & & & & & & & & & & & & \\
\hline SK & 33 & & 33 & & & & & & & 33 & & & & & & & & & & & & & & & & & & & \\
\hline EU & 33 & 64 & $\begin{array}{lllll}6 & 18\end{array}$ & 11 & 7 & 7 & 3 & 5 & 2 & 4 & 1 & 31 & 1 & 2 & 1 & 2 & 0 & 6 & 1 & 3 & 0 & 3 & 0 & 2 & 0 & 2 & \begin{tabular}{l|l} 
\\
\end{tabular} & 10 & $\begin{array}{ll}0 & 0 \\
\end{array}$ \\
\hline
\end{tabular}

Quelle: eigene Berechnungen

Die Verteilung der Konzerne nach Anzahl der Konzerngesellschaften erlaubt nur bedingt Rückschlüsse auf die Verteilung der Bemessungsgrundlage. Grundsätzlich harmoniert aber die in Abbildung 17 dargestellte Lorenzkurve mit

$487 \mathrm{Vgl}$. zu den unterschiedlichen Erklärungsansätzen von Direktinvestitionen Heiduk, G./Kerlen-Prinz, J., in: Döhrn/Heiduk, Direktinvestitionen, 1999, S. 23-54.

${ }^{488}$ Die stark verschobene Verteilung für die Slowakei ist auf die geringe Grundgesamtheit von nur drei Konzernen zurückzuführen. Vgl. auch Tabelle 25. 
den vorherigen Beobachtungen. So erwirtschaften in der Europäischen Union nur $20 \mathrm{v}$. H. der Konzerne fast die gesamte EU-Bemessungsgrundlage. Diese enorme Ungleichverteilung drückt sich auch in dem Gini-Koeffizienten von 0,937 aus. ${ }^{489}$ In Wirklichkeit dürfte dieser aber geringer ausfallen, da die Angaben auf einer einperiodigen Betrachtung beruhen und damit Konzerne miteinschließen, die in der entsprechenden Periode einen Verlust ausweisen, langfristig aber Gewinne erwirtschaften. ${ }^{490}$ Jedoch ist auch der Gini-Koeffizient der im Betrachtungszeitraum ertragreichen Konzerne mit 0,905 sehr hoch.

Abbildung 17: Verteilung der Bemessungsgrundlage auf die Konzerne - Lorenzkurve
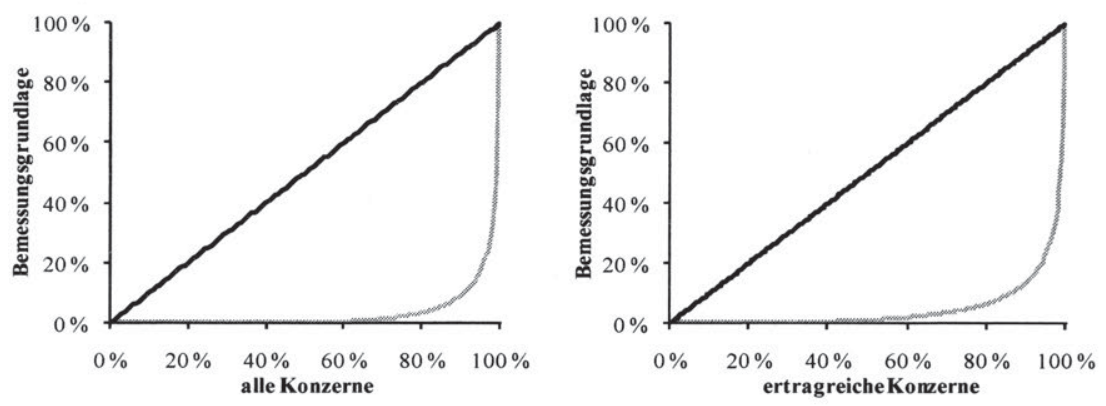

Quelle: eigene Berechnungen

Die Mehrzahl (58 v. H.) der Konzerngesellschaften werden direkt zu 100 v. H. von einer oder mehreren anderen Konzerngesellschaften gehalten. ${ }^{491}$ Eine Beteiligungshöhe von $98 \mathrm{v}$. H. wird bereits bei $87 \mathrm{v}$. H. der Konzerngesellschaften erreicht. ${ }^{492}$ Dieser starke Anstieg erklärt auch die hohe durchschnittliche Beteiligung in der Europäischen Union von über 95 v. H. Zwischen den Mitgliedstaaten zeigen sich kaum Unterschiede. In 18 der 24 betrachteten Ländern liegt die durchschnittliche Beteiligungshöhe bei über 90 v. H. Lediglich in Frankreich ist die durchschnittliche Beteiligungshöhe mit weniger als $80 \mathrm{v}$. H. sehr niedrig, was auf einen unverhältnismäßig hohen Anteil von Beteiligungen von knapp

489 Die Berechnung basiert ausschließlich auf den grenzüberschreitend tätigen Konzernen, die für über $50 \mathrm{v}$. H. der Konzerngesellschaften einen Jahresabschluss hinterlegt haben.

490 Die Bemessungsgrundlage wurde gleich null gesetzt, sofern die Konzerne in dem Betrachtungszeitraum einen Verlust erzielt haben.

491 In den meisten Fällen handelt es sich bei dem Anteilseigner um die Konzernmuttergesellschaft.

492 Dieser starke Anstieg zwischen 98 und 100 v. H. ist auf die Anpassung der Beteiligungsinformationen zurückzuführen, in deren Rahmen die Information „WO“ stets mit $98 \mathrm{v} . \mathrm{H}$. übersetzt wurde. Vgl. hierzu Kapitel D.IV.1. 
über 50 v. H. zurückzuführen ist. ${ }^{493}$ Auch die multiplikativ durchgerechneten Beteiligungshöhen liegen in der Mehrzahl der Mitgliedstaaten (17) deutlich über $90 \mathrm{v}$. H. Dieses ist bereits ein erster Hinweis darauf, dass die Beteiligungsketten kurz sind.

Tabelle 27: Summe der von anderen Konzerngesellschaften direkt an einer Konzerngesellschaft gehaltenen Beteiligung in v. $\mathbf{H}$.

\begin{tabular}{|c|c|c|c|c|c|c|c|c|c|c|c|c|}
\hline$>=$ & 55 & 60 & 65 & 70 & 75 & 80 & 85 & 90 & 95 & 100 & Durchschnitt & Standardabweichung \\
\hline AT & 3,80 & 2,24 & 1,16 & 2,30 & 3,53 & 2,77 & 1,05 & 2,90 & 2,00 & 78,25 & 93,94 & 13,06 \\
\hline $\mathrm{BE}$ & 2,80 & 1,93 & 1,27 & 2,52 & 2,70 & 2,86 & 1,64 & 3,32 & 3,81 & 77,17 & 94,01 & 12,19 \\
\hline CY & 30,77 & 15,38 & 15,38 & 7,69 & 15,38 & 0,00 & 0,00 & 0,00 & 0,00 & 15,38 & 66,08 & 17,00 \\
\hline $\mathrm{CZ}$ & 6,26 & 2,89 & 1,35 & 4,05 & 3,56 & 2,79 & 2,02 & 3,28 & 1,73 & 72,06 & 91,58 & 15,18 \\
\hline $\mathrm{DE}$ & 4,66 & 2,06 & 0,87 & 2,50 & 2,82 & 2,59 & 0,96 & 2,46 & 2,17 & 78,91 & 93,91 & 13,44 \\
\hline DK & 2,13 & 1,40 & 0,74 & 1,73 & 2,20 & 1,02 & 0,50 & 0,93 & 0,58 & 88,77 & 96,58 & 10,48 \\
\hline $\mathrm{EE}$ & 5,81 & 2,76 & 1,18 & 2,95 & 2,56 & 3,44 & 2,46 & 3,84 & 2,07 & 72,93 & 92,28 & 14,58 \\
\hline ES & 4,30 & 3,27 & 1,75 & 3,39 & 2,87 & 2,87 & 1,87 & 3,06 & 2,57 & 74,04 & 92,59 & 14,15 \\
\hline FI & 2,97 & 2,57 & 1,46 & 2,45 & 1,46 & 2,14 & 0,76 & 2,87 & 2,07 & 81,24 & 94,75 & 12,43 \\
\hline FR & 36,11 & 1,73 & 0,98 & 1,70 & 1,25 & 1,62 & 0,81 & 1,50 & 2,84 & 51,47 & 79,36 & 22,80 \\
\hline GB & 0,06 & 0,11 & 0,25 & 0,73 & 0,65 & 0,59 & 0,78 & 0,07 & 0,02 & 96,75 & 97,94 & 4,80 \\
\hline GR & 12,74 & 6,29 & 3,39 & 8,22 & 4,46 & 4,67 & 3,55 & 5,16 & 4,35 & 47,18 & 83,63 & 18,19 \\
\hline $\mathrm{HU}$ & 4,87 & 2,48 & 1,58 & 4,42 & 3,56 & 2,97 & 1,67 & 5,68 & 2,61 & 70,17 & 91,83 & 14,27 \\
\hline IE & 0,36 & 0,07 & 0,43 & 0,93 & 0,21 & 1,29 & 0,21 & 0,14 & 0,29 & 96,06 & 98,85 & 5,76 \\
\hline IT & 5,35 & 2,96 & 1,11 & 3,18 & 2,41 & 2,84 & 1,38 & 2,91 & 3,52 & 74,35 & 92,70 & 14,31 \\
\hline LT & 8,43 & 4,22 & 1,81 & 4,82 & 3,61 & 5,42 & 1,81 & 2,41 & 2,41 & 65,06 & 89,06 & 16,71 \\
\hline LU & 1,49 & 0,00 & 2,99 & 0,00 & 4,48 & 0,00 & 0,00 & 4,48 & 0,00 & 86,57 & 96,32 & 10,08 \\
\hline LV & 6,67 & 1,79 & 1,28 & 4,62 & 2,31 & 1,79 & 1,03 & 1,28 & 2,05 & 77,18 & 92,71 & 14,91 \\
\hline NL & 0,10 & 0,08 & 0,04 & 0,11 & 0,10 & 0,07 & 0,03 & 0,07 & 0,05 & 99,34 & 99,81 & 2,56 \\
\hline PL & 4,93 & 2,14 & 1,60 & 2,76 & 2,17 & 2,80 & 2,45 & 2,23 & 1,95 & 76,97 & 93,17 & 13,94 \\
\hline PT & 6,29 & 5,47 & 2,18 & 5,89 & 5,68 & 5,54 & 2,57 & 5,64 & 4,61 & 56,13 & 88,01 & 16,04 \\
\hline SE & 1,11 & 0,74 & 0,29 & 0,87 & 0,59 & 0,49 & 0,22 & 0,35 & 1,65 & 93,69 & 98,30 & 7,50 \\
\hline SI & 0,00 & 0,00 & 0,00 & 0,00 & 4,35 & 8,70 & 8,70 & 8,70 & 4,35 & 65,22 & 94,07 & 9,37 \\
\hline SK & 23,08 & 0,00 & 0,00 & 0,00 & 0,00 & 0,00 & 0,00 & 0,00 & 0,00 & 76,92 & 88,65 & 20,63 \\
\hline $\mathrm{EU}$ & 4,47 & 0,89 & 0,54 & 1,25 & 1,16 & 1,09 & 0,74 & 0,89 & 0,99 & 87,97 & 95,51 & 11,79 \\
\hline
\end{tabular}

Quelle: eigene Berechnungen

Tabelle 28: Länge der Beteiligungsketten

\begin{tabular}{|l|rrr|rrr|}
\hline & \multicolumn{3}{|c|}{ nationaler } & internationaler & \multicolumn{1}{|c|}{ nationaler internationaler } & $\Sigma$ \\
& Konzern & Konzern & \multicolumn{1}{c|}{ Konzern } & Konzern & \multicolumn{1}{c|}{} \\
\hline Direkt gehalten & 360.811 & 58.450 & 419.261 & $79,4 \%$ & $41,4 \%$ & $70,4 \%$ \\
Eine Zwischengesellschaft & 67.975 & 40.994 & 108.969 & $15,0 \%$ & $29,0 \%$ & $18,3 \%$ \\
Zwei Zwischengesellschaften & 16.258 & 22.149 & 38.407 & $3,6 \%$ & $15,7 \%$ & $6,4 \%$ \\
Drei Zwischengesellschaften & 5.332 & 10.484 & 15.816 & $1,2 \%$ & $7,4 \%$ & $2,7 \%$ \\
Vier und mehr Zwischengesellschaften & 3.941 & 9.115 & 13.056 & $0,9 \%$ & $6,5 \%$ & $2,2 \%$ \\
\hline
\end{tabular}

Quelle: eigene Berechnungen

Die Tabelle 28 bestätigt obigen Hinweis. ${ }^{494}$ Fast 90 v. H. der Tochtergesellschaften werden direkt von der Konzernmuttergesellschaft oder über eine

493 Die Beteiligungshöhen zyprischer Gesellschaften sind aufgrund der geringen Grundgesamtheit von 13 Unternehmen nicht aussagekräftig.

494 Werden lediglich Tochtergesellschaften deutscher Konzerne im Ausland betrachtet, die keine Holding (NACE 7415) sind, entsprechen die Ergebnisse denen von Weichenrieder 
Zwischengesellschaft gehalten. Jedoch unterscheiden sich nationale und internationale Konzerne wesentlich voneinander, was auf die größere Zahl an Tochtergesellschaften grenzüberschreitend tätiger Konzerne zurückzuführen ist (Tabelle 25).

Tabelle 29: Anzahl der Grenzübertritte ${ }^{495}$

\begin{tabular}{|c|cccc|}
\hline Grenzübertritte & 1 & 2 & 3 & 4 und mehr \\
\hline AT & $97,5 \%$ & $2,5 \%$ & & \\
BE & $95,0 \%$ & $4,7 \%$ & $0,3 \%$ & \\
DE (Bundesbank) & $94,8 \%(87,6 \%)$ & $5,1 \%(11,3 \%)$ & $0,1 \%(1,0 \%)$ & $0,0 \%(0,2 \%)$ \\
DK & $92,9 \%$ & $7,0 \%$ & $0,1 \%$ & \\
ES & $94,8 \%$ & $5,1 \%$ & $0,1 \%$ & \\
FI & $90,8 \%$ & $9,0 \%$ & $0,2 \%$ & \\
FR & $91,1 \%$ & $8,3 \%$ & $0,7 \%$ & \\
GB & $89,8 \%$ & $9,9 \%$ & $0,3 \%$ & \\
HU & $95,3 \%$ & $4,7 \%$ & & \\
IE & $90,1 \%$ & $9,9 \%$ & & \\
IT & $91,0 \%$ & $8,8 \%$ & $0,2 \%$ & \\
LT & $92,9 \%$ & $7,1 \%$ & & \\
LU & $70,8 \%$ & $27,4 \%$ & $1,8 \%$ & \\
NL & $94,2 \%$ & $5,5 \%$ & $0,2 \%$ & \\
PL & $95,6 \%$ & $4,4 \%$ & & \\
PT & $95,9 \%$ & $4,1 \%$ & & \\
SE & $90,4 \%$ & $9,3 \%$ & $0,2 \%$ & \\
SK & $88,9 \%$ & $11,1 \%$ & & \\
\hline EU & $92,7 \%$ & $7,0 \%$ & $0,3 \%$ & $0,0 \%$ \\
\hline
\end{tabular}

Quelle: eigene Berechnungen; Weichenrieder, A.Mintz, J.M., Ownerhip, 2006, S. 11

Obwohl nahezu $60 \mathrm{v}$. H. der Konzerngesellschaften internationaler Konzerne über eine Zwischengesellschaft gehalten werden, erfolgt innerhalb der Beteiligungsketten nur selten mehr als ein Grenzübertritt. Über 92 v. H. der ausländischen Konzerngesellschaften sind Bestandteil eines nationalen Teilkonzerns, der direkt von einer Gesellschaft mit Sitz im Stammland des internationalen Konzerns gehalten wird (Tabelle 29). Dies ist nicht verwunderlich, sind doch innerhalb der Europäischen Union durch die Mutter-TochterRichtlinie vielfach die Gründe für das Umleiten von Zahlungsströmen entfallen, da keine Quellensteuern mehr auf Gewinnausschüttungen erhoben werden. Damit ist für innereuropäische Sachverhalte die Etablierung von Zwischengesellschaften in einem Drittland im Rahmen eines „treaty shopping“ nicht mehr

und Mintz, die eine vergleichbare Analyse auf der Basis der Direktinvestitionsstatistik der Deutschen Bundesbank durchgeführt haben. Vgl. Wiechenrieder, A./Mintz, J.M., Ownerhip, 2006, S. 10; Tabelle 60 im Anhang. Die geringen Abweichungen können auf die besonderen Dokumentationspflichten der Direktinvestitionsstatistik zurückgeführt werden, wodurch Beteiligungsketten teilweise abgeschnitten werden. Vgl. Lipponer, A., in: Herrmann/Lipsey, Direct Investment, 2003, S. 216-218.

495 Nur Länder mit internationalen Konzernen, die mehr als einen Grenzübertritt aufweisen, sind abgebildet. Die Zeile „EU“ bezieht alle grenzüberschreitenden Konzerne mit ein. 
notwendig. ${ }^{496}$ Diese Beobachtung passt auch zu den empirischen Ergebnissen von Weichenrieder und Mintz, die die weltweiten Direktinvestitionen deutscher Konzerne analysieren und deutlich mehr Grenzübertritte innerhalb der $\mathrm{Be}$ teiligungsketten feststellen, obwohl die von ihnen betrachteten Unternehmensgruppen aufgrund der Dokumentationspflichten der deutschen Konzerne unvollständig abgebildet sind. ${ }^{497}$

Bisher wurde ausschließlich der statutarische Konzernaufbau beschrieben, nicht aber die Tätigkeit der Konzerne. Tabelle 30 zeigt die Verteilung der Konzernbranchen. Auf den Handel entfallen mit 21 v. H. die meisten Konzerne. Darauf folgen Konzerne zur Herstellung von Waren (18 v. H.) und Dienstleistungskonzerne (14 v. H.).

Der Vergleich zwischen nationalen und internationalen Konzernen verdeutlicht, dass die zuvor beschriebene Reihenfolge für internationale Konzerne nicht zutrifft. Zwar steigt sowohl der Anteil der Handelskonzerne als auch der Konzerne zur Herstellung von Waren an, doch ist die Veränderung für das verarbeitende Gewerbe größer, wodurch sich die Reihenfolge vertauscht. Mehr als jeder dritte grenzüberschreitend tätige Konzern ist diesem Gewerbe zuzuordnen, wobei der Anteil der internationalen Konzerne in den kapitalintensiven Branchen wie der Herstellung von chemischen Erzeugnissen (26 v. H.), der Mineralölverarbeitung (24 v. H.) und dem Maschinenbau (22 v. H.) am höchsten ist. Unter den Handelskonzernen ist vornehmlich der Großhandel grenzüberschreitend aktiv. Demgegenüber werden Konzerne des Baugewerbes sowie des Beherbergungsund Gaststättengewerbes selten mit einer Tochterkapitalgesellschaft im europäischen Ausland aktiv.

Werden die Sitze der einzelnen Gesellschaften und deren Funktionen einander gegenübergestellt, können Unterschiede zwischen den Mitgliedstaaten festgestellt werden (Tabelle 31). Während der Anteil der Dienstleistungskonzerne aller Unternehmensgruppen in den Mitgliedstaaten noch weitgehend gleich groß ist, zeigen sich bei Konzernen des verarbeitenden Gewerbes standortspezifische Unterschiede zwischen den Mitgliedstaaten. So sind Produktionsgesellschaften vermehrt in der Tschechischen Republik, Ungarn, Italien, Polen, Portugal, Slowenien und der Slowakei vorzufinden. Gleichzeitig zeichnen sich diese Länder durch ein vergleichsweise niedriges Lohnniveau aus. ${ }^{498}$

\footnotetext{
496 Auch Panayi geht in ihrem Beispiel für „treaty shopping“ innerhalb der Europäischen Union von einem Verfehlen der Anwendungsvoraussetzungen der Mutter-TochterRichtlinie aus. Gemäß der Konzerndefinition trifft das für keine der Gesellschaften zu. Vgl. Panayi, C., in: ET, 2006, S. 139.

$497 \mathrm{Vgl}$. Weichenrieder, A./Mintz, J.M., Ownerhip, 2006, S. 11. Zu den Dokumentationspflichten vgl. Lipponer, A., in: Herrmann/Lipsey, Direct Investment, 2003, S. 216-218. Siehe auch Tabelle 54 im Anhang.

498 Für eine Übersicht über das Lohnniveau in der Europäischen Union vgl. Abbildung 34.
} 
Tabelle 30: Verteilung der Konzernbranche ${ }^{499}$

\begin{tabular}{|c|c|c|c|c|c|}
\hline \multirow{2}{*}{$\begin{array}{l}\text { NACE - Abschnitt } \\
\text { Bau }\end{array}$} & \multicolumn{2}{|c|}{$\begin{array}{l}\text { nationale } \\
\text { Konzeme }\end{array}$} & \multicolumn{2}{|c|}{$\begin{array}{l}\text { internationale } \\
\text { Konzerne }\end{array}$} & \multirow{2}{*}{$\begin{array}{l}\Sigma \\
8 \%\end{array}$} \\
\hline & $7,9 \%$ & 17.396 & $3,0 \%$ & 517 & \\
\hline Beherbergungs- und Gaststätten & $2,3 \%$ & 5.037 & $0,9 \%$ & 152 & $2 \%$ \\
\hline Bergbau und Gewinnung v & $0,3 \%$ & 693 & $0,6 \%$ & 110 & $0 \%$ \\
\hline Energie- und Wasse & $0,4 \%$ & 854 & $0,5 \%$ & 83 & $0 \%$ \\
\hline lichen und persönlichen Dienstleistungen & $4,0 \%$ & 8.933 & $2,4 \%$ & 399 & $4 \%$ \\
\hline Erziehung und Unterricht & $0,7 \%$ & 1.501 & $0,3 \%$ & 43 & $1 \%$ \\
\hline Exten & e $11,3 \%$ & 25.093 & $6,7 \%$ & 1.144 & $11 \%$ \\
\hline Fisch & $0,1 \%$ & 285 & $0,2 \%$ & 28 & $0 \%$ \\
\hline Gesundheits-, Veterinär- und Sozialwesen & $1,4 \%$ & 3.022 & $0,3 \%$ & 54 & $1 \%$ \\
\hline $\begin{array}{l}\text { Grundstücks- und Wohnungswesen, Vermietung beweglicher } \\
\text { Erbringung von unternehmensbezogenen Dienstleistungen }\end{array}$ & $13,8 \%$ & 30.56 & $10,5^{\circ}$ & 1.788 & 14 \\
\hline Sonstige & $0,0 \%$ & 20 & $0,0 \%$ & 1 & $0 \%$ \\
\hline Daten & $3,6 \%$ & 7.951 & $5,8 \%$ & 981 & $4 \%$ \\
\hline Forscl & $0,4 \%$ & & $0,5 \%$ & 93 & $0 \%$ \\
\hline $\begin{array}{l}\text { Grundstücks- und Wohnungswesen/Erbringung von unternehmensbe- } \\
\text { zogenen Dienstleistungen }\end{array}$ & 9,1 & 20.225 & $3,4 \%$ & 573 & $9 \%$ \\
\hline Sachen ohne Bedienungsnersonal & $0,7 \%$ & 1.569 & $0,8 \%$ & 140 & $1 \%$ \\
\hline $\begin{array}{l}\text { andhaltung und Reparatur von Krafttahrzeugen un } \\
\text { m }\end{array}$ & 20 & 4 & 24 & 4.146 & 21 \\
\hline Sonstige & $0,0 \%$ & 70 & $0,1 \%$ & 20 & $0 \%$ \\
\hline $\begin{array}{l}\text { Einzelhandel (ohne Handel mit Kraftfahrzeugen und ohne Tank- } \\
\text { stellen); Reparatur von Gebrauchsgütern }\end{array}$ & $5,7 \%$ & 12.534 & $3,0 \%$ & 504 & 5 \\
\hline hne Handel mit Kfz) & $12,0 \%$ & 26.517 & $20,0 \%$ & 3.393 & $13 \%$ \\
\hline Kra & $3,1 \%$ & 6.840 & & 229 & $3 \%$ \\
\hline Hers & $16,4 \%$ & 36.347 & $38,0 \%$ & 5.438 & $18 \%$ \\
\hline Sons & $0,0 \%$ & 77 & $0,1 \%$ & 16 & $0 \%$ \\
\hline Fahr & $0,6 \%$ & 1.254 & $1,6 \%$ & 275 & $1 \%$ \\
\hline $\begin{array}{l}\text { Herstellung von Büromaschinen, Datenverarbeitungsgeräten und } \\
\text {-einrichtungen; Elektrotechnik, Feinmechanik und Optik }\end{array}$ & $1,7 \%$ & 3.665 & $5,8 \%$ & 986 & 2 \\
\hline en Erzeugnissen & $0,8 \%$ & 1.806 & $3,7 \%$ & 630 & $1 \%$ \\
\hline $\begin{array}{l}\text { Herstellung von Glas und Glaswaren, Keramik, Verarbeitung von Steinen } \\
\text { und Erden }\end{array}$ & $0,6 \%$ & 1.378 & $\%$ & 246 & $1 \%$ \\
\hline 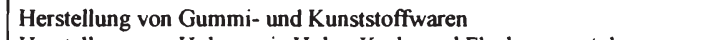 & $0,9 \%$ & 1.896 & $2,7 \%$ & 460 & $1 \%$ \\
\hline $\begin{array}{l}\text { Herstellung } \\
\text { Möbeln) }\end{array}$ & $6 \%$ & 1.337 & c & 129 & \\
\hline Herstellung $v$ & $0,1 \%$ & 268 & $0,3 \%$ & 47 & $0 \%$ \\
\hline $\begin{array}{l}\text { Schmuck, Musikinstrumenten, Sportgerăten, } \\
\text { Erzeugnissen; Rückgewinnung }\end{array}$ & 1 & .904 & 1 & 326 & \\
\hline Issmitteln, Tabakverarbeitung & 1, & 3.272 & & 537 & $2 \%$ \\
\hline $\mathbf{H}$ & $2,5 \%$ & 5.523 & & 494 & $3 \%$ \\
\hline sekleidung & $0,9 \%$ & 2.075 & $2,3 \%$ & 387 & $1 \%$ \\
\hline ineralölverarbeitung & $0,0 \%$ & 81 & $0,2 \%$ & 26 & $0 \%$ \\
\hline & $1,7 \%$ & 3.758 & & 1.056 & $2 \%$ \\
\hline $\mathrm{Me}$ & $3,2 \%$ & 7.053 & 4 & 823 & $3 \%$ \\
\hline (ohne Sozi & $15,0 \%$ & 33.159 & $5,9 \%$ & 997 & $14 \%$ \\
\hline & $1,2 \%$ & 2.755 & $0,5 \%$ & 89 & $1 \%$ \\
\hline Uar & $0,1 \%$ & 119 & $0,0 \%$ & 4 & \\
\hline shalte & $0,0 \%$ & 40 & $0,0 \%$ & 3 & $0 \%$ \\
\hline Verkehr ur & $4,3 \%$ & 9.419 & $5,7 \%$ & 967 & $4 \%$ \\
\hline Gesamtergebnis & $100 \%$ & 221.179 & $100 \%$ & 96 & 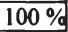 \\
\hline
\end{tabular}

Quelle: eigene Berechnungen

499 Für Erläuterungen zur Ermittlung der Konzernbranche vgl. Kapitel D.V.2.c). 
Die Dienstleistungsgesellschaften folgen einem entgegengesetzten Trend. Ihr Anteil ist besonders in Deutschland, Finnland, Großbritannien und Schweden sehr hoch. Kein eindeutiger Trend ist für die Handelsgesellschaften zu beobachten. Sie folgen vorrangig den zu bedienenden Märkten. Entsprechend gering fällt die Ungleichverteilung zwischen den Mitgliedstaaten aus.

Tabelle 31: Gesellschaften nach Funktionen ${ }^{500}$

\begin{tabular}{|c|crrrrrr|}
\hline Land & $\begin{array}{r}\text { Herstellung } \\
\text { von Waren }\end{array}$ & Handel & $\begin{array}{r}\text { Dienst- } \\
\text { leistung }\end{array}$ & Holding & Kreditwesen & Sonstige & k. A. \\
\hline AT & $22,2 \%$ & $34,3 \%$ & $15,8 \%$ & $13,8 \%$ & $0,7 \%$ & $13,2 \%$ & $0,2 \%$ \\
BE & $25,3 \%$ & $24,3 \%$ & $18,5 \%$ & $5,2 \%$ & $5,9 \%$ & $19,9 \%$ & $0,9 \%$ \\
CY & $9,1 \%$ & $9,1 \%$ & $0,0 \%$ & $0,0 \%$ & $9,1 \%$ & $72,7 \%$ & $0,0 \%$ \\
CZ & $43,2 \%$ & $27,3 \%$ & $11,1 \%$ & $0,3 \%$ & $1,1 \%$ & $17,0 \%$ & $0,0 \%$ \\
DE & $25,7 \%$ & $24,5 \%$ & $20,1 \%$ & $15,8 \%$ & $0,6 \%$ & $13,3 \%$ & $0,1 \%$ \\
DK & $18,0 \%$ & $23,4 \%$ & $11,5 \%$ & $12,3 \%$ & $12,6 \%$ & $16,0 \%$ & $6,2 \%$ \\
EE & $32,2 \%$ & $37,8 \%$ & $9,0 \%$ & $1,9 \%$ & $1,6 \%$ & $17,0 \%$ & $0,5 \%$ \\
ES & $31,4 \%$ & $23,5 \%$ & $15,1 \%$ & $2,9 \%$ & $2,1 \%$ & $24,8 \%$ & $0,2 \%$ \\
FI & $28,2 \%$ & $27,2 \%$ & $19,6 \%$ & $4,1 \%$ & $3,0 \%$ & $18,0 \%$ & $0,0 \%$ \\
FR & $28,3 \%$ & $23,1 \%$ & $14,9 \%$ & $9,5 \%$ & $3,3 \%$ & $20,6 \%$ & $0,3 \%$ \\
GB & $13,9 \%$ & $9,0 \%$ & $20,6 \%$ & $5,8 \%$ & $2,4 \%$ & $12,3 \%$ & $36,0 \%$ \\
GR & $32,5 \%$ & $32,5 \%$ & $14,2 \%$ & $1,8 \%$ & $0,3 \%$ & $18,7 \%$ & $0,0 \%$ \\
HU & $39,8 \%$ & $30,4 \%$ & $9,2 \%$ & $0,6 \%$ & $0,1 \%$ & $14,9 \%$ & $5,0 \%$ \\
IE & $10,2 \%$ & $6,6 \%$ & $7,0 \%$ & $2,9 \%$ & $3,2 \%$ & $3,3 \%$ & $66,8 \%$ \\
IT & $53,9 \%$ & $21,1 \%$ & $10,6 \%$ & $1,0 \%$ & $1,7 \%$ & $10,6 \%$ & $1,1 \%$ \\
LT & $31,4 \%$ & $20,6 \%$ & $2,9 \%$ & $0,0 \%$ & $2,0 \%$ & $13,7 \%$ & $29,4 \%$ \\
LU & $34,7 \%$ & $17,9 \%$ & $6,3 \%$ & $5,3 \%$ & $0,0 \%$ & $17,9 \%$ & $17,9 \%$ \\
LV & $24,6 \%$ & $50,2 \%$ & $11,3 \%$ & $0,3 \%$ & $0,7 \%$ & $13,0 \%$ & $0,0 \%$ \\
NL & $20,2 \%$ & $21,0 \%$ & $11,9 \%$ & $2,2 \%$ & $28,0 \%$ & $16,7 \%$ & $0,0 \%$ \\
PL & $49,1 \%$ & $29,6 \%$ & $7,1 \%$ & $0,3 \%$ & $1,0 \%$ & $12,8 \%$ & $0,1 \%$ \\
PT & $36,8 \%$ & $25,8 \%$ & $10,4 \%$ & $8,8 \%$ & $0,3 \%$ & $17,9 \%$ & $0,0 \%$ \\
SE & $20,3 \%$ & $20,0 \%$ & $18,9 \%$ & $5,4 \%$ & $1,9 \%$ & $17,3 \%$ & $16,3 \%$ \\
SI & $64,9 \%$ & $21,6 \%$ & $2,7 \%$ & $8,1 \%$ & $0,0 \%$ & $2,7 \%$ & $0,0 \%$ \\
SK & $37,5 \%$ & $31,3 \%$ & $18,8 \%$ & $0,0 \%$ & $0,0 \%$ & $6,3 \%$ & $6,3 \%$ \\
\hline EU & $22,2 \%$ & $18,5 \%$ & $17,2 \%$ & $7,0 \%$ & $4,9 \%$ & $15,6 \%$ & $14,5 \%$ \\
\hline
\end{tabular}

Quelle: eigene Berechnungen

Die Verteilung der Holdinggesellschaften lässt zunächst keine direkten Rückschlüsse zu. Hier sei angemerkt, dass auf der Basis des NACE Code keine Aussage über die einer Holding zugrunde liegende Intention möglich ist. Eine Vielzahl der Holdinggesellschaften kann also nicht auf steuerliche Gestaltungen zurückgeführt werden, sondern ist das Ergebnis einer diversifizierten Konzernorganisation. Dies in Verbindung mit einer möglichen Verzerrung aufgrund der kleinen Grundgesamtheit kann den vergleichsweise geringen Anteil an Holdinggesellschaften in Luxemburg erklären. ${ }^{501}$ Auch in den Niederlanden ist der An-

500 Die Zuordnung der Gesellschaften erfolgt nach dem NACE Code. Als Holdinggesellschaften gelten alle Unternehmen des Wirtschaftszweiges 7415. Zum Kreditwesen zählen Unternehmen, deren NACE Code mit 65 oder 671 beginnt, wobei Banken und Versicherungen nicht Bestandteil der Amadeus-Datenbank sind.

${ }^{501}$ Da nur elf luxemburgische Konzerne grenzüberschreitend tätig werden, steht diese Beobachtung nicht im Gegensatz zu der obigen Vermutung, dass die hohe Anzahl großer 
teil mit 2,2 v. H. erstaunlich niedrig. Doch ist wohl auch dieses Ergebnis verzerrt, was die folgende Spalte verdeutlicht. Über $28 \mathrm{v}$. H. der niederländischen Gesellschaften sind dem Kreditwesen zuzuordnen. Die nähere Analyse zeigt, dass diese Gesellschaften fast ausschließlich dem NACE Code 6523 (Finanzierungsinstitutionen a.n. g.) zugeordnet sind. Dass diese Gesellschaften auch Holdingfunktionen ausüben, ${ }^{502}$ bestätigt die Analyse der außereuropäischen Direktinvestitionen in die Niederlande. Sie erfolgen in der Mehrzahl (57,92 v. H.) über eine europäische Konzernmuttergesellschaft, die den NACE Code 6523 trägt. $^{503}$ Dass die Anzahl dieser Gesellschaften derart umfangreich ist, hängt auch damit zusammen, dass außereuropäische Investoren die Niederlande als Standort für eine Europa-Holdinggesellschaft bevorzugen (Tabelle 32). So liegen die Niederlande bei einem Vergleich der Summe der Bilanzsummen von nicht im Stammland der europäischen Konzernmuttergesellschaft ansässigen Tochtergesellschaften europäischer Konzerne, deren europäische Konzernmuttergesellschaft direkt mehrheitlich von einem außereuropäischen Anteilseigner gehalten wird, an vorderster Stelle.

Die allgemeine Beschreibung der auf der Basis der Amadeus-Datenbank nachgebildeten Konzerne macht auch standortspezifische Unterschiede im Konzernaufbau deutlich. Dies kommt insbesondere in Tabelle 31 Die Dienstleistungsgesellschaften folgen einem entgegengesetzten Trend. Ihr Anteil ist besonders in Deutschland, Finnland, Großbritannien und Schweden sehr hoch. Kein eindeutiger Trend ist für die Handelsgesellschaften zu beobachten. Sie folgen vorrangig den zu bedienenden Märkten. Entsprechend gering fällt die Ungleichverteilung zwischen den Mitgliedstaaten aus. zum Ausdruck. Worauf diese charakteristischen Merkmale zurückzuführen sind, lässt sich im Einzelnen nur vermuten. Um aber Rückschlüsse auf eventuelle Anpassungen der Konzernstruktur europäischer Konzerne bei Einführung der einheitlichen (konsolidierten) Körperschaftsteuer-Bemessungsgrundlage ziehen zu können, ist die Existenz von Gesellschaften nachzuweisen, die sich vornehmlich aus den bestehenden, mit Einführung der konsolidierten Besteuerung wegfallenden steuerrechtlichen Regelungen ableitet. ${ }^{504} \mathrm{Zu}$ den wichtigsten Elementen der Steuerplanung zählen in diesem Zusammenhang der Einsatz von Finanzierungs- und

Konzerne auch auf den bevorzugten Standort für Europa-Holdinggesellschaften zurückzuführen ist.

$502 \mathrm{Vgl}$. zur günstigen Besteuerung der niederländischen Finanzierungsgesellschaften und ihrer Holdingfunktion Peters, W., Holdinggesellschaften, 1998, S. 179-181.

${ }^{503}$ Bei der Berechnung wurden nur niederländische Konzernmuttergesellschaften berücksichtigt, an denen ein Anteilseigner mit über $50 \mathrm{v}$. H. beteiligt ist und der außerdem seinen Sitz außerhalb der Europäischen Union hat.

${ }^{504} \mathrm{Zu}$ den Ansatzpunkten einer Steuerplanung bei einer formelhaften Gewinnaufteilung vgl. Scheffler, W., in: Oestreicher, Konzernbesteuerung, 2005, S. 314-328. 
Holdinggesellschaften. Beide Gesellschaftsformen büßen mit Einführung der konsolidierten Körperschaftsteuer-Bemessungsgrundlage ihre Wirkungen ein, sodass erwartet werden kann, dass die identifizierten Gesellschaften langfristig aus dem Konzernverbund verschwinden werden, vorausgesetzt es stehen dem nicht triftige Gründe entgegen, wie z. B. das Vorhandensein qualifizierter Mitarbeiter am Standort der Finanzierungsgesellschaften.

Tabelle 32: Über welche Mitgliedstaaten fließen Direktinvestitionen nach Europa? ${ }^{505}$

\begin{tabular}{|c|c|c|c|c|c|c|}
\hline $\begin{array}{l}\text { Mutter- } \\
\text { gesellschaft }\end{array}$ & $\begin{array}{c}\text { Summe der } \\
\text { Bilanzsummen der } \\
\text { Tochtergesellschaften im } \\
\text { EU-Ausland in TEUR } \\
\end{array}$ & Rang & $\begin{array}{c}\text { Summe der Bilanzsummen } \\
\text { aller Tochtergesellschaften } \\
\text { in der EU in TEUR }\end{array}$ & Rang & $\begin{array}{l}\text { Anzahl der } \\
\text { Konzerne }\end{array}$ & Rang \\
\hline AT & 133.761 & 13 & 627.962 & 14 & 43 & 8 \\
\hline $\mathrm{BE}$ & 23.738 .117 & 3 & 86.257 .010 & 4 & 98 & 6 \\
\hline$C Z$ & 0 & 18 & 54.002 & 17 & 2 & 13 \\
\hline $\mathrm{DE}$ & 8.559 .852 & 7 & 41.390 .814 & 7 & 242 & 3 \\
\hline DK & 18.006 .661 & 4 & 40.644 .625 & 8 & 137 & 5 \\
\hline $\mathrm{EE}$ & 0 & 18 & 0 & 19 & 1 & 16 \\
\hline ES & 28.552 .874 & 2 & 52.160 .388 & 6 & 31 & 10 \\
\hline $\mathrm{FI}$ & 122.228 & 14 & 715.530 & 13 & 6 & 12 \\
\hline FR & 13.917 .369 & 6 & 126.007 .077 & 3 & 164 & 4 \\
\hline GB & 14.280 .235 & 5 & 692.556 .225 & 1 & 413 & 2 \\
\hline GR & 28.366 & 15 & 1.799 .445 & 11 & 1 & 16 \\
\hline $\mathrm{HU}$ & 0 & 18 & 0 & 19 & 1 & 16 \\
\hline IE & 626.944 & 10 & 3.913 .690 & 10 & 33 & 9 \\
\hline IT & 1.427 .875 & 9 & 6.825 .124 & 9 & 21 & 11 \\
\hline LU & 230.536 & 12 & 1.355 .345 & 12 & 1 & 16 \\
\hline LV & 1.760 & 17 & 21.762 & 18 & 2 & 13 \\
\hline NL & 146.050 .927 & 1 & 495.436 .847 & 2 & 415 & 1 \\
\hline PL & 6.550 & 16 & 264.864 & 16 & 2 & 13 \\
\hline PT & 527.944 & 11 & 527.944 & 15 & 1 & 16 \\
\hline SE & 6.508 .292 & 8 & 56.762 .822 & 5 & 74 & 7 \\
\hline
\end{tabular}

Quelle: eigene Berechnungen

\section{Analyse der Konzernstruktur unter steuerplanerischen Gesichtspunkten}

\section{Einsatz von Holdinggesellschaften}

\section{a) Eingrenzung des Untersuchungsgegenstandes}

Der betriebliche Hauptzweck einer Holdinggesellschaft ist das auf Dauer angelegte Halten von Beteiligungen an einem oder mehreren rechtlich selbstständigen Unternehmen. ${ }^{506}$ Die Beweggründe für die Integration einer Holding-

${ }^{505}$ Es wurden nur europäische Konzernmuttergesellschaften berücksichtigt, an denen ein ausländischer Anteilseigner direkt zu mehr als $50 \mathrm{v}$. H. beteiligt ist.

506 Vgl. Keller, T., Holdingkonzepte, 1990, S. 55; Kessler, W., Euro-Holding, 1996, S. 10. 
gesellschaft in den Konzernaufbau können dabei unterschiedlich sein. ${ }^{507}$ Häufig resultieren Holdinggesellschaften aus der Strukturierung größerer Unternehmensgruppen. Moderne Führungs- und Organisationskonzepte empfehlen zunehmend einen dezentralisierten und divisionalisierten Konzernaufbau, ${ }^{508}$ wodurch eine höhere Transparenz der Unternehmensstrukturen, eine Minimierung der Funktionsstellen, flachere Hierarchien, eine Minimierung des Kontroll- und Kostenaufwands und eine Erhöhung der Unternehmensflexibilität in Bezug auf zukünftige Strukturanpassungen erreicht werden soll. ${ }^{509}$ Dabei werden die einzelnen Geschäftsbereiche, die sowohl nach Sparten, Produktlinien und/oder Regionen abgegrenzt werden, ${ }^{510}$ der Dachholding in gesellschaftsrechtlich selbstständiger und betriebswirtschaftlich erfolgsverantwortlicher Form (Profit-Center) unterstellt. ${ }^{511}$

Während die dargelegten betriebswirtschaftlichen Gründe meistens zur Errichtung einer Dachholding führen, stehen im Rahmen der Steuerplanung die Zwischen- oder Landesholdings im Mittelpunkt der Betrachtung. ${ }^{512}$ Dabei lassen sich grundsätzlich drei Typen von Holdinggesellschaften in Abhängigkeit von ihrer Position innerhalb der Beteiligungskette eines grenzüberschreitend tätigen Konzerns unterscheiden (Abbildung 18): ${ }^{513}$ Erstens können Holdinggesellschaften im Land der Muttergesellschaft eingesetzt werden (Typ 1), um Beteiligungen an ausländischen Konzernunternehmen zu halten. Ein Vorteil dieser inländischen Auslandsholdings ist, dass im Falle eines Verkaufs anstelle der ausländischen Beteiligungen die Inlandsholding veräußert werden kann, wodurch jegliche Implikationen des ausländischen Steuerrechts vermieden

507 Für eine Übersicht über die unterschiedlichen Hauptinteressen der Gesellschafter vgl. Scheffler, E., in: Lutter, Holding, S. 30-32. Für eine tabellarische Darstellung der verschiedenen Typisierungsmerkmale von Holdinggesellschaften vgl. Jacobs, O.H. (Hrsg.), Unternehmensbesteuerung, 2002, S. 816.

$508 \mathrm{Vgl}$. Jacobs, O.H. (Hrsg.), Unternehmensbesteuerung, 2002, S. 817.

509 Vgl. Lutter, M., in: Lutter, Holding, 2004, S. 3.

510 Unter steuerlichen Gesichtspunkten können mit der Vernachlässigung einer regionalen Struktur gravierende Steuermehrbelastungen einhergehen, die beispielsweise durch fehlende Konsolidierung auf Landesebene oder eingeschränkte Fremdfinanzierungs- und Steuerplanungsmöglichkeiten entstehen. Vgl. Jacobs, O.H. (Hrsg.), Unternehmensbesteuerung, 2002, S. 819.

$511 \mathrm{Zu}$ den Vorteilen einer Holdingstruktur mit rechtlich verselbstständigten dezentralen Profit-Centern vgl. Lettl, T., in: DStR, 1997, S. 1016-1020; derselbe, in: DStR, 1996, S. 2020-2026.

512 Vgl. Jacobs, O.H. (Hrsg.), Unternehmensbesteuerung, 2002, S. 820.

513 Diese Differenzierung der Holdinggesellschaften wurde von Weichenrieder/Mintz übernommen. Vgl. Weichenrieder, A./Mintz, J.M., Ownership, 2006, S. 1. 
werden. ${ }^{514}$ Der zweite Typ umfasst Gesellschaften, die ihren Sitz weder im Stammland der Muttergesellschaft noch im Zielland der Investition haben. Der häufigste Grund für das Dazwischenschalten einer Drittlandholding kann unter dem Begriff ,treaty shopping“ zusammengefasst werden. ${ }^{515}$ Dabei versuchen die steuerlichen Gestalter durch die Kombination günstiger Abkommensvorschriften und/oder Richtlinienvergünstigungen eine niedrigere Besteuerung zu erlangen. ${ }^{516}$ Die dritte Variante hat ihren Sitz im Zielland der Investition. Als Landesholding hält diese Gesellschaft die Beteiligungen an den übrigen in diesem Land angesiedelten, meist operativen Gesellschaften. Der steuerliche Vorteil liegt beispielsweise in der Konsolidierung der Einzelergebnisse der Tochtergesellschaften.

Abbildung 18: Klassifizierung von Holdinggesellschaften

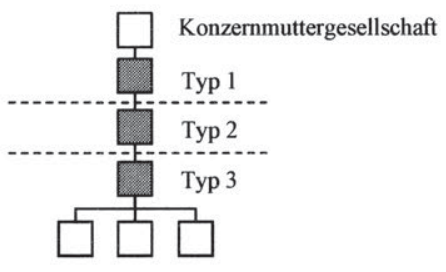

Quelle: eigene Abbildung

Im Folgenden sollen die aus steuerlichen Erwägungen eingeschalteten Holdinggesellschaften des dritten Typs analysiert werden. Da sich die primären Vorteile des ersten Typs nicht aus der laufenden Besteuerung ergeben, wird er im Folgenden vernachlässigt. Auch der zweite Typ von Zwischenholdings wird nicht näher analysiert, da im Betrachtungszeitraum 200315 der 25 betrachteten Länder bereits Mitglied der Europäischen Union waren, sodass gemäß der Mutter-Tochter-Richtlinie in diesen Ländern keine Quellensteuern mehr auf innergemeinschaftliche Gewinnausschüttungen erhoben wurden. Dadurch wurden auch steuerplanerische Gestaltungen innerhalb der Europäischen Union zur Reduktion bzw. Vermeidung der Quellensteuer überflüssig. Folglich können Holdinggesellschaften, die aufgrund eines "treaty shopping“ in den Konzernauf-

${ }^{514}$ Vgl. Weichenrieder, A./Mintz, J.M., Ownership, 2006, S. 1. Ist die Obergesellschaft ein Einzelunternehmen oder eine Personengesellschaft kann durch das Einschalten einer inländischen Auslandsholding und die Thesaurierung der Gewinne auf der Ebene der Kapitalgesellschaft die Steuerbelastung gesenkt werden. Vgl. Scheffler, W., in: FischerWinkelmann, Management-Consulting, 2003, S. 465-476.

515 Vgl. Kessler, W., Euro-Holding, 1996, S. 85-86.

516 Vgl. Jacobs, O.H. (Hrsg.), Unternehmensbesteuerung, 2002, S. 436-439, 862-867. 
bau integriert werden, nicht mittels der vorliegenden, auf Europa beschränkten Datenbasis analysiert werden. ${ }^{517}$

\section{b) Landesholding}

\section{( $\alpha$ ) Überblick}

Unter steuerplanerischen Gesichtspunkten gibt es mehrere Gründe, eine Landesholding zu errichten. ${ }^{518}$ Teilweise sind die Motive derart komplex, dass ihre Überprüfung an der restriktiven Datenlage der Amadeus-Datenbank scheitert. Dennoch soll der Einsatz von Landesholdings zur steuerlichen Optimierung der Konzernstruktur ökonometrisch nachgewiesen werden. Dazu werden zunächst die drei wesentlichen steuerlichen Beweggründe für das Integrieren einer Landesholding in den Konzernaufbau dargestellt. Im Einzelnen geht es um die Möglichkeit der Inanspruchnahme nationaler Gruppenbesteuerungssysteme, das Abschreiben von Beteiligungsbuchwerten und die Erhöhung des zulässigen Verhältnisses der Gesellschafter-Fremdfinanzierung zum Eigenkapital. Daran anschließend folgt die Definition von Landesholdings nach Maßgabe der in der Amadeus-Datenbank zur Verfügung stehenden Informationen. Schließlich geben sowohl deskriptive Statistiken als auch eine binär logistische Regression Aufschluss darüber, ob die dargelegten gesetzlichen Regelungen die Existenz der vorgefundenen Landesholdings erklären können.

\section{(B) Motive für den Einsatz von Landesholdings}

(aa) Anwendung von Gruppenbesteuerungssystemen

Landesholdings werden bevorzugt eingesetzt, um die Aktivitäten in einem Mitgliedstaat zu bündeln und damit die Voraussetzungen der nationalen Gruppenbesteuerungssysteme zu erfüllen. Die Motive der nationalen Teilkonzerne, sich für die Anwendung von Gruppenbesteuerungssystemen zu qualifizieren, sind eng mit dem Fehlen eines eigenständigen Konzernsteuerrechts verbunden. ${ }^{519}$ So folgen alle 25 Mitgliedstaaten der Europäischen Union dem Trennungsprinzip, das zwischen der Ebene der Gesellschaft und der der Gesellschafter unterscheidet. Die wirtschaftliche Verbundenheit der Konzerngesellschaften wird ignoriert, und steuerrechtlich stehen sich die einzelnen Konzerngesellschaften

517 Für eine umfangreiche empirische Analyse zum „treaty shopping“ auf der Basis der Direktinvestitionsstatistik der Deutschen Bundesbank vgl. Weichenrieder, A./Mintz, J.M., Ownerhip, 2006.

$518 \mathrm{Vgl}$. die Beispiele für den Einsatz von Holdinggesellschaften in Jacobs, O.H., Unternehmensbesteuerung, 2002, S. 821-852; Endres, D., in: WPg, 2003, S. S56-S63.

${ }^{519}$ Für eine detaillierte Darstellung alternativer Besteuerungskonzepte für Unternehmensgruppen vgl. die Ausführungen des Kapitels B. 
wie fremde Dritte gegenüber. Diese isolierte Betrachtungsweise der Konzerneinheiten kann mit systematischen Defiziten bei der Besteuerung von Unternehmensgruppen einhergehen, die nachfolgend dargestellt werden.

Erleiden einzelne Konzerngesellschaften einen Verlust, während andere ertragreich wirtschaften, übersteigt die Summe der Ertragsteuerbemessungsgrundlage daher per saldo den erwirtschafteten ökonomischen Gewinn des Konzerns. Folglich zahlen einzelne Konzerngesellschaften Körperschaftsteuer auf das von ihnen erwirtschaftete positive Teilergebnis, obwohl das wirtschaftliche Gesamtgebilde ein niedrigeres oder ausgeglichenes Ergebnis oder gar einen Verlust erzielt hat. ${ }^{520}$ Gewährt der Mitgliedstaat der verlustreichen Konzerngesellschaften einen unbegrenzten Verlustvortrag, ${ }^{521}$ handelt es sich unter der Annahme langfristig rentabler Gesellschaften zwar lediglich um einen temporären Effekt, doch besitzt dieser aufgrund des negativen Zinseffekts auch endgültigen Charakter. Ähnlich wirkt eine fehlende Zwischengewinneliminierung. Auch sie verlagert den Besteuerungszeitpunkt durch die steuerliche Erfassung der Gewinne aus internen Lieferungs- und Leistungstransaktionen zeitlich nach vorne. Die damit verbundenen Opportunitätskosten durch den vorzeitigen Abfluss liquider Mittel erhöhen die Steuerbelastung. Da es sich hierbei ausschließlich um temporäre Effekte handelt, ändert sich die nominale Steuerbelastung in der Totalperiode nicht. $^{522}$

Dies trifft nicht auf konzerninterne Gewinnausschüttungen zu. Als Nachsteuergröße stellen sie auch auf der Ebene der empfangenden Gesellschaft steuerpflichtiges Einkommen dar, wodurch eine wirtschaftliche Doppelbesteuerung droht. $^{523}$ Zudem kann sich durch Quellensteuern eine rechtliche Doppelbesteuerung der Gewinne ergeben. ${ }^{524}$ Mit Einführung der Mutter-TochterRichtlinie ist die Gefahr der Doppelbesteuerung von Gewinnausschüttungen innerhalb der Europäischen Union weitestgehend gebannt. Dividenden sind bei den empfangenden Gesellschaften freizustellen, oder aber es ist die im Ausland auf diese Einkünfte entrichtete Steuer anzurechnen. Stellen die Mitgliedstaaten die Gewinnausschüttungen von der Steuer frei, können sie auch den Abzug der

520 Vgl. Kessler, W., Euro-Holding, 1996, S. 30-31.

521 In Spanien dürfen Verluste nur 15 Jahre, in Finnland zehn Jahre, in Portugal sechs Jahre und in Tschechien, Griechenland, Italien, Lettland, Litauen, Polen, der Slowakei und Slowenien nur fünf Jahre vorgetragen werden. Vgl. Endres, D. et al., Taxable Income, 2006, S. 81.

522 Diese Aussage hat nur so lange Gültigkeit, wie die Mitgliedstaaten den Verlustvortrag nicht begrenzen. Vgl. a. FN 521.

${ }^{523} \mathrm{Zu}$ den konstitutiven Merkmalen einer rechtlichen und wirtschaftlichen Doppelbesteuerung vgl. Jacobs, O.H. (Hrsg.), Unternehmensbesteuerung, 2002, S. 3-4.

524 Vgl. a. die Darstellung und das Rechenbeispiel hinsichtlich einer potentiellen Steuermehrbelastung in einem mehrstufigen Konzern in Kessler, W., Euro-Holding, 1996, S. 24-26. 
mit der Beteiligung zusammenhängenden Verwaltungskosten versagen. Werden diese Kosten pauschal bestimmt, dürfen sie 5 v. H. der Gewinnausschüttungen nicht übersteigen. ${ }^{525}$ Diese pauschale Festlegung der nichtabzugsfähigen $\mathrm{Be}-$ triebsausgaben bewirkt eine fünf-prozentige Doppelbesteuerung der Dividendenerträge auf jeder Beteiligungsebene (Kaskadeneffekt). ${ }^{526}$ Obwohl die MutterTochter-Richtlinie ausschließlich grenzüberschreitende Gewinnausschüttungen regelt, behandelt die Mehrzahl der Mitgliedstaaten auch innerstaatliche Gewinnrepatriierungen entsprechend. ${ }^{527}$ Zum Zeitpunkt der Erhebung der Beteiligungsinformationen $(2003)^{528}$ besteuerten Frankreich, Belgien und Italien fünf Prozent der Dividendeneinnahmen auf jeder Konzernebene. In Deutschland wurden innerstaatlich Dividenden auf der Ebene der Muttergesellschaft noch in vollem Umfang freigestellt. ${ }^{529}$ Auch die zehn Mitgliedstaaten, die im Jahr 2003 noch nicht Mitglied in der Europäischen Union waren, stellten konzerninterne Gewinnausschüttungen auf der Ebene der Muttergesellschaft von der Steuer frei. ${ }^{530}$

Mit Einführung der Mutter-Tochter-Richtlinie gehören auch Quellensteuern auf zwischenstaatliche Gewinnausschüttungen innerhalb der Europäischen Union der Vergangenheit an. ${ }^{531}$ Dies gilt grundsätzlich nicht für innerstaatliche Gewinnausschüttungen. So erhoben 2003 Deutschland, Polen und Spanien, Tschechien und die Slowakei Quellensteuern unabhängig von der Höhe der gehaltenen Beteiligung, der Dauer des Bestehens der Beteiligung und der Rechtsform der beteiligten Gesellschaften. ${ }^{532}$ Außer in Tschechien und der Slowakei kann diese Steuer gegen die Körperschaftsteuerschuld der Muttergesellschaft angerechnet werden, doch erfolgt die Anrechnung erst in der Folgeperiode, sodass stets ein negativer Zinseffekt verbleibt. Je länger die Beteiligungsketten sind, desto größer ist dieser.

525 Art. 4 Abs. 2 Mutter-Tochter-Richtlinie.

526 Auch der deutsche Gesetzgeber folgt dieser Regelung. Für eine Darstellung der 2003 geltenden und der derzeitigen steuerlichen Behandlung der Beteiligungsaufwendungen und -erträge vgl. Winkeljohann, N., in: Oestreicher, Konzernbesteuerung, 2005, S. 65-81.

${ }^{527}$ Für eine Übersicht über die Behandlung von konzerninternen Dividenden in den jeweiligen Mitgliedstaaten vgl. Tabelle 1.

528 Die Mehrzahl der in der Amadeus-Datenbank hinterlegten Jahresabschlüsse stammen aus dem Jahr 2003. Dies gilt damit wohl auch für die Beteiligungsinformationen. Für eine Beschreibung Datenbasis vgl. Kapitel Vgl. D.IV.3.a).

529 Erst mit Wirkung zum 1. Januar 2004 hat der deutsche Gesetzgeber durch das sogenannte Korb II-Gesetz die unterschiedliche steuerrechtliche Behandlung von innerstaatlichen und grenzüberschreitenden Gewinnausschüttungen aufgegeben.

${ }^{530} \mathrm{Zu}$ den steuerrechtlichen Regelungen in den zehn neuen Mitgliedstaaten vgl. Kesti, J. (Hrsg.), Tax Handbook, 2003.

531 Art. 5 Mutter-Tochter-Richtlinie.

$532 \mathrm{Zu}$ den steuerrechtlichen Regelungen in den zehn neuen Mitgliedstaaten vgl. Kesti, J. (Hrsg.), Tax Handbook, 2003; Endres, D. et al., Taxable Income, 2006. 
Die dargelegten Probleme der Besteuerung von Beteiligungskonzernen werden von 17 Mitgliedstaaten teilweise dadurch gemindert, dass sie den Konzernen Gruppenbesteuerungssysteme zur Verfügung stellen. ${ }^{53}$ Diese ermöglichen grundsätzlich die Verrechnung von Gewinnen und Verlusten zwischen den qualifizierten Gesellschaften. Darüber hinaus entfallen innerhalb der steuerlichen Konzerngruppen etwaige Quellensteuern und eine fünf-prozentige Besteuerung der Beteiligungserträge auf der Ebene der empfangenden Konzerngesellschaft. ${ }^{534}$ Folglich sind die Teilkonzerne bestrebt, die Gruppenbesteuerungssysteme anzuwenden. ${ }^{535}$ Wie in Kapitel B.III bereits dargelegt, unterscheiden sich die Anwendungsvoraussetzungen der einzelnen Gruppenbesteuerungssysteme im Hinblick auf die territoriale Reichweite, die Mindestbeteiligungsquote, die qualifizierenden Rechtsformen und die Mindestbestandsdauer voneinander. ${ }^{536}$ Für die Existenz von steuerlich bedingten Landesholdings spielt dabei insbesondere die territoriale Reichweite der Gruppenbesteuerungssysteme eine Rolle. In Zypern, Irland, Lettland, Malta, Schweden und Großbritannien werden bei der Abgrenzung des Konsolidierungskreises auch ausländische Muttergesellschaften berücksichtigt, sodass Schwestergesellschaften eines ausländischen Anteilseigners eine nationale steuerliche Unternehmensgruppe bilden dürfen. Folglich ist eine nationale Dachgesellschaft, die die Beteiligungen an den übrigen Gliedgesellschaften hält, nicht zwingend erforderlich. In Österreich, Dänemark, Finnland, Frankreich, Deutschland, Luxemburg, den Niederlanden und Spanien können unmittelbar gehaltene Tochtergesellschaften eines ausländischen Anteilseigners nur dann ihre Ergebnisse verrechnen, wenn die Muttergesellschaft eine Betriebsstätte im Staat der Tochtergesellschaften unterhält. Existiert diese nicht, ist eine Dachgesellschaft zwingend erforderlich. In Polen, Portugal und Schweden ist eine Dachgesellschaft immer erforderlich. Zwar kann jede Gesellschaft, deren Rechtsform den Anforderungen des Gruppenbesteuerungssystems genügt, als Dachgesellschaft fungieren; sollen jedoch steuerinduzierte Holdinggesellschaften erklärt werden,

${ }^{533}$ Für eine Darstellung der unterschiedlichen Gruppenbesteuerungssysteme innerhalb der Europäischen Union vgl. Kapitel B.III. Für den Rechtsstand des Jahres 2003 siehe auch Kapitel D.V.3.a).

534 Gewinnabführungen des Organträgers an die Organgesellschaft stellen in Deutschland keine Dividendenzahlung dar, weswegen weder Kapitalertragsteuern zu entrichten sind, noch die Rechtsfolgen des $\S 8 \mathrm{~b} \mathrm{KStG}$ Anwendung finden.

535 Durch den zwingenden Abschluss eines Gewinnabführungsvertrages in Deutschland und Österreich sind mit der Errichtung einer Organschaft vor allem auch Haftungsrisiken verbunden, die Teilkonzerne von deren Anwendung abschrecken könnten.

${ }^{536}$ Im Jahr 2003 existierte in Italien noch kein Gruppenbesteuerungssystem. In Österreich galt noch das alte Organschaftssystem, und die Mindestbeteiligungsquote in Dänemark betrug $100 \mathrm{v}$. $\mathrm{H}$. 
ist ihre Existenz in den Mitgliedstaaten zu vermuten, deren Gruppenbesteuerungssystem eine Dachgesellschaft erfordert. Dies setzt aber voraus, dass die nationalen Teilkonzerne auch die übrigen Anforderungen der Gruppenbesteuerungssysteme erfüllen. ${ }^{537}$

(bb) Teilwertabschreibungen von Beteiligungsbuchwerten

Ein weiterer Einsatzbereich für Landesholdings ist die Geltendmachung bzw. Bewahrung von Teilwertabschreibungen und Liquidationsverlusten. ${ }^{538}$ Die Mehrzahl der Mitgliedstaaten stellt Dividendeneinkünfte frei. Dementsprechend sind regelmäßig auch Veräußerungserlöse von der Steuer befreit. So unterliegen diese Gewinne in vierzehn Mitgliedstaaten (Belgien, Dänemark, Deutschland, Finnland, Großbritannien, Irland, Italien, Luxemburg, Malta, den Niederlanden, Österreich, Schweden, Spanien und Zypern) gar nicht oder nur in sehr geringem Umfang der Steuer. ${ }^{539}$ Als Äquivalent dazu werden in vielen dieser Staaten Teilwertabschreibungen auf Tochtergesellschaften sowie Verluste aus der Veräußerung bzw. Liquidation einer Tochtergesellschaft steuerlich nicht anerkannt. Die Mitgliedstaaten, die eine Teilwertabschreibung grundsätzlich vorsehen, knüpfen an sie jedoch Bedingungen, die es Kapitalgesellschaften eines Unternehmensverbundes regelmäßig nicht erlauben, entsprechende Wertminderungen steuerlich geltend zu machen. ${ }^{540}$ Unter Berücksichtigung dieser Restriktionen kann das steuerliche Ergebnis lediglich in vier Mitgliedstaaten, Österreich, Ungarn, Estland und Tschechien, durch Teilwertabschreibungen geschmälert werden. Aufgrund des besonderen estnischen Steuersystems, das thesaurierte Gewinne nicht besteuert, haben dort Verluste keinen signifikanten Einfluss auf die Steuerzahllast. ${ }^{51}$ Für die verbleibenden drei Mitgliedstaaten gilt, dass der Einsatz einer Landesholding vorteilhaft ist, soweit der ausländische Anteilseigner nicht selbst seinen Sitz in einem dieser Mitgliedstaaten hat. Ist dies der Fall, sollte der Aufwand in dem Land mit dem höheren Gewinnsteuersatz ausgewiesen werden. Jedoch ist zu beachten, dass in Österreich nur Teilwertabschreibungen von Beteiligungsbuchwerten inländischer Tochtergesellschaften steuerlich geltend gemacht werden dürfen.

Die Höhe des zu verrechnenden Aufwands kann den Beteiligungsbuchwert nicht übersteigen. Damit aber eine Verrechnung überhaupt erfolgen kann, muss die Holding selbst Einkommen erwirtschaften. Eine reine Finanzholding erfüllt

\footnotetext{
537 Vgl. zur Gegenüberstellung der aufsummierten direkten Konzernbeteiligungshöhe und der Mindestbeteiligungsquote Tabelle 33.

538 Vgl. Jacobs, O.H. (Hrsg.), Unternehmensbesteuerung, 2002, S. 832-833.

539 Vgl. Endres, D. et al., Taxable Income, 2006, S. 67.

540 Vgl. hierzu die Tabelle 2.

541 Vgl. Endres, D. et al., Verlustberücksichtigung, 2006, S. 45.
} 
diese Bedingung regelmäßig nicht, da die Dividendeneinnahmen von der Steuer freigestellt sind oder aber die fällige Steuer mit zuvor auf diese Einkünfte entrichteten Steuern verrechnet werden kann.

(cc) Erhöhung des zulässigen Verhältnisses der GesellschafterFremdfinanzierung zum Eigenkapital

Drittens dienen Landesholdings der Sicherstellung einer steueroptimalen Finanz- und Kapitalstruktur innerhalb der Konzernunternehmung. Den Ausgangspunkt der Überlegung bildet die Tatsache, dass ein hoher Verschuldungsgrad steuerlich günstiger sein kann als eine hohe Eigenkapitalausstattung. ${ }^{542}$ So mindern die Zinsen im Land des Darlehensempfängers das zu versteuernde Einkommen, während sie im Staat des Fremdkapital gewährenden Unternehmens steuerpflichtig sind. Um den Steueraufwand des Konzerns zu minimieren, werden Unternehmen in Hochsteuerländer verstärkt fremdfinanziert, wobei die kapitalgebenden Gesellschaften ihren Sitz in Niedrigsteuerländern haben ${ }^{543} \mathrm{Um}$ einer Erosion des Steuersubstrats durch das Ersetzen von (nicht abziehbaren) Dividenden durch (abziehbare) Fremdkapitalvergütungen vorzubeugen, schränken die Mehrzahl der Mitgliedstaaten eine zu umfangreiche GesellschafterFremdfinanzierung durch sogenannte ,thin-capitalization rules" ein. ${ }^{544}$ Danach darf das Verhältnis des Eigenkapitals zum Fremdkapital bestimmte Schwellenwerte (,safe heaven“) nicht übersteigen. ${ }^{545}$ Mit Deutschland und Ungarn sehen aber zwei Mitgliedstaaten Sonderregelungen für Holdinggesellschaften vor. So galt 2003 in Deutschland für Holdinggesellschaften gemäß $\S 8 \mathrm{a}$ Abs. $4 \mathrm{KStG}$ noch ein erweiterter "safe heaven" von 3:1. In Ungarn werden Holdinggesellschaften explizit von den ,thin-capitalization rules“ ausgenommen. ${ }^{546}$ Dementsprechend ist in beiden Ländern das Etablieren einer Landesholding sinnvoll, wenn die auf das Fremdkapital entfallenden Zinsen im Staat des Empfängers einer niedrigeren Besteuerung als in Deutschland bzw. Ungarn unterliegen. ${ }^{547}$

542 Für einen Belastungsvergleich zwischen Eigen- und Fremdfinanzierung vgl. Jacobs, O.H. (Hrsg.), Unternehmensbesteuerung, 2002, S. 796-833.

543 Hierzu zählen auch Länder, die Finanzierungsgesellschaften ein spezielles Steuerregime einräumen. Vgl. die empirische Analyse zur Existenz von Konzernfinanzierungsgesellschaften in Kapitel E.II.2.

${ }^{544}$ Für eine detaillierte Übersicht über die ,thin-capitalization rules“ in den EUMitgliedstaaten vgl. Gouthière, B. (Hrsg.), in: ET, 2005, S. 367-441.

545 Für eine tabellarische Übersicht der „safe heavens“ in wichtigen Industrie- und EUMitgliedstaaten vgl. Tabelle 50 in Jacobs, O.H. (Hrsg.), Unternehmensbesteuerung, 2002, S. 807.

${ }^{546} \mathrm{Vgl}$. Gouthière, B. (Hrsg.), in: ET, 2005, S. 399.

547 Innerhalb Europas kann dies durch die Errichtung einer Finanzierungsgesellschaft, z. B. in Irland, gewährleistet werden. Vgl. hierzu die Ausführungen in Kapitel E.II.2. 
Unter Umständen kann aber auch in den übrigen Mitgliedstaaten der Einsatz einer Landesholding vorteilhaft sein, nämlich dann, wenn durch den konzerninternen Anteilserwerb der Beteiligungen von der Muttergesellschaft durch eine Holding das für die Ermittlung des „safe heaven“ herangezogene Eigenkapital aufgebläht wird. ${ }^{548}$

\section{( $\gamma$ Identifizierung von Landesholdings in der Amadeus-Datenbank und Beschreibung der Datenlage}

Sowohl die Beschreibung der Verbreitung von Landesholdings als auch die ökonometrische Analyse entsprechender Konzernstrukturen erfordert zunächst die Identifikation dieser Gesellschaften in der Amadeus-Datenbank.

Der allgemeinen Begriffsbestimmung folgend, ist die Grundvoraussetzung einer Holding, dass sie Beteiligungen an einer weiteren Konzerngesellschaft hält. ${ }^{549}$ Gemäß dieser Definition zählt jede Gesellschaft als Landesholding, die eine Tochtergesellschaft einer ausländischen Konzerngesellschaft ist und selbst an weiteren Unternehmen beteiligt ist. Dies schließt aber grundsätzlich auch operativ tätige Gesellschaften mit ein, deren primäre Tätigkeit nicht das Halten von Beteiligungen ist. Vor dem Hintergrund, dass Holdinggesellschaften identifiziert werden sollen, die primär aufgrund steuerplanerischer Überlegungen in den Konzernaufbau integriert wurden, ist die bisherige Definition zu grob. Zur weiteren Spezifizierung wird deshalb zusätzlich auf die Wirtschaftszweigklassifikation der entsprechenden Gesellschaften abgestellt. Grundsätzlich werden Holdinggesellschaften dem Code 7415 (Managementtätigkeiten von Holdinggesellschaften) zugeordnet. Jedoch zeigen die Erfahrungen, dass die $\mathrm{Zu}$ ordnungen stellenweise ungenau sind, weswegen auch eine weiter gefasste Definition in Betracht kommt, die alle Gesellschaften berücksichtigt, deren NACE Code mit den Ziffern 741 beginnt. ${ }^{550}$ Daneben hat die Beschreibung der Konzernstrukturen gezeigt, dass vorrangig in den Niederlanden auch Gesellschaften mit dem NACE Code 6523 Funktionen einer Holdinggesellschaft übernehmen. ${ }^{551}$

Bei den weiteren Analysen wird folgende Definition verwendet: Eine Landesholding ist jede Konzerngesellschaft, die (1) ihren Sitz im Zielland einer Direktinvestition hat, (2) die Tochter einer ausländischen Konzerngesellschaft ist,

548 Diese steuerplanerische Gestaltung ist zumindest in Deutschland nicht mehr möglich. Vgl. $\S 8 \mathrm{a} \mathrm{Abs} .6 \mathrm{KStG}$.

549 Vgl. zur Definition von Holdinggesellschaften Keller, T., Holdingkonzepte, 1990, S. 55; Kessler, W., Euro-Holding, 1996, S. 10.

550 Der NACE Code 741 umfasst folgende Wirtschaftszweige: Rechts-, Steuer- und Unternehmensberatung, Buchführung, Markt- und Meinungsforschung sowie Managementtätigkeiten von Holdinggesellschaften.

551 Vgl. hierzu die Ausführungen des Kapitels E.I und insbesondere die Tabelle 31. 
(3) dem NACE Code 7415 zugeordnet werden kann und (4) Beteiligungen an weiteren Konzerngesellschaften hält. Im Rahmen der Regressionsanalyse werden Variationsrechnungen mit abweichender Definition von Landesholdings vorgenommen. Vorausgesetzt die drei übrigen Kriterien sind erfüllt, gelten Gesellschaften auch dann als Landesholding, wenn sie einem NACE Code zugeordnet sind, der mit 741 beginnt, oder wenn sie neben der Wirtschaftszweigklasse 7415 auch der Klasse 6523 angehören.

Gemäß obiger Definition einer Landesholding sind die nationalen Unternehmensgruppen stets Teil eines internationalen Konzerns. Die 16.962 grenzüberschreitend tätigen Konzerne verfügen über 32.097 Teilkonzerne im Ausland, die zusammen aus 58.146 Konzerngesellschaften bestehen. ${ }^{552}$ Damit hat mehr als jede dritte Konzerngesellschaft (36,8 v. H.) ihren Sitz im Ausland. Die meisten Teilkonzerne bestehen aus drei oder weniger Gesellschaften (93,2 v. H.). Die Existenz einer Landesholding ist bei Unternehmensgruppen dieser Größe weniger wahrscheinlich. Sie in die Analyse einzubeziehen, würde das Ergebnis verzerren, da trotz entsprechender Rahmenbedingungen keine Landesholding in den Konzernaufbau integriert wird. Dementsprechend beschränkt sich die Analyse auf die 2.193 Teilkonzerne (6,8 v. H.), die aus mehr als drei Gesellschaften bestehen und zusammen 21.326 Konzerngesellschaften $(37,3 \mathrm{v} . \mathrm{H}$.) umfassen.

\section{(8) Deskriptive Statistiken}

Abbildung 19 verdeutlicht zunächst, dass der Anteil der Teilkonzerne, die auf erster Ebene über eine Landesholding verfügen, stark zwischen den Mitgliedstaaten schwankt. So ist der Anteil in Belgien, Tschechien, Ungarn, Irland, Italien, Luxemburg, den Niederlanden und Polen sehr gering. Zwar mag dieses Ergebnis durch die geringe Anzahl der Teilkonzerne in einzelnen Mitgliedstaaten verzerrt erscheinen, doch fällt bereits hier auf, dass von den acht Mitgliedstaaten vier den Konzernen kein Gruppenbesteuerungssystem zur Verfügung stellen. Der geringe Anteil in den Niederlanden kann mit der Definition der Landesholding zusammenhängen. Werden auch Dachgesellschaften des Teilkonzerns mit dem NACE Code 6523 berücksichtigt, erhöht sich der Anteil von 3 v. H. auf 55 v. H. In Deutschland, Dänemark, Frankreich, Österreich und Portugal verfügt ein hoher Anteil der nationalen Unternehmensgruppen über eine Dachholding.

Ein zentrales Motiv, eine Landesholding in den Konzernaufbau einzuschalten, ist die Bündelung der nationalen Aktivitäten unter einer Dachgesellschaft, um damit die Anforderungen der nationalen Gruppenbesteuerungssysteme zu er-

552 Zur Verteilung der Konzerne und Konzerngesellschaften nach Ländern vgl. Tabelle 25. 
füllen. Dies setzt jedoch voraus, dass die Teilkonzerne auch die übrigen Anforderungen der Gruppenbesteuerungssysteme erfüllen.

Abbildung 19: Anteil der Teilkonzerne mit Landesholding nach Ländern ${ }^{553}$

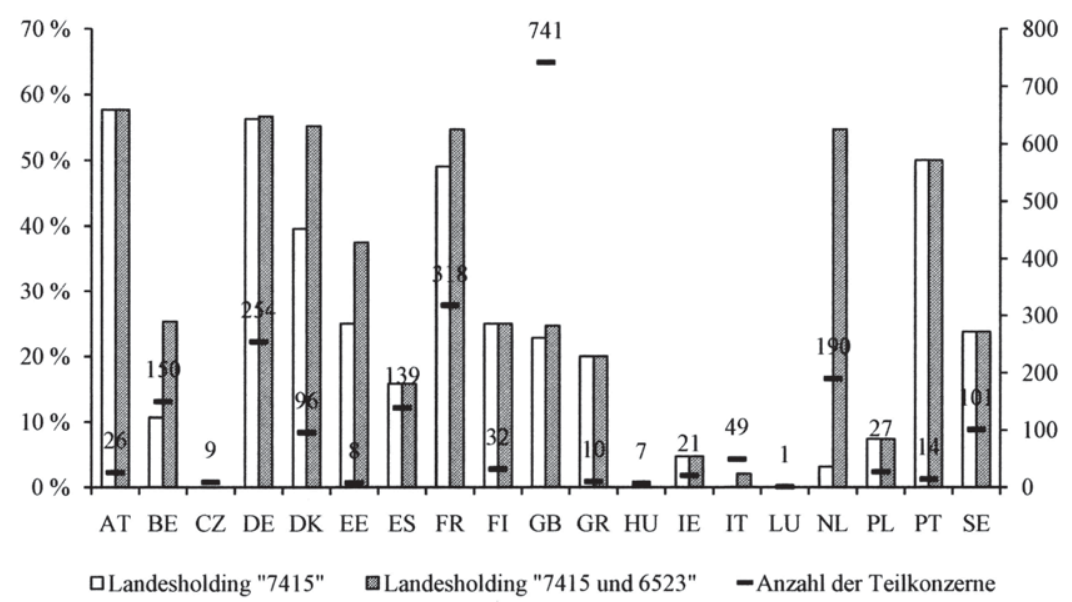

Quelle: eigene Darstellung

Hinsichtlich der Rechtsformen trifft dies zu, da sich die Gruppen fast ausschließlich aus Gesellschaften zusammensetzen, denen die Anwendung eines Gruppenbesteuerungssystems gestattet ist. ${ }^{554}$ Inwieweit die Anteilseigner die häufig geforderte Mindestbesitzdauer erfüllen, kann aufgrund der Zeitpunktbetrachtung der Konzernstrukturen nicht beobachtet werden, sie gilt deshalb als grundsätzlich erfüllt. Somit ist das entscheidende Qualifikationskriterium die Beteiligungshöhe. Ein Vergleich der Mindestbeteiligungsquoten der Gruppenbesteuerungssysteme mit der Beteiligungshöhe, mit der die jeweiligen Konzerngesellschaften gehalten werden, zeigt, dass die Mindestbeteiligungsquote bei fast 95 v. H. der Beteiligungen erreicht wird (Tabelle 33). Somit kann nahezu ausgeschlossen werden, dass einzelne Teilkonzerne keine Landesholding gründen, weil sie andere Anforderungen der Gruppenbesteuerungssysteme nicht erfüllen und sich damit ohnehin nicht für die steuerliche Behandlung als Unternehmensgruppe qualifizieren.

553 In der Abbildung sind nur die Mitgliedstaaten enthalten, in denen Teilkonzerne einer ausländischen Konzernmuttergesellschaft mit mehr als drei Konzerngesellschaften vorgefunden wurden.

${ }^{554} \mathrm{Zu}$ den im Rahmen der empirischen Analyse berücksichtigten Rechtsformen vgl. Kapitel D.IV.4. Eine Übersicht über die einzelnen Rechtsformen kann der Tabelle 55 entnommen werden. 
Tabelle 33: Durchschnittlich direkte Höhe der Beteiligung und Mindestbeteiligungsquote ${ }^{555}$

\begin{tabular}{|c|c|c|c|c|c|c|}
\hline \multirow{3}{*}{ Land } & \multirow{3}{*}{$\begin{array}{l}\text { Durchschnitt } \\
\text { (in v. H.) }\end{array}$} & \multirow{3}{*}{$\begin{array}{l}\text { Standard- } \\
\text { abweichung }\end{array}$} & \multirow{3}{*}{$\begin{array}{l}\text { Anzahl der Ge- } \\
\text { sellschaften }\end{array}$} & \multicolumn{3}{|c|}{ Mindestbeteiligungsquote } \\
\hline & & & & \multirow{2}{*}{ Höhe } & \multicolumn{2}{|c|}{ Gesellschaften, die diese erfüllen } \\
\hline & & & & & absolut & relativ \\
\hline$\overline{\mathrm{AT}}$ & 95,19 & 13,26 & 146 & 50.00 & $\overline{146}$ & $100,00 \%$ \\
\hline $\mathrm{DE}$ & 96,35 & 10,72 & 1.848 & 50.00 & $1.84 \varepsilon$ & $100,00 \%$ \\
\hline DK & 98,16 & 8,16 & 588 & 100.00 & 552 & $93,88 \%$ \\
\hline ES & 96,21 & 10,72 & 1.092 & 75.00 & 1.018 & $93,22 \%$ \\
\hline FI & 97,26 & 9,15 & 232 & 90.00 & 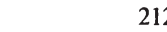 & $91,38 \%$ \\
\hline $\mathrm{FR}$ & 91,57 & 17,26 & 2.885 & 95.00 & 2.298 & $79,65 \%$ \\
\hline GB & 97,84 & 5,88 & 10.190 & 75.00 & 10.01 & $98,28 \%$ \\
\hline IE & 98,09 & 5,60 & 124 & 75.00 & 12 & $100,00 \%$ \\
\hline LU & 99,92 & 0,18 & 5 & 95.00 & & $100,00 \%$ \\
\hline $\mathrm{NL}$ & 98,86 & 6,24 & 1.281 & 95.00 & 1.236 & $96,49 \%$ \\
\hline PL & 93,35 & 13,29 & 175 & 95.00 & 136 & $77,71 \%$ \\
\hline PT & 96,44 & 10,21 & 73 & 90.00 & 6 & $91,78 \%$ \\
\hline SE & 98,62 & 6,63 & 901 & 90.00 & 868 & $96,34 \%$ \\
\hline $\bar{\Sigma}$ & & & 19.540 & & 18.52 & $94,81 \%$ \\
\hline
\end{tabular}

Quelle: eigene Berechnungen

Während sich die Vorteile der steuerlichen Behandlung in Bezug auf die Vermeidung einer Doppelbesteuerung der Gewinnausschüttungen und der Vermeidung von Quellensteuern unmittelbar ergeben, stellt sich der steuerliche Vorteil aus der Konsolidierung der Einzelergebnisse nur ein, wenn mindestens ein Unternehmen des Teilkonzerns einen Verlust erleidet. Demzufolge ist in Tabelle 34 die Existenz einer Landesholding in Abhängigkeit von der Verfügbarkeit und territorialen Reichweite der Gruppenbesteuerungssysteme und der Möglichkeit der Verlustverrechnung innerhalb des jeweiligen Teilkonzerns wiedergegeben. ${ }^{556}$ Der Anteil der Teilkonzerne mit einer Landesholding als Dachgesellschaft beträgt 9 v. H. in Ländern, die über kein Gruppenbesteuerungssystem verfügen. Mit $30 \mathrm{v}$. H. ist dieser Anteil in den anderen Mitgliedstaaten deutlich höher. Allerdings erfordert nicht jedes Gruppenbesteuerungssystem zwingend, dass alle Unternehmen von einer im Inland ansässigen Dachgesellschaft gehalten werden. Entsprechend ist der Anteil in Mitgliedstaaten höher (37 v. H.), in denen ausländische Anteilseigner entweder eine Betriebsstätte oder eine selbstständige Dachgesellschaft errichten müssen, um in den

555 In der Tabelle sind nur Mitgliedstaaten aufgeführt, die über ein Gruppenbesteuerungssystem verfügen und in denen Teilkonzerne mit mehr als drei Gesellschaften in der Datenbank vorliegen.

556 Die Möglichkeit der Verlustverrechnung stellt eine Ex-post-Analyse dar, während eine Landesholding ggf. ex ante zu etablieren ist. Jedoch kann aufgrund umfangreicher Planrechnungen der Unternehmen eine hohe Korrelation zwischen antizipierter und erzielter Ertragslage vermutet werden. 
Genuss der Gruppenbesteuerung zu kommen. ${ }^{557}$ Am höchsten sollte aber der Anteil in Mitgliedstaaten sein, die zwingend die Existenz einer Dachgesellschaft erfordern. Dies wird durch die Daten nicht bestätigt, doch sei angemerkt, dass diese Zahlen lediglich auf den Ausprägungen von 41 Teilkonzernen basieren. In Mitgliedstaaten, die grundsätzlich keine Verrechnung von Gewinnen und Verlusten zwischen Konzerngesellschaften vorsehen, zeigt sich kein Zusammenhang zwischen der Ertragslage und der Existenz von Holdinggesellschaften. So schwankt der Anteil zwischen 4 und 12 v. H., während in den übrigen Mitgliedstaaten tendenziell ein Anstieg in Abhängigkeit von der Anzahl möglicher Verlustverrechnungen in den letzten vier Jahren zu beobachten ist.

Tabelle 34: Verteilung der Landesholding in Abhängigkeit von der Verfügbarkeit der Gruppenbesteuerungssysteme und einer möglichen Verlustverrechnung innerhalb der letzten vier Jahre

\begin{tabular}{|l|ccccc|c|}
\hline $\begin{array}{l}\text { Anzahl möglicher Verrechnung von Verlusten } \\
\text { zwischen 2000 und 2003 }\end{array}$ & 0 & 1 & 2 & 3 & 4 & $\Sigma$ \\
\hline Kein Gruppenbesteuerungssystem vorhanden & $12 \%$ & $4 \%$ & $6 \%$ & $10 \%$ & $9 \%$ & $9 \%$ \\
\hline Gruppenbesteuerungssystem vorhanden & $30 \%$ & $18 \%$ & $26 \%$ & $29 \%$ & $38 \%$ & $30 \%$ \\
\hline keine Holding erforderlich & $10 \%$ & $17 \%$ & $21 \%$ & $21 \%$ & $34 \%$ & $22 \%$ \\
Holding erforderlich, wenn keine Betriebsstäte & $39 \%$ & $20 \%$ & $31 \%$ & $38 \%$ & $41 \%$ & $37 \%$ \\
vorhanden & $38 \%$ & $0 \%$ & $17 \%$ & $25 \%$ & $14 \%$ & $22 \%$ \\
\hline Holding zwingend erforderlich & & & & & & \\
\hline
\end{tabular}

Quelle: eigene Berechnungen

Ein zweiter Grund, eine Landesholding in den Konzernaufbau zu integrieren, ist die Sicherstellung einer indirekten Verlustverrechnung durch die Abschreibung von Beteiligungsbuchwerten. Folglich sollten Landesholdings verstärkt in Mitgliedstaaten vorzufinden sein, die auch steuerlich die Abschreibung des Beteiligungsbuchwertes unter die Anschaffungskosten erlauben. Tabelle 35 bekräftigt diese Vermutung. So liegt der Anteil der Teilkonzerne, der über eine Holding als Dachgesellschaft verfügt, in den entsprechenden Mitgliedstaaten bei 34 v. H. und damit 6 Prozentpunkte über dem Anteil in den übrigen Mitgliedstaaten.

Tabelle 35: Verteilung der Landesholdings in Abhängigkeit von einer möglichen Teilwertabschreibung von Beteiligungsbuchwerten

\begin{tabular}{|l|cc|}
\hline & Keine Landesholding & Landesholding \\
\hline Teilwertabschreibung nicht möglich & $72,28 \%$ & $27,72 \%$ \\
Teilwertabschreibung möglich & $66,00 \%$ & $34,00 \%$ \\
\hline
\end{tabular}

Quelle: eigene Berechnungen

557 Die Amadeus-Datenbank enthält keine Informationen über etwaige Betriebsstätten. Ob Schwestergesellschaften, die auf erster Ebene von einem ausländischen Anteilseigner gehalten werden, sich in Ländern, die mindestens das Vorliegen einer Betriebsstătte voraussetzen, ihre Ergebnisse konsolidieren dürfen, kann nicht eindeutig festgestellt werden. 
Ein weiteres Motiv für die Etablierung einer Landesholding ist die Sicherstellung einer steuerlich optimalen Kapitalstruktur innerhalb des Konzerns. Zur Gewinnverlagerung werden dabei Tochtergesellschaften häufig mit unverhältnismäßig viel Fremdkapital ausgestattet, weshalb Mitgliedstaaten entsprechende Gestaltungen durch vorgeschriebene Höchstverschuldungsgrade einschränken. Zwei Länder sehen begünstigende Regelungen für Holdinggesellschaften vor, sodass dort durch den Einsatz von Landesholdings ein höherer Verschuldungsgrad möglich ist. Tabelle 36 zeigt grundsätzlich ein kongruentes Bild. Jedoch sei darauf hingewiesen, dass nur Deutschland und Ungarn die dargelegten Begünstigungen kennen, wobei nur sieben ungarische Teilkonzerne in der Grundgesamtheit enthalten sind, sodass die Übersicht im Wesentlichen durch die Ausprägung deutscher Konzerne beeinflusst wird.

Tabelle 36: Verteilung der Landesholdings in Abhängigkeit von besonderen „thincapitalization rules" für Holdinggesellschaften

\begin{tabular}{|l|cc|}
\hline & Keine Landesholding & Landesholding \\
\hline $\begin{array}{l}\text { Gleiche „thin-capitalization rules“ für alle Gesell- } \\
\text { schaften }\end{array}$ & $75,78 \%$ & $24,22 \%$ \\
$\begin{array}{l}\text { Günstigere bzw. keine thin-capitalization rules“ für } \\
\text { Holdinggesellschaften }\end{array}$ & $45,21 \%$ & $54,79 \%$ \\
\hline
\end{tabular}

Quelle: eigene Berechnungen

Wie bereits bei der Auswahl der Datenbasis angedeutet, ist neben den steuerrechtlichen Rahmenbedingungen die Größe des Teilkonzerns eine entscheidende Einflussgröße für die Existenz einer Landesholding. Deren Etablierung verursacht Kosten, die durch den steuerlichen Vorteil kompensiert werden müssen. Dieser steigt tendenziell mit der Anzahl der Tochtergesellschaften eines Teilkonzerns, sodass die Existenz einer Landesholding bei größeren Teilkonzernen wahrscheinlicher ist. Diesen Zusammenhang verdeutlicht auch Abbildung 20. Die höhere Volatilität des Grafen für Teilkonzerne, die sich aus mehr als $29 \mathrm{Ge}-$ sellschaften zusammensetzen, resultiert aus der geringeren Anzahl großer nationaler Unternehmensgruppen. Nur 4,2 v. H. der in der Analyse betrachteten Teilkonzerne (92) verfügen über 30 oder mehr Konzerngesellschaften.

Abbildung 20: Anteil der Teilkonzerne mit Landesholding in Abhängigkeit von der Anzahl der Tochtergesellschaften

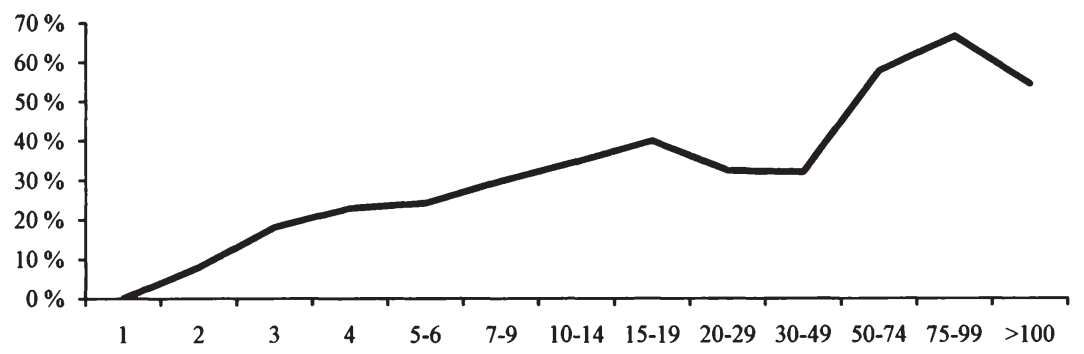

Quelle: eigene Darstellung 


\section{(ع) Logistische Regressionsanalyse}

Nachdem die deskriptiven Statistiken erste Hinweise auf einen Zusammenhang der entsprechenden steuerrechtlichen Regelungen mit der Existenz von Landesholdings gegeben haben, soll dies mittels einer multivariaten Analyse überprüft werden. Da die Ausprägungen der abhängigen Variablen, die Existenz bzw. das Fehlen einer Landesholding, binär sind, wird der Einfluss der steuerlichen Faktoren auf der Basis eines binär logistischen Regressionsmodells untersucht. ${ }^{558}$ Die Beteiligungsinformationen sind im Ownership-Modul der Amadeus-Datenbank ausschließlich für einen Zeitpunkt hinterlegt, sodass allein eine Querschnittanalyse durchgeführt wird.

Die abhängige Variable LANDESHOLDING nimmt den Wert eins an, wenn der Teilkonzern über eine Landesholding verfügt andernfalls ist der Wert null. Entsprechend den Überlegungen zur Identifikation von Landesholdings in der Datenbank kommen im Rahmen der Regressionsanalyse drei verschiedene, in Kapitel E.II.1.b)( $\gamma$ ) erläuterte Definitionen einer Landesholding zum Einsatz. Sie unterscheiden sich ausschließlich hinsichtlich des Branchencodes.

Die vorangegangene Diskussion zeigt, dass die Existenz von Landesholdings auf verschiedene Einflussgrößen zurückzuführen ist: Erstens wird die Variable GBS in das Regressionsmodell aufgenommen. Sie ist binär codiert und nimmt den Wert eins an, wenn der Mitgliedstaat, in dem sich der Teilkonzern befindet, den Gesellschaften ein Gruppenbesteuerungssystem zur Verfügung stellt. Wie die Darstellung des Rechtsrahmens gezeigt hat, ist aber für die Anwendung der Gruppenbesteuerungssysteme nicht zwangsweise eine Dachgesellschaft auf erster Ebene notwendig. Demzufolge wird in zwei weiteren Regressionsmodellen jeweils die Variable GBS durch GBS_BS bzw. GBS_KG ersetzt. Als Dummy-Variable nehmen sie nur dann den Wert eins an, wenn die steuerrechtlichen Regelungen hinsichtlich der territorialen Reichweite der Gruppenbesteuerungssysteme der Mitgliedstaaten mindestens eine Betriebsstätte (BS) bzw. eine Kapitalgesellschaft (KG) als Dacheinheit erfordern.

Der zweite Regressor (VERLUST) erfasst die Häufigkeit einer möglichen Verlustverrechnung in den vier dem Datensatz vorangegangenen Jahren. Da aber eine Verlustverrechnung nur möglich ist, wenn ein Gruppenbesteuerungssystem zur Verfügung steht, besteht zwischen VERLUST und GBS ein Interaktionseffekt, sodass in das Regressionsmodel nur die erklärende Variable VERLUST*GBS aufgenommen wird.

558 Die Modellparameter werden mittels der Maximum-Likelihood-Methode bestimmt. Vgl. zur Darstellung der binär logistischen Regressionsmethode Fromm, S., in: Bamberger Beiträge zur empirischen Sozialforschung, 2005, 11; Urban, D., Logit-Analyse, 1993; AndreB, H.-J./Hagenaars, J./Kühnel, S., Analyse, 1997, S. 261-299. 
Die Einschaltung einer Holdinggesellschaft zum Zweck der Sicherstellung einer indirekten Verlustverrechnung durch das Abschreiben von Beteiligungsbuchwerten wird durch die Variable AFA erfasst. Erneut handelt es sich hierbei um eine binäre Variable, die den Wert eins annimmt, wenn die Abschreibung durch den Einsatz einer Dachgesellschaft ermöglicht wird. In bestimmten Fällen, in denen bereits der Sitzstaat des Anteilseigners Wertminderungen der Beteiligungen steuerlich berücksichtigt, kann die Existenz einer Landesholding dennoch sinnvoll sein, und zwar dann, wenn der Steuersatz des Mitgliedstaates des Teilkonzerns über dem des Landes des Anteilseigners liegt. Dieser Effekt wird auch von der Variable AFA aufgefangen, indem sie in diesen Fällen trotz einer möglichen Abschreibung der Beteiligungsbuchwerte im Zielland der Investition den Wert null zugewiesen bekommt.

Die Variable TCR bezieht sich auf die Existenz besonderer „thin-capitalization rules" für Holdinggesellschaften. Sie ist binär codiert und nimmt den Wert eins an, sofern der Mitgliedstaat des Teilkonzerns einen erweiterten "safe heaven“ für Holdinggesellschaften vorsieht oder diese Gesellschaften explizit von der Anwendung etwaiger ,thin-capitaliziation rules" ausschließt.

Schließlich wird der Regressor STEUERSATZ in das Modell aufgenommen. Es wird vermutet, dass Konzerne verstärkt steuergestalterisch aktiv werden, je höher der Steuersatz in dem betreffenden Mitgliedstaat ist. Folglich wird ein positiver Zusammenhang zwischen der Höhe des Steuersatzes und der Existenz steuerplanerisch induzierter Holdinggesellschaften erwartet.

Neben den steuerspezifischen Einflussfaktoren wird die exogene Variable LN_NR_TK eingeführt, durch die die logarithmierte Anzahl an Gesellschaften des Teilkonzerns in das Modell einfließt. Zwischen der Existenz einer Landesholding und der Größe eines Teilkonzerns ist ein positiver Zusammenhang zu erwarten, da die Etablierung einer solchen Gesellschaft mit Kosten verbunden ist und die Vorteile einer Holdinggesellschaft mit der Größe der Teilkonzerne tendenziell zunehmen. ${ }^{559}$ Schließlich wird die Variable BIP in das Regressionsmodell aufgenommen. Als nicht steuerliche Größe soll die Berücksichtigung des Bruttoinlandsprodukts vermeiden, dass länderspezifische Effekte, die mit der Größe der Volkswirtschaft zusammenhängen, den steuerlichen Faktoren zugerechnet werden.

Bevor die Schätz- und Testergebnisse zum gesamten Modell bzw. zu einzelnen Regressoren interpretiert werden, wird zunächst der Erfolg der ModellSchätzung bezüglich einer effektiven Beschreibung der beobachteten Y-Werte mittels des Hosmer-Lemeshow-Anpassungstests bewertet (Tabelle 37). ${ }^{560}$ Dabei

559 Der Einfluss der Größe des Teilkonzerns wurde insofern entschärft, als nur Teilkonzerne in der Analyse berücksichtigt werden, die mindestens aus vier Gesellschaften bestehen.

${ }^{560}$ Liefert der Hosmer-Lemeshow-Anpassungstest einen signifikanten Wert, dann ist die Modellgültigkeit zu bezweifeln. Vgl. zum Test Hosmer, D./Lemeshow, S., Regression, 
wird mittels eines Chi-Quadrat-Tests die Nullhypothese überprüft, dass alle Differenzen zwischen den geschätzten und beobachteten Werten gleich null sind. ${ }^{561}$

Tabelle 37: Güte der Anpassung des logistischen Regressionsmodells

\begin{tabular}{|c|c|c|c|c|c|c|c|c|c|c|}
\hline & \multicolumn{3}{|c|}{$\begin{array}{l}\text { LANDESHOLDING } \\
(7415)\end{array}$} & \multicolumn{3}{|c|}{$\begin{array}{l}\text { LANDESHOLDING } \\
\text { (7415 und 6523) }\end{array}$} & \multicolumn{3}{|c|}{$\begin{array}{l}\text { LANDESHOLDING } \\
(741)\end{array}$} \\
\hline & & I & II & III & IV & $\mathrm{V}$ & VI & VII & VIII & IX \\
\hline & & GBS & GBS BS & GBS_KG & GBS & GBS_BS & $\begin{array}{c}\text { GBS_K } \\
\text { G }\end{array}$ & GBS & GBS_BS & GBS_KG \\
\hline \multirow{2}{*}{$\begin{array}{l}\text { Hosmer- } \\
\text { Lemeshow- } \\
\text { Test }\end{array}$} & $\begin{array}{l}\text { Chi- } \\
\text { Quadrat }\end{array}$ & 7,087 & 14,635 & 5,832 & 64,331 & 11,803 & 25,594 & 5,758 & 35,269 & 9,289 \\
\hline & Sig. & 0,527 & 0,067 & 0,666 & 0,000 & 0,160 & 0,001 & 0,674 & 0,000 & 0,319 \\
\hline \multicolumn{2}{|c|}{ Cox \& Snell R-Quadrat } & 0,114 & 0,126 & 0,111 & 0,070 & 0,098 & 0,059 & 0,081 & 0,084 & 0,081 \\
\hline \multicolumn{2}{|c|}{ Nagelkerkes R-Quadrat } & 0,164 & 0,182 & 0,160 & 0,096 & 0,135 & 0,081 & 0,113 & 0,117 & 0,113 \\
\hline
\end{tabular}

Quelle: eigene Berechnungen

Die Ergebnisse des Hosmer-Lemeshow-Tests zeigen, dass das gewählte Modell geeignet ist, Landesholdings mit dem NACE Code 7415 zu erklären. Für die beiden anderen Definitionen einer Landesholding liefert der Test für drei der sechs Modelle signifikante Ergebnisse, sodass die Nullhypothese nicht verworfen werden kann und ein signifikanter Unterschied zwischen den beobachteten und prognostizierten Werten auftritt. Die Signifikanz des Gesamtmodells wird mittels des generalisierten $\mathrm{R}^{2}$ nach Cox \& Snell und mittels des Pseudo- $\mathrm{R}^{2}$ nach Nagelkerke gemessen. ${ }^{562}$ Was das Modell (7415) betriff, so schwanken die Werte für Nagelkerkes $R^{2}$ zwischen 16,0 und 18,2 v. $H$. Zwar deuten bereits niedrige Werte auf eine hohe Anpassungsgüte hin ${ }^{563}$ dennoch geben die Werte zu bedenken, dass die Existenz von Landesholdings nur teilweise durch das Regressionsmodell erklärt wird. Für die Modelle IV-IX liegen die Werte der $\mathrm{R}^{2}$ deutlich niedriger.

Die bisherigen Ergebnisse zeigen, dass die Regression vordergründig geeignet ist, Landesholdings mit dem NACE Code 7415 zu erklären. Dies erscheint inso-

1989, S.140-145; Urban, D., Logit-Analyse, 1993, S. 65; Baltes-Götz, B.; Regressionsanalyse, 2006, S. 23-24.

561 Bei p-Werten unter 0,05 wird die Nullhypothese, dass kein Unterschied zwischen den beobachteten und geschätzten Werten existiert, abgelehnt. Weisen die Modelle hingegen ein nicht signifikantes Chi-Quadrat auf, kann die Nullhypothese nicht abgelehnt werden, was zugleich impliziert, dass die Schätzwerte des Modells sich den beobachteten Daten akzeptabel anpassen.

${ }^{562}$ Während der Höchstwert von Cox \& Snell $\mathrm{R}^{2}$ abhängig von der jeweiligen Stichprobe ist, ist das $\mathrm{Maß}$ von Nagelkerke auf die gewohnten Grenzen von 0 bis 1 normiert.

563 Bereits niedrige Werte zwischen 0,2 und 0,6 für Nagelkerkes $R^{2}$ repräsentieren eine sehr gute Anpassungsgüte. Für eine Erklärung vgl. Gujarati, D.N., Econometrics, 2003, S. 586. 
fern plausibel, als laut der Beschreibung des NACE Codes Holdinggesellschaften den Schlüssel 7415 tragen. ${ }^{564}$ Vor diesem Hintergrund werden in Tabelle 38 nur die Ergebnisse der Logit-Schätzungen für das Grundmodell (7415) mit den Variationen hinsichtlich der Abbildung der Gruppenbesteuerungssysteme wiedergegeben.

Da die abhängige Variable bei der Existenz einer Landesholding den Wert eins annimmt, bedeutet ein positives Vorzeichen eines Logit-Koeffizienten eine Erhöhung der Wahrscheinlichkeit, dass eine Landesholding in den Konzernaufbau integriert wird. Entsprechend den Erwartungen weisen in Modell I alle Koeffizienten ein positives Vorzeichen auf. Somit besteht zwischen den Werten der abhängigen und unabhängigen Variable für alle abgebildeten Regressoren ein positiver Zusammenhang. Dabei ist der Einfluss der in das Modell aufgenommenen Regressoren mindestens auf dem Niveau von 1 v. H. signifikant. ${ }^{565}$ Folglich zeigt das Regressionsmodell, dass die abgebildeten steuerlichen Regelungen den Einsatz von Holdinggesellschaften fördern. So ist beispielsweise die Wahrscheinlichkeit in Mitgliedstaaten, die Konzernen ein Gruppenbesteuerungssystem zur Verfügung stellen, eine Landesholding vorzufinden, um 110 v. H. höher als in Mitgliedstaaten, die über kein derartiges Steuersystem verfügen. ${ }^{566}$ Die Effektgrößen für AFA und TCR liegen mit 483 und 271 v. H. noch darüber. Auch der nichtsteuerliche Regressor LN_NR_TK beeinflusst positiv die Wahrscheinlichkeit, eine Landesholding im Teilkonzern beobachten zu können. Steigt LN_NR_TK um eine Einheit an und bleiben alle anderen Variablen konstant, so erhöht sich die Wahrscheinlichkeit, dass eine Landesholding an der Spitze des Teilkonzerns steht, um 28,2 v. H.

Das Modell I verwendet den Regressor GBS, der immer dann den Wert eins annimmt, sofern der Teilkonzern in einem Mitgliedstaat belegen ist, der über ein Gruppenbesteuerungssystem verfügt. Wie bereits mehrfach erwähnt, ist es in einigen Mitgliedstaaten nicht zwingend erforderlich, eine Landesholding in den Konzernaufbau zu integrieren, da auch Schwestergesellschaften einer ausländischen Muttergesellschaft ihre Ergebnisse verrechnen können. Infolgedessen wird im zweiten Regressionsmodell der Regressor GBS durch GBS_BS ersetzt.

564 Dieses Ergebnis mag insbesondere für die Berücksichtigung von Landesholdings mit dem NACE Code 6523 überraschen. Jedoch ist zu beachten, dass sich das Ergebnis für die Niederlande zwar verbessern mag, dem aber eine Verschlechterung in den anderen Mitgliedstaaten wie z. B. Belgien gegenübersteht. Auch dort steigt der Anteil an Landesholdings mit der Erweiterung des NACE Codes deutlich an, obwohl kein Gruppenbesteuerungssystem zur Verfügung steht (Abbildung 19).

${ }^{565}$ Zum Testen der Nullhypothese, dass ein Regressionskoeffizient gleich null ist, wird die Wald-Statistik verwendet. Vgl. hierzu Urban, D., Logit-Analyse, 1993, S. 58-59; BaltesGötz, B.; Regressionsanalyse, 2006, S. 38-39.

${ }^{566}$ Bei der Beurteilung der Effektgröße ist zu beachten, dass sich die Werte stets auf die Referenzkategorie beziehen. Vgl. Urban, D., Logit-Analyse, 1993, S. 40-44. 
Diese Dummy-Variable weist nur noch für die nationalen Gruppenbesteuerungssysteme eine Eins aus, die eine Betriebsstätte oder eine Kapitalgesellschaft im Zielland der Investition erfordern, welche an allen in den Konsolidierungskreis aufzunehmenden Gesellschaften beteiligt ist. Auch die Ergebnisse für das Modell II zeigen einen signifikanten positiven Einfluss der Regressionskoeffizienten auf die abhängige Variable. Lediglich der Regressor STEUERSATZ besitzt ein negatives Vorzeichen.

Tabelle 38: Ergebnisse der Regressionsanalyse - Landesholding (7415)

\begin{tabular}{|c|c|c|c|c|c|}
\hline Modell: & & & I & II & III \\
\hline Erklärende Variablen ( $\mathrm{Hy}_{\mathrm{F}}$ & pothesen) & & GBS & GBS BS & GBS KG \\
\hline GBS/GBS_BS/GBS_KG & Koeffizient B & + & 0,743 & 1,051 & 0,502 \\
\hline & Standardfehler & & 0,280 & 0,172 & 0,407 \\
\hline & Sig. & & $0,008 * *$ & $0,000^{* * *}$ & 0,217 \\
\hline & $\operatorname{Exp}(B)$ & & 2,103 & 2,861 & 1,652 \\
\hline VERLUST T*GBS & Koeffizient B & + & 0,286 & 0,243 & 0,326 \\
\hline & Standardfehler & & 0,042 & 0,042 & 0,039 \\
\hline & Sig. & & $0,000^{* * *}$ & $0,000^{* * *}$ & $0,000^{* * *}$ \\
\hline & $\operatorname{Exp}(B)$ & & 1,330 & 1,274 & 1,385 \\
\hline AFA & Koeffizient B & + & 1,763 & 1,562 & 1,680 \\
\hline & Standardfehler & & 0,347 & 0,343 & 0,343 \\
\hline & Sig. & & $0,000^{* * *}$ & $0,000 * * *$ & $0,000^{* * *}$ \\
\hline & $\operatorname{Exp}(B)$ & & 5,831 & 4,767 & 5,367 \\
\hline TCR & Koeffizient $B$ & + & 1,313 & 1,045 & 1,339 \\
\hline & Standardfehler & & 0,218 & 0,220 & 0,219 \\
\hline & Sig. & & $0,000^{* * *}$ & $0,000 * * *$ & $0,000^{* * *}$ \\
\hline & $\operatorname{Exp}(B)$ & & 3,717 & 2,844 & 3,814 \\
\hline STEUERSATZ & Koeffizient B & + & 0,039 & $-0,046$ & 0,039 \\
\hline & Standardfehler & & 0,014 & 0,019 & 0,014 \\
\hline & & & $0,004^{* *}$ & $0,014^{*}$ & $0,005^{* *}$ \\
\hline & $\operatorname{Exp}(B)$ & & 1,040 & 0,955 & 1,040 \\
\hline LN_NR_TK & Koeffizient $B$ & + & 0,249 & 0,327 & 0,226 \\
\hline & Standardfehler & & 0,080 & 0,082 & 0,079 \\
\hline & & & $0,002 * *$ & $0,000^{* * *}$ & $0,004^{* *}$ \\
\hline & $\operatorname{Exp}(B)$ & & 1,282 & 1,387 & 1,253 \\
\hline BIP & Koeffizient B & & 0,000 & 0,000 & 0,000 \\
\hline & Standardfehler & & 0,000 & 0,000 & 0,000 \\
\hline & Sig. & & $0,001^{* * *}$ & $0,000^{* * *}$ & $0,000 * * *$ \\
\hline & $\operatorname{Exp}(B)$ & & 1,000 & 1,000 & 1,000 \\
\hline Beobachtungen & & & 2.193 & 2.193 & 2.193 \\
\hline \begin{tabular}{|l} 
Trefferquote richtig Positi \\
\end{tabular} & & & 67,83 & 72,00 & 68,33 \\
\hline Trefferquote richtig Negat & & & 66,94 & 65,14 & 66,94 \\
\hline Gesamttrefferquote & & & 67,58 & 70,09 & 67,94 \\
\hline $\begin{array}{l}\text { Hinweis: Die dichotome a } \\
\text { den Hosmer-Lemeshow-T } \\
\text { zeichneten Teststatistiken } \\
\text { für die Klassifizierung bet } \\
\text { Die in das Regressionsm } \\
\text { einem Extremfall eine geri }\end{array}$ & $\begin{array}{l}\text { abhängige Variat } \\
\text { est und das Bes } \\
\text { sind auf einem N } \\
\text { rägt } 0,3 \text {. Eine Ko } \\
\text { odell aufgenomn }\end{array}$ & & $\begin{array}{l}\text { den Wert } \\
\text { smaß vgl. } \\
5 \mathrm{v} . \mathrm{H} ., 1 \\
\text { im Model } \\
\text { iablen sin }\end{array}$ & $\begin{array}{l}\text { lls eine } \mathrm{L} \\
\text { Die mi } \\
0,1 \mathrm{v} . \mathrm{H} \text {. } \\
\text { wird abe } \\
\text { end unko }\end{array}$ & $\begin{array}{l}\text { ing vorliegt. } \\
\text { w. *** gek } \\
\text { t. Der Trenn } \\
\text { wiedergege } \\
\text { d weisen nu }\end{array}$ \\
\hline
\end{tabular}

Quelle: eigene Berechnungen

Dass sowohl GBS als auch GBS_BS signifikant sind, deutet daraufhin, dass Teilkonzerne in Mitgliedstaaten, die es Schwestergesellschaften eines ausländischen Anteilseigners erlauben, Teil einer steuerlichen Gruppe zu sein, teilweise dennoch eine Landesholding errichten. Dies bietet sich beispielsweise aus 
Gründen der Vereinfachung an, beachtet man, dass die entsprechenden Mitgliedstaaten im Rahmen der Gruppenbesteuerung dem Konzept der Einzelveranlagung folgen, ${ }^{567}$ das eine Verlustverrechnung durch Übertrag oder Ausgleichszahlungen zwischen den Gruppenmitgliedern erreicht.

Das Modell III ändert den Koeffizienten GBS derart ab, dass die DummyVariable nur dann den Wert eins annimmt, wenn eine Kapitalgesellschaft als Landesholding zwingend erforderlich ist. Anders ausgedrückt geht dieses Regressionsmodell implizit davon aus, dass die Konzerne stets eine Betriebsstätte in den entsprechenden Mitgliedstaaten gründen, um das Einschalten einer Kapitalgesellschaft als Landesholding zu vermeiden. Die Ergebnisse zeigen, dass dies aber wohl nicht der Realität entspricht. Der Regressor GBS_KG besitzt zwar ein positives Vorzeichen, ist aber statistisch nicht signifikant.

Die dargelegten Ergebnisse der logistischen Regressionsanalyse verdeutlichen zweierlei: Erstens legt die Signifikanz der gewählten Regressionsfaktoren nahe, dass steuerliche Erwägungen bei der Gründung einer Landesholding eine entscheidende Rolle spielen. Da die dafür maßgeblichen steuerrechtlichen Regelungen mit Einführung der einheitlichen konsolidierten KörperschaftsteuerBemessungsgrundlage weitestgehend an Bedeutung verlieren, ist im Falle einer entsprechenden Gesetzesänderung mit einer Verschiebung der Bemessungsgrundlage und mit einem starken Rückgang von Landesholdings zu rechnen. ${ }^{568}$ Handelt es sich bei der Holding jedoch um eine reine Finanzholding, deren einzige Tätigkeit das Halten von Beteiligungen ist, wirkt sich ihre Existenz nicht oder nur in sehr geringem Maße auf die Körperschaftsteuer-Bemessungsgrundlage und deren Aufteilung aus, sodass aus steuerlicher Sicht die Existenz weder mit Vor- noch Nachteilen verbunden ist. Die Dividendeneinkünfte sind steuerfrei bzw. werden im Rahmen der Konsolidierung eliminiert. Gleiches gilt für das Vermögen und den Umsatz. Damit wird einer Finanzholding lediglich über den Lohnsummenfaktor eine Bemessungsgrundlage zugewiesen. Aufgrund der geringen Anzahl der Mitarbeiter dürfte der Anteil an der Gesamtbemessungsgrundlage des Konzerns aber gering sein.

Zweitens deuten sowohl die Angaben zur Modellrelevanz als auch die Klassifikationstabelle daraufhin, ${ }^{569}$ dass weitere Faktoren für die Existenz von Landesholdings maßgeblich sind und/oder dass die Datenbasis fehlerhaft ist. So kann beispielsweise eine Holdinggesellschaft bereits aus strategisch

${ }^{567}$ Für eine Übersicht über die Veranlagungstechnik der Gruppenbesteuerungssysteme vgl. Tabelle 4.

568 Die Verschiebung der Bemessungsgrundlage erfolgt unabhängig von der geänderten, nun pauschalen Gewinnabgrenzung nur dann, wenn zuvor Holdinggesellschaften auch eingesetzt wurden, um Steuersubstrat zu verlagern.

569 Der Anteil der richtig Positiven schwankt für die Modelle I-IIl zwischen 62,85 und 64,65 v. H. 
organisatorischen Gründen an der Spitze eines ausländischen Teilkonzerns stehen. ${ }^{570}$ Die zur Verfügung stehende Datenbasis erlaubt aber keine explizite Abbildung dieser organisatorischen Einflüsse; sie werden lediglich partiell durch die Anzahl der Gesellschaften in den Teilkonzernen berücksichtigt. Aber auch Fehler in der Datenbasis könnten für eine Verzerrung der Ergebnisse verantwortlich sein. Für 4,2 v. H. der Dachgesellschaften der ausländischen Teilkonzerne sind keine Angaben zum Wirtschaftszweig verfügbar (NACE CODE 9999). Annahmegemäß handelt es sich bei diesen Unternehmen nicht um Landesholdings, was aber grundsätzlich eine Fehlklassifikation sein kann. Daneben beruht auch die Abbildung der Beteiligungsketten auf Annahmen. So ist bei 43,8 v. H. der Gesellschaften in der Amadeus-Datenbank die Beteiligungshöhe unbekannt, sodass diese aus den zur Verfügung stehenden Angaben abgeleitet wurde. ${ }^{571}$ Dadurch kann die Zusammensetzung der Teilkonzerne teilweise fehlerhaft sein. Schließlich erweist sich die Ermittlung der detaillierten steuergesetzlichen Regelungen für 25 Mitgliedstaaten als schwierig, zumal zehn dieser Länder im Betrachtungszeitpunkt 2003 nicht Mitglied der Europäischen Union waren.

\section{Einsatz von Konzernfinanzierungsgesellschaften}

\section{a) Steuerliche Vorteile der Funktionsverlagerung und ihre Grenzen}

Neben Holdinggesellschaften sind es vor allem Konzernfinanzierungsgesellschaften, die sich als steuerplanerisches Element im statutarischen Konzernaufbau wiederfinden. ${ }^{572}$ Durch die Auslagerung der Finanzierungsfunktionen auf zwischengeschaltete Rechtsträger kann die Konzernsteuerbelastung gesenkt werden, indem Steuersubstrat in das niedriger besteuernde Ausland verlagert wird. ${ }^{573}$ Dazu wird eine Finanzierungsgesellschaft im Ausland errichtet, an der die Konzernmuttergesellschaft beteiligt ist. Die Finanzierungsgesellschaft stattet die EU-Tochtergesellschaften durch die Vergabe eines konzerninternen Darlehens mit Kapital aus. Die dafür benötigten finanziellen Mittel erhält die Finanzierungsgesellschaft entweder durch Aufnahme von Fremdkapital am

$570 \mathrm{Zu}$ den allgemeinen Vorteilen, die mit der Integration einer Holding in den Konzernaufbau einhergehen, vgl. Scheffler, E., in: Lutter, Holding, S. 34-35.

$571 \mathrm{Vgl}$. zur Methode der Ergänzung von Beteiligungsinformationen Kapitel D.IV.1.

$572 \mathrm{Vgl}$. zu den Kernbereichen internationaler Steuerplanung und insbesondere zu den strukturellen Reaktionsmöglichkeiten der Unternehmen bei der Planung der Gruppenstruktur, Jacobs, O.H. (Hrsg.), Unternehmensbesteuerung, 2002, S. 709-710.

$573 \mathrm{Vgl}$. für eine ausführliche Darstellung ausländischer Konzernfinanzierungsgesellschaften Burwirtz, G., Konzernfinanzierungsgesellschaften, 2005. Für eine Analyse unter steuerplanerischen Gesichtspunkten vgl. Potthof, A., Finanzierung, 1998; Spengel, C., Unternehmensbesteuerung, 2003, S. 164-168. 
Kapitalmarkt oder durch von der Muttergesellschaft zur Verfügung gestelltes Beteiligungskapital. Abbildung 21 verdeutlicht die Finanzierungsbeziehungen zwischen den beteiligten Gesellschaften.

Abbildung 21: Integration von Finanzierungs- und Koordinationszentren in den Konzernaufbau

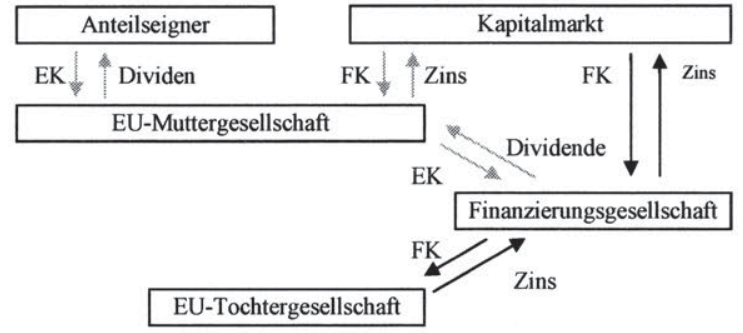

In Anlehnung an: Spengel, C., Unternehmensbesteuerung, 2003, S. 165

Aus Sicht des Gesamtkonzerns wird die Steuerbelastung nur reduziert, wenn (1) die im Land der Finanzierungsgesellschaft zu zahlenden Steuern geringer sind als im Land der Muttergesellschaft ${ }^{574}$ und (2) der Finanzierungsgewinn nicht in die inländische Bemessungsgrundlage einbezogen wird. ${ }^{575}$ Die erste Bedingung ist für viele Mitgliedstaaten der Europäischen Union leicht zu erfüllen. So verfügen Irland $(12,5 \text { v. H. })^{576}$ und Zypern $(10$ v. H.) über einen sehr niedrigen Gewinnsteuersatz. Daneben kann z. B. auch in Belgien eine niedrige Effektivbesteuerung durch eine vom Gewinn entkoppelte Besteuerung der Finanzerträge erreicht werden. ${ }^{577}$ Die zweite Bedingung zielt auf die „controlled foreign companies-rules“ der Mitgliedstaaten ab. ${ }^{578}$ Innerhalb der Europäischen Union

574 Neben den Ertragsteuern auf die Zinsmarge müssen auch etwaige Quellensteuern und Vermögensteuern berücksichtigt werden. Vgl. zu den Aspekten der steuerlichen Vorteilhaftigkeit Jacobs, O.H. (Hrsg.), Unternehmensbesteuerung, 2002, S. 1041-1042. Für eine Übersicht über die Ertragsteuersätze in der Europäischen Union vgl. Tabelle 47.

575 Vgl. Scheffler, W., in: Bank/Schiller, Finanzintermediation, 2004, S. 511.

576 Die Tarifbelastung der IFSC betrug bis 2005 lediglich $10 \mathrm{v}$. H. und wurde jetzt auf den regulären irischen Körperschaftsteuersatz von $12,5 \mathrm{v}$. H. angehoben.

577 Die Ermittlung der Bemessungsgrundlage nach der Kostenaufschlagsmethode („Costplus-Methode“) ist ausschließlich den „Belgien Coordination Centers“ vorbehaltend, zu deren Errichtung es einer Genehmigung durch die belgischen Behörden bedarf. Vgl. Burwirtz, G., Konzernfinanzierungsgesellschaften, 2005, S. 80-82.

578 Für einen Ländervergleich der „controlled foreign companies rules“ vgl. Brähler, K., $C F C, 2006, \mathrm{~S} .106-125$. Der EuGH hat bereits die britische Hinzurechnungsbesteuerung bedingt als Verstoß gegen die Niederlassungsfreiheit des Art. 43 EGV gewertet. EuGH v. 12. 9. 2006, Rs. C-196/04 - Cadbury-Schweppes, in: EuZW, 2006, S. 633-637. Daraufhin hat das BMF mit dem Schreiben vom 8. Januar 2007 die deutsche Hinzurechnungsbesteuerung teilweise außer Kraft gesetzt. Aber auch die deutsche Hinzurechnungs- 
kennen Deutschland, Finnland, Frankreich, Dänemark, Portugal, Schweden, Großbritannien und Italien eine Hinzurechnungsbesteuerung. ${ }^{579}$ Das Vorliegen einer entsprechenden Gesetzgebung lässt die Etablierung einer Finanzierungsgesellschaft in den Konzernaufbau aber nicht gleich unwirksam werden. Beispielsweise gelten in Deutschland Einkünfte als aktiv, sodass keine Hinzurechnungsbesteuerung vorgesehen ist, wenn die Finanzmittel auf einem ausländischen Kapitalmarkt aufgenommen und an im Ausland belegene aktive oder inländische Betriebe oder Betriebsstätten weitergeleitet werden. ${ }^{580}$ Bis 2003 konnte ein steuerlicher Vorteil auch bei einer asymmetrischen Finanzierungsstrategie durch die darlehensweise Weiterleitung von Eigenmitteln erreicht werden. Zwar waren auch damals die erzielten Einkünfte passiver Natur und unterlagen damit einer verschärften Hinzurechnungsbesteuerung, doch beschränkte $\S 10$ Abs. 6 bzw. Abs. 7 AStG den Hinzurechnungsbetrag auf 60 v. H. der Zwischeneinkünfte, sofern diese aus der Finanzierung aktiv tätiger, im Ausland ansässiger Konzernmitglieder stammten. ${ }^{581}$ Damit unterlagen im Ergebnis die Erträge aus der Konzernfinanzierung unabhängig vom Standort der Tochtergesellschaften einer einheitlichen Tarifbelastung von ungefähr $23,6 \mathrm{v} . \mathrm{H}^{582}$

Um als Standort für eine Finanzierungsgesellschaft in Frage zu kommen, müssen neben den steuerlichen Anforderungen einer günstigen Besteuerung auch außersteuerliche Faktoren wie die politische und wirtschaftliche Stabilität des Sitzlandes, die Leistungsfähigkeit des Bankwesens, eine moderate Währungs- und Zinsentwicklung, ein guter Zugang zu den Kapitalmärkten und eine generelle Liberalität des Handels- und Wirtschaftsrechts erfüllt werden. ${ }^{583}$ Als typische Standorte für Finanzierungsgesellschaften haben sich in den vergangenen Jahren Irland mit den „International Financial Service Centers“ (IFSC), die Niederlande mit den "Dutch Financial Companies“ (DFC) und Belgien mit den „Belgium Coordination Centers“ (BCC) herauskristallisiert.

besteuerung liegt dem EuGH bereits vor. Vgl. Vorlagebeschluss des Finanzgerichts Münster vom 5. 7. 2005, 15 K 1114/99 F, EW; beim EuGH anhängig unter Rs. C-298/05, Columbus Container Services B.V.B.A. \& Co. gegen Finanzamt Bielefeld-Innenstadt. Vgl. Wimpissinger, C., in: SWI, 2006, S. 559-564. Für eine Überprüfung der Vereinbarkeit der "controlled foreign companies rules" mit den Grundfreiheiten des EG-Vertrags vgl. Bille, S., Hinzurechnungsbesteuerung, 2004, S. 57-178; Helminen, M., in: intertax, 2005, S. 120-122.

579 Vgl. Wassermeyer, F., in: Wassermery/Pilz, Außensteuergesetz, 2006, Vor $§ \S 7-14$ AStG, Rz. 40; Kesti, J. (Hrsg.), Tax Handbook, 2003.

580 Siehe $\$ 8$ Abs. 1 Nr. 7 AStG.

581 Vgl. Spengel, C., Unternehmensbesteuerung, 2003, S. 48-51.

582 Vgl. Spengel, C., Unternehmensbesteuerung, 2003, S. 48-51. 23,6 = 39,35 × 0,6 (Tarifbelastung mit Körperschaftsteuer, Solidaritätszuschlag und Gewerbesteuer).

$583 \mathrm{Vgl}$. zu den außersteuerlichen Standortfaktoren Burwitz, G., Konzernfinanzierungsgesellschaften, 2005, S. 55-56; Jacobs, O.H. (Hrsg.), Unternehmensbesteuerung, 2002, S. 1042. 
Durch den Beitritt der zehn neuen Mitgliedstaaten sind Malta und Zypern als potenzielle Standorte hinzugekommen.

Charakteristisch für die speziellen Besteuerungsregime in den genannten Staaten ist, dass die Steuervergünstigungen nicht selten eine Diskrepanz zwischen Bemessungsgrundlage und wirtschaftlicher Substanz (Produktionsfaktoren) bewirken ${ }^{584}$ Dieses Missverhältnis zwischen wirtschaftlicher Aktivität und Bemessungsgrundlage wird im Rahmen der einheitlichen konsolidierten Körperschaftsteuer-Bemessungsgrundlage nivelliert, da nun die Bemessungsgrundlage auf der Basis der Aufteilungsfaktoren den einzelnen Konzerngesellschaften zugewiesen wird. Die Aufteilungsfaktoren entwickeln sich aber proportional zu den Produktionsfaktoren. Demzufolge ist nur noch eine zugewiesene Bemessungsgrundlage entsprechend der wirtschaftlichen Aktivität zu erwarten. Im Ergebnis wird mit Einführung der formelhaften Gewinnaufteilung den Finanzierungsgesellschaften ihr "Wettbewerbsvorteil“ genommen, sodass ein starker Rückgang des konzerninternen Outsourcings von Finanzierungsfunktionen zu erwarten ist. Die davon betroffenen Gesellschaften werden im folgenden Abschnitt identifiziert.

\section{b) Identifizierung der Finanzierungsgesellschaften}

Die Identifizierung der Konzernfinanzierungsgesellschaften in der AmadeusDatenbank erfolgt anhand des Unternehmenssitzes, des Firmennamens und der Tätigkeit der Gesellschaften. Die IFSC erhalten nur steuerliche Vorzüge, wenn sie ihren Sitz in der sogenannten Custom House Docks Area haben. ${ }^{585}$ Entsprechend erfolgt die Identifizierung dieser Gesellschaften anhand der Adresse und des Wirtschaftszweigs. Die BCC werden über den Firmennamen, der „Coordination“ beinhaltet, und den Wirtschaftszweig selektiert. Für die DFC kann kein geeignetes Verfahren gefunden werden, welches ausschließlich Finanzierungsgesellschaften herausfiltert, da die Auswahl nur anhand des Wirtschaftszweigs möglich ist. In den Niederlanden geben aber 55.154 (33,9 v. H.) der Unternehmen eine Tätigkeit an, die dem grundsätzlich für Finanzierungsgesellschaften in Frage kommenden NACE Code 6523 zugeordnet wird. ${ }^{586}$ Dass jede dritte niederländische Gesellschaft Finanzierungsaufgaben in der hier dargestellten Art und Weise ausübt, darf aber bezweifelt werden. Aus diesem

584 Vgl. Oestreicher, A. et al., Weiterentwicklung, 2006, S. 429.

585 Sec. 30 Finance Act 1987. Für eine ausführliche Darstellung vgl. Burwitz, G., Konzernfinanzierungsgesellschaften, 2005, S. 64-66.

${ }^{586} \mathrm{Vgl}$. auch die Übersicht über die Verteilung der Funktionen der Gesellschaften in Tabelle 31 . 
Grund beschränken sich die folgenden Ausführungen auf die Analyse der 122 IFSC und 67 BCC (Tabelle 39).

Der Vergleich des relativen Anteils der Bemessungsgrundlage mit der Anzahl der Gesellschaften verdeutlicht bereits die Bedeutung der Finanzierungsgesellschaften für das jeweilige nationale Steueraufkommen. Die IFSC machen, bezogen auf alle irischen Unternehmen grenzüberschreitend tätiger Konzerne, 9,3 v. H. der Gesellschaften und 34,7 v. H. der Bemessungsgrundlage aus. Dabei ist die Anzahl der IFSC unvollständig, da diese vornehmlich zum Konzernverbund von Geschäftsbanken gehören und damit nicht Bestandteil der AmadeusDatenbank sind. ${ }^{587}$ Für Belgien fällt der Vergleich weniger drastisch aus. Dort entfallen auf die BCC 7,4 v. H. der Bemessungsgrundlage und $0,6 \mathrm{v} . \mathrm{H}$. der Unternehmen.

Tabelle 39: Konzernfinanzierungsgesellschaften

\begin{tabular}{|llrrrr|}
\hline & & \multicolumn{2}{c}{ IFSC } & \multicolumn{2}{c|}{ BCC } \\
\cline { 2 - 6 } & absolut & $\begin{array}{c}\text { relativ zu irischen } \\
\text { Gesellschaften }\end{array}$ & absolut & $\begin{array}{c}\text { relativ zu belgischen } \\
\text { Gesellschaften }\end{array}$ \\
\hline alle & Gesellschaften & 122 & $5,4 \%$ & 67 & $0,2 \%$ \\
\hline $\begin{array}{l}\text { internationale Konzern- } \\
\text { gesellschaften mit Daten }\end{array}$ & \begin{tabular}{l} 
Gesellschaften \\
\cline { 2 - 6 }
\end{tabular} & 49 & $9,3 \%$ & 53 & $0,6 \%$ \\
\hline
\end{tabular}

Quelle: eigene Berechnungen

Die Auswahl der Finanzierungsgesellschaften nach den oben dargelegten Kriterien erscheint zwar plausibel, beweist aber nicht, dass in den entsprechenden Konzernen eine Funktionsverlagerung wirklich stattfindet. Da auf der Basis der Angaben der Amadeus-Datenbank nicht nachvollzogen werden kann, wer der Geber des den Konzerngesellschaften zur Verfügung stehenden Fremdkapitals ist, wird im Folgenden der Verschuldungsgrad der Gesellschaften analysiert. Ausgangspunkt bildet dabei die Überlegung, dass die oben dargestellte Gestaltung (Abbildung 21) stets eine Fremdfinanzierung der Konzerntochtergesellschaften vorsieht. Demzufolge sollte der Verschuldungsgrad der Tochtergesellschaften von Konzernunternehmungen, die über eine Finanzierungsgesellschaft verfügen, höher sein als bei den übrigen Unternehmensgruppen. Natürlich ist auch eine Fremdfinanzierung der Tochtergesellschaften ohne die Existenz einer Finanzierungsgesellschaft möglich und ggf. steuerlich vorteilhaft; im Mittel sollte jedoch der Verschuldungsgrad innerhalb der Konzerne höher sein, die über Finanzierungsgesellschaften ihre Tochtergesellschaften mit dem benötigten Kapital ausstatten.

In Tabelle 40 sind die Ergebnisse eines Vergleichs des durchschnittlichen Verschuldungsgrades und der durchschnittlichen Eigenkapitalquote von Konzernen mit und ohne Finanzierungsgesellschaften wiedergegeben. Der Vergleich des

587 Vgl. Kapitel D.III. 
Mittelwerts und des Medians der Eigenkapitalquote und des Verschuldungsgrads zeigt bei Konzernen mit Finanzierungsgesellschaften einen höheren durchschnittlichen Verschuldungsgrad. Im Mittel ist die Eigenkapitalquote der Konzerne mit einer Finanzierungsgesellschaft ungefähr 4 Prozentpunkte niedriger. Für die einhundert größten Konzerne in Europa beträgt die Differenz sogar fast 13 Prozentpunkte. Zwar sind Konzerne nicht direkt miteinander vergleichbar, da auch andere Faktoren wie z. B. der Sitz der Gesellschaften über die unterschiedlichen nationalen Regelungen zur Gesellschafter-Fremdfinanzierung den Verschuldungsgrad beeinflussen, ${ }^{588}$ doch sollten sich die externen Faktoren zwischen den analysierten Konzernen ausgleichen, sodass der signifikante Unterschied zwischen den beiden Gruppen auch auf den Einsatz von Finanzierungsgesellschaften zurückzuführen ist.

Tabelle 40: Verschuldungsgrad im Konzern

\begin{tabular}{|lccccc|}
\hline & Konzern- & \multicolumn{2}{c}{ Eigenkapitalquote } & \multicolumn{2}{c|}{ Verschuldungsgrad } \\
Top 100: & gesellschaften & Mittelwert & Median & Mittelwert & Median \\
\hline kein BCC/IFSC & 5.837 & 0,4744 & 0,4146 & $14.274,3825$ & 141,1902 \\
BCC/IFSC & 2.093 & 0,3487 & 0,2395 & $46.006,7576$ & 317,6190 \\
Top 500: & & & & & \\
\hline kein BCC/IFSC & 24.772 & 0,4610 & 0,4036 & $9.542,3954$ & 141,1902 \\
BCC/IFSC & 3.789 & 0,3975 & 0,3122 & $26.938,2977$ & 220,2614 \\
Alle: & & & & & \\
\hline kein BCC/IFSC & 71.551 & 0,4447 & 0,3928 & $4.360,4322$ & 154,6099 \\
BCC/IFSC & 4.145 & 0,4020 & 0,3189 & $24.825,9085$ & 213,5431 \\
\hline
\end{tabular}

Quelle: eigene Berechnungen

588 Vgl. für eine Übersicht über die unterschiedlich hohen „safe-heavens“ in den einzelnen Mitgliedstaaten Jacobs, O.H. (Hrsg.), Unternehmensbesteuerung, 2002, S. 807; Gouthière, B. (Hrsg.), in: ET, 2005, S. 367-451. 


\section{F. Quantitative Auswirkungen der Einführung der konsolidierten Besteuerung}

\section{I. Überblick}

Theoretische Überlegungen zur Ausgestaltung einer formelhaften Gewinnaufteilung können Empfehlungen nur unter Vorbehalt liefern. Insbesondere die Festlegung der Mindestbeteiligungsquote oder die Auswahl und Gewichtung der Formelfaktoren stellt auch eine politische Entscheidung dar. Gleichzeitig haben diese Parameter einen starken Einfluss auf die Veränderung der Bemessungsgrundlage in den jeweiligen Mitgliedstaaten, was eine Festlegung zusätzlich erschweren dürfte. Eine breitere auf empirischen Berechnungen basierende Entscheidungsgrundlage zur Verfügung zu stellen, ist Ziel dieses Kapitels.

Dazu werden im Rahmen einer statischen Mikrosimulation alternativen Optionen der konsolidierten Körperschaftsteuer-Bemessungsgrundlage mit dem geltenden Steuerrecht in den einzelnen Mitgliedstaaten verglichen, wobei als Zielgröße die Veränderung der Bemessungsgrundlage bzw. die Änderung der Steuereinnahmen herangezogen wird. ${ }^{589}$ Zunächst wird ein Basisfall definiert und die Änderung der Bemessungsgrundlage in drei Einzeleffekte zerlegt, die dann getrennt voneinander analysiert werden. Anschließend werden die dem Basisfall zugrunde liegende Annahmen bzgl. des interperiodischen Verlustausgleichs und der Behandlung der Finanzerträge kritisch hinterfragt. Im dritten Schritt werden die in Tabelle 41 aufgeführten Parameter ceteris paribus variiert und deren Einfluss auf die Änderung der Bemessungsgrundlage quantifiziert.

Tabelle 41: Variable Parameter bei der Analyse des Aufkommenseffekts der konsolidierten Körperschaftsteuer-Bemessungsgrundlage

\begin{tabular}{|c|c|c|c|c|}
\hline ZielgröBe & Parameter/Annahmen & mögliche Werte & Basisfall & Kapitel \\
\hline \multirow{4}{*}{$\begin{array}{l}\text { Änderung der } \\
\text { Bemessungs- } \\
\text { grundlage }\end{array}$} & $\begin{array}{l}\text { Höhe der } \\
\text { Mindestbeteiligung }\end{array}$ & 50,01 bis $100 \mathrm{v} . \mathrm{H}$. & 50,01 v. H. & F.III.2 \\
\hline & Aufteilungsformel: & & & \\
\hline & $\begin{array}{l}\text { Gewichtung der } \\
\text { Aufteilungsfaktoren }\end{array}$ & 0 bis $100 \mathrm{v} . \mathrm{H}$. & jeweils 33,33 v. $\mathrm{H}$. & F.III.3.a) \\
\hline & Aufteilungsfaktoren & $\begin{array}{l}\text { 1. Vermögen, Umsatz, Lohnsumme } \\
\text { 2. Vermögen, Umsatz, } \\
\text { Mitarbeiteranzahl }\end{array}$ & $\begin{array}{l}\text { Lohnsumme, } \\
\text { Vemögen, Umsatz }\end{array}$ & F.III.3.b) \\
\hline $\begin{array}{l}\text { Änderung der } \\
\text { Steuerzahllast }\end{array}$ & $\begin{array}{l}\text { Zugrunde liegendes } \\
\text { Besteuerungsprinzips }\end{array}$ & $\begin{array}{l}\text { 1. Quellenprinzip } \\
\text { 2. Wohnsitzprinzip }\end{array}$ & & F.IV \\
\hline
\end{tabular}

Quelle: eigene Darstellung

589 Zur Einordnung des gewählten Simulationsmodells vgl. Peichl, A., in: Finanzwissenschaftliche Diskussionsbeiträge, 2005, 1, S. 6. Für eine ausführliche Übersicht zur Mikrosimulation in der Steuerpolitik siehe Spahn, P.B. et al., Mikrosimulation, 1992. 


\section{Methodik der Berechnungen}

\section{Ermittlung der konsolidierten Bemessungsgrundlage}

\section{a) Ermittlung des aufzuteilenden Gewinns}

Der in den USA üblichen Differenzierung des Gewinns nach „business“ und „non-business income“ wird aus zwei Gründen nicht gefolgt. ${ }^{590}$ "Erstens hat die theoretische Analyse erhebliche Vorbehalte gegen eine Aufteilung des Einkommens deutlich gemacht. ${ }^{591}$ So werden bestehende unternehmensteuerliche Hindernisse mit einer Aufspaltung des Einkommens nicht vollständig beseitigt. Zudem bietet die Abgrenzung der Einkommensbestandteile erneut einen Angriffspunkt für steuerplanerische Gestaltungen. Zweitens genügen die Informationen der Amadeus-Datenbank nicht, um alle notwendigen Gewinnbestandteile separieren zu können. Die aggregierten Jahresabschlüsse weisen lediglich das Ergebnis der gewöhnlichen Geschäftstätigkeit, das Finanzergebnis und das außerordentliche Ergebnis getrennt aus. ${ }^{592}$ Aufteilungsfähiger Gewinn ist damit der gesamte Gewinn vor Steuern.

Tabelle 42: Anteil der Dividenden am Finanzergebnis

\begin{tabular}{|l|c|c|c|}
\hline Umsatz & weniger als 2,5 Mio. & 2,5 bis unter 50 Mio. & 50 Mio. und mehr \\
\hline $\begin{array}{l}\text { Anteil der Dividenden } \\
\text { am Finanzergebnis }\end{array}$ & $31,39 \mathrm{v} . \mathrm{H}$. & $38,28 \mathrm{v} . \mathrm{H}$. & $54,93 \mathrm{v} . \mathrm{H}$. \\
\hline
\end{tabular}

Quelle: eigene Berechnung, Deutsche Bundesbank, Verhältniszahlen, 2004, S. 18

In dem Gewinn vor Steuern sind noch Dividendeneinkünfte enthalten. Eine Doppelbesteuerung dieser Gewinne ist gemäß der Mutter-Tochter-Richtlinie zu vermeiden ${ }^{593}$ Die Mehrzahl der Mitgliedstaaten verhindert dies durch eine Freistellung der Dividendeneinkünfte. Damit ist der Gewinn vor Steuern um die Dividendeneinkünfte zu kürzen. Zwar enthält die Gewinn-und-VerlustRechnung der Amadeus-Datenbank einen Posten für Finanzerträge, darin enthalten sind aber auch Zinseinkünfte. Die Höhe der Dividendeneinkünfte ist damit zunächst unbekannt. Sie wird pauschal nach Angaben der Bundesbank gestaffelt nach der Höhe der Umsätze der Unternehmen geschätzt (Tabelle 42). Da die pauschale Kürzung der Bemessungsgrundlage um die Dividenden einem

590 Siehe zur Definition dieser Einkommensbestandteile Sec. 1 a) und e) UDITPA.

591 Vgl. hierzu die Ausführungen des Kapitels C.II.3.b).

592 Vgl. zur Gliederung der Gewinn-und-Verlust-Rechnung der Amadeus-Datenbank Abbildung 9.

593 Erfolgt eine Vollkonsolidierung der Einzelabschlüsse, betrifft dies nur Dividenden von Gesellschaften, die nicht Bestandteil des Konzernkreises sind. Konzerninterne Gewinnausschüttungen werden im Rahmen der Konsolidierung eliminiert. 
hohen Unsicherheitsfaktor unterliegt, wird im Rahmen einer Variationsrechnung nur der Gewinn der gewöhnlichen Geschäftstätigkeit als Aufteilungsmasse herangezogen. ${ }^{594}$

Unterschreitet die geforderte Mindestbeteiligungsquote $100 \mathrm{v}$. H., können auch Minderheitsgesellschafter an einem Unternehmen des Konsolidierungskreises beteiligt sein. In diesem Fall wird dennoch der gesamte Gewinn im Rahmen der konsolidierten Besteuerung berücksichtigt. Eine anteilige Zurechnung des Gewinns proportional zur Beteiligungshöhe ist nicht vorgesehen. Damit werden die Minderheitsgesellschafter den Mehrheitsbeteiligten gleichgestellt. D. h., sie profitieren einerseits von den Vorteilen einer konsolidierten Besteuerung, andererseits müssen sie aber auch für Steuerzahlungen aufkommen, die nach bisherigem Recht zu diesem Zeitpunkt noch nicht angefallen wären. ${ }^{595}$

Tabelle 43: Anpassung der Gewinn-und-Verlust-Rechnung bei Organträgern und Organgesellschaften

\begin{tabular}{|c|c|c|}
\hline & $\begin{array}{c}\text { Gewinn der gewöhnlichen } \\
\text { Geschäftstätigkeit }\end{array}$ & Gewinn vor Steuern \\
\hline $\begin{array}{l}\text { Separate } \\
\text { Accounting }\end{array}$ & $\begin{array}{l}\text { Organträger: } \\
\text { Der Gewinn der gewöhnlichen Geschäftstätig- } \\
\text { keit der Organgesellschaft wird dem direkten } \\
\text { Anteilseigner zugerechnet } \\
\text { Organgesellschaft: } \\
\text { Der Gewinn der gewöhnlichen Geschäftstätig- } \\
\text { keit der Organgesellschaft wird gleich null } \\
\text { gesetzt. }\end{array}$ & $\begin{array}{l}\text { Organträger: } \\
\text { Der Gewinn der Organgesellschaft ist bei dem } \\
\text { „unbekannten“ Organträger bereits enthalten, } \\
\text { sodass keine Anpassung notwendig ist. } \\
\text { Organgesellschaft: } \\
\text { Der Gewinn der Organgesellschaft wird in } \\
\text { Höhe der Gewinnabführung gekürzt. }\end{array}$ \\
\hline $\begin{array}{l}\text { Formula } \\
\text { Apportionment }\end{array}$ & $\begin{array}{l}\text { Keine Anpassungen notwendig, da der } \\
\text { Einzelabschluss des Organträgers und der } \\
\text { Organgesellschaft nur den ,eigenen“ Gewinn } \\
\text { der gewöhnlichen Geschäftstätigkeit enthält. }\end{array}$ & $\begin{array}{l}\text { Organträger: } \\
\text { Der Gewinn der Organgesellschaft ist in der } \\
\text { GuV des „unbekannten“ Organträgers bereits } \\
\text { enthalten, sodass keine Anpassung notwendig } \\
\text { ist. } \\
\text { Organgesellschaft: } \\
\text { Der Gewinn der Organgesellschaft wird in } \\
\text { Höhe der Gewinnabführung gekürzt. }\end{array}$ \\
\hline
\end{tabular}

Quelle: eigene Darstellung

Die Begründung einer Organschaft setzt bzw. setzte in Deutschland und Österreich einen Gewinnabführungsvertrag voraus. Aus dem Verweis des $\S 14$ Abs. 1 S. $1 \mathrm{KStG}$ auf $\S 291$ AktG ist bereits die Anknüpfung an das Gesellschafts- bzw. Zivilrecht ersichtlich. ${ }^{596}$ Dementsprechend geht mit der steuerlichen Organschaft immer eine gesellschaftsrechtliche Ergebnisübernahme einher, die sich in der handelsrechtlichen Bilanzierung widerspiegelt. Der Ausweis dieser Gewinnab-

\footnotetext{
594 Vgl. dafür Kapitel F.III.1.d). Mit der geänderten Gewinndefinition müssen auch die Aufteilungsfaktoren angepasst werden. Vgl. hierzu die Ausfuihrungen des Kapitels F.II.2.

595 Zur Problematik der Behandlung von Minderheitsgesellschaftern vgl. Kapitel C.II.2.a)( $\gamma$ ).

596 Vgl. Orth, M., in: Oestreicher, Konzernbesteuerung, 2005, S. 132.
} 
führung erfolgt bei dem Organträger und den Organgesellschaften an unterschiedlichen Positionen in der Gewinn-und-Verlust-Rechnung. ${ }^{57}$ In der verkürzten Gewinn-und-Verlust-Rechnung der Amadeus-Datenbank sind die Erträge aus Gewinnabführungsverträgen bzw. die Aufwendungen aus Verlustübernahmen Bestandteil des Finanzergebnisses des Organträgers. Bei der Organgesellschaft hingegen zählen die korrespondierenden Buchungen zum außerordentlichen Ergebnis, das nicht in die konsolidierte Bemessungsgrundlage einfließt. ${ }^{598}$ Diese Konstellation erfordert eine Anpassung der Ergebnisse der Mitglieder der Organschaften, um stets eine einmalige Berücksichtigung der Gewinne sicherzustellen. Tabelle 43 fasst die Anpassungsschritte bei dem Organträger und der Organgesellschaft in Abhängigkeit vom verwendeten Gewinnbegriff zusammen.

Problematisch ist die dargestellte Anpassung der Gewinn-und-Verlust-Rechnung von Mitgliedern einer Organschaft, wenn Organgesellschaft und Organträger nicht demselben Konsolidierungskreis angehören. Dies ist dann der Fall, wenn zwar die Anforderungen der finanziellen Eingliederung der Organschaft erfüllt werden, nicht aber die geforderte Mindestbeteiligungsquote der einheitlichen Körperschaftsteuer-Bemessungsgrundlage erreicht wird. Dadurch könnten Verluste zwischen zwei getrennten Konsolidierungskreisen verrechnet werden. Tabelle 44 zeigt jedoch, dass in über 85 v. H. der Fälle die Organgesellschaften vollständig oder fast vollständig finanziell eingegliedert sind. Im Umkehrschluss stellt damit eine falsche Verlustverrechnung die Ausnahme dar, die sich zudem lediglich bei der Variation der Mindestbeteiligungsquote auswirken kann.

Tabelle 44: Direkte Beteiligung der Organträger an den Organgesellschaften

\begin{tabular}{|l|cccccccccc|c|}
\hline Beteiligungsquote in v. H. $>$ & 95 & 90 & 85 & 80 & 75 & 70 & 65 & 60 & 55 & 50 & Summe \\
\hline Anzahl absolut & 994 & 45 & 26 & 7 & 21 & 23 & 9 & 7 & 11 & 21 & 1.164 \\
Anzahl relativ in v. H. & 85 & 4 & 2 & 1 & 2 & 2 & 1 & 1 & 1 & 2 & 100 \\
\hline
\end{tabular}

Quelle: eigene Darstellung

\section{b) Konsolidierung der Einzelabschlüsse}

Die Konsolidierung der Einzelabschlüsse ist wesentliches Merkmal der einheitlichen konsolidierten Bemessungsgrundlage. Zwar wird seitens der Europäischen Union keine Vollkonsolidierung angestrebt, eine Zwischenergebniseliminierung ist jedoch fester Bestandteil der einheitlichen (konsolidierten) Körperschaftsteuer-Bemessungsgrundlage. Schließlich garantiert sie, dass Ver-

\footnotetext{
597 Vgl. Tabelle 23.

598 Dadurch wird sichergestellt, dass bei einer einperiodigen Betrachtungsweise die Ergebnisse nicht durch Einzeleffekte verzerrt werden.
} 
rechnungspreisgestaltungen zwischen den Mitgliedern des Konsolidierungskreises keinen Effekt mehr auf das steuerliche Ergebnis haben. ${ }^{599}$

Dieser Vorgabe kann die empirische Analyse nicht gerecht werden. Sie muss sich mit dem Konzernsummenabschluss begnügen. Da die Amadeus-Datenbank nur Jahresabschlussinformationen zur Verfügung stellt, sind Angaben zu internen Lieferungs- und Leistungstransaktionen, Schuldverhältnissen und Beteiligungsbuchwerten nicht vorhanden. Eine Vollkonsolidierung, aber auch eine alleinige Zwischenergebniseliminierung der Einzelergebnisse ist somit ausgeschlossen. Alternativ wäre ein Rückgriff auf die in der Amadeus-Datenbank hinterlegten konsolidierten Konzernabschlüsse denkbar. ${ }^{600}$ Aber auch dieser Ansatz scheitert aus folgenden Gründen:

1. Der handelsrechtliche und der steuerliche Konsolidierungskreis weichen voneinander $a b .^{601}$ Gesellschaften mit Sitz außerhalb der Europäischen Union sind im handelsrechtlichen Konzernabschluss enthalten, dürfen aber bei einer formelhaften Gewinnaufteilung ,at water's edge“ nicht berücksichtigt werden. Darüber hinaus können in den handelsrechtlichen Konzernabschluss

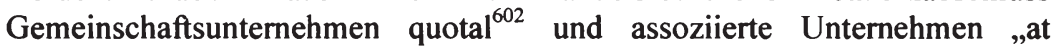
equity ${ }^{\text {“603 }}$ einbezogen werden.

2. Für europäische Teilkonzerne, die sich von der Aufstellung eines Teilkonzernabschlusses befreit haben, liegt kein Konzernabschluss vor. ${ }^{604}$

3. Die Anzahl der in der Amadeus-Datenbank hinterlegten Konzernabschlüsse ist gering. Insgesamt existieren nur 29.500 Konzernabschlüsse, von denen über 94 v. H. auf britische, niederländische und irische Konzernmuttergesellschaften entfallen.

4. Die Verwendung des handelsrechtlichen Konzernabschlusses würde eine Analyse unterschiedlicher Mindestbeteiligungsquoten ausschließen.

Dementsprechend werden die empirischen Berechnungen auf der Basis von Konzernsummenabschlüssen durchgeführt. Dies hat zur Folge, dass die ertragswirksamen Konsolidierungsschritte unterbleiben. Im Vergleich zu den Vorschlägen der Europäischen Kommission fehlt damit einzig die Eliminierung der

599 Vgl. zu den Vorschlägen der Europäischen Union Kapitel C.II.3.c).

${ }^{600}$ Diese Vorgehensweise wählten Sheffrin und Fulcher, um die Auswirkungen einer "worldwide unitary combination" in den USA zu untersuchen. Vgl. Sheffrin, S./Fulcher, J., in: McLure, State, 1984, S. 204-208.

$601 \mathrm{Vgl}$. zur Abgrenzung des handelsrechtlichen Konsolidierungskreises Küting, K./Weber, C.-P., Konzernabschluss, 2006, S. 129-150.

$602 \S 310 \mathrm{HGB}$, IAS 31 .

$603 \S 311$ HGB, IAS 28.

${ }^{604}$ In Deutschland ist der befreiende Konzernabschluss in $\S 292$ HGB i. V. m. der KonbefrV geregelt. 
Zwischenergebnisse. ${ }^{605}$ Dass dadurch nicht unbedingt große Verzerrungen der Ergebnisse zu erwarten sind, verdeutlicht die folgende Überlegung: Der Erfolg der wirtschaftlichen Einheit wird langfristig durch eine Zwischenergebniseliminierung nicht beeinflusst. Sie bewirkt lediglich eine spätere Realisierung der Gewinne. Dieser temporäre Effekt ist einmalig, wenn ein konstanter Warenfluss durch den Konzern erfolgt und sich die Verrechnungspreise im Zeitablauf nicht ändern. In diesem Fall weist die von den Produkten gleichmäßig durchlaufene Prozesskette einen konstanten Zwischengewinn auf, der nur eine einmalige Wirkung besitzt, da in den Folgeperioden der $\mathrm{zu}$ eliminierende Zwischengewinn dem anteiligen Gewinn entspricht, der durch den Verkauf der Waren an fremde Dritte erfolgswirksam realisiert wird. ${ }^{606}$ Auf den einzelnen Konzern mag die Annahme eines konstanten Warenflusses und gleich bleibender Verrechnungspreise nicht haltbar sein, doch sollten sich die Unterschiede über die Vielzahl der betrachteten Konzerne aus- bzw. angleichen.

\section{c) Einperiodige Betrachtungsweise}

Besonders für Gesellschaften aus Deutschland, Österreich und den Niederlanden ist die Verfügbarkeit von Jahresabschlüssen in der Amadeus-Datenbank schlecht. ${ }^{607}$ Um die Datenbasis zu verbreitern und aussagefähige Berechnungsergebnisse $\mathrm{zu}$ generieren, werden Jahresabschlüsse mehrerer Berichtsperioden miteinander kombiniert. ${ }^{608}$ Diese Vorgehensweise verhindert zugleich eine Längsschnittanalyse und damit die Abbildung der Verlustverrechnung in den Folgeperioden. Verluste, die in der laufenden Periode nicht verrechnet werden können, gelten damit als endgültig „verloren“. Dadurch wird die Abnahme der Bemessungsgrundlage bei Einführung der formelhaften Gewinnaufteilung tendenziell überbewertet. Denn mit der stark ausgeweiteten Verlustverrechnung, insbesondere über die Grenze hinweg, werden mehr Verluste in der laufenden Periode berücksichtigt, und zugleich gelten weniger Verluste als endgültig nicht verrechenbar. Um dieser Verzerrung entgegenzuwirken, werden die vorgetragenen Verluste abdiskontiert in der laufenden Periode berücksichtigt. Da die pauschale Verlustverrechnung ausschließlich auf Annahmen beruht, findet sie im Basisfall jedoch keine Anwendung und wird lediglich im Rahmen einer Variationsrechnung abgebildet.

605 Vgl. Europäische Kommission, Fragen zur Konzernbesteuerung, 2006, S. 9.

${ }^{606}$ Fasst man die unterschiedlichen Buchungssätze zusammen, wird der Zwischengewinn in den Folgejahren gegenüber dem Ergebnisvortrag immer erfolgsneutral eliminiert.

${ }^{607}$ Für niederländische Gesellschaften gilt diese Einschränkung nur für die $\mathrm{Ge}-$ winn-und-Verlust-Rechnung. Vgl. Tabelle 17.

${ }^{608}$ Vgl. zur Datenlage und Datenaufbereitung Kapitel D.III und D.IV. 


\section{Aufteilung des Gesamterfolgs}

Der Basisfall teilt die für den Gesamtkonzern ermittelte Bemessungsgrundlage anhand der Massachusetts-Formula auf, ${ }^{609}$ welche auch in den USA im Rahmen der Gewinnabgrenzung zwischen den Bundesstaaten vorgesehen ist. ${ }^{610}$ Davon abweichende Formeln werden im Rahmen der Variationsrechnungen analysiert, wobei die hier dargestellte Methodik der Auswahl und Bewertung der Formelfaktoren weiterhin Bestand hat. Die Massachusetts-Formula teilt die Bemessungsgrundlage den Konzerngesellschaften nach deren Anteil an dem Konzernvermögen, an der Konzernlohnsumme und an den Außenumsätzen zu. ${ }^{611}$ Jeder Aufteilungsfaktor geht mit gleichen Anteilen in diese Formel ein. Die Bewertung der Formelfaktoren hat sich grundsätzlich am aufzuteilenden Gewinn zu orientieren. Bestandteile der Aufteilungsfaktoren sollten nur das Vermögen, der Lohn und der Umsatz sein, der im Zusammenhang mit dem aufzuteilenden Gewinn steht. Da das US-amerikanische Recht grundsätzlich eine Trennung zwischen „bussiness“ und „non-business income“ vornimmt, dieser aber nicht gefolgt wird, ${ }^{612}$ weicht die hier vorgenommene Definition der Aufteilungsfaktoren zwangsweise von der amerikanischen ab. Neben diesen systembedingten Unterschieden existieren Differenzen, die auf die existierende Datenlage zurückzuführen sind. Denn der Wertansatz der Aufteilungsfaktoren unterscheidet sich grundsätzlich von dem in der Bilanz bzw. der Gewinn-undVerlust-Rechnung. Da aber nur Jahresabschlussinformationen zur Verfügung stehen, kann im Rahmen der empirischen Berechnungen nur auf diese zurückgegriffen werden. Im Folgenden werden die wesentlichen Unterschiede herausgearbeitet und die verzerrenden Effekte eingeschätzt.

Der Aufteilungsfaktor Umsatz unterscheidet sich in zwei wesentlichen Punkten von den in der handelsbilanziellen Gewinn-und-Verlust-Rechnung ausgewiesenen Umsatzerlösen. Erstens werden die Umsatzerlöse im Rahmen der Gewinnaufteilung nach dem Bestimmungslandprinzip („destination rule“) dem Land zugerechnet, indem sie erzielt werden. Liefert ein Konzernunternehmen Güter an einen fremden Dritten mit Sitz in einem anderen Mitgliedstaat der EU, in dem der Konzern einen steuerlichen Anknüpfungspunkt vorweist, müssen die erzielten Umsatzerlöse dem Staat des fremden Dritten und nicht dem Staat des liefernden Unternehmens zugeordnet werden. Ist das einheitliche Unternehmen

\footnotetext{
609 Vgl. a. Kapitel C.II.4.

${ }^{610}$ Siehe Sec. 9 UDITPA. Die Mehrzahl der Bundesstaaten jedoch folgt nicht mehr der Gleichgewichtung der drei Aufteilungsfaktoren. Vgl. hierzu Abbildung 4. Für eine Übersicht über die Regelungen in den einzelnen Bundesstaaten siehe American Chamber of Commerce/PricewaterhouseCoopers (Hrsg.), USA, 2001, S. 153-154.

611 Vgl. zur Auswahl und Bewertung der Formelfaktoren Kapitel C.II.4.

${ }^{612}$ Vgl. Kapitel F.II.1.a) und C.II.3.b).
} 
in diesem Staat jedoch nicht körperschaftsteuerpflichtig, greift die so genannte „throwback rule“, welche die Umsätze wieder dem Ursprungsland zuweist. Zweitens werden nur Außenumsätze der wirtschaftlichen Einheit berücksichtigt, denn der Umsatzfaktor dient der Berücksichtigung der Nachfrageseite der Wertschöpfung. ${ }^{613}$ Dementsprechend sind Umsätze, die aus internen Lieferungs- und Leistungsbeziehungen resultieren, $\mathrm{zu}$ vernachlässigen. Eine Unterscheidung zwischen Außen- und Innenumsätzen ist jedoch auf der Basis der Angaben der Gewinn-und-Verlust-Rechnung nicht möglich. Abbildung 22 verdeutlicht die aufgrund des Bestimmungslandprinzips und der alleinigen Berücksichtigung von Außenumsätzen abweichende Definition der Umsätze als Aufteilungsfaktor gegenüber der handelsrechtlichen Bilanzierung.

Abbildung 22: Abbildung des Aufteilungsfaktors Umsatz

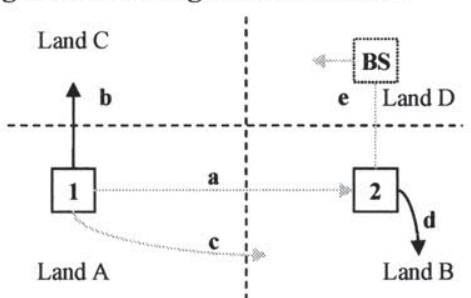

\footnotetext{
a falsch - Die Innenumsätze werden nicht berücksichtigt.

b richtig - Die Außenumsätze sind aufgrund der ,throwback rule“ steuerpflichtig in Land A.

c falsch - Die Außenumsätze sind nach dem Bestimmungslandprinzip in Land B zu versteuern.

d richtig - Die Außenumsätze sind in Land B zu versteuern.

e falsch - Außenumsatz, der aufgrund einer Betriebsstätte in Land D dort zu versteuern ist.
}

Quelle: eigene Darstellung

Im Rahmen der empirischen Berechnungen kann nur der Gesamtumsatz eines Konzernunternehmens herangezogen werden. Dies hat zur Folge, dass vertikal integrierte Gesellschaften, deren Wertschöpfung ausschließlich der Versorgung anderer Konzernunternehmen dient, Steuersubstrat auf der Basis des Aufteilungsfaktors Umsatz zugewiesen bekommen, obwohl sie effektiv keine Außenumsätze erwirtschaften. Klassische Produktionsstandorte bekämen zu viel Steuersubstrat zugewiesen, und die Zunahme (Abnahme) an Steuersubstrat aufgrund des Übergangs zur formelhaften Gewinnaufteilung würde überschätzt (unterschätzt) werden. ${ }^{614}$ Demgegenüber fällt der systematische Fehler aufgrund der Missachtung des Bestimmungslandprinzips wohl geringer aus. Denn bei Konzernen mit wenigen Tochtergesellschaften in unterschiedlichen Mitgliedstaaten werden die meisten Umsatzerlöse aufgrund der "throwback rule“ korrekt

$613 \mathrm{Vgl}$. die Ausführungen zum ,supply-demand approach“ in Kapitel C.II.4.b)( $\delta$ ).

${ }^{614}$ Eine Übersicht über die Verteilung der Gesellschaften hinsichtlich ihrer Funktionen in den einzelnen Mitgliedstaaten kann der Tabelle 31 entnommen werden. 
zugeordnet. Bei größeren Unternehmensverbunden ist ein Vertrieb über Tochterkapitalgesellschaften wahrscheinlich, sodass auch hier nur die Verzerrung aus der Berücksichtigung der Innenumsätze zum Tragen kommt.

Unter der Lohnsumme werden in den USA Löhne, Gehälter, Kommissionen und weitere Zuwendungen wie z. B. Pensionen verstanden. Darüber hinaus können auch bestimmte Sachbezüge in den Aufteilungsfaktor Lohnsumme einfließen. Zahlungen an unabhängige Kontraktoren und an Mitarbeiter, die nur in einem Staat tätig werden, in dem das betreffende Unternehmen nicht körperschaftsteuerpflichtig ist, werden hingegen in der Faktorgröße nicht berücksichtigt. Eine derart differenzierte Abbildung der Lohnsumme ist im Rahmen der empirischen Analyse nicht möglich. So entspricht der Lohnsummenfaktor dem Personalaufwand, der im Gesamtkostenverfahren ausgewiesen wird. ${ }^{615}$ Die größten Verzerrungen aufgrund der abweichenden Definition des Lohnsummenfaktors sind aus der Berücksichtigung nicht im Sitzstaat der Gesellschaft tätiger Mitarbeiter zu erwarten. Die Vernachlässigung ${ }^{616}$ oder zusätzliche Berücksichtigung ${ }^{617}$ von einzelnen Lohnbestandteilen sind im Vergleich zur gesamten Lohnsumme hingegen von untergeordneter Bedeutung.

Anstelle der Lohnsumme wird im Rahmen der Variationsrechnung auch die Mitarbeiteranzahl als Aufteilungsfaktor herangezogen. Damit wird zum einen eine Vergleichbarkeit der Ergebnisse mit anderen Studien sichergestellt, ${ }^{618}$ zum anderen sollen Verzerrungen aufgrund abweichender Lohnniveaus in den einzelnen Mitgliedstaaten ausgeblendet werden. ${ }^{619}$

Der Aufteilungsfaktor Vermögen schließt grundsätzlich alle Vermögenswerte ein, die das Unternehmen zur Einkommenserzielung einsetzt. ${ }^{620}$ Dabei ist es unerheblich, ob die Vermögensgegenstände im Eigentum des Unternehmens stehen oder auf der Basis eines Miet- und Pachtverhältnisses genutzt werden. Um Verzerrungen zu vermeiden, ist Vermögen, auf dessen Grundlage Einkommen erwirtschaftet wird, das aber einer direkten Zuweisung zu den Steuer-

\footnotetext{
615 Sind keine Informationen zur Mitarbeiteranzahl, der Lohnsumme oder beidem vorhanden, werden diese Angaben manuell ergänzt. Vgl. hierzu D.IV.3.b)( $\gamma)$.

616 Hier sind vor allem Pensionszahlungen oder Sachbezüge der Mitarbeiter angesprochen, die nicht im Personalaufwand enthalten sind.

${ }^{617}$ In einigen Bundesstaaten der USA fließen beispielsweise Zuwendungen an Vorstandsmitglieder nicht in die Lohnsumme mit ein.

618 Vgl. Fuest, C./Hemmelgarn, T./Ramb, F., Apportionment, 2006.

619 Vgl. McLure Jr., C.E./Weiner, J., in: Cnossen, Capital, 2000, S. 270. Vgl. hierzu auch Kapitel C.II.4.c)( $(\gamma)$.

${ }^{620}$ Vgl. Riecker, A., Körperschaftbesteuerung, 1996, S. 256.
} 
hoheiten unterliegt, nicht Bestandteil des Vermögensfaktors. ${ }^{621} \mathrm{Da}$ im Rahmen der empirischen Analyse eine Differenzierung zwischen „business“ und „nonbusiness income" nicht vorgenommen wird, zählen zu den Vermögenswerten das materielle und immaterielle Anlage- und Umlaufvermögen. Davon ausgenommen sind Zahlungsmittel und Forderungen, da sie nicht primär der Gewinnerzielung dienen. Zudem müssen die Beteiligungen an verbundenen Unternehmen herausgerechnet werden, da Dividendeneinkünfte steuerfrei vereinnahmt werden. Erneut lassen die aggregierten Jahresabschlussdaten eine eindeutige Identifizierung der einzelnen Bilanzpositionen nicht zu. Demzufolge werden die Beteiligungsbuchwerte pauschal herausgerechnet, indem das Vermögen um 86 v. H. der „other financial assets“ gekürzt wird. ${ }^{622}$

Eine territoriale Abgrenzung des Vermögens unterbleibt. Zwar dürften Vermögensgegenstände nur dem Staat ihrer Belegenheit bzw. Nutzung zugeordnet werden, doch ist diese Differenzierung nicht mit den empirischen Daten abzubilden.

Theoretisch korrekt wäre die Bewertung der Vermögensgegenstände zu Marktpreisen. ${ }^{623}$ Wegen der Schwierigkeiten bei der Bestimmung von Marktwerten treten in den USA an deren Stelle die historischen Anschaffungs- oder Herstellkosten. ${ }^{624}$ Im Rahmen der empirischen Analyse kann hingegen nur auf die Buchwerte zurückgegriffen werden. Zwar weichen diese unter Umständen erheblich von den Marktwerten der Vermögensgegenstände $a b$, doch entsprechen sie ihnen durchschnittlich eher als die historischen Anschaffungs- oder Herstellkosten. So bleiben Wertsteigerungen der Vermögensgegenstände bei dem Ansatz historischer Kosten stets unberücksichtigt. Bei dem Ansatz von Buchwerten hingegen ist teilweise ein Ansatz oberhalb der Herstell- und Anschaffungskosten möglich. ${ }^{65}$ Gleichzeitig werden Wertminderungen pauschal über Abschreibungen berücksichtigt.

Im Ergebnis umfasst das Vermögen das materielle und immaterielle Anlagevermögen abzüglich etwaiger Beteiligungen zuzüglich der Vorräte des Umlauf-

${ }^{621} \mathrm{Da}$ in den USA Lizenz- und Patentgebühren direkt zugerechnet werden, fließt auch das immaterielle Vermögen nicht in die Formel mit ein.

${ }^{622}$ Der Anteil der Beteiligungsbuchwerte an den „other financial assets“ wurde auf Basis der Verhältniszahlen aus Jahresabschlüssen deutscher Unternehmen von 2002 berechnet. Vgl. Deutsche Bundesbank, Verhältniszahlen, 2004, S. 18.

${ }^{623}$ Vgl. Kapitel C.II.4.c)( $\left.\alpha\right)$.

${ }^{624}$ Siehe Sec. 11 UDITPA.

${ }^{625}$ Für eine Übersicht über die wesentlichen Regeln für eine Fair-Value-Bewertung in den derzeit geltenden IFRS vgl. Wagenhofer, A., in: IRZ, 2006, S. 32; Hitz, J.-M., in: $W P g$, 2005, S. 1018-1021. 
vermögens zu Buchwerten. Nicht berücksichtigt werden geleaste oder gepachtete Vermögensgegenstände. ${ }^{626}$

\section{Datenlage}

Von den 833.650 Konzerngesellschaften verfügen lediglich $46,6 \mathrm{v}$. H. über Jahresabschlussinformationen. Diese Unternehmen sind aber nicht gleich über die 238.141 Konzerne verteilt. So setzen sich 36 v. H. der Konzerne ausschließlich aus Gesellschaften mit einem Jahresabschluss zusammen; in 41,7 v. H. der Konzerne verfügt kein Unternehmen über die erforderlichen Jahresabschlusspositionen. Zwar stellen 85.731 (36 v. H.) Konzerne mit vollständigen Daten noch eine breite Datenbasis dar, doch ist diese nicht unbedingt repräsentativ. ${ }^{627}$ Denn zum einem sind darin eine Vielzahl nationaler Konzerne enthalten, die vornehmlich ihren Sitz in Mitgliedstaaten haben, in denen die Datenlage überdurchschnittlich gut ist. Zum anderen steigt die Wahrscheinlichkeit, dass nicht alle Konzerngesellschaften Daten vorweisen können, mit der Größe der Konzerne an. Tabelle 45 stellt für die 16.962 grenzüberschreitend tätigen Konzerne dem Anteil der Konzernunternehmen mit Daten die Konzerngröße gemessen an der Anzahl der Konzerngesellschaften gegenüber.

Um die Verzerrungen zu minimieren, die aus der ungleichmäßigen Verteilung der Unternehmen mit Daten resultieren, werden auch Konzerne im Rahmen der empirischen Analyse berücksichtigt, zu deren Konsolidierungskreis Gesellschaften ohne hinterlegten Jahresabschluss gehören. Grundsätzlich besteht dabei die Gefahr, dass innerhalb eines grenzüberschreitenden Konzerns nur Unternehmen aus einem einzigen Mitgliedstaat über Angaben in der Bilanz und in der Gewinn-und-Verlust-Rechnung verfügen. Die Einführung der konsolidierten Körperschaftsteuer-Bemessungsgrundlage führt dann für diese Unternehmensverbunde zu keiner Umverteilung der Bemessungsgrundlage. Besonders drastisch wirkt sich dieser Effekt bei grenzüberschreitend tätigen Konzernen mit zwei Konzerngesellschaften aus. Um dieses zu verhindern, fließen in die Berechnungen nur Konzerne ein, deren Konzerngesellschaften zu über $50 \mathrm{v}$. H. Daten vorweisen können. Damit umfasst die Datenbasis der empirischen Analyse der Bemessungsgrundlagenänderung 8.346 Konzerne mit 82.925 Konzerngesellschaften. Tabelle 46 zeigt die Verteilung der Konzerne und Konzerngesellschaften in den einzelnen Mitgliedstaaten.

626 Von einem kostenorientierten Ansatz (,flow approach“) wird aufgrund der vielfach unvollständigen Gewinn-und-Verlust-Rechnung der Konzerngesellschaften abgesehen. Vgl. zu unterschiedlichen Bewertungsalternativen der Formelfaktoren Musgrave, P.B., in: McLure, State, 1984, S. 241; Oestreicher, A., Konzerngewinnabgrenzung, 1999, S. 190.

${ }^{627}$ Repräsentativ bezieht sich hier auf die 238.141 Konzerne. Für eine Beurteilung der Repräsentativität der in der Amadeus-Datenbank enthaltenen Unternehmen vgl. Kapitel D.III. 
Tabelle 45: Unternehmen mit Daten in v. H. nach Anzahl der Konzerngesellschaften

\begin{tabular}{|c|c|c|c|c|c|c|c|c|c|c|}
\hline \multirow{2}{*}{$\begin{array}{c}\text { Konzern- } \\
\text { gesellschaften }\end{array}$} & \multicolumn{10}{|c|}{ Anteil der Konzerngesellschaften mit Daten in v. H. } \\
\hline & $0-10$ & $>10-20$ & $>20-30$ & $>30-40$ & $>40-50$ & $>50-60$ & $>60-70$ & $>70-80$ & $>80-90$ & $>90-100$ \\
\hline 2 & $23 \%$ & & & & $45 \%$ & & & & & $31 \%$ \\
\hline 3 & $18 \%$ & & & $23 \%$ & & & $30 \%$ & & & $29 \%$ \\
\hline 4 & $13 \%$ & & $17 \%$ & & $17 \%$ & & & $27 \%$ & & $26 \%$ \\
\hline 5 & $11 \%$ & $13 \%$ & & $14 \%$ & & $18 \%$ & & $24 \%$ & & $20 \%$ \\
\hline 6 & $9 \%$ & $13 \%$ & & $15 \%$ & $12 \%$ & & $13 \%$ & & $19 \%$ & $19 \%$ \\
\hline 7 & $5 \%$ & $10 \%$ & $13 \%$ & & $11 \%$ & $12 \%$ & & $14 \%$ & $18 \%$ & $17 \%$ \\
\hline 8 & $6 \%$ & $9 \%$ & $10 \%$ & $10 \%$ & $7 \%$ & & $11 \%$ & $14 \%$ & $17 \%$ & $17 \%$ \\
\hline 9 & $4 \%$ & $8 \%$ & $9 \%$ & $8 \%$ & $8 \%$ & $8 \%$ & $11 \%$ & $12 \%$ & $18 \%$ & $13 \%$ \\
\hline 10 & $7 \%$ & $8 \%$ & $6 \%$ & $9 \%$ & $7 \%$ & $7 \%$ & $12 \%$ & $10 \%$ & $16 \%$ & $18 \%$ \\
\hline $11-15$ & $8 \%$ & $5 \%$ & $6 \%$ & $9 \%$ & $9 \%$ & $7 \%$ & $8 \%$ & $11 \%$ & $11 \%$ & $25 \%$ \\
\hline $16-20$ & $4 \%$ & $9 \%$ & $8 \%$ & $11 \%$ & $8 \%$ & $9 \%$ & $9 \%$ & $11 \%$ & $17 \%$ & $14 \%$ \\
\hline $21-30$ & $6 \%$ & $6 \%$ & $8 \%$ & $10 \%$ & $7 \%$ & $9 \%$ & $9 \%$ & $12 \%$ & $14 \%$ & $18 \%$ \\
\hline $31-50$ & $4 \%$ & $7 \%$ & $10 \%$ & $13 \%$ & $9 \%$ & $8 \%$ & $7 \%$ & $13 \%$ & $14 \%$ & $16 \%$ \\
\hline $51-100$ & $3 \%$ & $9 \%$ & $10 \%$ & $11 \%$ & $11 \%$ & $11 \%$ & $8 \%$ & $12 \%$ & $12 \%$ & $13 \%$ \\
\hline $101-500$ & $6 \%$ & $9 \%$ & $17 \%$ & $15 \%$ & $15 \%$ & $7 \%$ & $6 \%$ & $10 \%$ & $7 \%$ & $7 \%$ \\
\hline$>=501$ & & $29 \%$ & & & $14 \%$ & & $14 \%$ & $29 \%$ & $14 \%$ & \\
\hline Summe & $15 \%$ & $4 \%$ & $4 \%$ & $8 \%$ & $20 \%$ & $3 \%$ & $8 \%$ & $8 \%$ & $5 \%$ & $25 \%$ \\
\hline
\end{tabular}

Quelle: eigene Berechnungen

\section{Analyse der Bemessungsgrundlagenänderung}

\section{Basisfall}

\section{a) Gesamtergebnis}

Im Basisfall beträgt die geforderte Mindestbeteiligungsquote $>50 \mathrm{v} . \mathrm{H}$. Als Aufteilungsfaktoren werden zu gleichen Teilen das Vermögen, der Umsatz und die Lohnsumme herangezogen. Aufteilungsfähiger Gewinn ist der Gewinn vor Steuern. Maltesische Gesellschaften existieren in keinem Konzern. Die sechs zyprischen Gesellschaften weisen für 2003 in Summe unabhängig von der Ermittlungsmethode der Bemessungsgrundlage einen Verlust aus. Da im Basisfall ein intertemporaler Verlustausgleich nicht vorgesehen ist, entwickelt die konsolidierte Körperschaftsteuer-Bemessungsgrundlage für Zypern keine Aufkommenswirkung.

Die mit der Einführung der konsolidierten Körperschaftsteuer-Bemessungsgrundlage ausgeweitete Möglichkeit der Verlustverrechnung lässt die EUGesamtbemessungsgrundlage um 14,1 v. H. sinken (Abbildung 23, Tabelle 46). Von den 24 dargestellten Mitgliedstaaten kann lediglich Lettland einen Zuwachs verzeichnen. Auffällig ist, dass die Bemessungsgrundlage insbesondere der drei größten Volkswirtschaften der Europäischen Union, Deutschland, Frankreich und Großbritannien, nur unterdurchschnittlich sinkt. Ursächlich hierfür könnten die höheren effektiven Gewinnsteuersätze sein, die unter dem bestehenden System eine Gewinnverlagerung ins niedriger besteuerte Ausland be- 
günstigen. ${ }^{628}$ Außerdem könnte die Wahrscheinlichkeit, Verluste innerhalb einer großen Volkswirtschaft verrechnen zu können, steigen, wodurch der Effekt der ausgeweiteten Verlustverrechnungsmöglichkeit mit Einführung der konsolidierten Bemessungsgrundlage geringer ausfällt.

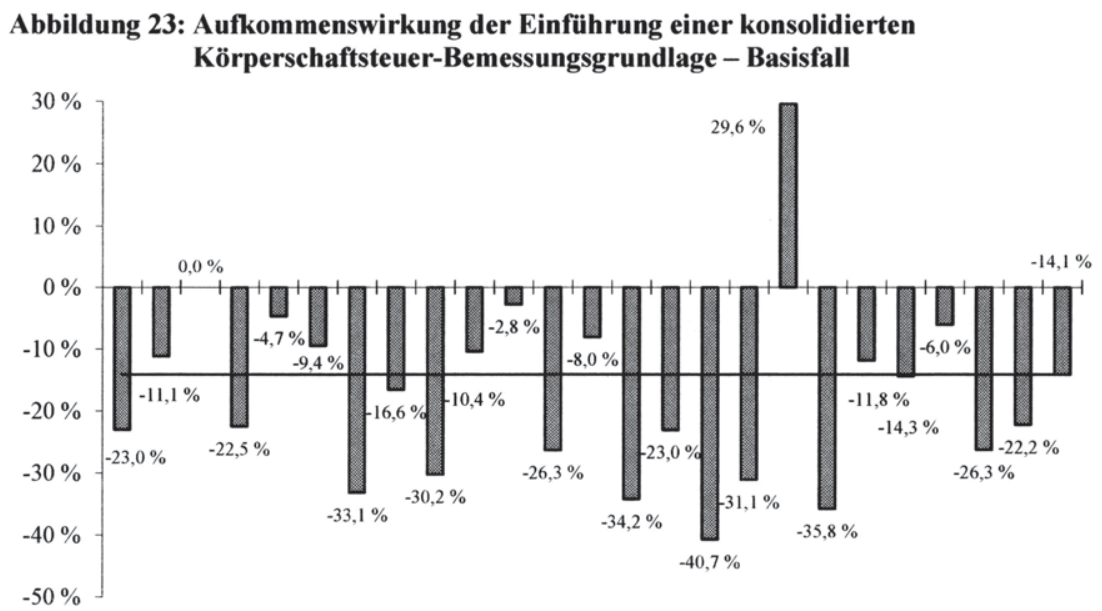

AT BE CY CZ DE DK EE ES FI FR GB GR HU IE IT LT LU LV NL PL PT SE SI SKEU

Quelle: eigene Darstellung

Verlierer der Einführung sind die Niederlande, Irland und Litauen. Die beiden zuerst genannten Länder sind dabei für ihre speziellen Besteuerungsregime bekannt ${ }^{629}$ sodass die Vermutung nahe liegt, die Abnahme sei vornehmlich auf die Umverteilung der Bemessungsgrundlage zwischen den Mitgliedstaaten zurückzuführen. In den acht Mitgliedstaaten, die den Unternehmen kein Gruppenbesteuerungssystem zur Verfügung stellen, sinkt die Bemessungsgrundlage durchschnittlich um 23,36 v. H. Die überdurchschnittlich hohe Abnahme mit der nun ermöglichten konzerninternen Verlustverrechnung zu erklären erscheint plausibel.

Wie bereits angedeutet, lassen sich die Veränderungen der Bemessungsgrundlage in den Mitgliedstaaten auf unterschiedliche Einflussfaktoren zurückführen. Im folgenden Kapitel werden die Einzeleffekte dargestellt und die Änderung der

${ }^{628}$ Dies gilt zumindest für Deutschland und Frankreich, deren effektive Gewinnsteuersätze den ersten und vierten Rang innerhalb der Europäischen Union belegen. Vgl. Devereux, M.P. et al., Tax Rates, 2006. Siehe a. Tabelle 47. Für eine Übersicht empirischer Studien zur Existenz von Steuerplanung in multinationalen Konzernen vgl. Oestreicher, A., in: Endres et al., Wandel, 2005, S. 80-81.

${ }^{629} \mathrm{Vgl}$. fur Irland die Analyse zum organisatorischen Konzernaufbau durch den Einsatz von IFSC-Gesellschaften im Kapitel E.II.2. 
Bemessungsgrundlage für eine eindeutige Zuordnung der Aufkommenswirkungen in ihre Einzeleffekte zerlegt.

Tabelle 46: Aufkommenswirkung der Einführung einer konsolidierten Körperschaftsteuer-Bemessungsgrundlage - Basisfall

\begin{tabular}{|c|c|c|c|c|c|c|}
\hline Land & $\begin{array}{l}\text { Anzahl der } \\
\text { Konzerne }\end{array}$ & $\begin{array}{l}\text { Anzahl der Konzern- } \\
\text { gesellschaften }\end{array}$ & $\begin{array}{l}\text { SA BMG } \\
\text { TEUR }\end{array}$ & $\begin{array}{l}\text { FA BMG } \\
\text { TEUR }\end{array}$ & \multicolumn{2}{|c|}{ Differenz } \\
\hline AT & 42 & 551 & 364.124 & 280.357 & -83.767 & $-23,0 \%$ \\
\hline $\mathrm{BE}$ & 1.269 & 6.977 & 13.096 .649 & 11.637 .498 & -1.459 .151 & $-11,1 \%$ \\
\hline $\mathrm{CY}$ & 5 & 6 & 0 & 0 & 0 & $0,0 \%$ \\
\hline $\mathrm{CZ}$ & 26 & 428 & 2.221 .674 & 1.722 .370 & -499.304 & $-22,5 \%$ \\
\hline $\mathrm{DE}$ & 352 & 4.514 & 10.359 .122 & 9.875 .849 & -483.273 & $-4,7 \%$ \\
\hline DK & 933 & 5.897 & 16.935 .900 & 15.336 .469 & -1.599 .431 & $-9,4 \%$ \\
\hline $\mathrm{EE}$ & 50 & 558 & 357.607 & 239.062 & -118.545 & $-33,1 \%$ \\
\hline ES & 684 & 8.082 & 26.496 .594 & 22.109 .614 & -4.386 .980 & $-16,6 \%$ \\
\hline $\mathrm{FI}$ & 269 & 2.249 & 10.295 .727 & 7.186 .864 & -3.108 .863 & $-30,2 \%$ \\
\hline FR & 1.310 & 17.566 & 40.546 .440 & 36.346 .642 & -4.199 .798 & $-10,4 \%$ \\
\hline GB & 533 & 16.016 & 31.556 .844 & 30.681 .327 & -875.517 & $-2,8 \%$ \\
\hline GR & 48 & 496 & 2.424 .880 & 1.786 .764 & -638.116 & $-26,3 \%$ \\
\hline $\mathrm{HU}$ & 7 & 427 & 751.934 & 691.438 & -60.496 & $-8,0 \%$ \\
\hline IE & 97 & 437 & 2.289 .118 & 1.506 .005 & -783.113 & $-34,2 \%$ \\
\hline IT & 743 & 3.027 & 19.403 .356 & 14.931 .588 & -4.471 .768 & $-23,0 \%$ \\
\hline LT & 7 & 78 & 44.996 & 26.676 & -18.320 & $-40,7 \%$ \\
\hline $\mathrm{LU}$ & 16 & 63 & 822.421 & 566.829 & -255.592 & $-31,1 \%$ \\
\hline LV & 10 & 238 & 47.842 & 61.996 & 14.154 & $29,6 \%$ \\
\hline NL & 610 & 3.575 & 14.766 .326 & 9.482 .845 & -5.283 .480 & $-35,8 \%$ \\
\hline PL & 29 & 1.071 & 1.403 .006 & 1.237 .137 & -165.869 & $-11,8 \%$ \\
\hline PT & 62 & 976 & 3.008 .043 & 2.577 .050 & -430.993 & $-14,3 \%$ \\
\hline SE & 1.240 & 9.673 & 13.656 .466 & 12.830 .810 & -825.656 & $-6,0 \%$ \\
\hline SI & 3 & 13 & 45.088 & 33.251 & -11.837 & $-26,3 \%$ \\
\hline SK & 1 & 7 & 94.705 & 73.694 & -21.011 & $-22,2 \%$ \\
\hline $\mathrm{EU}$ & 8.346 & 82.925 & 210.988 .860 & 181.222 .135 & -29.766 .725 & $-14,1 \%$ \\
\hline
\end{tabular}

Quelle: eigene Berechnungen

\section{b) Zerlegung der Aufkommenswirkung in ihre Einzeleffekte}

\section{(a) Beschreibung der Einzeleffekte}

Die Änderung der Bemessungsgrundlage in den jeweiligen Mitgliedstaaten ist auf zwei Effekte, die neue, pauschale Gewinnabgrenzung und die ausgeweitete Verlustverrechnung zurückzuführen.

Grundsätzlich ist zu erwarten, dass sich mit der Einführung einer indirekten Gewinnabgrenzung zwischen europäischen Konzerngesellschaften die Zuordnung von Steuersubstrat zwischen den Mitgliedstaaten verschiebt. Tendenziell sollten jene Mitgliedstaaten an Bemessungsgrundlage verlieren, die über spezielle Steuerregime verfügen. Denn diese Steuervergünstigungen bewirken in der Regel eine Diskrepanz zwischen Bemessungsgrundlage und wirtschaftlicher Substanz. Da die Faktoren der Massachusetts-Formula auch an den Produktionsfaktoren ansetzten, wird durch die pauschale Gewinnzuordnung das Missverhältnis beseitigt. Dieses ist gleichbedeutend mit einem Verlust an Steuersubstrat in den Mitgliedstaaten mit speziellem Steuerregime. 
Ein ähnlicher Effekt ist in Ländern mit niedrigem Steuerniveau zu erwarten. Auch hier wird den steuerplanerischen Gestaltungen ${ }^{630}$ der Konzerne durch Einführung der gemeinsamen (konsolidierten) Bemessungsgrundlage die Wirkung entzogen. Verrechnungspreisgestaltungen ${ }^{631}$ oder besondere Finanzierungsstrukturen ${ }^{632}$ haben keinen Einfluss mehr auf die Gewinnabgrenzung. Folglich sollten besonders Hochsteuerländer durch die Einführung der einheitlichen Bemessungsgrundlage mehr und Mitgliedstaaten mit einem geringen Steuerniveau weniger Steuersubstrat erhalten. ${ }^{633}$

Die Einführung der einheitlichen (konsolidierten) Bemessungsgrundlage weitet grundsätzlich die Möglichkeit der Verlustverrechnung aus, sodass die Gesamtbemessungsgrundlage in der Europäischen Union abnimmt. Voraussetzung für einen Aufkommenseffekt sind jedoch zuvor nicht verrechenbare Verluste innerhalb eines Konzerns, die im Rahmen der Konsolidierung jetzt mit Gewinnen ausgeglichen werden können. Dieser negative Aufkommenseffekt kann unterteilt werden in eine grenzüberschreitende und in eine rein nationale Komponente. Da im Veranlagungszeitraum 2003 nur Dänemark und Frankreich eine grenzüberschreitende Verlustverrechnung unter bestimmten Voraussetzungen vorsahen, diese aber bei den Berechnungen vernachlässigt wird, ${ }^{634}$ resultiert aus der Einführung der konsolidierten Bemessungsgrundlage stets ein negativer Aufkommenseffekt aufgrund der jetzt ermöglichten grenzüberschreitenden Verlustverrechnung. Im rein nationalen Fall hängt das Vorzeichen des Aufkommenseffekts von dem jeweilig geltenden Gruppenbesteuerungssystem ab. Wenn nämlich der Konsolidierungskreis des nationalen Gruppenbesteuerungssystems weiter gefasst ist als derjenige der einheitlichen (konsolidierten) Bemessungsgrundlage, kann die Möglichkeit des Ausgleichs

${ }^{630}$ Steuerplanerische Gestaltungen sind verstärkt von großen Konzernen zu erwarten. Zwar überwiegt die Anzahl kleinerer Unternehmensgruppen in der Analyse. Betrachtet man die Lorenzkurve (Abbildung 17) wird aber deutlich, dass die 93 v. H. der Gesamtbemessungsgrundlage von nur $20 \mathrm{v}$. H. der Konzerne erwirtschaftet werden.

631 Vgl. ausführlich zur Steuerplanung mit Verrechnungspreisen Jacobs, O.H. (Hrsg.), Unternehmensbesteuerung, 2002, S. 868-1087.

${ }^{632} \mathrm{Zu}$ den steuerlichen Aspekten von Finanzierungsgesellschaften siehe Potthof, A., Finanzierung, 1998, S. 65-126; Burwitz, G., Konzernfinanzierungsgesellschaften, 2005, S. 87-132; Gundel, G., in: Schaumburg, Konzern, 1998, S. 131-173. Vgl. a. E.II.2.

${ }^{633}$ Wie das jeweilige Steuerniveau der Mitgliedstaaten zu messen ist, hängt mit der zugrunde liegenden Fragestellung zusammen. Wird die Verlagerung von Gewinnen mittels Verrechnungspreisen untersucht, ist auf die effektive statutarische Gewinnsteuer abzustellen. Für Finanzierungsstrukturen spielt hingegen auch die Definition der jeweiligen Bemessungsgrundlagen eine entscheidende Rolle, sodass die EATR bzw. EMTR heranzuziehen sind.

${ }^{634} \mathrm{Zu}$ den Gründen, warum keine grenzüberschreitende Gruppenbesteuerung in Dänemark und Frankreich abgebildet werden, vgl. Kapitel D.V.3. 
von Gewinnen und Verlusten sogar eingeschränkt werden. Je enger der Konsolidierungskreis der konsolidierten Körperschaftsteuer-Bemessungsgrundlage abgegrenzt wird, desto geringer wird der negative Aufkommenseffekt aus der nationalen Verlustverrechnung ausfallen bzw. desto eher kann sich sogar ein positiver Effekt ergeben.

Lassen sich noch begründete Annahmen über die Vorzeichen der einzelnen Effekte formulieren, so hängt der Umfang der Verlustverrechnung von der Ertragslage der Unternehmen ab. Diese wird jedoch von einer Vielzahl von Faktoren bestimmt, welche in Summe nicht beobachtet werden können.

Um die hier beschriebenen Einzeleffekte näher analysieren zu können, wird der Aufkommenseffekt in drei Bestandteile zerlegt: den Formeleffekt, den nationalen Verlustverrechnungseffekt und den internationalen Verlustverrechnungseffekt. Wie die folgenden Ausführungen zeigen, bedarf es hierzu der Kenntnis der Gewinne auf der Grundlage „korrekter“ Verrechnungspreise. Da diese unbekannt sind, muss die Aufteilung auf der Basis nachfolgend erläuterter Annahmen erfolgen.

\section{(B) Methodik der Zerlegung}

Die indirekte Gewinnabgrenzung, wie sie die konsolidierte KörperschaftsteuerBemessungsgrundlage vorsieht, sorgt dafür, dass alle Konzerngesellschaften entweder einen Gewinn, einen Verlust oder ein Ergebnis von null ausweisen. Wird dieses Ergebnis als korrekte Gewinnabgrenzung akzeptiert, wäre jede Verlustverrechnung im Rahmen der Konsolidierung das Ergebnis zuvor falsch abgegrenzter Gewinne. Eine Unterteilung in Einzeleffekte wäre obsolet, da der gesamte Aufkommenseffekt der veränderten Gewinnabgrenzung zuzuschreiben wäre. Wird die einheitliche (konsolidierte) Bemessungsgrundlage jedoch als pauschale Methode aufgefasst, bei der die Gewinnabgrenzung lediglich im Mittel zum richtigen Ergebnis führt, sind Verluste einzelner Gesellschaften kurzfristig sehr wohl plausibel. Dabei können die Verluste jedoch auch ganz oder teilweise durch Gewinnverlagerungen entstanden sein. Für eine exakte Abgrenzung der drei Effekte voneinander müssten also die „wahren“ fremdvergleichskonformen Verrechnungspreise bekannt sein. ${ }^{635} \mathrm{Um}$ trotz der $\mathrm{Ab}$ grenzungsprobleme die Einzeleffekte analysieren zu können, werden diese wie folgt voneinander getrennt:

${ }^{635}$ Selbst wenn die korrekten Ergebnisse einer direkten Gewinnabgrenzung bekannt wären, kann die Zerlegung des Aufkommenseffektes fehlerhaft sein. Denn der Fremdvergleichsgrundsatz liefert keine Aufteilungsregel für Mehrgewinne, die auf Synergie- und Skaleneffekte von verbundenen Unternehmen basieren. Vgl. hierzu Oestreicher, A., in: Oestreicher, Verrechnungspreise, 2003, S. 35-40. 
Als Formeleffekt wird die Veränderung der Bemessungsgrundlage definiert, die bei Einführung der einheitlichen (konsolidierten) Bemessungsgrundlage erfolgen würde, ohne dabei die bestehenden Verlustverrechnungsmöglichkeiten zu erweitern. Dazu wird die indirekte Gewinnaufteilung nur zwischen ertragreichen Konzerngesellschaften vorgenommen, wodurch lediglich eine Umverteilung der Bemessungsgrundlage zwischen den Mitgliedstaaten erfolgt. Folglich ist der Formeleffekt europaweit ein Nullsummenspiel, bei dem die Gesamtbemessungsgrundlage unverändert bleibt. Die Differenz zwischen dem Formeleffekt und der gesamten Aufkommenswirkung entspricht dann der Veränderung der Bemessungsgrundlage aufgrund von Verlustverrechnungen. Diese kann weiter in einen nationalen und internationalen Anteil aufgegliedert werden. Als nationaler Verlustverrechnungseffekt gilt der Anteil, der dem zusätzlichen Verlustverrechnungspotenzial entspricht, wenn in allen Mitgliedstaaten nationale Gruppenbesteuerungssysteme eingeführt würden, deren Konsolidierungskreis analog zur einheitlichen Körperschaftsteuer-Bemessungsgrundlage abgrenzt würde, nur dass dieser auf das Inland beschränkt bliebe. Der internationale Verlustverrechnungseffekt ist die Residualgröße, die sich nach Abzug des nationalen Verlustverrechnungs- und Formeleffekts von der Gesamtänderung der Bemessungsgrundlagen ergibt. Abbildung 24 verdeutlicht die Vorgehensweise grafisch.

Abbildung 24: Zerlegung des Aufkommenseffekts der Einführung einer konsolidierten Körperschaftsteuer-Bemessungsgrundlage in drei Einzeleffekte

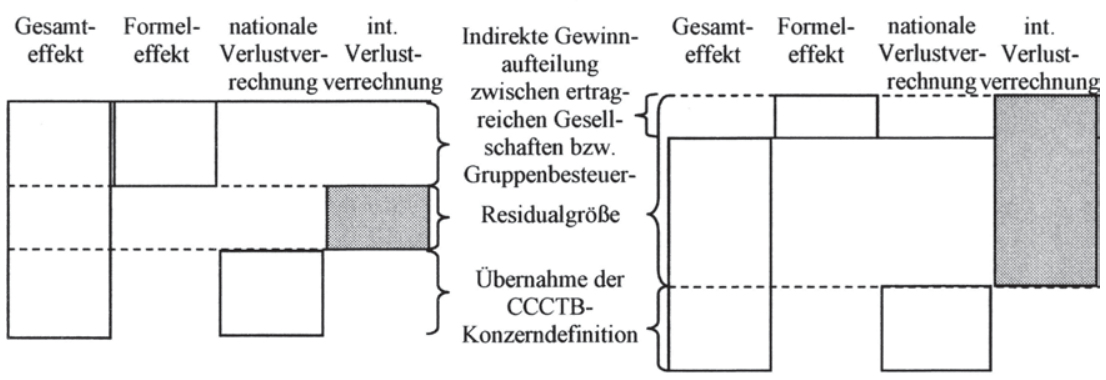

Quelle: eigene Darstellung

\section{( $\gamma$ ) Darstellung der Ergebnisse}

Abbildung 25 zeigt die Zusammensetzung der Aufkommenswirkung, untergliedert nach den oben definierten Effekten. Dabei fällt zunächst auf, dass die nationale Verlustverrechnung besonders in den Mitgliedstaaten einen großen Einfluss hat, die bisher kein Gruppenbesteuerungssystem kannten. Dies gilt in besonderem Maße für Italien und Belgien. Aber auch in Deutschland sinkt die Bemessungsgrundlage aufgrund der ausgeweiteten Verlustverrechnung um 10,8 v. H. Dieses Resultat muss in Verbindung mit den restriktiven Organschaftsvoraussetzungen gesehen werden. Dass sich ein ähnliches Bild nicht auch 
für Österreich zeigt, kann auf die sowohl absolut als auch relativ sehr geringe Datenbasis zurückgeführt werden. Erstaunlich ist, dass selbst in Mitgliedstaaten, die eine hohe Mindestbeteiligungsquote im Rahmen ihrer nationalen Gruppenbesteuerungssysteme voraussetzen (Slowenien, Dänemark, Frankreich, Luxemburg, Niederlande und Polen), ${ }^{636}$ der Anteil der im Inland verrechenbaren Verluste nach Einführung der einheitlichen konsolidierten Bemessungsgrundlage nicht maßgeblich zunimmt. Ursächlich dafür ist einerseits die hohe Konzernbeteiligungsquote an den Tochtergesellschaften. Über 89 v. H. der Gliedgesellschaften werden durch einen Anteilsbesitz von über $98 \mathrm{v} . \mathrm{H}$. gehalten. ${ }^{637}$ Andererseits ist anzunehmen, dass die Konzerne die Höhe der Beteiligung an den Tochtergesellschaften im Hinblick auf das geltende Gruppenbesteuerungssystem anpassen.

Abbildung 25: Aufkommenswirkung der Einführung einer konsolidierten Körperschaftsteuer-Bemessungsgrundlage - Zerlegung des Basisfalls in Einzeleffekte

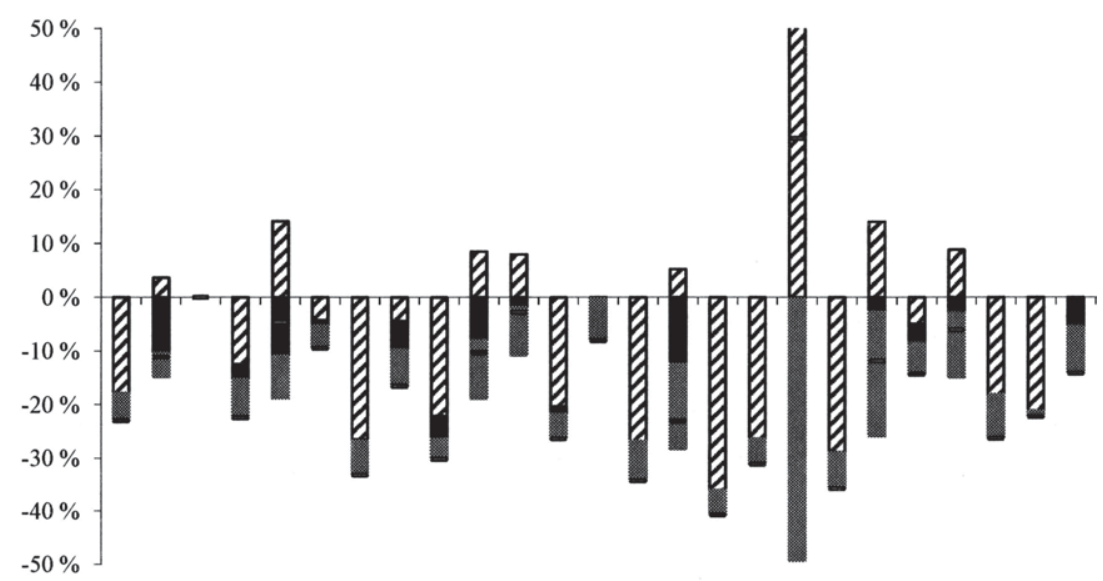

AT BE CY CZ DE DK EE ES FI FR GB GR HU IE IT LT LU LV NL PL PT SE SI SK EU

घFormel Dnationale Verlustverrechnung internationale Verlustverrechung -gesamt

Quelle: eigene Darstellung

Zwar beeinflussen die äußeren Rahmenbedingungen das zusätzliche Ausmaß der Verlustverrechnung mit Einführung der einheitlichen (konsolidierten) Bemessungsgrundlage, dennoch sind die Verluste zunächst exogen gegeben, sodass eine Erklärung dieses Effekts nur schwer möglich ist.

636 Vgl. Tabelle 21.

${ }^{637}$ Vgl. für eine Übersicht über die Verteilung der Höhe der Beteiligung der Muttergesellschaften an den Konzernunternehmen Tabelle 27. 
Anders verhält es sich beim Formeleffekt. Dieser beruht ausschließlich auf einer Umverteilung der Bemessungsgrundlage zwischen den Mitgliedstaaten. Dabei ist die Verschiebung der Bemessungsgrundlage aufgrund einer formelhaften Gewinnaufteilung keineswegs marginal. In über der Hälfte der Mitgliedstaaten übertrifft sie sogar die Aufkommenswirkung der ausgeweiteten Verlustverrechnung. Mit Einführung der einheitlichen konsolidierten Steuerbemessungsgrundlage sind Gewinnverlagerungen sowohl durch das Ausnutzen bestimmter Steuerregime als auch mittels Verrechnungspreisgestaltungen bei sämtlichen innereuropäischen Transaktionen ausgeschlossen, da an die Stelle der direkten Gewinnabgrenzung eine formelhafte Gewinnaufteilung tritt. ${ }^{638}$ Zugleich lässt eine Betrachtung der derzeitigen Rahmenbedingungen in der Europäischen Union Gewinnverlagerungen innerhalb transnationaler Unternehmen vermuten. Ein Vergleich der effektiven Gewinnsteuersätze verdeutlicht die großen Belastungsdifferenzen innerhalb der Europäischen Union (vgl. Tabelle 47). So beträgt die größte Streubreite zwischen Deutschland und Irland 30,66 Prozentpunkte. Im Durchschnitt weichen die effektiven Gewinnsteuersätze um 7,1 Prozentpunkte voneinander ab. Darüber hinaus steigt der Anteil der grenzüberschreitenden internen Lieferungs- und Leistungstransaktionen stetig an, ${ }^{639}$ wovon eine Vielzahl innerhalb des europäischen Binnenmarktes abgewickelt wird. ${ }^{640}$ Vor diesem Hintergrund ist zu erwarten, dass Hochsteuerländern im Rahmen einer einheitlichen konsolidierten Bemessungsgrundlage mehr und Niedrigsteuerländern weniger Steuersubstrat zugewiesen wird.

\section{(ס) Erklärung der Ergebnisse}

Im Folgenden soll der Zusammenhang zwischen dem Formeleffekt und dem geltenden Steuersatz in den einzelnen Mitgliedstaaten näher untersucht werden, wobei sich die Ausführungen auf Gewinnverlagerungen mittels der Gestaltung

${ }^{638} \mathrm{Vgl}$. hierzu ausführlich Abschnitt C.I. Werden die Einzelabschlüsse nicht konsolidiert, sondern lediglich ein Summenabschluss aufgestellt, besteht weiterhin für die Unternehmen ein Anreiz, durch Verrechnungspreisgestaltungen die Gewinnrealisation zeitlich nach hinten zu verschieben.

639 Die Anzahl der multinationalen Unternehmen ist seit Beginn der siebziger Jahre von 6.000 auf über $65.000 \mathrm{im}$ Jahr 2001 gestiegen. Vgl. UNCTAD, Investment Report, 2002, S. 14. Zur ökonomischen Bedeutung multinationaler Unternehmen vgl. Scherer, A.G., Globalisierung, 2003, S. 98-100. Schătzungen der OECD zufolge wurde bereits Anfang der 90er Jahre $60 \mathrm{v}$. H. des gesamten internationalen Handels zwischen verbundenen Unternehmen abgewickelt. Vgl. Europäische Kommission, Unternehmensbesteuerung, 2001, S. 24.

${ }^{640}$ Im Jahr 2003 betrug der Anteil der Direktinvestitionen deutscher Investoren im EU-Ausland 50,7 v. H. Umgekehrt stammen fast $70 \mathrm{v}$. H. der auslăndischen Direktinvestitionen in Deutschland aus einem Mitgliedstaat der Europäischen Union. 
von Verrechnungspreisen beschränken. ${ }^{641}$ Dazu sind zunächst Informationen über die internen Lieferungs- und Leistungsbeziehungen multinationaler Unternehmen notwendig. Da die Amadeus-Datenbank jedoch lediglich handelsrechtliche Jahresabschlüsse enthält, ist weder bekannt, welche Konzerngesellschaften interne Transaktionen abwickeln, noch, inwieweit die dabei vereinbarten Verrechnungspreise fremdvergleichskonform sind. Um dennoch Hypothesen über die Verschiebung der Bemessungsgrundlage zwischen den Mitgliedstaaten bei Einführung der einheitlichen konsolidierten Bemessungsgrundlage formulieren zu können, werden die Effekte vereinfacht hergeleitet.

Tabelle 47: Effektive steuerliche Tarifbelastung 2003 in der Europäischen Union in $\mathbf{v . ~} \mathbf{H}$.

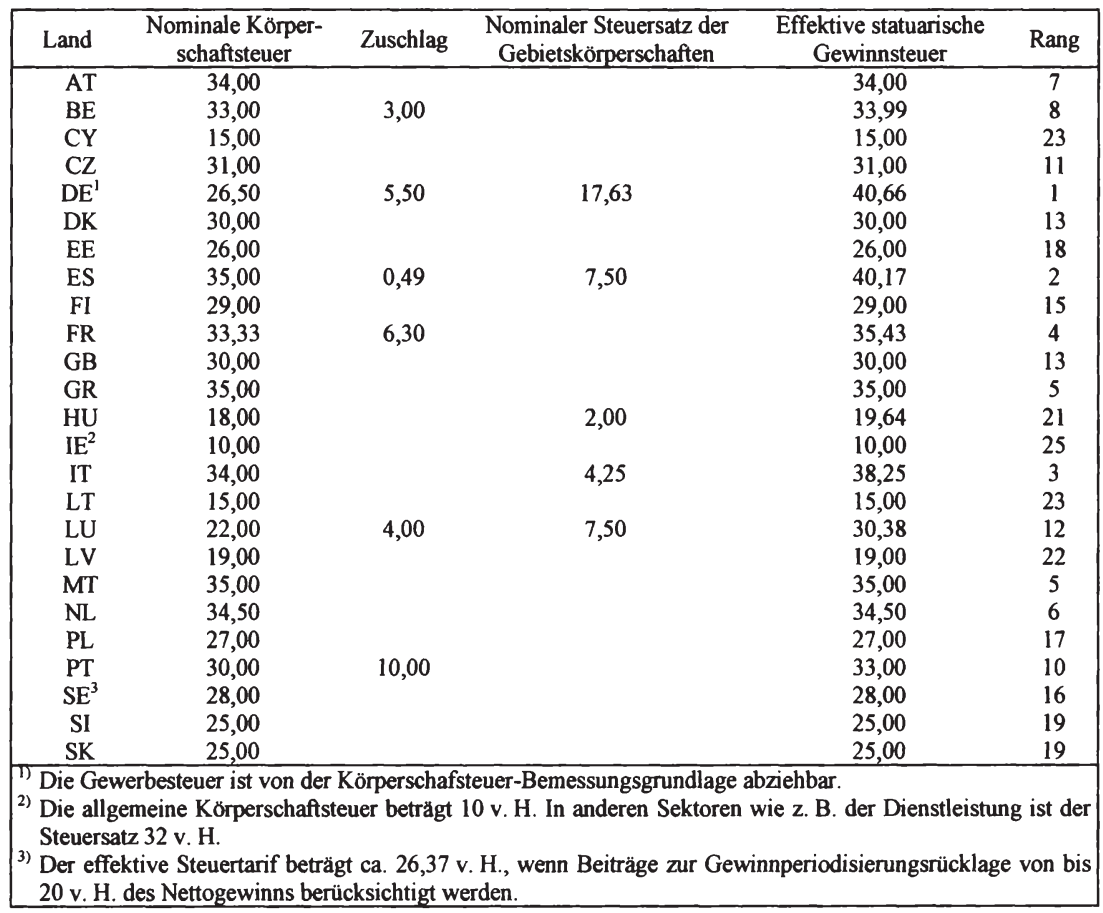

In Anlehnung an: Devereux, M.P. et al., Tax Rates, 2006

Grundsätzlich lassen sich drei Einflussfaktoren bestimmen, die Aufschluss über das Ausmaß der Gewinnverschiebungen zwischen den Mitgliedstaaten geben:

${ }^{641}$ Für Untersuchungen zu den speziellen Besteuerungsregimes in Europa vgl. Kapitel E.II.2. 
1. Damit die Unternehmen gezielt ihre Gewinne verlagern, muss zunächst eine Differenz der effektiven Gewinnsteuersätze existieren. ${ }^{642} \mathrm{Ob}$ ein Mitgliedstaat von der Gewinnverlagerung profitiert oder ihm Steuersubstrat entzogen wird, hängt von dem Vorzeichen der Differenz ab. Die Höhe der Differenz ist hingegen für den einzelnen Nationalstaat unerheblich. ${ }^{643}$

2. Das alleinige Betrachten der Steuersatzgefälle lässt nur Mutmaßungen zu, wie sich die Bemessungsgrundlage zwischen einzelnen Ländern verschiebt. Es erscheint plausibel, dass Deutschland mit dem höchsten Steuersatz von der Einführung der konsolidierten Körperschaftsteuer-Bemessungsgrundlage profitieren würde und Irland mit dem niedrigsten Steuersatz einen Verlust an Steuersubstrat zu verzeichnen hätte. Um aber beurteilen zu können, ob die übrigen Staaten in Summe an Steuersubstrat gewinnen oder verlieren, sind Informationen über den Umfang der Transaktionen zwischen Gesellschaften in den einzelnen Mitgliedstaaten notwendig.

3. Der Gewinnverlagerung anhand von Verrechnungspreisgestaltungen sind Grenzen gesetzt. Grundsätzlich müssen sämtliche Verrechnungspreise dem Fremdvergleich standhalten. Bei der Definition fremdvergleichskonformer Verrechnungspreise weichen die Interessen der Unternehmen und der Finanzverwaltung in Hochsteuerländern voneinander ab. Ob und in welchen Umfang die Unternehmen ihre Verrechnungspreise gestalten können, hängt damit auch von den nationalen Finanzbehörden und Dokumentationsvorschriften $a b{ }^{644}$

Um den Gesamteffekt zu ermitteln, müssen zuvor die drei dargestellten Einflussfaktoren quantifiziert werden. Der Einfluss der Dokumentationsvorschriften wird jedoch vernachlässigt. Zum einen fällt er vergleichsweise gering aus, zum anderen ist eine objektive Bewertung dieses Effekts nur schwer bzw. gar nicht möglich. Die jeweilige Differenz der effektiven Gewinnsteuersätze zwischen den Mitgliedstaaten ergibt sich aus den Angaben der Tabelle 47. Der Umfang der Transaktionen der einzelnen Gesellschaften untereinander kann nur pauschal

${ }^{642}$ Entscheidend für die Gewinnverlagerung von Verrechnungspreisen ist die Differenz der effektiven Gewinnsteuerbelastung für Kapitalgesellschaften, da die Abnahme der Bemessungsgrundlage in einem Land der Zunahme in dem anderen Land entspricht.

${ }^{643}$ Es ist anzunehmen, dass der Anreiz zur Gewinnverlagerung für die Unternehmen bei einer größeren Differenz der effektiven Gewinnsteuern zunimmt. Dieser Effekt wird hier ausgeblendet.

${ }^{644}$ Zum Vergleich der nationalen Dokumentationsvorschriften mit dem EU Code of Conduct vgl. Schnorberger, S./Rosenkranz, J./Garcia, M., in: intertax, 2006, S. 305-313, 406-417.

Zum Code of Conduct on Transfer Pricing documentation for associated enterprises in the E.U. siehe a. Jie-A-Joen, C./Weenink, M., in: TPITP, 2005, 12, S. 11-13; Hickman, A., in: TPITP, 2005, 11, S. 8-9. 
ermittelt werden. Ausgangspunkt bildet dabei die Überlegung, dass interne Lieferungs- und Leistungsbeziehungen vornehmlich in vertikal integrierten Konzernen auftreten. Es wird unterstellt, dass die Produktionsgesellschaften ihre Erzeugnisse an die Vertriebsgesellschaften veräußern und diese dann den Endabnehmer bedienen. Eine Gewinnverlagerung zwischen zwei Steuerhoheiten erfolgt demnach nur, wenn die Vertriebsgesellschaft und die Produktionseinheit ihren Sitz in unterschiedlichen Mitgliedstaaten haben. In der Datenbank wurden 5.687 Konzerne identifiziert, die diese Voraussetzungen erfüllen. Zusammen besitzen diese Konzerne 67.714 Konzerngesellschaften, wovon $66 \mathrm{v}$. H. Vertriebs- oder Produktionsgesellschaften sind (vgl. Tabelle 48). Gemeinsam erzielen die ausgewählten Produktions- und Vertriebsgesellschaften 49 v. H. der Gesamtumsätze der grenzüberschreitend tätigen Konzerne.

Tabelle 48: International vertikal integrierte Konzerne

\begin{tabular}{|c|c|c|c|c|c|c|}
\hline & \multicolumn{2}{|c|}{ Konzerne } & \multicolumn{2}{|c|}{ Gesellschaften } & \multicolumn{2}{|l|}{ Umsatz } \\
\hline internationale Konzerne & 16.962 & & 158.154 & & 9.838 .626 .451 & \\
\hline $\begin{array}{l}\text { internationale Konzerne mit Produktions- } \\
\text { gesellschaft und Vertriebsgesellschaft }\end{array}$ & 5.989 & $35 \%$ & 95.396 & $60 \%$ & 7.510 .058 .157 & $76 \%$ \\
\hline $\begin{array}{l}\text { Vertriebsgesellschaften und Produktions- } \\
\text { gesellschaften in unterschiedlichen Ländern }\end{array}$ & 5.687 & $34 \%$ & 67.714 & $43 \%$ & 7.183 .925 .075 & $73 \%$ \\
\hline $\begin{array}{l}\text { davon Produktions- und Vertriebsgesell- } \\
\text { schaften }\end{array}$ & & & 44.525 & $28 \%$ & 4.856 .888 .933 & $49 \%$ \\
\hline
\end{tabular}

Quelle: eigene Berechnungen

Des Weiteren wird unterstellt, dass jede Produktionsgesellschaft eines Konzerns mit jeder Vertriebsgesellschaft der Unternehmensgruppe eine Lieferungs- und Leistungsbeziehung unterhält. Der Umfang der Lieferungen und Leistungen richtet sich nach der Größe der Produktionsgesellschaften und der Größe der Vertriebsgesellschaften. Alle übrigen Unternehmen des jeweiligen Konzerns finden keine weitere Beachtung. Diese Vorgehensweise wird an folgendem Beispiel nochmals verdeutlicht.

\section{Beispiel 3:}

Ein Konzern mit Sitz in Deutschland besitzt fün Tochtergesellschaften. Jeweils eine Produktionsgesellschaft hat ihren Sitz in Tschechien (A), Polen (B) und Österreich (C). Daneben unterhält der Konzern eine Vertriebsgesellschaft in Deutschland (D) und in Frankreich (E). Die Produktionsgesellschaften setzen gemeinsam Waren im Wert von $450 \mathrm{GE}$ um. Davon entfallen auf Gesellschaft A 22,2 v. H., auf Gesellschaft B 33,3 v. H. und auf Gesellschaft C 44,4 v. H. Annahmegemäß liefern die Produktionsgesellschaften ihre Ware ausschließlich an die Vertriebsgesellschaften D und E, wobei sich der Umfang der Lieferung auch nach dem Umfang der Umsatzerlöse der Vertriebsgesellschaft richtet. Die Produktionsgesellschaft A liefert folglich $8 \mathrm{v}$. H. der Gesamtumsatzerlöse der Produktionsgesellschaften an die Vertriebsgesellschaft $\mathrm{D}$, was $36 \mathrm{GE}$ entspricht. ${ }^{645}$ Das grau unterlegte Feld gibt die Lieferbeziehungen zwischen den Mitgliedstaaten für dieses Beispiel wieder. Die Differenz 
zwischen den Umsatzerlösen der Produktions- und Vertriebsgesellschaft hat im Rahmen dieses Modells drei mögliche Ursachen. Erstens wird der Verkaufspreis der Vertriebsgesellschaften über dem der Produktionsgesellschaften liegen, sodass diese selbst bei direkter Weiterverăußerung der Waren höhere Umsätze erzielen. Zweitens können Differenzen aus dem Verkauf von Lagerbeständen der Vertriebsgesellschaften herrühren, oder drittens verkaufen letztere zusätzlich Produkte, die sie von Drittanbietern bezogen haben.

\begin{tabular}{|c|c|c|c|c|c|c|c|c|}
\hline & & \multirow[t]{2}{*}{ Land } & \multirow[t]{2}{*}{ Umsatz } & \multirow[t]{2}{*}{ Anteil } & \multicolumn{2}{|c|}{$\begin{array}{c}\text { Anteil am gesamten } \\
\text { Produktionsumsatz, den A, } \\
\mathrm{B}, \mathrm{C} \text { an } \mathrm{D} \text { und } \mathrm{E} \text { liefern }\end{array}$} & \multicolumn{2}{|c|}{$\begin{array}{l}\text { Monetäre Bewertung mit } \\
\text { den Umsätzen der } \\
\text { Produktionsgesellschafter }\end{array}$} \\
\hline & & & & & & E & $\mathrm{D}$ (DE) & $\mathrm{E}(\mathrm{FR})$ \\
\hline \multirow{4}{*}{$\begin{array}{l}\text { Produktions- } \\
\text { gesellschaften }\end{array}$} & A & $\mathrm{CZ}$ & 100 & $22,2 \%$ & $8 \%$ & $14 \%$ & 36 & 64 \\
\hline & B & PL & 150 & $33,3 \%$ & $12 \%$ & $21 \%$ & 55 & 95 \\
\hline & $\mathrm{C}$ & AT & 200 & $44,4 \%$ & $16 \%$ & $28 \%$ & 73 & 127 \\
\hline & $\sum$ & & 450 & $100,0 \%$ & $36 \%$ & $64 \%$ & 164 & 286 \\
\hline \multirow{3}{*}{$\begin{array}{l}\text { Vertriebs- } \\
\text { gesellschaften }\end{array}$} & D & $\mathrm{DE}$ & 200 & $36,4 \%$ & & & & \\
\hline & E & FR & 350 & $63,6 \%$ & & & & \\
\hline & $\Sigma$ & & 550 & $100,0 \%$ & & & & \\
\hline Konzernmutter & $\mathrm{F}$ & $\mathrm{DE}$ & 300 & & & ht weiter & eachtet & \\
\hline
\end{tabular}

Quelle: eigene Darstellung

Für Gewinnverlagerungen durch Verrechnungspreisgestaltungen ist es unerheblich, in welche Richtung der eigentliche Güterstrom fließt. Folglich werden in Tabelle 49 für den einzelnen Mitgliedstaat alle Umsatzerlöse ausgewiesen, die aus dem Handel von Gesellschaften mit Sitz in den jeweiligen Mitgliedstaaten resultieren. ${ }^{646}$ Die Summe der so für jede Länderkombination ermittelten Umsatzerlöse wird anschließend am Gesamtumsatz jedes Landes relativiert. Das Ergebnis für Deutschland zeigt, dass 48,8 v. H. der Transaktionen mit französischen, britischen, italienischen oder niederländischen Konzerngesellschaften abgewickelt werden. Innerdeutsch werden 21,6 v. H. der Transaktionen durchgeführt. Betrachtet man die Verteilung der Transaktionen in Tabelle 49, lassen sich zwei Tendenzen erkennen. Erstens schwankt der Anteil der innerstaatlichen Transaktionen erheblich zwischen den einzelnen Mitgliedstaaten und fällt bei größeren Mitgliedstaaten höher aus. So liegt der Anteil der innerstaatlichen Transaktionen nur in Deutschland, Spanien, Frankreich und Großbritannien über $20 \mathrm{v}$. H. Zweitens wird bevorzugt in große Volkswirtschaften und/oder in die Nachbarländer investiert. So machen beispielsweise nur in Dänemark, Estland, Finnland, Litauen und Lettland die Transaktionen mit Schweden über $10 \mathrm{v} . \mathrm{H}$. aus. Demgegenüber nehmen Transaktionen, an denen eine deutsche Gesellschaft beteiligt ist, in fast $80 \mathrm{v} . \mathrm{H}$. der Mitgliedstaaten einen Anteil von mehr als 10 v. H. ein. Diese Ergebnisse sind wenig überraschend und entsprechen grund-

646 Absolut gemessen entsprechen für die jeweiligen Lånderkombinationen die Summen der Umsätze einander. D. h., den 3,5 v. H. für Österreich und den $0,9 \mathrm{v}$. H. für Belgien liegen jeweils Umsätze in Höhe von 2.915.268 TEUR zugrunde. 
sätzlich den Erkenntnissen der Direktinvestitionsforschung. ${ }^{647}$ Insofern untermauern sie das pauschale Herleiten der Lieferungs- und Leistungsbeziehungen.

Tabelle 49: Lieferungs- und Leistungsbeziehung zwischen den Mitgliedstaaten

in $\mathbf{v} . \mathbf{H}$.

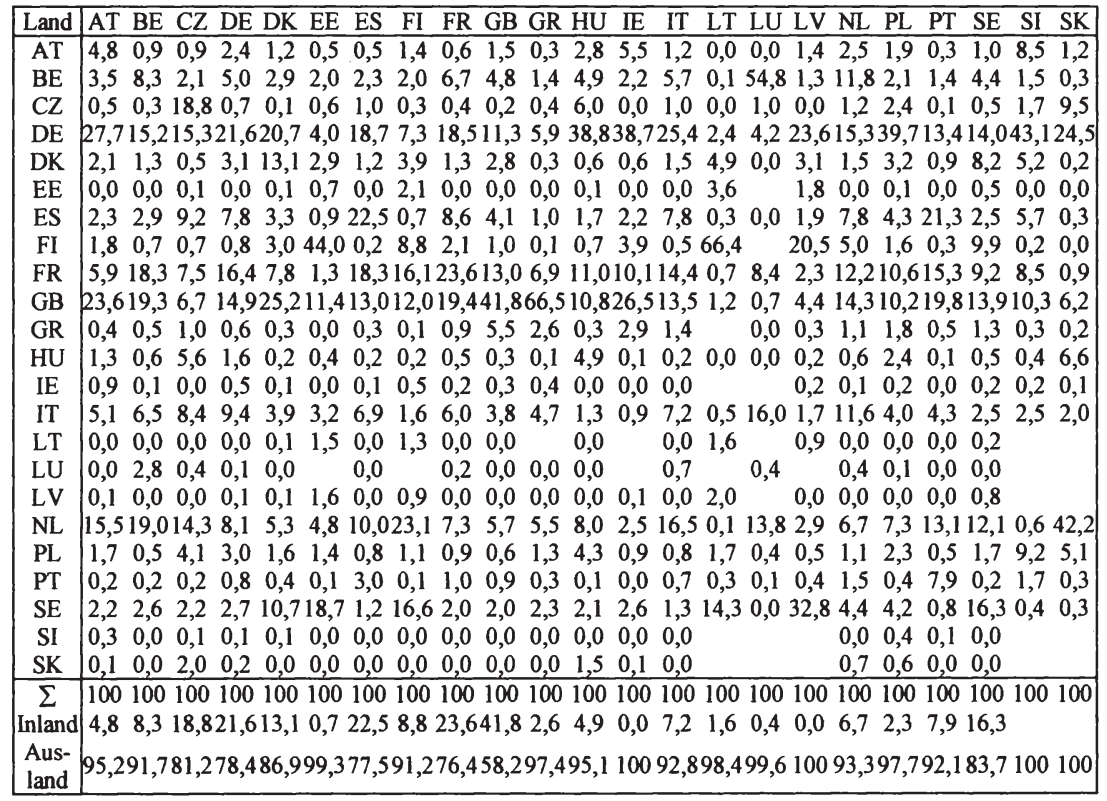

Quelle: eigene Berechnungen

Im zweiten Teil werden die zuvor quantifizierten Effekte in mehreren Schritten zusammengeführt (vgl. Tabelle 50). Zunächst werden die Umsatzerlöse aller Lieferungen und Leistungen, an denen Gesellschaften eines Mitgliedstaates beteiligt sind, für jeden Staat aufsummiert (1). Im zweiten Schritt wird das Gesamtumsatzvolumen unterteilt, je nachdem ob die korrespondierenden Handelspartner in ihrem Sitzstaat einer höheren (2a), niedrigeren (2b) oder der gleichen (2c) effektiven Gewinnsteuerbelastung unterliegen. Transaktionen, die mit Konzerngesellschaften in einem höher (niedriger) besteuernden Staat abgewickelt werden, lassen die Vermutung zu, dass Gewinne ins Inland (Ausland) verlagert werden. Die Differenz (3) fasst beide Effekte (2a-2b) zusammen und

${ }^{647}$ Einen negativen Zusammenhang zwischen den Direktinvestitionen und der Entfernung sowie eine positive Beziehung zwischen den Direktinvestitionen und dem Bruttoinlandsprodukt finden beispielsweise Görg, H./Greenaway, D., in: Herrmann/Lipsey, Direct Investment, 2003, S. 165-183; Slaughter, M., in: Herrmann/Lipsey, Direct Investment, 2003, S. 7-32. 
drückt in ihrem Vorzeichen den potenziellen Gesamteffekt der Gewinnverlagerung des jeweiligen Staates aus. Nicht möglich sind insoweit Aussagen über das Ausmaß dieses Effekts. Dazu muss die Differenz erst in Relation zu dem Gesamtumsatzvolumen gesetzt werden (4). Dadurch wird berücksichtigt, dass bei Transaktionen im Inland oder zwischen Ländern mit gleichem effektiven Gewinnsteuersat $\mathrm{z}^{648}$ keine Gewinnverlagerungen möglich sind. Zudem fließt über die Höhe der Differenz in die Relation mit ein, um wie viel sich die Umsatzvolumina (a) und (b) untereinander und im Verhältnis zur Gesamtsumme unterscheiden. Die ermittelte Verhältniszahl gibt an, welcher Anteil des Gesamtumsatzvolumens nach Nivellierung positiver und negativer Effekte zu einer potenziellen Gewinnverlagerung genutzt werden kann, wobei ein positives Vorzeichen bedeutet, dass im Falle der Einführung der einheitlichen konsolidierten Besteuerung das entsprechende Land von der indirekten Gewinnabgrenzung profitieren würde.

\section{Tabelle 50: Zusammenführung der Einzeleffekte der Gewinnverlagerung}

\begin{tabular}{|c|c|c|c|c|c|c|c|c|c|}
\hline \multirow[t]{2}{*}{ Land } & \multirow[t]{2}{*}{$\begin{array}{c}\text { effektiver } \\
\text { Gewinn- } \\
\text { steuersatz }\end{array}$} & \multirow{2}{*}{$\begin{array}{l}\text { Gesamt- } \\
\text { umsatz- } \\
\text { volumen } \\
\text { TEUR (1) }\end{array}$} & \multicolumn{3}{|c|}{$\begin{array}{l}\text { Effektiver Gewinnsteuersatz } \\
\text { des Handelspartners TEUR (2) }\end{array}$} & \multirow[t]{2}{*}{$\begin{array}{c}\text { Differenz } \\
\text { TEUR } \\
\text { (3) }\end{array}$} & \multirow{2}{*}{$\begin{array}{c}\text { Differenz } \\
\text { relativ } \\
\text { in } \mathrm{v} . \mathrm{H} . \\
(4)\end{array}$} & \multirow[t]{2}{*}{ Rang } & \multirow{2}{*}{\begin{tabular}{|c|} 
Rang \\
effekti- \\
ver Ge- \\
winn- \\
steuersatz
\end{tabular}} \\
\hline & & & höher (a) & niedriger (b) & gleich (c) & & & & \\
\hline AT & 34 & 83.675 .243 & 47.677 .085 & 531.971 .689 & 4.026 .469 & .396 & $-18,77$ & 7 & \\
\hline BE & S & 316.0 & 199.717 .298 & 90.145 .835 & 26.205 .007 & -109.57 & & 9 & \\
\hline $\mathrm{CZ}$ & 31 & .268 & 25.311 .009 & 9.642 .556 & 8.087 .702 & -15.6 & & 10 & 10 \\
\hline $\mathrm{DE}$ & 41 & 965.639 .890 & & 757.479 .032 & 208.160 .859 & 757.479 .032 & 78,44 & 1 & l \\
\hline DK & 30 & & 337 & 722.801 .084 & 54.559 .158 & -42 & & 8 & 12 \\
\hline EE & 2 & & 4.96 & 92 & & -4.7 & & 17 & 17 \\
\hline ES & 40 & 400 & 75.062 .812 & 235.5 & 90.211 .602 & 160.52 & 05 & 3 & 1 \\
\hline FI & & & 75.4 & & & & & 11 & 14 \\
\hline FR & 3 & & 283.3 & 371. & 202.3 & & & 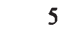 & \\
\hline GB & 30 & 1.27 & 648.698 .437 & 76.242.084 & 567.157 .289 & -592.456 .353 & $-46,57$ & 12 & 12 \\
\hline GR & 3 & & & 16 & & & & 2 & \\
\hline $\mathrm{HU}$ & 20 & & 38. & 25.640 & 5.833 & -38 & & 18 & 20 \\
\hline IE & 10 & & 13.555 .274 & 0 & 561 & -13 & $-100,00$ & 23 & 23 \\
\hline IT & 38 & 35 & 118.751 .126 & 792 & 2.843 & 1.666 & & 4 & 3 \\
\hline LT & & & & 0 & & & & 21 & 22 \\
\hline LU & 3 & & 15. & & 65.175 & -15 & & 19 & 11 \\
\hline LV & 19 & 463 & 4.713 .329 & 53.482 & 1.652 & 847 & $-97,72$ & 20 & 21 \\
\hline NL & & 509.728 .891 & 244.991 .446 & 230 & 76.988 & & & 6 & \\
\hline PL & 27 & & 69.2 & & 30 & & & 16 & 16 \\
\hline PT & 33 & 56.569 .891 & 39.287 .646 & $\begin{array}{ll}5 & 12.817 .276\end{array}$ & 4.464 .969 & -26.470 .370 & $-46,79$ & 13 & \\
\hline SE & 28 & 185.4 & 147.972 .140 & 7.187 .615 & 30.265 .189 & & & 14 & 15 \\
\hline SI & 2 & & & 17.717 & & & & 22 & 18 \\
\hline SK & 25 & 9.042 .002 & 8.435 .433 & 606.569 & 0 & -7.828 .865 & $-86,58$ & 15 & 18 \\
\hline
\end{tabular}

Quelle: eigene Berechnungen

Der Vergleich der Rangfolgen der Länder hinsichtlich des effektiven Gewinnsteuersatzes und der relativen Differenz (4) zeigt, dass jener zwar einen maßgeb-

${ }^{648}$ Hierzu zählen Großbritannien und Frankreich sowie die Slowakei und Slowenien. 
lichen Einfluss hat, die Rangfolge sich nach der Berücksichtigung der Handelsvolumina aber leicht verschiebt. Am drastischsten ist die Veränderung der Rangfolge (+8) in Luxemburg, das mit $30 \mathrm{v}$. H. den elfthöchsten effektiven Gewinnsteuersatz aufweist und dennoch $98 \mathrm{v}$. H. der Transaktionen mit Mitgliedstaaten abwickelt, die eine noch höhere Besteuerung von Gewinnen vorsehen. Gemäß den Berechnungen in der Tabelle 50 profitieren von der Einführung der konsolidierten Körperschaftsteuer-Bemessungsgrundlage besonders Deutschland, Großbritannien, Spanien, Frankreich und Italien. ${ }^{649}$ Einen hohen Verlust an Steuersubstrat müssen aufgrund der geänderten Gewinnabgrenzung Irland, Litauen und Lettland hinnehmen.

Tabelle 51: Vergleich des Formelefiekts mit dem Indikator für potenzielle Gewinnverlagerung

\begin{tabular}{|c|cccccc|}
\hline Land & $\begin{array}{c}\text { effektiver Gewinn- } \\
\text { steuersatz }\end{array}$ & $\begin{array}{c}\text { Formeleffekt } \\
\text { Berechnung }\end{array}$ & $\begin{array}{c}\text { Rang } \\
\text { Berechnung }\end{array}$ & $\begin{array}{c}\text { Differenz } \\
\text { relativ (4) }\end{array}$ & $\begin{array}{c}\text { Rang } \\
\text { Differenz }\end{array}$ & $\begin{array}{c}\text { Abweichung } \\
\text { Rang }\end{array}$ \\
\hline AT & 34 & $-17,81 \%$ & 14 & $-18,77 \%$ & 7 & -7 \\
BE & 34 & $3,65 \%$ & 8 & $-34,67 \%$ & 9 & 1 \\
CZ & 31 & $-12,43 \%$ & 13 & $-36,40 \%$ & 10 & -3 \\
DE & 41 & $14,14 \%$ & 2 & $78,44 \%$ & 1 & -1 \\
DK & 30 & $-4,26 \%$ & 10 & $-29,85 \%$ & 8 & -2 \\
EE & 26 & $-26,46 \%$ & 20 & $-92,13 \%$ & 17 & -3 \\
ES & 40 & $-4,39 \%$ & 11 & $40,05 \%$ & 3 & -8 \\
FI & 29 & $-22,07 \%$ & 18 & $-45,83 \%$ & 11 & -7 \\
FR & 35 & $8,51 \%$ & 5 & $10,29 \%$ & 5 & 0 \\
GB & 30 & $7,98 \%$ & 6 & $-46,57 \%$ & 12 & 6 \\
GR & 35 & $-20,31 \%$ & 16 & $60,52 \%$ & 2 & -14 \\
HU & 20 & $-0,14 \%$ & 9 & $-94,97 \%$ & 18 & 9 \\
IE & 10 & $-26,72 \%$ & 21 & $-100,00 \%$ & 23 & 2 \\
IT & 38 & $5,25 \%$ & 7 & $26,43 \%$ & 4 & -3 \\
LT & 15 & $-36,04 \%$ & 23 & $-98,44 \%$ & 21 & -2 \\
LU & 30 & $-26,33 \%$ & 19 & $-97,33 \%$ & 19 & 0 \\
LV & 19 & $78,84 \%$ & 1 & $-97,72 \%$ & 20 & 19 \\
NL & 35 & $-28,89 \%$ & 22 & $-2,85 \%$ & 6 & -16 \\
PL & 27 & $14,05 \%$ & 3 & $-90,21 \%$ & 16 & 13 \\
PT & 33 & $-4,98 \%$ & 12 & $-46,79 \%$ & 13 & 1 \\
SE & 28 & $8,84 \%$ & 4 & $-75,93 \%$ & 14 & 10 \\
SI & 25 & $-18,08 \%$ & 15 & $-98,81 \%$ & 22 & 7 \\
SK & 25 & $-21,06 \%$ & 17 & $-86,58 \%$ & 15 & -2 \\
\hline
\end{tabular}

Quelle: eigene Berechnungen

Tabelle 51 stellt die Rangfolgen der auf dem Formeleffekt basierenden Verschiebung der Bemessungsgrundlage dem oben dargestellten Indikator für potenzielle Gewinnverlagerungen (Differenz relativ) gegenüber. Ein Vergleich der Rangfolgen der einzelnen Länder zeigt, dass in $78 \mathrm{v}$. H. der Fälle deren Verschiebung weniger als zehn Plätze beträgt. Die größten Ausreißer sind Griechenland, die Niederlande und Lettland. Zumindest für Lettland erscheinen auch die Ergebnisse des Formeleffekts $(+78,8$ v. H.) unrealistisch, was auf die geringe

649 Entsprechend der Analyse bezieht sich diese Aussage nur auf den Formeleffekt. 
Datenbasis von nur 10 Konzernen und 238 Konzerngesellschaften zurückzuführen ist.

Einen detaillierteren Aufschluss erlaubt der Vergleich der Aufkommensänderung aufgrund der formelhaften Gewinnaufteilung (Formeleffekt) mit dem potenziellen Indikator für Gewinnverlagerungen. Die jeweiligen Werte werden dazu in einem Streudiagramm abgetragen. Für 16 der 23 betrachteten Mitgliedstaaten, die sich im ersten und dritten Quadranten des Koordinatensystems befinden, stimmt das Vorzeichen des Formeleffekts mit dem des Gewinnverlagerungsindikators überein. Dieses trifft für $65 \mathrm{v} . \mathrm{H}$. der Staaten zu (Abbildung 26, links). Dieser Zusammenhang drückt sich auch anhand der positiven, wenn auch geringen Steigung der Trendlinie aus. In deren Berechnung gehen alle Länder zu gleichen Anteilen ein. Zwar ist diese Vorgehensweise methodisch korrekt, missachtet aber, dass insbesondere für kleine Länder nur wenige Beobachtungen vorhanden sind und diese deshalb einer großen Schwankungsbreite unterliegen. Wie oben bereits erwähnt, erscheinen die Ergebnisse für Lettland wenig plausibel. Vernachlässigt man diesen Ausreißer, zeigt sich ein deutlicherer Zusammenhang (Abbildung 26, rechts).

Abbildung 26: Gegenüberstellung des Formeleffekts und der potenziellen

Gewinnverlagerung
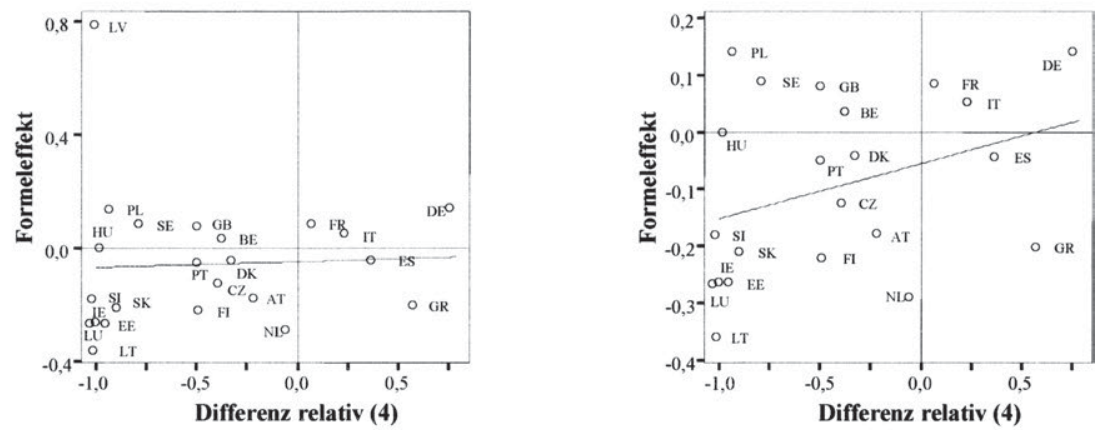

Quelle: eigene Darstellung

\section{c) Berücksichtigung einer pauschalen Verlustverrechnung}

Der Basisfall ignoriert die nicht verrechenbaren Verluste der einzelnen Gesellschaften. Ein intertemporaler Verlustausgleich findet nicht statt, wodurch vorgetragene Verluste als wertlos erachtet werden. Diese Vorgehensweise führt, bezogen auf die EU-Gesamtbemessungsgrundlage, zu einer Überschätzung des mit der Einführung der einheitlichen (konsolidierten) Bemessungsgrundlage verbundenen negativen Aufkommenseffekts. ${ }^{650}$ Denn der weiter gefasste Konso-

${ }^{650}$ Vgl. hierzu Kapitel F.II.1.c). 
lidierungskreis bewirkt, dass mehr Verluste in der laufenden Periode verrechnet werden können als nach bestehendem Recht. Damit gelten zugleich weniger Verluste als endgültig nicht verrechenbar. Diese Verzerrung lässt sich pauschal beseitigen, indem vorgetragene Verluste positiv bewertet werden, da sie zukünftig die Steuerzahllast der Unternehmen mindern. Einen im Zeitverlauf konstanten effektiven Gewinnsteuersatz ${ }^{651}$ und keine zeitliche Beschränkung der Verlustvortragsdauer ${ }^{652}$ vorausgesetzt, ist das steuerliche Einkommen der laufenden Periode um die abgezinsten Verlustvorträge zu mindern. Für den zugrunde zu legenden Diskontierungsfaktor wird der im Steuergesetz übliche Zinssatz von 5,5 v. H. angenommen. Die Nutzungsdauer richtet sich nach dem Zeitpunkt der Nutzung der Verlustvorträge. Untersuchungen haben ergeben, dass jährlich $8 \mathrm{v}$. H. der Verlustvorträge genutzt werden und $50 \mathrm{v}$. H. der Verluste niemals verrechnet werden können. Damit ist der steuerliche Gewinn der laufenden Periode um 41,3 v. H. der Verlustvorträge zu mindern. ${ }^{653}$ In Abbildung 31 sind die auf diesen Annahmen beruhenden Berechnungen dem Basisfall gegenübergestellt.

Abbildung 27: Aufkommenswirkung der Einführung einer konsolidierten

Körperschaftsteuer-Bemessungsgrundlage - Berücksichtigung einer pauschalen Verlustverrechnung

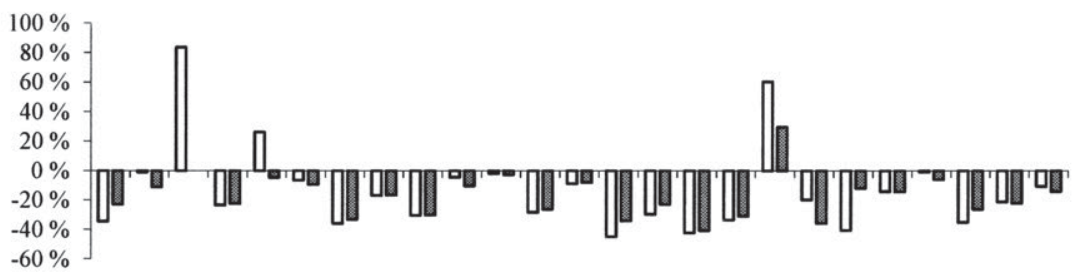

AT BE CY CZ DE DK EE ES FI FR GB GR HU IE IT LT LU LV NL PL PT SE SI SK EU $\square$ mit pauschaler Verlustverrechnung $\square$ Basisfall

Quelle: eigene Darstellung

${ }^{651}$ Entgegen des vielfach zitierten „race to the bottom“ war die Steuerbelastung in den Mitgliedstaaten in den letzten Jahren relativ konstant. Der Abnahme der Steuersätze stand eine Ausweitung der Bemessungsgrundlage gegenüber. Vgl. Büttner, T., in: Müller, Systemwettbewerb, 2001, S. 62-63; Boss, A., Steuerwettbewerb, 2003, S. 10-16; anderer Auffassung Sørensen, P., in: CESifo Forum, 2002, 1, S. 34. Da die Verlustvorträge in ihrer Höhe bereits festgestellt sind, ist für ihre Bewertung lediglich der effektive Gewinnsteuersatz relevant.

652 Einige Mitgliedstaaten beschränken den Verlustvortrag zeitlich. So dürfen in Spanien Verluste nur 15 Jahre, in Finnland zehn Jahre, in Portugal sechs Jahre und in Tschechien, Griechenland, Italien, Lettland, Litauen, Polen, der Slowakei und Slowenien nur fünf Jahre vorgetragen werden. Vgl. Endres, D. et al., Taxable Income, 2006, S. 81; Scheunemann, M., in: IStR, 2006, S. 148.

$653 \frac{1,055^{6}-1}{1,055^{6} \times 0,055} \times 0,08+\frac{0,02}{1,055}=0,413$. 
Abbildung 27 bestätigt die oben genannte Vermutung. Die EU-Gesamtbemessungsgrundlage sinkt nur noch um 10,8 v. H. Damit fällt die Abnahme gegenüber dem Basisfall um 3,3 Prozentpunkte geringer aus. Gleichwohl ist in einigen Mitgliedstaaten der relative Verlust an Steuersubstrat höher als im Basisfall. Dieses ist mit der sich auch ändernden Bezugsgröße des relativen Aufkommenseffekts zu erklären. Grundsätzlich reduziert die Berücksichtigung der Verlustvorträge unabhängig von der Ermittlungsmethode die Bemessungsgrundlage. Lediglich in Slowenien und der Slowakei konnten im Fall der direkten bzw. indirekten Gewinnabgrenzung bereits im Basisfall alle Verluste verrechnet werden (Tabelle 52). Der Umfang, in dem die Berücksichtigung der Verlustvorträge die Bemessungsgrundlage reduziert, fällt nach geltendem Recht und im Rahmen einer formelhaften Gewinnaufteilung unterschiedlich hoch aus. Ist die Abnahme der SA-Bemessungsgrundlage gegenüber dem Basisfall geringer (stärker) als die der FA-Bemessungsgrundlage, wird der Aufkommenseffekt bei Einführung der letzteren verstärkt (abgeschwächt). Besonders drastisch zeigt sich dieser Effekt in Deutschland $(+30,9)$. Die Berücksichtigung der pauschalen Verlustverrechnung bewirkt, dass Deutschland von der Einführung der konsolidierten Körperschaftsteuer-Bemessungsgrundlage sogar absolut profitiert. Ursächlich hierfür ist das große, im Rahmen der separaten Gewinnermittlung zuvor gar nicht berücksichtigte Verlustvolumen. So sinkt die SA-Bemessungsgrundlage um 39,6 v. H. Demgegenüber nimmt die Deutschland zugewiesene konsolidierte Bemessungsgrundlage nur um 20,1 v. H. ab, da die nach geltendem Recht nicht verrechenbaren Verluste bereits durch den weiter gefassten Konsolidierungskreis zum Teil ausgeglichen werden konnten. ${ }^{654}$

Als mögliche Erklärung für die Unterschiede der Veränderung des Aufkommenseffekts zwischen den Mitgliedsstaaten gegenüber dem Basisfall könnte die unterschiedliche Steuerbelastung in den jeweiligen Mitgliedstaaten herangezogen werden. So erscheint es plausibel, dass durch steuerplanerische Gestaltungen Gewinne vornehmlich in Mitgliedstaaten realisiert werden, in denen diese einer niedrigeren Steuerbelastung unterliegen. Umgekehrt würden Gesellschaften in Ländern mit einer hohen Steuerbelastung eher Verluste ausweisen. Folglich wären in diesen Staaten auch höhere Verlustvorträge zu erwarten, wodurch auch die im Basisfall endgültig nicht verrechenbaren Verluste höher ausfielen. Die positive Steigung der Trendlinie in Abbildung 28 deutet zwar auf einen entsprechenden Zusammenhang hin, doch erweist sich die Korrelation zwischen beiden Größen als nicht signifikant.

${ }^{654}$ Dieses Ergebnis ist deckungsgleich mit den Erkenntnissen des Kapitels F.III.1.e). Der starke negative Aufkommenseffekt der Untersuchung von Fuest/Hemmelgarn/Ramb ist auch durch die Beschränkung ihrer Analyse auf deutsche grenzüberschreitende Konzerne zu erklären. 
Abbildung 28: Änderung des Aufkommenseffekts der Einführung einer konsolidierten Körperschaftsteuer-Bemessungsgrundlage unter Berücksichtigung einer pauschalen Verlustverrechnung gegenüber dem effektiven Gewinnsteuersatz

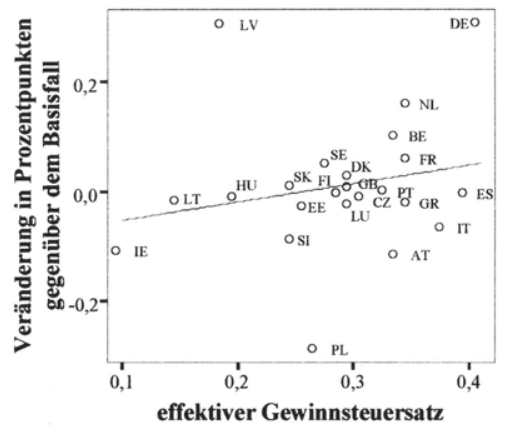

Quelle: eigene Darstellung

Tabelle 52: Aufkommenswirkung der Einführung einer konsolidierten Körperschaftsteuer-Bemessungsgrundlage - Berücksichtigung einer pauschalen Verlustverrechnung

\begin{tabular}{|c|c|c|c|c|c|c|c|}
\hline Land & SA Verlust & $\begin{array}{c}\text { Differenz SA Verlust } \\
\text { - SA Basisfall }\end{array}$ & FA Verlust & $\begin{array}{c}\text { Differenz FA Verlust } \\
\text {-FA Basisfall }\end{array}$ & $\begin{array}{r}\text { Differenz SA } \\
\text { - FA Ve }\end{array}$ & $\begin{array}{l}\text { Verlust } \\
\text { rlust }\end{array}$ & \begin{tabular}{|c|} 
Ver- \\
änderung
\end{tabular} \\
\hline AT & 341.667 & $-22.457 \quad-6,2 \%$ & 223.604 & $-56.753-20,2 \%$ & -118.063 & $-34,6 \%$ & $-11,5 \%$ \\
\hline $\mathrm{BE}$ & 9.907 .220 & $-3.189 .429-24,4 \%$ & 9.811 .245 & $-1.826 .253-15,7 \%$ & -95.975 & $-1,0 \%$ & $10,2 \%$ \\
\hline $\mathrm{CZ}$ & 2.110 .113 & $-111.560-5,0 \%$ & 1.613 .929 & $-108.441-6,3 \%$ & -496.185 & $-23,5 \%$ & $-1,0 \%$ \\
\hline $\mathrm{DE}$ & 6.254 .559 & $-4.104 .563-39,6 \%$ & 7.893 .718 & $-1.982 .130-20,1 \%$ & 1.639 .159 & $26,2 \%$ & $30,9 \%$ \\
\hline DK & 14.840 .799 & $-2.095 .100-12,4 \%$ & 13.871 .697 & $-1.464 .771 \quad-9,6 \%$ & -969.102 & $-6,5 \%$ & $2,9 \%$ \\
\hline EE & 346.707 & $-10.900 \quad-3,0 \%$ & 222.233 & $-16.829 \quad-7,0 \%$ & -124.474 & $-35,9 \%$ & $-2,8 \%$ \\
\hline ES & 25.017 .519 & $-1.479 .075 \quad-5,6 \%$ & 20.820 & $-1.289 .555 \quad-5,8 \%$ & -4.19 & $-16,8 \%$ & $-0,2 \%$ \\
\hline FI & 9.668 .910 & $-626.816-6,1 \%$ & 6.721 .746 & $-465.118-6,5 \%$ & -2.9 & $-30,5 \%$ & $-0,3 \%$ \\
\hline FR & 25.533 .112 & $-15.013 .329-37,0 \%$ & 24.389 .924 & $-11.956 .719-32,9 \%$ & -1.143 .188 & $-4,5 \%$ & $5,9 \%$ \\
\hline GB & 24.7 & $-6.853 .706-21,7 \%$ & 24.2 & $-6.443 .765-21,0 \%$ & 76 & $-1,9 \%$ & $0,9 \%$ \\
\hline GR & 289 & $591-4,5 \%$ & 1. & $-125.584-7,0 \%$ & 109 & $-28,3 \%$ & $-2,0 \%$ \\
\hline $\mathrm{HU}$ & 132 & $803-2,2 \%$ & & $-22.019 \quad-3,2 \%$ & & $-8,9 \%$ & $-0,9 \%$ \\
\hline IE & 292 & $-556.826-24,3 \%$ & 72 & $-552.733-36,7 \%$ & 020 & $-45,0 \%$ & $-10,8 \%$ \\
\hline IT & 13.079 .683 & $-6.323 .673-32,6 \%$ & 203.781 & $-5.727 .807-38,4 \%$ & -3.875 .902 & $-29,6 \%$ & $-6,6 \%$ \\
\hline LT & 42.135 & $-2.861 \quad-6,4 \%$ & 24.328 & $-2.349-8,8 \%$ & & $-42,3 \%$ & $-1,5 \%$ \\
\hline LU & 776.660 & $-45.761 \quad-5,6 \%$ & 51 & $-49.865 \quad-8,8 \%$ & 696 & $-33,4 \%$ & $-2,4 \%$ \\
\hline LV & 35.144 & $-12.698-26,5 \%$ & 56 & $-5.669-9,1 \%$ & 21.183 & $60,3 \%$ & $30,7 \%$ \\
\hline NL & 9.697 .121 & $-5.069 .205-34,3 \%$ & 7.78 & $-1.701 .265-17,9 \%$ & -1.9 & -19 & $16,0 \%$ \\
\hline PL & 655 & $51-16,7 \%$ & & $04-43,9 \%$ & & $-40,6 \%$ & $-28,8 \%$ \\
\hline PT & 2.820 .550 & $-187.493 \quad-6,2 \%$ & 2.423 .945 & $-153.104 \quad-5,9 \%$ & -396.605 & $-14,1 \%$ & $0,3 \%$ \\
\hline SE & 10.503 .112 & $-3.153 .353-23,1 \%$ & 10.406 .445 & $-2.424 .365-18,9 \%$ & 668 & & $5,1 \%$ \\
\hline SI & 45.088 & $0 \quad 0,0 \%$ & 29.251 & $-4.001-12,0 \%$ & 837 & $-35,1 \%$ & $-8,9 \%$ \\
\hline SK & 93.526 & $-1.179-1,2 \%$ & 73.694 & $0 \quad 0,0 \%$ & -19.832 & $-21,2 \%$ & $1,0 \%$ \\
\hline $\mathrm{EU}$ & 61.764 .985 & $-49.223 .875-23,3 \%$ & 144.291 .917 & $-36.930 .218-20,4 \%$ & -17.473 .068 & $-10,8 \%$ & $3,3 \%$ \\
\hline
\end{tabular}

Quelle: eigene Berechnungen 


\section{d) Operativer Gewinn als aufzuteilende Bemessungsgrundlage}

Die Amadeus-Datenbank weist zwar die Finanzerträge und die Finanzaufwendungen separat aus, eine Unterscheidung zwischen Zins- und Dividendenerträgen ist jedoch nicht möglich. Im Basisfall wird dieses Informationsdefizit durch eine pauschale Aufteilung der Finanzerträge in Zins- und Dividendenerträge gelöst. Letztere sind steuerfrei und damit nicht Bestandteil der aufzuteilenden Bemessungsgrundlage. ${ }^{655}$ Analog dazu wird das Finanzanlagevermögen pauschal um die Beteiligungsbuchwerte der verbundenen Unternehmen gekürzt. Dass diese Arbeitshypothese nicht auf jedes Unternehmen gleichermaßen zutrifft, stellt dabei noch kein Problem dar, da sich die Differenzen innerhalb der großen Stichprobe zumindest teilweise ausgleichen. Existieren hingegen systematische Unterschiede zwischen den einzelnen Mitgliedstaaten, wird das Ergebnis verzerrt dargestellt. So ist anzunehmen, dass der Anteil der Dividenden am Finanzergebnis an typischen Holdingstandorten erheblich höher ist. Zudem können im Finanzanlagevermögen stille Reserven gebunden sein. Beides bewirkt, dass der negative Aufkommenseffekt der Einführung der konsolidierten Körperschaftsteuer-Bemessungsgrundlage in diesen Ländern überschätzt wird, da Dividendeneinkünfte als steuerpflichtiges Einkommen betrachtet werden, denen nicht im gleichen Maße Aufteilungsfaktoren gegenüberstehen, sodass vermehrt Steuersubstrat durch die indirekte Gewinnabgrenzung aus den Holdingstandorten abgezogen wird.

Um diese Verzerrung auszuschließen, wird der Aufkommenseffekt der Einführung der konsolidierten Körperschaftsteuer-Bemessungsgrundlage auf der Basis des operativen Gewinns ermittelt. Somit werden weder die Finanzerträge noch die Finanzaufwendungen weiter beachtet. Damit sind aber auch die Ergebnisse der formelhaften Gewinnaufteilung des operativen Gewinns nur eingeschränkt mit dem Basisfall vergleichbar, weswegen lediglich die Änderungen der Rangfolgen des Aufkommenseffekts betrachtet werden.

Tabelle 53 zeigt, dass sich die Rangfolge für die Mehrheit der Mitgliedstaaten kaum verändert. Deutlich dagegen rutschen die Niederlande $(-16)$, Slowenien $(-8)$ sowie Estland und Luxemburg $(-4)$ ab. Vor allem die Niederlande und Luxemburg sind als bevorzugter Holdingstandort in der Europäischen Union bekannt. ${ }^{656}$ Für diese Länder ist anzunehmen, dass im Basisfall der negative Aufkommenseffekt tendenziell überschätzt wurde. Diese Vermutung wird zudem durch die Aussage der Abbildung 25 erhärtet, die im Wesentlichen die starke Abnahme der Bemessungsgrundlage in Luxemburg und den Niederlanden auf einen negativen Formeleffekt zurückführt.

655 Vgl. zu der Ermittlung des aufzuteilenden Gewinns Kapitel F.II.1.a).

656 Zur Standortwahl für eine europäische Holdinggesellschaft vgl. Günkel, M., in: $W P g$, 2003, S. S40-S56. 
Tabelle 53: Vergleich der Rangfolgen des Aufkommenseffekts der Einführung einer konsolidierten Körperschaftsteuer-Bemessungsgrundlage bei direkter Zurechnung des Finanzergebnisses mit dem Basisfall

\begin{tabular}{|c|cc|cr|r|}
\hline Land & $\begin{array}{c}\text { Differenz SA BMG - FA } \\
\text { BMG (operativer Gewinn) }\end{array}$ & Rangfolge & $\begin{array}{c}\text { Differenz SA BMG -FA } \\
\text { BMG (Gewinn vor Steuern) }\end{array}$ & $\begin{array}{r}\text { Rangfolge } \\
\text { BT }\end{array}$ & $\begin{array}{c}\text { Rangfolgen- } \\
\text { änderung }\end{array}$ \\
\hline BE & $-19,0 \%$ & 19 & $-23,0 \%$ & 15 & 4 \\
CY & $-16,5 \%$ & 18 & $-11,1 \%$ & 9 & 9 \\
CZ & $0,0 \%$ & 3 & $0,0 \%$ & 2 & 1 \\
DE & $-26,8 \%$ & 21 & $-22,5 \%$ & 14 & 7 \\
DK & $-2,9 \%$ & 6 & $-4,7 \%$ & 4 & 2 \\
EE & $-5,3 \%$ & 8 & $-9,4 \%$ & 7 & 1 \\
ES & $-15,4 \%$ & 17 & $-33,1 \%$ & 21 & -4 \\
FI & $-10,3 \%$ & 13 & $-16,6 \%$ & 12 & 1 \\
FR & $-30,0 \%$ & 24 & $-30,2 \%$ & 19 & 5 \\
GB & $-7,4 \%$ & 10 & $-10,4 \%$ & 8 & 2 \\
GR & $8,8 \%$ & 2 & $-2,8 \%$ & 3 & -1 \\
HU & $-26,8 \%$ & 22 & $-26,3 \%$ & 18 & 4 \\
IE & $-1,9 \%$ & 4 & $-8,0 \%$ & 6 & -2 \\
IT & $-28,9 \%$ & 23 & $-34,2 \%$ & 22 & 1 \\
LT & $-10,7 \%$ & 14 & $-23,0 \%$ & 16 & -2 \\
LU & $-53,1 \%$ & 25 & $-40,7 \%$ & 24 & 1 \\
LV & $-12,2 \%$ & 16 & $-31,1 \%$ & 20 & -4 \\
NL & $9,9 \%$ & 1 & $29,6 \%$ & 1 & 0 \\
PL & $-5,1 \%$ & 7 & $-35,8 \%$ & 23 & -16 \\
PT & $-9,8 \%$ & 12 & $-11,8 \%$ & 10 & 2 \\
SE & $-11,3 \%$ & 15 & $-14,3 \%$ & 11 & 4 \\
SI & $-1,9 \%$ & 5 & $-6,0 \%$ & 5 & 0 \\
SK & $-6,0 \%$ & 9 & $-26,3 \%$ & 17 & -8 \\
\hline EU & $-17,6 \%$ & $-22,2 \%$ & 13 & 6 \\
\hline
\end{tabular}

Quelle: eigene Berechnung

\section{e) Vergleich mit auf anderen Datenbasen beruhenden empirischen Studien}

Die Motivation der vorliegenden Arbeit leitete sich auch aus den fehlenden ausführlichen empirischen Analysen zu den Aufkommenswirkungen der Einführung einer einheitlichen (konsolidierten) Körperschaftsteuerbemessungsgrundlage ab. Bis heute sind nur vereinzelte Studien hierzu erschienen. ${ }^{657}$ Weiner zeigt die Auswirkungen der Einführung einer formelhaften Gewinnaufteilung, indem sie den Anteil des Nettoeinkommens US-amerikanischer Konzerne in den Mitgliedstaaten der Europäischen Union mit deren Aktivität vergleicht. Diese wird gemäß der Massachusetts-Formula mit dem gewichteten Anteil der Formelfaktoren gemessen. ${ }^{658}$ Im Ergebnis sind die Einkommen in Irland, den Niederlanden und Luxemburg unverhältnismäßig hoch. Deutschland,

${ }^{657} \mathrm{Vgl}$. für Berechnungen, die auch auf den Daten von Bureau van Dijk basieren, sich aber stellenweise einer anderen Methodik bedienen, Devereux, M.P./Loretz, S., Apportionment, 2007.

Vgl. Weiner, J.M., Reform, 2006, S. 7-8. 
Frankreich und Großbritannien hingegen weisen im Vergleich zur Aktivität ein zu geringes Einkommen auf. Dies ist ein Indiz für potenzielle Gewinner einer konsolidierten Besteuerung.

Fuest/Hemmelgarn/Ramb haben die Aufkommenswirkung der Einführung einer konsolidierten Körperschaftsteuer-Bemessungsgrundlage auf der Basis der Daten der Bundesbank-Direktinvestitionsstatistik berechnet. ${ }^{69}$ Sie betrachten demzufolge ausschließlich europäische Konzerne, die eine Direktinvestition in Deutschland tätigen, oder deutsche Unternehmensgruppen, die mit einer $\mathrm{Be}-$ triebsstätte oder einer Tochterkapitalgesellschaft im Ausland aktiv sind. ${ }^{660}$ Wesentlicher Nachteil dieser Datenbasis ist die von den gängigen steuerlichen Gruppendefinitionen abweichende Abgrenzung des Konzernkreises. ${ }^{661}$ So tauchen ausländische Tochtergesellschaften schon ab einer Beteiligung von 10 v. H. in der Statistik auf. Auch die Abbildung deutscher Konzerne erfolgt unvollständig. Konzerngesellschaften ohne Direktinvestitionen sind nicht in der Statistik enthalten. Zudem kann kein Bezug zwischen verschiedenen deutschen Tochtergesellschaften eines Konzerns hergestellt werden, die jeweils eine ausländische Direktinvestition halten. Damit zerbrechen größere Konzerne in kleinere Teileinheiten. Trotz der unterschiedlichen Daten und Berechnungsmethoden werden in Abbildung 29 die Ergebnisse von Fuest/Hemmelgarn/Ramb mit denen der vorliegenden Arbeit verglichen. Ihrer Vorgehensweise folgend wird die Lohnsumme in der Aufteilungsformel durch die Anzahl der Mitarbeiter ersetzt. ${ }^{662}$ Wird Österreich als extremer Ausreißer (+ 122 v. H.) vernachlässigt, beträgt der mittlere Betrag der Abweichung der Ergebnisse 17,6 Prozentpunkte. Für die Mehrzahl der Mitgliedstaaten ermitteln Fuest/Hemmelgarn/Ramb eine stärkere Abnahme der Bemessungsgrundlage, ${ }^{663}$ sodass die Ergebnisse dennoch eine signifikante mittlere Korrelation $(0,526)$ aufweisen. ${ }^{664}$ Diesen Zusammenhang verdeutlicht auch das Diagramm der Abbildung 29. Beide Untersuchungen identifizieren Irland und die Niederlande als große Verlierer der Einführung einer konsolidierten Körperschaftsteuer-Bemessungsgrundlage in der Europäischen Union. Demgegenüber weisen Deutschland, Großbritannien und Spanien in beiden Berechnungen eine unterdurchschnittliche Abnahme des Steuersubstrats auf. Neben Österreich, das nicht im Diagramm auftaucht, weichen ins-

659 Vgl. Fuest, C./Hemmelgarn, T./Ramb, F., Apportionment, 2006.

${ }^{660}$ Für eine Erläuterung der Grundlagen der Bestandsstatistik, insbesondere der Meldefreigrenzen, vgl. Lipponer, A., in: Herrmann/Lipsey, Direct Investment, 2003, S. 216-218.

661 Vgl. hierzu a. Oestreicher, A. et al., Weiterentwicklung, S. 386-387; siehe a. Kapitel D.II.

662 Vgl. hierzu a. Kapitel F.III.3.b).

${ }^{663}$ Die stärkere Abnahme ist auf eine erweiterte Verlustverrechnung zurückzuführen, was sich auch in der um acht Prozentpunkte höheren Abnahme der EU-Gesamtbemessungsgrundlage ausdrückt.

664 Die Korrelation nach Pearson ist auf dem Niveau von 0,05 (1-seitig) signifikant. 
besondere die Ergebnisse für Schweden, Griechenland und Portugal stark voneinander $a b$.

Abbildung 29: Vergleich der Untersuchungsergebnisse des Basisfalls mit Berechnungen auf der Basis der Direktinvestitionsstatistik

\begin{tabular}{|c|cc|}
\hline Land & $\begin{array}{c}\text { Eigene } \\
\text { Berechnungen }\end{array}$ & $\begin{array}{c}\text { Fuest/Hemmelgarn/ } \\
\text { Ramb }\end{array}$ \\
\hline AT & $-21 \%$ & $112 \%$ \\
BE & $-15 \%$ & $-41 \%$ \\
DE & $-8 \%$ & $-17 \%$ \\
DK & $-9 \%$ & $-24 \%$ \\
ES & $-17 \%$ & $-16 \%$ \\
FI & $-30 \%$ & $-25 \%$ \\
FR & $-12 \%$ & $-31 \%$ \\
GB & $1 \%$ & $-8 \%$ \\
GR & $-17 \%$ & $15 \%$ \\
IE & $-32 \%$ & $-51 \%$ \\
IT & $-23 \%$ & $-26 \%$ \\
LU & $-34 \%$ & $-39 \%$ \\
NL & $-45 \%$ & $-74 \%$ \\
PT & $-9 \%$ & $13 \%$ \\
SE & $-5 \%$ & $-57 \%$ \\
\hline EU & $-14 \%$ & $-22 \%$ \\
\hline
\end{tabular}

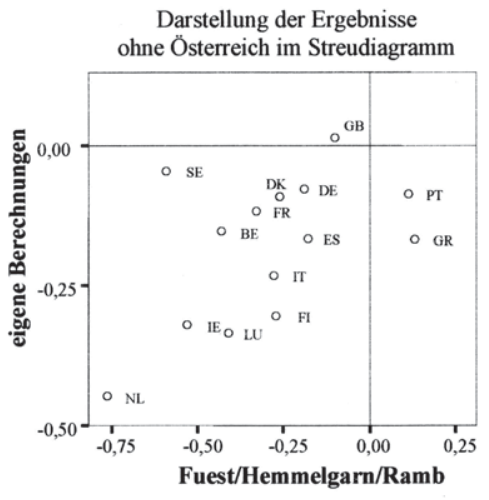

Quelle: Fuest, C./Hemmelgarn, T./Ramb, F., Apportionment, 2006, S. 18; eigene Berechnungen

\section{Alternative Mindestbeteiligungshöhen}

Die Berechnungen des Basisfalls beruhen auf einer Abgrenzung des Konsolidierungskreises, die eine Mindestbeteiligungsquote von $>50 \mathrm{v}$. H. voraussetzt. Sowohl in der politischen als auch in der wissenschaftlichen Diskussion werden jedoch häufig höhere Mindestbeteiligungsquoten gefordert. ${ }^{665}$ Dadurch soll erstens die politische Akzeptanz der konsolidierten Körperschaftsteuer-Bemessungsgrundlage erhöht werden, denn der durchschnittliche Schwellenwert der bestehenden europäischen Gruppenbesteuerungssysteme liegt bei 77,2 v. ${ }^{666}$ Zweitens sollen strengere Qualifikationskriterien missbräuchliche steuerplanerische Gestaltungen verhindern. Dies gilt insbesondere für einen ausschließlich nach rechtlichen Kriterien abgegrenzten Konsolidierungskreis.

Auf der Basis der empirischen Daten lässt sich zunächst ein linearer Zusammenhang zwischen der EU-Gesamtbemessungsgrundlage und der Mindestbeteiligungsquote feststellen. Ein Anheben der Mindestbeteiligungsquote schwächt den negativen Aufkommenseffekt der Einführung der konsolidierten Körperschaftsteuer-Bemessungsgrundlage ab. Ursächlich hierfür ist das Zer-

${ }^{665}$ Vgl. hierzu a. Kapitel C.II.2.

${ }^{666}$ Vgl. hierzu Tabelle 8 und FN 251. 
fallen der Konsolidierungskreise in Teileinheiten, wodurch die Möglichkeit der Verlustverrechnung innerhalb der Konzerne eingeschränkt wird. ${ }^{667}$ Im Durchschnitt bewirkt eine Anhebung der Mindestbeteiligungsquote um 10 Prozentpunkte einen Anstieg der EU-Gesamtbemessungsgrundlage um 1,12 Prozentpunkte (Abbildung 30). Damit streut die FA-Bemessungsgrundlage je nach festgelegter Mindestbeteiligungsquote um 5,58 Prozentpunkte.

Abbildung 30: Entwicklung der gesamten konsolidierten Körperschaftsteuer-

Bemessungsgrundlage in v. H. SA in Abhängigkeit von der

Mindestbeteiligungsquote

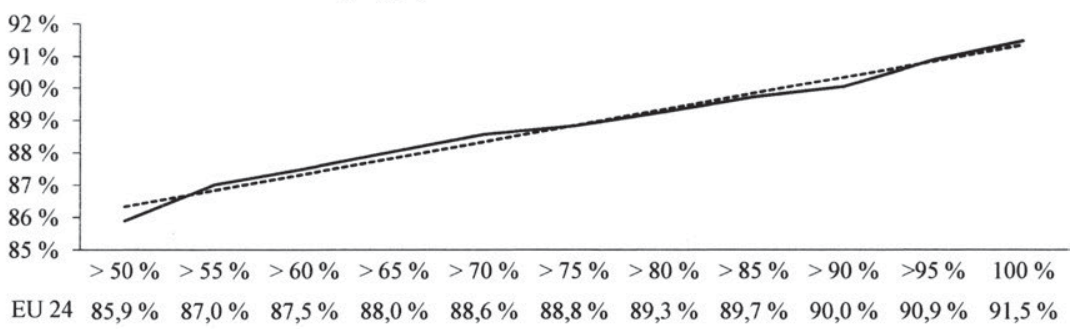

Quelle: eigene Darstellung

Dieser leichte, kontinuierliche Anstieg der Bemessungsgrundlage ist nicht für alle Mitgliedstaaten zu beobachten (Abbildung 31). Besonders in den neuen Mitgliedstaaten steigt die Bemessungsgrundlage mit dem Anheben der Mindestbeteiligungsquote teilweise sprunghaft an. Diese Entwicklung ist vornehmlich auf die sehr begrenzte Anzahl von Konzernen in der Untersuchung für die entsprechenden Länder zurückzuführen. ${ }^{668}$ Dadurch wirkt sich bereits das „Zerschlagen" eines einzelnen Konzerns direkt auf die Gesamtbemessungsgrundlage des einzelnen Staates aus. Aber auch für die großen Volkswirtschaften der „alten“ EU-15-Mitgliedstaaten kann der Anstieg der Bemessungsgrundlage teilweise auf einen einzelnen Konzern zurückgeführt werden. So ist für die Entwicklung der Bemessungsgrundlage in den Niederlanden fast ausschließlich der Zerfall der Shell Gruppe in neun Teilkonzerne verantwortlich.

Zwischen dem Schwellenwert der bestehenden Gruppenbesteuerungssysteme und der Entwicklung der Bemessungsgrundlage kann kein Zusammenhang beobachtet werden. Zwar könnte man vermuten, dass sich die Höhe der Beteiligung an den Tochtergesellschaften auch nach den Anwendungsvoraussetzungen der Gruppenbesteuerungssysteme richtet, sodass in den entsprechenden Ländern nach dem Überschreiten der entsprechenden Schwellenwerte

${ }^{667}$ Für eine Übersicht über die Entwicklung der Anzahl der Konsolidierungskreise in Abhängigkeit von der Mindestbeteiligung vgl. Abbildung 2.

${ }^{668}$ Für eine Übersicht über die Anzahl der in die Berechnungen eingehenden Konzerne und Konzerngesellschaften siehe Tabelle 46. 
mit einem verstärkten Anstieg der Bemessungsgrundlage zu rechnen wäre, doch werden 87 v. H. der Konzerngesellschaften direkt von anderen Konzerngesellschaften zu $98 \mathrm{v}$. H. oder mehr gehalten. ${ }^{669}$

Abbildung 31: Aufkommenswirkung der Einführung einer konsolidierten Körperschaftsteuer-Bemessungsgrundlage bei alternativen Mindestbeteiligungsquoten in v. H. SA

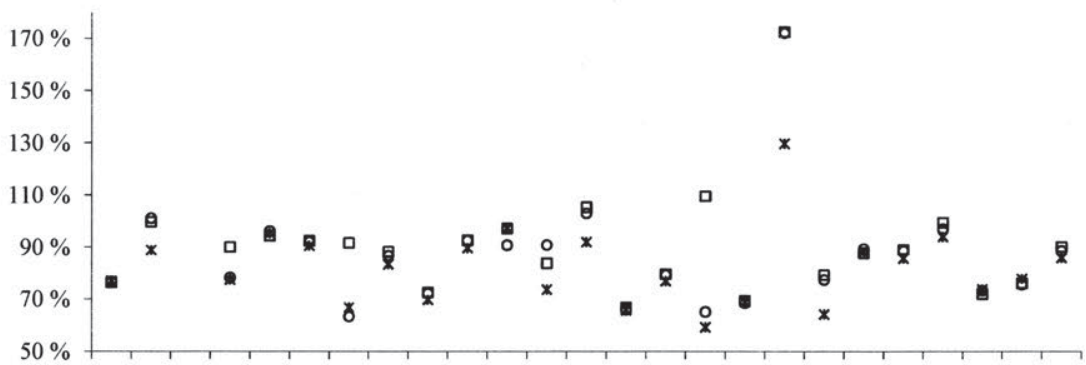

AT BE CY CZ DE DK EE ES FI FR GB GR HU IE IT LT LU LV NL PL PT SE SI SK EU $\times 50 \circ 70 \square 90$

Quelle: eigene Darstellung

\section{Alternative mikroökonomische Aufteilungsformeln}

\section{a) Alternative Gewichtung der Formelfaktoren}

Die Massachusetts-Formula, die im Basisfall zur Anwendung kam, ist die bekannteste Formel im Rahmen der indirekten Gewinnabgrenzung. Zwar ist ihre Zusammensetzung theoretisch fundiert, ${ }^{670}$ doch gilt dieses in gleichem Maße für andere mikroökonomische Aufteilungsformeln. ${ }^{671}$ So existieren sowohl in der Literatur als auch in der Praxis Vorschläge bzw. Beispiele für eine alternative Gewichtung ${ }^{672}$ der Formelfaktoren Vermögen, Umsatz und Lohnsumme, die auf unterschiedlichen Überlegungen basieren.

669 Vgl. hierzu Tabelle 27.

${ }^{670} \mathrm{Vgl}$. zu den theoretischen Überlegungen zur Aufteilung der Bemessungsgrundlage Kapital C.II.4.

${ }^{671}$ Vgl. Weiner, J.M., in: ET, 2001, S. 384. Die Anwendung makroökonomischer Aufteilungsformeln ist aufgrund ihrer systematischen Defizite sehr unwahrscheinlich (vgl. Kapitel C.II.4.b)( $\beta)$ ). Aufgrund dessen werden sie im Rahmen der empirischen Analyse nicht behandelt.

${ }^{672}$ Eine alternative Gewichtung beinhaltet auch das Weglassen von einem oder zwei Formelfaktoren (Gewichtung mit 0 v. H.). Beispielsweise wird im Rahmen der deutschen Gewerbesteuer der Steuermessbetrag auf die Gemeinden der einzelnen Betriebsstătten mittels der Lohnsumme zerlegt ( $\$ 29 \mathrm{GewStG})$. Vgl. hierzu ausfuihrlich Oestreicher, A., Konzerngewinnabgrenzung, 1999, S. 145-148. 
Beispielsweise weist Oestreicher auf den der Massachusetts-Formula zugrunde liegenden Ansatz hin. Dieser „supply-demand approach“ betrachtet den Gewinn als das Ergebnis des Zusammenspiels von Angebot und Nachfrage. Folglich sollten die Angebotsseite und die Nachfrageseite gleichberechtigt nebeneinanderstehen, was eine Gewichtung des Umsatzfaktors von $50 \mathrm{v}$. H. voraussetzt. ${ }^{673}$ Obwohl das UDIPTA eine Gleichgewichtung der Formelfaktoren empfiehlt, ${ }^{674}$ ist eine starke Tendenz der US-amerikanischen Bundesstaaten $\mathrm{zu}$ erkennen, die Außenumsätze innerhalb der Aufteilungsformel stärker zu gewichten. ${ }^{675}$ Diese Entwicklung geht aber nicht auf den von Oestreicher vorgebrachten Einwand zurück, sondern hängt mit der Wirkung einer formelhaften Gewinnaufteilung zusammen, die auf der Lohnsumme und dem Vermögen basiert. Diese wirkt wie eine Steuer auf die Faktoren, ${ }^{676}$ weshalb die Bundesstaaten zur Förderung von Beschäftigung und Investitionen den Umsatzfaktor stärker gewichten. ${ }^{677}$ Zwar ist bereits, um eine Doppel- bzw. Minderbesteuerung zu vermeiden, eine einheitliche Formel innerhalb der Europäischen Union vorgesehen, ${ }^{678}$ doch wird auch hier jeder Mitgliedstaat diejenige Formel favorisieren, die ihm den größten Anteil an der EU-Gesamtbemessungsgrundlage verspricht. ${ }^{679}$

Abbildung 32 zeigt den Aufkommenseffekt bei Einführung der einheitlichen konsolidierten Bemessungsgrundlage, würde die Aufteilung wahlweise allein nach der Lohnsumme, dem Vermögen oder dem Umsatz erfolgen. Unten in der Grafik sind die Abweichungen vom Basisfall in Prozentpunkten dargestellt. Zunächst ist festzustellen, dass die Wahl der Gewichtung der Formelfaktoren den Aufkommenseffekt erheblich beeinflusst. Zwar zeigt Abbildung 32 die Extrempunkte auf, dennoch ist eine Spannweite der Ergebnisse von über 45 Prozentpunkten in Irland und Litauen beachtlich. Die Unterschiede zwischen dem Aufteilungsfaktor Vermögen und Umsatz sind für die Mehrzahl der Mitgliedstaaten gering. Demgegenüber liefert die Aufteilung der Bemessungsgrundlage auf Basis der Lohnsumme ein gänzlich anderes Bild. Länder mit einem

\footnotetext{
${ }^{673} \mathrm{Vgl}$. hierzu Oestreicher, A., Konzernbesteuerung, 1999, S. 182. Siehe auch Kapitel C.II.4.b)(ס). Die Fälle, in denen diese Forderung erfüllt ist, sind in der Abbildung 33 grau markiert.

674 Siehe Sec. 9 UDITPA.

675 Siehe Abbildung 4.

676 Vgl. McLure Jr., C.E., in: Aaron/Boskin, Economics, 1980, S. 327-334.

677 Goolsbee und Maydew liefern einen empirischen Beweis für die US-amerikanischen Bundesstaaten, dass ein Absenken des Lohnsummenfaktors von $1 / 3$ auf $1 / 4$ zu einem Anstieg der Beschäftigung im verarbeitenden Gewerbe um 1,1 v. $\mathrm{H}$. führt. Vgl. Goolsbee, A./Maydew, E.L., in: JoPE, 2000, S. 126.

${ }^{678} \mathrm{Vgl}$. Europäische Kommission, Mechanismus, 2006, S. 7.

$679 \mathrm{Vgl}$. McLure Jr., C.E., in: BIFD, 2002, S. 594.
} 
niedrigen Lohnniveau verlieren deutlich an Bemessungsgrundlage, was insbesondere die osteuropäischen Mitgliedstaaten betrifft. Mit Deutschland, den Niederlanden und Großbritannien zählen Mitgliedstaaten mit einem hohen Lohnniveau zu den Gewinnern einer solchen Verteilung der Bemessungsgrundlage. Dieses Ergebnis ist ein weiteres Indiz für die im Schrifttum geäußerten Bedenken, dass die Lohnsumme aufgrund der verschiedenen Lohnniveaus in den einzelnen Mitgliedstaaten keinen geeigneten Aufteilungsfaktor darstellt und demzufolge durch die Anzahl der Mitarbeiter ersetzt werden sollte. ${ }^{680}$ Ein Vergleich der beiden Aufteilungsfaktoren erfolgt in Kapitel F.III.3.b).

Abbildung 32: Aufkommenseffekt der Einführung einer konsolidierten

Körperschaftsteuer-Bemessungsgrundlage auf der Basis des Vermögens, der Lohnsumme und des Umsatzes in v. H. SA und Abweichung vom Basisfall in Prozentpunkten ${ }^{681}$

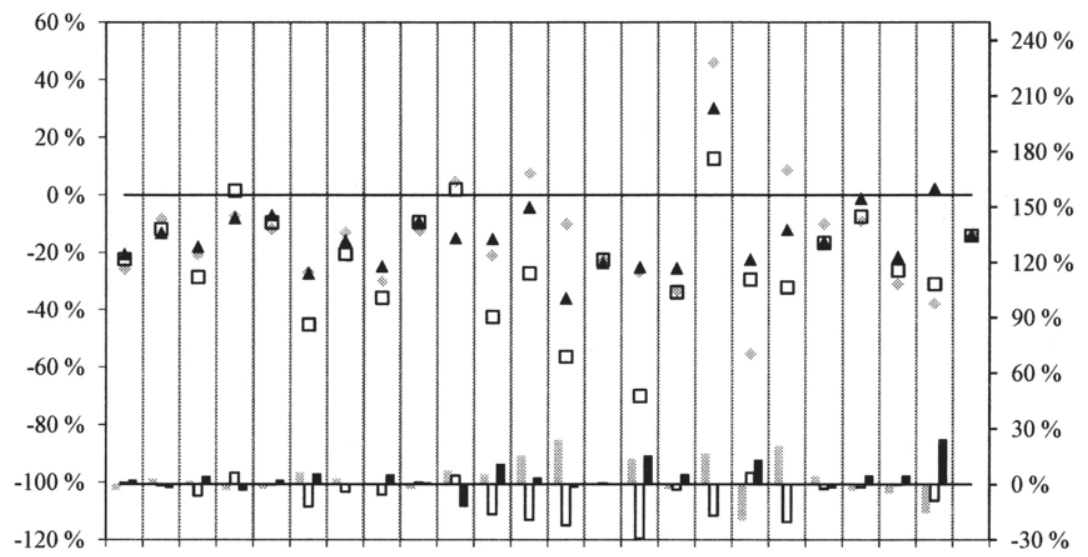

AT BE CZ DEDK EE ES FI FR GBGRHU IE IT LT LULVNL PL PT SE SI SK EU

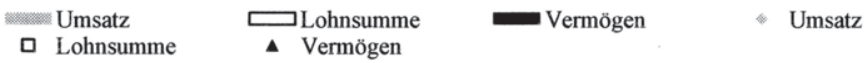

Quelle: eigene Darstellung

Die starken Auswirkungen einer unterschiedlichen Gewichtung der Formelfaktoren verdeutlichen, dass die Festlegung auf eine einheitliche Aufteilungsformel ein schwieriger politischer Auftrag ist. ${ }^{62}$ Dabei erscheint theoretisch diejenige Formel am vielversprechendsten, die im Durchschnitt die geringste Ver-

${ }^{680}$ Vgl. McLure Jr., C.E./Weiner, J., in: Cnossen, Capital, 2000, S. 270; Wellisch, D., in: StuW, 2004, S. 273; kritisch Oestreicher, A., Konzerngewinnabgrenzung, 1999, S. 184190.

${ }^{681}$ Die dazugehörige Datentabelle befindet sich im Anhang in Tabelle 61.

${ }^{682}$ Vgl. Radulescu, D.M., in: ifo Schnelldienst, 2002, 2, S. 15-16; Hey, J., in: StuW, 2004, S. 206. 
änderung der Bemessungsgrundlage der Mitgliedstaaten in der Europäischen Union bewirkt.
Abbildung 33: Durchschnittliche quadrierte Abweichung des Aufkommenseffekts der Einführung einer konsolidierten Körperschaftsteuer-
Bemessungsgrundlage in Abhängigkeit von der Gewichtung der Formelfaktoren in v. H. SA $\mathbf{S B 3}^{683}$

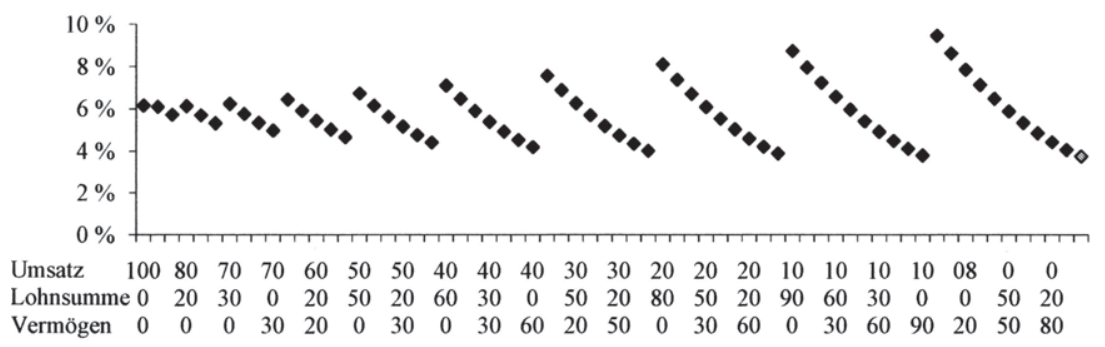

Quelle: eigene Darstellung

Abbildung 33 zeigt die durchschnittliche quadrierte Abweichung für unterschiedliche Gewichtungen der Formelfaktoren. Danach entwickelt im Vergleich zum bestehenden Steuerrecht eine indirekte Gewinnabgrenzung ausschließlich auf der Basis des Vermögens im Mittel die geringste Aufkommenswirkung für die einzelnen Mitgliedstaaten. Dieses Ergebnis ist wenig verwunderlich, kommt doch nur in dem Vermögensfaktor und dem Lohnsummenfaktor das Quellenverständnis der direkten Methode zum Ausdruck. ${ }^{684} \mathrm{Da}$ aber der Lohnsummenfaktor nur eine Erweiterung der Vermögensformel darstellt und der oben dargelegten Verzerrung aufgrund abweichender Lohnniveaus unterliegt, ${ }^{685}$ kommt eine Aufteilung der Bemessungsgrundlage anhand des Vermögens der direkten Gewinnabgrenzung am nächsten. ${ }^{686}$

Ein Vergleich der indirekten Gewinnabgrenzung nach dem vorhanden Vermögen und mittels der Massachusetts-Formula kann dem unteren Teil der Abbildung 32 entnommen werden.

683 Die dazugehörige Datentabelle befindet sich im Anhang in Tabelle 62.

${ }^{684} \mathrm{Vgl}$. Oestreicher, A., Konzerngewinnabgrenzung, 1999, S. 184; Musgrave, P.B., in: McLure, State, 1984, S. 238-240.

685 Hier sei angemerkt, dass Oestreicher beispielhaft für sechs Länder zeigt, dass die bestehenden Lohnsatzunterschiede nicht zu einer Benachteiligung der Länder mit niedrigem Lohnniveau füren. Vgl. Oestreicher, A., Konzerngewinnabgrenzung, 1999, S. 184-190.

$686 \mathrm{Vgl}$. hierzu auch die theoretische Beweisführung von Musgrave, P.B., in: McLure, State, 1984, S. 238-240. 


\section{b) Alternativer Aufteilungsfaktor}

Wie bereits mehrfach angesprochen, werden im Schrifttum Bedenken geäußert, die Aufteilung der Bemessungsgrundlage auf der Basis der Lohnsumme führe aufgrund des unterschiedlichen Lohnniveaus in den einzelnen Mitgliedstaaten zu Verzerrungen. Mitgliedstaaten mit einem niedrigen Lohnniveau erhielten einen vergleichsweise geringen Anteil am Konzerneinkommen, obwohl oftmals das niedrige Lohnniveau in diesen Ländern für höhere Gewinne im Konzern verantwortlich ist und Unternehmensgruppen veranlasst, bestimmte Funktionen dorthin zu verlagern. ${ }^{67}$ Stattdessen wird der Ersatz der Lohnsumme durch die Anzahl der Mitarbeiter diskutiert. ${ }^{688}$ Zwar ist damit das Lohnniveau ausgeblendet, dafür geht aber auch die Produktivität der Arbeit und damit deren Beitrag zur Gewinnerzielung nicht mehr in die Aufteilungsformel mit ein, was ebenfalls Verzerrungen zur Folge hat. ${ }^{689}$

Abbildung 34 verdeutlicht zunächst die großen Differenzen zwischen dem Lohnniveau der einzelnen Mitgliedstaaten. Abgebildet sind sowohl die Daten der Eurostat Lohn- und Gehaltsstrukturstatistik als auch eigene Berechnungen auf der Basis der Amadeus-Datenbank. Beide Balkenpaare zeigen jeweils die gleiche Tendenz. Besonders in den osteuropäischen Mitgliedstaaten ist das Lohnniveau niedrig. Folglich profitieren insbesondere diese Staaten von der Anzahl der Mitarbeiter als Aufteilungsfaktor.

Abbildung 34: Lohnniveau und Arbeitsproduktivität in der Europäischen Union ${ }^{690}$

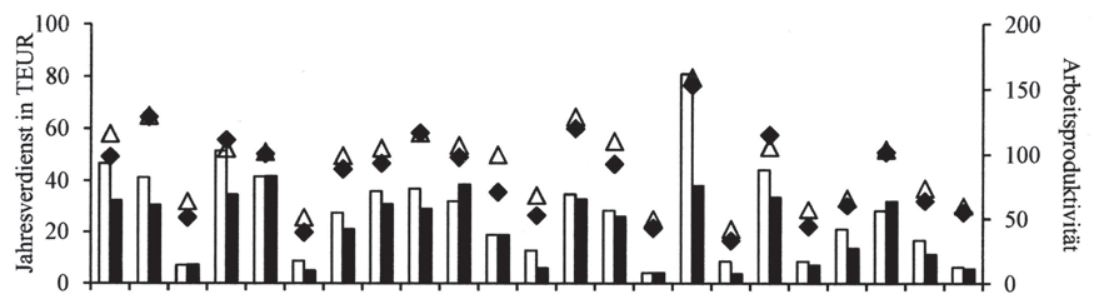

AT BE CZ DE DK EE ES FI FR GB GR HU IE IT LT LU LV NL PL PT SE SI SK

口Jahresverdienst in TEUR Amadeus-Datenbank

- Jahresverdienst in TEUR Eurostat

$\triangle$ Arbeitsproduktivität je Beschäftigten EU $25=100$

- Arbeitsproduktivität je geleisteter Arbeitsstunde EU $15=100$

Quelle: eigene Darstellung, Eurostat Lohn- und Gehaltsstrukturstatistik

${ }^{687}$ Vgl. Wellisch, D., in: StuW, 2004, S. 273.

688 Vgl. Agúndez-García, A., Apportionment, 2006, S. 47-48; Europäische Kommission, Mechanismus, 2006, S. 7-8.

${ }^{689} \mathrm{Vgl}$. McLure Jr., C.E./Weiner, J., in: Cnossen, Capital, 2000, S. 270; Wellisch, D., in: StuW, 2004, S. 273.

${ }^{690}$ Die dazugehörige Datentabelle befindet sich im Anhang in Tabelle 63. 

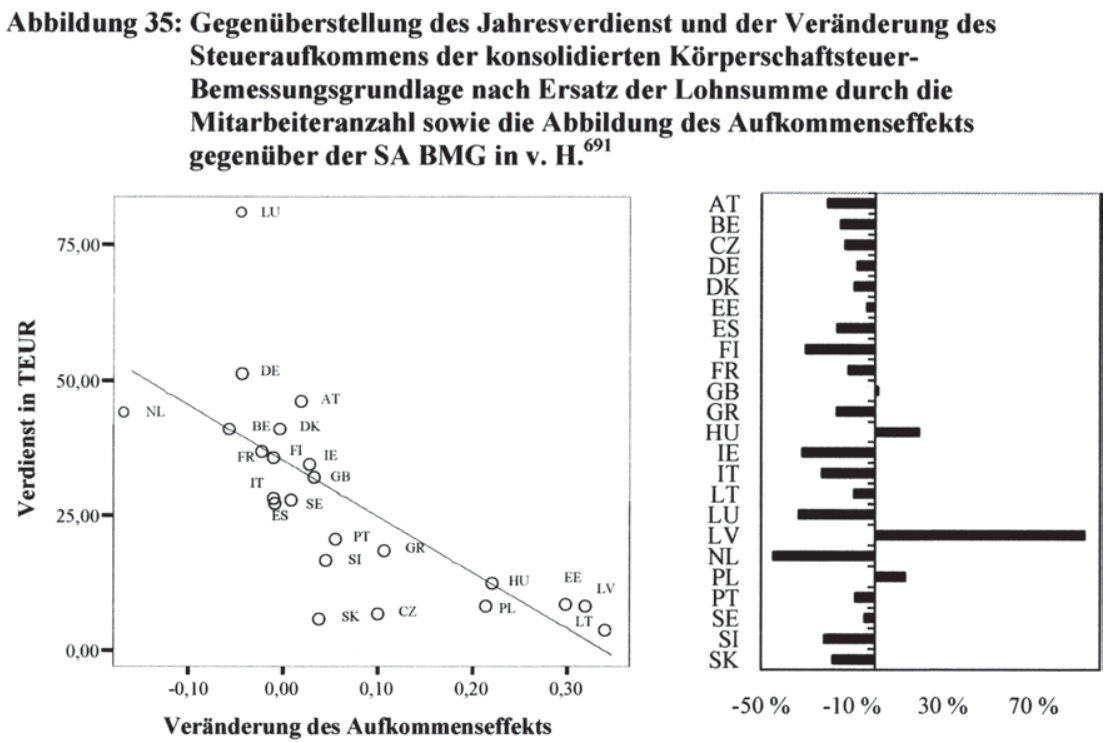

Quelle: eigene Darstellung

Abbildung 35 stellt die Änderung der Bemessungsgrundlage durch die Berücksichtigung der Mitarbeiteranzahl anstelle der Lohnsumme in der MassachusettsFormula dem durchschnittlichem Jahresverdienst gegenüber. Erwartungsgemäß zeigt sich ein signifikanter negativer Zusammenhang. So erhalten im Wesentlichen die osteuropäischen Mitgliedstaaten auf Kosten von Deutschland, Belgien, Frankreich, Luxemburg und den Niederlanden mehr Steuersubstrat zugewiesen. Die im Schrifttum geäußerten Bedenken sind damit zunächst einmal berechtigt. Jedoch weisen Oestreicher und auch Wellisch zu Recht auf die unterschiedliche Produktivität der Arbeitskräfte in den Mitgliedstaaten hin. ${ }^{692}$ In Abbildung 34 ist der Zusammenhang zwischen dem Lohnniveau und der Arbeitsproduktivität deutlich zu erkennen. Damit erscheint die angebliche Verzerrung durch die Berücksichtigung der Lohnsumme in der Aufteilungsformel zumindest teilweise gerechtfertigt. Zwar wäre auch eine Berücksichtigung der Produktivität durch die Bildung unterschiedlicher Kategorien von Arbeitskräften ${ }^{693}$ möglich; vor dem Hintergrund der Zielsetzung, eine einfache, überschaubare Formel zur

${ }^{691}$ Die dazugehörige Datentabelle befindet sich im Anhang in Tabelle 64.

692 Vgl. Oestreicher, A., Konzerngewinnabgrenzung, 1999, S. 184-190; Wellisch, D., in: StuW, 2004, S. 273.

${ }^{693}$ Zum Beispiel Arbeiter, einfache Angestellte, einfaches Management, Top-Management. 
Aufteilung der Bemessungsgrundlage zu benutzen, stellt die Verwendung der Lohnsumme jedoch einen vertretbaren Kompromiss dar. ${ }^{694}$

\section{Analyse der Steuerzahllaständerung}

Die verursachungsgerechte Aufteilung der einheitlichen konsolidierten Körperschaftsteuer-Bemessungsgrundlage mittels eines Schlüssels beinhaltet die Grundidee des Quellenprinzips. Die zugewiesene Bemessungsgrundlage wird in jedem Mitgliedstaat abschließend besteuert. Eine Vermeidung der Doppelbesteuerung durch die Freistellung der Gewinne im Ausland ist nicht notwendig, da diese aufgrund der Konsolidierung der Einzelabschlüsse erst gar nicht ausgewiesen werden. Somit garantiert eine indirekte Gewinnabgrenzung auf der Basis des Prinzips des einheitlichen Unternehmens eine kapitalimportneutrale Besteuerung, sofern eine Konsolidierung der Einzelergebnisse erfolgt.

Alternativ ist auch das Wohnsitzprinzip als Ausgangspunkt der einheitlichen konsolidierten Körperschaftsteuerbemessungsgrundlage denkbar. Dazu sind sämtliche Einkünfte europäischer Tochtergesellschaften im Land der Muttergesellschaft steuerpflichtig und unterliegen dem dort geltenden Steuersatz. Die im Ausland bereits auf diese Einkünfte entrichtete Körperschaftsteuer ist im Inland anzurechnen. Dies stellt eine kapitalexportneutrale Besteuerung sicher, sofern die Anrechnung unabhängig von der Höhe der inländischen Steuerschuld erfolgt. ${ }^{695}$ Aus fiskalpolitischen Gründen wird jedoch die Anrechnung der ausländischen Steuern vielfach begrenzt, um eine Erstattung über die anteilig auf die ausländischen Einkünfte entfallende Inlandssteuer hinaus zu vermeiden. Die Berechnung dieses Höchstbetrags kann dabei entweder für jedes Unternehmen (Per-Company Limitation) oder für jeden Mitgliedstaat (Per-Country Limitation) separat erfolgen oder aber alle ausländischen Einkünfte (Overall Limitation) miteinbeziehen. ${ }^{696}$ Im Rahmen der grenzüberschreitenden Gruppenbesteuerungssysteme wenden Dänemark und Frankreich eine Per-Country Limitation an, Italien hingegen ermittelt den Höchstbetrag für jedes Unternehmen getrennt. Auch außerhalb der Gruppenbesteuerungssysteme ist die Per-Country Limitation das vorherrschende Konzept in der Europäischen Union. ${ }^{697}$ Trotz der weiten Verbreitung verstößt es nach herrschender Meinung gegen den Grund-

\footnotetext{
694 Vgl. Wellisch, D., in: StuW, 2004, S. 273.

${ }^{695} \mathrm{Vgl}$. Oestreicher, A. et al., Weiterentwicklung, 2006, S. 278.

${ }^{696}$ Für eine Gegenüberstellung der Per-Country- und der Overall Limitation siehe Jacobs, O.H. (Hrsg.), Unternehmensbesteuerung, 2002, S. 51-54.

697 Von den 25 EU-Mitgliedstaaten sehen Irland und die Niederlande die Abzugsmethode vor, Schweden, Slowenien, Spanien und Zypern wenden keine Per-Country Limitation an. In den verbleibenden 19 Mitgliedstaaten wird der Höchstbetrag nach der Per-Country Limitation berechnet. Vgl. Oestreicher, A. et al., Weiterentwicklung, 2006, S. 281.
} 
gedanken der allgemeinen Binnenmarktgarantie ${ }^{698}$ da der Steuerpflichtige dazu gedrängt wird, seine Aktivitäten auf möglichst wenige Mitgliedstaaten zu konzentrieren. ${ }^{699}$ Dagegen erlaubt eine Overall Limitation den Unternehmen, etwaige Anrechnungsüberhänge mit nicht ausgeschöpften Anrechnungshöchstbeträgen zu verrechnen. ${ }^{700}$

Voraussetzung für das dargestellte Besteuerungskonzept ist der Zugriff auf die thesaurierten ausländischen Gewinne. Dem steht aber das in allen europäischen Mitgliedstaaten verfolgte Trennungsprinzip entgegen. Um eine transparente Besteuerung zu gewährleisten, ist es aufzuheben, damit der Mitgliedstaat der Konzernmuttergesellschaft das Einkommen der ausländischen Tochterkapitalgesellschaften besteuern kann. Somit ist das Konzept mit einer grenzüberschreitenden Verlustverrechnung nach der Zurechnungsmethode vergleichbar, ${ }^{701}$ die sich aber zugleich der indirekten Gewinnabgrenzung bedient. Die Zurechnungsmethode wird wiederum als eine allgemeine Hinzurechnungsbesteuerung charakterisiert, wobei sie unabhängig von der Aktivität, des ausländischen Steuerniveaus und der Erfolgssituation der Tochtergesellschaften angewendet wird. ${ }^{702}$ Die dafür notwendige Abschaffung des Trennungsprinzips ist aber bereits aus gesellschaftsrechtlicher Sicht problematisch ${ }^{703}$ und dürfte mit dem völkerrechtlichen Interventionsverbot kollidieren, ${ }^{704}$ gegen geltendes $\mathrm{Ge}$ meinschaftsrecht verstoßen und zudem das Abkommensrecht verletzen. ${ }^{705}$

Trotz der skizzierten Vorbehalte sollen im Rahmen einer komparativ-statischen Analyse beide Besteuerungsalternativen miteinander verglichen werden. Der relative Aufkommenseffekt einer auf dem Quellenprinzip basierenden Be-

${ }^{698}$ Art. 3 Abs. 1 c und Art. 14 Abs. 2 EGV.

699 Vgl. Cordewener, A./Schnittger, A., in: StuW, 2006, S. 73, m. w. N.

${ }^{700}$ Mehrbelastungen im Verlustfall einzelner Gesellschaften entstehen nicht, da aufgrund der indirekten Gewinnabgrenzung im Rahmen der konsolidierten KörperschaftsteuerBemessungsgrundlage entweder alle Konzerngesellschaften einen Gewinn oder einen Verlust ausweisen.

701 Vgl. Herzig, N./Englisch, J./Wagner, T., in: Der Konzern, 2005, S. 317; ausführlich Oestreicher, A. et al., Weiterentwicklung, 2006, S. 137-146.

702 Vgl. Oestreicher, A. et al., Weiterentwicklung, 2006, S. 137-138.

${ }^{703} \mathrm{Vgl}$. Herzig, N./Englisch, J./Wagner, T., in: Der Konzern, 2005, S. 317; Hahn, H., in: IStR, 1999, S. 615-616.

704 Vgl. Spengel, C., Unternehmensbesteuerung, 2003, S. 303. Anderer Auffassung Wissenschaftlicher Beirat beim BMF, Kapitaleinkommensbesteuerung, 1999, S. 41. Vor dem Hintergrund dieser Einwände sieht Spengel bei seiner auf dem Wohnsitzprinzip basierenden Weiterentwicklung der internationalen Unternehmensbesteuerung von einer Durchbrechung des Trennungsprinzips ab.

705 Vgl. Herzig, N./Englisch, J./Wagner, T., in: Der Konzern, 2005, S. 317; Spengel, C., Unternehmensbesteuerung, 2003, S. 303. 
steuerung entspricht dem Basisfall in Kapitel III.1, da lediglich die den einzelnen Konzerngesellschaften zugewiesene Bemessungsgrundlage mit dem geltenden Körperschaftsteuersatz multipliziert wird (Tabelle 65). Insofern haben auch die weiterführenden Analysen jenes Kapitels weiterhin Bestand.

Eine auf dem Wohnsitzprinzip beruhende Umsetzung der konsolidierten Körperschaftsteuer-Bemessungsgrundlage rechnet die im Ausland gezahlte Körperschaftsteuer im Inland an, wobei im Rahmen der empirischen Analyse der Anrechnungshöchstbetrag für jeden Mitgliedstaat separat ermittelt wird (PerCountry Limitation). Durch die Verwendung eines Anrechnungshöchstbetrags ist das Steueraufkommen jedes Mitgliedstaates mindestens gleich hoch oder höher als zuvor. Profitieren werden vor allem Mitgliedstaaten, die über einen hohen nominalen Gewinnsteuersatz verfügen. Dieser allein reicht aber noch nicht aus. Vielmehr müssen in den jeweiligen Mitgliedstaaten Konzernmuttergesellschaften ansässig sein, deren Tochtergesellschaften ihren Sitz in einem niedriger besteuernden Staat haben. ${ }^{706}$

Für die Berechnungen wird lediglich der nominale Körperschaftsteuersatz herangezogen, da regelmäßig nur Auslandssteuern angerechnet werden können, die der inländischen Steuer entsprechen, ihr also gleichartig sind. ${ }^{707} \mathrm{Die} \mathrm{Zu}$ rechnung der ausländischen Ergebnisse zur Mutterkapitalgesellschaft in Verbindung mit der Anrechnung der an der Quelle entrichteten Körperschaftsteuer lässt die Steuerzahllast in der Europäischen Union um 1,6 v. H. steigen. Die Hälfte dieses Anstiegs entfällt auf die Niederlande (+ 14,3 v. H.). In allen übrigen Mitgliedstaaten liegt die Zunahme unter 5 v. H. In acht Mitgliedstaaten hat die Wahl des Besteuerungskonzeptes keinen Aufkommenseffekt, da keine ausländischen Einkünfte auf ein höheres Steuerniveau hochgeschleust werden (Abbildung 36). Die Muttergesellschaften mit Sitz in diesen Staaten sind also nur an Tochtergesellschaften beteiligt, die in ihrem Ansässigkeitsstaat einer geringeren Steuerbelastung unterliegen, oder aber die betrachteten Konzerne erzielen einen Verlust. ${ }^{708}$

Ein Vergleich der relativen Änderung des Steueraufkommens mit dem nominalen Gewinnsteuersatz verdeutlicht den Zusammenhang zwischen der

706 Diese Aussage gilt natürlich nur ceteris paribus und vernachlässigt die dynamischen Anpassungsmaßnahmen der Konzerne. So ist vergleichbar zur Home State Taxation ein „country-shopping“ zu erwarten, nur mit dem Unterschied, dass allein die Höhe des Gewinnsteuersatzes die entscheidungsrelevante Zielgrőe darstellt. Vgl. zum "countryshopping" Mintz, J.M., in: CESifo Forum, 2002, 1, S. 7; Sánchez, G.E./Fluxà, J.F., in: ET, 2006, S. 203-206.

707 Vgl. für Deutschland Jacobs, O.H. (Hrsg.), Unternehmensbesteuerung, 2002, S. 41.

708 Im laufenden Jahr ungenutzte Verluste des gesamten Konsolidierungskreises werden im Rahmen der empirischen Analyse nicht weiter beachtet. 
Höhe des Steuersatzes und der Entwicklung des Steueraufkommens (Abbildung 36).

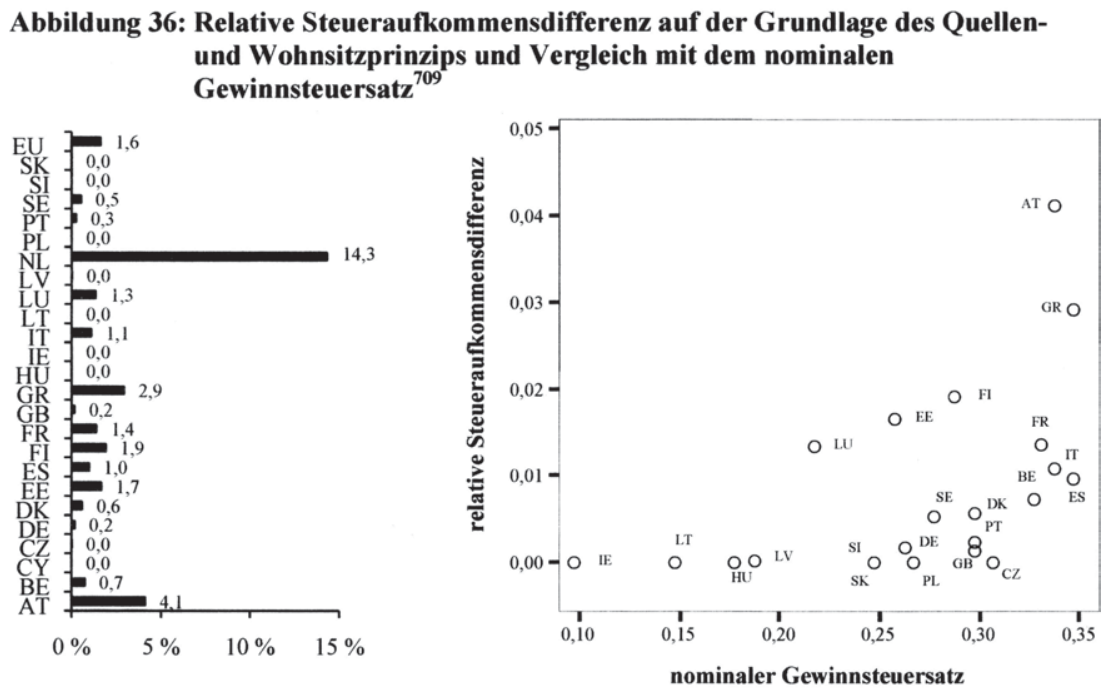

Quelle: eigene Berechnung

Dass dieses Ergebnis weniger eindeutig als erwartet ausfällt, kann mit der Verteilung der Direktinvestitionen in Verbindung gebracht werden. So ist zu beobachten, dass die Entfernung zwischen Heimatland und Zielland einen signifikanten Faktor bei Investitionsentscheidungen darstellt. ${ }^{710}$ Zugleich besteht innerhalb der Europäischen Union ein Gefälle der nominalen Gewinnsteuersätze nach Osten hin. ${ }^{711}$ Beide Effekte bewirken, dass die relative Steueraufkommens-

709 Die dazugehörige Datentabelle befindet sich im Anhang in Tabelle 65. In der rechten Abbildung wurden die Niederlande aufgrund der hohen relativen Steueraufkommensdifferenz vernachlässigt. Da die Niederlande den dritthöchsten nominalen Gewinnsteuersatz in der Europäischen Union besitzen, weist auch dieses Ergebnis dieselbe Tendenz auf.

710 Beispielsweise zeigen Görg und Greenaway, dass der Umfang britischer Direktinvestitionen auch signifikant von der Entfernung der Investitionsobjekte bestimmt wird. Vgl. Görg, H./Greenaway, D., FDI, 2002. Siehe a. Deutsche Bundesbank, in: Monatsbericht, 2002, 6, S. 82-83; Heiduk, G., Außenwirtschaft, 2005, S. 326. Vgl. a. die Angaben in Tabelle 49.

711 Der durchschnittliche nominale Gewinnsteuersatz der EU-15-Mitgliedstaaten liegt bei 29,1 v. H. und ist damit 6,7 Prozentpunkte höher als in den zehn neuen osteuropäischen Mitgliedstaaten. Die Standardabweichung der nominalen Gewinnsteuersätze in der 
differenz geringer als erwartet ausfällt, also die Trendlinie in Abbildung 36 eine flachere Steigung aufweist. Demgegenüber verlagern aber Konzerne auch ihre Aktivitäten gezielt in niedriger besteuernde Mitgliedstaaten, die zudem vielfach ein niedrigeres Lohnniveau vorweisen (Abbildung 34). Zwar verursachen diese Funktionsverlagerungen eine höhere relative Steueraufkommensdifferenz, doch werden sie vorwiegend nur von größeren Konzernen genutzt.

Der starke relative Aufkommenseffekt für die Niederlande kann darauf zurückgeführt werden, dass sie für Europa-Holdinggesellschaften als Standort bevorzugt werden. ${ }^{712}$ So zeigen die empirischen Daten, dass die Niederlande neben Großbritannien der Mitgliedstaat sind, über den die meisten Direktinvestitionen nach Europa fließen (Tabelle 32). Damit ist gemessen an der Zahl der Konzerngesellschaften der Anteil an Konzernmuttergesellschaften in den Niederlanden sehr hoch (Tabelle 25) ${ }^{713}$ Daneben verfügen die Niederlande nach Griechenland und Spanien über den dritthöchsten nominalen Gewinnsteuersatz (Tabelle 47). Somit sind die Rahmenbedingungen dort besonders günstig, um von der Umsetzung des Wohnsitzprinzips bei der Einführung der konsolidierten Körperschaftsteuer-Bemessungsgrundlage zu profitieren.

Europäischen Union beträgt 7,1 Prozentpunkte. Die durchschnittliche Standardabweichung zwischen Nachbarstaaten liegt lediglich bei 6,0 Prozentpunkten.

712 Dass die Niederlande ein bevorzugter Standort für eine Europa-Holding sind, macht auch die empirische Analyse auf der Basis der Direktinvestitionsstatistik der Deutschen Bundesbank von Weichenrieder und Mintz deutlich. Vgl. Weichenrieder, A./Mintz, J.M., Ownership, 2006, S. 19, Siehe a. Grundy, M., Offshore, 1997, S. 155.

713 Die Definition der Konzerne stellt europäische Teilkonzerne ausländischer Anteilseigner europäischen Konzernen, deren Sitz in Europa ist, gleich. Vgl. zur Definition der Konzerne Kapitel D.V. 


\section{G. Zusammenfassung der zentralen Untersuchungsergebnisse}

In der von der Europäischen Kommission in Auftrag gegebenen Binnenmarktstudie aus dem Jahr 2001 kommen die Autoren zu dem Schluss, dass die Unternehmensbesteuerung in der Europäischen Union den Anforderungen an einen Gemeinsamen Binnenmarkt nicht mehr gerecht wird. Erstens verzerrt das Nebeneinander der mittlerweile 27 europäischen Steuersysteme Standort-, Investitions- und Finanzierungsentscheidungen und behindert die grenzüberschreitende Geschäftstätigkeit von Unternehmen. Zweitens resultiert aus der räumlichen Abgrenzung nationaler Besteuerungshoheiten eine zusätzliche Behinderung der grenzüberschreitenden Unternehmenstätigkeiten, mit der ebenfalls Wohlfahrtsverluste einhergehen. Zur Beseitigung dieser Hindernisse werden in der Binnenmarktstudie vier umfassende Lösungsansätze angeführt. Sie bilden die Ausgangsbasis der vorliegenden Arbeit, deren wesentlichen Ergebnisse sich wie folgt zusammenfassen lassen:

1. Von den vier umfassenden Lösungsansätzen stellt sich die einheitliche (konsolidierte) Körperschaftsteuer-Bemessungsgrundlage als der geeignetste Ansatz heraus. Zum einem ist die Anwendung sowohl für die Unternehmen als auch für die sich beteiligenden Mitgliedstaaten fakultativ, was eine politische Durchsetzbarkeit realistisch erscheinen lässt. Zum anderen beseitigt dieser Ansatz die wesentlichen unternehmenssteuerlichen Hindernisse bei grenzüberschreitender Geschäftstätigkeit in der Europäischen Union.

2. Die Erörterung der einheitlichen (konsolidierten) Körperschaftsteuer-Bemessungsgrundlage liefert zwar keinen konkreten Lösungsvorschlag, jedoch erlaubt sie Empfehlungen auszusprechen hinsichtlich der Abgrenzung des Konsolidierungskreises, der Ermittlung Konzernbemessungsgrundlage und ihrer Aufteilung.

3. Um den Eingriff in die Fiskalautonomie der Mitgliedstaaten möglichst gering zu halten, ist eine Beschränkung auf körperschaftsteuerpflichtige Konzerngesellschaften geboten. Der Konsolidierungskreis sollte aus Gründen der Objektivität und der Praktikabilität nur auf der Basis rechtlicher Kriterien abgegrenzt werden und sich auf die teilnehmenden Mitgliedstaaten beschränken. Eine wirksame Beseitigung der bestehenden unternehmenssteuerlichen Hindernisse ist nur gewährleistet, wenn sämtliche Einkommensbestandteile anhand einer Formel aufgeteilt werden. Bei der Ermittlung der Konzernbemessungsgrundlage ist gezwungenermaßen eine Zwischenergebniseliminierung vorzunehmen, denn nur dadurch verlieren Verrechnungspreisgestaltungen endgültig ihre Wirkung. Die Aufteilung der Bemessungsgrundlage sollte anhand einer Formel mit den Faktoren Vermögen, Lohnsumme und Außenumsatz erfolgen, wobei unterschiedliche Gewichtungen der Faktoren denkbar sind.

4. Auch wenn aus der Datenauswahl und -aufbereitung kein direkter mit der Zielsetzung der Arbeit zusammenhängender Erkenntrisgesvinm 6 inhergeht, 
soll nicht unerwähnt bleiben, dass vielfach die restriktive Datenlage einer detaillierteren Analyse entgegensteht.

5. Die Mehrzahl der auf der Basis der Amadeus-Datenbank abgebildeten Konzerne ( $84 \mathrm{v} . \mathrm{H}$.) besitzen weiniger als fünf Tochtergesellschaften, die zudem ihren Sitz ausschließlich im Stammland der Muttergesellschaft haben. Für die EU-Gesamtbemessungsgrundlage sind sie von untergeordneter Bedeutung. Sie wird fast ausschließlich von $20 \mathrm{v}$. H. der Konzerne erwirtschaftet.

6. Zwischen den Mitgliedstaaten existieren systematische Unterschiede hinsichtlich der Verteilung der Funktionen der ansässigen Gesellschaften. Dafür sind auch die steuerlichen Rahmenbedingungen in den einzelnen Mitgliedstaaten ausschlaggebend. So kann mittels einer logistischen Regressionsanalyse verdeutlicht werden, dass die Existenz von Landesholdings auch das Ergebnis steuerlich induzierter Gestaltungen ist. Für Konzernfinanzierungsgesellschaften zeigt die Analyse des durchschnittlichen Verschuldungsgrades, dass Konzerne mit Finanzierungsgesellschaften ihre Tochtergesellschaften vermehrt mit Fremdkapital ausstatten, was ebenfalls das Ergebnis steuerlicher Überlegungen ist. Mit Einführung der einheitlichen (konsolidierten) Körperschaftsteuer-Bemessungsgrundlage verlieren die den beiden Gesellschaftstypen zugrunde liegenden steuerlichen Gestaltungen ihre Wirkung, weshalb mit strukturellen Anpassungsmaßnahmen seitens der Konzerne zu rechnen ist.

7. Durch die Einführung der einheitlichen (konsolidierten) KörperschaftsteuerBemessungsgrundlage in der Europäischen Union sinkt die EUGesamtbemessungsgrundlage um 14,1 v. H. Diese Abnahme ist zu 2/3 auf eine dann mögliche grenzüberschreitende Verlustverrechnung zurückzuführen. Wird eine zukünftige Verrechnung der vorgetragenen Verluste der laufenden Periode pauschal abgebildet, sinkt die Abnahme der Gesamtbemessungsgrundlage um 3,3 Prozentpunkte auf 10,8 v. H. Der Vergleich der Verschiebung der Bemessungsgrundlage durch die indirekte Abgrenzung der Gewinne verdeutlicht, dass besonders Mitgliedstaaten mit einer hohen Steuersatzdifferenz zwischen ihnen und ihren Handelspartnern von der Einführung einer harmonisierten Unternehmensbesteuerung profitieren.

8. Verschiedene Variationsrechnungen zeigen, dass ein Anheben der geforderten Mindestbeteiligungsquote um 10 Prozentpunkte eine Zunahme der EU-Gesamtbemessungsgrundlage um 1,12 Prozentpunkte bewirkt. Eine Aufteilung der Bemessungsrundlage ausschließlich nach Maßgabe des Vermögens verursacht durchschnittlich die geringste Veränderung gegenüber dem Status quo. Außerdem wird deutlich, dass die Gewichtung des Lohnsummenfaktors maßgeblich die zugewiesene Bemessungsgrundlage der einzelnen Mitgliedstaaten beeinflusst. In diesem Zusammenhang muss auch die Diskussion über den Ersatz des Lohnsummenfaktors durch die Anzahl 
der Mitarbeiter verstanden werden, der besonders für osteuropäische Staaten wegen des niedrigen Lohnniveaus vorteilhaft ist. 
Andreas Poppe - 978-3-631-75688-1

Downloaded from PubFactory at 01/11/2019 02:57:41AM

via free access 


\section{Anhang}

Tabelle 54: Meldeangaben der Direktinvestitionsstatistik

Inländische Direktinvestition im Ausland

Der inländische Meldepflichtige (Investor) meldet

a) Über sich:

- Name, Branche, Rechtsform, Sitz

- seit Berichtsjahr 2002 Umsatz, Zahl der Beschäftigten und Bilanzsumme.

b) Über seine Beteiligung im Ausland:

- Name, Branche, Rechtsform, Sitz

- Art der Beteiligung (unmittelbar/mittelbar)

- Anteil der Stimmrechte (nicht in Datenbank enthalten)

- Umsatz

- Beschäftigte

- Bilanzstichtag

- Bilanzangaben gemäß Meldebogen K3

Ausländische Direktinvestitionen im Inland

Der inländische Meldepflichtige (Investitionsobjekt) meldet

a) Über sich (Überblick):

- Name, Branche, Rechtsform, Sitz

b) Über die ausländische Mutter (Investor):

- Name, Sitz, Land

- ggf. Land der Obergesellschaft

c) Über seine und damit für den Ausländer indirekten Beteiligungen im Inland: - Name, Branche, Rechtsform, Sitz.

d) Im Detail über sich und seine Beteiligungen:

- Art der Beteiligung (unmittelbar/mittelbar)

- Anteil der Stimmrechte (nicht in Datenbank enthalten)

- Umsatz

- Zahl der Beschäftigten

- Bilanzstichtag

- Bilanzangaben gemäB Meldebogen K4 (siehe Anhang A)

- seit Berichtsjahr 2002 für börsennotierte Gesellschaften den Börsenwert - seit Berichtsjahr 2002 für börsennotierte Gesellschaften den Börsenwert 
Tabelle 55: Rechtsformen in der Datenbank

\begin{tabular}{|c|c|c|c|c|c|c|}
\hline & Rechtsform gemäß Amadeus & Anzahl & Land & Summe Land & $\begin{array}{c}\text { relativer } \\
\text { Anteil/Land }\end{array}$ & $\begin{array}{c}\text { M-T- } \\
\text { Richtlinie }\end{array}$ \\
\hline 1 & AG \& Co KG & 1 & AT & 22.200 & $0,00 \%$ & \\
\hline 2 & BGB & 13 & AT & & $0,06 \%$ & \\
\hline & Not classified & 20 & AT & & $0,09 \%$ & \\
\hline 3 & Einzelfirma & 39 & $\mathrm{AT}$ & & $0,18 \%$ & \\
\hline 4 & $\mathrm{eG}$ & 88 & AT & & $0,40 \%$ & ja \\
\hline 5 & BGB - Arbeitsgemeinschaft & 109 & AT & & $0,49 \%$ & \\
\hline 6 & e. V. & 140 & AT & & $0,63 \%$ & ja \\
\hline 7 & $\mathrm{OHG}$ & 163 & AT & & $0,73 \%$ & \\
\hline 8 & KG & 577 & AT & & $2,60 \%$ & \\
\hline 9 & AG & 819 & AT & & $3,69 \%$ & ja \\
\hline 10 & $\mathrm{GmbH} \& \mathrm{Co} \mathrm{KG}$ & 3.663 & AT & & $16,50 \%$ & \\
\hline 11 & $\mathrm{GmbH}$ & 16.568 & AT & & $74,63 \%$ & ja \\
\hline 12 & European Economic Interest Group & 5 & $\mathrm{BE}$ & 51.669 & $0,01 \%$ & \\
\hline 13 & Public institution & 7 & $\mathrm{BE}$ & & $0,01 \%$ & ja \\
\hline & Not classified & 148 & $\mathrm{BE}$ & & $0,29 \%$ & - \\
\hline 14 & GCV / VOF, SCS / SNC & 870 & $\mathrm{BE}$ & & $1,68 \%$ & ja \\
\hline 15 & Cooperative company & 1.809 & $\mathrm{BE}$ & & $3,50 \%$ & ja \\
\hline 16 & (E)BVBA / SPRL(U) & 12.029 & $\mathrm{BE}$ & & $23,28 \%$ & ja \\
\hline 17 & NV / SA & 36.801 & $\mathrm{BE}$ & & $71,22 \%$ & ja \\
\hline 18 & Limited company & 132 & CY & 132 & $100,00 \%$ & ja \\
\hline 19 & Spol.S.R.O. & 1.197 & $\mathrm{CZ}$ & 2.913 & $41,09 \%$ & ja \\
\hline 20 & AS & 1.716 & $\mathrm{CZ}$ & & $58,91 \%$ & ja \\
\hline 21 & AG \& CO. OHG & 1 & $\mathrm{DE}$ & 248.049 & $0,00 \%$ & \\
\hline & Not classified & 1 & $\mathrm{DE}$ & & $0,00 \%$ & - \\
\hline 22 & e. V. & 1 & $\mathrm{DE}$ & & $0,00 \%$ & ja \\
\hline 23 & EG \&CO. KG & 1 & $\mathrm{DE}$ & & $0,00 \%$ & \\
\hline 24 & $A G \& C O K G$ aA & 3 & $\mathrm{DE}$ & & $0,00 \%$ & ja \\
\hline 25 & GMBH \& $\mathrm{CO} O \mathrm{OHG}$ & 7 & $\mathrm{DE}$ & & $0,00 \%$ & \\
\hline 26 & GMBH \& CO. Kgaa & 10 & $\mathrm{DE}$ & & $0,00 \%$ & ja \\
\hline 27 & $A G \&$ Co KG & 12 & $\mathrm{DE}$ & & $0,00 \%$ & \\
\hline 28 & $\mathrm{KGaA}$ & 14 & $\mathrm{DE}$ & & $0,01 \%$ & ja \\
\hline 29 & $\mathrm{eG}$ & 26 & $\mathrm{DE}$ & & $0,01 \%$ & ja \\
\hline 30 & Einzelfirma & 669 & $\mathrm{DE}$ & & $0,27 \%$ & \\
\hline 31 & $\mathrm{OHG}$ & 1.855 & $\mathrm{DE}$ & & $0,75 \%$ & \\
\hline 32 & KG & 3.664 & $\mathrm{DE}$ & & $1,48 \%$ & \\
\hline 33 & AG & 5.485 & $\mathrm{DE}$ & & $2,21 \%$ & ja \\
\hline 34 & $\mathrm{GmbH} \& \mathrm{Co} \mathrm{KG}$ & 79.555 & $\mathrm{DE}$ & & $32,07 \%$ & \\
\hline 35 & $\mathrm{GmbH}$ & 156.745 & $\mathrm{DE}$ & & $63,19 \%$ & ja \\
\hline 36 & Assurance company & 2 & DK & 76.662 & $0,00 \%$ & ja \\
\hline 37 & Association & 7 & DK & & $0,01 \%$ & ja \\
\hline 38 & Limited Partnership by shares & 51 & DK & & $0,07 \%$ & \\
\hline 39 & Company with limited liability & 52 & DK & & $0,07 \%$ & ja \\
\hline 40 & Cooperative with limited liability & 108 & DK & & $0,14 \%$ & ja \\
\hline 41 & Private limited company (Faroe Islands) & 227 & DK & & $0,30 \%$ & ja \\
\hline 42 & Limited company (Faroe Islands) & 297 & DK & & $0,39 \%$ & ja \\
\hline 43 & General partnership & 378 & DK & & $0,49 \%$ & \\
\hline 44 & Profit foundation & 435 & DK & & $0,57 \%$ & ja \\
\hline 45 & Limited partnership & 1.756 & DK & & $2,29 \%$ & \\
\hline 46 & Limited company & 27.740 & DK & & $36,18 \%$ & ja \\
\hline 47 & Private limited company & 45.609 & DK & & $59,49 \%$ & ja \\
\hline 48 & Affiliate & 1 & $\mathrm{EE}$ & 2.379 & $0,04 \%$ & \\
\hline 49 & Limited partnership & 1 & $\mathrm{EE}$ & & $0,04 \%$ & ja \\
\hline 50 & Profit oriented co-operatives & 12 & $\mathrm{EE}$ & & $0,50 \%$ & ja \\
\hline 51 & Limited liability company & 1.097 & $\mathrm{EE}$ & & $46,11 \%$ & ja \\
\hline 52 & Share company & 1.268 & $\mathrm{EE}$ & & $53,30 \%$ & ja \\
\hline 53 & Corporaciones locales & 1 & $\mathrm{ES}$ & 80.860 & $0,00 \%$ & ja \\
\hline 54 & Cong.relig., Org.auton., Asim. & 1 & ES & Pas & $309 \%$ & ja \\
\hline
\end{tabular}




\begin{tabular}{|c|c|c|c|c|c|c|}
\hline & Rechtsform gemäß Amadeus & Anzahl & Land & Summe Land & $\begin{array}{c}\text { relativer } \\
\text { Anteil/Land }\end{array}$ & $\begin{array}{c}\text { M-T- } \\
\text { Richtlinie }\end{array}$ \\
\hline 55 & Sociedad regular colectiva & 15 & ES & & $0,02 \%$ & ja \\
\hline 56 & Sociedad comanditaria & 41 & ES & & $0,05 \%$ & ja \\
\hline 57 & UTE & 75 & ES & & $0,09 \%$ & \\
\hline 58 & FIM & 164 & ES & & $0,20 \%$ & ja \\
\hline & Not classified & 213 & ES & & $0,26 \%$ & - \\
\hline 59 & AIE & 344 & ES & & $0,43 \%$ & \\
\hline 60 & Cooperative company & 574 & ES & & $0,71 \%$ & \\
\hline 61 & Sociedad anonima (SA) & 38.395 & ES & & $47,48 \%$ & ja \\
\hline 62 & Sociedad limitada & 41.037 & ES & & $50,75 \%$ & ja \\
\hline & Not classified & 3 & FI & 10.280 & $0,03 \%$ & - \\
\hline 63 & State company & 3 & FI & & $0,03 \%$ & \\
\hline 64 & Foundation & 10 & FI & & $0,10 \%$ & \\
\hline 65 & Partnership & 15 & FI & & $0,15 \%$ & \\
\hline 66 & KY - Limited Partnership & 36 & FI & & $0,35 \%$ & \\
\hline 67 & Cooperative company & 59 & FI & & $0,57 \%$ & ja \\
\hline 68 & OYJ & 170 & FI & & $1,65 \%$ & ja \\
\hline 69 & OY & 9.984 & FI & & $97,12 \%$ & ja \\
\hline 70 & Société en Participation & 1 & FR & 119.502 & $0,00 \%$ & \\
\hline 71 & Association & 2 & FR & & $0,00 \%$ & \\
\hline 72 & Branch of a foreign company & 4 & FR & & $0,00 \%$ & \\
\hline 73 & EARL & 5 & FR & & $0,00 \%$ & \\
\hline 74 & EPIC & 9 & FR & & $0,01 \%$ & \\
\hline 75 & Société en commandite simple & 94 & FR & & $0,08 \%$ & \\
\hline 76 & European Economic Interest Group & 118 & FR & & $0,10 \%$ & \\
\hline 77 & $\mathrm{SCA}$ & 198 & FR & & $0,17 \%$ & ja \\
\hline & Not classified & 260 & FR & & $0,22 \%$ & - \\
\hline 78 & Société civile & 370 & FR & & $0,31 \%$ & ja \\
\hline 79 & Cooperative company & 845 & FR & & $0,71 \%$ & ja \\
\hline 80 & EURL & 2.912 & FR & & $2,44 \%$ & \\
\hline 81 & SA Directoire & 3.199 & FR & & $2,68 \%$ & \\
\hline 82 & Société en nom collectif & 4.655 & FR & & $3,90 \%$ & \\
\hline 83 & SA & 33.583 & FR & & $28,10 \%$ & ja \\
\hline 84 & Société en action simple & 34.804 & FR & & $29,12 \%$ & ja \\
\hline 85 & SARL & 38.443 & FR & & $32,17 \%$ & ja \\
\hline 86 & Assurance company & 2 & GB & 475.071 & $0,00 \%$ & ja \\
\hline 87 & Not Companies Act & 19 & GB & & $0,00 \%$ & \\
\hline 88 & Industrial/Provident & 24 & GB & & $0,01 \%$ & ja \\
\hline 89 & Limited partnership & 192 & GB & & $0,04 \%$ & ja \\
\hline & Not classified & 0 & GB & & $0,00 \%$ & - \\
\hline 90 & Public, investment trust & 308 & GB & & $0,06 \%$ & ja \\
\hline 91 & Public, A.I.M. & 677 & GB & & $0,14 \%$ & ja \\
\hline 92 & Public, quoted & 791 & GB & & $0,17 \%$ & ja \\
\hline 93 & Unlimited & 2.273 & GB & & $0,48 \%$ & ja \\
\hline 94 & Public, not quoted & 5.968 & GB & & $1,26 \%$ & ja \\
\hline 95 & Guarantee & 56.717 & GB & & $11,94 \%$ & ja \\
\hline 96 & Private & 408.100 & GB & & $85,90 \%$ & ja \\
\hline 97 & $\begin{array}{l}\text { Sole shareholder limited liability com- } \\
\text { pany }\end{array}$ & 107 & GR & 5.904 & $1,81 \%$ & ja \\
\hline 98 & Limited liability company & 484 & GR & & $8,20 \%$ & ja \\
\hline 99 & SA & 5.313 & GR & & $89,99 \%$ & ja \\
\hline 100 & $\begin{array}{l}\text { Economic association operating by the } \\
\text { responsibility of a legal person }\end{array}$ & 1 & $\mathrm{HU}$ & 9.634 & $0,01 \%$ & \\
\hline 101 & Proprietary company & 1 & $\mathrm{HU}$ & & $0,01 \%$ & \\
\hline 102 & $\begin{array}{l}\text { Society of management of water sup- } \\
\text { plies }\end{array}$ & 1 & $\mathrm{HU}$ & & $0,01 \%$ & \\
\hline 103 & $\begin{array}{l}\text { Direct commercial representation of } \\
\text { foreigners in Hungary }\end{array}$ & 2 & $\mathrm{HU}$ & & $0,02 \%$ & \\
\hline 104 & $\begin{array}{l}\text { Joint venture } \\
\text { Not classified }\end{array}$ & $\begin{array}{r}3 \\
A^{3}\end{array}$ & $\begin{array}{l}\mathrm{HU} \\
\mathrm{dH} \text { HS }\end{array}$ & Poppe - 97 & $\begin{array}{r}0,03 \% \\
3-60,03 \% 6\end{array}$ & ja \\
\hline
\end{tabular}




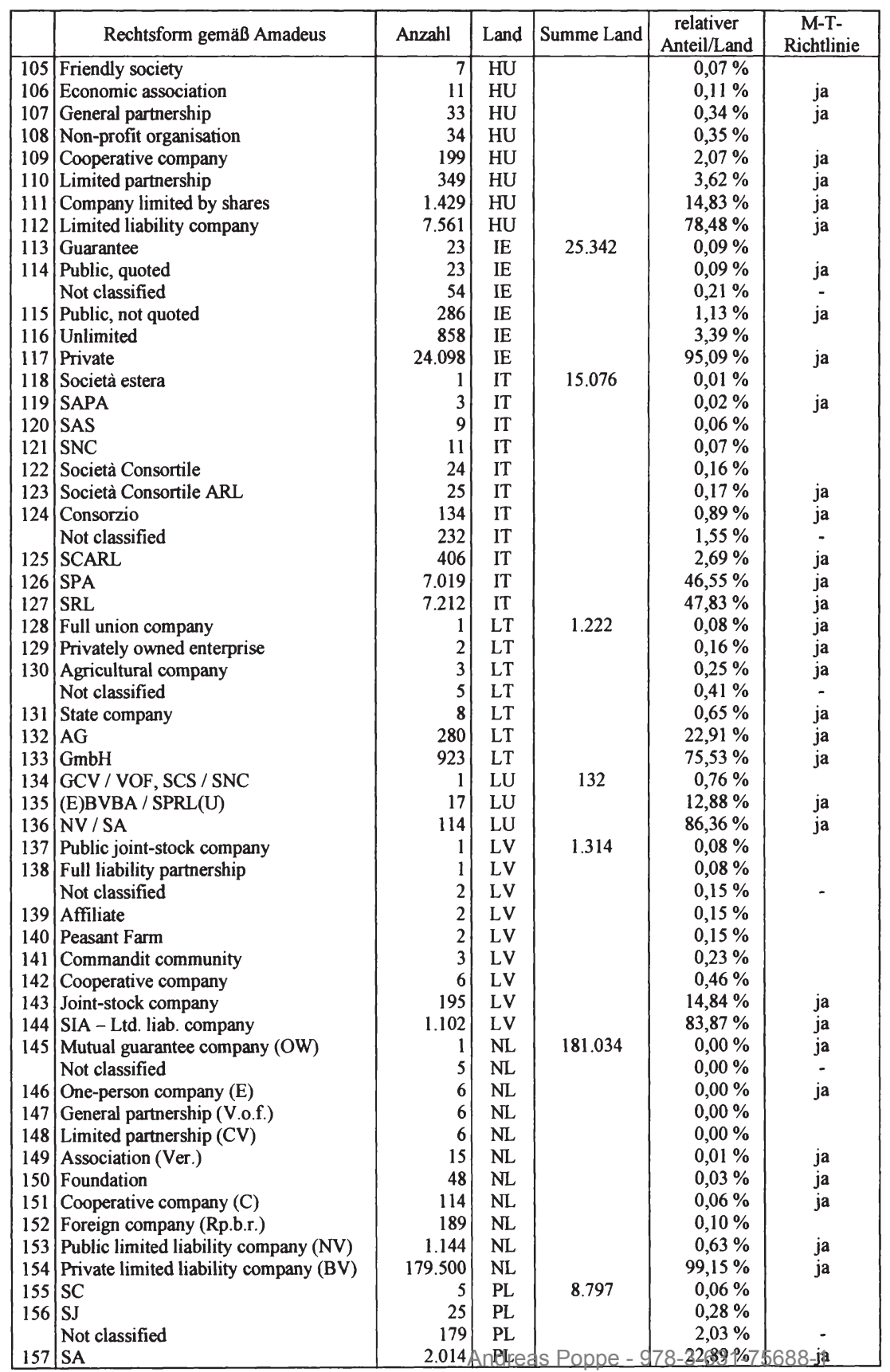




\begin{tabular}{|c|c|c|c|c|c|c|}
\hline & Rechtsform gemäß Amadeus & Anzahl & Land & Summe Land & $\begin{array}{c}\text { relativer } \\
\text { Anteil/Land }\end{array}$ & $\begin{array}{c}\text { M-T- } \\
\text { Richtlinie }\end{array}$ \\
\hline 158 & Sp. z.o.o. & 6.574 & PL & \multirow{7}{*}{9.588} & $74,73 \%$ & ja \\
\hline 159 & Sociedade em comandita & 1 & PT & & $0,01 \%$ & ja \\
\hline 160 & Sociedade em nome Colectivo & 1 & PT & & $0,01 \%$ & ja \\
\hline 161 & ACE & 20 & PT & & $0,21 \%$ & \\
\hline 162 & Cooperative company & 27 & PT & & $0,28 \%$ & ja \\
\hline & Not classified & 58 & PT & & $0,60 \%$ & \\
\hline 163 & Sociedade an $\not$ nima $[!]$ (SA) & 3.518 & PT & & $36,69 \%$ & ja \\
\hline 164 & Sociedade por cuotas & 5.963 & PT & \multirow{3}{*}{67.736} & $62,19 \%$ & ja \\
\hline 165 & $\mathrm{AB}$ - Public limited & 775 & SE & & $1,14 \%$ & Ja \\
\hline 166 & AB - Private limited & 66.961 & SE & & $98,86 \%$ & $\mathrm{Ja}$ \\
\hline 167 & $\begin{array}{l}\text { Cessation of business with legal succes- } \\
\text { sor }\end{array}$ & 1 & SI & \multirow[t]{4}{*}{80} & $1,25 \%$ & \\
\hline 168 & Cooperative with limited liability & 1 & SI & & $1,25 \%$ & \\
\hline 169 & Limited liability company & 30 & SI & & $37,50 \%$ & $\mathrm{Ja}$ \\
\hline 170 & Joint-stock company & 48 & SI & & $60,00 \%$ & $\mathrm{Ja}$ \\
\hline 171 & Limited liability company & 6 & SK & \multirow[t]{2}{*}{25} & $24,00 \%$ & $\mathrm{Ja}$ \\
\hline 172 & Joint-stock company & 19 & SK & & $76,00 \%$ & Ja \\
\hline & Summe & 1.415 .601 & & 1.415 .601 & & \\
\hline
\end{tabular}

Quelle: eigene Berechnungen

Tabelle 56: Angepasste NACE-Abschnitte und -Abteilungen in der Datenbank

\begin{tabular}{|c|c|c|c|}
\hline $\begin{array}{l}\text { Abschnitts- } \\
\text { kürzel neu }\end{array}$ & $\begin{array}{l}\text { Abteilungs- } \\
\text { kürzel neu }\end{array}$ & $\begin{array}{c}\text { NACE } \\
\text { Abteilung/ } \\
\text { Gruppe/Klasse }\end{array}$ & Bezeichnung \\
\hline $\begin{array}{l}\mathrm{A} / \mathrm{B} \\
\mathrm{C} \\
\mathrm{D}\end{array}$ & $\begin{array}{l}11 \\
12 \\
13 \\
14 \\
15 \\
16 \\
17 \\
18 \\
19 \\
20\end{array}$ & $\begin{array}{c}01,02,05 \\
10-14 \\
15-37 \\
15 \\
16 \\
17 \\
18 \\
19 \\
20 \\
21 \\
22\end{array}$ & $\begin{array}{l}\text { Land- und Forstwirtschaft / Fischerei und Fischzucht } \\
\text { Bergbau und Gewinnung von Steinen und Erden } \\
\text { Herstellung von Waren } \\
\text { Ernährungsgewerbe } \\
\text { Tabakverarbeitung } \\
\text { Textilgewerbe } \\
\text { Bekleidungsgewerbe } \\
\text { Ledergewerbe } \\
\text { Holzgewerbe (ohne Herstellung von Möbeln) } \\
\text { Papiergewerbe } \\
\text { Verlagsgewerbe, Druckgewerbe, Vervielfaltigung von bespielten } \\
\text { Trägem } \\
\text { Kokerei, Mineralölverarbeitung, Herstellung und Verarbeitung } \\
\text { von Spalt- und Brutstoffen } \\
\text { Chemische Industrie } \\
\text { Herstellung von Gummi- und Kunststoffwaren } \\
\text { Glasgewerbe, Keramik, Verarbeitung von Steinen und Erden } \\
\text { Metallerzeugung und -bearbeitung } \\
\text { Stahl- und Leichtmetallbau, Herstellung von Metallerzeugnissen } \\
\text { Maschinenbau } \\
\text { Herstellung von Büromaschinen, Datenverarbeitungsgeräten und } \\
\text {-einrichtungen } \\
\text { Herstellung von Geräten der Elektrizitätserzeugung, -verteilung } \\
\text { Nachrichtentechnik, Herstellung von Rundfunk- und Fernseh- } \\
\text { geräten und elektronischen Bauelementen } \\
\text { Herstellung von Medizin-, Mess-, Steuer- und Regelungstechnik; } \\
\text { Optik } \\
\text { Herstellung von Kraftwagen und Kraftwagenteilen } \\
\text { Sonstiger Fahrzeugbau } \\
\text { Herstellung von Möbeln, Schmuck, Musikinstrumenten, Sport- } \\
\text { geräten, Spielwaren und sonstige Erzeugnissen } \\
\text { Rückgewinnung } \\
\text { Energie- und Wasseryersergung }\end{array}$ \\
\hline
\end{tabular}




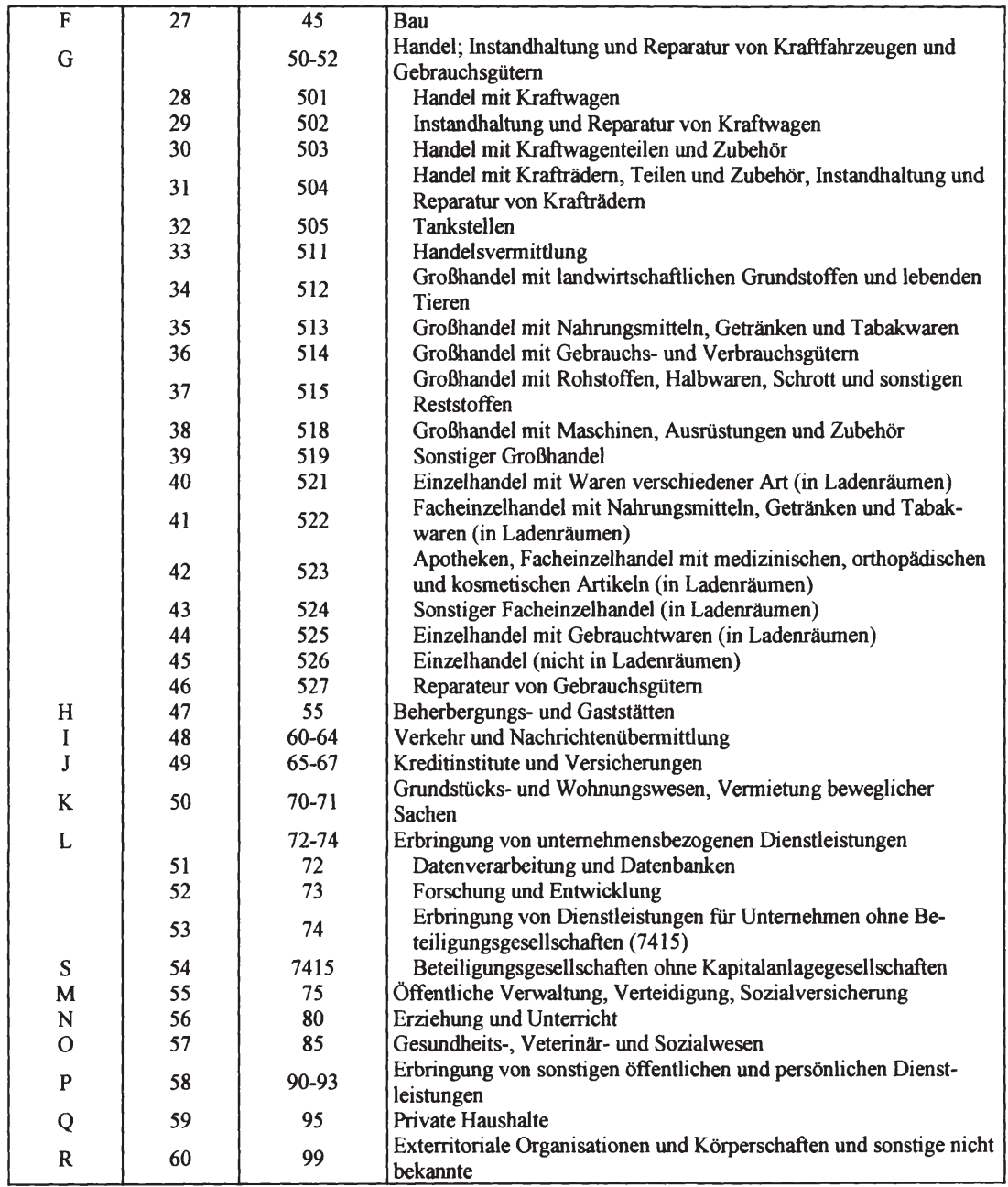

Quelle: eigene Darstellung 
Tabelle 57: Anpassen der Aktivseite der Bilanz ${ }^{714}$

\begin{tabular}{|c|c|c|c|c|c|c|c|c|c|c|c|c|c|c|c|}
\hline $\begin{array}{l}\text { Anzahl der } \\
\text { Unbekannten }\end{array}$ & 0 & \multicolumn{4}{|c|}{1} & \multicolumn{5}{|c|}{2} & \multicolumn{4}{|c|}{3} & 4 \\
\hline $\begin{array}{l}\text { fias } \\
\text { ifas } \\
\text { tfas } \\
\text { ofas }\end{array}$ & $\begin{array}{l}\mathrm{nn} \\
\mathrm{nn} \\
\mathrm{nn} \\
\mathrm{nn}\end{array}$ & $\begin{array}{l}\mathrm{nn} \\
\mathrm{nn} \\
\mathrm{nn}\end{array}$ & $\begin{array}{l}\mathrm{nn} \\
\mathrm{nn} \\
\mathrm{nn}\end{array}$ & $\begin{array}{l}\mathrm{nn} \\
\mathrm{nn} \\
\mathrm{nn}\end{array}$ & $\begin{array}{l}\mathrm{nn} \\
\mathrm{nn} \\
\mathrm{nn}\end{array}$ & $\begin{array}{ll}\mathrm{nn} & \mathrm{nn} \\
\mathrm{nn} & \\
& \mathrm{nn}\end{array}$ & $\begin{array}{l}\mathrm{nn} \\
\mathrm{nn}\end{array}$ & $\begin{array}{l}\mathrm{nn} \\
\mathrm{nn}\end{array}$ & $\begin{array}{l}\mathrm{nn} \\
\mathrm{nn}\end{array}$ & $\begin{array}{l}\mathrm{nn} \\
\mathrm{nn}\end{array}$ & $\mathrm{nn}$ & $\mathrm{nn}$ & $\mathrm{nn}$ & $\mathrm{nn}$ & \\
\hline $\begin{array}{l}\text { vorher } \\
\text { nachher }\end{array}$ & $\begin{array}{l}530.942 \\
709.215 \\
\end{array}$ & $\begin{array}{c}14.481 \\
0 \\
\end{array}$ & $\begin{array}{c}709 \\
0\end{array}$ & $\begin{array}{c}3.509 \\
0\end{array}$ & $\begin{array}{l}0 \\
0\end{array}$ & $\begin{array}{cc}35 & 268 \\
0 & 0\end{array}$ & $\begin{array}{c}62 \\
0 \\
\end{array}$ & $\begin{array}{l}0 \\
0\end{array}$ & $\begin{array}{l}0 \\
0\end{array}$ & $\begin{array}{l}0 \\
0\end{array}$ & $\begin{array}{c}158.345 \\
54 \\
\end{array}$ & $\begin{array}{l}0 \\
0\end{array}$ & $\begin{array}{l}0 \\
0\end{array}$ & $\begin{array}{l}0 \\
0\end{array}$ & $\begin{array}{l}125.299 \\
124.381\end{array}$ \\
\hline
\end{tabular}

\begin{tabular}{|c|c|c|c|c|c|c|c|c|c|c|c|c|c|c|c|c|}
\hline $\begin{array}{l}\text { Anzahl der } \\
\text { Unbekannten }\end{array}$ & 0 & \multicolumn{4}{|c|}{1} & \multicolumn{6}{|c|}{2} & \multicolumn{4}{|c|}{3} & 4 \\
\hline $\begin{array}{l}\text { cuas } \\
\text { stok } \\
\text { debt } \\
\text { ocas }\end{array}$ & $\begin{array}{l}\mathrm{nn} \\
\mathrm{nn} \\
\mathrm{nn} \\
\mathrm{nn}\end{array}$ & $\begin{array}{l}\mathrm{nn} \\
\mathrm{nn} \\
\mathrm{nn}\end{array}$ & $\begin{array}{l}\mathrm{nn} \\
\mathrm{nn} \\
\mathrm{nn}\end{array}$ & $\begin{array}{l}\mathrm{nn} \\
\mathrm{nn} \\
\mathrm{nn}\end{array}$ & $\begin{array}{l}\mathrm{nn} \\
\mathrm{nn} \\
\mathrm{nn}\end{array}$ & $\begin{array}{l}\mathrm{nn} \\
\mathrm{nn}\end{array}$ & $\begin{array}{l}\mathrm{nn} \\
\mathrm{nn}\end{array}$ & $\begin{array}{l}\mathrm{nn} \\
\mathrm{nn}\end{array}$ & & $\begin{array}{l}\mathrm{nn} \\
\mathrm{nn}\end{array}$ & $\begin{array}{l}\mathrm{nn} \\
\mathrm{nn}\end{array}$ & $\mathrm{nn}$ & $\mathrm{nn}$ & $\mathrm{nn}$ & $\mathrm{nn}$ & \\
\hline $\begin{array}{l}\text { vorher } \\
\text { nachher }\end{array}$ & $\begin{array}{l}685.871 \\
709.247\end{array}$ & $\begin{array}{c}4.214 \\
0\end{array}$ & $\begin{array}{l}0 \\
0\end{array}$ & $\begin{array}{l}0 \\
0\end{array}$ & $\begin{array}{l}1 \\
0\end{array}$ & $\begin{array}{l}2 \\
0\end{array}$ & $\begin{array}{l}2 \\
0\end{array}$ & $\begin{array}{c}6.700 \\
0\end{array}$ & $\begin{array}{l}1 \\
0\end{array}$ & $\begin{array}{l}0 \\
0\end{array}$ & $\begin{array}{l}0 \\
0\end{array}$ & $\begin{array}{c}11.547 \\
16\end{array}$ & $\begin{array}{l}0 \\
0\end{array}$ & $\begin{array}{l}0 \\
0\end{array}$ & $\begin{array}{c}506 \\
0\end{array}$ & $\begin{array}{l}124.806 \\
124.387\end{array}$ \\
\hline
\end{tabular}

\begin{tabular}{|l|c|ccc|ccc|c|}
\hline $\begin{array}{l}\text { Anzahl der } \\
\text { Unbekannten }\end{array}$ & 0 & & 1 & & & 2 & 3 \\
\hline toas & $\mathrm{nn}$ & & $\mathrm{nn}$ & $\mathrm{nn}$ & $\mathrm{nn}$ & & & \\
fias & $\mathrm{nn}$ & $\mathrm{nn}$ & & $\mathrm{nn}$ & & $\mathrm{nn}$ & \\
cuas & $\mathrm{nn}$ & $\mathrm{nn}$ & $\mathrm{nn}$ & & & & $\mathrm{nn}$ & \\
\hline vorher & 708.223 & 20 & 92 & 106 & 1 & 2 & 1 & 125.205 \\
nachher & 709.269 & 0 & 0 & 0 & 138 & 0 & 0 & 124.243 \\
\hline
\end{tabular}

Quelle: eigene Berechnungen

714 Die konsolidierten Konzernabschlüsse sind in dieser Übersicht noch enthalten. 
Tabelle 58: Anpassen der Passivseite der Bilanz ${ }^{715}$

\begin{tabular}{|l|c|ccc|ccc|c|}
\hline Anzahl der & 0 & \multicolumn{3}{|c|}{1} & \multicolumn{3}{|c|}{2} & 3 \\
Unbekannten & & & & & & \\
\hline shfd & $\mathrm{nn}$ & & $\mathrm{nn}$ & $\mathrm{nn}$ & $\mathrm{nn}$ & & \\
capi & $\mathrm{nn}$ & $\mathrm{nn}$ & & $\mathrm{nn}$ & & $\mathrm{nn}$ & \\
osfd & $\mathrm{nn}$ & $\mathrm{nn}$ & $\mathrm{nn}$ & & & & $\mathrm{nn}$ & \\
\hline vorher & 704.351 & 5.212 & 546 & 1 & 14.855 & 20.613 & 42 & 88.030 \\
nachher & 710.849 & 0 & 0 & 0 & 14.155 & 20.613 & 0 & 88.033 \\
\hline
\end{tabular}

\begin{tabular}{|c|c|c|c|c|c|c|c|c|}
\hline $\begin{array}{l}\text { Anzahl der } \\
\text { Unbekannten }\end{array}$ & 0 & \multicolumn{3}{|c|}{1} & \multicolumn{3}{|c|}{2} & 3 \\
\hline $\begin{array}{l}\text { ncli } \\
\text { ltdb } \\
\text { oncl }\end{array}$ & $\begin{array}{l}\text { nn } \\
\text { nn } \\
\text { nn }\end{array}$ & $\begin{array}{l}\mathrm{nn} \\
\mathrm{nn}\end{array}$ & $\begin{array}{l}\mathrm{nn} \\
\mathrm{nn}\end{array}$ & $\begin{array}{l}\mathrm{nn} \\
\mathrm{nn}\end{array}$ & nn & nn & $\mathrm{nn}$ & \\
\hline $\begin{array}{l}\text { vorher } \\
\text { nachher }\end{array}$ & \begin{tabular}{|l|}
429.314 \\
702.080
\end{tabular} & $\begin{array}{c}109 \\
0\end{array}$ & $\begin{array}{c}16 \\
0\end{array}$ & $\begin{array}{c}24 \\
0\end{array}$ & $\begin{array}{c}255.777 \\
8.255\end{array}$ & $\begin{array}{l}0 \\
0\end{array}$ & $\begin{array}{l}0 \\
0\end{array}$ & \begin{tabular}{|l|}
148.410 \\
123.315 \\
\end{tabular} \\
\hline
\end{tabular}

\begin{tabular}{|c|c|c|c|c|c|c|c|c|c|c|}
\hline $\begin{array}{l}\text { Anzahl der } \\
\text { Unbekannten }\end{array}$ & 0 & \multicolumn{3}{|c|}{1} & \multicolumn{4}{|c|}{2} & 3 & 4 \\
\hline $\begin{array}{l}\text { culi } \\
\text { loan } \\
\text { cred } \\
\text { ocli }\end{array}$ & $\begin{array}{l}\mathrm{nn} \\
\mathrm{nn} \\
\mathrm{nn} \\
\mathrm{nn}\end{array}$ & $\begin{array}{l}\mathrm{nn} \\
\mathrm{nn} \\
\mathrm{nn}\end{array}$ & $\begin{array}{l}\mathrm{nn} \\
\mathrm{nn} \\
\mathrm{nn}\end{array}$ & $\begin{array}{l}\mathrm{nn} \\
\mathrm{nn} \\
\mathrm{nn}\end{array}$ & $\begin{array}{l}\mathrm{nn} \\
\mathrm{nn}\end{array}$ & $\begin{array}{l}\mathrm{nn} \\
\mathrm{nn}\end{array}$ & $\begin{array}{l}\mathrm{nn} \\
\mathrm{nn}\end{array}$ & $\begin{array}{l}\mathrm{nn} \\
\mathrm{nn}\end{array}$ & $\mathrm{nn}$ & \\
\hline vorhe & 561.276 & 1.358 & 1.231 & 24 & 25 & 29.457 & 174 & 2 & $124.137 \quad 1$ & 115.96 \\
\hline ach & 696.540 & 0 & 0 & 0 & 0 & 0 & 0 & 0 & $21.666 \quad 0$ & 115.44 \\
\hline
\end{tabular}

\begin{tabular}{|c|c|c|c|c|c|c|c|c|c|c|c|c|c|c|}
\hline $\begin{array}{l}\text { Anzahl der } \\
\text { Unbekannten }\end{array}$ & 0 & \multicolumn{4}{|c|}{1} & \multicolumn{5}{|c|}{2} & \multicolumn{3}{|c|}{3} & 4 \\
\hline $\begin{array}{l}\text { tshf } \\
\text { shfd } \\
\text { ncli } \\
\text { culi }\end{array}$ & $\begin{array}{l}\mathrm{nn} \\
\mathrm{nn} \\
\mathrm{nn} \\
\mathrm{nn}\end{array}$ & $\begin{array}{l}\mathrm{nn} \\
\mathrm{nn} \\
\mathrm{nn}\end{array}$ & $\begin{array}{l}\mathrm{nn} \\
\mathrm{nn} \\
\mathrm{nn}\end{array}$ & $\begin{array}{l}\mathrm{nn} \\
\mathrm{nn} \\
\mathrm{nn}\end{array}$ & $\begin{array}{l}\mathrm{nn} \\
\mathrm{nn} \\
\mathrm{nn}\end{array}$ & $\begin{array}{l}\mathrm{nn} \\
\mathrm{nn}\end{array}$ & $\begin{array}{l}\text { nn } \\
\text { nn }\end{array}$ & $\begin{array}{l}\mathrm{nn} \\
\mathrm{nn}\end{array}$ & $\begin{array}{l}\mathrm{nn} \\
\mathrm{nn}\end{array}$ & $\begin{array}{l}\mathrm{nn} \\
\mathrm{nn}\end{array}$ & $\mathrm{nn}$ & nn & $\mathrm{nn}$ & \\
\hline $\begin{array}{l}\text { vorher } \\
\text { nachher }\end{array}$ & \begin{tabular}{|l|}
682.428 \\
709.155
\end{tabular} & $\begin{array}{c}16 \\
0\end{array}$ & $\begin{array}{c}24.990 \\
0\end{array}$ & $\begin{array}{c}522 \\
0\end{array}$ & $\begin{array}{c}979 \\
0\end{array}$ & $\begin{array}{l}422 \\
249 \\
\end{array}$ & $\begin{array}{l}6 \\
0 \\
\end{array}$ & $\begin{array}{c}36 \\
0\end{array}$ & $\begin{array}{l}9.233 \\
9.229 \\
\end{array}$ & $\begin{array}{l}1.179 \\
1.180\end{array}$ & $\begin{array}{l}2 \\
2 \\
\end{array}$ & $\begin{array}{c}0 \\
5.191 \\
\end{array}$ & $\begin{array}{l}1 \\
0\end{array}$ & \begin{tabular}{|l|}
113.836 \\
108.644 \\
\end{tabular} \\
\hline
\end{tabular}

Quelle: eigene Berechnungen

715 Die konsolidierten Konzernabschlüsse sind in dieser Übersicht noch enthalten. 
Tabelle 59: Anpassen der Gewinn-und-Verlust-Rechnung ${ }^{716}$

\begin{tabular}{|c|c|c|c|c|c|c|c|c|}
\hline $\begin{array}{l}\text { Anzahl der } \\
\text { Unbekannten }\end{array}$ & 0 & \multicolumn{3}{|c|}{1} & \multicolumn{3}{|c|}{2} & 3 \\
\hline $\begin{array}{l}\text { fire } \\
\text { fiex } \\
\text { fipl }\end{array}$ & $\begin{array}{l}\mathrm{nn} \\
\mathrm{nn} \\
\mathrm{nn}\end{array}$ & $\begin{array}{l}\mathrm{nn} \\
\mathrm{nn}\end{array}$ & $\begin{array}{c}\mathrm{nn} \\
\mathrm{nn}\end{array}$ & $\begin{array}{l}\mathrm{nn} \\
\mathrm{nn}\end{array}$ & $\mathrm{nn}$ & $\mathrm{nn}$ & $\mathrm{nn}$ & \\
\hline $\begin{array}{l}\text { vorher } \\
\text { nachher }\end{array}$ & $\begin{array}{l}296.931 \\
403.978\end{array}$ & $\begin{array}{c}36.184 \\
0\end{array}$ & $\begin{array}{c}50.716 \\
0\end{array}$ & $\begin{array}{c}225 \\
0\end{array}$ & $\begin{array}{l}3 \\
1 \\
\end{array}$ & $\begin{array}{l}1 \\
0\end{array}$ & $\begin{array}{c}24.776 \\
6.193\end{array}$ & $\begin{array}{l}424.814 \\
423.478\end{array}$ \\
\hline
\end{tabular}

\begin{tabular}{|l|c|ccc|ccc|c|c|}
\hline $\begin{array}{l}\text { Anzahl der } \\
\text { Unbekannten }\end{array}$ & 0 & \multicolumn{3}{|c|}{1} & \multicolumn{3}{|c|}{2} & 3 \\
\hline exre & $\mathrm{nn}$ & \multicolumn{3}{|c|}{$\mathrm{nn}$} & $\mathrm{nn}$ & $\mathrm{nn}$ & & \\
exex & $\mathrm{nn}$ & $\mathrm{nn}$ & & $\mathrm{nn}$ & \multicolumn{2}{c|}{$\mathrm{nn}$} & & \\
extr & $\mathrm{nn}$ & $\mathrm{nn}$ & $\mathrm{nn}$ & & & $\mathrm{nn}$ & \\
\hline vorher & 125.721 & 17.266 & 9.785 & 10 & 0 & 1 & 258.798 & 422.069 \\
nachher & 371.930 & 0 & 0 & 0 & 0 & 1 & 40.103 & 421.616 \\
\hline
\end{tabular}

\begin{tabular}{|c|c|c|c|c|c|c|c|c|}
\hline $\begin{array}{l}\text { Anzahl der } \\
\text { Unbekannten }\end{array}$ & 0 & \multicolumn{3}{|c|}{1} & \multicolumn{3}{|c|}{2} & 3 \\
\hline $\begin{array}{l}\text { opre } \\
\text { cost } \\
\text { gros }\end{array}$ & $\begin{array}{l}\mathrm{nn} \\
\mathrm{nn} \\
\mathrm{nn}\end{array}$ & $\begin{array}{l}\text { nn } \\
\text { nn }\end{array}$ & $\begin{array}{l}\mathrm{nn} \\
\mathrm{nn}\end{array}$ & $\begin{array}{l}\mathrm{nn} \\
\mathrm{nn}\end{array}$ & $\mathrm{nn}$ & nn & $\mathrm{nn}$ & \\
\hline $\begin{array}{l}\text { vorher } \\
\text { nachher }\end{array}$ & $\begin{array}{l}85.803 \\
89.701\end{array}$ & $\begin{array}{c}1.655 \\
0\end{array}$ & $\begin{array}{c}1.641 \\
0\end{array}$ & $\begin{array}{c}448 \\
0\end{array}$ & $\begin{array}{l}239.729 \\
239.642\end{array}$ & $\begin{array}{l}713 \\
682\end{array}$ & $\begin{array}{l}32.625 \\
40.996\end{array}$ & $\begin{array}{l}471.036 \\
462.629\end{array}$ \\
\hline
\end{tabular}

\begin{tabular}{|c|c|c|c|c|c|c|c|c|}
\hline $\begin{array}{l}\text { Anzahl der } \\
\text { Unbekannten }\end{array}$ & 0 & \multicolumn{3}{|c|}{1} & \multicolumn{3}{|c|}{2} & 3 \\
\hline $\begin{array}{l}\text { gros } \\
\text { oope } \\
\text { oppl }\end{array}$ & $\begin{array}{l}\mathrm{nn} \\
\mathrm{nn} \\
\mathrm{nn}\end{array}$ & $\begin{array}{l}\mathrm{nn} \\
\mathrm{nn}\end{array}$ & $\mathrm{nn}$ & $\begin{array}{l}\mathrm{nn} \\
\mathrm{nn}\end{array}$ & nn & $\mathrm{nn}$ & $\mathrm{nn}$ & \\
\hline $\begin{array}{l}\text { vorher } \\
\text { nachher }\end{array}$ & $\begin{array}{l}121.120 \\
130.670 \\
\end{array}$ & $\begin{array}{c}8.815 \\
0\end{array}$ & $\begin{array}{c}48 \\
0\end{array}$ & $\begin{array}{c}485 \\
0\end{array}$ & $\begin{array}{l}71 \\
27 \\
\end{array}$ & $\begin{array}{l}243 \\
118 \\
\end{array}$ & $\begin{array}{l}273.965 \\
279.893\end{array}$ & $\begin{array}{l}428.903 \\
422.942 \\
\end{array}$ \\
\hline
\end{tabular}

\begin{tabular}{|c|c|c|c|c|c|c|c|c|}
\hline $\begin{array}{l}\text { Anzahl der } \\
\text { Unbekannten }\end{array}$ & 0 & \multicolumn{3}{|c|}{1} & \multicolumn{3}{|c|}{2} & 3 \\
\hline $\begin{array}{l}\text { oppl } \\
\text { fipl } \\
\text { plbt }\end{array}$ & $\begin{array}{l}\mathrm{nn} \\
\mathrm{nn} \\
\mathrm{nn}\end{array}$ & $\begin{array}{l}\mathrm{nn} \\
\mathrm{nn}\end{array}$ & $\begin{array}{l}\mathrm{nn} \\
\mathrm{nn}\end{array}$ & $\begin{array}{l}\mathrm{nn} \\
\mathrm{nn}\end{array}$ & $\mathrm{nn}$ & nn & $\mathrm{nn}$ & \\
\hline $\begin{array}{l}\text { vorher } \\
\text { nachher }\end{array}$ & $\begin{array}{l}400.336 \\
409.871\end{array}$ & $\begin{array}{c}6.093 \\
0\end{array}$ & $\begin{array}{c}1.461 \\
0\end{array}$ & $\begin{array}{c}1.883 \\
0\end{array}$ & $\begin{array}{l}705 \\
692\end{array}$ & $\begin{array}{l}295 \\
300\end{array}$ & $\begin{array}{l}2.632 \\
3.315\end{array}$ & $\begin{array}{l}420.245 \\
419.472\end{array}$ \\
\hline
\end{tabular}

\begin{tabular}{|l|c|ccc|ccc|c|}
\hline $\begin{array}{l}\text { Anzahl der } \\
\text { Unbekannten }\end{array}$ & 0 & \multicolumn{3}{|c|}{1} & \multicolumn{3}{|c|}{2} & 3 \\
\hline plat & $\mathrm{nn}$ & & $\mathrm{nn}$ & $\mathrm{nn}$ & $\mathrm{nn}$ & & & \\
taxa & $\mathrm{nn}$ & $\mathrm{nn}$ & & $\mathrm{nn}$ & & $\mathrm{nn}$ & & \\
plbt & $\mathrm{nn}$ & $\mathrm{nn}$ & $\mathrm{nn}$ & & & & $\mathrm{nn}$ & \\
\hline vorher & 410.293 & 165 & 43 & 968 & 635 & 0 & 21 & 421.525 \\
nachher & 411.694 & 0 & 0 & 0 & 510 & 0 & 1.492 & 419.954 \\
\hline
\end{tabular}

\begin{tabular}{|l|c|ccc|ccc|c|}
\hline $\begin{array}{l}\text { Anzahl der } \\
\text { Unbekannten }\end{array}$ & 0 & & 1 & & & 2 & & 3 \\
\hline $\mathrm{pl}$ & $\mathrm{nn}$ & & $\mathrm{nn}$ & $\mathrm{nn}$ & $\mathrm{nn}$ & & & \\
extr & $\mathrm{nn}$ & $\mathrm{nn}$ & & $\mathrm{nn}$ & & $\mathrm{nn}$ & & \\
plat & $\mathrm{nn}$ & $\mathrm{nn}$ & $\mathrm{nn}$ & & & & $\mathrm{nn}$ & \\
\hline vorher & 411.355 & 78 & 441 & 127 & 99 & 10 & 65 & 421.475 \\
nachher & 412.023 & 0 & 0 & 0 & 80 & 10 & 181 & 421.356 \\
\hline
\end{tabular}

Quelle: eigene Berechnungen

${ }^{716}$ Die konsolidierten Konzernabschlüsse sind in dieser Übersicht noch enthalten. 
Tabelle 60: Länge der Beteiligungsketten - Vergleich der Amadeus-Datenbank mit der Direktinvestitionsstatistik der Deutschen Bundesbank

\begin{tabular}{|l|rr|}
\hline & Amadeus & Bundesbank \\
\hline direkt gehalten & $61,9 \%$ & $69,8 \%$ \\
Eine Zwischengesellschaft & $20,6 \%$ & $20,7 \%$ \\
Zwei Zwischengesellschaften & $10,3 \%$ & $5,8 \%$ \\
Drei Zwischengesellschaften & $5,0 \%$ & $2,0 \%$ \\
Vier Zwischengesellschaften & $1,8 \%$ & $0,9 \%$ \\
Fünf Zwischengesellschaften & $0,3 \%$ & $0,3 \%$ \\
Sechs und mehr Zwischengesellschaften & $0,1 \%$ & $0,6 \%$ \\
\hline Summe & $100 \%$ & $100 \%$ \\
\hline
\end{tabular}

Quelle: eigene Berechnungen, Weichernrieder, A./Mintz, J.M., Ownership, 2006, S. 10

Tabelle 61: Vergleich des Aufkommenseffekts der Einführung einer konsolidierten Körperschaftsteuer-Bemessungsgrundlage für die Formelfaktoren Vermögen, Lohnsumme und Umsatz

\begin{tabular}{|c|c|c|c|c|c|c|}
\hline \multirow[t]{2}{*}{ Land } & \multicolumn{3}{|c|}{ Abweichung gegenüber $\mathrm{SA}$ in $\mathrm{v} . \mathrm{H}$. } & \multicolumn{3}{|c|}{$\begin{array}{l}\text { Abweichung gegenüber dem Basisfall } \\
\text { in Prozentpunkten }\end{array}$} \\
\hline & Umsatz & Lohnsumme & Vermögen & Umsatz & Lohnsumme & Vermögen \\
\hline AT & $-26 \%$ & $-22 \%$ & $-21 \%$ & $-3 \%$ & $1 \%$ & $2 \%$ \\
\hline $\mathrm{BE}$ & $-8 \%$ & $-12 \%$ & $-13 \%$ & $3 \%$ & $-1 \%$ & $-2 \%$ \\
\hline $\mathrm{CZ}$ & $-21 \%$ & $-29 \%$ & $-18 \%$ & $2 \%$ & $-6 \%$ & $4 \%$ \\
\hline $\mathrm{DE}$ & $-8 \%$ & $2 \%$ & $-8 \%$ & $-3 \%$ & $6 \%$ & $-3 \%$ \\
\hline DK & $-12 \%$ & $-9 \%$ & $-7 \%$ & $-2 \%$ & $0 \%$ & $2 \%$ \\
\hline $\mathrm{EE}$ & $-27 \%$ & $-45 \%$ & $-27 \%$ & $6 \%$ & $-12 \%$ & $6 \%$ \\
\hline ES & $-13 \%$ & $-21 \%$ & $-16 \%$ & $3 \%$ & $-4 \%$ & $1 \%$ \\
\hline FI & $-30 \%$ & $-36 \%$ & $-25 \%$ & $0 \%$ & $-6 \%$ & $5 \%$ \\
\hline $\mathrm{FR}$ & $-12 \%$ & $-9 \%$ & $-9 \%$ & $-2 \%$ & $1 \%$ & $1 \%$ \\
\hline GB & $5 \%$ & $2 \%$ & $-15 \%$ & $7 \%$ & $5 \%$ & $-12 \%$ \\
\hline $\mathrm{GR}$ & $-21 \%$ & $-43 \%$ & $-15 \%$ & $5 \%$ & $-16 \%$ & $11 \%$ \\
\hline $\mathrm{HU}$ & $7 \%$ & $-27 \%$ & $-4 \%$ & $16 \%$ & $-19 \%$ & $4 \%$ \\
\hline IE & $-10 \%$ & $-56 \%$ & $-36 \%$ & $24 \%$ & $-22 \%$ & $-2 \%$ \\
\hline IT & $-23 \%$ & $-23 \%$ & $-24 \%$ & $0 \%$ & $1 \%$ & $-1 \%$ \\
\hline LT & $-27 \%$ & $-70 \%$ & $-25 \%$ & $14 \%$ & $-29 \%$ & $16 \%$ \\
\hline $\mathrm{LU}$ & $-34 \%$ & $-34 \%$ & $-26 \%$ & $-3 \%$ & $-3 \%$ & $6 \%$ \\
\hline LV & $46 \%$ & $13 \%$ & $30 \%$ & $17 \%$ & $-17 \%$ & $0 \%$ \\
\hline NL & $-55 \%$ & $.29 \%$ & $-23 \%$ & $-20 \%$ & $6 \%$ & $13 \%$ \\
\hline PL & $9 \%$ & $-32 \%$ & $-12 \%$ & $21 \%$ & $-20 \%$ & $0 \%$ \\
\hline PT & $-10 \%$ & $-17 \%$ & $-16 \%$ & $4 \%$ & $-2 \%$ & $-2 \%$ \\
\hline $\mathrm{SE}$ & $-9 \%$ & $-8 \%$ & $-1 \%$ & $-3 \%$ & $-2 \%$ & $5 \%$ \\
\hline SI & $-31 \%$ & $.26 \%$ & $-22 \%$ & $-5 \%$ & $0 \%$ & $5 \%$ \\
\hline SK & $-38 \%$ & $-31 \%$ & $2 \%$ & $-15 \%$ & $-9 \%$ & $24 \%$ \\
\hline $\mathrm{EU}$ & $-14 \%$ & $-14 \%$ & $-14 \%$ & $0 \%$ & $0 \%$ & $0 \%$ \\
\hline
\end{tabular}

Quelle: eigene Berechnungen 
Tabelle 62: Durchschnittliche quadrierte Abweichung des Aufkommenseffekts der Einführung einer konsolidierten Körperschaftsteuer-

Bemessungsgrundlage in Abhängigkeit von der Gewichtung der

Formelfaktoren in v. H. SA

\begin{tabular}{|c|c|c|c|c|c|c|c|c|c|c|}
\hline Umsatz & 100 & 90 & 90 & 80 & 80 & 80 & 70 & 70 & 70 & 70 \\
\hline Lohnsumme & 0 & 10 & 0 & 20 & 10 & 0 & 30 & 20 & 10 & 0 \\
\hline Vermögen & 0 & 0 & 10 & 0 & 10 & 20 & 0 & 10 & 20 & 30 \\
\hline Abweichung & $6,2 \%$ & $6,1 \%$ & $5,7 \%$ & $6,1 \%$ & $5,7 \%$ & $5,3 \%$ & $6,2 \%$ & $5,8 \%$ & $5,3 \%$ & $5,0 \%$ \\
\hline Umsatz & 60 & 60 & 60 & 60 & 60 & 50 & 50 & 50 & 50 & 50 \\
\hline Lohnsumme & 40 & 30 & 20 & 10 & 0 & 50 & 40 & 30 & 20 & 10 \\
\hline Vermögen & 0 & 10 & 20 & 30 & 40 & 0 & 10 & 20 & 30 & 40 \\
\hline Abweichung & $6,4 \%$ & $5,9 \%$ & $5,4 \%$ & $5,0 \%$ & $4,7 \%$ & $6,7 \%$ & $6,2 \%$ & $5,6 \%$ & $5,2 \%$ & $4,8 \%$ \\
\hline Umsatz & 50 & 40 & 40 & 40 & 40 & 40 & 40 & 40 & 30 & 30 \\
\hline Lohnsumme & 0 & 60 & 50 & 40 & 30 & 20 & 10 & 0 & 70 & 60 \\
\hline Vermögen & 50 & 0 & 10 & 20 & 30 & 40 & 50 & 60 & 0 & 10 \\
\hline Abweichung & $4,4 \%$ & $7,1 \%$ & $6,5 \%$ & $5,9 \%$ & $5,4 \%$ & $4,9 \%$ & $4,5 \%$ & $4,2 \%$ & $7,6 \%$ & $6,9 \%$ \\
\hline Umsatz & 30 & 30 & 30 & 30 & 30 & 30 & 20 & 20 & 20 & 20 \\
\hline Lohnsumme & 50 & 40 & 30 & 20 & 10 & 0 & 80 & 70 & 60 & 50 \\
\hline Vermögen & 20 & 30 & 40 & 50 & 60 & 70 & 0 & 10 & 20 & 30 \\
\hline Abweichung & $6,3 \%$ & $5,7 \%$ & $5,2 \%$ & $4,8 \%$ & $4,4 \%$ & $4,0 \%$ & $8,1 \%$ & $7,4 \%$ & $6,7 \%$ & $6,1 \%$ \\
\hline Umsatz & 20 & 20 & 20 & 20 & 20 & 10 & 10 & 10 & 10 & 10 \\
\hline Lohnsumme & 40 & 30 & 20 & 10 & 0 & 90 & 80 & 70 & 60 & 50 \\
\hline Vermögen & 40 & 50 & 60 & 70 & 80 & 0 & 10 & 20 & 30 & 40 \\
\hline Abweichung & $5,5 \%$ & $5,0 \%$ & $4,6 \%$ & $4,2 \%$ & $3,9 \%$ & $8,7 \%$ & $8,0 \%$ & $7,2 \%$ & $6,6 \%$ & $6,0 \%$ \\
\hline Umsatz & 10 & 10 & 10 & 10 & 10 & 0 & 0 & 0 & 0 & 0 \\
\hline Lohnsumme & 40 & 30 & 20 & 10 & 0 & 100 & 90 & 80 & 70 & 60 \\
\hline Vermögen & 50 & 60 & 70 & 80 & 90 & 0 & 10 & 20 & 30 & 40 \\
\hline Abweichung & $5,4 \%$ & $4,9 \%$ & $4,5 \%$ & $4,1 \%$ & $3,8 \%$ & $9,5 \%$ & $8,6 \%$ & $7,9 \%$ & $7,1 \%$ & $6,5 \%$ \\
\hline Umsatz & 0 & 0 & 0 & 0 & 0 & 0 & & & & \\
\hline Lohnsumme & 50 & 40 & 30 & 20 & 10 & 0 & & & & \\
\hline Vermögen & 50 & 60 & 70 & 80 & 90 & 100 & & & & \\
\hline Abweichung & $5,9 \%$ & $5,3 \%$ & $4,9 \%$ & $4,4 \%$ & $4,1 \%$ & $3,8 \%$ & & & & \\
\hline
\end{tabular}

Quelle: eigene Berechnungen 
Tabelle 63: Lohnniveau und Arbeitsproduktivität in der Europäischen Union

\begin{tabular}{|c|cc|cc|}
\hline Land & $\begin{array}{c}\text { Jahresverdienst } \\
\text { in TEUR } \\
\text { Amadeus- } \\
\text { Datenbank }\end{array}$ & $\begin{array}{c}\text { Jahresver- } \\
\text { dienst in } \\
\text { TEUR } \\
\text { Eurostat }\end{array}$ & $\begin{array}{c}\text { Arbeitsproduktivität je Be- } \\
\text { schäftigten - BIP in KKS je Be- } \\
\text { schäftigten im Vergleich zu EU- } \\
\text { 25 (EU-25=100) }\end{array}$ & $\begin{array}{c}\text { Arbeitsproduktivität je geleisteter } \\
\text { Arbeitsstunde - BIP in KKS je } \\
\text { geleisteter Arbeitsstunde im Ver- } \\
\text { gleich zu EU-15 (EU-15=100) }\end{array}$ \\
\hline AT & 46,5 & 32,43 & 116 & 98,4 \\
BE & 41,3 & 30,69 & 129,8 & 128,9 \\
CZ & 7,1 & 7,21 & 63,9 & 51 \\
DE & 51,5 & 34,62 & 104,5 & 111,2 \\
DK & 41,5 & 41,74 & 102 & 100,6 \\
EE & 8,7 & 4,93 & 51,1 & 39,4 \\
ES & 27,4 & 21,06 & 99,1 & 88,9 \\
FI & 36,0 & 30,97 & 104,9 & 93,4 \\
FR & 37,0 & 29,08 & 116,5 & 116,8 \\
GB & 32,2 & 38,54 & 107 & 98 \\
GR & 18,8 & 18,75 & 99,8 & 71,2 \\
HU & 12,7 & 5,91 & 68,7 & 52,7 \\
IE & 34,8 & 32,91 & 128,9 & 120 \\
IT & 28,3 & 25,81 & 110,2 & 92,7 \\
LT & 4,0 & 4,10 & 49,7 & 42,6 \\
LU & 80,9 & 38,10 & 159,1 & 153,1 \\
LV & 8,4 & 3,62 & 41,9 & 33 \\
NL & 44,2 & 33,68 & 105,7 & 115,1 \\
PL & 8,5 & 7,06 & 57,3 & 44,2 \\
PT & 21,0 & 13,61 & 66,3 & 60,5 \\
SE & 28,2 & 32,06 & 103,9 & 101,9 \\
SI & 16,8 & 11,27 & 74,1 & 64,2 \\
SK & 6,2 & 5,71 & 60,1 & 55 \\
\hline
\end{tabular}

Quelle: eigene Berechnungen 
Tabelle 64: Vergleich des Aufkommenseffekts der Einführung einer konsolidierten Körperschaftsteuer-Bemessungsgrundlage bei Verwendung der Lohnsumme und Mitarbeiteranzahl als Aufteilungsfaktor

\begin{tabular}{|c|c|c|c|c|c|c|c|}
\hline \multirow{2}{*}{$\begin{array}{l}\text { Land } \\
\text { AT }\end{array}$} & \multirow{2}{*}{\begin{tabular}{r|} 
SA BMG \\
364.124
\end{tabular}} & \multirow{2}{*}{$\begin{array}{r}\begin{array}{c}\text { FA BMG } \\
\text { Mitarbeiter }\end{array} \\
287.773\end{array}$} & $\begin{array}{l}\text { Differenz SA BMG - } \\
\text { FA BMG Mitarbeiter }\end{array}$ & $\begin{array}{c}\text { FA BMG } \\
\text { Lohnsumme }\end{array}$ & \multicolumn{2}{|c|}{$\begin{array}{c}\text { Differenz FA BMG } \\
\text { Mitarbeiter - FA BMG } \\
\text { Lohnsumme }\end{array}$} & \multirow[t]{2}{*}{\begin{tabular}{|c|}
$\begin{array}{c}\text { Jahresverdienst } \\
\text { in TEUR } \\
\text { Amadeus- } \\
\text { Datenbank }\end{array}$ \\
46,5129
\end{tabular}} \\
\hline & & & $-76.351-20,97 \%$ & 280.357 & 7.416 & $2,58 \%$ & \\
\hline $\mathrm{BE}$ & 13.096 .649 & 11.091 .573 & $-2.005 .075-15,31 \%$ & 11.637 .498 & .545 .924 & $2 \%$ & 41,25 \\
\hline $\mathrm{CZ}$ & 674 & .504 & $170-13,24 \%$ & & & & 7,08 \\
\hline $\mathrm{DE}$ & 10.359 .122 & 9.535 .402 & $-823.720-7,95 \%$ & 9.875 .849 & -34 & $7 \%$ & 51,4706 \\
\hline DK & 16.935 .900 & 15.389 .632 & $-1.546 .267 \quad-9,13 \%$ & 15.336 .469 & & $5 \%$ & 41,5375 \\
\hline $\mathrm{EE}$ & & & $-13.200-3,69 \%$ & & & & \\
\hline ES & 26.496 .594 & 22.070 .775 & $-4.425 .819-16,70 \%$ & 22.1 & 39 & $8 \%$ & 27,3524 \\
\hline $\mathrm{FI}$ & 10.295 .727 & & $-3.136 .187-30,46 \%$ & & & & 36 \\
\hline FR & 40.5 & 35 & $43-11$ & 36 & 6 & & 37 \\
\hline GB & 844 & & 1.0431 & 30. & & & 32,168 \\
\hline GR & .880 & .041 & $-408.839-16,86 \%$ & 964 & & $\%$ & 18,751 \\
\hline $\mathrm{HU}$ & & & 7 & & & & 12,7464 \\
\hline IE & 118 & & $-730.370-31,91 \%$ & & & & 34,764 \\
\hline IT & 19.403.356 & 14.882 .730 & $-4.520 .626-23,30 \%$ & 14.931 .588 & -48.857 & $-0,33 \%$ & 28,3115 \\
\hline LT & & & 1172 & & & & 4,039 \\
\hline LU & 822.421 & 546. & $-275.622-33,51$ & 566 & & & 80,8967 \\
\hline LV & 47.842 & 91.985 & $44.143 \quad 92,27 \%$ & 61.996 & 89 & $32,60 \%$ & 8,4467 \\
\hline NL & 14.766 .326 & & $-6.591 .033-44$, & & & & 44,1749 \\
\hline PL & & & 185.41713 , & & & & 8,5015 \\
\hline PT & 3.008 .043 & 2.746 .637 & $-261.406-8,69 \%$ & 2.577 .050 & & $17 \%$ & 20,98 \\
\hline SE & 13.656 .466 & 13.037 .597 & $-618.868-4,5$ & 12.830 & & & 28,1769 \\
\hline SI & & & $-10.022-22,23 \%$ & & & & 16,7537 \\
\hline SK & 94.705 & 77.151 & $-17.554-18,54 \%$ & 73.694 & 3.456 & $4,48 \%$ & 6,2453 \\
\hline $\mathrm{EU}$ & 210.988 .860 & 181.222 .135 & $-29.766 .725-14,11 \%$ & 181.222 .135 & 0 & $0,00 \%$ & \\
\hline
\end{tabular}

Quelle: eigene Berechnungen 
Tabelle 65: Vergleich des Aufkommenseffekts der Einführung einer konsolidierten Körperschaftsteuer-Bemessungsgrundlage bei Anwendung der Anrechnungsmethode und der Freistellungsmethode

\begin{tabular}{|c|c|c|c|c|c|c|c|c|c|c|}
\hline \multirow[t]{2}{*}{ Land } & \multirow[t]{2}{*}{$\mathbf{S}$} & \multirow{2}{*}{\begin{tabular}{|c|} 
Separate \\
Accounting \\
TEUR
\end{tabular}} & \multicolumn{2}{|c|}{$\begin{array}{c}\text { Formulary Apportionment } \\
\text { TEUR } \\
\end{array}$} & \multicolumn{2}{|c|}{\begin{tabular}{|c|} 
Differenz SA - FA \\
Wohnsitz
\end{tabular}} & \multicolumn{2}{|c|}{$\begin{array}{c}\text { Differenz SA - FA } \\
\text { Quelle }\end{array}$} & \multicolumn{2}{|c|}{\begin{tabular}{|c|} 
Differenz \\
Quelle/Wohnsitz
\end{tabular}} \\
\hline & & & Quelle & Woh & $\begin{array}{l}\text { absolut } \\
\text { TEUR }\end{array}$ & relativ & $\begin{array}{l}\text { absolut } \\
\text { TEUR }\end{array}$ & relativ & \begin{tabular}{|c|} 
absolut \\
TEUR
\end{tabular} & relativ \\
\hline AT & 34,0 & 302 & & & -28.481 & & -24.541 & & 3.940 & $\%$ \\
\hline $\mathrm{BE}$ & 33,0 & 4.32 & .868 .653 & & -481.520 & & -453.241 & $-10,5 \%$ & 28.279 & $0,7 \%$ \\
\hline CY & 15,0 & & 0 & 0 & 0 & $0,0 \%$ & & $0,0 \%$ & 0 & \\
\hline $\mathrm{CZ}$ & 31,0 & 719 & 008 & 935 & -154.784 & $-22,5 \%$ & -154.710 & $-22,5 \%$ & 74 & $0,0 \%$ \\
\hline $\mathrm{DE}$ & 26,5 & 2.74 & 2.621 .710 & 100 & -128.067 & $-4,7 \%$ & -123.457 & $-4,5 \%$ & 4.610 & $0,2 \%$ \\
\hline DK & 30,0 & 5.080 .770 & 4.627 .834 & 4.60 & -479.829 & $-9,4 \%$ & -452.936 & -8, & 26.893 & $0,6 \%$ \\
\hline $\mathrm{EE}$ & 26,0 & 978 & & & 22 & & -29.794 & & 1.028 & $1,7 \%$ \\
\hline ES & 35,0 & 9.273 .808 & 7.813 .724 & 365 & -1.535 .443 & $-16,6 \%$ & -1.460 .084 & $-15,7 \%$ & 75.359 & $1,0 \%$ \\
\hline FI & 29,0 & 2.98 & .013 & 91 & -901.570 & -30 & -86 & -28 & 39.822 & $1,9 \%$ \\
\hline FR & 33,3 & 13.51 & 12.2 & 36 & -1.399 & & -1.23 & & 166.315 & 1, \\
\hline GB & 30,0 & 9.467 .053 & 218.592 & 398 & -262.655 & $\%$ & -248 & $-2,6 \%$ & 14.195 & $0,2 \%$ \\
\hline GR & 35,0 & 708 & 34 & 57 & -223.341 & -26 & -20 & $-24,2$ & 18.266 & $2,9 \%$ \\
\hline $\mathrm{HU}$ & 18,0 & 348 & & & 389 & & 389 & & d & $0,0 \%$ \\
\hline IE & 10,0 & .912 & 0.600 & 600 & -78.311 & $-34,2 \%$ & 311 & $-34,2 \%$ & 0 & $0,0 \%$ \\
\hline IT & 34,0 & 6.597 .141 & 0.131 .927 & 5.076 .740 & .520 .401 & $-23,0 \%$ & .465 .214 & $-22,2$ & 55.188 & $1,1 \%$ \\
\hline LT & 15,0 & 749 & 4.001 & 4.001 & -2.748 & $-40,7 \%$ & -2.748 & $-40,7$ & 0 & $0,0 \%$ \\
\hline LU & 22,0 & 180.933 & 126.368 & 124.702 & -56.230 & $-31,1 \%$ & -54.565 & $-30,2$ & 1.665 & $1,3 \%$ \\
\hline LV & 19,0 & 9.090 & & & 2.689 & $29,6 \%$ & 2.692 & $29,6 \%$ & 4 & $0,0 \%$ \\
\hline $\mathrm{NL}$ & 34,5 & 5.094 .382 & 3.739 .536 & 3.271 .582 & -1.822 .801 & $-35,8 \%$ & -1.354 .847 & $-26,6 \%$ & 467.954 & $14,3 \%$ \\
\hline PL & 27,0 & 378.812 & 334.047 & 334.027 & -44.785 & $-11,8 \%$ & -44.765 & $-11,8 \%$ & 20 & $0,0 \%$ \\
\hline PT & 30,0 & 902.413 & 775.073 & 115 & -129.298 & $-14,3 \%$ & -127.340 & $-14,1 \%$ & 1.958 & $0,3 \%$ \\
\hline SE & 28,0 & 3.823 .810 & 3.611 .578 & 3.592 .627 & -231.184 & $-6,0 \%$ & -212.232 & $-5,6 \%$ & 18.952 & $0,5 \%$ \\
\hline SI & 25,0 & 11.272 & & & -2.959 & $-26,3 \%$ & -2.959 & $-26,3$ & 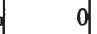 & $0,0 \%$ \\
\hline SK & 25,0 & 23.676 & 18.424 & 18.424 & -5.253 & $-22,2 \%$ & -5.253 & $-22,2 \%$ & $y$ & $0,0 \%$ \\
\hline $\mathrm{EU}$ & & 66.535 .327 & 57.931 .372 & 57.006 .853 & -9.528 .474 & $-14,3 \%$ & -8.603 .955 & $-12,9 \%$ & 924.519 & $1,6 \%$ \\
\hline
\end{tabular}

Quelle: eigene Berechnungen 


\section{Literaturverzeichnis}

Aaron, H.J./Boskin, M.J. (Hrsg.); (Economics, 1980), The Economics of Taxation, Washington D.C.

Agúndez-García, A.; (Apportionment, 2006), The Delineation and Apportionment of an Consolidated Tax Base for Multi-jurisdictional Corporate Income Taxation: a Review of Issues and Options, European Commission Working Paper, Luxemburg

Albers, J./Klapper, D./Konradt, U./Walter, A./Wolf, J. (Hrsg.); (Forschung, 2006), Methodik der empirischen Forschung, Wiesbaden

Althuber, F./Mang, M.; (IWB, Fach 5, Gruppe 2, 8. 9. 2004), Ausgewählte Fragen zur neuen Gruppenbesteuerung in Österreich, in: Internationale Wirtschafts-Briefe, S. 607-620

Altman, G.T./Keesling, F.M.; (Income, 1946), Allocation of Income in State Taxation, New York

American Chamber of Commerce/PricewaterhouseCoopers (Hrsg.); (USA, 2001), Steuern in den USA - Ein Leitfaden für deutsche Investoren, Frankfurt am Main

Andreß, H.-J./Hagenaars, J./Kühnel, S.; (Analyse, 1997), Analyse von Tabellen und kategorialen Daten - Log-lineare Modelle, latente Klassenanalyse, logistische Regression und GSK-Ansatz, Berlin, Heidelberg, New York

Arbeitskreis Professor Dr. Krähe; (Konzernorganisation, 1964), Konzernorganisation, Köln, Opladen

Arbeitskreis „Steuern und Revision“ im Bund der Wirtschaftsakademiker (BWA) e. V.; (DStR, 2004), Maßgeblichkeit im Wandel der Rechnungslegung - Die Maßgeblichkeit im System internationaler Steuerbemessungsgrundlagen vor dem Hintergrund aktueller Herausforderungen, in: Deutsches Steuerrecht, S. 1267-1268

Balmes, A./Brück, M./Ribbrock, M.; (BB, 2006), Das EuGH-Urteil Marks \& Spencer: Grenzüberschreitende Verlustnutzung kommt voran!, in: Betriebs-Berater, S. 186-189

Baltes-Götz, B.; (Regressionsanalyse, 2006), Logistische Regressionsanalyse mit SPSS, URL: http://www.uni-trier.de/urt/user/baltes/docs/logist/ logist.pdf, 29. 4. 2007

Bank, M./Schiller, B. (Hrsg.); (Finanzintermediation, 2004), Finanzintermediation - Theoretische, wirtschaftspolitische und praktische Aspekte aktueller Entwicklungen im Bank- und Börsenwesen, Stuttgart

Baretti, C./Huber, B./Lichtblau, K.; (ITPF, 2002), A Tax on Tax Revenue: The Incentive Effects of Equalizing Transfers: Evidence from Germany, in: International Tax and Public Finance, S. 631-649 
Bartelsman, E./Beetsma, R.; (JoPE, 2003), Why pay more? Corporate tax avoidance through transfer pricing in OECD countries, in: Journal of Public Economics, S. 2225-2252

Beiser, R./Pülzl, P.; (Weber/Wimmer, Verfassungsstaat, 2005), Harmonisierung der direkten Steuern in der EU. Rechtsgrundlagen, Stand und Perspektiven, in: K. Weber, N. Wimmer, (Hrsg.), Vom Verfassungsstaat am Scheideweg, Wien, New York, S. 43-65

Benecke, A.; (IStR, 2005), Änderungsrichtlinie zur Fusionsrichtlinie: Vermeidung der wirtschaftlichen Doppelbesteuerung und Aufnahme transparenter Gesellschaften - Zwei unvereinbare Ziele?, in: Internationales Steuerrecht, S. 606-612 (Teil I) und Seite 641-649 (Teil II)

Bieber, R./Epiney, A./Haag, M.; (Union, 2005), Die Europäische Union Europarecht und Politik, Baden-Baden

Bille, S.; (Hinzurechnungsbesteuerung, 2004), Hinzurechnungsbesteuerung in Europa, Aachen

Bird, R.M./Head, J.G. (Hrsg.); (Fiscal, 1972), Modern Fiscal Issues, Essays in Honor of Carl S. Shoup, Toronto

Bjørnholm, N./Becker-Christensen, A.; (ET, 2006), The New Danish Tax Consolidation Regime, in: European Taxation, S. 47-49

Bleicher, K.; (Organisation, 1991), Organisation - Strategien - Strukturen Kulturen, Wiesbaden

Blumenthal, M./Slemrod, J.; (ITPF, 1995), The Compliance Cost of Taxing Foreign- Source Income: Its Magnitude, Determinants, and Policy Implications, in: International Tax and Public Finance, S. 37-53

Bökelmann, J.; (Gewinnzurechnung, 1997), Gewinnzurechnung im Körperschaftsteuerrecht - Die Worldwide Unitary Taxation US-amerikanischer Einzelstaaten und ihre Anwendbarkeit durch die Europäische Union, Konstanz

Boss, A.; (Steuerwettbewerb, 2003), Steuerharmonisierung oder Steuerwettbewerb?, Kieler Arbeitspapier Nr. 1178; Kiel

Brähler, K.; (CFC, 2006), Controlled Foreign Companies-Rules - eine steuersystematische Analyse im Rahmen eines Ländervergleichs unter Berücksichtigung der Vereinbarkeit mit den Doppelbesteuerungsabkommen und dem Europäischen Gemeinschaftsrecht, Frankfurt am Main

Bravenec, L.; (ET, 2000), Corporate Income Tax Coordination in the 21 st Century, in: European Taxation, S. 450-463

Bravenec, L.; (ET, 2001), Corporate Income Tax Coordination - Current Practitioner Options, in: European Taxation, S. 428-429

Brügelmann, R.; (ifo Schnelldienst, 2004, 11), Eine Vereinheitlichung der Körperschaftsteuer schafft mehr Probleme, als sie löst, in: ifo Schnelldienst, Nr. 11, S. 5-8 
Büttner, T.; (Müller, Systemwettbewerb, 2001), Empirie des Steuerwettbewerbs: Zum Stand der Forschung, in: W. Müller (Hrsg.), Regeln für den europäischen Systemwettbewerb - Steuern und soziale Sicherungssysteme, Marburg, S. 53-70

Bullinger, P.; (IStR, 2004), Änderungen der Mutter-Tochter-Richtlinie ab 2005: Erweiterung des Anwendungsbereiches und verbleibende Probleme, in: Internationales Steuerrecht, S. 406-412

Burwitz, G.; (Konzernfinanzierungsgesellschaften, 2005), Ausländische Konzernfinanzierungsgesellschaften im Internationalen Vergleich Zugleich eine Analyse von Dublin-Docks-Gesellschaften deutscher Konzernmütter, Köln

Buzzel, R.D./Bradley, T.G.; (PIMS, 1989), Das PIMS-Programm - Strategie und Unternehmenserfolg, Wiesbaden

Carlson, G.N./Galper, H.; (McLure, State, 1984), Water's Edge Versus Worldwide Unitary Combination, in: C.E. McLure Jr. (Hrsg.); The State Corporate Income Tax - Issues in Worldwide Unitary Combination, Stanford, S. $1-40$

Cerioni, L.; (ET, 2005/2006), The Introduction of Comprehensive Approaches to Business Taxation: At the Root of Competition and Discrimination or... The Long and Winding Road to a Solution?, in: European Taxation, 2005, S. 541-559 (Teil I) und 2006, S. 13-27 (Teil II)

Cerioni, L.; (ET, 2006), The Possible Introduction of Common Consolidated Base Taxation via Enhanced Cooperation: Some Open Issues, in: European Taxation, S. 187-196

Cnossen, S. (Hrsg.); (Capital, 2000), Taxing Capital Income in the European Union: Issues and Options for Reform, Oxford

Cnossen, S.; (FA, 2001), Tax Policy in the European Union - A Review of Issues and Options, in: Finanzarchiv, S. 466-558

Cordewener, A.; (Grundfreiheiten, 2002), Europäische Grundfreiheiten und nationales Steuerrecht - „Konvergenz" des Gemeinschaftsrechts und „Kohärenz" der direkten Steuern in der Rechtssprechung des EuGH, Köln

Cordewener, A./Schnitger, A.; (StuW, 2006), Europarechtliche Vorgaben für die Vermeidung der internationalen Doppelbesteuerung im Wege der Anrechnungsmethode, in: Steuern und Wirtschaft, S. 50-78

Corrigan, E.; (TN, 1980), Toward Uniformity in Interstate Taxation, in: Tax Notes, S. 507-514

Derpa, U.; (Zusammenarbeit, 2003), Die verstärkte Zusammenarbeit im Recht der Europäischen Union, Stuttgart, München, Hannover, Berlin, Weimar, Dresden 
Desai, M./Foley. F./Hines Jr. J.; (Hermann/Lipsey, Direct Investment, 2003), Chains of Ownership, regionla Tax Competition and Foreign Investment, in: H. Herrmann/R. Lipsey (Hrsg.), Foreign Direct Investment in the Real and Financial Sector of Industrial Countries, Heidelberg, S. 61-98.

Deutsche Bundesbank; (Monatsbericht, 2002, 6), Direktinvestitionen im realen und finanziellen Sektor: Die Bundesbank-Frühjahrskonferenz 2002, in: Deutsche Bundesbank Monatsbericht Juni 2002, S. 79-84

Deutsche Bundesbank; (Verhältniszahlen, 2004), Verhältniszahlen aus Jahresabschlüssen deutscher Unternehmen von 2000 bis 2002, Statistische Sonderveröffentlichung 6, Frankfurt am Main

Devereux, M.P./Elschner/C., Overesch/M., Schreiber, U./Spengel, C.; (Tax Rates, 2006), Effective tax rates for companies in the European Union, Project for the European Commission TAXUD /2005/DE/3 10, Inception Report, Mannheim and Warwick 2006

Devereux, M.P./Loretz, S., (Apportionment, 2007), The Effects of EU Formula Apportionment on Corporate Tax Revenues, Working Paper, Oxford University

Diemer, R.; (DSWR, 2003), EU-Pilotprojekt zur KMU-Besteuerung, in: Datenverarbeitung, Steuern, Wirtschaft, Recht, S. 151-153

Diemer, R./Mors, M.; (ITPJ, 2006), Outline for a Possible Home State Taxation Pilot Project for SMEs, in: International Transfer Pricing Journal, S. 111116

Dietel, M.; (Gewinnermittlung, 2004), International Accounting Standards/International Financial Reporting Standards und steuerliche Gewinnermittlung - Möglichkeiten für eine modifizierte Maßgeblichkeit, Wien

Döhrn, R./Heiduk, G. (Hrsg.); (Direktinvestitionen, 1999), Theorie und Empirie der Direktinvestitionen, Berlin

Dörr, I.; (DSWR, 2004), Gruppenbesteuerung in Italien - Die internationale „consolidato mondiale“, in: Datenverarbeitung, Steuern, Wirtschaft, Recht, S. 248-249

Eden, L.; (Multinationals, 1998), Taxing Multinationals, Transfer Pricing and Corporate Income Taxation in North America, Toronto

Eggert, W./Haufler, A.; (Coordination, 2006), Company tax coordination cum tax rate competition in the European Union, Discussion Paper LudwigMaximilians-Universität München, Nr. 11

Endres, D.; (Herzig, Organschaft, 2003), Konzernbesteuerung in wichtigen Industriestaaten, in: N. Herzig (Hrsg.), Organschaft: laufende und aperiodische Besteuerung, nationale und internationale Aspekte, Stuttgart, S. 461-482 
Endres, D.; (WPg, 2003), Typische Holdingstrukturen anhand von Beispielfällen, in: Die Wirtschaftsprüfung, Sonderheft, S. S56-S63

Endres, D./Lüdicke, J./Brink, T./Naumburg, S./Suhrbier-Hahn, U./Zuber, B./Welling, B./Schmid, C.; (Verlustberücksichtigung, 2006), Verlustberücksichtigung über Grenzen hinweg - Vergleichende Gegenüberstellung der Verlustverrechnungsmöglichkeiten in 33 Ländern, Frankfurt am Main

Endres, D./Oestreicher, A./Scheffler, W./Schreiber, U./Spengel, C. (Hrsg.); (Wandel, 2005), Die internationale Unternehmensbesteuerung im Wandel, Symposium für Otto H. Jacobs zum 65. Geburtstag, München

Endies, D./Oestreicher, A./Scheffler, W./Spengel, C.; (Taxable Income, 2006), The Determination of Corporate Taxable Income in the EU Member States, Amsterdam

English, J.; (StuW, 2003), Zur Dogmatik der Grundfreiheiten des EGV und ihren ertragsteuerlichen Implikationen, in: Steuern und Wirtschaft, S. 88-97

Ernst \& Young; (Pricing, 1999), Transfer Pricing - 1999 Global Survey, o. O.

Esterer, F.; (Endres et al., Wandel, 2005), Maßgeblichkeit der IAS/IFRS oder eigenständige steuerrechtliche Gewinnermittlung?, in: D. Endres/A. Oestreicher/W. Scheffler/U. Schreiber/C. Spengel (Hrsg.), Die internationale Unternehmensbesteuerung im Wandel, München, S. 110-126

Europäische Kommission; (Sachverständigenausschuß, 1992), Bericht des unabhängigen Sachverständigenausschuß zur Unternehmensbesteuerung, Brüssel

Europäische Kommission; (Unternehmensbesteuerung, 2001), Mitteilung der Kommission an den Rat, das Europäische Parlament und den Wirtschaftsund Sozialausschuß: Ein Binnenmarkt ohne steuerliche Hindernisse Strategie zur Schaffung einer konsolidierten KörperschaftsteuerBemessungsgrundlage für die grenzüberschreitende Unternehmenstätigkeit in der EU, KOM (2001) 582 endgültig, Mitteilung vom 23. 10. 2001

Europäische Kommission; (Vorschläge, 2001), Mitteilung der Kommission: Vorschläge, die von der Kommission zurückgezogen werden - Überholte Vorschläge, KOM (2001), 763 endgültig, Mitteilung vom 21. 12. 2001

Europäische Kommission; (Binnenmarkt, 2003), Mitteilung der Kommission an den Rat, das europäische Parlament und den europäischen Wirtschaftsund Sozialausschuss: Ein Binnenmarkt ohne unternehmenssteuerliche Hindernisse. Ergebnisse Initiativen, Herausforderungen, KOM (2003) 726 endgültig, Mitteilung vom 24. 11. 2003 
Europäische Kommission; (Ergebnisse, 2003), Zusammenfassung der Ergebnisse der von der GD Steuern und Zollunion durchgeführten Konsultation zum Thema „Anwendung der International Accounting Standards (IAS) und ihre Implikationen für die Schaffung einer konsolidierten Steuerbemessungsgrundlage für die grenzüberschreitende Unternehmenstätigkeit in der EU", URL: http://ec.europa.eu/taxation_customs/resources/ documents/ias_summary_de.pdf, 15.2.2007

Europäische Kommission; (European Economy, 2003, 6), The EU economy: 2003 review, in: European Economy, Nr. 6

Europäische Kommission; (Konsultationspapier, 2003), Die Anwendung der International Accounting Standards (IAS) ab 2005 und ihre Implikationen für die Schaffung einer konsolidierten Steuerbemessungsgrundlage für die grenzüberschreitende Unternehmenstätigkeit in der EU, URL: http://ec.europa.eu/taxation_customs/resources/documents/iaspaper de.pdf, 15. 2. 2007

Europäische Kommission; (Non-Paper, 2004a), Non-Paper der Kommission für die informelle Tagung des Rates ,Wirtschaft und Finanzen" am 10. und 11. September 2004, Einheitliche konsolidierte Körperschaftsteuerbemessungsgrundlage, URL: http://ec.europa.eu/taxation_customs/ resources/documents/taxation/company_tax/common_tax_base/CCTBWP Non_Paper.pdf, 15.2. 2007

Europäische Kommission; (Activities, 2004), Activities of the European Union (EU) in the tax field in 2003, URL: http://ec.europa.eu/taxation_customs/ resources/documents/report_activities_2003_annex.pdf, 17. 3. 2007

Europäische Kommission; (Non-Paper, 2004b), Non-Paper der Kommission für die informelle Tagung des Rates "Wirtschaft und Finanzen" am 10. und 11. September 2004, Sitzlandbesteuerung bei kleinen und mittleren Unternehmen, URL: http://ec.europa.eu/taxation_customs/resources/ documents/HST_Non-Paper_DE.pdf, 15.2. 2007

Europäische Kommission; (Pilotprojekt, 2004), Versuchsweise Anwendung der "Sitzlandbesteuerung" auf KMU - Skizzierung eines möglichen Pilotprojekts, URL: http://ec.europa.eu/taxation_customs/resources/ documents/HST_Paper_June_04_DE.pdf, 15.2.2007

Europäische Kommission; (Survey, 2004), European Tax Survey, SEC (2004) $1128 / 2$ vom 10.9. 2004 
Europäische Kommission; (Verrechnungspreisforum, 2004), Mitteilung der Kommission an den Rat, das europäische Parlament und den europäischen Wirtschafts- und Sozialausschuss: über die Tätigkeit des Gemeinsamen EU-Verrechnungspreisforums im Bereich der Unternehmensbesteuerung von Oktober 2002 bis Dezember 2003 und über den Vorschlag eines Verhaltenskodexes zur effektiven Durchführung des Schiedsübereinkommens (90/436/EWG vom 23.7. 1990), KOM (2004) 297 endgültig, Mitteilung vom 23. 4. 2004

Europäische Kommission; (Arbeitsleitlinien, 2004), Entwurf Arbeitsleitlinien und Geschäftsordnung, URL: http://ec.europa.eu/taxation_customs/ resources/documents/taxation/company_tax/common_tax_base/CCTBTO RFinal_de.pdf, 17.3. 2007

Europäische Kommission; (Neubeginn, 2005), Mitteilung für die Frühjahrstagung des Europäischen Rates - Zusammenarbeit für Wachstum und Arbeitsplätze. Ein Neubeginn für die Strategie von Lissabon - Mitteilung von Präsident Barroso im Einvernehmen mit Vizepräsident Verheugen, KOM (2005) 24 endgültig, Mitteilung vom 2.2. 2005

Europäische Kommission; (Lissabon-Programm), Mitteilung der Kommission an den Rat und das Europäische Parlament-Gemeinsame Maßnahmen für Wachstum und Beschäftigung: Das Lissabon-Programm der Gemeinschaft, KOM (2005) 330 endgültig, Mitteilung vom 20. 7. 2005

Europäische Kommission; (Herausforderungen, 2005), Umsetzung des Lissabon-Programms der Gemeinschaft - Mitteilung der Kommission an den Rat und das Europäische Parlament - Der Beitrag der Steuer- und Zollpolitik zur Lissabon-Strategie, KOM (2005), 532 endgültig, Mitteilung vom 25. 10. 2005

Europäische Kommission; (Schiedsübereinkommen, 2005), Gemeinsame EUVerrechnungspreisforum - Bericht über das Wiederinkraftreten des Schiedsübereinkommens, DOK: JTPF/019/REV5/ 2004/DE, vom 30.5. 2005

Europäische Kommission; (Sitzlandbesteuerung, 2005), Mitteilung der Kommission an den Rat, das europäische Parlament und den europäischen Wirtschafts- und Sozialausschuss: Sitzlandbesteuerung Skizzierung eines möglichen Pilotprojekts zur Beseitigung unternehmenssteuerlicher Hindernisse für kleine und mittlere Unternehmen im Binnenmarkt, KOM (2005) 702 endgültig, Mitteilung vom 23. 12. 2005

Europäische Kommission; (Treaties, 2005), EC Law an Tax Treaties, DOC (05) 2306, vom 9. 6. 2006 
Europäische Kommission; (Verhaltenskodex, 2005), Mitteilung der Kommission an den Rat, das europäische Parlament und den europäischen Wirtschafts- und Sozialausschuss: über die Tätigkeit des Gemeinsamen EU-Verrechnungspreisforums im Bereich der Verrechnungspreisdokumentation für verbundene Unternehmen in der EU-Vorschlag für einen Verhaltenskodex zur Verrechnungspreisdokumentation für verbundene Unternehmen in der EU, KOM (2005) 543 endgültig, Mitteilung vom 23. 4.2004

Europäische Kommission; (Comments, 2006), Comments on document CCCTB $|W P| 035$, Common Consolidated Corporate Tax Base Working Group - Issues related to Group Taxation, URL: http://ec.europa.eu/ taxation_customs/resources/documents/taxation/company_tax/common_ta x_base/CCCTBWP035_comments_en.pdf, 15. 2. 2007

Europäische Kommission; (Fragen zur Konzernbesteuerung, 2006), Fragen zur Konzernbesteuerung, URL: http://ec.europa.eu/taxation_customs/resour ces/documents/taxation/company_tax/common_tax_base/CCCTBWP035 _consolidationFINAL_de.pdf, 15.2. 2007

Europäische Kommission; (Implementation, 2006), Planned Implementation of the IAS Regulation (1606/2002) in the EU and EEA, URL: http://ec.europa.eu/internal_market/accounting/docs/ias/ias-use-of-options _en.pdf; 15. 2. 2007

Europäische Kommission; (Internationale Apekte, 2006), Zusammenfassung der wichtigsten, auf der Sitzung der Unterarbeitsgruppe „Internationale Aspekte" herausgearbeitete Fragestellungen, URL: http://ec.europa.eu/ taxation_customs/resources/documents/taxation/company_tax/common_ta x_base/CCCTBWP029_overviewSG4_de.pdf, 15.2.2007

Europäische Kommission; (Mechanismus, 2006), Mechanismus zur Aufteilung $\operatorname{der} G K K B$, URL: http://ec.europa.eu/taxation_customs/resources/docu ments/taxation/company_tax/common_tax_base/CCCTBWP47_sharing_ mechanism_de.pdf, 19.2. 2007

Europäische Kommission; (Persönlicher Anwendungsbereich, 2006), Persönlicher Anwendungsbreich der GKKB, URL: http://ec.europa. eu/taxation_customs/resources/documents/taxation/company_tax/common tax_base/CCCTBWP040_scope_de.pdf, 15. 2. 2007

Europäische Kommission; (Räumlicher Anwendungsbereich, 2006), Räumlicher Anwendungsbereich der GKKB, URL: http://ec.europa.eu/taxation customs/resources/documents/taxation/company_tax/common_tax_base/ $\overline{\mathrm{C}}$ CCTBWP026_territorial_scope_de.pdf, 15.2.2007 
Europäische Kommission; (Umsetzung, 2006), Mitteilung der Kommission an den Rat, das Europäische Parlament und den Europäischen Wirtschaftsund Sozialausschuss: Umsetzung des Lissabon-Programms der Gemeinschaft: Bisherige Fortschritte und weitere Schritte zu einer gemeinsamen konolidierten Körperschaftsteuer-Bemessungsgrundlage (GKKB), KOM (2006), 157 endgültig, Mitteilung vom 5. 4. 2006

Europäische Kommission; (Verluste, 2006), Mitteilung der Kommission an den Rat, das europäische Parlament und den europäischen Wirtschafts- und Sozialauschuss: Steuerliche Behandlung von Verlusten bei grenzübergreifenden Sachverhalten, KOM (2006), 824 endgültig, Mitteilung vom 19. 12. 2006

Europäische Zentralbank; (Monatsbericht, 2004, 7), Entwicklung der Arbeitsproduktivität im Euro-Währungsgebiet: Gesamtwirtschaftliche und sektorale Trends, in: Monatsbericht, Nr. 7, S. 49-61

Eurostat; (NACE, 2002), Statistische Systematik der Wirtschaftszweige in der Europäischen Gemeinschaft, Rev. 1.1 (NACE Rev. 1.1, 2002) Methodological Introduction, URL: http://ec.europa.eu/eurostat/ramon/ documents/nace_1_1/doc/intro_de.zip, 15.2. 2007

Eurostat; (Unternehmensdemografie, 2006), Statistiken zur Unternehmensdemografie - Länderspezifische Hinweise zur Methodik, URL: http://europa. eu.int /estatref/info/sdds/de/bd/bd_countries_de.pdf, 15.2.2007

Fischer-Winkelmann, W. (Hrsg.); (Management-Consulting, 2003), MC - Management-Consulting \& Controlling, Hamburg

Forster, K.H./Gierdeler, R./Lanfermann, J./Müller, H.P./Siepe, G./Stolberg, K. (Hrsg.); (ADS, 1994), Rechnungslegung und Prüfung der UnternehmenKommentar zum HGB, AktG, GmbHG, PublG nach den Vorschriften des Bilanzrichtlinien-Gesetzes, Aller/Düring/Schmaltz, Stuttgart

Fox, W.F./Murray, M.N./Luna, L.; (NTJ, 2005), How Should a Subnational Corporate Income Tax on Multistate Business Be Structured, in: National Tax Journal, S. 139-159

Frank, R.; (Option, 2002), Die Option der Personengesellschaften, sich wie Kapitalgesellschaften besteuern zu lassen, Klagenfurt

Fromm, S.; (Bamberger Beiträge zur empirischen Sozialforschung, 2005, 11), Binäre logistische Regressionsanalyse - Eine Einführung für Sozialwissenschaftler mit SPSS für Windows, in: Bamberger Beiträge zur empirischen Sozialforschung, Nr. 11, S. 1-35

Fuest, C.; (Coordination, 2002), Corporate Tax Coordination in the European Internal Market and the Problem of ", Harmful Tax competition", Working Paper Universität Köln, Köln 
Fuest, C./Hemmelgarn, T./Ramb, F.; (Apportionment, 2006), How would formula apportionment in the EU affect the distribution and the size of the corporate tax base? An analysis based on German multinationals, Deutsche Bundesbank Discussion Paper, Frankfurt am Main

Fuest, C./Huber, B.; (Vierteljahrhefte zur Wirtschaftsforschung, 2003), Zur Koordinierung der Unternehmensbesteuerung in Europa, in: Vierteljahrhefte zur Wirtschaftsforschung, S. 378-390

Fuest, W.; (Steuerharmonisierung, 2006), Steuerharmonisierung und Steuerwettbewerb - Zur Unternehmensbesteuerung in der Europäischen Union, Köln

Gammie, M./Radaelli, C.M./Klemm, A.; (Reform, 2001), EU Corporate Tax Reform-Report of a CEPS Task Force, Brüssel

Gammie, M./Giannini, S./Klemm, A./Oestreicher, A./Parascandolo, P./Spengel, C.; (Achieving, 2005), Achieving a Common Consolidated Tax Base in the EU-Report of a CEPS Task Force, Brüssel

Gassner, W.; (ET, 2002), Company Taxation in the Internal Market, in: European Taxation, S. 317-321

Giannini, S.; (CESifo Forum, 2002, 1), Home State Taxation vs. Common Base Taxation, in: CESifo Forum, Nr. 1, S. 24-30

Görg, H./Greenaway, D.; (FDI, 2002), Is there a potential for increase in FDI for Central and Eastern European countries following EU accession?, Research Paper 2002/31, University of Nottingham

Göthlich, S.; (Albers et al., Forschung, 2006), Zum Umgang mit fehlenden Daten in großzahligen empirischen Erhebungen, in: J. Albers/D. Klapper/U. Konradt/A. Walter/J. Wolf (Hrsg.), Methodik der empirischen Forschung, Wiesbaden, S. 133-150

Goolsbee, A./Maydew, E.L.; (JoPE, 2000), Coveting thy neighbor's manufacturing: the dilemma of state income apportionment, in: Journal of Public Economics, S. 125-143

Gordon, R./Wilson, J.D.; (Econometrica, 1986), An Examination of Multi-Jurisdictional Corporate Income Taxation Under Formula Apportionment, in: Econometrica, S. 1357-1373

Görg, H./Greenaway, D.; (Herrmann/Lipsey, Direct Investment, 2003), Is there a Potential for Increases in FDI for Central and Eastern European Countries Following EU Accession?, in: H. Herrmann/R. Lipsey (Hrsg.), Foreign Direct Investment in the Real and Financial Sector of Industrial Countries, Heidelberg, S. 165-183

Gouthière, B. (Hrsg.); (ET, 2005), A Comparative Study of the Thin Capitalization Rules in the Member States of the European Union and Certain Other States, in: European Taxation, S. 367-451 
Greulich, M.; (WuS, 2004), Revision von Wirtschaftsklassifikationen bis 2007 ein Zwischenbericht, in: Wirtschaft und Statistik, S. 381-390

Grotherr, S.; (StuW, 1996), Die unterschiedlichen Körperschaftsteuersysteme in den Mitgliedstaaten der Europäischen Union - Eine steuersystematische Analyse im Hinblick auf Reformüberlegungen beim steuerlichen Organkonzept, in: Steuern und Wirtschaft, S. 356-378

Grundy, M.; (Offshore, 1997), Offshore Business Centres: A World Survey, London

Gujarati, D.N.; (Econometrics, 2003), Basic Econometrics, 4. Aufl., Boston

Gundel, G.; (Schaumburg, Konzern, 1998), Steuergestaltungen bei der Einschaltung internationalen Finanzierungsgesellschaften in die Finanzierung deutscher internationaler Konzerne, in: H. Schaumburg (Hrsg.), Steuerrecht und steuerorientierte Gestaltungen im Konzern, Köln, S. 131-173

Günkel, M.; (WPg, 2003), Standortwahl für eine europäische Holdinggesellschaft, in: Die Wirtschaftsprüfung, Sonderheft, S. S40-S56

Hagen, A.; (indirekte Steuern, 2000), Harmonisierung der indirekten Steuern in Europa - Grenzen und Umfang unter besonderer Berücksichtigung von Art. 33 der 6. MWSt-RL und Art. 3 der System-RL, Frankfurt am Main, Berlin, Bern, Brüssel, New York, Oxford, Wien

Hahn, H.; (IStR, 1999), Das ICI-Urteil des EuGH und die Hinzurechnungsbesteuerung gemäß $\S \oint 7$ ff. AStG, in: Internationales Steuerrecht, S. 609617

Hahner, A.; (WIRO, 2002), Einkommensteuerrecht in Estland, in: Wirtschaft und Recht in Osteuropa, S. 143-145

Harvard Note; (HLR, 1976), Multinational Corporations and Income Allocation under Section 482 of the Internal Revenue Code, in: Harvard Law Review, S. 1202-1238

Hasse, R./Schenk, K.-E./Graf Wass von Czege, A. (Hrsg.); (Wettbewerb, 2002), Europa zwischen Wettbewerb und Harmonisierung, Baden-Baden

Hebig, M./Kaiser, K./Koschmieder, K./Oblau, M. (Hrsg.); (Entwicklungsaspekte, 2006), Aktuelle Entwicklungsaspekte der Unternehmensbesteuerung, Berlin

Heiduk, G.; (Außenwirtschaft, 2005), Außenwirtschaft - Theorie, Empirie und Politik der interdependenten Weltwirtschaft, Heidelberg.

Heiduk, G./Kerlen-Prinz, J.; (Döhrn/Heiduk, Direktinvestitionen, 1999), Direktinvestitionen in der Außenwirtschaftstheorie, in: R. Döhrn/G. Heiduk (Hrsg.), Theorie und Empirie der Direktinvestitionen, Berlin, S. 23-54

Hellerstein, J.R.; (NTJ, 1968), Recent Developments in State Tax Apportionment and the Circumstances of Unitary Business, in: National Tax Journal, S. 487-503 
Hellerstein, J.R.; (TN, 1982), Allocation and Apportionment of Dividends and the Delineation of the Unitary Business, in: Tax Notes, S. 155-168

Hellerstein, W.; (STN, 2001), The Business/Non-Business Income Distinction and the Case for Its Abolition, in: State Tax Notes, S. 725-739

Hellerstein, W./McLure Jr., C.E.; (BIFD, 2004), Lost in Translation: Contextual Considerations in Evaluating the Relevance of US Experience for the European Commission's Company Taxation Proposal, in: Bulletin for International Fiscal Documentation, S. 86-98

Hellerstein, W./McLure Jr., C.E.; (ITPF, 2004), The European Commission's Report on company Income Taxation: What the EU Can Learn from the Experience of the US States, in: International Tax and Public Finance, S. $199-220$

Helminen, M.; (intertax, 2005), Is There a Future for CFC-regimes in the EU?, in: intertax, S. 117-123

Hernler, J.; (DB, 2003), ETAS - European tax allocation system, ein Lösungsvorschlag für eine einheitliche Ertragsbesteuerung europäischer Unternehmen, in: Der Betrieb, S. 60-65

Hernler, J.; (ET, 2004), European Tax Allocation System (ETAS): A Proposal for a Consolidated European Tax System, in: European Taxation, S. 246251

Herrmann, H./Lipsey, R. (Hrsg.); (Direct Investment, 2003), Foreign Direct Investment in the Real and Financial Sector of Industrial Countries, Heidelberg

Herzig, N. (Hrsg.); (Organschaft, 2003), Organschaft: laufende und aperiodische Besteuerung, nationale und internationale Aspekte, Stuttgart

Herzig, N.; (IAS/IFRS, 2004), IAS/IFRS und steuerliche Gewinnermittlung, Düsseldorf

Herzig, N.; (WPg, 2005), IAS/IFRS und steuerliche Gewinnermittlung, in: Die Wirtschaftsprüfung, S. 211-235

Herzig, N.; (Endres et al., Wandel, 2005), Maßgeblichkeit der IAS/IFRS oder eigenständige steuerrechtliche Gewinnermittlung?, in: D. Endres/A. Oestreicher/W. Scheffler/U. Schreiber/C. Spengel (Hrsg.), Die internationale Unternehmensbesteuerung im Wandel, München, S. 127-135

Herzig, N.; (Hebig et al., Entwicklungsaspekte, 2006), Die steuerliche Gewinnermittlung in der Europäischen Union, in: M. Hebig/K. Kaiser/K. Koschmieder/M. Oblau (Hrsg.), Aktuelle Entwicklungsaspekte der Unternehmensbesteuerung, Berlin, S. 265-282

Herzig, N.; (DB, 2006), Reform der Unternehmensbesteuerung - Zwischenbericht zum Konzept der Stiftung Marktwirtschaft, in: Der Betrieb, S. 1-7

Herzig, N.; (IStR, 2006), Steuerliche Gewinnermittlung und handelsrechtliche

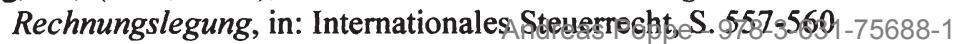


Herzig, N./Wagner, T.; (DB, 2005), Zukunft der Organschaft im EGBinnenmarkt, in: Der Betrieb, S. 1-9

Herzig, N./Englisch, J./Wagner, T.; (Der Konzern, 2005), Steuerliche Berücksichtigung von Verlusten ausländischer Konzerntöchter - Überlegungen nach den Schlussanträgen in der Rechtssache Marks \& Spencer, in: Der Konzern, S. 298-318

Herzig, N./Wagner, T.; (DStR, 2006), EuGH-Urteil „,Marks \& Spencer“-Begrenzter Zwang zur Öffnung nationaler Gruppenbesteuerungssysteme für grenzüberschreitende Sachverhalte, in: Deutsches Steuerrecht, S. 1-11

Hey, J.; (Harmonisierung, 1997), Harmonisierung der Unternehmensbesteuerung in Europa, Köln

Hey, J.; (StuW, 2004), Perspektiven der Unternehmensbesteuerung in Europa, in: Steuern und Wirtschaft, S. 193-211

Hickman, A.; (TPITP, 2005, 11), European Union Transfer Pricing Documentation (, E.U. TPD“) - BSF or JAA?, in: Tax Planning International Transfer Pricing, Nr. 11, S. 8-10

Higinbotham, H.N./Asper, D.W./Stoffregen, P.A./Raymond, P.W.; (TLR, 1987), Effective Application of the Section 482 Transfer Pricing Regulations, in: Tax Law Review, S. 295-379

Hirschler, K./Schindler, C.; (IStR, 2004), Die österreichische Gruppenbesteuerung als Vorbild für Europa?, in: Internationales Steuerrecht, S. 505-512

Hitz, J.-M.; (WPg, 2005), Fair value in der IFRS-Rechnungslegung Konzeption, Inhalt und Zweckmäßigkeit, in: Die Wirtschaftsprüfung, S. 1013-1027

Hoffmann, F. (Hrsg.), (Konzernhandbuch, 1993), Konzernhandbuch - Recht Steuern - Rechnungslegung - Führung - Organisation - Praxisfälle, Wiesbaden

Hörnschemeyer, A.; (GmbHR, 2005), Gestaltungsüberlegungen zur internationalen Rechtsformwahl in der EU nach der Änderung der MutterTochter-Richtlinie, in: GmbH-Rundschau, S. 1397-1409

Homburg, S.; (Endres et al., Wandel, 2005), Internationale Kapitaleinkommensbesteuerung nach dem Wohnsitzprinzip oder dem Quellenprinzip?, in: D. Endres/A. Oestreicher/W. Scheffler/U. Schreiber/C. Spengel (Hrsg.), Die internationale Unternehmensbesteuerung im Wandel, München, S. 14-27

Hosmer, D./Lemeshow, S.; (Regression, 1989), Applied logistic regression, New York

House of Representatives; (Report, 1964), State Taxation of Interstate Commerce, Report of the Special Subcommittee on State Taxation of Interstate Commerce, H.R. Rep. No. 1480, 88th Cong., 2d Sess. 
Hrehorovska, L.; (intertax, 2006), Tax Harmonisation in the European Union, in: intertax, S. 158-166

Hübl, L.; (DStZ, 1965), Die Organtheorien im Körperschaftsteuerrecht, in: Deutsche Steuerzeitung, S. 17-25

Jacobs, O.H. (Hrsg.); (Besteuerung, 1983), Internationale Unternehmensbesteuerung, 1. Aufl., München

Jacobs, O.H. (Hrsg.); (Bilanzanalyse, 1994), Bilanzanalyse - EDV-gestützte Jahresabschlussanalyse als Planungs- und Entscheidungsrechnung, 2. neu bearbeitete und erweiterte Aufl., München

Jacobs, O.H. (Hrsg.); (Unternehmensbesteuerung, 2002), Internationale Unternehmensbesteuerung, 5. Aufl., München

Jacobs, O.H./Spengel, C./Schäfer, A.; (intertax, 2004), ICT and Profit Allocation within Multinational Groups, in: intertax, S. 268-283

Jacobs, O.H./Spengel, C./Stetter, T./Wendt, C.; (intertax, 2005), EU Company Taxation in Case of a Common Tax Base: A Computer-based Calculation and Comparison Using the Enhanced Model of the European Tax Analyser,in: intertax, S. 414-428

Jensen, J./Svensson, P.; (Cooperation, 2004), Economic effects of tax cooperation in an enlarged European Union, Kopenhagen

Jie-A-Joen, C./Weenink, M.; (TPITP, 2005, 12), E.U. Commission Issues Proposal for Code of Conduct on Transfer Pricing Documentation, in: Tax Planning International Transfer Pricing, Nr. 12, S. 11-13

Jurkat, W.; (Organschaft, 1975), Die Organschaft im Körperschaftsteuerrecht, Heidelberg

Keller, T.; (Holdingkonzepte, 1990), Unternehmensführung mit Holdingkonzepten, Köln

Kellersmann, D./Treisch, C.; (Europäische Unternehmensbesteuerung, 2002), Europäische Unternehmensbesteuerung, Wiesbaden

Kessler, W.; (Euro-Holding, 1996), Die Euro-Holding - Steuerplanung, Standortwahl, Länderprofile, München

Kessler, W./Kröner, M./Köhler, S. (Hrsg.); (Konzernsteuerrecht, 2004), Konzernsteuerrecht, Organisation - Recht - Steuern, München

Kessler, W./Daller, R.; (IStR, 2005), Dänemark: Verschärfung der Gruppenbesteuerung, in: Internationales Steuerrecht, Beihefter 15, S. 2*-3*

Kesti, J. (Hrsg.); (Tax Handbook, 2003), European Tax Handbook, Amsterdam

Kilches, R.; (FJ, 2001), Künftige Steuerpolitik der Gemeinschaft, in: FinanzJournal, S. 173-175

Kinzel, U.-P.; (BB, 2005), Änderungen der Fusionsrichtlinie: Warten auf den $E u G H$, in: Betriebs-Berater, S. 971-975 
Klemm, R.J./Dwyer, D.W./Brewer, T.L.; (GFJ, 1995), Determining appropriate international transfer prices: Economic and administrative rationales for using asset-based profit splits under section 482 of the U.S. tax code, in: Global Finance Journal, S. 111-119

König, R./Wosnitza, M.; (Steuerwirkungslehre, 2004), Betriebswirtschaftliche Steuerplanungs- und Steuerwirkungslehre, Heidelberg

Krause-Jung, G.; (ifo Schnelldienst, 2002, 2), Besteuerung multinationaler Unternehmen - wie sind die Vorschläge der EU-Kommission zu bewerten?, in: ifo Schnelldienst, Nr. 2, S. 3-5

Kromer, C.; (Kessler/Kröner/Köhler, Konzernsteuerrecht, 2004), Konzerninterner Lieferungs- und Leistungsverkehr, in: W. Kessler/M. Kröner/S. Köhler (Hrsg.), Konzernsteuerrecht, Organisation - Recht Steuern, München, S. 263-321

Küting, K./Weber, C.-P.; (Konzernabschluss, 2006), Der Konzernabschluss, 10. Aufl., Stuttgart

Kuiper, G.W.; (ET, 1988), EC Commission proposes a directive on the harmonization of rules for the determination of taxable profits of enterprises, in: European Taxation, S. 319-329

Kußmaul, H./Schäfer, R.; (IstR, 2000), Die Option von Personengesellschaften für eine Besteuerung durch die Körperschaftsteuer im französischen Steuerrecht - Voraussetzungen und steuerliche Wirkungen im Optionszeitpunkt, in: Internationales Steuerrecht, S. 161-166

Lang, J.; (GmbHR, 2000), Die Unternehmenssteuerreform - eine Reform pro $\mathrm{GmbH}$, in: GmbH-Rundschau, S. 453-462

Lang, M.; (IStR, 2006), Gemeinschaftsrechtliche Verpflichtung zur Rechtsformneutralität im Steuerrecht?, in: Internationales Steuerrecht, S. 397-402

Lang, M./Loukota, H./Reich, M./Wassermeyer, F./Zorn, N.; (SWI, 2002), Konsequenzen der neuen Judikatur des Verwaltungsgerichtshofs zu den Auslandsverlusten - Podiumsdiskussion, in: Steuern und Wirtschaft International, S. 428-440

Lannoo, K./Levin, M.; (EU Tax, 2002), An EU Company without an EU Tax? A Corporate Tax Action Plan for Advancing the Lisbon Process, Brüssel

Lehis, L.; (IStR 2001), Die Einkommensteuerreform in Estland - Steuerfreiheit thesaurierter Gewinne, in: Internationales Steuerrecht, S. 707-713

Lehner, M.; (ifo Schnelldienst, 2002, 2), Besteuerung multinationaler Unternehmen - wie sind die Vorschläge der EU-Kommission zu bewerten?, in: ifo Schnelldienst, Nr. 2, S. 6-8

Lettl, T.; (DStR, 1996), Das Holding-Konzept als Instrument zur erfolgreichen Neuausrichtung von Unternehmen, in: Deutsches Steuerrecht, S. 2020-2026 
Lettl, T.; (DStR, 1997), Betriebswirtschaftliche Vor- und Nachteile bzw. Gefahren der Unternehmensorganisation in Form der Holding-Struktur, in: Deutsches Steuerrecht, S. 1016-1020

Levin, M.; (Harmonising, 2003), Harmonising corporate tax bases in the EU, Prospectus for CEPS Task Force, URL: http://www.ceps.be/files/TF/ ProspectusTFTaxHarmo.doc, 15. 2. 2007

Li, J.; (CTJ, 2002), Global Profit Split: An Evolutionary Approach to International Income Allocation, in: Canadian Tax Journal, S. 823-883

Lipponer, A.; (Herrmann/Lipsey, Direct Investment, 2003), $A$ "new" Micro Database for German FDI, in: H. Herrmann/R. Lipsey (Hrsg.), Foreign Direct Investment in the Real and Financial Sector of Industrial Countries, Heidelberg, S. 215-244

Lodin, S.-O./Gammie, M.; (ET, 1999), The Taxation of the European Company, in: European Taxation, S. 286-294

Lodin, S.-O./Gammie, M.; (Home, 2001), Home state taxation - tax treaty aspects, Amsterdam

Lüdemann, P./Hruschka, F.; (IStR, 2000), Die Behandlung von Personengesellschaften im Verhältnis Deutschland - Spanien, in: Internationales Steuerrecht, S. 25-31

Lüdicke, J. (Hrsg.); (Steuerrecht, 2004), Deutsches Steuerrecht im europäischen Rahmen, Köln

Lühn, A.; (Rechtsformwahl, 2004) Rechtsformwahl im nationalen und transnationalen Konzern, Herne/Berlin

Lutter, M. (Hrsg.); (Holding, 2004), Holding Handbuch, 4. Aufl., Köln

Lutter, M.; (Lutter, Holding, 2004), Begriff und Erscheinungsformen der Holding, in: M. Lutter (Hrsg.), Holding Handbuch, 4. Aufl., Köln, S. 1-29

Luttermann, C.; (IStR, 1994), Unitary Taxation und U.S. Supreme Court, in: Internationales Steuerrecht, S. 489-493

Mai, H.; (WuS, 1991), Die neue europäische Wirtschaftszweigsystematik, in: Wirtschaft und Statistik, S. 7-15

Matiaske, W./Mellewigt, T. (Hrsg.); (Organisationsforschung, 2000), Empirische Organisations- und Entscheidungsforschung - Ansätze, Befunde, Methoden, Heidelberg

Matiaske, W./Mellewigt, T.; (Matiaske/Mellewigt, Organisationsforschung, 2000), Empirische Organisationsforschung - State of the Art und künftiger Forschungsbedarf, in: W. Matiaske/T. Mellewigt (Hrsg.), Empirische Organisations- und Entscheidungsforschung - Ansätze, Befunde, Methoden, Heidelberg, S. 9-25

Maiterth, R.; (DStR, 2006), Das EuGH-Urteil „Marks \& Spencer" und die grenzüberschreitende Verlustverrechnung aus ökonomischer Sicht, in: Deutsches Steuerrecht, S. 915-919 Andreas Poppe - 978-3-631-75688-1 
Mazerov, M.; (Loopholes, 2003), Closing Three Common Corporate Income Tax Loopholes Could Raise Additional Revenue for Many States, Center on Budget and Policy Priorities, Washington D.C.

McDaniel, P.R.; (TLR, 1994), Formulary Taxation in the North American Free Trade Zone, in: Tax Law Review, S. 691-744

McKinsey Global Institute; (Productivity, 1996), Capital Productivity, Washington D.C.

McLure Jr., C.E.; (Aaron/Boskin, Economics, 1980), The State Corporate Income Tax: Lambs in Wolves' Clothing, in: H.J. Aaron/M.J. Boskin (Hrsg.), The Economics of Taxation, Washington D.C., S. 327-346

McLure Jr., C.E. (Hrsg.); (State, 1984), The State Corporate Income Tax - Issues in Worldwide Unitary Combination, Stanford

McLure Jr., C.E.; (McLure, State, 1984), Defining a Unitary Business, in: C.E. McLure Jr. (Hrsg.), The State Corporate Income Tax - Issues in Worldwide Unitary Combination, Stanford, S. 228-246

McLure Jr., C.E.; (TN, 1986), State Taxation of Foreign-Source Dividends: Starting from First Principles, in: Tax Notes, S. 975-989

McLure Jr., C.E.; (BIFD, 2002), Replacing Separate Entity Accounting and the Arm's Length Principle with Formulary Apportionment, in: Bulletin for International Fiscal Documentation, S. 586-599

McLure Jr., C.E.; (TNI, 2004), Corporate Tax Harmonization in the European Union: The Commission's Proposals, in: Tax Notes International, S. 45-69

McLure Jr., C.E.; (CESifo Forum, 2005, 1), The European Commission Proposals for Corporate Tax Harmonization, in: CESifo Forum, Nr. 1, S. 32-41

McLure Jr., C.E.,/Weiner, J.M.; (Cnossen, Capital, 2000), Deciding whether the European Union should adopt formula apportionment of company income, in: S. Cnossen (Hrsg.), Taxing Capital Income in the European Union: Issues and Options for Reform, Oxford, S. 243-292

Miller, B.F.; (McLure, State, 1984), Worldwide Unitary Combination: The California Practice, in: C.E. McLure Jr. (Hrsg.), The State Corporate Income Tax - Issues in Worldwide Unitary Combination, Stanford, S. 132-166

Mintz, J.M.; (FA, 1999), Globalization of the Corporate Income Tax: The Role of Allocation, in: Finanzarchiv, S. 389-423

Mintz, J.M.; (CESifo Forum, 2002, 1), Company Taxation and the Internal Market, in: CESifo Forum, Nr. 1, S. 3-9

Mintz, J.M.; (ITPF, 2004), Corporate Tax Harmonisation in Europe: It's All About Compliance, in: International Tax and Public Finance, S. 221-234

Mintz, J.M./Weiner, J.M.; (ITPF, 2003), Exploring Formula Allocation for the European Union, in: International Tax and Public Finance, S. 695-711 
Mors, M.; (TNI, 2002); eForum: Company Taxation in the European Union, in: Tax Notes International, S. 153-174

Mors, M.; (CESifo Forum, 2002, 1), Roundtable Discussion on the European Commission's Study on Company Taxation, in: CESifo Forum, Nr. 1, S. 21-23

Multistate Tax Commission; (Compact, 2003), The Multistate Tax Compact Suggested State Legislation and Enabling Act, Washington D.C.

Müller, W. (Hrsg.); (Systemwettbewerb, 2001), Regeln für den europäischen Systemwettbewerb - Steuern und soziale Sicherungssysteme, Marburg

Musgrave, P.B.; (Public Finance, 1972), International Tax Base Division and the Multinational Corporation, in: Public Finance, S. 394-413

Musgrave, P.B.; (McLure, State, 1984), Principles for Dividing the State Corporate Tax Base, in: C.E. McLure Jr. (Hrsg.), The State Corporate Income Tax - Issues in Worldwide Unitary Combination, Stanford, S. 228-246

Musgrave, R.A./Musgrave, P.B.; (Bird/Head, Fiscal, 1972), Inter-nation equity, in: R.M. Bird/J.G. Head (Hrsg.), Modern Fiscal Issues, Essays in Honor of Carl S. Shoup, Toronto, S. 63-85

Neumark, F. (Hrsg.); (Finanzwissenschaft, 1980), Handbuch der Finanzwissenschaft, Band II, 3. Aufl., Tübingen

OECD; (Guidelines, 2001), Transfer Pricing Guidelines for Multinational Enterprises and Tax Administrations 2001 Update, Paris

Oestreicher, A.; (Konzerngewinnabgrenzung, 1999), Konzerngewinnabgrenzung - Gewinnabgrenzung - Gewinnermittlung - Gewinnaufteilung, München

Oestreicher, A.; (StuW, 2002), Konzernbesteuerung in Europa-Zum Vorschlag einer konsolidierten körperschaftsteuerlichen Bemessungsgrundlage für die grenzüberschreitende Unternehmenstätigkeit in der EU, in: Steuern und Wirtschaft, S. 342-356

Oestreicher, A.; (Handelsbilanzen, 2003), Handels- und Steuerbilanzen, HGB, IAS/IFRS, US-GAAP, EStG und BewG, 6. völlig neu konzipierte Aufl., Heidelberg

Oestreicher, A. (Hrsg.); (Verrechnungspreise, 2003), Internationale Verrechnungspreise - Beiträge zu einer Ringveranstaltung an der Universität Göttingen im Sommersemester 2002, Herne, Berlin

Oestreicher, A.; (Oestreicher, Verrechnungspreise, 2003), Entwicklung und Rechtfertigung des Arms's length principle im Rahmen der Gewinnabgrenzung internationaler Konzerne, in: A. Oestreicher (Hrsg.), Internationale Verrechnungspreise - Beiträge zu einer Ringveranstaltung an der Universität Göttingen im Sommersemester 2002, Herne, Berlin, S. 1-51 
Oestreicher, A.; (Endres et al., Wandel, 2005), Gewinnabgrenzung bei EUKonzernen auf der Basis von Verrechnungspreisen oder durch formelhafte Gewinnaufteilung, in: D. Endres/A. Oestreicher/W. Scheffler/U. Schreiber/C. Spengel (Hrsg.), Die internationale Unternehmensbesteuerung im Wandel, München, S. 73-81

Oestreicher, A. (Hrsg.); (Konzernbesteuerung, 2005), Konzernbesteuerung Beiträge zu einer Ringveranstaltung an der Universität Göttingen im Sommersemester 2004, Herne, Berlin

Oestreicher, A.; (Oestreicher, Konzernbesteuerung, 2005), Konzernbesteuerung im Spannungsfeld zwischen wirtschaftlicher Einheit und rechtlicher Vielheit, in: A. Oestreicher (Hrsg.), Konzernbesteuerung - Beiträge zu einer Ringveranstaltung an der Universität Göttingen im Sommersemester 2004, Herne, Berlin, S. 1-31

Oestreicher, A.; (Hebig et al., Entwicklungsaspekte, 2006), Vergleichende Analyse der Steuerbemessungsgrundlagen in Europa, in: M. Hebig/K. Kaiser/K. Koschmieder/M. Oblau (Hrsg.), Aktuelle Entwicklungsaspekte der Unternehmensbesteuerung, Berlin, S. 283-291

Oestreicher, A./Koch, R.; (Organschaft, 2007), Steuerliche Bedeutung und empirische Relevanz der ertragsteuerlichen Organschaft, Working Paper, Universität Göttingen, Göttingen

Oestreicher, A./Spengel, C.; (Maßgeblichkeit, 1999), Maßgeblichkeit der International Accounting Standards für die steuerliche Gewinnermittlung?, Baden-Baden

Oestreicher, A./Scheffler, W./Spengel, C./Wellisch, D.; (Weiterentwicklung, 2006), Weiterentwicklung der Konzernbesteuerung in Deutschland und Europa, unveröffentlichter Bericht eines Dienstleistungsauftrags des Bundesministeriums der Finanzen

Oppermann, T.; (Europarecht, 2005), Europarecht, München

Orth, M.; (Oestreicher, Konzernbesteuerung, 2005), Die Bedeutung des Gewinnabführungsvertrags für die Besteuerung des Konzernerfolgs, in: A. Oestreicher (Hrsg.), Konzernbesteuerung - Beiträge zu einer Ringveranstaltung an der Universität Göttingen im Sommersemester 2004, Herne, Berlin, S. 129-195

Osterweil, E.; (ET, 2002), Reform of Company Taxation in the EU Internal Market, in: European Taxation, S. 271-275

Ottosen, A./Nørremark, M.; (BIFD, 2005), Denmark: New Joint Taxation Regime, Territoriality Principle and Reduction of the Corporate Tax Rate, in: Bulletin for International Fiscal Documentation, S. 471-475

Paetsch, R.; (Verlustberücksichtigung, 2004), Grenzüberschreitende Verlustberücksichtigung im Europäischen Binnenmarkt, Köln 
Panayi, C.; (ET, 2006), Treaty Shopping and Other Tax Arbitrage Opportunities in the European Union: A Reassessment, in: European Taxation, S. 104110 (Teil I) und 2006, S. 139-155 (Teil II)

Peichl, A.; (Finanzwissenschaftlichen Diskussionsbeiträge, 2005, 1), Die Evaluation von Steuerreformen durch Mikrosimulationsmodelle, in: Finanzwissenschaftliche Diskussionsbeiträge, Nr. 1, 2005

Pellens, B./Fülbier, R.U./Gassen, J.; (Rechnungslegung, 2004), Internationale Rechnungslegung - IFRS/IAS mit Beispielen und Fallstudien, 5. überarbeitete und erweiterte Aufl., Stuttgart

Peters, W.; (Holdinggesellschaft, 1998), Die Besteuerung von internationalen Holdinggesellschaften unter volkswirtschaftlichen Aspekten - dargestellt unter Bezugnahme auf jüngere deutsche Steuerrechtsänderungen, Hamburg

Plasschaert, S.; (ET, 1997), An EU tax on the consolidated profits of multinational enterprises, in: European Taxation, S. 2-13

Plasschaert, S.; (ET, 2002), Further Thoughts on the "European Union Company Income Tax" and its First Cousins, in: European Taxation, S. 336-345

Plasschaert, S.; (Income Tax, 2002), The EU consolidated Income Tax revisited, CESifo Working Paper, München

Plasschaert, S.; (ET, 2004), Towards an Own Tax Resource for the European Union? Why? How? And When?, in: European Taxation, S. 470-479

Potthof, A.; (Finanzierung, 1998), Finanzierung ausländischer Unternehmenseinheiten - Steuerliche Aspekte von Finanzierungsgesellschaften, Wiesbaden

Rädler, A.; (TNI, 2002); eForum: Company Taxation in the European Union, in: Tax Notes International, S. 153-174

Radulescu, D.M.; (ifo Schnelldienst, 2002, 2), Besteuerung von Kapitalgesellschaften im Binnenmarkt - Reformvorschlag der Europäischen Kommission, in: ifo Schnelldienst, Nr. 2, S. 12-17

Redig, K./Müller, W.; (Steuerlehre, 1999), Einführung in die Allgemeine Steuerlehre, München

Reinhardt, F.; (Erfolgsabgrenzung, 2003), Erfolgsabgrenzung im Global Trading: Ein Beispiel für die Gewinnabgrenzung in unvollkommenen Märkten, Hamburg

Richard, J.; (intertax, 2003), Comparison between UK and French Taxation of Groups of Companies, in: intertax, S. 20-39

Rickmann, J.; (TNI, 2006), Tax Commissioner Kovács Doubts Prospects of Tax to Fund EU, in: Tax Notes International, S. 959 
Riecker, A.; (Körperschaftbesteuerung, 1996), Körperschaftbesteuerung in der Europäischen Union und das US-amerikanische Modell der Unitary Taxation, Konstanz

Romani, B./Strnad, O./Grabbe, C.; (IStR, 2004), Italien - Große Steuerreform $a b$ 2004, in: Internationales Steuerrecht, S. 155-161

Ruding, O.; (ET, 2002), The Long Way to Removing Obstacles in Company Taxation in Europe, in: European Taxation, S. 3-5

Ruding, O.; (EC Tax Review, 2005), The past and the future of EU corporate tax, in: EC Tax Review, S. 2-3

Sánchez, G.E./Fluxà, J.F.; (ET, 2006), Problems and Options in Calculating the Tax Base of Companies in the European Union under Home State Taxation, in: European Taxation, S. 197-207

Schäfer, A./Spengel, C.; (ICT, 2003), The Impact of ICT on Profit Allocation within Multinational Groups: Arm's Length Pricing or Formulary Apportionment?, ZEW Discussion Paper, Mannheim

Schaumburg, H. (Hrsg.); (Konzern, 1998), Steuerrecht und steuerorientierte Gestaltungen im Konzern, Köln

Scheffler, E.; (Lutter, Holding, 2004), Vor- und Nachteile der Holding, in: M. Lutter (Hrsg.), Holding Handbuch, 4. Aufl., Köln, S. 30-42

Scheffler, W.; (DBW, 1991), Grundzüge der Besteuerung von inländischen Konzernen, in: Die Betriebswirtschaft, S. 701-717

Scheffler, W.; (Fischer-Winkelmann, Management-Consulting, 2003), Die inländische Auslandsholding als Steuerplanungsinstrument für mittelständische Unternehmen, in: W. Fischer-Winkelmann (Hrsg.), MC Management-Consulting \& Controlling, Hamburg, S. 447-478

Scheffler, W.; (Bank/Schiller, Finanzintermediation, 2004), Möglichkeiten der Steuerplanung durch Errichtung einer Finanzierungsgesellschaft, in: M. Bank, B. Schiller (Hrsg.), Finanzintermediation - Theoretische, wirtschaftspolitische und praktische Aspekte aktueller Entwicklungen im Bank- und Börsenwesen, Stuttgart, S. 507-528

Scheffler, W.; (BFuP, 2005), Grenzüberschreitende Verlustverrechnung Nachversteuerungsmethode im Vergleich zum geltenden Recht und zur konsolidierten Körperschaftsteuer-Bemessungsgrundlage, in: Betriebswirtschaftliche Forschung und Praxis, S. 156-175

Scheffler, W.; (Endres et al., Wandel, 2005), Maßgeblichkeit der LAS/IFRS oder eigenständige steuerrechtliche Gewinnermittlung?, in: D. Endres/A. Oestreicher/W. Scheffler/U. Schreiber/C. Spengel (Hrsg.), Die internationale Unternehmensbesteuerung im Wandel, München, S. 105-109 
Scheffler, W.; (Oestreicher, Konzernbesteuerung, 2005), Gemeinsame konsolidierte Steuerbemessungsgrundlage (CCTB) in der EU - Das Ende der Steuerplanung mit Verrechnungspreisen?, in: A. Oestreicher (Hrsg.), Konzernbesteuerung - Beiträge zu einer Ringveranstaltung an der Universität Göttingen im Sommersemester 2004, Herne, Berlin, S. 305-331, Scheffler, W.; (Unternehmen, 2006), Besteuerung von Unternehmen I Ertrags-, Substanz- und Verkehrssteuern, Heidelberg, München, Landsberg, Berlin

Scherer, A.G.; (Globalisierung, 2003), Multinationale Unternehmen und Globalisierung: Zur Neuorientierung der Theorie der Multinationalen Unternehmung, Heidelberg

Scheuchzer, M.; (Konzernbesteuerung, 1994), Konzernbesteuerung in der Europäischen Union, Bielefeld

Scheunemann, M.; (Konzernbesteuerung, 2005), Grenzüberschreitende konsolidierte Konzernbesteuerung, Paris, Osnabrück

Scheunemann, M.; (IStR, 2006), Praktische Anforderungen einer grenzüberschreitenden Verlustberücksichtigung im Konzern in Inbound- und Outboundfällen nach der Entscheidung Marks \& Spencer, in: Internationales Steuerrecht, S. 145-154

Schipporeit, E.; (StuW, 1980), Ziele und Möglichkeiten einer Unternehmungsteuer: Grundzüge eines Reformvorschlags, in: Steuern und Wirtschaft, S. 190-199

Schmalwasser, O.; (WuS, 2001), Revision der Anlagevermögensrechnung 1991 bis 2001, in: Wirtschaft und Statistik, S. 342-356

Schneider, D.; (StuW, 1975), Körperschaftsteuerreform und Gleichmäßigkeit der Besteuerung, in: Steuern und Wirtschaft, S. 97-112

Schneider, D.; (Neumark, Finanzwissenschaft, 1980), Körperschaftsteuer, in: F. Neumark (Hrsg.), Handbuch der Finanzwissenschaft, Band II, 3. Aufl., Tübingen, S. 509-563

Schneider, D.; (Grundzüge, 1994), Grundzüge der Unternehmensbesteuerung, 6. Aufl., Wiesbaden

Schneider, D.; (BB, 2003), Konzernrechnungslegung nach IAS als Besteuerungsgrundlage, in: Betriebs-Berater, S. 299-304

Schnorberger, S./Rosenkranz, J./Garcia, M.; (intertax, 2006), Transfer Pricing Documentation: The EU Code of Conduct Compared with Member States Rules, in: intertax, S. 305-313 (Teil I) und S. 406-417 (Teil II)

Schön, W.; (ET, 2002), The European Commission's Report on Company Taxation: Magic Formula for European Taxation?, in: European Taxation, S. 276-286 
Schön, W.; (ET, 2004), International Accounting Standards - A „Starting Point" for a Common European Tax Base?, in: European Taxation, S. 426-440

Schreiber, U.; (StuW, 2004), Unternehmensbesteuerung im Binnenmarkt. Angleichung der Gewinnermittlung und des Satzes der Körperschaftsteuer?, in: Steuern und Wirtschaft, S. 212-226

Selling, H.-J.; (IStR, 2000), Steuerharmonisierung im europäischen Binnenmarkt - Ein Überblick, in: Internationales Steuerrecht, S. 417-424

Sepp, J./Wrobel, R.; (Hasse/Schenk/Graf Wass von Czege, Wettbewerb, 2002), Das Steuersystem in Estland im Spannungsfeld zwischen Transformationserfordernissen und EU-Harmonisierung, in: R. Hasse/K.-E. Schenk/A. Graf Wass von Czege (Hrsg.), Europa zwischen Wettbewerb und Harmonisierung, Baden-Baden, S. 69-75

Shackelford, D./Slemrod, J.; (ITPF, 1998), The Revenue Consequences of Using Formula Apportionment to Calculate U.S. and Foreign Source Income: A Firm-Level Analysis, in: International Tax and Public Finance, S. 41-59

Sheffrin, S./Fulcher, J.; (McLure, State, 1984), Alternative Divisions of the Tax Base: How much is a Stake?, in: C.E. McLure Jr. (Hrsg.), The State Corporate Income Tax - Issues in Worldwide Unitary Combination, Stanford, S. $192-213$

Sievert, E.; (Konzernbesteuerung, 2006), Konzernbesteuerung in Deutschland und Europa - Ertragsteuerliche und betriebswirtschaftliche Analyse der europäischen Gruppenbesteuerungssysteme, Düsseldorf

Slaughter, M.; (Herrmann/Lipsey, Direct Investment, 2003), Host-Country Determninants of U.S. Foreign Direct Investment into Europe, in: $\mathrm{H}$. Herrmann/R. Lipsey (Hrsg.), Foreign Direct Investment in the Real and Financial Sector of Industrial Countries, Heidelberg, S. 7-23

Smith, E.H.; (CTJ, 1976), Allocating to Provinces the Taxable Income of Corporations: How the Federal-Provincial Allocation Rules Evolved, in: Canadian Tax Journal, S. 545-571

Sørensen, P.; (CESifo Forum, 2002, 1), To Harmonise or not to Harmonise?, in: CESifo Forum, Nr. 1, S. 31-35

Sørensen, P.; (ITPF, 2004), Company Tax Reform in the European Union, in: International Tax and Public Finance, S. 91-114

Spahn, P.B./Galler, H.P./Kaiser, H./Kassella, T./ Merz, J.; (Mikrosimulation, 1992), Mikrosimulation in der Steuerpolitik, Heidelberg

Spengel, C.; (IStR, 2003), International Accounting Standards und Unternehmensbesteuerung in der Europäischen Union, in: Internationales Steuerrecht, S. 29-36 (Teil I) und S. 67-72 (Teil II) 
Spengel, C.; (Unternehmensbesteuerung, 2003), Internationale Unternehmensbesteuerung in der Europäischen Union - Steuerwirkungsanalyse empirische Befunde - Reformüberlegungen, Düsseldorf

Spengel, C.; (ifo Schnelldienst, 2004, 13), Sollte die Unternehmensbesteuerung innerhalb der EU harmonisiert werden?, in: ifo Schnelldienst, Nr. 13, S. 3-6

Spengel, C.; (Lüdicke, Steuerrecht, 2004), Unternehmensbesteuerung in der EU - quo vadis?, in: J. Lüdicke (Hrsg.), Deutsches Steuerrecht im europäischen Rahmen, Köln, S. 109-155

Spengel, C.; (DB, 2006), IFRS als Ausgangspunkt der steuerlichen Gewinnermittlung in der Europäischen Union - Steuerbelastungskonsequenzen im Länder und Branchenvergleich, in: Der Betrieb, S. 681-687

Spengel, C./Braunagel, R.U.; (StuW, 2006), EU-Recht und Harmonisierung der Konzernbesteuerung in Europa, in: Steuern und Wirtschaft, S. 34-49

Statistisches Bundesamt; (Körperschaftsteuerstatistik, 2005), Körperschaftsteuerstatistik 2001, Wiesbaden

Stefaner, M./Weninger, P.; (SWI, 2005), Gruppenbesteuerung; Unbeschränkte Verwertung ausländischer Verluste durch Cross-Over-Kaskaden, in: Steuern Wirtschaft International, S. 133-141

Steuerreformkommission; (Gutachten, 1971), Gutachten der Steuerreformkommission, Schriftenreihe des BMF, Heft 17, Bonn

Thömmes, O./Rasch, S./Koch, T.; (ITPJ, 2006), Commission Proposal for Home State Taxation, in: International Transfer Pricing Journal, S. 117-123

Tipke, K.; (Steuerrechtsordnung, 2003), Die Steuerrechtsordnung, Köln

Treisch, C.; (Unternehmensbesteuerung, 2004), Europataugliche Ausgestaltung der Unternehmensbesteuerung - Anforderungen, Probleme und Lösungsmöglichkeiten, Wiesbaden

United Nations Conference on Trade and Development; (Investment Report, 2002), World Investment Report 2002 - Transnational Corporations and Export Competitiveness, New York

Union of Industrial and Employers' Confederations of Europe; (Memorandum, 2000), UNICE Memorandum on Cross-Border Company Taxation Obstacles in the Single Market, Brüssel

Urban, D.; (Logit-Analyse, 1993), Logit-Analyse - Statistische Verfahren zur Analyse von Modellen mit qualitativen Response-Variablen, Stuttgart, Jena, New York

Vodrazka, K.; (StuW, 1971), Die Körperschaftsbesteuerung der Kapitalgesellschaften - Argumente und Folgerungen, in: Steuern und Wirtschaft, S. 234-246 
Vormoor, C.; (Unternehmensdatenbanken, 2005), Die Eignung öffentlicher Unternehmensdatenbanken zur Bestimmung von Verrechnungspreisen im Rahmen der Einkunftsabgrenzung im internationalen Konzern, Hamburg

Wagenhofer, A.; (IRZ, 2006), Fair Value-Bewertung im IFRS-Abschluss und Bilanzanalyse, in: Zeitschrift für internationale Rechnungslegung, S. 31-37

Wagner, A.; (Gebührenlehre, 1890), Finanzwissenschaft - 2. Theil: Theorie der Besteuerung. Gebührenlehre und allgemeine Steuerlehre, Leipzig

Wagner, F.; (StuW, 2006), Was bedeutet und wozu dient Rechtsformneutralität der Unternehmensbesteuerung, in: Steuern und Wirtschaft, S. 101-114

Wassermey, F.; (Wassermery/Pilz, Außensteuergesetz, 2006), Vor $\S \S 7-14$ $A S t G$, in: F. Wassermeyer/D. Pilz (Hrsg.), Außensteuergesetz Kommentar, Köln, Lfg. 50, Oktober 2002

Wassermeyer, F./Pilz, D. (Hrsg.); (Außensteuergesetz, 2006), Außensteuergesetz - Kommentar, Köln, Lfg. 59, November 2006

Watrin, C./Sievert, E./Strom, C.; (FR, 2004), Reform der Konzernbesteuerung in Deutschland und Europa, in: Finanz-Rundschau, S. 1-11

Weber, K./Wimmer, N. (Hrsg.); (Verfassungsstaat, 2005), Vom Verfassungsstaat am Scheideweg, Wien, New York

Wehrheim, M.; (IStR, 2003), Die Vorschläge der Europäischen Kommission zur Unternehmensbesteuerung im Binnenmarkt - Ein Überblick, in: Internationales Steuerrecht, S. 14-19

Weichenrieder, A.; (ifo Schnelldienst, 2002, 2), Das Kommissionsgutachten zur Unternehmensbesteuerung: Mehr Fragen als Antworten, in: ifo Schnelldienst, Nr. 2, S. 9-11

Weichenrieder, A./Mintz, J.M.; (Ownership, 2006), What Determines the Use of Holding Companies and Ownership Chains?, Working Paper, Workshop der Bundesbank und des NBER zum Thema: Mikrodatenbasierte Forschung zu Direktinvestitionen, URL: http:/www.bundesbank.de/down load/vfz/konferenzen/20070329_cambridge/paper_weichenrieder.pdf, 30. 3. 2007

Weiner, J.M.; (TNI, 1996), Using the Experience in the U.S. States to Evaluate Issues in Implementing Formula Apportionment at the International Level, in: Tax Notes International, S. 2113-2144

Weiner, J.M.; (Implementing, 1999), Using the Experience in the U.S. States to Evaluate Issues in Implementing Formula Apportionment at the International Level, OTA Paper 83, Washington D.C.

Weiner, J.M.; (TNI, 2000), Globalization and the Taxation of Foreign Investment: A Symposium in Honor of Professor Hugh J. Ault, in: Tax Notes International, S. 1112-1118 
Weiner, J.M.; (ET, 2001), The European Union and Formula Apportionment: Caveat Emptor, in: European Taxation, S. 380-388

Weiner, J.M.; (Apportionment, 2002), Formula Apportionment in the EU: A Dream Come True or the EU's Worst Nightmare, CESifo Working Paper, München

Weiner, J.M.; (CESifo Forum, 2002, 1), Formulary Apportionment and the Future of Company Taxation in the European Union, in: CESifo Forum, Nr. 1, S. 10-20

Weiner, J.M.; (ifo Studien, 2002), Would introducing Formula Apportionment in the European Union Be a Dream Come True or the EU's Worst Nightmare, in: ifo Studien, S. 519-532

Weiner, J.M.; (TNI, 2002), EU Commission, Member State Commit to EU-Wide Company Taxation, Formulary Apportionment, in: Tax Notes International, S. 515-520

Weiner, J.M.; (Group Taxation, 2005), Formulary Apportionment and Group Taxation in the European Union: Insights from the United States and Canada, European Commission Working Paper, Luxemburg

Weiner, J.M.; (Company, 2006), Company Tax Reform in the European UnionGuidance from the United States and Canada on Implementing Formulary Apportionment in the EU, New York

Weiner, J.M.; (Reform, 2006), A New Way of Thinking about Company Tax Reform in the European Union, URL: http://www.iiea.com/images/managed/ events_attachments/ WeinerPaper.pdf, 15. 2. 2007

Wellisch, D.; (FA, 2004), Taxation under formula apportionment - tax competition, tax incidence, and the choice of apportionment factors, in: Finanzarchiv, S. 24-41

Wellisch, D.; (StuW, 2004), Maßstäbe zur indirekten Gewinnaufteilung im Rahmen einer neuen Konzernbesteuerung in der EU: Möglichkeiten und Grenzen, in: Steuer und Wirtschaft, S. 267-276

Westberg, B.; (ET, 2002), Consolidated Corporate Tax Bases for EU-Wide Activities: Evaluation of Four Proposals Presented by the European Commission, in: European Taxation, S. 322-330

Wildasin, D.E.; (CTJ, 2000), State and Provincial Corporation Income Taxation: Current Practice and Policy Issues for the US and Canada, in: Canadian Tax Journal, S. 424-441

Wimpissinger, C.; (SWI, 2006), Gemeinschaftsrechtswidrigkeit der Hinzurechnungsbesteuerung ausländischer Betriebsstätteneinkünfte in Deutschland: Neue Erkenntnisse aus Cadbury Schweppes für Columbus Container?, in: Steuern und Wirtschaft International, S. 559-564 
Winkeljohann, N.; (Oestreicher, Konzernbesteuerung, 2005), Die steuerliche Behandlung der Beteiligungsaufwendungen und -erträge im Spiegel der Steuerplanung, in: A. Oestreicher (Hrsg.), Konzernbesteuerung - Beiträge zu einer Ringveranstaltung an der Universität Göttingen im Sommersemester 2004, Herne, Berlin, S. 67-81

Wissenschaftlicher Beirat beim BMF, (Kapitaleinkommensbesteuerung, 1999), Reform der internationalen Kapitaleinkommensbesteuerung - Gutachten erstattet vom Wissenschaftlichen Beirat beim Bundesministerium der Finanzen, Schriftenreihe des BMF, Heft 65, Bonn

Zanotti, E.; (ET, 2005), Taxation of Inter-Company Dividends in the Presence of a PE: The Impact of the EC Fundamental Freedoms, in: European Taxation, S. 493-505 (Teil I) und S. 535-546 (Teil II) 


\section{Verzeichnis der Entscheidungen}

\section{Bundesfinanzhof}

\begin{tabular}{|r|c|l|}
\hline 9. 9. 1986 & VIII R 20/85 & BFH/NV, 1987, S. 442 \\
\hline 14.4 .1992 & VIII R $149 / 86$ & BStBl II, 1992, S. 817 \\
\hline
\end{tabular}

\section{Europäischer Gerichtshof}

\begin{tabular}{|r|c|l|}
\hline 28.1 .1986 & $\begin{array}{c}\text { Rs. 270/83 } \\
\text { (Avoir-Fiscal) }\end{array}$ & EuGHE 1986, I-273 \\
\hline 15.5 .1997 & $\begin{array}{c}\text { Rs. C-250/95 } \\
\text { (Futura/Singer) }\end{array}$ & EuGHE 1997, I-2471 \\
\hline 28.4 .1998 & $\begin{array}{c}\text { Rs. C-118/96 } \\
\text { (Safir) }\end{array}$ & EuGHE 1998, I-1897 \\
\hline 21.9 .1999 & $\begin{array}{c}\text { Rs. C-307/97 } \\
\text { (Saint-Gobain) }\end{array}$ & EuGHE 1999, I-6161 \\
\hline 6.6 .2000 & $\begin{array}{c}\text { Rs. C-35/98 } \\
\text { (Verkooijen) }\end{array}$ & EuGHE 2000, I-4071 \\
\hline 14.12. & $\begin{array}{c}\text { Rs. C-141/99 } \\
\text { (AMID) }\end{array}$ & EuGHE 2000, I-11619 \\
\hline 12.9 .2002 & $\begin{array}{c}\text { Rs. C-431/01 } \\
\text { (Mertens) }\end{array}$ & EuGHE 2002, I-7073 \\
\hline 13.12. & $\begin{array}{c}\text { Rs. C-446/03 } \\
\text { (Marks \& Spencer) }\end{array}$ & IStR 2006, S. 19-23 \\
\hline 21.2 .2006 & $\begin{array}{c}\text { Rs. C-152/03 } \\
\text { (Ritter-Coulais) }\end{array}$ & HFR 2006, S. 525 \\
\hline 12.9 .2006 & $\begin{array}{c}\text { Rs. C-196/04 } \\
\text { (Cadbury-Schweppes) }\end{array}$ & $\begin{array}{l}\text { EuZW 2006, S. 633- } \\
\text { 637 }\end{array}$ \\
\hline
\end{tabular}

\section{III. Österreichischer Verwaltungsgerichtshof}

\begin{tabular}{|l|l|l|}
\hline 25.9 .2001 & Rs. 99/14/0217 & AnwBl., 2002, S. 285 \\
\hline
\end{tabular}

\section{US Supreme Court}

\begin{tabular}{|r|c|l|}
\hline 1911 & $\begin{array}{c}\text { Flint v. } \\
\text { Stone Tracy Co }\end{array}$ & 220 U.S. 107 \\
\hline 1965 & $\begin{array}{c}\text { General Motors Corp. v. } \\
\text { District of Columbia }\end{array}$ & 380 U.S. 553 \\
\hline 1978 & $\begin{array}{c}\text { Moorman Manufacturing Company v. } \\
\text { Bair }\end{array}$ & 437 U.S. 267 \\
\hline 1980 & $\begin{array}{c}\text { Mobil Oil Corp. v. } \\
\text { Commissioner of Taxes of Vermont }\end{array}$ & 445 U.S. 425 \\
\hline 1982 & $\begin{array}{c}\text { Asarco Inc. v. } \\
\text { Idaho State Tax Commission }\end{array}$ & 458 U.S. 307 \\
\hline
\end{tabular}




\begin{tabular}{|r|c|l|}
\hline 1982 & $\begin{array}{c}\text { F.W. Woolworth Corp. v. } \\
\text { Taxation and Revenue Department of } \\
\text { New Mexico }\end{array}$ & 458 U.S. 354 \\
\hline 1983 & $\begin{array}{c}\text { Container Corp. of America v. } \\
\text { Franchise Tax Board }\end{array}$ & 463 U.S. 159 \\
\hline 1992 & $\begin{array}{c}\text { Allied-Signal Inc. v. } \\
\text { Director, Division of Taxation }\end{array}$ & 504 U.S. 768 \\
\hline 1994 & $\begin{array}{c}\text { Barclays Bank PLC v. } \\
\text { Franchise Tax Board }\end{array}$ & 512 U.S. 298 \\
\hline
\end{tabular}

V. California Supreme Court

\begin{tabular}{|r|c|l|}
\hline 1942 & Butler Brothers v. McColgan & $\begin{array}{l}17 \text { Cal. 2d 664, 111 P. } \\
2 \mathrm{~d} \mathrm{334}\end{array}$ \\
\hline 1947 & Edison California Stores v. McColgan & $\begin{array}{l}30 \text { Cal. 2d 480, 183 P. } \\
2 \mathrm{~d} \mathrm{16}\end{array}$ \\
\hline
\end{tabular}




\title{
Verzeichnis der Drucksachen, Richtlinien, Verordnungen
}

\author{
BMF-Schreiben vom 8. 1. 2007, IV B 4-S 1351-1/07
}

Bundesrats-Drucksache 281/1/06 vom 6. 6. 2006

Empfehlung der Kommission vom 6.5. 2003 betreffend die Definition der Kleinstunternehmen sowie der kleinen und mittleren Unternehmen (2003/361/EC), Abl. Nr. L 124, vom 20. 5. 2003, S. 36-41

Siebente Richtlinie 83/349/EWG des Rates vom 13.6. 1983 aufgrund von Artikel 54 Absatz 3 Buchstabe g) des Vertrages über den konsolidierten Abschluss, Abl. Nr. L 193 vom 18. 7. 1983, S. 1-17

Richtlinie 90/434/EWG des Rates vom 23.7. 1990 über das gemeinsame Steuersystem für Fusionen, Spaltungen, die Einbringung von Unternehmensteilen und den Austausch von Anteilen, die Gesellschaften verschiedener Art betreffen, Abl. Nr. L 225 vom 20. 8. 1990, S. 1-5

Richtlinie 90/435/EWG des Rates vom 23.7. 1990 über das gemeinsame Steuersystem der Mutter- und Tochtergesellschaften verschiedener Mitgliedstaaten, Abl. EG Nr. L 225 vom 20. 8. 1990, S. 6-9

Richtlinie 2003/49/EG des Rates vom 3.6. 2003 über eine gemeinsame Steuerregelung für Zahlungen von Zinsen und Lizenzgebühren zwischen verbundenen Unternehmen verschiedener Mitgliedstaaten, Abl. Nr. L 157 vom 26. 6. 2003, S. 49-54

Richtlinie 2003/123/EG des Rates vom 22. 12. 2003 zur Änderung der Richtlinie 90/435/EWG über das gemeinsame Steuersystem der Mutter- und Tochtergesellschaften verschiedener Mitgliedstaaten, Abl. Nr. L 007 vom 3. 1. 2004, S. 41-44

Richtlinie 2005/19/EG des Rates vom 17. 2. 2005 zur Änderung der Richtlinie 90/434/EWG über das gemeinsame Steuersystem für Fusionen, Spaltungen, die Einbringung von Unternehmensteilen und den Austausch von Anteilen, die Gesellschaften verschiedener Art betreffen, Abl. Nr. L 58 vom 4. 3. 2005 , S. 19-27

Vorschlag einer Richtlinie des Rats über eine Regelung für Unternehmen zur Berücksichtigung der Verluste ihrer in anderen Mitgliedstaaten belegenen Betriebsstätten und Tochtergesellschaften vom 28.11. 1990, KOM (90) 595 endgültig, Abl. Nr. C 53 vom 28. 2. 1991, S. 30

Vorschlag für eine Richtlinie des Rates zur Änderung der Richtlinie 90/434/EWG des Rates vom 23.7. 1990 über das gemeinsame Steuersystem für Fusionen, Spaltungen, die Einbringung von Unternehmensteilen und den Austausch von Anteilen, die Gesellschaften verschiedener Mitgliedstaaten betreffen, KOM (2003) 613 endgültig, vom 17. 10.2003 
Übereinkommen über die Beseitigung der Doppelbesteuerung im Falle von Gewinnberichtigungen zwischen verbundenen Unternehmen (90/436/EWG), Abl. Nr. L 225 vom 20. 8. 1990, S. 10-24

Verordnung (EG) Nr. 1606/2002 des europäischen Parlaments und des Rates vom 19.7. 2002 betreffend die Anwendung internationaler Rechnungslegungsstandards, Abl. Nr. L 243, vom 11. 9. 2002, S. 1-4

Verordnung (EWG) Nr. 3037/90 des Rates vom 9.10. 1990 betreffend die statistische Systematik der Wirtschaftszweige in der Europäischen Gemeinschaft, Abl. Nr. L 293, vom 24. 10. 1990, S. 1-26

Verordnung (EWG) Nr. 761/93 der Kommission vom 24. 3. 1993 zur Änderung der Verordnung (EWG) Nr. 3037/90 des Rates betreffend die statistische Systematik der Wirtschaftszweige in der Europäischen Gemeinschaft, Abl. Nr. L 83 vom 3. 4. 1993, S. 1-24, Berichtigung Abl. Nr. L 159, vom 11. 7. 1995 , S. 31

Verordnung (EG) Nr. 2700/98 der Kommission vom 17. 12. 1998 betreffend die Definitionen von Merkmalen der strukturellen Unternehmensstatistik, Abl. Nr. L 344, vom 18. 12. 1998, S. 49-80

Verhaltenskodex zur Verrechnungspreisdokumentation für verbundene Unternehmen in der Europäischen Union, Abl. Nr. C 176, vom 28. 7. 2006, S. 1-7

Verhaltenskodex zur wirksamen Durchführung des Übereinkommens über die Beseitigung der Doppelbesteuerung im Falle von Gewinnberichtigungen zwischen verbundenen Unternehmen, Abl. Nr. C 176, vom 28. 7. 2006, S. 8-12 
Andreas Poppe - 978-3-631-75688-1

Downloaded from PubFactory at 01/11/2019 02:57:41AM

via free access 
cege-Schriften

Das cege - Centrum für Europa-, Governance- und Entwicklungsforschung - wurde 1999 von der Wirtschaftswissenschaftlichen Fakultăt der Georg-August-Universităt gegründet. Das cege dient als Forum zur internationalen und interdisziplinăren Forschungszusammenarbelt in den Themenbereichen Europäische Integration, Governance und Entwicklungsökonomik. In den cege-Schriften werden Forschungsergebnisse aus Dissertationen, Habilitationen oder anderen Forschungsprojekten des cege veröffentlicht.

Band 1 Axel Gerloff: Wechselkurspolitik in Mittel- und Osteuropa. Eine theoretische Analyse unter besonderer Berücksichtigung der Erfahrungen der zehn Kandidaten für eine EU-Ostenwejterung. 2001.

Band 2 Ingo Konrad: Zur Integration ausgewăhlter mittel- und osteuropäischer Lănder in die wăhrungspolitische Ordnung Europas. 2002.

Band 3 Axel Hennighausen: Wegekostenfinanzierung und Lenkung im deregulierten europäischen Verkehrsmarkt. 2002.

Band 4 Wolfgang Münch: Effects of EU Enlargement to the Central European Countries on Agricultural Markets. 2002.

Band 5 Holger Niermann: Welchen Finanzausgleich braucht Europa? Ein konkreter Vorschlag zur Neugestaltung der europăischen Finanzbeziehungen mit Simulationsmodell. 2002.

Band 6 Jens Südekum: Agglomeration and Regional Unemployment Disparities. A Theoretical Analysis with Relerence to the European Union. 2003.

Band 7 Christoph Schinke: Der Geldmarkt im Euro-Wăhrungsraum. Geldmarktgeschăfte, Zinsbildung und die Taylor Rule. 2004.

Band 8 Kai Stukenbrock: The Stability of Currency Boards. 2004.

Band 9 Harald Grethe: Effects of Including Agricultural Products in the Customs Union between Turkey and the EU. A Partial Equllibrium Analysis for Turkey. 2004.

Band 10 Götz Zeddles: Perspektiven der Gemeinschaftspolltiken in der enwelterten EU. 2005.

Band 11 Ingmar Kumpmann: Systemwettbewert und Umverteilung. Gefăhrdet die Globalisienung den Sozlalstaat? 2005.

Band 12 Katarzyna Haverkamp: Bestimmungsfaktoren der Vertragsforschung in Deutschland. Eine theoretische und Okonometrische Analyse. 2007.

Band 13 Peter Schwarz: Kapitalsteuenwettbewerb zwischen Nationalstaaten unter besonderer Borücksichtigung Deutschlands. Eine empirische Analyse. 2007.

Band 14 Andreas Poppe: Auswirkungen der Einführung einer konsolidierten Körperschaftsteuer-Bomessungsgrundlage in der Europăischen Union. Eine empirieche Analyse der Konzernstrukturen und des Steueraufikommens. 2008.

uww.peterlang.de 
Andreas Poppe - 978-3-631-75688-1

Downloaded from PubFactory at 01/11/2019 02:57:41AM

via free access 


\section{Kapitalsteuerwettbewerb zwischen Nationalstaaten unter besonderer Berücksichtigung Deutschlands}

\section{Eine empirische Analyse}

Frankfurt am Main, Berlin, Bern, Bruxelles, New York, Oxford, Wien, 2007. XIV, 236 S., zahlr. Tab. und Graf.

CeGE-Schriften. Verantwortlicher Herausgeber: Robert Schwager. Bd. 13 ISBN 978-3-631-56834-7 · br. $€ 45.50$ *

Gegenstand der Untersuchung ist der zwischen Nationalstaaten sich intensivierende Steuerwettbewerb um mobiles Kapital. Es wird die Besteuerung von Kapitalgesellschaften sowie die Besteuerung von Zinserträgen privater Haushalte ökonometrisch analysiert. Zudem untersucht die Studie speziell am Beispiel Deutschlands Gewinnverlagerungsaktivitäten multinationaler Unternehmen. Ergebnis der Untersuchung ist, dass Steuenwettbewerb um Unternehmen vor allem um mobile Unternehmensgewinne geführt wird, während Steueroasen im Bereich der Zinsbesteuerung aufgrund der Bedeutung ihres Bankensektors keinen Anreiz zur grenzüberschreitenden Kooperation haben.

Aus dem Inhalt. Steuerwettbewerb um Unternehmen - Steuerwettbewerb um Finanzkapitalanlagen privater Haushalte - Methoden zur Ermittlung der Steuerbelastung von Kapitalgesellschaften - Untersuchung der Unternehmenssteuerbelastung - Steuervermeidungsstrategien multinationaler Unternehmen: Der Fall Deutschland · Untersuchung zur Zinsbesteuerung

Frankfurt am Main - Berlin - Bern - Bruxelles - New York - Oxford - Wien

Auslieferung: Verlag Peter Lang AG

Moosstr. 1, CH-2542 Pieterlen

Telefax $0041(0) 32 / 3761727$

*inklusive der in Deutschland gültigen Mehnwertsteuer

Preisánderungen vorbehalten 
Andreas Poppe - 978-3-631-75688-1

Downloaded from PubFactory at 01/11/2019 02:57:41AM

via free access 